\title{
An Experimental Study of a
}

\section{Three-Dimensional Pressure-Driven Turbulent}

\section{Boundary Layer}

by

\author{
Semih M. Ölçmen
}

Thesis submitted to the Faculty of the

Virginia Polytechnic Institute and State University

in partial fulfillment of the requirements for the degree of

Doctor of Philosophy

in

Aerospace and Ocean Engineering

APPROVED:

Whoger L. Simpsón, Chairm

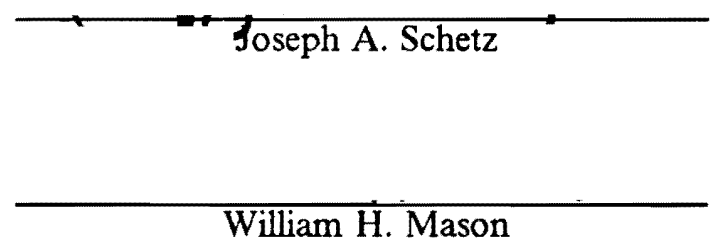

William H. Mason

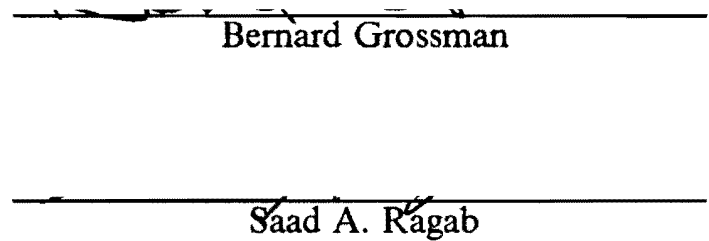

Saad A. Ragab

June, 1990

Blacksburg, Virginia 


\author{
An Experimental Study of a \\ Three-Dimensional Pressure-Driven Turbulent \\ Boundary Layer \\ by \\ Semih M. Ölçmen \\ Roger L. Simpson, Chairman \\ Aerospace and Ocean Engineering
}

(ABSTRACT)

A three dimensional, pressure driven turbulent boundary layer created by an idealized wing-body junction flow is experimentally studied. The body used is a $3: 2$ elliptical nosed NACA 0020 tailed symmetric profile which has a chord length of $30.5 \mathrm{~cm}$ (12 inches), maximum thickness of $7.17 \mathrm{~cm}$ (2.824 inches), heigth of $22.9 \mathrm{~cm}$ (9.016 inches). The body was sitting on a flat plate. The nominal reference velocity of the flow is $27 \mathrm{~m} / \mathrm{sec}$ and the Reynolds number based on the momentum thickness at 0.75 chord upstream of the body on the centerline of the tunnel is $\simeq 5936$. The data presented include time-mean static pressure, skin friction magnitude and direction on the wall, as well as the mean velocity and all Reynolds stresses at several stations on a line determined with the mean velocity vector component parallel to the wall in the layer where the $\overline{u^{2}}$ normal stress is maximum. The mean velocity and stress data were obtained both with hot-wire ( HW) and laser-Doppler-velocimeter ( LDV) techniques. The LDV measurements were taken twice due to the differences observed between the HW and LDV data, which is also shown with the present study. This gave a chance to study the uncertainties on the mean velocity and the stresses extensively. Pressure distributions on the wing and the on the bottom plate were obtained with a Scanivalve and an inclined manometer. Skin friction vectors at several locations on the wall were measured in another study done by Ailinger ( 1990 ) with a laser interferometer technique. The data show that the eddy viscosity of the flow is not isotropic, but the ratio of eddy viscosities perpendicular and parallel to the direction of the mean velocity vector component parallel to the wall at the point in the layer where $\overline{u^{2}}$ is maximum is close to unity, and the shear-stress vector 
direction in the flow lags behind the flow gradient vector direction. $A_{1}$, Townsend's structural parameter is not a constant of 0.15 as expected. The production of the turbulent kinetic energy and shear stresses are important below the logarithmic regions of the $U$ axial velocity component profiles. The skin friction velocity is not the scale of the turbulence in such a flow. Further, a collection of 3-D turbulent boundary layer data including the present study is used to investigate the concept of the Law of the Wall velocity profile and the limitations of eddy-viscosity turbulence models in 3-D flows. For this purpose, several Law-of-the-Wall velocity profile models and eddy-viscosity models were tested. Johnston's Law-of-the-Wall relation and, for the pressure-driven flows the Johnson-King eddy-viscosity model and for the shear-driven flows Patel's eddy-viscosity model are most promising. 


\section{Dedication}

To the memory of my father 


\section{Acknowledgements}

I would like to thank to my advisor Dr. Roger L. Simpson for sharing his own ideas and experience not only in the research area but also in the general realm of life itself. My special thanks goes to Dr. Bernard Grossman for his support and guidance in the second year of my studies, which I believe without that guidance there would not be any of this study done. I also would like to thank to Dr. Joseph A. Schetz, Dr. William H. Mason, Dr. Saad A. Ragab for serving in my committee and for editing my dissertation.

Several fellow students and research associates need to be thanked. I would like to thank especially to Dr. Naval K. Agarwal for being friendly and helpful, even in personal problems, and to Dr. William J. Devenport for preparing much of the instrumentation that $I$ used in the research. Patience of a good friend, Fei T. Kwok, in proof reading my entire dissertation is unforgettable. I would like to thank to Seungki Ahn, Kevin A. Shinpaugh, and Richard B. Mays for being friends. I also would like thank to my parents my mother Melâhat Ölçmen, my sister Merih Özgūr and my niece Burcu Özgür for just being there on Earth even though far away.

This work was supported by DARPA/Office of Naval Research Grant N00014-89-J-1275. 


\section{Table of Contents}

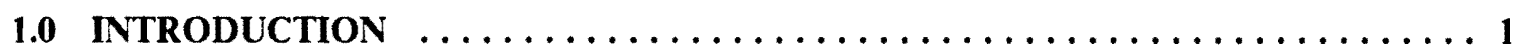

1.1 NEED FOR EXPERIMENTAL DATA $\ldots \ldots \ldots \ldots \ldots \ldots \ldots \ldots \ldots \ldots$

1.2 REVIEW OF THE GOVERNING EQUATIONS $\ldots \ldots \ldots \ldots \ldots \ldots \ldots \ldots$

1.3 REVIEW OF SOME TURBULENCE MODELS $\ldots \ldots \ldots \ldots \ldots \ldots \ldots \ldots$

1.4 OUTLINE OF THE PRESENT WORK $\ldots \ldots \ldots \ldots \ldots \ldots \ldots \ldots \ldots \ldots \ldots \ldots$

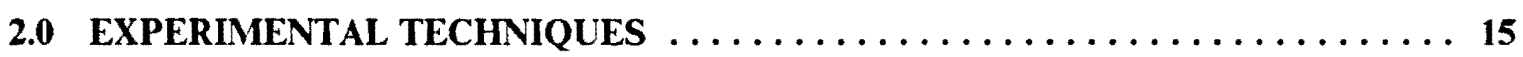

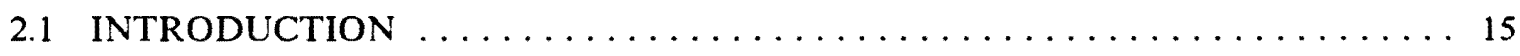

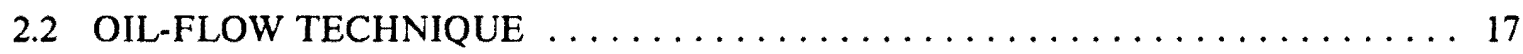

2.3 SKIN-FRICTION INTERFEROMETER $\ldots \ldots \ldots \ldots \ldots \ldots \ldots \ldots \ldots \ldots$

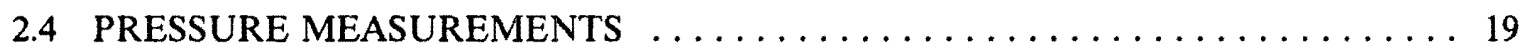

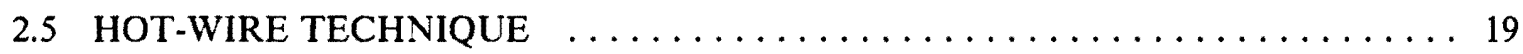

2.6 LASER-DOPPLER VELOCIMETER (LDV) TECHNIQUE $\ldots \ldots \ldots \ldots \ldots 21$

3.0 EXPERIMENTAL RESULTS $\ldots \ldots \ldots \ldots \ldots \ldots \ldots \ldots \ldots \ldots \ldots \ldots \ldots \ldots$

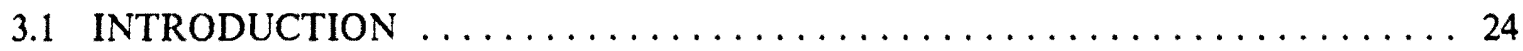

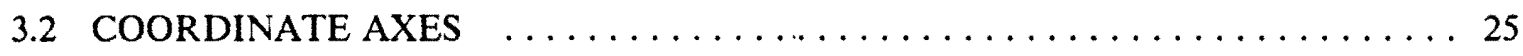




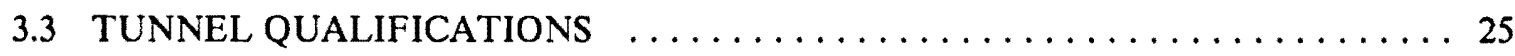

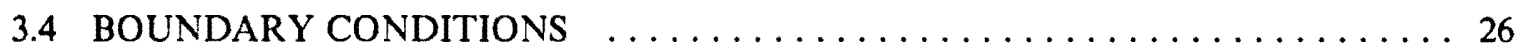

3.5 OIL-FLOW VISUALIZATION RESULTS $\ldots \ldots \ldots \ldots \ldots \ldots \ldots \ldots \ldots \ldots \ldots \ldots$

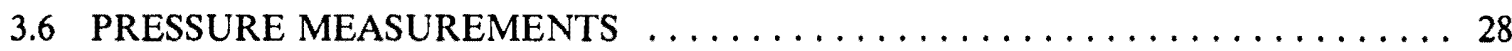

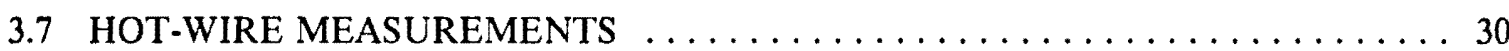

3.8 VELOCITY AND STRESS MEASUREMENTS $\ldots \ldots \ldots \ldots \ldots \ldots \ldots \ldots$

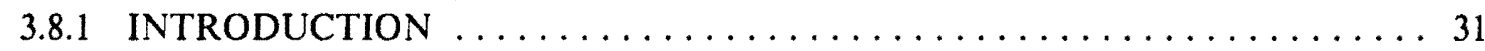

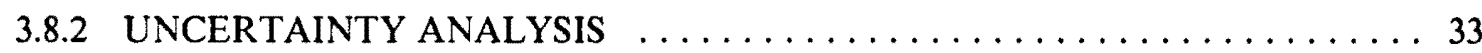

3.8.3 MEAN FLOW MEASUREMENT RESULTS $\ldots \ldots \ldots \ldots \ldots \ldots \ldots \ldots$

3.8.3.1 IN TUNNEL COORDINATES $\ldots \ldots \ldots \ldots \ldots \ldots \ldots \ldots \ldots \ldots \ldots \ldots \ldots \ldots$

3.8.3.2 IN FREE-STREAM COORDINATES $\ldots \ldots \ldots \ldots \ldots \ldots \ldots \ldots, 40$

3.8.3.3 IN MAXIMUM-NORMAL-STRESS COORDINATES $\ldots \ldots \ldots \ldots \ldots 41$

3.8.4 FLUCTUATION VELOCITIES $\ldots \ldots \ldots \ldots \ldots \ldots \ldots \ldots \ldots \ldots \ldots \ldots \ldots \ldots \ldots$

3.8.4.1 IN TUNNEL COORDINATES $\ldots \ldots \ldots \ldots \ldots \ldots \ldots \ldots \ldots \ldots, 42$

3.8.4.2 IN FREE-STREAM COORDINATES $\ldots \ldots \ldots \ldots \ldots \ldots \ldots \ldots \ldots 46$

3.8.4.3 IN MAXIMUM-NORMAL-STRESS COORDINATES $\ldots \ldots \ldots \ldots \ldots 47$

3.8.5 REYNOLDS SHEAR-STRESSES $\ldots \ldots \ldots \ldots \ldots \ldots \ldots \ldots \ldots \ldots \ldots$

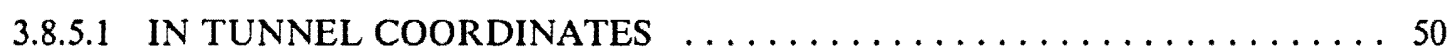

3.8.5.2 IN FREE-STREAM COORDINATES $\ldots \ldots \ldots \ldots \ldots \ldots \ldots \ldots \ldots, 52$

3.8.5.3 IN MAXIMUM-NORMAL-STRESS COORDINATES $\ldots \ldots \ldots \ldots \ldots 54$

4.0 EXAMINATION OF THE FLOW STRUCTURE $\ldots \ldots \ldots \ldots \ldots \ldots \ldots \ldots \ldots$

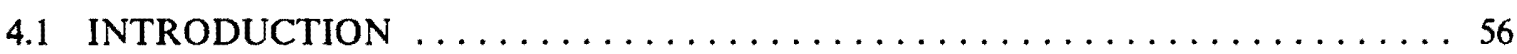

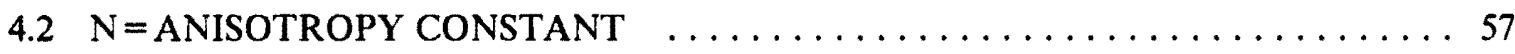

$4.3 \mathrm{~A} 1=$ TOWNSEND'S STRUCTURAL PARAMETER $\ldots \ldots \ldots \ldots \ldots \ldots$

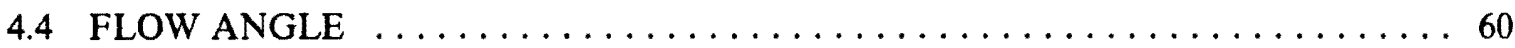

4.5 FLOW GRADIENT ANGLE $\ldots \ldots \ldots \ldots \ldots \ldots \ldots \ldots \ldots \ldots \ldots \ldots \ldots \ldots \ldots \ldots \ldots$

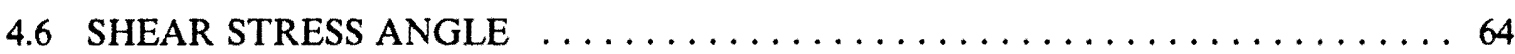


4.7 FLUCTUATION VELOCITIES AND SHEAR STRESSES NORMALIZED WITH THE SKIN-FRICTION VELOCITY $\ldots \ldots \ldots \ldots \ldots \ldots \ldots \ldots \ldots \ldots \ldots$

4.8 EDDY VISCOSITIES IN X AND $Z$ DIRECTIONS $\ldots \ldots \ldots \ldots \ldots \ldots \ldots$

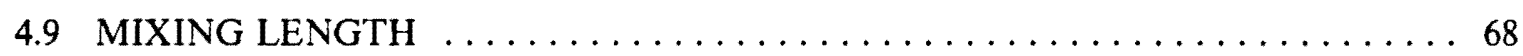

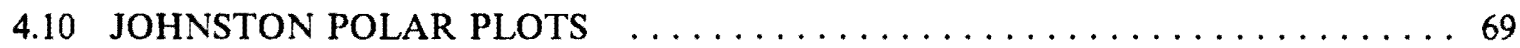

4.11 TURBULENT KINETIC ENERGY $\ldots \ldots \ldots \ldots \ldots \ldots \ldots \ldots \ldots \ldots \ldots$

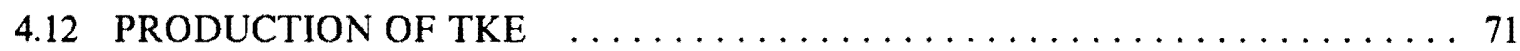

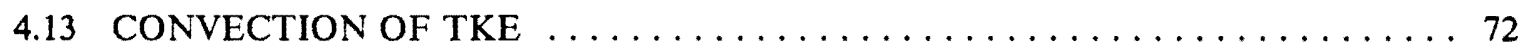

4.14 PRODUCTION AND CONVECTION OF NORMAL AND SHEAR STRESSES . 73

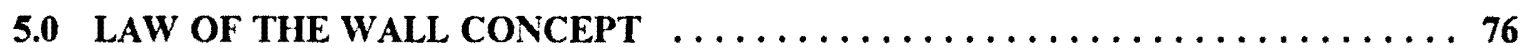

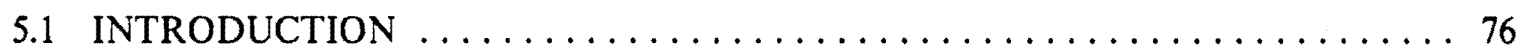

5.2 LAW-OF-THE-WALL RELATION REVIEW $\ldots \ldots \ldots \ldots \ldots \ldots \ldots \ldots$

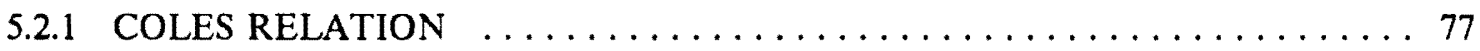

5.2 .2 JOHNSTON RELATION $\ldots \ldots \ldots \ldots \ldots \ldots \ldots \ldots \ldots \ldots \ldots \ldots$

5.2 .3 HORNUNG-JOUBERT RELATION $\ldots \ldots \ldots \ldots \ldots \ldots \ldots \ldots \ldots$

5.2.4 PIERCE-KROMMENHOEK AND CHANDRASHEKHAR-SWAMY

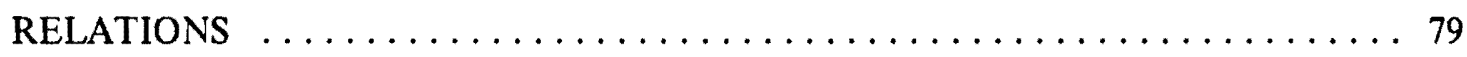

5.2 .5 EAST AND HOXEY RELATION $\ldots \ldots \ldots \ldots \ldots \ldots \ldots \ldots \ldots \ldots \ldots$

5.2 .6 PERRY-JOUBERT RELATION $\ldots \ldots \ldots \ldots \ldots \ldots \ldots \ldots \ldots \ldots \ldots \ldots \ldots \ldots \ldots$

5.2 .7 WHITE-LESSMANN-CHRISTOPH RELATION $\ldots \ldots \ldots \ldots \ldots \ldots \ldots 8$

5.2 .8 VAN DEN BERG RELATION $\ldots \ldots \ldots \ldots \ldots \ldots \ldots \ldots \ldots \ldots \ldots$

5.3 EXPERIMENTAL DATA $\ldots \ldots \ldots \ldots \ldots \ldots \ldots \ldots \ldots \ldots \ldots \ldots \ldots$

5.3 .1 ANDERSON AND EATON FLOW $\ldots \ldots \ldots \ldots \ldots \ldots \ldots \ldots \ldots$

5.3 .2 FERNHOLZ AND VAGT FLOW $\ldots \ldots \ldots \ldots \ldots \ldots \ldots \ldots \ldots$

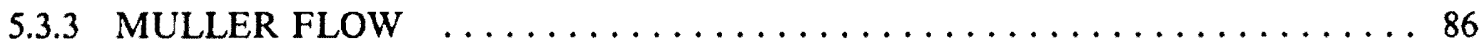

5.3 .4 DECHOW AND FELSCH FLOW $\ldots \ldots \ldots \ldots \ldots \ldots \ldots \ldots \ldots \ldots$

5.3 .5 ELSENAAR AND BOELSMA FLOW $\ldots \ldots \ldots \ldots \ldots \ldots \ldots \ldots$ 
5.3.6 BISSONNETTE AND MELLOR FLOW $\ldots \ldots \ldots \ldots \ldots \ldots \ldots \ldots \ldots$

5.3 .7 LOHMANN FLOW $\ldots \ldots \ldots \ldots \ldots \ldots \ldots \ldots \ldots \ldots \ldots, \ldots$

5.4 RESULTS AND DISCUSSION $\ldots \ldots \ldots \ldots \ldots \ldots \ldots \ldots \ldots \ldots \ldots$

6.0 TURBULENCE MODELING $\ldots \ldots \ldots \ldots \ldots \ldots \ldots \ldots \ldots \ldots \ldots \ldots \ldots$

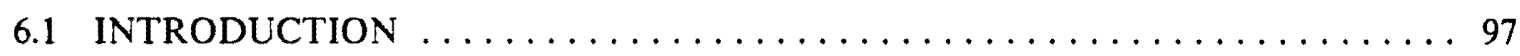

6.2 SELECTED ALGEBRAIC TURBULENCE MODELS $\ldots \ldots \ldots \ldots \ldots$

6.2 .1 CEBECI-SMITH MODEL $\ldots \ldots \ldots \ldots \ldots \ldots \ldots \ldots \ldots \ldots \ldots \ldots$

6.2 .2 ROTTA'S MODEL $\ldots \ldots \ldots \ldots \ldots \ldots \ldots \ldots \ldots \ldots \ldots \ldots \ldots \ldots$

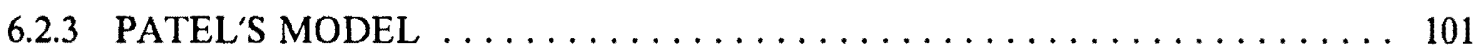

6.2 .4 JOHNSON-KING MODEL $\ldots \ldots \ldots \ldots \ldots \ldots \ldots \ldots \ldots \ldots \ldots \ldots \ldots$

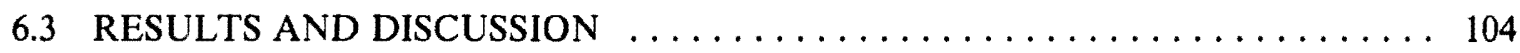

7.0 CONCLUSIONS $\ldots \ldots \ldots \ldots \ldots \ldots \ldots \ldots \ldots \ldots \ldots \ldots \ldots \ldots \ldots \ldots \ldots, 116$

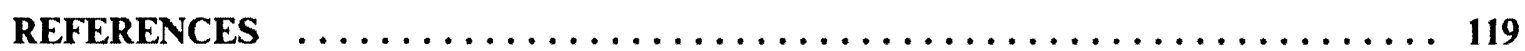

TABLES $\ldots \ldots \ldots \ldots \ldots \ldots \ldots \ldots \ldots \ldots \ldots \ldots \ldots \ldots \ldots \ldots \ldots, 128$

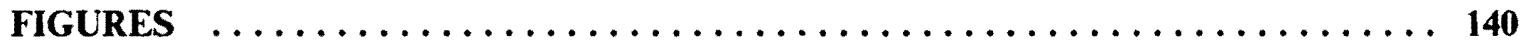

Appendix A. DATA REDUCTION FORMULAE $\ldots \ldots \ldots \ldots \ldots \ldots \ldots \ldots \ldots \ldots, 450$

A.1 HOT-WIRE DATA REDUCTION EQUATIONS $\ldots \ldots \ldots \ldots \ldots \ldots \ldots 450$

A.2 CONFIGURATION OF LDV BEAMS $\ldots \ldots \ldots \ldots \ldots \ldots \ldots \ldots \ldots \ldots, \ldots \ldots \ldots$

A.3 LDV DATA REDUCTION EQUATIONS $\ldots \ldots \ldots \ldots \ldots \ldots \ldots \ldots \ldots 453$

Appendix B. GRADIENT AND FINITE TRANSIT TIME BROADENING

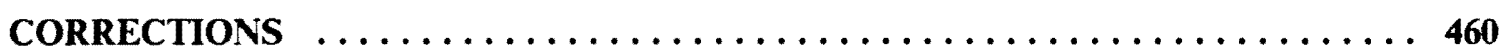


B.1 GRADIENT BROADENING $\ldots \ldots \ldots \ldots \ldots \ldots \ldots \ldots \ldots \ldots \ldots \ldots \ldots$

B.2 FINITE TRANSIT TIME BROADENING CORRECTION .......... 463

Appendix C. TENSOR TRANSFORMATION AND PARABOLA FITTING PROGRAMS 465

C.1 TENSOR TRANSFORMATION $\ldots \ldots \ldots \ldots \ldots \ldots \ldots \ldots \ldots \ldots \ldots$

C.2 PARABOLA FITTING $\ldots \ldots \ldots \ldots \ldots \ldots \ldots \ldots \ldots \ldots \ldots \ldots \ldots \ldots \ldots \ldots \ldots \ldots$

Appendix D. DATA FOR PRESENT WORK $\ldots \ldots \ldots \ldots \ldots \ldots \ldots \ldots \ldots$

Vita $\ldots \ldots \ldots \ldots \ldots \ldots \ldots \ldots \ldots \ldots \ldots \ldots \ldots \ldots \ldots \ldots \ldots \ldots \ldots \ldots \ldots \ldots \ldots$ 


\section{List of Tables}

Table 1. LEFT-HAND-SIDE HOT-WIRE LOCATIONS. $\ldots \ldots \ldots \ldots \ldots \ldots \ldots$

Table 2. 0.75 CHORD UPSTREAM HOT-WIRE PROFILE LOCATIONS. $\ldots \ldots 130$

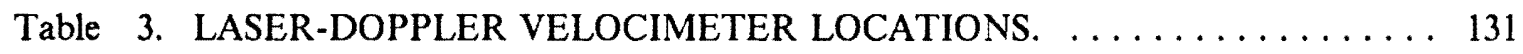

Table 4. RIGHT-HAND-SIDE HOT-WIRE LOCATIONS. $\ldots \ldots \ldots \ldots \ldots \ldots$

Table 5. AVERAGED NOMINAL VALUES OF THE UNCERTAINTIES AT DIFFERENT REGIONS OF THE BOUNDARY LAYER. . . . . . . . . . 133

Table 6. SEVERAL THICKNESSES COMPUTED USING THE LDV DATA IN

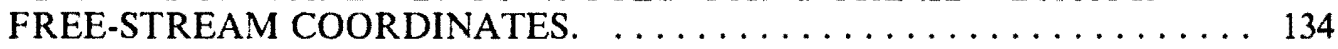

Table 7. SUMMARY OF EXPERIMENTAL DATA SETS. $\ldots \ldots \ldots \ldots \ldots \ldots \ldots$

Table 8. SUMMARY OF SEMI-LOGARITHMIC y + REGIONS OF FIVE

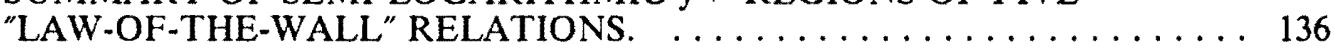

Table 9. OVERLAP y + RANGES FOR THE LEFT-HAND SIDE OF THE PROPOSED MODELS FOR PRESSURE-DRIVEN DATA SETS FOR THE AXIAL COMPONENT. $\ldots \ldots \ldots \ldots \ldots \ldots \ldots \ldots \ldots \ldots \ldots \ldots \ldots$

Table 10a. THE RANGES AND ABSOLUTE RANGES OF ANGLE DIFFERENCES $\left(\alpha_{m}-\alpha_{c}\right.$ ) in degrees CALCULATED WITH SEVERAL TURBULENCE MODELS AT

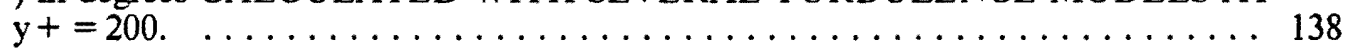

Table 10b. THE RANGES AND ABSOLUTE RANGES OF MAGNITUDE RATIOS $\left(\tau_{m}\right.$ $\left.1 \tau_{c}\right)$ CALCULATED WITH SEVERAL TURBULENCE MODELS AT

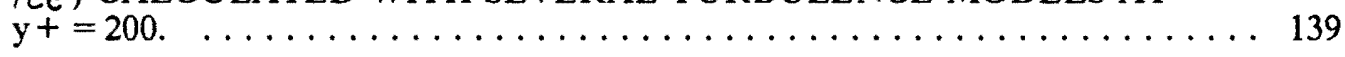




\section{List of Illustrations}

Figure 1. Mean velocity, normal and shear stress measurement locations. . . . . . 141

Figure 2a. Oil flow visualization picture taken on the test wall surrounding the wing, at 27 $\mathrm{m} / \mathrm{sec}$ nominal reference velocity. $\ldots \ldots \ldots \ldots \ldots \ldots \ldots \ldots \ldots \ldots \ldots$

Figure $2 \mathrm{~b}$. Oil flow visualization picture taken on the test wall surrounding the wing, at 27 $\mathrm{m} / \mathrm{sec}$ nominal reference velocity.

Figure 3a. Positions of pressure tappings on the test wall surrounding wing. $\ldots \ldots \ldots 144$

Figure 3b. Positions of pressure tappings on the wing surface projected on to an XY plane. 145

Figure 4. Hot-wire probe, in the tunnel. $\ldots \ldots \ldots \ldots \ldots \ldots \ldots \ldots \ldots \ldots \ldots$

Figure 5. Probe holder designed to reach to measurement locations. $\ldots \ldots \ldots \ldots \ldots 147$

Figure 6. Definition of the angles and the coordinate axes used in the present study. . . 148

Figure 7. Sideview of the wind-tunnel test section for the wing-body junction experiments. 149

Figure 8. Free-stream velocity distribution along the axis of the tunnel test section at 27

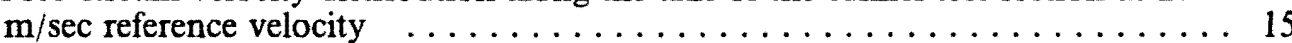

Figure 9a. U velocity component measured with the hot-wire technique at .75 chord length ( chord $=12$ inches upstream of the body expressed in tunnel coordinates. ) . .

Figure $9 \mathrm{~b}$. U velocity component measured with the hot-wire technique at .75 chord length upstream of the body continued

Figure 9c. W velocity component measured with the hot-wire technique at .75 chord length ( chord $=12$ inches upstream of the body expressed in tunnel coordinates. . . . 153

Figure 9d. $u^{\prime}$ fluctuation velocity component measured with the hot-wire technique at .75 chord length ( chord $=12$ inches upstream of the body expressed in tunnel coordinates.

Figure 9e. w' fluctuation velocity component measured with the hot-wire technique at .75 chord length ( chord $=12$ inches upstream of the body expressed in tunnel coordinates. 
Figure 9f. uw shear stress component measured with the hot-wire technique at .75 chord length $($ chord $=12$ inches thickness. $\ldots \ldots \ldots \ldots \ldots \ldots \ldots \ldots \ldots \ldots \ldots \ldots \ldots$

Figure 10. Characteristic dimensions visualized with the oil flow technique. $\ldots \ldots \ldots 157$

Figure 11. Distribution of time-mean static pressure on the test wall surrounding the wing at Reynolds number based on momentum thickness at 0.75 upstream . . . . . 158

Figure 12a. Distribution of time-mean static pressure on the wall surrounding the wing at Reynolds number based on momentum thickness of $7000 . \ldots \ldots \ldots \ldots 159$

Figure $12 \mathrm{~b}$. Distribution of time-mean static pressure on the wall surrounding the wing at Reynolds number based on momentum thickness of $7000 . \ldots \ldots \ldots \ldots$

Figure 13. Comparison of surface pressure distributions in tunnel $\ldots \ldots \ldots \ldots \ldots \ldots 1$

Figure 14. Comparison of surface pressure distributions on wing. $\ldots \ldots \ldots \ldots \ldots \ldots 2$

Figure 15a. Contours of measured mean static pressure on the test wall. $\ldots \ldots \ldots \ldots 3$

Figure 15b. Contours of computed mean static pressure on the test wall. . . . . . . 164

Figure 16a. Scheamatic figure of the histogram noise level determining routine. $\ldots \ldots \ldots 165$

Figure $16 \mathrm{~b}$. Scheamatic figure of the histogram noise level determining routine. $\ldots \ldots, 167$

Figure 16b. Comparison of $U$ and $W$ mean velocity components measured with hot-wire technique using two different set of equipments and reduction programs. . . . .

Figure 17b. Comparison of $u^{\prime}$ and $w^{\prime}$ fluctuation velocity and uw shear stress components measured with hot-wire technique using two different set of equipments and reduction programs $\ldots \ldots \ldots \ldots \ldots \ldots \ldots \ldots \ldots$

Figure 18. Probe volume formed by two intersecting laser beams. . . . . . . . . . 169

Figure 19. Comparison of station 0 u'fluctuation velocity LDV profile with Klebanoff's two-dimensional turbulent boundary layer data.

Figure 20a. U,V,W mean velocity components measured with two different $\mathrm{LDV}$ techniques presented in tunnel coordinates.

Figure 20b. $\mathrm{u}^{\prime}, \mathrm{v}^{\prime}, \mathrm{w}^{\prime}$ fluctuation velocity components measured with two different LDV

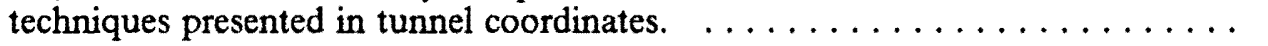

Figure $20 \mathrm{c} .-\overline{\mathrm{uv}},-\overline{\mathrm{uw}},-\overline{\mathrm{vw}}$ shear stress components measured with two different LDV techniques presented in tunnel coordinates.

Figure 20d. U,V,W mean velocity components measured with two different LDV techniques presented in tunnel coordinates.

Figure 20e. $u^{\prime}, v^{\prime}, w^{\prime}$ fluctuation velocity components measured with two different LDV techniques presented in tunnel coordinates.

Figure $20 \mathrm{f} .-\overline{\mathrm{uv}},-\overline{\mathrm{uw}},-\overline{\mathrm{vw}}$ shear stress components measured with two different LDV techniques presented in tunnel coordinates. 
Figure 20g. U,V,W mean velocity components measured with two different LDV techniques presented in tunnel coordinates.

Figure 20h. $u^{\prime}, v^{\prime}, w^{\prime}$ fluctuation velocity components measured with two different LDV techniques presented in tunnel coordinates.

Figure 20i. $-\overline{u v},-\overline{u w},-\overline{v w}$ shear stress components measured with two different LDV techniques presented in tunnel coordinates.

Figure $20 \mathrm{j}$. U,V,W mean velocity components measured with two different LDV techniques presented in tunnel coordinates.

Figure $20 \mathrm{k} . \mathrm{u}^{\prime}, \mathrm{v}^{\prime}, \mathrm{w}^{\prime}$ fluctuation velocity components measured with two different LDV techniques presented in tunnel coordinates.

Figure 201. $-\overline{\mathrm{uv}},-\overline{\mathrm{uw}},-\overline{\mathrm{vw}}$ shear stress components measured with two different LDV techniques presented in tunnel coordinates.

Figure $20 \mathrm{~m}$. U,V,W mean velocity components measured with two different LDV techniques presented in tunnel coordinates.

Figure 20n. $u^{\prime}, v^{\prime}, w^{\prime}$ fluctuation velocity components measured with two different LDV techniques presented in tunnel coordinates.

Figure 200. $-\overline{u v},-\overline{u w},-\overline{v w}$ shear stress components measured with two different LDV techniques presented in tunnel coordinates.

Figure 20p. U,V,W mean velocity components measured with two different LDV techniques presented in tunnel coordinates.

Figure 20q. $u^{\prime}, v^{\prime}, w^{\prime}$ fluctuation velocity components measured with two different LDV techniques presented in tunnel coordinates.

Figure 20r. $-\overline{u v},-\overline{u w},-\overline{v w}$ shear stress components measured with two different LDV techniques presented in tunnel coordinates.

Figure 20s. U,V,W mean velocity components measured with two different LDV techniques presented in tunnel coordinates.

Figure 20t. $\mathrm{u}^{\prime}, \mathrm{v}^{\prime}, \mathrm{w}^{\prime}$ fluctuation velocity components measured with two different LDV techniques presented in tunnel coordinates.

Figure 20u. $-\overline{u v},-\overline{u w}, \overline{v w}$ shear stress components measured with two different LDV techniques presented in tunnel coordinates.

Figure 20w. U,V,W mean velocity components measured with two different LDV techniques presented in tunnel coordinates.

Figure 20x. $\mathrm{u}^{\prime}, \mathrm{v}^{\prime}, \mathrm{w}^{\prime}$ fluctuation velocity components measured with two different LDV techniques presented in tunnel coordinates.

Figure $20 \mathrm{y} .-\overline{\mathrm{uv}},-\overline{\mathrm{uw}},-\overline{\mathrm{vw}}$ shear stress components measured with two different LDV techniques presented in tunnel coordinates.

Figure 21. U component of the mean velocity vector measured with LDV technique presented in tunnel coordinates. 
Figure 22a. U component of the mean velocity vector measured with hot-wire technique on the left-hand side of the wing presented in tunnel coordinates. . . . . . . . 196

Figure 22b. U component of the mean velocity vector measured with hot-wire technique on the left-hand side of the wing presented in tunnel coordinates.

Figure 23. V component of the mean velocity vector measured with LDV technique presented in tunnel coordinates.

Figure 24. W component of the mean velocity vector measured with LDV technique presented in tunnel coordinates.

Figure 25a. W component of the mean velocity vector measured with hot-wire technique on the left-hand side of the wing presented in tunnel coordinates.

Figure $25 \mathrm{~b}$. W component of the mean velocity vector measured with hot-wire technique on the left-hand side of the wing presented in tunnel coordinates.

Figure 26. U component of the mean velocity vector measured with LDV technique presented in free-stream coordinates.

Figure 27a. U component of the mean velocity vector measured with hot-wire technique on the left-hand side of the wing presented in free-stream coordinates. . . . . . . 203

Figure 27b. U component of the mean velocity vector measured with hot-wire technique on the left-hand side of the wing presented in free-stream coordinates.

Figure 28. W component of the mean velocity vector measured with LDV technique presented in free-stream coordinates.

Figure 29a. W component of the mean velocity vector measured with hot-wire technique on the left-hand side of the wing presented in free-stream coordinates.

Figure $29 \mathrm{~b}$. W component of the mean velocity vector measured with hot-wire technique on the left-hand side of the wing presented in free-stream coordinates.

Figure 30. U component of the mean velocity vector measured with LDV technique presented in maximum normal stress coordinates.

Figure 31a. U component of the mean velocity vector measured with hot-wire technique on the left-hand side of the wing presented in normal stress coordinates.

Figure 31b. U component of the mean velocity vector measured with hot-wire technique on the left-hand side of the wing presented in normal stress coordinates. . . . . . 210

Figure 32. W component of the mean velocity vector measured with LDV technique presented in maximum normal stress coordinates.

Figure 33a. W component of the mean velocity vector measured with hot-wire technique on the left-hand side of the wing presented in normal stress coordinates.

Figure 33b. W component of the mean velocity vector measured with hot-wire technique on the left-hand side of the wing presented in normal stress coordinates. . . . . . .

Figure 34a. $\mathrm{u}^{\prime}$, fluctuating velocity component measured with LDV technique presented in tunnel coordinates. 
Figure $34 \mathrm{~b} . \mathrm{u}^{\prime}$, fluctuating velocity component measured with LDV technique presented in tunnel coordinates.

Figure 35a. $\mathrm{u}^{\prime}$, fluctuating velocity component measured with hot-wire technique on the left-hand side of the wing presented in tunnel coordinates.

Figure $35 \mathrm{~b} . \mathrm{u}^{\prime}$, fluctuating velocity component measured with hot-wire technique on the left-hand side of the wing presented in tunnel coordinates.

Figure 36a. $\mathbf{v}^{\prime}$, fluctuating velocity component measured with LDV technique presented in tunnel coordinates.

Figure $36 \mathrm{~b} . \quad \mathrm{v}^{\prime}$, fluctuating velocity component measured with LDV technique presented in tunnel coordinates.

Figure 37a. w' fluctuating velocity component measured with LDV technique presented in tunnel coordinates.

Figure $37 \mathrm{~b}$. w' fluctuating velocity component measured with LDV technique presented in tunnel coordinates.

Figure 38a. w', fluctuating velocity component measured with hot-wire technique on the left-hand side of the wing presented in tunnel coordinates.

Figure 38b. w', fluctuating velocity component measured with hot-wire technique on the left-hand side of the wing presented in tunnel coordinates.

Figure 39. u', fluctuating velocity component measured with LDV technique presented in free-stream coordinates.

Figure 40a. $u^{\prime}$, fluctuating velocity component measured with hot-wire technique on the left-hand side of the wing presented in free-stream coordinates.

Figure 40b. $\mathrm{u}^{\prime}$, fluctuating velocity component measured with hot-wire technique on the left-hand side of the wing presented in free-stream coordinates.

Figure 41 . w' fluctuating velocity component measured with LDV technique presented in free-stream coordinates.

Figure 4la. w', fluctuating velocity component measured with hot-wire technique on the left-hand side of the wing presented in free-stream coordinates.

Figure $41 \mathrm{~b} . \quad w^{\prime}$, fluctuating velocity component measured with hot-wire technique on the left-hand side of the wing presented in free-stream coordinates.

Figure 43. $u^{\prime}$, fluctuating velocity component measured with LDV technique presented in maximum normal stress coordinates. . . . . . . . . . . . . . . .

Figure 44a. $u^{\prime}$, fluctuating velocity component measured with hot-wire technique on the left-hand side of the wing presented in normal stress coordinates.

Figure $44 \mathrm{~b} . \mathrm{u}^{\prime}$, fluctuating velocity component measured with hot-wire technique on the left-hand side of the wing presented in normal stress coordinates.

Figure 45. w' fluctuating velocity component measured with LDV technique presented in maximum normal stress coordinates. 
Figure 46a. $w^{\prime}$, fluctuating velocity component measured with hot-wire technique on the left-hand side of the wing presented in normal stress coordinates.

Figure $46 \mathrm{~b}$. w', fluctuating velocity component measured with hot-wire technique on the left-hand side of the wing presented in normal stress coordinates.

Figure 47a. - - $\overline{\mathrm{vv}}$, shear stress component measured with LDV technique presented in tunnel coordinates.

Figure $47 \mathrm{~b} .-\overline{\mathrm{uv}}$, shear stress component measured with LDV technique presented in tunnel

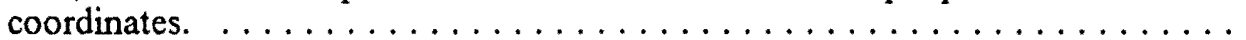

Figure 48a. - - $\overline{\mathrm{uw}}$, shear stress component measured with LDV technique presented in tunnel

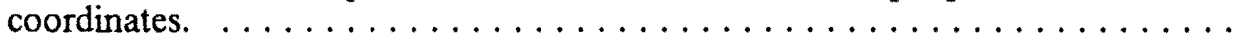

Figure 48b. - - $\mathrm{uw}$, shear stress component measured with LDV technique presented in tunnel coordinates.

Figure 49a. - - $\overline{\mathrm{uw}}$, shear stress component measured with hot-wire technique on the left-hand side of the wing presented in tunnel coordinates.

Figure 49b. - - $\overline{\mathrm{uw}}$, shear stress component measured with hot-wire technique on the left-hand side of the wing presented in tunnel coo rdinates.

Figure 50a. $-\overline{\mathrm{vw}}$, shear stress component measured with LDV technique presented in tunnel coordinates.

Figure 50b. $-\overline{\mathrm{vw}}$, shear stress component measured with LDV technique presented in tunnel coordinates.

Figure 51. - - $\mathrm{uv}$, shear stress component measured with LDV technique presented in free-stream coordinates.

Figure 52. $-\overline{\mathrm{uw}}$, shear stress component measured with LDV technique presented in free-stream coordinates.

Figure 53. $-\overline{\mathrm{vw}}$, shear stress component measured with LDV technique presented in free-stream coordinates.

Figure 54a. - $\overline{u w}$, shear stress component measured with hot-wire technique on the left-hand side of the wing presented in free-stream coordinates.

Figure 54b. $-\overline{u w}$, shear stress component measured with hot-wire technique on the left-hand side of the wing presented in free-stream coordinates.

Figure 55. $-\overline{\mathrm{uv}}$, shear stress component measured with LDV technique presented in maximum normal stress coordinates.

Figure 56. - $\overline{u w}$, shear stress component measured with LDV technique presented in maximum normal stress coordinates.

Figure 57a. $-\overline{u w}$, shear stress component measured with hot-wire technique on the left-hand side of the wing presented in normal stress coordinates.

Figure 57b. $-\overline{u w}$, shear stress component measured with hot-wire technique on the left-hand side of the wing presented in normal stress coordinates. 
Figure 58. $-\overline{\mathrm{vw}}$, shear stress component measured with LDV technique presented in maximum normal stress coordinates. . . . . . . . . . . . . . . .

Figure 59a. N, anisotropy constant computed using LDV data in tunnel coordinates. . . . 254

Figure 59b. N, anisotropy constant computed using LDV data in tunnel coordinates. . . . 255

Figure 59c. N, anisotropy constant computed using LDV data in tunnel coordinates. . . . 256

Figure 60a. N, anisotropy constant computed using LDV data in free-stream coordinates. 257

Figure $60 \mathrm{~b}$. N, anisotropy constant computed using LDV data in free-stream coordinates. 258

Figure 60c. N, anisotropy constant computed using LDV data in free-stream coordinates. 259

Figure 61a. $\mathrm{N}$, anisotropy constant computed using LDV data in maximum normal stress coordinates. . . . . . . . . . . . . . . . . . . . . . . . 260

Figure 61b. $\mathrm{N}$, anisotropy constant computed using LDV data in maximum normal stress coordinates. ........................... 261

Figure 61c. N, anisotropy constant computed using LDV data in maximum normal stress

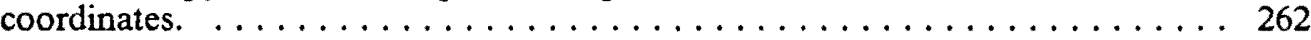

Figure 62a. A1, Townsend's structural parameter computed using LDV data. . . . . . 263

Figure 62b. Al, Townsend's structural parameter computed using LDV data. . . . . . . 264

Figure 62c. Al, Townsend's structural parameter computed using LDV data. . . . . . 265

Figure 63a. Flow angle computed using LDV data in tunnel coordinates. . . . . . 266

Figure 63b. Flow angle computed using LDV data in tunnel coordinates. . . . . . 267

Figure 63c. Flow angle computed using LDV data in tunnel coordinates. . . . . . . 268

Figure 64a. Flow angle in tunnel coordinates computed using hot-wire data taken on the left-hand side of the wing. . . . . . . . . . . . . . . . . . 269

Figure $64 \mathrm{~b}$. Flow angle in tunnel coordinates computed using hot-wire data taken on the

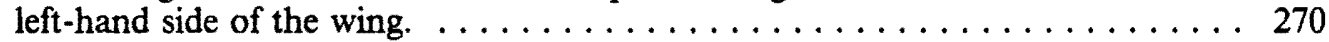

Figure 64c. Flow angle in tunnel coordinates computed using hot-wire data taken on the left-hand side of the wing. . . . . . . . . . . . . . . 271

Figure 65a. Flow angle computed using LDV data in free-stream coordinates. . . . . 272

Figure $65 \mathrm{~b}$. Flow angle computed using LDV data in free-stream coordinates. . . . . 273

Figure 65c. Flow angle computed using LDV data in free-stream coordinates. . . . . 274

Figure 66a. Flow angle in free-stream coordinates computed using hot-wire data taken on the

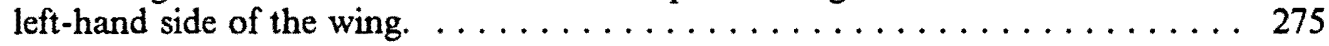

Figure 66b. Flow angle in free-stream conrdinates computed using hot-wire data taken on the

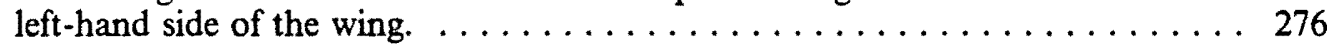


Figure 66c. Flow angle in free-stream coordinates computed using hot-wire data taken on the

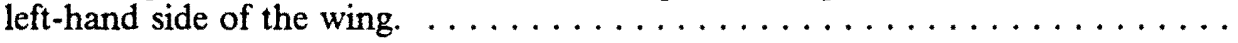

Figure 67a. Flow angle computed using LDV data in maximum normal stress coordinates. 278

Figure $67 \mathrm{~b}$. Flow angle computed using LDV data in maximum normal stress coordinates. 279

Figure 67c. Flow angle computed using LDV data in maximum normal stress coordinates. 280

Figure 68a. Flow angle in maximum normal stress coordinates computed using hot-wire data taken on the left-hand side of the wing. $\ldots \ldots \ldots \ldots \ldots \ldots \ldots \ldots \ldots$

Figure $68 \mathrm{~b}$. Flow angle in maximum normal stress coordinates computed using hot-wire data

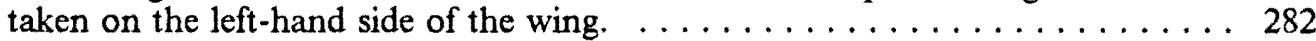

Figure 68c. Flow angle in maximum normal stress coordinates computed using hot-wire data taken on the left-hand side of the wing. . . . . . . . . . . . . 283

Figure 69a. Flow gradient angle computed using LDV data in free-stream coordinates. . . 284

Figure $69 \mathrm{~b}$. Flow gradient angle computed using LDV data in free-stream coordinates. . . 285

Figure 69c. Flow gradient angle computed using LDV data in free-stream coordinates. . . 286

Figure 70a. Shear stress angle computed using LDV data in free-stream coordinates. $\ldots 287$

Figure $70 \mathrm{~b}$. Shear stress angle computed using LDV data in free-stream coordinates. . . 288

Figure 70c. Shear stress angle computed using LDV data in free-stream coordinates. . . . 289

Figure 7la. $u^{\prime}$, fluctuating velocity component of LDV data in free-stream coordinates normalized with the skin friction velocity. . . . . . . . . . . . . . . . . 290

Figure $71 \mathrm{~b} . \mathrm{u}^{\prime}$, fluctuating velocity component of LDV data in free-stream coordinates normalized with the skin friction velocity. . . . . . . . . . . . . . 291

Figure 72a. $\mathrm{v}^{\prime}$, fluctuating velocity component of LDV data in free-stream coordinates normalized with the skin friction velocity.

Figure 72b. $\mathrm{v}^{\prime}$, fluctuating velocity component of LDV data in free-stream coordinates normalized with the skin friction velocity.

Figure 73a. w', fluctuating velocity component of LDV data in free-stream coordinates normalized with the skin friction velocity.

Figure 73b. $w^{\prime}$, fluctuating velocity component of LDV data in free-stream coordinates normalized with the skin friction velocity.

Figure 74a. $-\overline{u v}$, shear stress of LDV data in free-stream coordinates normalized with the square of the skin friction velocity.

Figure $74 \mathrm{~b} .-\overline{\mathrm{uv}}$, shear stress of LDV data in free-stream coordinates normalized with the square of the skin friction velocity.

Figure 75a. $-\overline{u w}$, shear stress of LDV data in free-stream coordinates normalized with the square of the skin friction velocity. 
Figure $75 b,-\overline{u w}$, shear stress of LDV data in free-stream coordinates normalized with the square of the skin friction velocity.

Figure 76a. $-\overline{\mathrm{VW}}$, shear stress of LDV data in free-stream coordinates normalized with the square of the skin friction velocity.

Figure $76 \mathrm{~b} .-\overline{\mathrm{vw}}$, shear stress of LDV data in free-stream coordinates normalized with the square of the skin friction velocity.

Figure 77a. $\mathrm{x}$ eddy viscosity computed using the LDV data in free-stream coordinates. . . 302

Figure $77 \mathrm{~b}$. $\mathrm{x}$ eddy viscosity computed using the LDV data in free-stream coordinates. . . 303

Figure 78a. $z$ eddy viscosity computed using the LDV data in free-stream coordinates. . . 304

Figure 78b. $z$ eddy viscosity computed using the LDV data in free-stream coordinates. . . 305

Figure 79a. Mixing length nondimensionalized with boundary layer thickness $\ldots \ldots \ldots 6$

Figure $79 \mathrm{~b}$. Mixing length nondomensionalized with boundary layer thickness . . . . . 307

Figure $80 \mathrm{a}$. Johnston's polar plots of the present data. $\ldots \ldots \ldots \ldots \ldots \ldots \ldots$

Figure $80 \mathrm{~b}$. Johnston's polar plots of the present data. . . . . . . . . . . . . 309

Figure 81a. Turbulent kinetic energy profiles obtained using LDV data. . . . . . . 310

Figure $81 \mathrm{~b}$. Turbulent kinetic energy profiles obtained using LDV data. . . . . . 311

Figure 82a. Production of turbulent kinetic energy profiles obtained using LDV data in

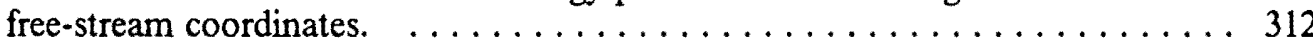

Figure $82 \mathrm{~b}$. Production of turbulent kinetic energy profiles obtained using LDV data in free-stream coordinates.

Figure 83a. Convection of turbulent kinetic energy profiles obtained using LDV data in free-stream coordinates.

Figure 83b. Convection of turbulent kinetic energy profiles obtained using LDV data in free-stream coordinates.

Figure $84 a$. Production of $\overline{\mathrm{u} 2}$ normal stress profiles computed using LDV data in free-stream coordinates.

Figure $84 \mathrm{~b}$. Production of $\overline{\mathrm{u} 2}$ normal stress profiles computed using LDV data in free-stream coordinates.

Figure $85 \mathrm{a}$. Production of $\overline{\mathrm{v} 2}$ normal stress profiles computed using LDV data in free-stream coordinates.

Figure $85 \mathrm{~b}$. Production of $\overline{\mathrm{v}}$ normal stress profiles computed using LDV data in free-stream coordinates.

Figure 86a. Production of $\overline{\mathrm{w} 2}$ normal stress profiles computed using LDV data in free-stream coordinates. 
Figure $86 \mathrm{~b}$. Production of $\overline{\mathrm{w} 2}$ normal stress profiles computed using LDV data in free-stream coordinates.

Figure 87a. Production of -uv shear stress profiles computed using LDV data in free-stream coordinates.

Figure $87 \mathrm{~b}$. Production of $-\overline{\mathrm{uv}}$ shear stress profiles computed using LDV data in free-stream coordinates.

Figure 88a. Production of - $\overline{\mathrm{uw}}$ shear stress profiles computed using LDV data in free-stream coordinates.

Figure $88 \mathrm{~b}$. Production of $-\overline{\mathrm{uw}}$ shear stress profiles computed using LDV data in free-stream coordinates.

Figure 89a. Production of $-\overline{\mathrm{VW}}$ shear stress profiles computed using LDV data in free-stream coordinates.

Figure $89 \mathrm{~b}$. Production of $-\overline{\mathrm{vw}}$ shear stress profiles computed using LDV data in free-stream coordinates.

Figure 90a. Convection of $-\overline{\mathrm{u} 2}$ normal stress profiles computed using LDV data in free-stream coordinates.

Figure 90b. Convection of $\overline{\mathrm{u} 2}$ normal stress profiles computed using LDV data in free-stream coordinates.

Figure $90 \mathrm{c}$. Convection of $\overline{\mathrm{v} 2}$ normal stress profiles computed using LDV data in free-stream coordinates.

Figure 90d. Convection of $\overline{\mathrm{v}} \mathrm{n}$ normal stress profiles computed using LDV data in free-stream coordinates.

Figure 90e. Convection of $\overline{\mathrm{w} 2}$ normal stress profiles computed using LDV data in free-stream coordinates.

Figure 90f. Convection of $\overline{\mathrm{w} 2}$ normal stress profiles computed using LDV data in free-stream coordinates.

Figure 90g. Convection of - $\overline{\mathrm{uV}}$ shear stress profiles computed using LDV data in free-stream coordinates.

Figure $90 \mathrm{~h}$. Convection of $-\overline{\mathrm{uv}}$ shear stress profiles computed using LDV data in free-stream coordinates.

Figure 90i. Convection of - $\overline{\mathrm{uw}}$ shear stress profiles computed using LDV data in free-stream coordinates.

Figure $90 \mathrm{j}$. Convection of $-\overline{\mathrm{uw}}$ shear stress profiles computed using LDV data in free-stream coordinates.

Figure 90k. Convection of $-\overline{\mathrm{vw}}$ shear stress profiles computed using LDV data in free-stream coordinates.

Figure 901. Convection of $-\overline{\mathrm{vw}}$ shear stress profiles computed using LDV data in free-stream coordinates. 
Figure 91a. Schematic figure of Anderson's experimental set up. $\ldots \ldots \ldots \ldots \ldots \ldots 340$

Figure 92b. Flow angles of Anderson's Case I, Fernholz-Vagt, Muller, Dechow-Felsch, Elsenaar-Boelsma, Bissonnette-Mellor, Lohmann flows. . . . . . . . . . 341

Figure 93. Schematic figure of Fernholz-Vagt experimental set up. . . . . . . . . . 342

Figure 94. Schematic figure of Muller's experimental set up. $\ldots \ldots \ldots \ldots \ldots \ldots \ldots 34$

Figure 95. Schematic figure of Dechow-Felsch experimental set up. . . . . . . . . 344

Figure 96. Schematic figure of Elsenaar-Boelsma experimental set up. $\ldots \ldots \ldots \ldots \ldots 345$

Figure 97. Schematic figure of Bissonnette-Mellor experimental set up. $\ldots \ldots \ldots \ldots \ldots 346$

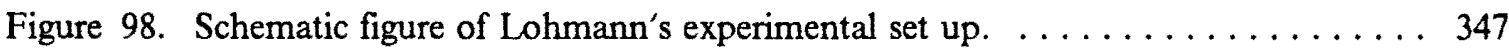

Figure 99a. Pressure-driven data in the Law-of-the-Wall coordinate system of Coles. . . . 348

Figure 99b. Shear-driven data in the Law-of-the-Wall coordinate system of Coles. . . . . 349

Figure 100a. Pressure-driven data in the Law-of-the-Wall coordinate system of Johnston. . 350

Figure 100b. Shear-driven data in the Law-of-the-Wall coordinate system of Johnston. . . 351

Figure 101a. Pressure-driven data in the Law-of-the-Wall coordinate system of Hornung-Joubert. ...................... 352

Figure 10lb. Shear-driven data in the Law-of-the-Wall coordinate system of Hornung-Joubert.

Figure 102a. Pressure-driven data in the Law-of-the-Wall coordinate system of Pierce-Krommenhoek, Chandrashekhar-Swamy. 354

Figure 102b. Shear-driven data in the Law-of-the-Wall coordinate system of Pierce-Krommenhoek, Chandrashekhar-Swamy.

Figure 103a. Pressure-driven data in the Law-of-the-Wall coordinate system of Chandrashekhar-Swamy for the lateral component.

Figure 103b. Shear-driven data in the Law-of-the-Wall coordinate system of Chandrashekhar-Swamy for the lateral component.

Figure 104a. Pressure-driven data in the Law-of-the-Wall coordinate system of East-Hoxey. 358

Figure 104b. Shear-driven data in the Law-of-the-Wall coordinate system of East-Hoxey. . 359

Figure 105ai. Pressure-driven data in the Law-of-the-Wall coordinate system of Perry-Joubert.

Figure 105aii. Shear-driven data in the Law-of-the-Wall coordinate system of Perry-Joubert. 361

Figure 105bi. Pressure-driven data in the Law-of-the-Wall coordinate system of Perry-Joubert.

Figure 105bii. Shear-driven data in the Law-of-the-Wall coordinate system of Perry-Joubert. 363 
Figure 106ai. Pressure-driven data in the Law-of-the-Wall coordinate system of

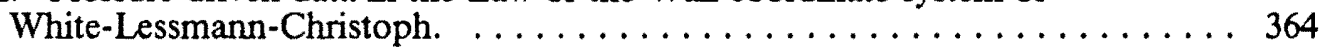

Figure 106aii. Shear-driven data in the Law-of-the-Wall coordinate system of White-Lessmann-Christoph.

Figure 106bi. Pressure-driven data in the Law-of-the-Wall coordinate system of

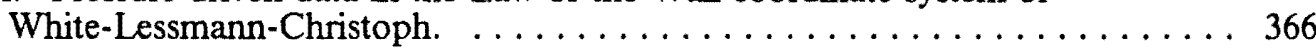

Figure 106bii. Shear-driven data in the Law-of-the-Wall coordinate system of White-Lessmann-Christoph.

Figure 106ci106di. Pressure-driven data in the Law-of-the-Wall coordinate system of White-Lessmann-Christoph.

Figure 106cii. Shear-driven data in the Law-of-the-Wall coordinate system of White-Lessmann-Christoph.

Figure 106dii. Shear-driven data in the Law-of-the-Wall coordinate system of White-Lessmann-Christoph.

Figure 107ai. Pressure-driven data in the Law-of-the-Wall coordinate system of Van Den Berg.

Figure 107aii. Shear-driven data in the Law-of-the-Wall coordinate system of Van Den Berg. 372

Figure 107bi. Pressure-driven data in the Law-of-the-Wall coordinate system of Van Den Berg.

Figure 107bii. Shear-driven data in the Law-of-the-Wall coordinate system of Van Den Berg. 374

Figure 107ci. Pressure-driven data in the Law-of-the-Wall coordinate system of Van Den Berg.

Figure 107cii. Pressure-driven data in the Law-of-the-Wall coordinate system of Van Den Berg. . . . . . . . . . . 377

Figure 107ciii. Shear-driven data in the Law-of-the-Wall coordinate system of Van Den Berg. 140

Figure 107di. Pressure-driven data in the Law-of-the-Wall coordinate system of Van Den

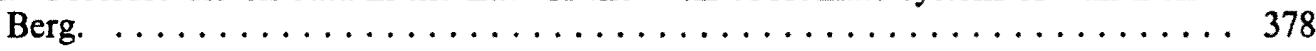

Figure 107dii. Pressure-driven data in the Law-of-the-Wall coordinate system of Van Den

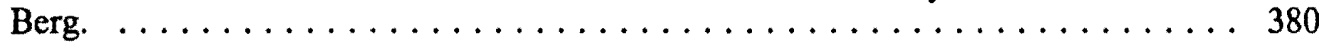

Figure 107diii. Shear-driven data in the Law-of-the-Wall coordinate system of Van Den Berg. 140

Figure 108a. $Q+$ range at $y+=70$ for various law of the wall profiles using various data sets. 381

Figure $108 \mathrm{~b}$. $Q+$ range at $y+=70$ for various Law-of-the-Wall profiles using various data sets.

Figure 109a. $Q+$ range at $y+=40$ and $y+=100$ for various "Law-of-the-Wall" profiles using various data sets. 
Figure 109b. $Q+$ range at $y+=40$ and $y+=100$ for various "Law-of-the-Wall" profiles using various data sets.

Figure 110a. Present data in the Law-of-the-Wall coordinate systems of Coles (left) and

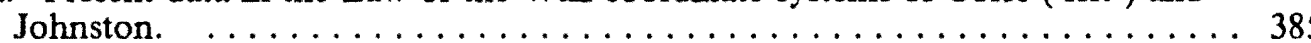

Figure $110 \mathrm{~b}$. Present data in the Law-of-the-Wall coordinate systems of Hornung-Joubert ( left ) and Pierce-Krommenhoek and Chandrashekhar-Swamy (right ). . . . 386

Figure 110c. Present data in the Law-of-the-Wall coordinate systems of Chandrashekhar-Swamy (left, lateral component ) and East-Hoxey ( right ). . 387

Figure 110d. Present data in the Law-of-the-Wall coordinate system of Perry-Joubert. . . 388

Figure 110ei. Present data in the Law-of-the-Wall coordinate system of White-Lessmann-Christoph ( Axial component ).

Figure 110eii. Present data in the Law-of-the-Wall coordinate system of White-Lessmann-Christoph. ( Axial component)

Figure 110eiii. Present data in the Law-of-the-Wall coordinate system of White-Lessmann-Christoph. ( Lateral component )

Figure 110eiiii. Present data in the Law-of-the-Wall coordinate system of White-Lessmann-Christoph. ( Lateral component )

Figure 110fi. Present data in the Law-of-the-Wall coordinate system of Van Den Berg. (Axial component )

Figure 110fii. Present data in the Law-of-the-Wall coordinate system of Van Den Berg. ( Axial component )

Figure 110fiii. Present data in the Law-of-the-Wall coordinate system of Van Den Berg. ( Lateral component )

Figure 110fiii. Present data in the Law-of-the-Wall coordinate system of Van Den Berg. ( Lateral component $) \ldots \ldots \ldots \ldots \ldots \ldots \ldots \ldots \ldots \ldots \ldots \ldots \ldots \ldots$

Figure 111. Computed values for different various laws of the wall for Present data at $y+=40,70,100,250$, with the uncertainty bands

Figure 112a. Present data in Cebeci-Smith model. $\ldots \ldots \ldots \ldots \ldots \ldots \ldots$

Figure 112b. Present data in Cebeci-Smith model. . . . . . . . . . . . . . . 399

Figure $112 \mathrm{c}$. Present data in Cebeci-Smith model. $\ldots \ldots \ldots \ldots \ldots \ldots \ldots \ldots \ldots$

Figure 112d. Present data in Cebeci-Smith model. $\ldots \ldots \ldots \ldots \ldots \ldots \ldots \ldots \ldots$

Figure 113a. Present data in Rotta's model with anisotropy constant $T=0.3 \ldots \ldots \ldots 4$

Figure 113b. Present data in Rotta's model with anisotropy constant $T=0.3 . \ldots \ldots 403$

Figure 113c. Present data in Rotta's model with anisotropy constant $T=0.3, \ldots \ldots 404$

Figure 113d. Present data in Rotta's model with anisotropy constant $T=0.3 \ldots \ldots 405$ 
Figure 114a. Present data in Rotta's model with anisotropy constant $\mathrm{T}=0.5 \ldots \ldots \ldots 4$

Figure 114b. Present data in Rotta's model with anisotropy constant $T=0.5$. . . . 407

Figure 114c. Present data in Rotta's model with anisotropy constant $\mathrm{T}=0.5 \ldots \ldots \ldots 4$

Figure 114d. Present data in Rotta's model with anisotropy constant $\mathrm{T}=0.5 \ldots \ldots \ldots 409$

Figure 115a. Present data in Rotta's model with anisotropy constant $T=0.7 \ldots \ldots \ldots 410$

Figure 115b. Present data in Rotta's model with anisotropy constant $T=0.7, \ldots \ldots 411$

Figure 115c. Present data in Rotta's model with anisotropy constant $\mathrm{T}=0.7 \ldots \ldots \ldots 412$

Figure 115d. Present data in Rotta's model with anisotropy constant $T=0.7 \ldots \ldots \ldots 413$

Figure 116a. Present data in Patel's model. .................... 414

Figure 116b. Present data in Patel's model. $\ldots \ldots \ldots \ldots \ldots \ldots \ldots \ldots \ldots \ldots \ldots$

Figure 116c. Present data in Patel's model. .................. 416

Figure 116d. Present data in Patel's model. $\ldots \ldots \ldots \ldots \ldots \ldots \ldots \ldots \ldots \ldots$

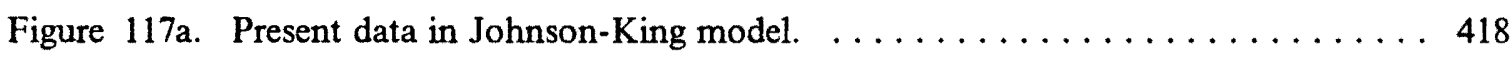

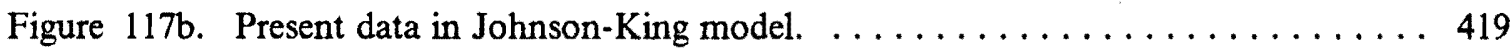

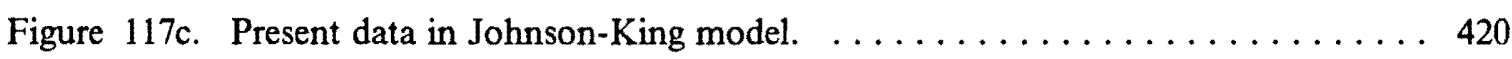

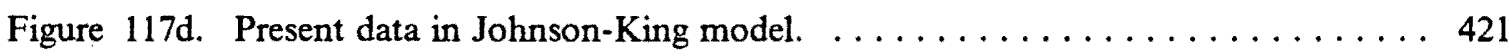

Figure 118a. Elsenaar-Boelsma data in Cebeci-Smith model ( left ) and in Rotta's model with anisotropy constant $\mathrm{T}=0.3$ ( right $). \ldots \ldots \ldots \ldots \ldots \ldots \ldots \ldots \ldots \ldots \ldots \ldots \ldots \ldots$ 422

Figure 118b. Elsenaar-Boelsma data in Rotta's model with anisotropy constant $T=0.5$ (left ) and with anisotropy constant $\mathrm{T}=0.7$ ( right ). ................

Figure 118c. Elsenaar-Boelsma data in Johnson-King ( left) and in Patel's ( right ) models. 424

Figure 118d. Dechow's data in Cebeci-Smith model ( left ) and in Rotta's model with anisotropy constant $\mathrm{T}=0.3$ ( right ).

Figure 119a. Dechow's data in Rotta's model with anisotropy constant $\mathrm{T}=0.5$ ( left ) and

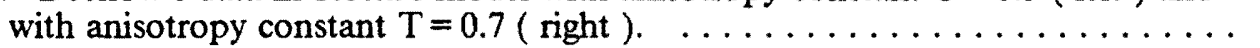

Figure 119b. Dechow's data in Johnson-King ( left ) and in Patel's ( right ) models. .... 426

Figure 120a. Müller's data in Cebeci-Smith model (left ) and in Rotta's model with anisotropy constant $\mathrm{T}=0.3$ ( right ).

Figure 120b. Müller's data in Rotta's model with anisotropy constant $T=0.5$ ( left) and with

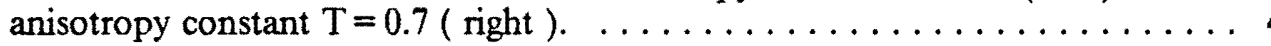

Figure 120c. Müller's data in Johnson-King ( left ) and in Patel's ( right ) models. 
Figure 121a. Anderson's data in Cebeci-Smith model ( left ) and in Rotta's model with

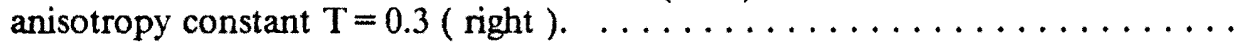

Figure 12lb. Anderson's data in Rotta's model with anisotropy constant $\mathrm{T}=0.5$ ( left ) and

Figure 121c. Anderson's data in Johnson-King ( left ) and in Patel's ( right ) models. ...

Figure 122a. Lohmann's data in Cebeci-Smith model ( left ) and in Rotta's model with

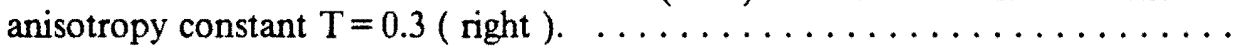

Figure 122b. Lohmann's data in Rotta's model with anisotropy constant $T=0.5$ (left) and with anisotropy constant $T=0.7$ ( right ).

Figure 122c. Lohmann's data in Rotta's model with anisotropy constant $T=1.2$ ( left ) and with anisotropy constant $\mathrm{T}=1.5$ ( right ).

Figure 122d. Lohmann's data in Johnson-King ( left ) and in Patel's ( right) models. ...

Figure 123a. Bissonnette-Mellor low Reynolds number data in Cebeci-Smith model (left) and in Rotta's model with anisotropy constant $T=0.3$ ( right ).

Figure 123b. Bissonnette-Mellor low Reynolds number data in Rotta's model with anisotropy constant $T=0.5$ (left) and with anisotropy constant $T=0.7$ ( right ). 439

Figure 123c. Bissonnette-Mellor low Reynolds number data in Rotta's model with anisotropy constant $T=1.2$ ( left ) and with anisotropy constant $T=1.5$ ( right ). . . . .

Figure 123d. Bissonnette-Mellor low Reynolds number data in Johnson-King ( left ) and in Patel's ( right ) models.

Figure 124a. Bissonnette-Mellor high Reynolds number data in Cebeci-Smith (left) and in Johnson-King (right) models.

Figure 124b. Bissonnette-Mellor high Reynolds number data in Rotta's model with anisotropy constant $T=0.3$ (left) and with anisotropy constant $T=0.5$ (right). 443

Figure 124c. Bissonnette-Mellor high Reynolds number data in Rotta's model with anisotropy constant $T=1.2$ (left) and with anisotropy constant $T=1.5$ ( right ). 444

Figure 124d. Bissonnette-Mellor high Reynolds number data in Rotta's model with anisotropy constant $T=0.7$ ( left ) and in Patel's ( right ) models.

Figure 125. Single hot-wire in the $\mathrm{x}, \mathrm{z}$ plane 446

Figure 126. Schematic figure of UV system beams of the LDV set up. 447

Figure 127. Schematic figure of UW system beams of the LDV set up 448

Figure 128. Schematic figure of UW system beams of the LDV set up 449 


\section{LIST OF SYMBOLS}

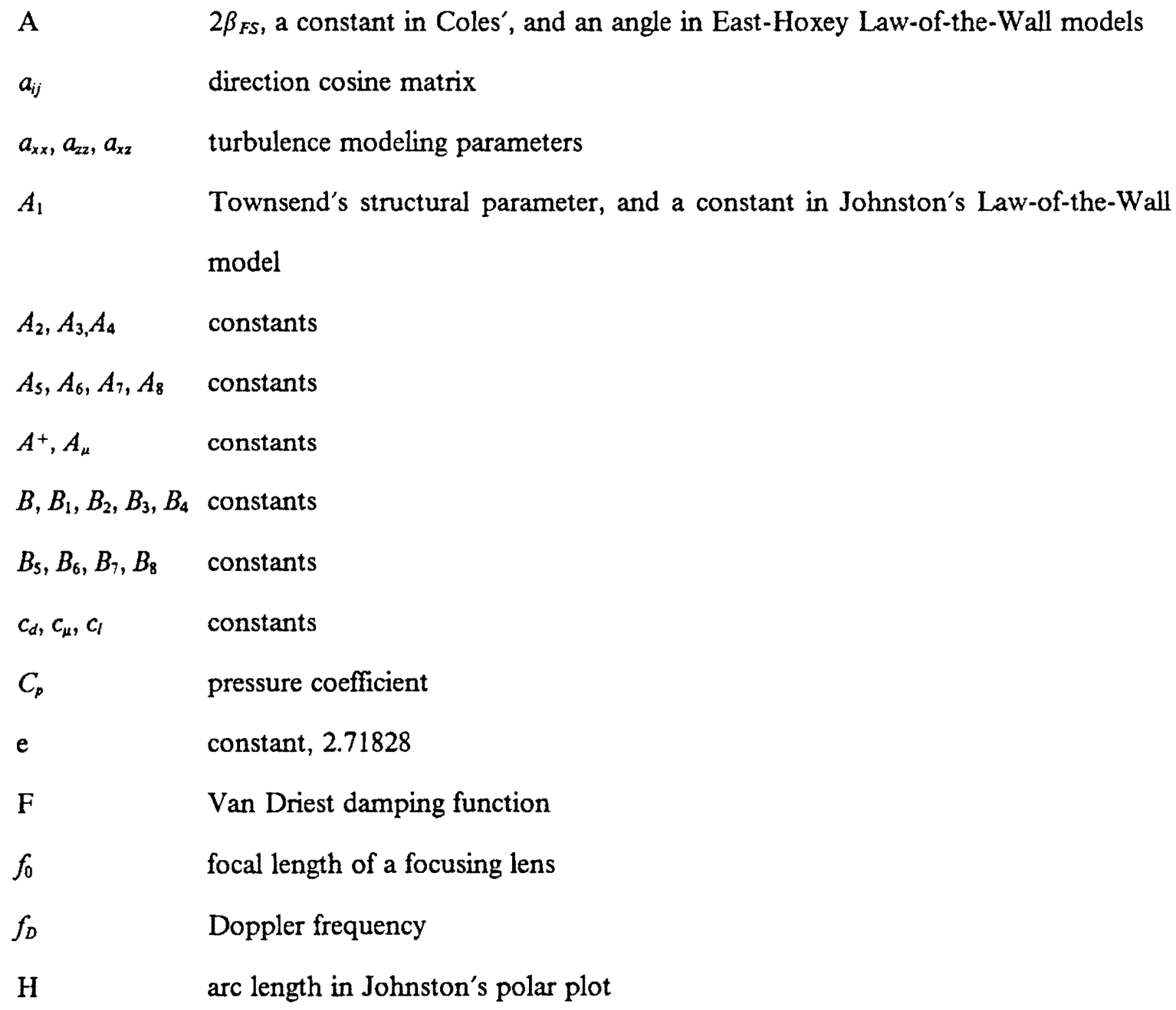


ratio of cooling effect of the tangential flow to normal flow on the hot wire

$\mathrm{I}(\mathrm{x}, \mathrm{y}, \mathrm{z}) \quad$ intensity distribution in the probe volume

$I_{\text {center }} \quad$ intensity at the center of the probe volume

$h_{1} \quad$ metric coefficient

$\mathrm{k}, \mathrm{TKE} \quad$ turbulent kinetic energy, $\frac{1}{2}\left(\overline{u^{2}}+\overline{v^{2}}+\overline{w^{2}}\right)$

K

a constant

L dissipation length scale and body length

$L_{m}, l \quad$ mixing length

N

anisotropy constant

$\mathrm{p}$

static pressure

$p_{0} \quad$ reference total pressure

$\bar{p}$

mean static pressure

$p^{\prime}$

fluctuating static pressure

$p_{\text {ref }}$

reference static pressure

q

mean velocity magnitude $\mathrm{q}=\sqrt{U^{2}+W^{2}}$

$Q^{+}$

wall law parameter

$q$.

dynamic pressure

R

Reynolds stress tensor

$R_{y}$

turbulent Reynolds number

$\operatorname{Re}$

Reynolds number

$R e_{\theta}$

Reynolds number based on momentum thickness

$\mathrm{S}, S_{0} \quad$ wall law parameters

$\delta^{+} \quad \delta u_{T} / v$

$S_{i j}$

$\mathbf{t}$

rate of strain tensor

time and maximum body thickness ( 2.824 inches)

T

anisotropy constant in Rotta's model

$\mathrm{U}, \mathrm{V}, \mathrm{W}$ mean velocity components

$u^{\prime}, v^{\prime}, w^{\prime} \quad$ fluctuation velocities 

$\mathrm{u}, \mathrm{v}, \mathrm{w} \quad$ instantaneous fluctuating velocity components
U. free-stream velocity at the boundary layer edge
$U_{\text {off }} \quad$ effective velocity which cools the hot wire
$u_{i} \quad$ instantaneous velocity components
$U_{i}, V_{i}, W_{i} \quad$ instantaneous velocity components
$U_{\text {ins }} \quad$ instantaneous velocity seen by the hot wire
$U_{x}^{+} \quad$ wall law parameter
$U_{2}^{+} \quad$ wall law parameter
$U^{+} \quad$ wall law parameter, $\frac{U}{u_{r}}$
$u_{r} \quad$ skin friction velocity
$\overline{u_{i} u_{l}} \quad$ Reynolds-averaged stress tensor
$\overline{u^{2}}, \overline{v^{2}}, \overline{w^{2}} \quad$ kinematic normal stresses
$U_{\text {ref }} \quad$ reference velocity
$\vec{V} \quad$ velocity vector in the probe volume of two intersecting laser beams
$\vec{V}_{0} \quad$ velocity vector at the center of the probe volume formed by two intersecting laser beams
$W_{0} \quad$ surface velocity of rotating cylinder
$-\overline{u v},-\overline{u w} \quad$ kinematic shear stresses
$-\overline{v w} \quad$ kinematic shear stress
$\mathrm{x}, \mathrm{y}, \mathrm{z} \quad$ coordinate axes
$x_{0}, y_{0}, z_{0} \quad$ center of the probe volume formed by two intersecting laser beams
$y^{+} \quad$ wall law parameter, $\frac{y u_{r}}{v}$
$\alpha \quad$ pressure gradient and shear stress angle with respect to the $\mathrm{x}$ axis of the free-stream coordinates
$\alpha_{c} \quad$ computed shear stress vector angle with respect to the $\mathrm{x}$ axis of the free-stream coordinates 
measured shear stress vector angle with respect to the $\mathrm{x}$ axis of the free-stream coordinates

pressure gradient parameter in the direction of the wall stress vector

pressure gradient parameter perpendicular to the direction of the wall stress vector angles between the intersecting beams in several optical systems

flow angle with respect to $\mathrm{x}$ axis of the free-stream coordinates angle between $x$ axes of the tunnel and free-stream coordinates angle between $\mathrm{x}$ axes of tunnel and normal stress coordinates wall shear stress direction with respect to $\mathrm{x}$ free-stream coordinate. wall stress gradient parameter in the wall stress vector direction wall stress gradient parameter perpendicular to the wall stress vector an angle in East and Hoxey Law-of-the-Wall model an angle

Klebanoff intermittency function boundary layer thickness, point in the layer where $\sqrt{U^{2}+W^{2}} / U_{c}=0.995$ displacement thickness $=\int_{0}^{\delta}\left(1-U / U_{0}\right) d y$

displacement thickness $=\int_{0}^{\delta}\left(1-\sqrt{U^{2}+W^{2}} / U_{e}\right) d y$

momentum thickness $=\int_{0}^{\delta}\left(1-U / U_{0}\right) U / U_{e} d y$

lateral displacement thickness $=\int_{0}^{\delta}\left(-W / U_{e}\right) d y$

dissipation

$\tan \left(\beta_{w}\right)$

angle between the pressure gradient and wall shear stress vectors and momentum thickness $\delta_{3}$

Von Karman constant

pressure gradient parameter and laser wave length

dynamic viscosity

kinematic viscosity

turbulent eddy viscosity 


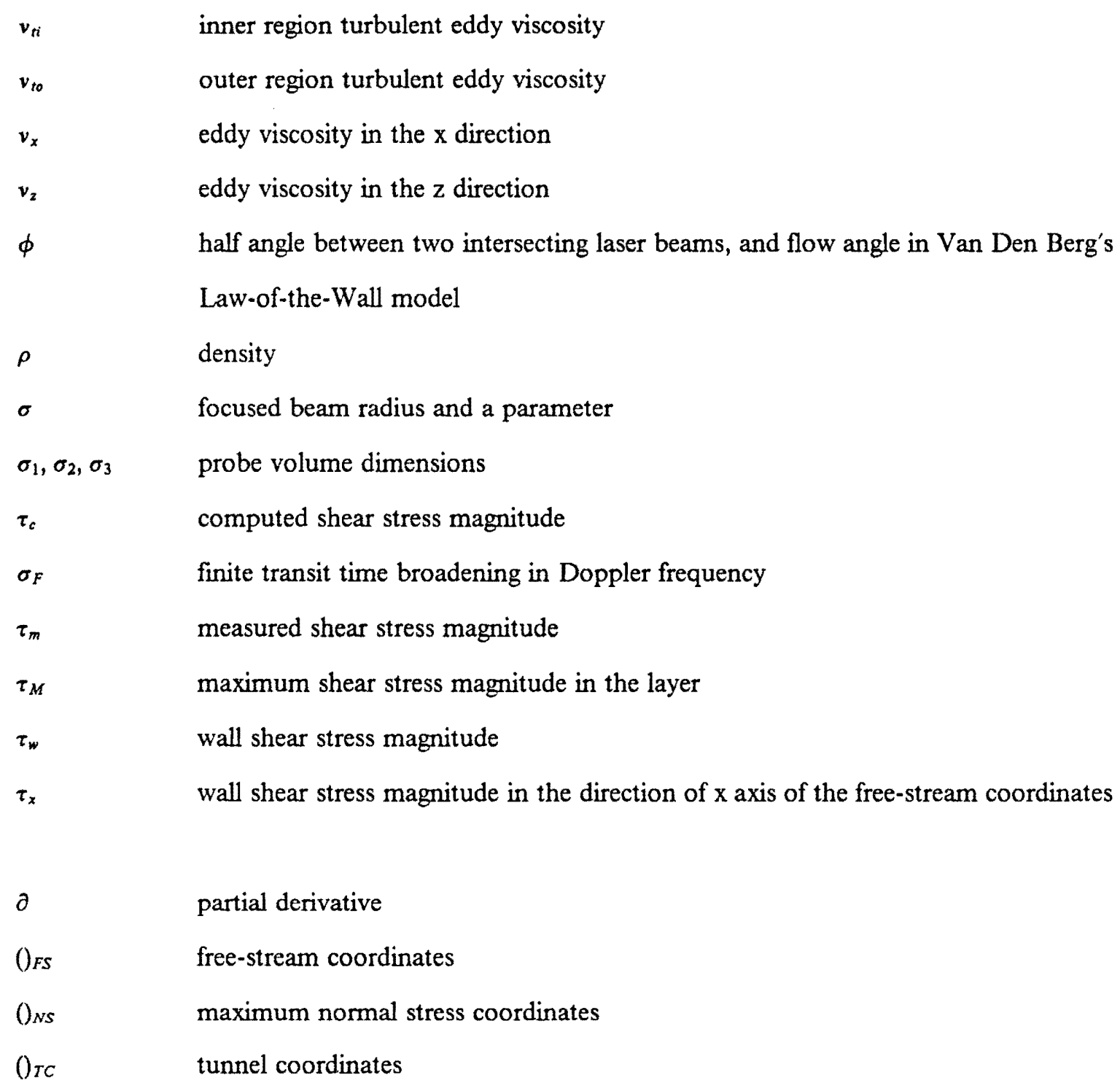




\subsection{INTRODUCTION}

\subsection{NEED FOR EXPERIMENTAL DATA}

The present study was directed towards experimentally investigating the characteristics of an incompressible three-dimensional turbulent boundary layer ( 3-D TBL) generated by a wing-junction flow. The existence of a Law-of-the-Wall velocity profile was extensively studied with the aid of some existing 3-D TBL data. The validity of the turbulence models for 3-D TBLs was tested within the limits of the available data.

As pointed out by Bradshaw (1987), the study of the effect of the three-dimensionality of the flow on the turbulence structure still necessitates further reliable data sets which include all terms of the Reynolds stress tensor. Previous reviews on the available 3-D TBL data were made by several authors, including Johnston (1976), Fernholz and Vagt (1982), Anderson and Eaton (1987), Van Den Berg ( 1987 ).

The necessity and importance of the quantities to be measured and how the data sets would be qualified to be useful was discussed in Van Den Berg ( 1987 ). Van Den Berg pointed out three 
important aspects: the reliability of the data, the necessity of sufficient data to make the calculations possible, and the flow studied having application in real life. The reliability or consistency of data could be verified by examining the Reynolds shear stresses outside the viscous sublayer but very near the wall to see if they extrapolate to the skin friction value measured, by checking if the global flow laws such as the momentum integral balance is satisfied or not, and by using different experimental techniques. The second aspect questions the completeness of the data for computations. Data sets which document the Reynolds number of the flow, the initial conditions including the turbulence quantities along the initial line where the computations start, the pressure distribution on the wall closely enough so that the pressure gradients can be computed and the necessary boundary conditions for the computations, were seen as adequate. Since the objective of the measurements is to check the validity of the empirical assumptions which are used in the computational methods and since these empirical assumptions have only limited validity, the experiments should be designed by keeping this in mind. This third aspects imposes the conditions on the experiments that,

* the inital turbulence properties of the shear layer in experiment should be similar to those flows encountered in engineering practice,

* $R e_{\theta}$ of the measurements should be greater than 5000 ,

* Mach number should be in the correct range,

* the pressure gradient parameter $\lambda=\left(\delta_{1} / q_{e}\right) \times(\partial p / \partial x)$ should be on the order of magnitude encountered in the real life flows, and

* $\delta_{1}\left(\frac{\partial \lambda}{\partial x}\right)$ the flow development rate should be comparable with that of the flows used in practice.

In the rest of the Chapter, first the governing equations of the fluid flow for a Newtonian fluid will be given, then the turbulence closure problem will be addressed and some general rules and methods to develop these models will be briefly discussed. The available turbulence models used 
for the mathematically closure of the of equations, assumptions made for each model and the quantities to be measured for the further development of these models are also going to be pointed out.

\subsection{REVIEW OF THE GOVERNING EQUATIONS}

For a Newtonian viscous fluid with constant density and viscosity, the momentum equation in the divergence form, neglecting the body forces, buoyancy, rotation and streamline curvature effects, can be written as

$$
\frac{1}{\rho}\left[\frac{\partial \rho u_{i}}{\partial t}+\frac{\partial\left(\rho u_{i} u_{l}\right)}{\partial x_{l}}\right]=-\frac{1}{\rho} \frac{\partial p}{\partial u_{i}}+\frac{\mu}{\rho} \frac{\partial^{2} u_{i}}{\partial x_{l}^{2}}
$$

( Bradshaw, 1978) or the ensemble-averaged version is

$$
\frac{\partial U_{l}}{\partial t}+U_{l} \frac{\partial U_{l}}{\partial x_{l}}=-\frac{1}{\rho} \frac{\partial \bar{p}}{\partial x_{i}}+v \frac{\partial^{2} U_{l}}{\partial x_{l}^{2}}-\frac{\partial^{2} \overline{u_{l} u_{l}}}{\partial x_{l}^{2}}
$$

The transport equations for the Reynolds stresses can be written by taking the $x_{i}$ component of the instantaneous Navier-Stokes equation, and multiplying it with the $u_{j}$ instantaneous velocity, and adding the instantaneous Navier-Stokes equation written for $x_{j}$ component multiplied by the $u_{i}$ instantaneous velocity, and then applying ensemble averaging to read as 


$$
\begin{aligned}
& \frac{\partial \overline{u_{i} u_{j}}}{\partial t}+U_{l} \frac{\partial \overline{u_{i} u_{j}}}{\partial x_{l}}=-\left(\overline{u_{i} u_{l}} \frac{\partial U_{j}}{\partial x_{l}}+\overline{u_{j} u_{l}} \frac{\partial U_{i}}{\partial x_{l}}\right) \\
& +\overline{\frac{p^{\prime}}{\rho}\left(\frac{\partial u_{i}}{\partial x_{j}}+\frac{\partial u_{j}}{\partial x_{i}}\right)} \\
& \text { redistribution } \\
& \left.-\frac{\partial}{\partial x_{l}}\left(\overline{u_{i} u_{j} u_{l}}\right)-\frac{1}{\rho}\left(\frac{\partial}{\partial x_{i}}\left(\overline{p^{\prime} u_{j}}\right)+\frac{\partial}{\partial x_{j}} \overline{\left(p^{\prime} u_{i}\right.}\right)\right)+v \frac{\partial^{2} \overline{u_{i} u_{j}}}{\partial x_{l}^{2}} \\
& \text { turbulent diffusion pressure diffusion viscous diffusion } \\
& -2 v \overline{\frac{\partial u_{i}}{\partial x_{l}} \frac{\partial u_{j}}{\partial x_{l}}} \\
& \text { viscous dissipation }
\end{aligned}
$$

( Launder,Reece and Rodi, 1975; Schumann, 1977)

The turbulent kinetic energy $\left(\operatorname{TKE}=u_{i} u_{i} / 2\right)$ equation can be obtained by multiplying the instantaneous Navier-Stokes equation with the instantaneous velocity $u_{i}$ and applying ensemble-averaging to the resultant equation and then subtracting the kinetic energy of the mean flow :

$$
\begin{array}{r}
\underbrace{\frac{\partial}{\partial t}\left(\frac{1}{2} \overline{u_{i} u_{i}}\right)+U_{j} \frac{\partial}{\partial x_{j}}\left(\frac{1}{2} \overline{u_{i} u_{i}}\right)}_{\text {convection }}=-\underbrace{\frac{\partial}{\partial x_{j}}\left(\frac{\overline{u_{j} p^{\prime}}}{\rho}+\frac{1}{2} \overline{u_{i} u_{i} u_{j}}-v u_{i}\left(\frac{\partial u_{i}}{\partial x_{j}}+\frac{\partial u_{j}}{\partial x_{i}}\right)\right)}_{\text {diffusion }} \\
-\frac{1}{2} \overline{u_{i} u_{j}}\left(\frac{\partial U_{i}}{\partial x_{j}}+\frac{\partial U_{j}}{\partial x_{i}}\right) \\
\text { production } \\
-\frac{1}{2} v\left(\frac{\partial u_{i}}{\partial x_{j}}+\frac{\partial u_{j}}{\partial x_{i}}\right)\left(\frac{\partial u_{i}}{\partial x_{j}}+\frac{\partial u_{j}}{\partial x_{i}}\right) \\
\text { viscous dissipation }
\end{array}
$$

( Tennekes and Lumley, 1972 ) 
The dissipation $(\varepsilon)$ equation may be written by differentiating the equation of motion for $u_{i}$ with respect to $x_{l}$ and multiplying through by $2 v \frac{\partial u_{i}}{\partial x_{i}}$. After averaging the equation can be written as

$$
\begin{aligned}
\left.\frac{\partial \varepsilon}{\partial t}+U_{k} \frac{\partial \varepsilon}{\partial x_{k}}=-\frac{\partial^{2} U_{j}}{\partial x_{l} \partial x_{k}} \overline{\left(u_{k} \frac{\partial u_{j}}{\partial x_{l}}\right.}\right)-\frac{\partial U_{j}}{\partial x_{k}}\left(\overline{\frac{\partial u_{j}}{\partial x_{l}} \frac{\partial u_{k}}{\partial x_{l}}}\right) \\
-\frac{\partial U_{k}}{\partial x_{l}}\left(\overline{\frac{\partial u_{j}}{\partial x_{l}} \frac{\partial u_{j}}{\partial x_{k}}}\right)-\frac{\partial u_{j}}{\partial x_{l}} \frac{\partial u_{k}}{\partial x_{l} \frac{\partial u_{j}}{\partial x_{k}}} \\
-\frac{1}{2} \frac{\partial}{\partial x_{k}}\left(\overline{\left.u_{k}\left(\frac{\partial u_{i}}{\partial x_{l}}\right)\right)-\frac{\partial}{\partial x_{j}}\left(\frac{\partial u_{j}}{\partial x_{l}} \frac{\partial p^{\prime}}{\partial x_{l}}\right.}\right) \\
+v \frac{\partial^{2} \varepsilon}{\partial x_{k}^{2}}-v\left(\frac{\partial^{2} u_{j}}{\partial x_{k} \partial x_{l}}\right)
\end{aligned}
$$

where,

$$
\varepsilon=\frac{1}{2} \overline{\left(\frac{\partial u_{i}}{\partial x_{l}}\right)\left(\frac{\partial u_{i}}{\partial x_{l}}\right)}
$$

(Daly and Harlow, 1970)

\subsection{REVIEW OF SOME TURBULENCE MODELS}

The main problem in solving these equations lies in the modeling of turbulence. The exact 3D, unsteady Navier-Stokes equations can not be solved yet due mainly to limited capacity and speed of present computers which are necessary in taking into account the fine scales of the flows. Since the Reynolds-averaged equations have more unknowns than the number of equations, the approach to overcome the difficulty has been to develop new equations for the unknowns in terms of the other unknowns so that the problem can be mathematically closed. 
The methods of analyzing the turbulence for modeling was briefly given by Tennekes and Lumley (1972). These methods include dimensional analysis, asymptotic invariance and local invariance. Dimensional analysis was explained as useful in the cases where it is plausible to assume that there are only a few independent variables and parameters which control the structure of the turbulence. When the Reynolds number approaches infinity, the turbulent flows were described as almost independent of viscosity, and the asymptotic behavior observed was shown as leading to similarity in flows with Reynolds number being equal. The last method needs the assumption that turbulence time scales are small enough to permit adjustment to gradually changing environment. Once this condition is satisfied or assumed, it may be possible to accept that the turbulence is dynamically similar everywhere if nondimensionalized with local length and time scales. Even though this method seems very attractive in 3D flows this condition is rarely satisfied due to history effects.

The general rules of constructing models still seem to be unclear, as pointed out by many authors. Some general rules were suggested by Lumley ( 1983 ). These may be summarized as :

a) The models should have the same tensor structure as terms they replace, to make transformations to other coordinate systems possible.

b) The symmetry in the various indices for $u_{i} u_{j}$ modeling should be ensured and, to satisfy the incompressible continuity equation the moments formed with $u_{i, i}$ should Vanish.

c) The realizability conditions should not be violated. These conditions for the Reynolds stress tensor were studied by Schumann (1977). The conditions

$$
\begin{aligned}
& R_{\alpha \beta} \geq 0 \phi o \rho \alpha \neq \beta \\
& R_{\alpha \beta}^{2} \leq R_{\alpha \alpha} R_{\beta \beta} \quad \text { for } \quad \alpha \neq \beta \\
& \operatorname{det}\left(R_{\alpha \beta}\right) \geq 0
\end{aligned}
$$

in which the summation convention is not used ensure that the kinetic energy of turbulence should be greater than zero and the correlation coefficients should be less or equal to 1 . 
The currently used models for turbulence closure were reviewed by several authors. (Rodi, 1982; Lakshminarayana, 1986; Humphreys and Lindhout, 1988 ). According to Lakshminarayana (1986) they are classified as:

a) Zero equation models, such as the algebraic eddy viscosity and mixing length models,

b) One equation models, such as the $\mathrm{k}$ model

c) Two equation models, such as the $k-\varepsilon$

d) Algebraic Reynolds stress models

e) Reynolds stress models

f) Large eddy simulation

Also, the formulation of the various models and their advantages and shortcomings were described in that paper.

For detailed modeling and development of the models, several books and review papers may be referred to: Launder and Spalding, 1972; Cebeci and Smith, 1974; Bradshaw, Cebeci and Whitelaw, 1981; Rodi, 1980, Launder, Reece and Rodi, 1975; Lakshminarayana, 1986; Humphreys and Lindhout, 1988.

In the present study, the difficulties in terms of the measurements necessary for the modeling are pointed out. The models are listed in order of increasing complexity.

a) A zero equation model relates the turbulent stresses to the mean quantities in the

$$
\begin{aligned}
-\rho \overline{u_{i} u_{j}} & =\rho v_{t}\left(U_{i, j}+U_{j, i}\right) \\
v_{t} & =2 L_{m}^{2} \sqrt{S_{i j} S_{i j}}
\end{aligned}
$$


form where

$$
S_{i j}=\frac{1}{2}\left(U_{i, j}+U_{j, i}\right) \text { and } L_{m}=\text { mixing length }
$$

( Lakshminarayana, 1986)

The idea behind this model is that the turbulent shear stresses could be related to the mean flow quantities in a similar fashion as in laminar or molecular stresses. But, since the eddy viscosity is not a property of the fluid, but of the flow and also changes from point to point in the flow, it was seen as not suitable for the closure of turbulence (Tennekes and Lumley, 1972). The necessary assumption to derive equation ( 6 ) is that the turbulence energy produced at each point in the flow is dissipated with the same rate, or in other words that the flow is in equilibrium neglecting history effects. Another disadvantage of the model is observed when buoyancy, rotation or streamline curvature are taken into account. For these effects, the model can only be devised in an entirely empirical way ( Rodi, 1982 ). Most of the available computational schemes tend to use this model ( Humphreys and Lindhout, 1988 ) due to its programing simplicity and speed. However, the results are inadequate or are only in qualitative agreement with Reynolds shear stress data ( Kline, Cantwell and Lilley, 1982a; Kline, Cantwell and Lilley, 1982b; Marvin, 1983; Wilcox and Rubesin, 1980; Cebeci, 1977; Baldwin and Lomax, 1978; Visbal and Knight, 1984; Kirtley and Lakshminarayana, 1985; Sugavanam, 1985, Horstman and Hung, 1979; Kussoy, Horstman and Viegas, 1980).

The eddy viscosity was further developed by several researchers to take into account the differences between the measured and predicted shear stresses. The anisotropic eddy viscosity of Rotta ( 1977 ), Rhyming and Fannelop ( 1982 ), and Humphreys ( 1986 ) used were seen to be calculating some flows rather well and some rather poorly (Humphreys, 1986; Cousteix, 1982; Van Den Berg, 1988 ). 
b) To remedy the lack of theoretical base that the eddy viscosity model can only be empirically defined or its being only a result of phenomenological reasoning by analogy to laminar stress so that the turbulent quantities are not used in the development, and difficulty in defining the mixing length in some cases, the modeling of turbulent kinetic energy was introduced. Eq. (3) describes the transport equation for the turbulent kinetic energy. Even though the TKE is the energy of the turbulence, and it explains how the energy is distributed among various terms so that it gives more insight to the understanding of turbulence, it still needs to be modeled or related to the shear stresses to close the mathematical formulation. The modeling necessitates quantities like the pressure fluctuations, triple correlations, or derivatives of the fluctuations to be measured and eventually related to shear stresses themselves. Two of the models which stand out are briefly discussed below. Bradshaw (1967) chose to use the formulation in which the dissipation and diffusion are empirical functions of $y / \delta$ and kinematic shear stress $-\overline{u v}$, and the ratio of shear stress to kinetic energy is a constant, where $y$ is the direction of the largest gradient and $\delta$ is the boundary layer thickness. The functions were empirically defined with the use of the two-dimensional data on a flat plate. He further solved this equation together with the momentum and continuity equations and previously defined functions for the development of the shear stress.

Another approach to relate the shear stress to the turbulent kinetic energy was given by Prandtl ( 1945 ) and Kolmogorov ( 1968 )

$$
v_{t}=c_{\mu} \sqrt{k} L
$$

where $\mathrm{L}$ is the dissipation length scale and $c_{\mu}$ is a constant. Dissipation, with dimensional reasoning is usually modeled as

$$
\varepsilon=c_{d}\left(\frac{k^{3 / 2}}{L}\right)
$$


and diffusion is modeled as a gradient of $k$. The representation of $L$ length scale was seen as the most defective part of the model and Rodi ( 1980 ), tried to extend it. The difficulty arises due to defining $\mathrm{L}$ in complex flows.

c) Work towards overcoming the difficulty of defining an appropriate $L$ directed researchers to write an equation for the $L$ length scale itself. But, since the transport of a length did not have any physical meaning, the equation for the dissipation itself was used with the relation $\varepsilon \simeq \frac{k^{3 / 2}}{L}$. The $\varepsilon$ equation was given as eq.(4). At high turbulent Reynolds numbers $\left(R e_{t}=\frac{v_{t}}{v}\right)$, the equation could be further simplified to read as,

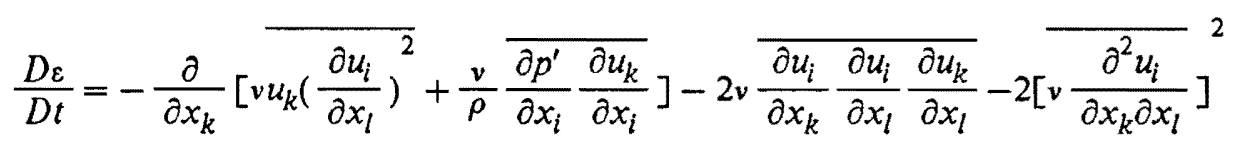

where,

$$
\varepsilon=v \overline{\left(\frac{\partial u_{i}}{\partial x_{k}}\right)^{2}}
$$

( Launder, Reece and Rodi, 1975 )

The difficulty of modeling results from the lack of experimental data for the terms on the right-hand side of the equation. Pressure fluctuations within a flow can not be measured sufficiently well with the available experimental techniques. Simultaneous velocity fluctuation gradients and second derivatives may perhaps be measured if difficult experimental techniques are applied, but they are not yet available. The validation of the model therefore is done comparing the computed $\varepsilon$ with the measured $\varepsilon$ from available data; and from the energy balance. The assumptions applied makes the model equation highly empirical in character. The discussion on the details of the modeling can be found in ( Launder, Reece and Rodi, 1975; Hanjalic and Launder, 1972 ).

Once the $\mathrm{k}$ and $\varepsilon$ are computed, the shear stress in this model can be computed from relations 


$$
-\overline{u_{i} u_{j}}=v_{t}\left(\frac{\partial U_{i}}{\partial x_{j}}+\frac{\partial U_{j}}{\partial x_{i}}\right)-\frac{2}{3} k \delta_{i j}
$$

and

$$
v_{t}=c_{\mu} \frac{k^{2}}{\varepsilon}
$$

A thorough survey of the application of the model for free shear flows can be found in (Launder, Morse, Rodi and Spalding, 1972 ). Problems concerning the near wall character or the $c_{\mu}$ constant were further addressed by Bernard ( 1986 ) and Jones, Launder (1972, 1973). The applications and limitations of the model were thoroughly discussed by Lakshminarayana ( 1986 ). The predictions with this model for 3-D flows was found to be poor due to the pressure strain term, the assumption of isotropy for $\varepsilon$, the eddy viscosity, and the low Reynolds number formulation in the near-wall vicinity. Still, the application of the model for wing-body junction flow gave better predictions than the algebraic eddy viscosity formulation (Gorski, Govindan and Lakshminarayana, 1985 ).

d) The k- $\varepsilon$ model depends on an isotropy assumption and that the turbulence can be represented by one velocity scale $\sqrt{k}$. This seemed to be unsatisfactory for complex flows where the eddy viscosity would depend on the stress ( Rodi, 1982 ). This is observed in 3-D boundary layer flows and is an indication that the $\overline{u_{i} u_{j}}$ stresses would develop separately. This necessitates that the transport equation for the $\overline{u_{i} u_{j}}$ given as eq.(2) to be included to the set of equations to be solved. For this reason, the redistribution, diffusion, viscous dissipation terms need to be expressed in terms of the other unknowns. Due to the terms to be measured this does not seem to be a trivial problem (Bradshaw, 1987 ). The model was called as "Algebraic Reynolds stress " to emphasize that, the equations for the transport of shear stresses after the simplifications are algebraic expressions. As described by Rodi, if the diffusion and convection terms could be related to the kinetic energy of turbulence, then the transport equation' for the stresses would yield algebraic expressions. This 
was done by assuming the proportionality of $\overline{u_{i} u_{j}}$ to $\mathrm{k}$. This model was also called as an extension to the $\mathrm{k}-\varepsilon$, model since it still uses the $\mathrm{k}$ and $\varepsilon$ equations in conjunction with this equation.

e) Further extension of the turbulence modeling of the Reynolds stress equations can be found in Launder, Reece and Rodi ( 1975 ). The superiority of their model comes from using more generalized equations for the pressure strain and diffusion terms. The study of Abid and Schmitt ( 1984 ) on a 3-D boundary layer under infinite swept wing conditions revealed that the flow characteristics could be better predicted with increasing degree of complexity for the turbulence closure. At station 7 of Van Den Berg and Elsenaar ( 1972) data used in that study, at the points where the maximum stresses were observed, while $\overline{w v}$ stress was overpredicted by $67 \%, 37 \%, 13 \%$ with the algebraic eddy viscosity, $\mathrm{k}-\varepsilon$, and the algebraic Reynolds stress models respectively, the models overpredicted the $-\overline{v w}$ kinematic stress as much as $130 \%, 86 \%, 44 \%$.

\subsection{OUTLINE OF THE PRESENT WORK}

In the present study, a three-dimensional boundary layer ( $3 \mathrm{D}$ TBL ) formed due to a cylinder protruding from a plate was studied. The pressure, skin-friction, mean velocity, fluctuating velocity and Reynolds stresses were measured and used in the investigation of the existence of a Law-of-the-Wall velocity profile for three-dimensional ( 3D ) boundary layer flows and in examining the validity of some turbulence models in the prediction of the turbulence structure of these types of flows. As summarized in the previous sections, the development of the turbulence models still necessitates reliable, complete data sets of flows which are encountered in engineering practice. This study was intended to satisfy all the conditions by suggested Van Den Berg ( 1987 ) and discussed in section 1.1 above. The test flow is encountered in many flows of engineering interest, for example in the wing/body junction on aeroplanes and the ship appendage and hull junction on submarines. The reliability of the data was guaranteed by taking the velocity and stress 
measurements with two different techniques, namely the laser-Doppler velocimetry and hot-wire anemometry techniques. The boundary conditions and the characteristics of the approaching boundary layer to the wing were established. Pressure data were taken at closely enough spaced locations to obtain pressure gradients from the data. The flow studied had a $R e_{\theta}$ of $\simeq 5936$ on the plate at 0.75 chord upstream of the body on the centerline of the tunnel. Another important aspect of the present study is that it is the unique data set which documents the flow characteristics on a line which is found by translating in the direction of the mean velocity vector component parallel to the floor at a point in the boundary layer where $\overline{u^{2}}$ is maximum. Since most of the turbulent kinetic energy of the flow is stored in the $\overline{u^{2}}$ normal stress, this study gives a chance to investigate the effect of the turbulent kinetic energy of the flow on the flow characteristics. Compared to the previous measurements of wing/body junction flows, the present work differs mainly due to the use of both LDV and hot-wire techniques for the measurements and for the locations of the measurements being directed to investigate the effect of the turbulent kinetic energy in the flow development. The previous studies of Shabaka (1981, 1979 ) and Mc Mahon, Hubbarrt and Kubendran ( 1982 ) dealt with constant thickness bodies with $6: 1$ and $3: 2$ elliptical noses, respectively. Shabaka measured the double and triple velocity correlations at 3 different streamwise planes and the fluctuating velocity and mean velocities at 8 streamwise planes. He studied the complete balances for the turbulent kinetic energy and momentum equations. Mc Mahon, Hubbarnt and Kubendran used horizontal and slanted hot wires supported on needles which projected into the boundary layer from the flat plate that the body was on. The study included all six Reynolds stresses in 2 streamwise planes. The same shaped symmetric body as used in the present study, 3:2 elliptical nose/ NACA 0020 tail, body was tested by Merati, Mc Mahan and Yoo ( 1988 ), and by Dickinson ( 1986 ). In those studies Merati et al. used the same two hot-wire probes as used by Mc Mahon et al., and measurements of all Reynolds stresses at 5 streamwise planes were made. Dickinson used X configuration hot films and made the measurements of all Reynolds stresses except the $-\overline{v w}$ at 7 streamwise planes. Those studies were directed mostly towards understanding the nature of the wing/body junction flow globally and investigating the size of the horse-shoe vortex formed around the body and the wake flow. In all these earlier studies, 
the prongs which held the hot-wire and hot-film sensors produced interference with the flow. The main goal was to investigate flow features along the selected $\mathrm{x}-\mathrm{z}$ path mentioned above, not a global characterization as in earlier studies. Such a path resembles the one studied by Dechow and Felsch ( $1977 \mathrm{a}$ ) in front of their cylinder/flat plate junction flow, although their path was along a free-stream streamline.

The experimental techniques and, the measurement results and associated uncertainties may be found in Chapters 2 and 3, respectively. The discussion on the quantities which question the existing assumptions on the structure of 3D boundary layers is the subject of chapter 4 . Research on the Law of the Wall for 3D boundary layers which includes comparison of the nine existing 3D Laws of the Wall with nine data sets is discussed in Chapter 5. Comparison of the data of shear stress magnitudes and directions with the predictions of the eddy-viscosity models reveals the shortcomings of the models in 3D flows. The comparison of four different models with 8 existing data sets is presented in Chapter 6. Chapter 7 is contains to the conclusions of this study. The figures 9 to 58 present the data taken in this study. The flow characteristics of the present flow were investigated with the help of figures 59 to 90 . The figures 91 to 111 cover the analysis of the Law-of-the-Wall research. Examination of the selected Algebraic eddy-viscosity models was carried out with the aid of figures 112 to 124 . 


\subsection{EXPERIMENTAL TECHNIQUES}

\subsection{INTRODUCTION}

The measurements in a 3-D turbulent boundary layer created by a wing-body junction flow performed in this study include single hot-wire measurements at 39 locations and Laser-Doppler Velocimeter measurements at 8 locations with two different data processing units. The wing used was a $3: 2$ elliptical nose, NACA 0020 tail symmetric profile which had maximum thickness of 7.17 $\mathrm{cm}$ and chord length of $30 \mathrm{~cm}$ and heigth of $22.9 \mathrm{~cm}$. The measurements were carried out at a nominal speed of $27 \mathrm{~m} / \mathrm{sec}$ and Reynolds number of the flow based on the momentum thickness at 0.75 chord upstream of the body on the centerline of the tunnel was $\simeq 5936$. The main objective of these measurements was to observe how the flow characteristics changed in a 3-D pressure-driven boundary layer type flow along a line whose $\mathrm{x}-\mathrm{z}$ direction is given by the $\mathrm{U}, \mathrm{W}$ velocities where $\overline{u^{2}}$ normal stress is maximum. The measurement locations can be seen in ( Fig 1 ).

Hot-wire measurements done were aimed to find this line and to compare with the LDV measurements, besides revealing some other flow quantities. They were done at 18 locations on the left side of the model, looking upstream ( Table 1 ). The location of the first profile was chosen 
with the information gathered from the oil-flow visualization pictures ( Fig 2 ). The pictures will be discussed in Chapter III of the present study. The equations used for the hot wire data reduction are derived (Appendix I ) assuming that the velocity component perpendicular to the wall, therefore, $\mathrm{V}$ mean velocity component and $\mathrm{v}^{\prime}$ fluctuating velocity component are negligible. Therefore, the point was chosen such that the governing equations of the hot-wire reduction programs would not be violated throughout the determination process of the expected line but yet the effect of the pressure on making the flow 3-D would be felt as much as possible. The most downstream point was the first point. The next 17 points were found by finding the direction of the maximum normal stress point obtained in each profile and translating in the direction of the mean velocity component parallel to the wall at that location for 0.5 inches. At some locations near the leading edge or ahead of the body where the effect of three dimensionality started diminishing, the distance translated was different than 0.5 inches. The measurement locations were also away from the region where the bimodal characteristic of the velocity field is seen (Devenport and Simpson, 1987a ). Another 12 profiles of hot-wire profiles 0.75 chord upstream of the body on a crosswise line were taken to define the inlet boundary conditions of the flow (Table 2 ).

Skin friction at the wall was obtained by Alinger ( 1990 ) using a laser interferometer technique at the same conditions. The use of the technique supplies the wall friction independently which is crucially important in the Law-of-the-Wall research in 3-D flows.

Time averaged surface pressures were obtained by a Scanivalve and a pressure transducer couple, and flow visualization on the tunnel floor was done by using a mixture of $\mathrm{TiO}_{2}$, oleic acid, and kerosene.

Purpose of the 3-D LDV measurements were to find the flow characteristics. The experimental data gathered includes the mean velocities and all six components of the Reynolds stress tensor. The LDV measurements were done on the right-hand side of the body at the symmetric locations of the previous hot-wire points but only at 8 points ( Table 3 ). To check the symmetry of the flow, before LDV measurements, 7 more hot-wire profiles were taken ( Table 4 ). The gathered data 
from pressure measurements, surface oil-flow visualization patterns and from these hot-wire measurements shows that the flow around the body is symmetric.

\subsection{OIL-FLOW TECHNIQUE}

The oil-flow technique was used to reveal the limiting streamline or surface skin friction line structure on the surface. Self-adhesive black glossy plastic sheet was laid on the surface (Frisk Coverseal from Cambridge University, England ). A mixture of kerosine, $\mathrm{TiO}_{2}$ and oleic acid was applied on this surface and several runs with different percentages of components were tested to obtain the most clear picture. It was observed that a lesser amount of $\mathrm{TiO}_{2}$ resulted in lower contrast but finer structure could be seen more clearly. The mixture used in this study is $20 \mathrm{ml}$ of $\mathrm{TiO}_{2}$, some amount of kerosene so that the total adds upto $55 \mathrm{ml}$ and $9 \mathrm{ml}$ of oleic acid. This was different than the original ratio of $5 \mathrm{ml} \mathrm{TiO}, 15 \mathrm{ml}$ of kerosine, $1 \mathrm{ml}$ of oleic acid proposed by Sutton ( 1985 ). Once the mixture was applied, the tunnel was run until there was not any visible movement of the mixture, then the plastic sheet was taken out of the tunnel and further dried with a hair dryer. To be able to make photocopies directly from the original sheet, painter's fixer was used to fix and preserve the picture obtained.

\subsection{SKIN-FRICTION INTERFEROMETER}

The wall skin-friction magnitudes and directions used in this study were measured by $\mathrm{K}$. Ailinger ( 1990 ) using the skin-friction interferometry technique described below. 
The technique relates the thinning rate of an oil film applied on the surface to the skin friction at that point by using the lubrication theory for thin films. The thinning rate of oil was found with the use of an interferometer using a $5 \mathrm{~mW}$ Helium-Neon laser and photodiodes (Monson, Higuchi, $1981)$.

The laser beam directed with an angle close to the normal angle to the surface of the oil applied to a body surface reflects back from that body surface and also from the surface of the oil. Once the two reflected waves interfere with each other either constructively when they are in phase or destructively when they are out of phase to form interference fringes, which is directly related to the thickness of the oil, the variation of the intensity in time can be used to measure the thinning of the oil in time. But, since the thinning of the oil film is a linear function of the shear stress acting on it, this information could be used to find this stress. As discussed by Monson, the thinning rate and thus the shear stress may be found easier if two beams with a known spacing in between them are used to measure the thinning rates at two points on the oil surface.

Dow-Corning ( 50 centi-Stokes ) oil was laid on the floor with a razor blade and the useful data were taken after it thinned down to $20-50$ microns. The beams were directed with a spacing of 4.5 $\mathrm{mm}$ between them and they were at 1.5 to 2 beam spacing behind the film leading edge. The direction of the stresses were found by measuring the stresses in 3 different selected directions, by rotating the two incident beams so that they would focus on the defined directions on the oil. Each of these measurements were repeated 3 times, and the direction and magnitude were found by averaging these values. More detail on the sources of difficulties and solutions to them can be found in Ailinger ( 1990 ). 


\subsection{PRESSURE MEASUREMENTS}

The pressure measurements were made with a $\mathrm{J}$ type Scanivalve and a conventional inclined manometer. The locations of the measurements both on the body and the surface are shown in ( Fig 3 ). The pressure taps used were $1 \mathrm{~mm}$ in diameter.

The pressure coefficient was calculated using

$$
C_{p}=\frac{\left(p-p_{r e f}\right)}{\left(p_{0}-p_{r e f}\right)}
$$

The reference dynamic head and the reference static pressure were obtained from the pitot static tube ( United Sensor Probe, type PAC-8-KL ) located at $1.55 \mathrm{~m}$ downstream of the tunnel entrance.

\subsection{HOT-WIRE TECHNIQUE}

The single hot-wire technique used in this study was described also in Devenport and Simpson, ( 1986 ). The technique is designed to measure $\mathrm{U}$ and $\mathrm{W}$ components of the mean velocity and $\overline{u^{2}}, \overline{w^{2}}, \overline{u w}$ components of the Reynolds' stress tensor with the assumption that $\mathrm{V}$ and $\mathrm{v}^{\prime}$ are small compared to the velocity magnitude in the $\mathrm{x}, \mathrm{z}$, i.e. in (U, W) plane ( Fig 4 ). For the flow studied, this assumption restricts the use of the technique away from the wing-body junction, since either flow ahead of the body separates and/or flows backwards and since the flow wraps around the wing close to the body to form a horse-shoe vortex producing non-negligible $\mathrm{V}$ and $\mathrm{v}^{\prime}$ mean and fluctuation velocity component. The reduction equations used are presented in Appendix I. The 
probe used was a boundary layer type probe ( TSI 1218T1.5). The tungsten wire of the probe was $4 \mu \mathrm{m}$ in diameter and $1.5 \mathrm{~mm}$ in length.

The constant temperature anemometer bridge used was designed by Miller ( 1976) and modified by Simpson et al. ( 1979 ). With an overheat ratio of 0.7 , the frequency response of the hot-wire was flat up to $12 \mathrm{kHz}$. The linearized output of the anemometer was fed into a universal waveform analyzer (DATA 6000, Data Precision Corporation, Analogic Corporation).

The summing amplifier and Hewlett-Packard power supply were used to adjust the hot-wire anemometer's linearized output voltage which changed 0 to $10 \mathrm{~V}$ to vary from $-5 \mathrm{~V}$ to $5 \mathrm{~V}$ so that the full range of the DATA 6000 could be used. The power supply's drift after a day of experiments was always less than $1 \mathrm{mV}$.

The DATA 6000 was used to gather the 10000 velocity samples in 20 seconds and form a histogram from it, dividing the range into 256 bins. These 256 values were transferred to the computer by the A/D converter ( Data Translation Inc. ) to further compute the mean velocity and r.m.s. value. Even though 10000 values could directly be transferred into the computer which would eliminate the histogram formation step, this would increase the time of processing at least 10 times. The computer used was an IBM-PC. The necessary cable connections to the probe which sits at the end of a dogleg were done through the roof of the test section ( Fig 4 ).

Calibration of the wire was done using the previously described instrumentation and TSI calibrator ( model 1125 ) but by taking only 1000 samples of each velocity setting. Calibrations were carried out daily, just before the experiments and calibration constants were found by a least-square line fitting to the measured output voltages of the linearizer of the anemometer. To ensure that the calibrations were performed at the running temperature of the experiments the tunnel was first allowed to run for 15 minutes, and the calibrations were done just after the drift in the room settled down. The temperature in the laboratory could be kept constant within $\pm 2^{\circ}$ around the set temperature with an air conditioner. 
Due to physical obstructions to reach some of the parts of the test section another probe holder was designed. The orientation of the probe was found by a voltage divider to obtain resolution of $0.5^{\circ}$ or less. The voltage divider was sitting on the probe holder ( Fig 5 ). First a look up table for the voltages corresponding to the direction of the probe was prepared by setting the probe at known directions and then reading the output voltages of the voltage divider. Later this table was used to find the direction of the probe by finding the angle corresponding to the voltage read. The probe could be oriented from outside of the tunnel with a tacheometer wire, which is used in the cars in measuring the speed of the vehicle, worm gear and anti-backlash gear system. A pin connected to an anti-backlash gear traveling on a $10 \mathrm{~K}$ circular resistance was used as the arm for the voltage divider. The cable connections for the voltage divider were also attached to the holder and they were extending to the outside of the tunnel for the voltage readings. The wire sitting in a dog-leg holder was attached to the shaft of the anti-backlash gear.

\subsection{LASER-DOPPLER VELOCIMETER (LDV)}

\section{TECHNIQUE}

The LDV uses a Coherent Innova 90 argon-ion laser operated at $514.5 \mathrm{~nm}$ with a power output of $1.5 \mathrm{~W}$. The smoke necessary for the light scattering particles was generated by an aeresol generator originally designed by Echols and Young ( 1963 ). The fluid used in the generator to produce the smoke was dioctal phatalate. The smoke was injected into the flow either through the air intake of the tunnel or at the entrance of the tunnel test section.

Three different optical measurement systems each composed of 3 beams were used to measure the 3 components of the mean velocity vector and 6 Reynolds stresses. The optical systems were named as UV, UW and VW systems to distinguish what are measured by each system. The UV system 
was designed to measure nominally the $\mathrm{U}$ and $\mathrm{V}$ components of the mean velocity vector and $\overline{u^{2}}, \overline{v^{2}}, \overline{u v}$ components of the Reynolds stress tensor. The UW system nominally measures the $\mathrm{U}$ and $\mathrm{W}$ components of the mean velocity vector and $\overline{u^{2}}, \overline{w^{2}}, \overline{w w}$ components of the Reynolds stress tensor. With the same thinking, VW system nominally measures the $\mathrm{V}$ and $\mathrm{W}$ components of the mean velocity vector and $\overline{v^{2}}, \overline{w^{2}}, \overline{v w}$ components of the Reynolds stress tensor. The description of the measurement systems and the derivation of the reduction equations used, can be found in Appendix 1. The each couple of the three beams in each optical system would define a probe volume and the velocity component perpendicular to the bisector of these two beams could be measured. First the measurements with the UV system then with the UW system and then with the VW system were taken. By doing so, the mean velocity components and the normal stress components were measured two times. Even though this was necessary to do so for the shear stress measurements, it also increased the confidence to the data.

The signal processing with fast-sweep-rate sampling spectrum analysis used in this study was described by Simpson and Barr (1974, 1975) and Simpson and Chew (1979). The technique uses a swept spectrum analyzer and peak detection and sample and hold electronics to obtain the Doppler frequency and A/D converter and computer to store and process the gathered data. The swept spectrum analyzer sweeps a certain range of frequencies chosen with a chosen band-width at a rate of 1000 times per second. The location of the band-width within the range can be known by the calibration of the spectrum analyzer with a known frequency. Within a given range, the relation between the horizontal voltage output of the spectrum analyzer and the frequency is linear. This calibration factor changes from range to range, since the sweep rate is constant. When the bandwidth and the frequency of the input current from the photomultiplier tube to the swept spectrum analyzer coincide, a peak is generated at the vertical voltage of the analyzer. This voltage is then used to gate the peak detector circuit and the pulse generated by this circuit is fed into a sample and hold device for the horizontal voltage. The sampled voltage is held until another peak is detected. Once the sampled voltage value is converted into frequency by the calibration factor to obtain the Doppler frequency, the velocity can be computed by multiplying it with the fringe 
spacing of the beams. In this study, two spectrum analyzers, HP8557A and HP8558B, were used simultaneously. The ranges of the analyzers were spanning between $0.01-350 \mathrm{MHz}$ and $0.0-1500$ $\mathrm{MHz}$, respectively.

Another frequency analysis signal processing technique used in this study was Burst-spectrum analysis. The technique uses a Burst Spectrum Analyzer (BSA type $57 \mathrm{~N} 10$ ) developed by DANTEC and a host computer for the processing of the data. The BSA extracts the Doppler frequency by a hardwired Fast Fourier Transform developed by DANTEC. In order to do this the BSA takes 8, 16, 32 or 64 samples with a chosen band-width and performs a Discrete Fourier Transform (DFT) on them to find the frequency spectrum and hence the power spectrum. If the ratio between the two largest maxima of the power spectrum is greater than 4 , the burst is validated. The BSA can be used to sample frequencies up to $80 \mathrm{MHz}$ with high resolution due to the zooming technique used, and it was designed to extract frequency information from only one sampled data in time. 


\subsection{EXPERIMENTAL RESULTS}

\subsection{INTRODUCTION}

In this chapter, the data obtained with several experimental techniques described before will be discussed. The coordinate systems used in the study, the pressure distribution resulting due to the existence of the wing, the limiting streamline structure on the wall are going to be given. The boundary conditions for the studied flow will be defined and it will be shown with the oil flow technique, pressure, hot-wire measurement results that, the flow structure around the wing is symmetric. Mean velocity, fluctuating velocity and shear stress data obtained, and the uncertainties of these quantities will be studied.

The locations of the mean velocity and Reynolds stress measurements can be seen in Fig 1 . The hot-wire profile locations on the left-hand side of the wing looking upstream are tabulated in Table 1. Table 2 gives the 0.75 chord upstream measurement locations. The laser-Doppler-velocimeter ( LDV) and hot wire ( HW ) measurement locations on the right-hand side of the wing are given in Tables 3 and 4 respectively. 


\subsection{COORDINATE AXES}

The axes used in this study are defined in Fig 6. TC stands for tunnel coordinates. In this coordinate system, the $\mathrm{x}$ axis is parallel to the tunnel centerline pointing downstream as positive, and, the $y$ axis is perpendicular to the tunnel floor. In all axes, the $y$ axis was identically defined. The $\mathrm{z}$ axis in TC was defined to complete a right handed coordinate system. FS stands for free-stream coordinate system. Positive $\mathrm{x}$ axis in this coordinate system is in the direction of the mean velocity at the boundary layer edge, parallel to the tunnel floor. The $\mathrm{z}$ axis is formed to define a right handed coordinate system. NS denotes maximum $\overline{u^{2}}$ normal stress direction coordinate system. It is defined such that the positive $\mathrm{X}$ axis is in the direction of the local mean velocity vector component which is parallel to the tunnel floor at the profile point where the normal stress obtained is maximum.

\subsection{TUNNEL QUALIFICATIONS}

The measurements were carried out in the Virginia Tech. low speed boundary layer tunnel, which has been used in much previous work at Virginia Tech and at Southern Methodist University ( Fig 7 ). The air enters through the air filter and the blower increases the pressure, passes through a honeycomb and 7 screens which are used to remove the swirl of the mean flow and the turbulence in the flow. The flow accelerates through a 2-D four to one contraction nozzle before going into the tunnel test section. The measurements were carried out at a nominal speed of $27 \mathrm{~m} / \mathrm{sec}$. Since the open-circuit tunnel was especially designed to study two dimensional boundary layer separation, the shape of it was configured to create an adverse pressure gradient to induce separation. In the present study, the tunnel roof was repiced with a Plexiglass roof which was reinforced with 
aluminum so that, when the model was removed a two dimensional boundary layer with zero pressure gradient could be obtained. The test section is $0.91 \mathrm{~m}$ wide and $8 \mathrm{~m}$ long.

The detailed study of the tunnel characteristics can be found in Ahn ( 1986 ). That work was directed to study the characteristics of two dimensional boundary layers subject to zero and favorable pressure gradients with steady free stream and the development of the boundary layers in

unsteady flows in various Reynolds numbers. His data of power spectra, mean flow, and $u^{\prime}, v^{\prime}, \overline{u v}$ data showed good agreement with the measurements of previous researchers. The free-stream velocity along the axis of the tunnel is shown in ( Fig 8 ). At $27 \mathrm{~m} / \mathrm{sec}$ the turbulence intensity observed in the tunnel was $0.2 \%$ and the potential core was uniform within $0.5 \%$ in the spanwise and $1 \%$ in the vertical directions, respectively (Devenport and Simpson, 1987 ).

\subsection{BOUNDARY CONDITIONS}

The inlet flow, is tripped by the $0.63 \mathrm{~cm}$ blunt leading edge of the tunnel floor to ensure that the boundary layer forming is turbulent.

The two dimensionality of flow when the model is not present was mentioned before. The flow 0.8 chord length ahead of the body, as seen from the hot wire measurements made at the most upstream, eighteenth hot wire station on the left-hand side, was again 2-D since the W component of the mean velocity component and $\overline{u w}$ shear stress component were fluctuating around 0.0 . Therefore, the flow may be computed by assuming the flow as a two dimensional turbulent boundary layer on a flat plate approaching a cylindrical protuberance. The hot-wire measurement results at 0.75 chord upstream of the wing are plotted in Fig 9. Locations of these profiles, boundary layer thicknesses, displacement and momentum thicknesses calculated with trapezoidal rule, skin 
friction coefficients computed using the 2-D law of the wall and 6 point line fit to the logarithmic regions of the $\mathrm{U} / U_{\text {ref }}$ profiles are tabulated in Table 4.

The restriction of the flow in the spanwise direction due to the tunnel side walls (as will be clear with the computed and measured pressure distributions and oil flow pictures) was negligible.

A gap of $37 \mathrm{~mm}$ between the model and the tunnel roof was left to avoid the interaction of the roof boundary layer and the model, so as not to generate a second horse-shoe vortex, which could interact with the studied flow.

\subsection{OIL-FLOW VISUALIZATION RESULTS}

The oil flow visualization pictures of the flow studied were taken at $27 \mathrm{~m} / \mathrm{sec}$ nominal reference velocity.

The limiting streamline structure of the flow field developed ahead and around the body are shown in Fig 2 and Fig 10. The effect of the lateral and streamwise pressure gradients on skewing the flow field developed, is clearly seen from these figures. The separation line which forms due to the adverse pressure gradient, the line of low shear where the mixture accumulates more due to the lower skin friction, the fish tail shaped wake flow limiting streamline characteristics and the separation of the flow on the wing very close to the floor are also clearly observable. Same type of characteristics was also observed by Dickinson ( 1986 ) in his study of a wing-body junction flow using a body same in shape with the body used in the present study. Some of the characteristic lengths measured from these figures are presented in Fig 10.

It can be postulated from these figures that the spanwise vorticity in the 2-D turbulent boundary layer transforms into streamwise vorticity which forms the horseshoe-vortex. Between the 
separation line and the body, existence of a low shear line suggests the existence of a counter rotating flow with respect to the horseshoe vortex, which is in the $+z$ direction. The reversal or back flow close to the leading edge of the body is also observed. The primary separation line which was observed to be at 0.47 body thickness ( $t$ ) away from the leading edge continues to be at the same perpendicular distance away from the body until it joins with the low shear line at $1.48 \mathrm{t}$ downstream of the leading edge. Following the body contour, the perpendicular distance between the body and the low shear line which was observed as $0.28 \mathrm{t}$ away at the leading edge, continues to grow until the trailing edge is reached. At the trailing edge, the distance between the trailing edge and the low shear line is observed as $1.99 \mathrm{t}$ in the $+\mathrm{z}$ direction.

The figures presented also indicate that the flow is symmetric. This could be done by comparing the major distances at several $\mathrm{x} / \mathrm{t}$ stations. The low shear line locations away from the body measured on both sides of the model show good agreement and are shown in Fig 10.

\subsection{PRESSURE MEASUREMENTS}

The perspective view of the measured $C_{p}$ values are plotted in Fig 11. Positive $C p$ values correspond to slower flow regions while the negative $\mathrm{Cp}$ values show faster flow regions than the reference flow region. The measured pressure distributions on both sides of the reference plane are plotted in ( Fig 12 ). The symbols indicate the negative $z$ values; lines indicate the $+z$ axes side values. The pressure cofficients on both sides of the model show good agreement at every $x / t$ station. Along the centerline the flow decelerates towards a stagnation point on the leading edge $(\mathrm{Cp}=+1)$.

The lateral pressure gradients seen ahead of the wing at $x / t=-2$ are close to zero. The acceleration of the flow around the nose part of the wing results in the sharp drop in the $\mathrm{Cp}$ and high positive 
lateral pressure gradients seen at $x / t=0.25,0.5$ and 0.75 . In the tail part of the airfoil until $x / t=3.25$, the positive pressure gradients observed are milder. After this point the gradient changes sign.

The streamwise pressure gradients at $z / t=0.5$ are observed to be positive till $x / t=-0.5$, after this station, the gradients not only changed sign but were higher in magnitude till $x / t=0.756$. The lesser positive gradients observed start after this station. The magnitude of the gradients away from the body are lower than the near body values.

Overall, the $\mathrm{Cp}$ values on the flat plate where the wing is sitting on, show a gradual increase with a peak at the leading edge when the flow is slowed down approaching to the wing At the sides, when the flow is going around the wing, encountered higher velocities than the free stream result in Cps below zero, and after $\mathrm{x} / \mathrm{t}=3.25, \mathrm{Cp}$ values again start to increase at both sides of the wing when the flow slows down.

The measured pressure values on both sides of the model, on the model, and 9 inches $(z / t=3.187)$ away from the model at several $x / t$ locations also show the symmetry of the flow. The measurements at these locations were compared with results from a potential-flow code, which was selected to have 100 source and sink panels for each side of the model (Fig 13 ) and (Fig 14 ). The source density distribution code was written using the formulation presented in Bertin and Smith ( 1979 ).

The potential-flow results and the data are observed to have differences. While the maximum difference between the computed and measured $C p$ values at $z / t=3.187$ is 0.04 , the measured quantities on both sides of the body show a maximum difference of 0.01 . At this station, while the $\max \mathrm{Cp}$ read is 0.015 , the minimum is -0.09 . While the computed and measured values on the body had a difference of 0.085 , the measured pressures show perfect agreement. The contour plot of the measured and computed values of $\mathrm{Cp}$ also displays the difference ( Fig 15). The differences on and near the body may be attributed to the horse-shoe vortex and to the separation of the flow on the model before the trailing edge. In a boundary layer code, if the pressure distibution around the 
body is prescribed from a solution of a potential-flow code, as pointed out by Müller ( 1982a ), may effect the results of the computations. The best remedy to the problem that the difference might cause in the boundary layer computations may be the use of the measured presure instead of the computed pressure.

\subsection{HOT-WIRE MEASUREMENTS}

Hot-wire measurements were made on both sides of the wing. Right-hand side hot-wire measurements were done at the same locations of the laser measurements, except at the most upstream laser location where no hot-wire measurement was made, since the flow here was almost 2-D and the rest of the data were seem to be satisfactory for the purposes intended. The mean velocities and turbulence quantities measured with the hot-wire technique on both sides of the wing show good agreement throughout the layers (figures 21-25, 35-38, 47-50). In the figures, the lines show the results of the measurements on the right-hand side of the wing and the symbols denote the left-hand side hot-wire ( HW) profiles.

First comparison of the data showed that the data taken on both sides of the wing had minor differences near the wall. To see if the \pm 0.003 inches uncertainty in the probe location adjustment at the first points of the profiles could be the reason for these differences, the hot-wire profiles on the right-hand side of the wing were shifted upwards at the most by 0.005 inches $(0.127 \mathrm{~mm})$. Further comparision resulted in better agreement except at one location which needed a shift of 0.01 inches. This location which corresponds to the fifth LDV location was therefore discarded. The comparison of these six profiles with the corresponding location left-hand side profiles show that the mean and turbulence quantities measured agree well, which in turn show that the flow is symmetric. 


\subsection{VELOCITY AND STRESS MEASUREMENTS}

\subsubsection{INTRODUCTION}

After the measurements both with the hot-wire system and fast-sweep-rate sampling spectrum analysis LDV techniques were completed, it was observed that some turbulence quantities reduced from these two measurement techniques did not match in the inner layer and in the logarithmic region of the profiles. These discrepancies were originally attributed to the gradient broadening in the inner region. A simple formulation for the gradient broadening corrections suggested by George and Lumley ( 1973 ) was applied. Yet, the results were seen to be unsatisfactory, since either the normal stresses at a few points near the wall were less than zero, the probe volume dimensions were too big to be acceptable, or the corrections were too small.

The next choice to explain these discrepancies was to examine the measurement electronics and equipment. With this in mind, the photomultiplier tube and the acquisition cables were replaced; the spectrum analysers were checked to see if they satisfied the manufacturer's standards; the smoke level was changed; and even the collecting lens itself and its location were changed. The results obtained showed that there was not any error due to these possibilities. Yet, since the source of the discrepancy could not be located and since the experimenter was not satisfied with the existing data, the whole acquisition system was replaced.

Instead of using swept spectrum analyzers, samplers and A/D converter, the BSA was used since the software and hardware were readily available. The whole LDV measurements were repeated as if there were no LDV data taken before.

Since the data acquisition system and the program were changed, for the last step of reducing the data, the reduction program was also changed. This was done by developing an objective procedure 
to distinguish the data from the noise in the histograms instead of judging each histogram one by one as done previously. This empirical procedure basically fits a parabola to each side of the peak of the logarithm of the histogram values and finds the noise level with an iterative procedure ( Fig 16). In the parabola fit, first, all the data including the noise are used and the parabolas found are checked if they are crossing the $\log (1)=0$ line. If the parabolas were crossing this line, the iteration was stopped, otherwise the amount of the data used was reduced by discarding the data below some percentage of the peak value. The percentage discarded was increased in each iteration until the $\log (1)=0$ line was crossed by both parabolas. Once these crossing points were defined, the maximum value within the 10 bins from each point within the crossing points was seen to be the best way of assessing the noise level. The data taken underneath this noise level and the regions outside of these two maximum points found was discarded. The reason to fit a parabola to each side of the logarithm of the histogram values was that if the distribution were Gaussian, the logarithm of it would be a parabola. Even though the distributions were not Gaussian, they were close to it, and that was also the reason why two separate parabolas were fit to each side of the peak of the histograms.

After the measurements with the BSA were completed it was seen that the stresses measured with these 3 different techniques were different from each other. The discrepancy had increased. Yet,this was seen to be due to judging on the noise level of the each histogram one by one for the data taken with the swept spectrum analyzer technique. Once all the data taken with the swept spectrum analyzer technique was re-reduced by using the new reduction program, it was observed that the swept spectrum analyzer and BSA measurements' results were very close to each other, yet now they were both different from the hot-wire technique results.

The next step in resolving the discrepancy was to recheck the hot-wire technique. Profiles taken on both sides of the wing show that the system measured the same quantities at symmetric locations within a reasonable range. Additional measurements were made using the same type of hot-wire probe and same anemometer but using the non-linearized output of the anemometer as input to the computer through an $A / D$ converter and using another reduction program for the calibration 
of the wire and sampling of the data to measure one of the hot-wire station's profiles again. The measurements were taken by Fleming ( 1990 ). Since these new profiles overlapped for the mean and turbulent quantities with the previously taken data, the reason of the dicrepancy could not be attributed to the hot-wire technique ( Fig 17 ).

The final situation was that both the hot wire and LDV technique measurement results were consistent in themselves, yet they were contradicting each other. The gradient broadening and finite transit time broadening which are applied to LDV data were no longer for overcoming the discrepancy but to subtract the effects of these from the LDV data. The gradient broadening correction equations were rederived to take into account all the existing gradients that may contribute to the broadening, instead of taking only one gradient along the major axis of the probe volume as suggested by George and Lumley ( Fig 18 ). These equations may be found in Appendix II. Even though the probe volume dimensions given in Fig 18 suggests that the focused beam diameter can be computed if the focal length of the focusing lens and the unfocused beam diameter is known, the measurement of the beam diameter with the definition of it as the points where the intensity of the beam drops down to the $1 / e^{2}$ of the $I_{\text {center }}$ raises difficulty in measuring it in the reality. The way the focused beam diameter is found will be discussed in the next section.

The difference between the hot wire and LDV results, especially for the $\overline{u^{2}}$ normal stress, can be attributed to the hot wire interference with the wall. This should be adressed in a later study.

\subsubsection{UNCERTAINTY ANALYSIS}

The uncertainties in the measured quantities with the LDV technique were expected to be due to the uncertainties in the angles between the intersecting beams, focused beam radii used, and how the histogram edges (noise levels) were defined. 
The uncertainty in the angles were estimated to be less than $\pm 2 \%$ and cutting the histograms from different points of its skirts showed that the uncertainty in the mean quantity computed from the histogram could vary as much as $\pm 0.5 \%$ and the turbulence quantity not more than $\pm 4 \%$.

The definition and assessment of the uncertainty in the focused beam radii was reached in the following way. As a criteria to be used first, the result of the analysis of Rotta ( 1962 ) in two-dimensional boundary layers about how the normal stresses very near the wall would vary was chosen. The analysis shows that very near the wall while $\overline{u^{2}}$ and $\overline{w^{2}}$ vary as a cubic function of the $\mathrm{y}$ distance, the $\overline{v^{2}}$ vary with the fourth power of the distance. A least-squares, $c_{1} y^{2}+c_{2} y^{3}+c_{3}$ type of function as suggested by Rotta was fitted to the 4 points of the $\overline{u^{2}}$ and $\overline{w^{2}}$ normal stresses starting from the 2 nd point of each profile. Further changing the beam radius standard deviation (the effective beam radius) $\sigma$ for UV and UW system beam couples to recalculate the $\overline{u^{2}}$ and $\overline{w^{2}}$ stresses and reapplying the function fit until the minimum total error between the measured and computed values was found revealed the effective beam radius that could be used. This approach could not be accepted as totally satisfactory, since the criteria defined by Rotta (1962) is effective only for y values of $y^{+}=y u_{4} / v \leq 7$, since the first 4 points to which the criteria was applied were located between $y^{+}=8$ and $y^{+}=23$ and, since the corrected data did not follow the 2-D flow characteristics for the close Reynolds number based on momentum thickness data of Klebanoff ( 1955 ), ( Fig $19)$.

Another approach that was tested was based on subjective judgment on the measured quantities so that using the same beam diameter for the two different LDV techniques would result in the fairly close values used and the normal stresses at the first point of the profiles would be close to zero but non-negative. Since the resultant diameters found with this approach were not highly different from each other, this approach was used, but since the reasoning was not a result of a rigorous theory, the uncertainty on the beam diameter was kept as $50 \%$. Finally, $\sigma$ the effective beam radius, used for UV system was $0.078 \mathrm{~mm}$ and $0.009 \mathrm{~mm}$ for the UW system. As described in Appendix 1 the VW system uses the same beams of the UV and UW systems and an additional beam which has a radius of $0.0088 \mathrm{~mm}$. 
The uncertainties in the mean and turbulence quantities were computed using the technique given in (Kline, McClintock, 1953; Holman, 1984 ) The technique can be described as follows: If R, the result, is a function of $x_{1}, x_{2}, x_{3}, \ldots, x_{n}$ independent measured quantities in the form of

$$
R=R\left(x_{1}, x_{2}, \ldots, x_{n}\right)
$$

and if the uncertainty in each of the independently measured quantities are $w_{1}, w_{2}, \ldots, w_{n}$ with the same odds then the uncertainty in the result can be found with using

$$
w_{r}=\left[\left(\frac{\partial R}{\partial x_{1}}\right)^{2} w_{1}^{2}+\left(\frac{\partial R}{\partial x_{2}}\right)^{2} w_{2}^{2}+\ldots\left(\frac{\partial R}{\partial x_{n}}\right)^{2} w_{n}^{2}\right]^{1 / 2}
$$

and using

$$
\frac{\partial R}{\partial x_{i}} \simeq \frac{R\left(x_{i}+\Delta x_{i}\right)-\left(x_{i}\right)}{\Delta x_{i}}
$$

if the functional form is not explicit.

Once the uncertainty estimates for the beam radius, angles and histogram edge definition were found, the uncertainty in the mean and turbulence quantities were computed using the same program but running it 22 times consecutively by taking each disturbance as 0.1 of the corresponding uncertainty value. This was due to each uncertainty, separately, in the beam diameters, in the beam angles, cutting the histogram noise for each measured quantity could effect the outcome of the each measured quantity. The program was first run by assuming all the beam angles were uncertain with the uncertainty values estimated. Secondly the beam diameters were assumed as $50 \%$ smaller than the estimated values. The uncertainty in the each histogram was changed separately for the nine quantities measured by the each LDV system; the ones taken with the BSA and for the ones taken with swept spectrum analyzers. The uncertainty in each measured quantity due to individual uncertainties was found by finding the difference between the computed value without any uncertainty included, and dividing it with the uncertainty used in the 
computation for each individual uncertainty contributor; therefore using the approximate equation for the derivatives. These uncertainty values computed were compared to the uncertainties computed in another way which will be discussed shortly, and the bigger uncertainty value was accepted as the uncertainty value in the associated quantity.

Final LDV data after the gradient broadening and finite transit time broadening corrections (Appendix II) were carried out was found in the following way: since all mean velocity components and normal stresses were measured four times, an averaging process was applied. The data before the averaging at each station location can be found in Fig 20. The four profiles did not have equal number of points. The averaging was done by taking the maximum of the number of points in each four profiles and linearly interpolating the other 3 profiles in eachselves to find the corresponding data to the locations of the selected profile. Once four values for each y location were obtained, Chauvenet's criteria was applied to discard the data points which did not satisfy

maximum acceptable difference / standard deviation $\leq 1.4$

The standard deviation for the shear stresses which were measured only two times were found by using

maximum acceptable difference/standard deviation $\leq 1.15$

The acceptable points were then averaged to find the data value at that point. Shear stresses presented as the data were computed using only the BSA data, since the stresses are the measured quantities which are smallest in magnitude which makes them hard to measure and since the BSA data were taken after the data with swept spectrum analyzer technique were taken. In author's opinion since the two data sets were essentially same, the most recent set was chosen. The further computed standard deviation with the acceptable data values were used to define the uncertainty for the averaged data values using the equation

uncertainty in the quantity $= \pm 2 *$ standard deviation 
The data presented in Appendix IV also include these uncertainty analysis results. The uncertainties of the mean velocity and Reynolds stress data, as will be discussed in the later chapters, were used to find the uncertainties of the parameters which include these measured quantities. The nominal values of the mean velocity and stress data uncertainties are given in Table 5.

In the following sections of this chapter the measured mean and fluctuating velocities, and the shear stresses will be discussed. Even though in three-dimensional turbulent boundary layer research mostly the data are presented in local free-stream coordinates, one of the main reasons for the present measurements were to reveal the characteristics of the flow field on line where the $\overline{u^{2}}$ normal stress is maximum. Therefore, to see if the presentation of the data in different coordinate systems supplied more information, the quantities were presented in three different coordinate systems. First the discussion in tunnel coordinates will be given. Then the observed differences in free-stream and maximum-normal-stress coordinates will be comparatively discussed.

\subsubsection{MEAN FLOW MEASUREMENT RESULTS}

\subsubsection{IN TUNNEL COORDINATES}

In the tunnel coordinates, the $U$ mean velocity profiles are plotted in $\log (y / t)$ v.s. $U / U_{\text {ref }}$ coordinates, in which $t$ is the maximum body thickness and $U_{\text {ref }}$ is the reference velocity obtained at the tunnel test section entrance. The abscissa for each profile is shifted by a decade, and station numbering for the laser-Doppler-velocimeter technique (LDV) profiles starts by zero being the zeroth station. The symbols are used to denote the measured quantities and smaller symbols are used to show the uncertainty band at each measurement point. The solid lines on the LDV data, and on the hot-wire (HW) data taken at the left-hand side of the model looking upstream, are the data taken on the right-hand-side of the model with the HW at the same locations of the LDV, and 
at symmetric locations with respect to the tunnel axis of the left-hand-side HW data profiles. The $\mathrm{U}$ mean velocity component in tunnel coordinates obtained with LDV are shown in (Fig 21 ).

The observed deceleration of the flow up to station 2 is followed by an accelerating flow region. Starting with station 3 , the profiles get fuller. The logarithmic regions in the profiles are clearly observed. The slopes of these regions all with respect to the slope of the zeroth station first show an increase at the first location, a constancy at the second location, and continuous decrease till the sixth station, which in turn shows that the skin friction at the wall is increasing with the decreasing slope. At the seventh location a slight increase with respect to the sixth station is observed.

The $18 \mathrm{HW}$ profiles taken on the left-hand side of the wing were plotted in the same format, with eighteenth profile being the most upstream profile ( Fig 22 ). The symmetric HW locations start with the one corresponding to the fifteenth profile location, and the other profiles were taken at every other profile location. Due to the systematic error of 0.010 inches made at the first measurement point, the profile at fifth station location at the right-hand side was omitted. The regions where the flow first experiences a deceleration and then an acceleration and then another slight deceleration are more clearly observable from these $18 \mathrm{HW}$ profiles. The first 7 profiles, starting from the eighteenth profile location which is the most upstream location and including the eleventh station profile, form the first deceleration region. The maximum $U$ velocity component observed was $\sim 0.915 U_{\text {ref }}$ at the eleventh station. The next 9 profiles show an accelerating flow region where the maximum $U$ observed reaches to $\sim 1.12 U_{\text {ref }}$ at the third station. The next 2 profiles show slight deceleration. In all the profiles the existence of the logarithmic regions are clearly observed.

The $\mathrm{U} / U_{\text {ref }}$ profiles obtained with the HW and LDV techniques show good agreement, since the measured quantities with two systems overlap within the uncertainty bands. The HW profiles on both sides of the body also agree, which shows that the flow is symmetric around the wing. 
The $\mathrm{V}$ velocity component presented in $\mathrm{V} / U_{\text {ref }} \mathrm{v} . \mathrm{s} . \log (\mathrm{y} / \mathrm{t})$ coordinates have the scale of the plots as one tenth of the $\mathrm{U}$ and $\mathrm{W}$ components (Fig 23 ). The results were obtained with the LDV technique, and since the transformation to the freestream and normal stress coordinates were done only by a rotation along the $y$ axis, they will be presented only once.

The magnitude of the maximum observed at the first 5 stations show a gradual decrease, and starting with the fifth station profile, the maximum observed increases. In these last three stations, the maximum observed is at 0.56 inches away from the wall. In all the profiles approaching to the boundary layer edge the magnitude observed decrease. This results in the hill-shaped profiles which are clearer at the last 5 stations. Overall, the maximum V component measured was $\simeq 0.022 U_{\text {ref }}$, which is an order of magnitude smaller than the $U$ component. This in turn shows that the negligence of the $\mathrm{V}$ component in the HW reduction equations is acceptable.

The $\mathrm{W}$ component of the velocity profiles were plotted in $\mathrm{W} / U_{\text {ref }} \mathrm{vs} . \log (\mathrm{y} / \mathrm{t})$ coordinate system (Fig 24 ). All the measurements are presented as the top profile being the most upstream profile. Both the LDV and the HW measurements on the left-hand side of the wing are shown with symbols, and the solid lines are drawn to emphasize the HW measurements done on the right-hand side of the wing. The HW measurements on the left-hand side were plotted in $-\mathrm{W} / U_{\text {rof }} \mathrm{vs} . \log (\mathrm{y} / \mathrm{t})$ coordinates to be able to make the comparison easier ( Fig 25 ).

The development of the $-\mathrm{W}$ component measured is displayed more completely with the profiles taken on the left-hand side of the wing. The most upstream location profile shows that the approaching flow at that location is $2-\mathrm{D}$ in the mean. The first 3 profiles show that $\mathrm{W}$ component are slightly effected by the body ahead. Starting with the fifteenth station profile, in the next 5 profiles the slowing down of the flow to the zero value at the wall occurs within 0.010 inches away from the wall. The maximum velocity observed at the first location is at 0.155 inches away from the wall, and the position of the maxima in the intermediate profiles gradually increases until this location is reached. The logarithmic rions in the profiles span a shorter distance in the more 
developed profiles, and the overlapped portions of the $\mathrm{U}$ and $\mathrm{W}$ profiles' logarithmic regions are only at a couple of points in this coordinate system.

The comparison of the profiles taken on the right-hand side, both with LDV and HW techniques, show that at third and sixth station locations, the W component measured with HW was slightly less in magnitude. HW measurements done on both sides show that except for the fifth station of the left-hand side HW profile location, they are in good agreement.

The free-stream direction was found separately from each profile, by taking the average of the last three points in each profile. The transformations from tunnel coordinates to the free-stream coordinates were done by using the same angles throughout the each profile.

\subsubsection{IN FREE-STREAM COORDINATES}

The $\mathrm{U}$ velocity profiles in the freestream coordinates show the same kind of development as seen in TC except at the seventh station ( Fig 26 ). The seventh station profile looks like the sixth station profile, and it is not fuller than the sixth station profile as observed in tunnel coordinates. Compared to the profiles in the tunnel coordinates, $U$ profiles on the left-hand side of the wing are observed to be less full, however the slopes of the logarithmic regions do not change ( Fig 27 ).

The $\mathrm{W}$ component shows the same features as in the tunnel coordinates ( Fig 28 ). The profiles taken with LDV technique develop until the fourth station is reached. The fourth and fifth station profiles closely resemble each other; sixth and seventh station profiles show a decrease in the maximum velocity observed. It is also clearly observable that the maxima of the profiles shift to a higher $\mathrm{y}$ location in each successive location. The W component for the HW measurements done on the left-hand side of the body in the free-stream coordinates also show the same characteristics previously discussed ( Fig 29 ). Clearly observable logarithmic regions within the first 7 profiles span throughout the inner and logarithmic regions of the $U$ profiles, which may be attributed to the 
small velocities and initial development. Starting with the eleventh station location, the logarithmic regions observed begin only at the end of the logarithmic regions for the $U$ velocity and span into the outer regions.

\subsubsection{IN MAXIMUM-NORMAL-STRESS COORDINATES}

The measurements done were originally designed to investigate the characteristics of the flow on a line following the maximum normal stress direction. Transformation of quantities into the normal stress coordinates was done using the mean flow direction at the maximum normal stress point in each profile obtained from the HW measurements done on the left-hand side of the wing. The angles used both for the LDV and right-hand side HW profiles were the same, but the signs were changed.

The $\mathrm{U}$ profiles within the first three stations (zeroth, 1st and 2nd) show a gradual decrease in fullness and in the freestream velocity ( Fig 30 ). Starting from third station, the profiles become fuller, and free-stream velocity increases. The slope of the logarithmic region stays constant till the third station location is reached. From the fourth station till the seventh, the last station, the slope is observed to be constant. Compared to the LDV profiles in tunnel coordinates, the profiles starting with station 3 are fuller, and also the slopes of the logarithmic regions become steeper. The hot-wire profiles on the left-hand side are plotted in Fig 31.

The effect of the pressure gradient was seen to be least effective in the normal stress coordinate system. This can also be observed from the $\mathrm{W}$ component profiles in this coordinate system ( Fig 32 ). Since the free-stream angle is always smaller than the maximum normal stress direction, the $\mathrm{W}$ component at the boundary layer edge appears as positive. The near wall structure of the $\mathrm{W}$ profiles shows that since the lateral pressure gradients in NS coordinates is smaller than in FS ( freestream ) coordinates or TC ( tunnel coordinates ), the W component is smaller with respect to the other corresponding $\mathrm{W}$ components in the other coordinate systems. The existence of a 
logarithmic region in the profiles, even though it gets shorter proceeding downstream, is clearly observable. When compared to the other coordinate system plots, the logarithmic regions in NS system are observed to be longer. The logarithmic regions as also observed from the left-hand side profiles show that until the fifth station for the HW profiles or sixth station of the LDV profiles are reached they overlap in almost all the logarithmic regions of the $U$ profiles plotted in this coordinate system ( Fig 33 ).

\subsubsection{FLUCTUATION VELOCITIES}

\subsubsection{IN TUNNEL COORDINATES}

The characteristics of the $u^{\prime}, v^{\prime}$, and $w^{\prime}$ fluctuation velocities will also be discussed in 3 different coordinate systems. In the tunnel coordinates in the first three stations, the $\mathrm{u}^{\prime}$ profiles show the same characteristics ( Fig 34 ). The magnitude increases very sharply within 0.010 to 0.014 inches from the zero value at the wall to 0.09 of the reference velocity. This region is followed by a region where the magnitude drops to a value of 0.08 of the reference velocity. Starting from 0.22 $\sim 0.25$ inches away from the wall, the magnitude drops to a value of 0.005 of the reference velocity at the boundary layer edge. In the third and fourth stations, this maximum very near the wall is not observed. Instead, the $\mathrm{u}^{\prime}$ rapidly rises to a value of $0.075 \sim 0.08 U_{\text {ref }}$, then forms a plateau at this value which is followed by a region until it reaches to $\sim 0.005$ of the reference velocity at the edge of the layer. Starting with the fifth station, sixth and seventh stations show a maximum near the wall, and even though the maximum occurs at the same $\mathrm{y}$ in the profiles, the magnitude increases proceeding downstream. At the seventh station, the maximum reaches to 0.1 of the reference velocity which is higher than the value observed in the first three profiles. The rapid rise up to the maximum value observed in the fifth, sixth, and seventh stations is followed by a rapid drop region to a plateau and this region is followed by a drop to the boundary layer edge value. 
Beginning with the third station profile, the maxima observed in the profiles drop successively till the sixth station. Even though sixth and seventh stations have the maxima equivalent to the first three stations, the magnitude observed for the plateau region values still show a decrease. At the y location of 0.045 inches, the magnitude drop is $\sim 21 \%$ of the zeroth location profile. The plateau region was seen to be lying roughly between $y / t=0.01 \sim 0.1$, which corresponds to the regions where the logarithmic variation for the $\mathrm{U} / U_{\text {rof }}$ was observed. In the fifth, sixth, and seventh stations, the beginning of the plateau regions roughly match with the beginning of the logarithmic regions in $\mathrm{W} / U_{\text {ref }}$ profiles.

The HW profiles taken at the locations of LDV profiles were shown with lines for comparison. The HW profiles near the wall and in most part of the logarithmic regions for the $U / U_{\text {ref }}$ underestimate the $u^{\prime}$ velocity. Within the first 4 stations where the comparison is possible, the maximum difference between the LDV and HW results was seen to be $\sim 13 \%$ of the LDV value. The difference between the measured values diminishes away from the wall. Even though it seems that the HW values tend to be within the uncertainty band of LDV, it is believed that the HW values are consistently less than the LDV values. In the last two profiles at the sixth and seventh stations, the difference is more restricted to the near wall and the beginning of the logarithmic regions, but still consistent. The maximum difference observed is on the order of $\sim 20 \%$.

The second set of LDV data were taken because of this discrepancy between the first set of LDV and HW data. Since the second set of LDV data confirmed the results of the first LDV data and since the repeated measurements of the HW technique also consistently showed this difference, the difference between the measurements is believed to be true. This difference is attributed to the interference of the wire with the wall, which needs to be further investigated.

The hot-wire profiles on the left-hand side in the three most upstream profiles show the rapid rise of the $u^{\prime}$ fluctuating velocity and the transition to the formation of the flat plateau region ( Fig 34 ). These profiles are the ones in which the effect of three-dimensionality is least felt. The formation of the plateau region is due to the increase in the fluctuating velocity away from the wall and 
decrease of the fluctuating velocity near the wall. At station $13, \mathrm{u}^{\prime} / U_{\text {ref }}$ is nearly constant at 0.076 till the end of the $\log$ region for $\mathrm{U} / U_{\text {ref. }}$. Further downstream within the next four profiles, an overall reduction is observed. Starting with the eigth station profile which also corresponds to the station where the maximum of the $\mathrm{W} / U_{\text {ref }}$ profiles start shifting away from the wall, the fluctuating velocity near the wall begins to increase while the fluctuating velocity which corresponds to the logarithmic region of the $\mathrm{W} / U_{\text {ref }}$ profiles continues to decrease. For $\mathrm{y} / \mathrm{t}=0.1$ while $\mathrm{u}^{\prime} / U_{\text {ref }}$ is $\sim 0.076$ for station 13 , it reduces $24 \%$ to 0.0575 at station 1 . The $u^{\prime}$ velocity measured on the right-hand side of the wing with the hot-wire was plotted with the full lines for comparison. All the stations except the left-hand side fifth station location profile show that the inner and logarithmic regions of both sides agree fairly well. The maximum difference observed at the fifth station was $\sim 16 \%$ of the maximum value at that station. The maximum difference for the points where $y / t>0.1$ was at station 13 and was computed as $16 \%$ of the maximum value at that station. The other stations show good agreement with differences less than $10 \%$ of the maxima observed at those stations.

The $\mathrm{v}^{\prime}$ velocity profiles show almost the same characteristics in the six profiles obtained ( Fig 36 ). The magnitude of the $v^{\prime}$ fluctuation velocity reaches up to a value of $0.04 \sim 0.045$ of the reference velocity at a y location of $\sim 0.035$ inch and forms a plateau region which is followed by a region starting from 0.35 inch where the magnitude drops down to a value of $\sim 0.005$ at the boundary layer edge. At the sixth and seventh stations the plateau region is replaced by a short plateau and a hill region where a maxima of the profiles can be defined. Still the maxima stays within $0.04 \sim$ 0.045 of the reference velocity range.

The $w^{\prime}$ fluctuating velocity is also presented in $\log (y / t)$ v.s. $w^{\prime} / U_{\text {ref }}$ coordinate system ( Fig 37 ). Unlike the $u^{\prime}$ or $v^{\prime}$ in all the eight profiles observed with the LDV technique, $w^{\prime}$ profiles show somewhat the same characteristics. The rise of the velocity to the maxima of the profiles within $y / t=\sim 0.01 \sim 0.02$ is continued with a semi-log region which corresponds to the logarithmic region of the $\mathrm{U} / U_{\text {ref }}$ profiles and a region where the $\mathrm{w}^{\prime}$ drops to its value at the boundary layer edge. The maximum reached at the zeroth station seem to be slightly reduced to $\sim 0.055$ at the first station from the value of $\sim 0.058$ of the reference velocity. Within the next six locations, the maximum 
observed increases to a value of $\sim 0.066 U_{\text {rof. }}$. The hot-wire profiles at the locations of LDV show a difference between the two measurement techniques, mostly at first, second and third stations. Even though the fourth, sixth and seventh station profiles follow each other very closely, the difference at the previous three locations is on the order of $25 \sim 40 \%$ at the points where maximum differences occurred. At station 4 the difference is $\sim 15 \%$.

The hot-wire profiles taken on the left-hand side of the wing show a continuous increment in the first seventeen profiles in the average value observed within the near wall and $y / t=0.01-0.1$ region which corresponds to the logarithmic region for the $\mathrm{U} / U_{\text {ref }}$ profiles ( Fig 38 ). The increment in $\mathrm{w}^{\prime}$ velocity in the second station is on the order of $\sim 25 \%$ of the $\sim 0.0475 U_{\text {ref }}$ average value estimated at the eighteenth, the most upstream location. The high scatter of the data within the most upstream five profiles makes it hard to judge the profile shapes. Starting with the thirteenth station profile, the formation of a semi-logarithmic region spanning to the end of the log region of the $\mathrm{U} / U_{\text {esf }}$ profiles, preceded by the quick rise near the wall, is seen. In the most downstream location a slight drop of the average value within $y / t=0.01-0.1$ with respect to the seventeenth station profile is observed. The hot-wire profiles obtained on both sides of the body show good agreement for all the profiles taken, which in turn shows that not only the mean velocity components but also the turbulence structures on both sides of the body are in symmetry.

The $u^{\prime}$ and $w^{\prime}$ fluctuating velocities in the free-stream coordinates were found with a tensor transformation which was applied to the Reynolds stress tensor, using the appropriate angles obtained from each profile separately (Appendix III and Table 3 ). Both the LDV and HW measurements of both fluctuating velocities seem to follow the quantities presented in tunnel coordinates. This is due to the small free-stream angles encountered. The maximum free-stream angle observed for the LDV measurements was at station 4 as $10.16^{\circ}$ and for the left-hand side HW measurements it was $13.87^{\circ}$ at station 8 . 


\subsubsection{IN FREE-STREAM COORDINATES}

The presentation of $u^{\prime}$ fluctuating velocity measured with the LDV technique in the free-stream coordinates follows the presentation in tunnel coordinates very closely except with minor differences at two stations ( Fig 39 ). At station 4 slight decrease within $y / t=\sim 0.01-0.1$ range, and at station 5 very slight increase till $y / t=0.01$ and small decrease within $y / t=0.01-0.1$ range, are observed. The order of increments and decrements are less than 0.005 in the vicinity of $7 \%$ of the observed quantities in tunnel coordinates.

The HW profiles on the left-hand side of the body show the same characteristics in the FS coordinates as in TC for the first eight most upstream profiles ( Fig 40). In the next four profiles the $u^{\prime}$ velocity is higher for $y / t \simeq 0.008$ to 0.01 and lower within $y / t=\sim 0.01-0.1$. Even though the increment near the wall is decreased in y direction from its initial value of $5 \%$ difference, the decrement in the $\log$ region of the $U / U_{\text {ref }}$ reaches to a minimum around $y / t=\sim 0.02$ to the $5 \%$ of the 0.062 at the seventh station. For $w^{\prime}$ profiles of LDV, the most significant difference is observed near the wall before $y / t=0.01$ where the FS values which are on the order $16 \%$ of the maximum $\sim 0.062$ smaller than TC values increase till they start following the quantities in TC

( Fig 41 ). This was mostly significant at the sixth station. For the first eight profiles starting from the most upstream, the HW profiles do not show any significant change than the profiles in TC ( Fig 42 ). Starting from tenth station, the near wall $w^{\prime}$ in FS coordinates is observed to be less till $y / t=0.01$ and seen to be higher till $y / t \simeq 0.04$, with respect to the TC profiles. This difference due to the presentation in different coordinate systems is seen to be practically non-existent at station 3 . 


\subsubsection{IN MAXIMUM-NORMAL-STRESS COORDINATES}

The fluctuating velocities in normal stress coordinates were found by applying the tensor transformation to the Reynolds-stress tensor with the angles found in each left-hand side HW profile. These angles were also used for the right-hand side HW and LDV profiles at the symmetric locations with a sign change.

In the first two profiles, $u^{\prime}$ follows closely the values in TC ( Fig 43 ) Starting with the second station profile, the coordinate transformation seems to be reducing the $u^{\prime}$ fluctuating velocity. In the second station, except at the few points very close to the wall, above $y / t \simeq 0.005$, an overall reduction on the order of $\sim 0.006$ or $7 \%$ of the maximum 0.087 was observed. In the third station profile, this reduction is on the order of $\sim 0.004$ and seems to be reducing to $\sim 0.003$ after $y / t=0.1$. The gradually achieved reduction of $u^{\prime}$ in the fourth station profile reaches to a maximum of $\sim 0.011$ at $y / t=0.03$ and reduces further in the profile till $y / t=\sim 0.1$ is reached. The reduction after this point is $\sim 0.005$. The maximum in TC at this station was read as $\sim 0.0755$. The fifth station profile points below $y / t=\sim 0.01$ were observed to have higher $u^{\prime}$ by as much as 0.006 which gradually reduced down to zero. Above $y / t=\sim 0.01$ overall reduction till the edge, within the order of 0.008 below $y / t=0.1$ and $\sim 0.005$ above $y / t=\sim 0.1$ is noticed. The $u^{\prime}$ at station 6 which are observed to be higher in NS coordinates than the TC near the wall below $y / t=0.002$ at station 6 gradually decrease to the values of the TC presentation at this y location. The difference of 0.03 in the third point in the profile is reduced to 0.008 at the 6 th point. Between $y / t=0.01-0.1$, NS values are seen to be smaller; the maximum difference measured is on the order of 0.004 . Above $y / t \simeq 0.1$, the differences further reduce. Except at the first three points in the profile, seventh station profile presentation in NS follows closely the one in TC.

The first 5 most upstream HW profiles of the left-hand side follow the presentation in TC closely ( Fig 44 ). Starting with the thirteenth station profile, overall reduction in the $u^{\prime}$ is observed. In station 13 starting from $y / t=0.007$, the difference was $\sim 0.003, \sim 4 \%$ of the maximum 0.08 in 
TC. The distinction between the two presentations become more clear starting with the zeroth station profile. Except the points below $y / t \simeq 0.006, u^{\prime}$ in NS coordinates is observed to be lower in the whole layer in the next four profiles including the zeroth station. The differences which gradually increase till $y / t \simeq 0.02$ decrease towards the edge of the layers. The difference within the $y / t \simeq 0.01-0.1$ range is seen to be increasing proceeding downstream. At station 7 this process is reversed. The difference in this range is on the order of $\sim 0.006$ at station 10 , where the maximum is $\sim 0.073$, and $\sim 0.009$ in station 8 , where the maximum is $\sim 0.072$. In the next four profiles starting with the sixth station, the reduction in the difference between the presentations between $y / t=0.01-0.1$ is accompanied by the increased values below $y / t \simeq 0.01$. Proceeding downstream, both the increments and decrements gradually diminish. The increments near the wall is on the order of 0.07 at station 6 , where maximum is 0.076 and 0.005 at station 3 where the maximum is $\sim 0.082$. The decrements at these stations are 0.003 and 0.002 succesively.

Contrary to what is observed for $\mathrm{u}^{\prime}, \mathrm{w}^{\prime}$ fluctuating velocity in NS coordinates show an increase in most of the profiles almost in the whole layers ( Fig 45 ). For the LDV profiles in the first two stations, the values follow closely the TC presentation with a maximum increment of 0.002 . The second station profile reaches to its peak at $y / t \simeq 0.01$ with an increment of $\sim 0.01,17 \%$ of the maximum value $\sim 0.059$. This positive difference reduces down to 0.005 at $y / t \simeq 0.1$. Same characteristics are also seen in the third station, but the increment is seen to be constant on the order of $0.006, \sim 10 \%$ of the maximum $\sim 0.061$ in TC in between $y / t \simeq 0.01-0.08$. Out in the layer, the quantities are seen to be following each other closely. At fourth station w' shows a peak at $y / t \simeq 0.02$, on the order of $0.012, \sim 19 \%$ of the maximum 0.062 in TC which is gradually achieved starting from $y / t \simeq 0.007$. In the outer layer above $y / t \simeq 0.3$ where two presentations have the same value, the $w^{\prime}$ in NS coordinates are observed to be slightly less than in TC. Within the next three stations, near wall $w^{\prime}$ values below $y / t \simeq 0.01$ are observed to be quite lower. The differences which are observed to be $50 \%$ or more at some points are believed to be mostly due to the uncertainties. Above $y / t \simeq 0.01$ proceeding downstream, the higher values observed in NS 
decrease down to the TC values. The maximum difference at station 5 is on the order of $\sim 0.011$ and the average is $\sim 0.003$ in the $\mathrm{y} / \mathrm{t} \simeq 0.01-0.1$ range.

The first four profiles of $w^{\prime}$ in NS follow closely the values observed in TC ( Fig 46). Starting with the thirteenth profile $w^{\prime}$ in NS is seen to increase. thirteenth profile is observed to have increments in the order of $\sim 0.006, \sim 11 \%$ of the maximum 0.053 within $y / t \simeq 0.01-0.2$ range. Twelfth and eleventh station profiles do show same characteristics as the thirteenth station, in which the increments are on the order of 0.004 . Beginning with tenth station $w^{\prime}$ values reduce proceeding downstream. At station 7 the maximum difference is on the order of $0.006, \sim 10 \%$ of the maximum 0.062 . The increments are mostly observed within $y / t=0.01-0.1$ range. Below this range, $w^{\prime}$ in NS is lower as much as 0.004 , and in the outer layer it was higher with the same increment. Proceeding downstream starting with the third station, the two presentations of $\mathrm{w}^{\prime}$ follow each other closely except below $y / t \simeq 0.007$, the NS values are seen to be higher by about 0.003 .

\subsubsection{REYNOLDS SHEAR-STRESSES}

The $-\overline{w v},-\overline{u w},-\overline{w w}$ kinematic Reynolds shear stresses will also be discussed in three coordinate systems. $-\overline{u v}$ ' and ' $-\overline{v w}$ stresses were obtained only by LDV technique. The different symbols at the points very close to the wall and at the outer edge show where the realizability conditions are not satisfied. The points where these conditions were not satisfied will not be included in the discussion. The quantities are discussed in terms of ( kinematic stress $/ U_{\text {ref }}{ }^{2}$ ). Small symbols in TC designates the uncertainty bands. The shear stress components at the wall in the $\mathrm{x}$ and $\mathrm{z}$ coordinates of the TC are also going to be shown in the figures of these quantites. The stress magnitude in the zeroth station was found using Clauser 2-D Law of the Wall, and at station 1 Johnston's 3-D Law of the Wall was used. At other stations, shear stress magnitudes and directions are taken from Ailinger's data ( 1990 ). 


\subsubsection{IN TUNNEL COORDINATES}

The $-\bar{w}$ kinematic stresses in TC in the first four most upstream profiles show the same form ( Fig 47 ). The negative stresses very near the wall were followed by a region where the stress changes sign. This region was followed by a plateau region and a region where the stresses decrease to zero value outside the boundary layers. At station 0 , the plateau forms at $\sim 0.0017$. This region includes most of the logarithmic region of $\mathrm{U} / U_{\text {ref }}$ profile. At station 1 the minimum reached is slightly less negative than zeroth station. The increase up to $\sim 0.0009$ was complete at $y / t \simeq 0.01$, and until $y / t \simeq 0.2$, the stress is seen to be constant around this value. Minimum observed in the second station is lower than the previous two profiles. The value measured at $y / t \simeq 0.01$ is $\sim 0.0016$ and at $y / t \simeq 0.08$ is $\sim 0.002$. In the $y / t \simeq 0.01-0.1$ region, the average is $\sim 0.0019$. Quantities observed in the third station are lower than the zeroth station values. Plateau formed in the $y / t \simeq$ $0.01-0.1$ is seen to be $\sim 0.0015$. In all of the profiles discussed, the very near wall stress was on the order of $\sim-0.0005$. In the fourth station, the increase near the wall was followed by a constant stress region on the order of 0.0012 . In the fifth station, the increase near the wall and in the $\log$ region is followed by a region where the stresses decrease until the layer edge. Station 6 shows same form of a profile. Near wall values at station 7 show a maximum at $y / t \simeq 0.01$. The decrease in the stresses until $y / t \simeq 0.03$ is further seen to be increasing until $y / t \simeq 0.2$. The stresses further decrease to the zero value at the layer edge. No specific relation between the $-\overline{w v}$ kinematic shear stress and the mean velocity profiles could be observed.

Development of the $-\overline{u w}$ kinematic shear stress in TC in all profiles observed to be restricted within $y / t$ less than 0.2 except at station 7 where a variation until $y / t \simeq 0.5$ is observed ( Fig 48 ). Near wall characteristics were seen to be developing with the development of the $W / U_{\text {ref }}$ mean flow component. In the zeroth station, $-\overline{u w}$ decreased to -0.0014 near the wall at $\mathrm{y} / \mathrm{t} \simeq 0.006$, and was seen to be linearly increasing to the zero value outside the boundary layer. Starting with the first station profile near the wall, $-\overline{w w}$ are observed to be positive. At first and second stations, the gradient of $-\overline{u w}$ above $y / t=0.01$ was observed to be less than the zeroth station. Near wall value 
at station 2 at $y / t \simeq 0.004$ is $\sim 0.0016$. Starting with the zeroth profile proceeding downstream, a decrease in region $y / t \simeq 0.01-0.1$ was observed, which in the fourth profile shows a peak at $y / t \simeq$ $0.004, \sim-0.0011$. The peak formed is seen to decay downstream of this station, while its location in the profiles shifted up. Also, proceeding downstream starting with the fourth station, the maximum observed near the wall gradually increases. At station 6, the maximum was as high as $\sim 0.0036$. At station 7 , the decrease in the $-\overline{w w}$ up to $\sim-0.0005$ at $y / t \simeq 0.08$ shows an increase until $y / t \simeq 0.2$, which further decreases to a value of zero at the layer edge.

The HW profiles taken in the locations of the LDV profiles are seen to be following the LDV profiles closely with slightly lower values in magnitude especially in the negative side up to station 4. The profiles at sixth and seventh stations agree in the whole layer.

The HW profiles obtained on the left-hand side of the body are plotted in $\log (\mathrm{y} / \mathrm{t})$ v.s. $-\overline{u w} / U_{\text {ref }}^{2}$ coordinates to be able to compare with the HW profiles on the right-hand side, which are in the $\overline{u w} / U_{\text {ref }}$ sup 2 coordinates in the ordinate (Fig 49). The kinematic stresses observed in the eighteenth station are virtually zero, which shows in fact that the approaching boundary layer is two-dimensional not only in the mean but also in the turbulence quantities, too. Within the next 7 profiles downstream, while $-\overline{u w}$ observed near the wall gradually decreases, the stress in each profile in the $y$ direction gradually increases to the zero value at the layer edge. Starting with the tenth station, above $y / t \simeq 0.01$ development of the positive stresses begin. Until station 6 , while the near wall stresses reduce slightly in the negative side, the increase of the peak at the positive side and the following semi-logarithmically decrease to the zero value at the edge are continued. At station 6 while the peak value reaches to its maximum of 0.001 at $y / t \simeq 0.03$, the near wall value further decreases. Starting with the sixth station profile, development of a region where the reduction of the stresses after the maximum of the peak takes place is seen. The reduction of the kinematic shear stresses reaches to a maximum near the wall at station 4 at a value of $\sim-0.0019$. In the further downstream profiles, near-wall values gradually relax. The peak values observed on the positive side gradually reduce starting with the sixth station, while the point in each profile where the peak occurs gradually shifts up from $y / t \simeq 0.03$ at sixth station to $y / t \simeq 0.06$ at the first 
station. In the region where the reduction of the kinematic stresses are observed, the minimum kinematic shear stresses observed are seen to be around $y / t \simeq 0.1 \sim 0.2$. These points in the profiles also indicate the end of the logarithmic region. Kinematic shear stresses further in the profiles increase to zero value at the layer edge. The comparison between the both side profiles showed that the $-\overline{u w}$ stresses agree well with each other, which in turn verifies that the flow is not only symmetric in the mean and fluctuating velocities but also in the stresses formed.

Among the kinematic Reynolds shear stresses measured, $-\overline{v w}$ is seen as the smallest in magnitude. At all stations the range of the data is between 0.0006 to -0.0005 ( Fig 50 ). In the first station except slight decrease near the wall, the stress profiles are seen to be fluctuating around the value of zero. The slight decrease on the order of 0.0002 increased down to zero around $y / t \simeq 0.006$. First station shows an decrease in the $y / t \simeq 0.04$ to 0.08 . Starting with the second station, the change in the sign of $-\overline{v w}$ near the wall is observed. The kinematic stress at station 2 was seen to be gradually decreasing, starting from the maximum of 0.0005 at the first point of the profile, till $y / t \simeq 0.01$, where it reached zero. The third station values are seen to be forming a flat region which spans between $y / t \simeq 0.02-0.09$ at a value of 0.0004 . Starting with the fourth station, $-\overline{\nu w}$ profiles look similar to those of $-\overline{u w}$. The stresses near the wall start decreasing from a positive maximum to a negative minimum and then increase above zero and further in the profile decreases to zero value at the layer edge. Also, the maximum reached near the wall at station 5 was followed by a decrease at station 6 and further decrease in station 7. The peak in the negative side observed to be maximum at station 7 , and the maximum obtained at the outer region was seen to be increasing until station 7. At station 7, the maximum was at the level of that of station 4 .

\subsubsection{IN FREE-STREAM COORDINATES}

The presentation of $-\bar{w}$ stresses in the FS coordinates, in general, follow the TC presentation due to the small free-stream angles realized ( Fig 51 and Fig 52 ). 
The $-\overline{u w}$ stresses in general were observed to be shifting down in all the stations ( Fig 53 ). The decrements were more clearly distinguishable near the wall region and were seen to diminish towards the layer edges. At zeroth and first stations, the shifting down is on the order of 0.00025 near the wall region until $y / t \simeq 0.1$. Downstream, the decrements are seen to gradually decrease, while second station decrements are $\sim 0.0005$. It is $\sim 0.0006$ in the third station. In both stations, the decrements seem to be gradually increasing above $y / t \simeq 0.1$. Beginning with the fourth station, the decrease of the - $\overline{\tau w}$ kinematic stresses in FS with respect to the ones in TC starts to gradually diminish in the following stations. In station 4 , almost constant amount of decrease at each point of the profile on the order of 0.0005 is seen to gradually increase above $y / t \simeq 0.1$. This type of difference is also observed at station 5. At station 6 and 7 the maximum difference between two presentations are seen near the wall and are seen to decrease in the layer. Maximum difference at station 7 was $\sim 0.0003$.

The presentation of the profiles taken on the left-hand side of the wing in FS coordinates show distinguishable differences, starting with the sixteenth station profile ( Fig 54 ). Even though the differences increase in the downstream direction until the tenth station, the increments were seen to be decreasing in the $y$ direction starting from the wall until the eleventh station. Starting with the tenth profile proceeding downstream, the differences start to decrease. After station 6 , the differences were mostly seen near the wall below $y / t \simeq 0.01$. Between the eleventh and eigth stations, the differences between $y / t \simeq 0.02-0.1$ were seen to be higher than the near wall differences. In all the profiles, the values in both coordinate systems near the outer edge above $y / t \simeq 0.3$ follow each other closely. While the difference observed at station 16 was on the order of 0.0001 , it was increased to $\sim 0.0005$ by station 11 . Overall, the $-\overline{u w}$ kinematic stresses in FS coordinates show higher stresses in the negative side of the scale, although the shapes of the profiles are similar.

The effect of coordinate transformation on $-\overline{w w}$ kinematic stress is felt starting with the second station profile of the LDV profiles. Starting from $y / t \simeq 0.007$ up to $y / t \simeq 0.2$, the $-\overline{v w}$ kinematic stresses in the second station were seen to be shifted up as much as 0.0002 . The increase in the third station results in $-\overline{w w}$ kinematic stresses on the order of $\simeq 0.0005$ in the range $y / t \simeq 0.02-0.2$ which 
is a part where the log region of $U / U_{\text {ref }}$ is seen. The increase near the wall is seen to be less. In the following profiles, the same type of increase was observed, which, overall, results in more distinguishable stresses away from zero. Starting with fourth station proceeding downstream, the differences are observed to decrease.

\subsubsection{IN MAXIMUM-NORMAL-STRESS COORDINATES}

The distinguishable difference between two presentations of the $-\bar{w}$ stresses in TC and NS coordinates were observed at station 2. At this station, $-\bar{w}$ in NS coordinates is seen to shift down on the order of 0.0002 in the whole layer below $y / t \simeq 0.5$ ( Fig 55 ). The decrement at the fourth station in NS coordinates with respect to the ones in TC was seen to be highest. In the following stations the decrements reduce. The decrements near the wall are seen to gradually increase until $y / t \simeq 0.07$. The difference which increase up to $y / t \simeq 0.2$ seen gradually decrease until $y / t \simeq 0.5$. The most observable differences are seen between $y / t \simeq 0.02-0.15$, which also corresponds to log region. The maximum difference at station 4 was on the order of 0.0005 ; by station 7 this was seen to drop down to 0.0001 .

The most observable differences between two coordinate system presentations occur for $-\overline{u w}$ stresses. In all the stations, NS coordinate $-\overline{u w}$ stresses were seen to be lower than TC values (Fig 56 ). At the zeroth station, maximum decrement was on the order of 0.0007 at $y / t \simeq 0.008$. By station 4, the maximum decrement reaches to 0.0015 . In all the profiles, the almost constant decrement until $y / t \simeq 0.08$ is seen to decrease further in the profiles, and above $y / t=0.4$, the difference seems negligible. The decrements observed decrease in downstream stations. At station 4 and 5 , a decrease in the decrement until $y / t \simeq 0.02$ and further increase until $y / t \simeq 0.07$ are observed. At station 6 the decrements near the wall and in the outer region were higher than the region between $y / t \simeq 0.02-0.07$. At station 7 , differences were mostly below $y / t \simeq 0.02$. 
For the $-\overline{w w}$ measurements on the left-hand side of the wing, all the stresses including the eigth station values are seen to be positive ( Fig 57 ). The increase in the $-\overline{2 w}$ stresses proceeding downstream were seen to be reaching to their maxima in each profile around $y / t \simeq 0.1$ until station 6. The maximum $-\overline{w w}$ reached occurs in station 10 , with a value of $\sim 0.0013$. Further downstream the maximum reached decreases. By station 1 the maximum reduced to 0.0005 . Starting with the seventh station profile near wall, values start to be observed as negative. At station 7 , the point where maximum is reached in the profile shifted down to $y / t \simeq 0.05$. By station 1 , the maximum shifts up to $y / t \simeq 0.07$. The minimum reached near the wall at station 6 is on order of $\sim-0.0013$. Above $y / t \simeq 0.1$, starting with station 9 till station 3, stresses above this height are almost zero. In the further downstream profiles,stresses are below zero. In all the profiles, near wall values are seen to be gradually increasing to the maximum value observed in the profiles, and, as expected, the values at the layer edges are zero.

The observable differences between TC and NS coordinate system presentations of $-\overline{v w}$ stresses start with the zeroth station ( Fig 58 ). At station 0 , the difference is seen as an increase above $y / t$ $\simeq 0.02$. In station 1 the $-\overline{v w}$ in NS coordinates is observed to be higher between $y / t \simeq 0.02-0.2$. In station 2 , the increase in $-\overline{v w}$ on the order of 0.0009 between $y / t \simeq 0.01-0.2$ is seen. In station 3 , the increase seen is on the order of 0.0006 . In the further downstream profiles, increased $-\overline{v w}$ profiles result in more observable profiles away from zero. The differences between two presentations decrease proceeding downstream. The range where the increase occured observed to be in the same range as the previous stations. At station 4 , the increase was on the order of $\sim$ 0.0006 while at station 7 it was only about $\sim 0.0002$. 


\subsection{EXAMINATION OF THE FLOW STRUCTURE}

\subsection{INTRODUCTION}

The derived quantities are the computed values which appear in the form of relations of the previously discussed measured quantites. These quantites appeared through the ongoing discussion about the 3-D boundary-layer research, either from the forms of the equations used or by the assumptions and approaches made for solving the governing equations. These quantities were, $\mathrm{N}=$ anisotropy constant, $A_{1}=$ Townsend's constant, flow angle, velocity gradient angle, shear stress angle, convection and production profiles for the stresses and kinetic energy of turbulence, eddy viscosities, mixing length, and kinetic energy of turbulence.

Due to the encountered uncertainties in the $\overline{w v}$ and $\overline{v w}$ shear stresses, especially, the shear stress angle, $A_{1}$, Townsend's constant, and $\mathrm{N}$, anisotropy constant quantities were seen necessary to be smoothed. To be able to do this task, first the $\overline{w v}$ and $\overline{w w}$ stress profiles were smoothed with a least-squares parabola fitting to the 5 successive points in the profiles and computing the value at 
the third point location. For the first two points in the profile, the same parabola fitted for the first 5 points was used. The parabola fittings were done for the profiles in free-stream coordinates for the computation of $A_{1}$ and shear stress values and in the three coordinate systems for the anisotropy constant, N. The length scales of the flow studied; boundary layer thickness, displacement thickness, and momentum thicknesses at each station were computed using the trapezoidal rule for the integrations. The definition and the values for these lengths may be found in Table 6. The boundary layer thickness is defined as the point in the layer where the velocity magnitude reaches to the 0.995 of the free-stream velocity magnitude. Free stream velocity magnitude was found by taking the average of the last three points in the profiles at the layer edge.

\section{2 $N=$ ANISOTROPY CONSTANT}

Anisotropy constant is defined as

$$
N=\frac{\left(-\frac{\overline{v w}}{\partial W / \partial y}\right)}{\left(-\frac{\overline{u v}}{\partial U / \partial y}\right)}
$$

In the approach relating the shear stresses to the mean flow quantities, isotropic eddy viscosity models make use of a single eddy viscosity value with the assumption that the transfer of momentum by the fluctuating velocities in any direction in the flow can be related to the local mean flow gradients in the coordinate system defined, by a single eddy viscosity term as in the laminar flow with the use of the kinematic viscosity of the fluid. Yet, since the eddy viscosity is not a property of the fluid but the flow, and turbulence in 3-D flows appears as not only a function of the local variables, the assumption of isotropy of the eddy viscosity is questionable. The anisotropy constant makes it possible to judge this issue clearly. 
With this in mind, the quantity $\mathrm{N}$ was plotted in three different coordinate systems to see even if in a preferrred direction the assumption could be validated. If the assumption is validated the value would be computed as 1 . The shear stresses in different coordinate systems were computed with a tensor transformation given in Appendix III. The gradients for the mean flow quantities were computed with the same type of curve fitting to the profiles in each coordinate system. Furthermore, not to be misguided with the uncertain quantities, the uncertainties were computed and the values with uncertainty levels within \pm 1 are presented. The band of the values is also kept as \pm 2 , since the assumption to be valuable the parameter $N$ would be close to 1 . The uncertainty bands of the quantity are presented as bars. For the coordinate system other than TC, the uncertainty values for individual stresses were kept same as ones in the TC. The uncertainty values for the gradients are $10 \%$ of $\partial W / \partial y$ and $5 \%$ of the $\partial U / \partial y$.

Overall, the $\mathrm{N}$ values are observed to be mostly less than 1 and sometimes negative, with no distinct form of variation in the profiles ( Fig 59,60,61). In tunnel coordinates none of the zeroth station values had less than \pm 1 of uncertainty. Also in all the coordinate systems, starting with station 2 the station where the $\mathrm{W}$ and $\overline{v w}$ profiles start to develop more points in the profiles are seen. The available data within the chosen uncertainty band are clustered within $y / t \simeq 0.01-0.3$ region. The data in TC ( Fig 59 ) were seen to be more scattered with respect to the data in FS (Fig 60 ), and data in NS coordinates ( Fig 61 ) were seen to be in a narrower $\mathrm{N}$ range than the data in FS coordinates. While most of the data in TC is in between $-0.5-1$, it is seen to be within $0-1$ in NS coordinates. Even though the shapes of the profiles do not follow a trend, the data in NS coordinates seem to be fluctuating in a band of 0.5-1 around the value of 1 . Once the uncertainties are included except at stations 1 and 7 where the values observed are closer to 0.5 , the $\mathrm{N}$ anisotropy constant is close to 1.0 . It could be concluded with the data that $N=1$ in such a $3-\mathrm{D} T B L$ was an exception, but not the rule. Data also clearly show that the eddy viscosity is not isotropic, but rather close to being isotropic in a coordinate system defined in the mean flow direction in the profile where the $\overline{u^{2}}$ stress is maximum. 


\section{$4.3 A 1=$ TOWNSEND'S STRUCTURAL PARAMETER}

The structural parameter $A_{1}$

$$
A_{1}=\frac{\sqrt{\left(\overline{w w}^{2}+\overline{u v}^{2}\right)}}{\left(\overline{u^{2}}+\overline{v^{2}}+\overline{w^{2}}\right)}
$$

was used by Bradshaw in his 1967 paper in the formulation to relate the transport of the turbulent kinetic energy to the Reynolds shear stresses. Later on, he solved the momentum, continuity and turbulent kinetic energy equations, which formed a hyperbolic set of equations, for 2-D TBL. The value for $A_{1}$, as presented in that paper was $A_{1}=0.15=\overline{w w} /(2 k)$, a constant. If this were a constant in 3-D TBL, still the magnitude of the stress in a plane parallel to the wall could be related to the TKE. Thus, the TKE transport equation could be used to represent the transport of the magnitude of the shear stress.

The magnitude of the shear stress parallel to the wall and the turbulent kinetic energy do not change with the tensor transformation. Therefore, the quantity is presented only once in the tunnel coordinates. With the known uncertainties of the individual stresses, the uncertainty of the $A_{1}$ was computed. Since the quantity defined by Bradshaw was 0.15 , the data in a band of $0-0.3$ with the uncertainty less than of \pm 0.15 were presented, and the rest was discarded.

Starting with the zeroth station, the form of the $A_{1}$ profiles show that the assumption of constancy of $A_{1}$ as 0.15 for the present 3-D data is questionable ( Fig 62 ). In the zeroth station, by taking into account the uncertainty of the quantity in most of the layer, the assumption could be validated, especially in the logarithmic and outer regions of $U / U_{\text {ref }}$ profile; but the actual values computed are lower than 0.15 , near the wall region and higher in the log and outer regions. At station 1 , the values are mostly less than 0.15 until the end of the $\log$ region. At station 2 , if the uncertainty is included, 
the structural parameter $A_{1}$ again is observed to be $\sim 0.15$, in the whole region. In the stations downstream beginning with the third station the structural parameter was seen to be $\sim 0.15$ within the logarithmic and outer regions of the profiles including uncertainty bands. In all the profiles, near wall values were seen to be less than 0.15 including the uncertainty bands.

\subsection{FLOW ANGLE}

Mean flow angle which is

$$
\text { Flow Angle }=\operatorname{Arctan}\left(\frac{W}{U}\right)
$$

was computed in three different coordinate systems. The uncertainty for the computed flow angles were found using the individual uncertainties for $\mathrm{W}$ and $\mathrm{U}$ observed in tunnel coordinates, and these individual uncertainties were kept the same for the other coordinate systems. The computed uncertainty is denoted with bars for the LDV measrurements. The hot-wire values computed at the same profile locations for the LDV profiles were plotted with lines. Even though the transformation into coordinate systems other than TC only shifts the profiles by the transformation angle used, the profiles are still discussed in three different coordinate systems to emphasize some important aspects seen.

The profiles in TC starting with the zeroth, including the second station profiles, show a semi-logarithmic variation almost in the whole layers (Fig 63 ). The maximum angle reached very near the wall was $-21^{\circ}$ in station 2 . In station 3 and 4 , near the wall flow is observed to be collateral, but the uncertainties near the wall are much higher than the uncertainties observed in the rest of the profiles. At station 3, the semi-logarithmic variation is seen to be starting at $y / t \simeq 0.08$; at station 4 it is shifted up to $y / t \simeq 0.014$. The minimum angle at station 4 is $\sim-37^{\circ}$, but the 
uncertainty is $\pm 25^{\circ}$. In the further downstream locations, the near wall flow angle is seen to be increasing, thus decreasing in magnitude, and the points in the profiles where the semi-log variations start are observed as near to the points where the minimum flow angles are reached. This point in the seventh station is at $y / t \simeq 0.03$. The gradual decrease in the magnitude of the minimum flow angle results in $-13^{\circ}$ observed at station 7 . In all the profiles, the semi-log variation of the flow angle is seen to end at $y / t \simeq 0.4$, and the points above are observed to form a region where the angle slowly decrease to the layer edge values.

Except at station 1, the flow angles computed for the hot-wire profiles at the same location of the LDV profiles show the same form as the LDV profiles. At station 1, the flow angles seen as low as $-10^{\circ}$ for the LDV values could not be observed for the HW values, which are down to $-5^{\circ}$ only. The agreement for the other profiles is within the uncertainty limits.

The development of the flow angle is better observed with the computed values for the HW profiles on the left-hand side of the wing. Since the flow angle is positive, it is plotted in $\log (\mathrm{y} / \mathrm{t})$ vs $-1^{*}$ ( the flow angle) for easier comparison with the right-hand side profiles which are plotted with lines.

The first eight profiles including the eleventh station profile show semi-logarithmic variation in the whole layer up to $y / t \simeq 0.4$, starting from the minimum flow angle observed near the wall (Fig 64 ). In these profiles, the gradual decrease in the minimum observed proceeding downstream results in $\sim-33^{\circ}$ at station 11 . In the further downstream profiles, the starting point of the semi-logarithmic variation starts shifting up, and the minimum observed in the profiles close to this point starts decreasing in the magnitude. The minimum observed in tenth station was $\sim-32^{\circ}$ at $y / t \simeq 0.004$ and $\sim 9^{\circ}$ at $y / t \simeq 0.04$ at the first station. The end of the log regions are observed to be at $y / t \simeq 0.3$ including the fourth station and further downstream profiles, which results in shortened regions. 
The HW profiles on both sides of the wing show reasonable agreement except at station 5 . At this station the right-hand side profiles show flow angles $\sim 12^{\circ}$ less at the first 4 points in the profile. In the rest of the profile, the difference is $\sim 6^{\circ}$. In the other profiles, the difference is less than $\sim 6^{\circ}$ at the few points of discrepancy. This agreement is further validation of the symmetry of the flow around the body.

The presentation of the profiles in the FS coordinates shows the same type of variation in the layers ( Fig 65 ). The gradual decrease in the flow direction near the wall including the third station, gradually increases in the further downstream stations. Starting with the fifth station, the location in the layers where the minimum flow angle reached is observed as shifting up away from the wall. For the HW profiles taken on the left-hand side of the body, the near-wall flow angle is observed to be reducing until eleventh station ( Fig 66 ). The minimum at the eleventh station is $-23^{\circ}$. The profiles in the further downstream show a gradual increase not only near the wall but in the whole layers. The points where the minimums are reached in the profiles start shifting up with the tenth station profile, while the minimums themselves decrease in magnitude.

Most of the LDV and HW profiles in NS coordinates were observed to be on the positive side of the plots ( Fig 67 and Fig 68 ). This is due to the transformation applied. For the LDV profiles except at station 3 , the minimum of the profiles are seen to be very close to $0^{\circ}$. All the previously discussed characteristics of the flow angle profiles are also observed in this coordinate system, without any specific difference. 


\subsection{FLOW GRADIENT ANGLE}

Flow gradient angle was computed using the gradients computed perpendicular to the wall and

$$
\text { Gradient Angle }=\operatorname{Arctan}\left(\frac{\partial W / \partial y}{\partial U / \partial y}\right)
$$

relation. The gradients in the $\mathrm{y}$ direction were computed by fitting parabolas to the $\mathrm{W}$ and $\mathrm{U}$ velocity component profiles in that particular coordinate system at each successive five points in the profiles, and the derivatives were computed from the derivatives of the parabolas. The uncertainty of the gradient angle was computed using the estimated uncertainties on the individual gradients as $5 \%$ of the $\partial U / \partial y$ for $\partial U / \partial y$ and $10 \%$ of $\partial W / \partial y$ for $\partial W / \partial y$, and plotted as bars for each individual point. The quantities were computed only for the LDV profiles, since the main reason was to investigate if the gradient angle and shear stress angle were aligned with each other. The full stress tensor was only measured with the LDV.

In the first three profiles, while the gradient angle gradually decreases to $\sim-20^{\circ}$ below $y / t \simeq 0.005$, it is seen to be increasing up to $\sim 10^{\circ}$ above $y / t \simeq 0.02$ (Fig 69 ). The sudden increment between the two heights is seen to be at the beginning of the log layer for U/Uref. Within these profiles the very near wall and above $y / t \simeq 0.02$ values are seen to be nearly constant. At station 4 , while the values below $\mathrm{y} / \mathrm{t}=\sim 0.006$ are nearly constant around $\sim-30^{\circ}$, the peak value reached is $\sim 20^{\circ}$ at $y / t=\sim 0.02$. Further values in the profile are seen to be decreasing down to a 0 value at the layer edge. Starting with the fourth station profile, not only the away from wall values but also the near wall values are seen to be changing with the distance. After forming a lower peak, the reducing values very near the wall increase to form a peak on the positive side. Further in the profiles, the values drop down to $\sim 0$ value near the layer edge. While the magnitude of the lower and higher peaks increase in magnitude till station 6 , at station 7 , the lower peak is seen to be decreasing in magnitude, and the higher peak is observed to continue to increase. Within these stations, also the 
locations where the peaks are observed are seen to be shifting up in the profiles proceeding downstream.

Gradient angle in other coordinate systems show the same characteristics, since the only difference in the presentations are due to the shifts resulting by the transformation. The amount of shifts are same as free-stream and maximum normal stress point flow angles in the other two coordinate systems.

\subsection{SHEAR STRESS ANGLE}

Shear stress angle is the angle which the shear stress vector component in the $\mathrm{x}, \mathrm{z}$ plane makes with the $\mathrm{x}$ coordinate and is computed using

$$
\text { Stress angle }=\operatorname{Arctan}\left(\frac{\overline{v w}}{\overline{u v}}\right)
$$

Since the transformations into different coordinate systems only shift the plots the same as the rotation applied, the shear stress angle will only be discussed in the free-stream coordinates. The uncertainty analysis on the angles was done using the individual uncertainties for the $\overline{w v}$ and $\overline{v w}$ calculated in tunnel coordinates. Results of the analysis of the quantity is plotted as bars in the figures ( Fig 70 ). Exceptionally high uncertainties were mostly due to the uncertainties in the $\overline{v w}$ being on the order of $\bar{w}$ measured. This uncertainty and scatter in the data makes the judgment on the shape of the profiles difficult.

In the first two stations, the stress angle is seen to be fluctuating around $\sim 5^{\circ}$ zero. In station 1 , the increase up to $\sim 22^{\circ}$ till $y / t \simeq 0.02$ is followed with a region where the stress angle decreased to $-22^{\circ}$ at $y / t=0.04$. In the outer layer or above $y^{+}>250$, the stress angle was seen to be zero. At station 2 , the increase near the wall below $y^{+}=40$ was seen to have decreased in the log layer and 
seen to be around $7^{\circ}$ till the layer edge. At station 3, the stress angle reaches a peak of $\sim 30^{\circ}$ at $y / t \simeq 0.02$, which further reduces down to $\sim 5^{\circ}$ at $y / t \simeq 0.2$. The increase of the stress angles in the whole layers continue proceeding downstream. At station 4 , near wall values which reach a maximum of $\sim 73^{\circ}$ at $y / t \simeq 0.006$, reduce to $\sim 10^{\circ}$ at $y / t \simeq 0.01$. Further increase in the layer results in higher stress angles on the order of $\sim 30^{\circ}$ in the whole layer. The station 5 profile shows resemblance to the fourth station profile. The angles observed near the wall at station 6 show a minimum at $\sim 26^{\circ}$ at $y / t \simeq 0.005$. The almost semi-log increase in the profile results in a peak of $\sim 40^{\circ}$ at $y / t \simeq 0.04$. The variations in the profile after this point are similar to the variations seen in the previous two profiles. The seventh station profile shows a decrease in the stress angle in the whole layer. While the minimum reached in the profile is at $y / t \simeq 0.015$ at a value of $\sim-23^{\circ}$, the maximum is at $y / t \simeq 0.06$ with a value of $27^{\circ}$. The further decrease in the profiles result in stress angles $\sim 0^{\circ}$ at $\mathbf{y} / \mathrm{t} \simeq 0.3$.

Comparison between shear stress and flow gradient angle reveals that the shear stress angle is varying in the same form as the flow gradient angle in the layers, but is smaller in magnitude, i.e, lagging behind the flow gradient angle. The lags are more distinguishable below $y / t \simeq 0.01$ in the profiles. In the outer regions above $y / t \simeq 0.01$ in the zeroth station the angles are seen to be following each other. The difference generated at the first station is seen to be decreasing proceeding downstream. At station 3 while the angles below $y / t \simeq 0.01$ are following each other closely at station 4 , the outer region values are seen to be practically the same. Starting with station 5 , the station where the flow gradient angles show sharp changes, the stress angles are seen to be lagging more than the other stations. The lag at station 7 , at the outer region is seen to be $\sim 20^{\circ}$ 


\subsection{FLUCTUATION VELOCITIES AND SHEAR}

\section{STRESSES NORMALIZED WITH THE}

\section{SKIN-FRICTION VELOCITY}

Fluctuation velocities and shear stresses measured were non-dimensionalized with $u_{\tau}$ and $u_{\tau}^{2}$ respectively to see if the skin-friction velocity defined could be used as the parameter to collapse the individual profiles into one profile. The skin friction velocity is defined as $u_{4}=U_{e} \sqrt{\left(C_{f} / 2\right)}$, where $U_{e}$ is the layer edge velocity magnitude and $C_{f}$ is the skin-friction coefficient.

The $u^{\prime}$ profiles in the overall were seen to be reducing in magnitude proceeding downstream. At the sixth and seventh stations while the peaks near the wall reached were higher than the previous 2 stations, starting from $y / t \simeq 0.02$ the profile values were again to be reducing proceeding downstream ( Fig 71 ).

The $\mathrm{v}^{\prime}$ profiles in the first and last four profiles were seen to be collapsed on top of each other, but the difference between two groups was noticeable ( Fig 72 ). Same kind of structure was also observed for the $w^{\prime}$ profiles ( Fig 73 ).

While the first four station profiles of the $-\overline{u v}$ stresses nondimensionalized with $u_{\tau}^{2}$ show no match of the profiles, the last four station profiles show fair agreement in the whole layers ( Fig 74 ). Except at station 2, the nondimensionalized profiles are seen to be decreasing in magnitude in the whole layers proceeding downstream.

Within the first four profiles, $-\overline{u w} / u_{\tau}^{2}$ above $y / t \simeq 0.01$ are observed to be increasing in magnitude in the downstream direction ( Fig 75 ). Starting with the fourth station profile, the process reversed, and a decrease in the magnitude is seen. Due to the small magnitude of the $-\overline{v w} / u_{\tau}^{2}$ values and the 
form of the profiles, it was hard to judge on this quantity's profiles. While the first 4 profiles do not collapse on each other, the next three profiles are seen to be following each other closely. The last station is also seen to be not following these 3 profiles ( Fig 76 ). In general, the $u_{r}$ parameter could not be observed as the necessary parameter to collapse the fluctuating velocities and Reynolds stresses into one profile.

\subsection{EDDY VISCOSITIES IN X AND Z DIRECTIONS}

The eddy viscosity in the mean flow direction at the outer edge of the layer is computed using

$$
v_{x f s}=-\frac{\overline{w v} /(\partial U / \partial y)}{\rho \delta U_{e}}
$$

and the measured shear stresses, density, external velocity and boundary layer thickness and computed $\partial U / \partial y$, by fitting a parabola for each successive five points and taking the derivative of this parabola at the third location point.

For the first 4 stations, the profiles start with small negative values on the order of $\sim-0.000003$ below $y / t \simeq 0.009$ ( Fig 77 ). Above this height the $x$ eddy viscosity was observed to be positive and seen to be parabolically changing with its apex at $y / t \simeq 0.001$ and $v_{x}=-0.000003$ for the first four stations. In the last four stations the apex is of the parabola is $v_{x}=-0.000005$ at $y / t \simeq 0.001$. In all the stations, the maximum is reached at $y / t \simeq 0.2$ and is seen to be reducing further in the profiles. Also the last 4 profiles below $y / t \simeq 0.06$ follow each other closely.

The eddy viscosity in the $z$ direction of the FS coordinates is defined as

$$
v_{z j s}=-\frac{\overline{v w} /(\partial W / \partial y)}{\rho \delta U_{e}}
$$


In the first three stations since the $\mathrm{W}$ profiles are just starting to develop, the scatter in the computed profiles are high ( Fig 78 ). In the third station, even though the scatter is also high, the values are seen to be positive above $y / t \simeq 0.01$ and a semi-logarithmic variation till $y / t \simeq 0.07$ is observed. In the last four profiles except at station 7 , the profiles above $y / t \simeq 0.02$ were observed to be on the positive side. At station 7, the change in the sign of $\partial W / \partial y$ results in negative values to be computed besides the positive ones within the layer. Overall, the $v_{z f s}$ profiles seemed to have no specific form, and the changes in the $\partial W / \partial y$ and the small magnitude of the $-\overline{w w}$ stress are suspected to be the reasons for the scatter.

\subsection{MIXING LENGTH}

Mixing length is computed using

$$
L_{m}=\frac{\frac{\left(\frac{|\vec{\tau}|}{\rho}\right)^{1 / 2}}{\left[\left(\frac{\partial U}{\partial y}\right)^{2}+\left(\frac{\partial W}{\partial y}\right)^{2}\right]}}{\delta}=\frac{\frac{\left[(\bar{w})^{2}+(\overline{v w})^{2}\right]^{1 / 2}}{\left[\left(\frac{\partial U}{\partial y}\right)^{2}+\left(\frac{\partial W}{\partial y}\right)^{2 / 2}\right]^{1 / 2}}}{\delta}
$$

and presented in $L_{m} / \delta$ v.s. $\log (\mathrm{y} / \mathrm{t}$ ) coordinates ( Fig 79 ). The mixing length values were observed to be very close to zero very near the wall below $y / t \simeq 0.01$. The variation suggested by many researchers as $L_{m} / \delta=0.41^{*} \mathrm{y} / \delta$ near the wall is plotted with the lines in the figures, using the boundary layer thickness found for the zeroth station. This variation is observed within $y / t \simeq$ 0.02-0.07 of all the profiles. The suggested change as $L_{m} / \delta=0.09$ in the outer region could not be verified in most of the profiles. While the variation at station 0 the most upstream station is as $L_{m} / \delta=0.09$ in the other profiles, the values were either continuously increasing till the layer edge or leveling at values different than 0.09 . Some other length scales of the flow may be found in Table 6. 


\subsection{JOHNSTON POLAR PLOTS}

The existence of a relation between the lateral and axial components of the mean flow field was questioned by Johnston ( 1960 ). Johnston suggests the existence of two linearly varying regions for the mean velocity component in free-stream coordinates plotted in a polar plot; one for the inner region near the wall and one for the outer region. (Even though the idea was popularized by Johnston ( 1960 ), it was attributed to Gruschwitz ( 1935 ) by him. ) In the inner region

$$
\frac{W}{U_{e}}=\zeta \frac{U}{U_{e}} \quad \text { where, } \zeta=\tan \left(\beta_{w}\right)
$$

and in the outer region

$$
\frac{W}{U_{e}}=A\left(1-\frac{U}{U_{e}}\right) \text { where, } A=2 \beta_{F S}
$$

relations are supposed to hold. In his study Johnston ( 1960 ) found good agreement when he compared his own data (Johnston, 1957 ) of a two dimensional air jet forced to flow against a perpendicular back wall, Gruschwitz's ( 1935 ) data of well-developed, collateral, turbulent boundary layer becoming 3-D under the influence of a turning main flow, and the data of Kuethe, et. al. ( 1949 ) on a yawed wing of elliptical planform.

Figure $80 \mathrm{a}, \mathrm{b}$ show the polar plots of the present data and the suggested relations by Johnston. From the figure it is seen that, even though the relation seems to hold for the zeroth station, in the next four stations the location of the peak values are predicted at points closer to the wall in the profiles, and the magnitude of the peak values are overestimated as much as $20 \%$. While in the fifth station the position of the peak in the profile was correctly predicted the magnitude was underestimated. In the last two profiles neither the position nor the magnitude of the peaks were correctly estimated. The peak value at station 6 was $\sim 60 \%$ below the data. 


\subsection{TURBULENT KINETIC ENERGY}

Turbulent kinetic energy is computed with the relation,

$$
T K E=\frac{\left(\overline{u^{2}}+v^{2}+\overline{w^{2}}\right)}{2}
$$

The TKE profiles were nondimensionalized with the $U_{\text {ref. }}^{2}$ The equation which describes the transport of the TKE was given in the Introduction chapter. To be able to follow the change in the TKE within successive stations, the profiles at each four stations were plotted on top of each other ( Fig 81).

The plots at the first four profiles show that the change in TKE is not significant in these stations. In these profiles the increment near the wall up to $y / t \simeq 0.01$ goes to a value of 0.00575 and is followed by a region where it is seen to be constant around this value. Above $y / t \simeq 0.06$ the decrease until the layer edge is seen. In the next two profiles ( 4 and 5), the plateau region values are observed to be lower, $\sim 0.0053$. Last two station profiles are observed to be higher near the wall, but lower in the plateau region with respect to the previous two profiles. While the maximum reached in station 6 is 0.0062 at $y / t \simeq 0.008$, the maximum at station 7 at the same height is $\sim 0.007$. The plateau region for both profiles seems to be starting at $y / t \simeq 0.03$, and it is seen to be $\sim 0.00475$. The profiles show that TKE in the successive locations in the studied flow do not change significantly overall, which suggests that the production and dissipation at each location was on the same order, and convection is negligible. This can further be seen from the convection and production profiles obtained. 


\subsection{PRODUCTION OF TKE}

Production of TKE as given in the Introduction chapter is expressed with equation

$$
-\frac{1}{2} \overline{u_{i} u_{j}}\left(\frac{\partial U_{i}}{\partial x_{j}}+\frac{\partial U_{j}}{\partial x_{i}}\right)
$$

After expanding this equation, the derivatives with respect to $x$ and $z$ were neglected, since the data taken did not give chance to compute these derivatives. The resultant equation which was used to compute the profiles of TKE production terms reads as

$$
-\left(\overline{w w} \frac{\partial U}{\partial y}+\overline{v^{2}} \frac{\partial V}{\partial y}+\overline{v w} \frac{\partial W}{\partial y}\right)
$$

Production profiles were plotted as TKE Production over $U_{r e f}^{3}$ v.s. $\log (\mathrm{y} / \mathrm{t})$ coordinate systems ( Fig 82 ). The mean velocity derivatives and shear and normal stresses were the values computed in the FS coordinates.

All the profiles presented have the same form. Very near wall values in all the profiles are observed to be negative, and a very sharp rise to a positive peak value below $y / t \simeq 0.01$ is followed by a region where the production decays down to the zero value at the layer edge. The negative values near the wall are seen to be higher in magnitude than the positive peak values. The two positive peak values were observed to be higher than the others, and they are seen to be at station 2 and station 7 . While the peak at station 2 is $\sim 0.18$, it is $\sim 0.36$ at station 7 . In other stations peaks are observed to be between 0.08-0.12. 


\subsection{CONVECTION OF TKE}

Convection term in the transport equation for TKE as given in the Introduction chapter

$$
U_{j} \frac{\partial}{\partial x_{j}}\left(\frac{1}{2} u_{i} u_{i}\right)
$$

was further simplified to read as

$$
V \frac{\partial}{\partial y}\left(\frac{1}{2}\left(\overline{u^{2}}+v^{2}+\overline{w^{2}}\right)\right)
$$

and the plots of the profiles were done using this equation. Neglecting the derivatives other than in the y direction was necessary since not enough data were gathered to compute these derivatives.

Likewise for the production terms, the convection terms are also seen to be significant below $y / t \simeq$ 0.01 ( Fig 83 ). In all the profiles, the maxima reached near the wall seem to have decayed below this y point and are observed as close to zero. The only significant convection term observed above this height is at station 7 and is $\sim-0.006$.In all the profiles above $y / t \simeq 0.01$, the convection terms are between -0.004 and 0.003 values.

The comparison between production and convection terms show that the convection terms are an order of magnitude lower than the production terms. 


\subsection{PRODUCTION AND CONVECTION OF}

\section{NORMAL AND SHEAR STRESSES}

Production and convection terms of the governing equations for the transport of the normal and shear stresses were given in the first chapter. The production term in the tensor form for the $\overline{u_{i} u_{j}}$ component $(i, j=1,2,3)$ is given as

$$
-\left(\overline{u_{i} u_{l}} \frac{\partial U_{j}}{\partial x_{l}}+\overline{u_{j} u_{l}} \frac{\partial U_{i}}{\partial x_{l}}\right)
$$

and the convection term as

$$
U_{l} \frac{\partial}{\partial x_{l}}\left(\widetilde{u_{i} u_{j}}\right)
$$

The derivatives were computed in the manner previously described in several discussions. Since the derivatives with respect to $x$ and $z$ could not be computed due to the way the measurements were designed, the derivatives only in the y direction were considered. Due to this shortcoming, even though the discussions will be restricted, especially for the convection terms, since the mean velocity is a direct multiplier, it is believed that it may be useful for the stress production terms.

Within the profiles, $\overline{u^{2}}$ production is seen to be the highest ( Fig 84 ). Even though the production very near the wall is seen to be negative, a sharp rise to a positive peak was completed before $y / t$ $\simeq 0.01$. The peaks of the $\overline{u^{2}}$ production are seen to be of the same order of magnitude except at the zeroth and second stations. At station 0 , the production seems close to zero, and at station 2 the production is higher than the other stations in the whole layer. The profiles after the peak is reached are seen to be decaying to the zero production at the layer edge. 
The ordinate of the $\overline{v^{2}}$ production plots was chosen to be 40 times smaller than the $\overline{u^{2}}$ production plots. This was due to the small magnitudes observed, which in general makes the production of $\overline{v^{2}}$ insignificant ( Fig 85 ). The values in the profiles seem to be mostly negative except near the wall. The scatter of the data is mostly due to the small scale of the plots. The production of $\overline{v^{2}}$ is seen to be increasing in magnitude on the negative side proceeding downstream.

The $\overline{w^{2}}$ production is plotted with an absissa scale 4 times smaller than the $\overline{u^{2}}$ (Fig 86 ). In the first two profiles, while the production very near the wall seems positive and reaching to a peak, the values above $y / t \simeq 0.01$ are close to zero. Also in the next profile, while the production is $\sim 0$ above this height, near the wall the production is seen to be negative. In the next four profiles, while the production is negative below $y / t \simeq 0.01$, it is seen to be increasing in magnitude on the positive side for stations 3, 4 and 5. Starting with station 6, the magnitude observed in the whole layer is seen to be decreasing. In the seventh station, the production below $y / t \simeq 0.01$ is again seen to be positive and the values above this height is close to zero.

In all the profiles of $\overline{w v}$ production, the values are seen to be on the negative side ( Fig 87 ). In the first four profiles the values near the wall form a negative peak at around $y / t \simeq 0.006$ to 0.009 . In the next 4 profiles, values are observed to be reducing towards the wall. Further in the profiles, production decays down to a zero value at the layer edge. In the first profile, the peak observed is seen to be higher than the next three station peaks. The production of $\bar{w}$ in these four profiles near the wall and in the whole layers except at station 2 were seen to be comparable in magnitude to the production of $\overline{u^{2}}$. In the second station, $\overline{u^{2}}$ production was higher. In the next four profiles, the production was seen to be increasing in magnitude downstream. Also the production in these profiles were seen to be comparable to the $\overline{u^{2}}$ production terms in the same locations.

Within the first four stations, $\overline{w w}$ production profiles seen to be increasing in magnitude above $y / t$ $\simeq 0.01$ ( Fig 88 ). Below this point, they were observed to be negative. The peaks formed at stations 2 and 3 were seen to be located at $y / t \simeq 0.03$. While in the next two stations the peaks observed at same location are on the same order as seen in station 3 , a decrease in the peak at 
station 6 , is observed, and a decrease and a shift in the peak value and location is seen in station 7. The peak of seventh station profile is at 0.07 . In the near wall region, while all the values of the fourth station are positive, they are less than zero below $y / t \simeq 0.015$. At station 7 , the values below $y / t \simeq 0.04$ are negative, and this is seen the case for station 6 below $y / t \simeq 0.02$.

The $\overline{v w}$ production in the first four profiles were seen to be increasing in magnitude both below and above $y / t \simeq 0.008$ and proceeding downstream, in the whole layers ( Fig 89 ). The peak formed at station 3 was located at $y / t \simeq 0.02$. In the next four stations, the zero production height within the layer was seen to be shifting up in the layers proceeding downstream. While the maximum reached in the production near the wall increase within the first 3 locations, the last station shows a decrease in the peak reached. While the minimums reached at station 4 and 5 are on the same order, it is seen to be reducing in magnitude starting with station 6.

In all profiles obtained and for all the stresses, the convection terms computed were seen to be very close to zero above $y / t \simeq 0.01$. The abscissa of the scales of the convection term plots were at least an order of magnitude smaller than the corresponding production plots ( Fig 90). 


\subsection{LAW OF THE WALL CONCEPT}

\subsection{INTRODUCTION}

The existence of the similarity "Law-of-the-Wall" velocity profile in the form of $\frac{U}{u_{r}}=f\left(y u_{r} / v\right)$ in two dimensional turbulent boundary layers has been well established ( Schetz, 1984). The existence of such a similarity "Law-of-the-Wall" reduces the time necessary for the boundary layer computation codes to calculate the flows studied by reducing the domain of the calculations and gives the necessary information between the mean velocity profile and the wall shear stress. Existence of such a "Law-of-the-Wall" in three-dimensional boundary layers was investigated by several authors (Coles, 1956; Johnston, 1960; Hornung-Joubert 1963; Perry-Joubert, 1965; Pierce-Krommenhoek, 1968; Chandrashekhar-Swamy, 1975; East-Hoxey, 1969; White-Lessmann-Christoph, 1975; Van Den Berg, 1979; Pierce-McAllister-Tenant, 1982).

Nine existing Law-of-the-Wall similarity profiles are compared here with nine different sets of 3-D turbulent boundary layer data available to further investigate if such a law existed.The relations chosen included Coles, Johnston, Hornung-Joubert, Pierce-Krommenhoek, and Chandrashekhar-Swamy, East-Hoxey, Perry-Joubert, White-Lessmann-Christoph, and Van Den 
Berg. The pressure-driven experimental data were of Anderson and Eaton ( 1987a, 1987b) , Dechow-Felsch ( 1977a, 1977b), Müller (1982b), Fernholz-Vagt ( 1981, 1978 ), Elsenaar-Boelsma ( 1972, 1974), and the present data. The shear driven data were taken from Bissonnette-Mellor ( 1974 ) and Lohmann ( 1976 ). A summary of the flows included in this study, the techniques used to measure the stress and mean velocity in the flow and the skin friction at the wall may bay be found in Table 7. Before the comparison, all the data sets used were first expressed in free-stream coordinates, and, $\mathrm{U}$ and $\mathrm{W}$ in this chapter denote the mean velocity components in the $x_{F S}$ and $z_{F S}$ directions, respectively.

\subsection{LAW-OF-THE-WALL RELATION REVIEW}

\subsubsection{COLES RELATION}

The equation proposed by Coles in 1956 had the form of

$$
q \frac{\cos \left(\beta_{w}-\beta\right)}{u_{\tau}}=A \ln \left(\frac{y u_{\tau}}{v}\right)+B
$$

in which he assumed that (i) the velocity vector throughout the layer could be written as a sum. of near surface and wake vectors and (ii) the magnitude of the wake vector near the wall should be small, so that the direction of the near surface velocity vector should have the direction of the shear stress vector on the wall. He also presumed that the velocity vector component in the wall shear stress vector direction versus $y^{+}$would give the Law of the Wall. 


\subsubsection{JOHNSTON RELATION}

The second relation was proposed in 1960 by Johnston. His reasoning was based on his "triangular model" ( U/q vs. W/q, which was popularized by Johnston but attributed by him to Gruschwitz (1935) ) which points to the existence of a region near the wall where the flow angle is constant and the fact that the direction of the velocity vector in this region is coincident with the shear stress vector. Furthermore, he assumed that the fictituous velocity component in the direction of the shear stress direction defined as $\frac{U}{\cos \left(\beta_{w}\right)}$ obeyed the 2-D Law of the Wall of Clauser ( 1956 ), resulting in a Law of the form of

$$
\frac{U}{u_{\tau} \cos \left(\beta_{w}\right)}=A_{1} \ln \left(\frac{y u_{\tau}}{v}\right)+B_{1}
$$

He compared his Law of the Wall with the measurements of Gruschwitz ( 1935 ), Kuethe (1949) and his own data (Johnston, 1957 ) and found good agreement.

\subsubsection{HORNUNG-JOUBERT RELATION}

A third relation was proposed by Homung and Joubert. They wished to see if Clauser's ( 1956 ) Law of the Wall for 2-D turbulent boundary layers applied to 3-D turbulent boundary layers. They chose to nondimensionalize the magnitude of the velocity vector $\mathrm{q}$ with $u_{4}$. Their relation reads

$$
\frac{q}{u_{\tau}}=A_{2} \ln \left(\frac{y u_{\tau}}{v}\right)+B_{2}
$$

from which they had actually computed $u_{c}$ by fitting their measured velocity profiles with equation (3). They made no comparison using other data sets. 


\subsubsection{PIERCE-KROMMENHOEK AND}

\section{CHANDRASHEKHAR-SWAMY RELATIONS}

The Pierce-Krommenhoek ( 1968 ) and Chandrashekhar-Swamy ( 1975 ) relations have the same form for the streamwise velocity. The latter relation also has a Law of the Wall in the cross-wise direction. The validity of the 2-D Law of the Wall was assumed with the nondimensionalizing skin-friction velocity derived from the shear stress vector component in the streamwise direction. For the streamwise velocity, they propose

$$
\frac{U}{u_{\tau}\left(\cos ^{1 / 2}\left(\beta_{w}\right)\right)}=A_{3} \ln \left(\frac{y u_{\tau}\left(\cos ^{1 / 2}\left(\beta_{w}\right)\right)}{v}\right)+B_{3}
$$

The latter relation suggests

$$
\frac{W}{u_{\tau}\left(\sin ^{1 / 2}\left(\beta_{w}\right)\right)}=A_{4} \ln \left(\frac{y u_{\tau}\left(\sin ^{1 / 2}\left(\beta_{w}\right)\right)}{v}\right)+B_{4}
$$

for the cross-wise velocity component.

The main feature which distinguishes these relations from the previous three is that, the very near wall velocity vector direction is not assumed to be coincident with the wall shear stress vector direction, although $\beta_{w}$ is the direction of the shear stress vector at the wall with respect to the streamwise axis. When there were available data for the shear stress direction, this information was used for the $\beta_{w}$. Pierce and East ( 1972 ) and Kliensiek and Pierce ( 1973 ) state that their finite difference solutions very near the wall did not predict any collateral velocity region, but that the velocity vector changed direction down to the wall. 


\subsubsection{EAST AND HOXEY RELATION}

The relation proposed by East and Hoxey in 1969 can be defined as an effort to improve Johnston's relation. They reasoned that since the $U^{+}$in 2-D flows was a function of $y^{+}$, the same relation in 3-D flows would hold, taking into account the vectorial nature of the local velocity. Their relation reads

$$
\frac{U}{u_{\tau}\left(\cos \left(\beta_{0}\right)\right)}=A_{6} \ln \left(y^{+}\right)+B_{6}
$$

in which $\beta_{0}$ is a modified version of $\beta_{w}$. $\beta_{0}$ is derived from Johnston's $U, W$ polar plot. Johnston's polar plot in the viscous sublayer or close to the wall represents the cross-flow as $\frac{W}{U_{e}}=\frac{U}{U_{e}} \tan \left(\beta_{w}\right)$ and in the outer region as $\frac{W}{U_{e}}=A\left(1-\frac{U}{U_{e}}\right)$. The apex of the $\frac{W}{U_{e}}$ profile where these two relations meet can be found from the relation that $\left(\frac{q}{U_{e}}\right)_{a p e x}=K \frac{u_{r}}{U_{e}}$.

By using the trigonometric sine rule for the Johnston's triangular polar plot $\beta_{w}$ could be written as,

$$
\beta_{w}=\operatorname{Arcsin}\left\{\frac{\sin (\gamma)}{\left(K u_{\tau} / U_{e}\right)}\right\}-\gamma
$$

To compute the angle $\gamma$, East and Hoxey used the approximation that the whole polar plot could be represented only with the outer region using

$$
\begin{aligned}
\delta_{1} & =\int_{0}^{\delta}\left(1-\frac{U}{U_{e}}\right) d y \\
\delta_{4} & =\int_{0}^{\delta}-\left(W / U_{e}\right) d y \\
A & =\frac{-\delta_{4}}{\delta_{1}}
\end{aligned}
$$


they found $\gamma$ as

$$
\gamma=\operatorname{Arctan}(A)
$$

To reduce scatter in $\mathrm{K}$ from their data, which could be computed from the equation for $\beta_{w}$ above, they nondimensionalized the friction velocity with $U_{0}$ which was defined as the "working section reference velocity". The computed $\mathrm{K}$ from their data which is used is 19.45 .

The choice of one $\mathrm{K}$ and $U_{0}$ resulted in different values for the $\beta_{w}$ than the measured values. The equation they used is

$$
\beta_{0}=\operatorname{Arcsin}\left\{\frac{\sin (\gamma)}{\left(K u_{\tau} / U_{0}\right)}\right\}-\gamma
$$

The use of this angle instead of $\beta_{w}$ in Johnston's relation results in the East-Hoxey relation (eq.6).

\subsubsection{PERRY-JOUBERT RELATION}

The relation proposed by Perry and Joubert ( 1965 ) takes into account the effect of the pressure gradient near the wall region. They assumed that the near wall region could be treated as an equilibrium layer and that mixing length theory was valid. They further assumed that, the maximum shear stress acts in the same direction as the maximum rate of strain, which in turn assumes that the turbulence is isotropic. After algebraic manipulation of the momentum equations near the wall, their final equation reads 


$$
\begin{aligned}
\frac{H}{u_{\tau}} & =\frac{1}{u_{\tau}} \int_{0}^{y^{+}}\left\{1+\left(\frac{d w}{d u}\right)^{2}\right\}^{1 / 2} d u \\
& =\frac{1}{\kappa} \int_{0}^{y^{+}} \frac{1}{y^{+}}\left\{1-2 \cos \theta\left(\frac{\alpha v}{u_{\tau}^{3}}\right) y^{+}+\left(\frac{\alpha v}{u_{\tau}^{3}}\right)^{2} y^{+2}\right\}^{1 / 4} d y^{+}+\text {constant }
\end{aligned}
$$

in which;

$\alpha=\left[\left(\frac{\partial p}{\partial x}\right)^{2}+\left(\frac{\partial p}{\partial z}\right)^{2}\right]^{1 / 2}=$ pressure gradient

$\theta=$ the angle between the pressure gradient vector and the wall shear stress vector.

$\mathrm{H}=$ arc length on Johnston's polar plot.

They compared their Law of the Wall formulation with the Hornung-Joubert data and found good agreement.

\subsubsection{WHITE-LESSMANN-CHRISTOPH RELATION}

The relation proposed by White, Lessmann and Christoph ( 1975 ) also takes into account the effect of the pressure gradient on the streamwise velocity component. It was part of an integral method for the analysis of 3-D incompressible turbulent boundary layers. The key assumption in the development of the relation is that the shear stress component in the local free-stream direction could be written as a sum of the shear stress component at the wall in the local free-stream direction and the pressure force in the direction of the local free stream direction. The relation between the shear stress and velocity was established by the mixing length theory, yielding 


$$
\tau_{x} \simeq \tau_{x w}+\frac{1}{h_{1}} \frac{\partial p}{\partial x} y \simeq \rho \kappa^{2}\left|\frac{\partial U}{\partial y}\right| \frac{\partial U}{\partial y}
$$

The skin-friction velocity defined in the direction of the outer streamline is

$$
u_{\tau} \cos ^{1 / 2}\left(\beta_{w}\right)=\left(\frac{\tau_{w} \cos \left(\beta_{w}\right)}{\rho}\right)^{1 / 2}
$$

Equation (9) was integrated in the y direction to obtain

$$
U^{+}=\frac{1}{\kappa}\left[2\left(S-S_{0}\right)+\ln \left[\frac{(S-1)\left(S_{0}+1\right)}{(S+1)\left(S_{0}-1\right)}\right]\right]
$$

where,

$$
\begin{aligned}
S & =\left(1+\alpha y^{+}\right)^{1 / 2} \\
S_{0} & =(1+0.1108 \alpha)^{1 / 2} \\
\alpha & =\frac{v}{\rho\left(u_{\tau} \cos ^{1 / 2}\left(\beta_{w}\right)\right)^{3} h_{1}} \frac{\partial p}{\partial x}
\end{aligned}
$$

$S_{0}$ was chosen such that in the zero pressure gradient limit $U^{+}=\frac{1}{\kappa} \ln \left(y^{+}\right)+5.5$ would be obtained.

To supply the necessary $\mathrm{W}(\mathrm{y})$ velocity profile for the integral method, the unilateral hodograph proposed by Mager ( 1951 ) was used

$$
W^{+}=U^{+} \zeta\left(1-\frac{y^{+}}{\delta^{+}}\right)^{2}
$$

where,

$\zeta=\tan \left(\beta_{w}\right)$

$\beta_{w}=$ angle between the resultant surface shear $\tau_{w}$ direction with the streamwise direction. 
$h_{1}=$ metric coefficient since the derivative is taken along the streamwise direction. They compared the predictions of their Law of the Wall with the data of Kliensiek and Pierce ( 1970 ) and found only fair agreement.

\subsubsection{VAN DEN BERG RELATION}

The relation proposed in 1975 by Van Den Berg was designed to take into account both pressure gradient and inertial force effects. The relation was developed as a part of a calculation method for 3-D turbulent boundary layers. The relation assumes that mixing length theory holds outside the viscous sublayer and the shear stress direction coincides with the direction of the maximum rate of deformation in the region where the Law of the Wall is supposed to hold. The correction of the logarithmic Law of the Wall for the shear stress variation due to inertial effects was done using the log-law form in the equations of motion for thin layers.

Van Den Berg preferred to formulate his equations in a right-handed coordinate system in which $\mathrm{x}$ lies in the direction of the wall shear stress, $\mathrm{z}$ is perpendicular to this direction lying on the floor plane, and $\mathrm{y}$ is perpendicular to both directions. His final equations after algebraic manipulations and simplifications are

$$
\begin{gathered}
U_{x}^{+}=\frac{1}{\kappa}\left[\ln \left(y^{+}\right)+\frac{1}{2} \alpha_{x} y^{+}+\frac{1}{2} \beta_{x} \frac{\left(\ln \left(y^{+}\right)\right)^{2} y^{+}}{\kappa^{2}}\right]+B_{7} \\
U_{z}^{+}=\frac{1}{\kappa}\left[\alpha_{z}\left(y^{+}\right)+\beta_{z} \frac{\left(\ln \left(y^{+}\right)\right)^{2} y^{+}}{\kappa^{2}}\right]+B_{8}
\end{gathered}
$$

in which, 


$$
\begin{aligned}
\alpha_{x} & =\frac{v}{\rho u_{\tau}^{3}} \frac{\partial p}{\partial x} \quad ; \quad \alpha_{z}=\frac{v}{\rho u_{\tau}^{3}} \frac{\partial p}{\partial z} ; \\
\beta_{x} & =\frac{v}{u_{\tau}^{2}} \frac{\partial u_{\tau x}}{\partial x} \quad ; \quad \beta_{z}=\frac{v}{u_{\tau}^{2}} \frac{\partial u_{\tau z}}{\partial z} ; \\
U_{x}^{+} & =\frac{q \cos \left(\beta_{w}-\beta\right)}{u_{\tau}} \\
U_{z}^{+} & =\frac{q \sin \left(\beta_{w}-\beta\right)}{u_{\tau}}
\end{aligned}
$$

He compared his calculations from equations (12) and (13) with Van Den Berg and Elsenaar's measurements at two stations in terms of the flow angle $\phi$ relative to the wall shear stress angle which can be written as

$$
\phi=\frac{\alpha_{z}\left(y^{+}+\kappa \frac{B_{8}}{\alpha_{z}}\right)+\beta_{z}\left(\ln \left(y^{+}\right)\right)^{2} y^{+} / \kappa^{2}}{\ln \left(y^{+}\right)+\kappa B_{7}}
$$

and found good agreement within $8 \%$ up to $y^{+}=400$.

\subsection{EXPERIMENTAL DATA}

\subsubsection{ANDERSON AND EATON FLOW}

Anderson and Eaton at Stanford University studied flow around a wedge facing into the flow with a 90 degrees included angle ( Fig 91 ). The approach 2-D turbulent boundary layer was subjected to transverse streamwise pressure gradients which made it three dimensional. The freestream velocity in the inlet section was $16 \mathrm{~m} / \mathrm{sec}$. Data taken include measurements at five different locations which were on a free-stream streamline ( Fig 91 ). This flow was chosen such that a 
separation region near the wedge was avoided but yet it was representative of the highly skewing effects on the development of the shear layer ( Fig 92 ).The flow showed highly anisotropic features. The ratio of streamwise eddy viscosity to cross stream eddy viscosity $N=\frac{-(\overline{v w}) /(\partial W / \partial y)}{-(\overline{u v}) /(\partial U / \partial y)}$ changed from 0.1 to 1.0 Also the structural parameter of Townsend

defined as $A_{1}=\frac{\sqrt{\left(\overline{w^{2}}\right)+\left(\overline{v w^{2}}\right)}}{\left(\overline{u^{2}}+\overline{v^{2}}+\overline{w^{2}}\right)}$ was lower and not a constant of about 0.15 as in 2-D boundary layers.

\subsubsection{FERNHOLZ AND VAGT FLOW}

Fernholz and Vagt at the Technical University of Berlin studied 3-D flow created by a downstream back plate on a cylinder with an elliptical nose at a nominal speed of $18 \mathrm{~m} / \mathrm{sec}$ corresponding to $\frac{U_{\infty}}{v}$ of $1.23 \times 10^{6} / \mathrm{m}$ ( Fig 93). Data taken include mean velocities and six Reynolds stress tensor components along 3 generators of the cylinder at 24 locations. The streamwise pressure gradient was dominant in the development, so that the effect of the lateral pressure gradient was only observed in the downstream part of the test section. One important conclusion of this study was that the mean-velocity profiles near the wall showed skewing all the way to the wall ( Fig 92 ). It also seemed that the shear stress vector led the velocity gradient vector. As in Anderson's data, Townsend's structural parameter $A_{1}$ was not a constant of 0.15 .

\subsubsection{MÜLLER FLOW}

Müller studied an initially 2-D turbulent boundary layer on a flat plate subjected to lateral and streamwise pressure gradients produced by turning vanes, which caused wall skin friction lines to turn up to 50 degrees ( Fig 94 and Fig 92 ). The unit Reynolds number $\frac{U_{\infty}}{v}$ was $1.95 \times 10^{6} / \mathrm{m}$ throughout the measurements. Data included measurements of mean velocity and turbulent 
quantities at 21 locations. The data employed here include the measurements on a free-stream streamline which is closest to the separation line.

He concluded that the shear stress vector direction led the velocity gradient vector direction; the velocity continued to skew all the way to the wall. However, the structural parameter $A_{1}$ was approximately 0.15 as proposed by Bradshaw.

\subsubsection{DECHOW AND FELSCH FLOW}

Dechow and Felsch studied the 3-D turbulent boundary layers in front of a cylinder standing on a flat plate ( Fig 95 ). An initially 2-D turbulent boundary layer was subjected to the transverse and streamwise pressure gradients which caused three dimensionality ( Fig 92 ).The data included mean velocities and six Reynolds shear stress tensor components at 10 locations along a free-stream streamline, 2 on another streamline and on the centerline of the tunnel. Data used in the current investigation include 7 profiles which were upstream of the 3-D separation line. Their investigation also pointed out that the flow was anisotropic and that the stress vector direction lagged the velocity gradient vector.

\subsubsection{ELSENAAR AND BOELSMA FLOW}

Elsenaar and Boelsma studied an incompressible turbulent boundary layer on an infinite swept wing in an adverse pressure gradient ( Fig 96 ). A quasi-two dimensional boundary layer as found on an infinite swept wing was simulated on a flat plate, swept at an angle of 35 degrees. Measurements

were carried out at $\frac{U_{\infty}}{v}$ of $2.42 \times 10^{6} / \mathrm{m}$. Measurements included the mean flow and Reynolds shear stress tensor components at 8 locations ( Fig 92 ). 
The anisotropic character of the 3-D turbulent boundary layers was also noted in these measurements. The shear stress vector direction lagged behind the direction of the velocity gradient. The parameter $A_{1}$ decreased from 0.15 to 0.11 moving downstream; a value of 0.08 was observed in the wall region.

\subsubsection{BISSONNETTE AND MELLOR FLOW}

Bissonnette and Mellor studied 3-D turbulent boundary layers formed on a rotating cylinder with a sudden circumferential strain ( Fig 97 ). Measurements of the shear driven boundary layers were carried out at 8 stations with two different axial velocities. In the present study 4 stations were selected where the skin friction coefficients are available ( Fig 92). The cylinder used had a diameter of 5 inches and the Reynolds numbers based on the radius and the free-stream velocity were $4.16 \times 10^{6}$ and $7.95 \times 10^{6}$ for the low and the high Reynolds number cases, respectively. $W_{0}$ was kept constant throughout these measurements. It was observed by those authors that the mean rate of strain vector assumed a constant direction equal to the wall shear stress vector, which meant that the flow was collateral in the near wall region in a rotating frame of reference. It was also noted that $\mathrm{N}$ was less than 1 , which meant that the flow is anisotropic and shear stress vector direction is lagging behind the velocity gradient vector direction.

\subsubsection{LOHMANN FLOW}

Lohmann studied the 3-D turbulent boundary layers formed on a rotating cylinder ( Fig 98 ). He carried out the measurements at a free-stream velocity of $16.8 \mathrm{~m} / \mathrm{sec}$ at a nominal Reynolds number of $2.9 \times 10^{5}$ based on $25.4 \mathrm{~cm}$ diameter of the rotating body and free-stream velocity. Surface velocity to freestream velocity $W_{0} / U_{\infty}=1.65$. Data at 6 stations were used in the current analysis 
(Fig 92 ). This flow indicated that near the wall the flow was collateral and that Reynolds shear stress vector direction was leading the mean velocity gradient vector direction.

\subsection{RESULTS AND DISCUSSION}

Data taken from the nine selected data sets were plotted in the nondimensional coordinates of nine selected Law-of-the-Wall relations in order to investigate the applicability and the range of validity of these relations ( Fig 99-107). The range of validity of these relations is summarized in Table 8 . Figure 108 shows the difference of maximum and minimum values for each relation for the different sets of data at $y^{+}=70$, which is a point well out of the viscous sublayer and presumably within any semi-logarithmic region. Inner ticks show the difference when the least fitting profiles were excluded. This figure may also be interpreted as an evaluation of the method of skin friction measurement or the accuracy of the measured skin friction. Also, to see if the slopes of the curves predicted by each relation differed from one data set to the other, a plot of the scatter of the data at $y^{+}=40$ and at $y^{+}=100$ is shown in Figure 109.

The less complicated relations which do not include the effect of the pressure gradient near the wall are discussed separately for each flow.

All of the examined relations were seen to be equally applicable for the Elsenaar-Boelsma flow. The semi-logarithmic region was well defined for all relations. Based on the scatter of the data at $y^{+}=70$, the Hornung-Joubert relation was seen to be slightly better than the rest (Fig 108 ), with $A_{2}=2.37$ and $B_{2}=4.715$. ( Fig 100 ) shows a Johnston relation plot for comparison. Van Den Berg-Elsenaar had plotted the data in Clauser's 2-D Law-of-the-Wall coordinates with the velocity magnitude and had found good agreement. It was observed from ( Fig 101 ) that if the profile at 
the first position was omitted, the $y^{+}$range for Hornung-Joubert relation would extend up to $y^{+}=450$.

For Müller's data it was not easy to see the existence of a logarithmic region in the Chandrashekhar-Swamy and Pierce-Krommenhoek relations at stations D42, E53 and F65. Scatter of the $Q^{+}$at different $y^{+}$values for Coles and Hornung-Joubert relations was around $10 \%$ of the overall $Q^{+}$range of their relations. The Johnston relation was seen to be the most promissing for this flow for $20 \leq y^{+} \leq 200$. None of the relations except Coles' relation was able to predict a logarithmic region for station E53. Excluding this station, the Johnston relation ( Fig 100) may be approximately fit with $A_{1}=2.922$ and $B_{1}=4.557$. Müller ( $1982 \mathrm{~b}$ ) plotted his data using the $u_{\uparrow}$ that he computed using Spalding's formula with Coles' Law of the Wall constants vs. using $u_{\uparrow}$ he measured with the Preston tubes and found the difference to be about $10 \%$.

In most of the stations of the Fernholz-Vagt flow, the turning angle of the flow was less than $15^{\circ}$. Since the distinction between the proposed relations depended on how they treated this angle, all of the relations seemed to be performing equally well within $7.5 \%$ scatter among the profiles within each non-dimensional coordinate. The distinction was clear at the station St 802 where the flow angle was 22.5 degrees in turn. For this station Johnston's relation was able to include this station within $6 \%$ scatter for $15 \leq y^{+} \leq 60$ with $A_{1}=3.514$ and $B_{1}=1.367$.

Fernholz-Vagt ( 1981 ) discussed the possibility of a Law-of-the-Wall with three different relations including Hornung-Joubert, $\frac{U}{u_{4}}$ vs. $y^{+}, U / u_{\tau} \cos (\beta)$ vs. $y^{+} \cos (\beta)$, and Van Den Berg's Law of the Wall. They concluded that Hornung-Joubert relation was in much better agreement with the data than the others. They also favored the validity of use of the Law of the Wall for 2-D boundary layers in 3-D boundary layers.

For the Dechow-Felsch data, the flow skewed up to $50^{\circ}$, which resulted in a good test case for the relations. Even though the Coles and Johnston relations predict logarithmic regions, the profiles diverge from each other starting from $y^{+}=20$, with $20 \%$ scatter for Coles and $9.5 \%$ for Johnston 
of the overall range of these plots, $20 \leq y^{+} \leq 150$. Pierce-Krommenhoek and ChandrashekharSwamy relations predict a Law of the Wall for this flow except at station St 7. When this station is excluded, these relations show a good semi-logarithmic fit within $4 \%$ of their overall range.

The Hornung-Joubert relation shows a semi-logarithmic fit within $9 \%$ overall of the range including St7, in contrast to $11.5 \%$ scatter of the Chandrashekhar- Swamy and Pierce-Krommenhoek relations. Excluding station St 7 the latter relations produce a fit ( Fig 102 ) with $A_{3}=3.025$ and $B_{3}=3.06$. The Hornung-Joubert relation including St 7 fits the data with $A_{2}=2.502$ and $B_{2}=4.167 \leq 5.21$.

Due to the measurement techniques the Anderson flow did not have any data at less than $y^{+}=55$ which made it hard to investigate the range and applicability of the proposed relations. The flow had turning angles up to 45 degrees. For this flow, Coles, Hornung-Joubert, Pierce-Krommenhoek and Chandrashekhar-Swamy relations showed $37.5 \%, 20 \%, 18 \%$ and $18 \%$ scatter of the data, respectively, up to $y^{+}=150$. For the last station, S5, Coles' relation had almost zero slope. In this severe test case, Johnston's relation with $A_{1}=2.91$ and $B_{1}=3.357$ seemed to be the only one working up to $y^{+}=250$ with scatter of $12.5 \%$. Scatter up to $y^{+}=150$ was $7.5 \%$ ( Fig 100 ).

The East and Hoxey relation performs in an almost identical manner to Johnston's relation, but with a few exceptions. Even though it collapses Müller's data set better than Johnston's relation, it performs poorer for the other data sets. This may be due to the choice of $\mathrm{K}$ and $U_{0}$ constants. In their analysis, East and Hoxey also mentioned that different choices of $\mathrm{K}$ resulted in better agreement for the subsets of their own data ( Fig 104 ).

The rest of the discussion in terms of the simple Law-of-the-Wall relations deals with the shear-driven data in which the direction of the shear stress at the wall was used for $\beta_{w}$. For the Bissonnette-Mellor low Reynolds number data, the Coles and Hornung-Joubert relations did not work since the slope of Coles' relation was negative, and Hornung-Joubert relation was resulting in slopes close to zero, especially for the high Reynolds number data. Among the other proposed 
simple relations, Johnston's relation was superior. Plots of the data in Chandrashekhar-Swamy and Pierce-Krommenhoek relation coordinates had scatter of $18.5 \%$, in contrast to $11 \%$ of overall range of Johnston's relation ( Fig 100 ). Within a $y^{+}$range of $45 \leq y^{+} \leq 250$, Johnston's relation could be approximated with $A_{1}=1.78$ and $B_{1}=9.378$.

For the high Reynolds number data of Bissonnette-Mellor, the Coles' relation showed approximately zero slope, but the Hornung-Joubert relation was able to capture the semi-logarithmic region with a reasonable slope. The scatter of data was least in Johnston's relation coordinates, $7 \%$ of the overall range ( Fig 100 ) with $A_{1}=1.741$ and $B_{1}=7.554$. The scatter in the Pierce-Krommenhoek, Chandrashekhar- Swamy, and Hornung-Joubert relations was 9\%, 9\%, $16.5 \%$, respectively, in the overall range. Bissonnette-Mellor ( 1974 ) discussed the possibility of a law of the wall in a coordinate system of $\frac{\sqrt{U^{2}+\left(W_{0}-W\right)^{2}}}{u_{r}}$ vs. $y^{+}$in order to test the applicability of Clauser's proposition for such a flow. Even though Clauser's law of the wall did not fit the data, they still had shown existence of a Law of the Wall in such a coordinate system.

For Lohmann's data surprisingly none of the relations seemed to collapse the data. The Hornung-Joubert relation had approximately zero slope and scatter in Coles' coordinates was $\sim 90 \%$. Even though the remaining three simple relations merged the data into a Law of the Wall, the slopes of the individual profiles were different. If stations St 1 and St 2 were excluded, Johnston's relation was able to collapse the data within $60 \leq y^{+} \leq 150$ (Fig 100 ) and $A_{1}=2.182$ and $B_{1}=6.213$. Lohmann ( 1976 ) plotted his velocity data relative to the moving wall $W_{0}$, divided by friction velocity $u_{\uparrow}$ versus $y^{+}$and found good agreement except at stations 1 and 2 , where data followed a the Law-of-the-Wall for $40 \leq y^{+} \leq 80$ range only.

The Law of the Wall proposed by Chandrashekhar-Swamy for the W velocity component was observed as not working for the pressure-driven data ( Fig 103 ). For the low Reynolds number case, scatter was in $6.5 \%$ for the overall range and for the high Reynolds number case, scatter was in $11.5 \%$ ( Fig 103 ). 
Predictions of the Law-of-the-Wall relations which also include the effect of the pressure gradient near the wall are discussed below. The White-Lessmann-Christoph Law of the Wall was investigated only for the pressure driven data since in the limit of zero pressure gradient it returned back to the 2-D Law of the Wall. The W-L-C Law of the Wall employed the streamwise shear stress component at the wall to obtain the skin-friction velocity used to nondimensionalize the streamwise velocity component. The $Q^{+}$defined in this manner was the same as the Chandrashekhar-Swamy or Pierce-Krommenhoek laws of the wall. For the profiles in which $\alpha y^{+}$ was below (-1), it was not possible to compute the square-root terms in equation (10). This kind of profiles were observed for Anderson's fourth and fifth stations and Dechow's sixth and seventh stations, which were excluded from the comparison. For Dechow's data set $Q^{+}$vs. $\log _{10}\left(\frac{y}{v} \sqrt{\tau_{w} \cos \left(\beta_{w}\right) / \rho}\right)$ variables performed better than Johnston's relation ( Fig $106 \mathrm{a}$ ).

The computed values for the streamwise velocity component from the derived W-L-C equation (10) measured $Q^{+}$seemed to overlap each other reasonably well, but only in a short $y^{+}$range for the Dechow, Anderson, and Elsenaar-Boelsma flows. For Müller's data, computed values could not resemble the wide range of $Q^{+}$observed, and, for Fernholz-Vagt data, the resemblance was in a very short range of $y^{+}$( Table 9 ). W-L-C method for the shear driven data for the U component was seen to be working as good as the Perry-Joubert or Johnston relations. Scatter of the data at $y^{+}=70$ was $6.25 \%, 3.9 \%, 2.86 \%$, for Bissonnette-Mellor, low and high Reynolds number cases and Lohmann flow respectively of the overall $Q^{+}$range of 25 .

The lateral flow component nondimensionalized with the same skin-friction velocity used for the streamwise component versus $\log _{10}\left(\frac{y}{v} \sqrt{\tau_{w} \cos \left(\beta_{w}\right) / \rho}\right)$ plots showed that the choice of these variables did not produce a Law of the Wall for the crosswise velocity. The computed values for the crosswise velocity in terms of the wall law variables versus $\frac{W}{\left(u_{\tau}\left(\cos ^{1 / 2}\left(\beta_{w}\right)\right)\right)}$ were much higher than the data (Fig $106 \mathrm{c}$ and $\mathrm{d}$ ).

The Perry-Joubert relation was also designed to take into account the effect of the pressure gradient. The arc length $\mathrm{H}$ in the polar plot of Johnston, nondimensionalized with the skin-friction velocity, 
vs. $y^{+}$could be tested separately for the shear-driven data. For the pressure-driven and shear-driven data these variables were seen to be performing as well as Johnston's Law of the Wall. For Dechow's data set, the performance was the same as the W-L-C relation which was also better than Johnston's relation. The computed values with the use of, $\theta, \alpha, u_{\uparrow}$ and $y^{+}$were compared with the $Q^{+}=\frac{H}{u_{r}}$ values discussed above. In a limited $y^{+}$range, a match of $Q^{+}$with the computed values were observed ( Table 9 ). Also see (Fig $105 \mathrm{a}$ and b ).

Van Den Berg chose the velocity component in the direction of the wall shear stress and the component perpendicular to it in the $\mathrm{x}-\mathrm{z}$ plane to be nondimensionalised with the $u_{r}$. This equation in the shear stress direction reads the same as Coles' Law of the Wall. The comparison was done only for the pressure-driven data sets, since shear-driven flows cannot be described, as was the case for Coles' Law of the Wall.

The comparison for the computed values vs. the variables $U_{x}^{+}$and $U_{z}^{+}$(equations 12 and 13 ) was done for $\beta_{x}=\beta_{2}=0$, since the extraction of these terms from the data necessitated either more information or the neglect of some terms whose order of magnitude could not be determined. For the Fernholz-Vagt data $\beta_{x}$ and $\beta_{z}$ were computed by neglecting the terms mentioned above, with little effect on the computed $U_{x}^{+}$and $U_{z}^{+}$.

For the Van Den Berg relation, it was observed from these comparisons that computed and measured values overlapped within a limited $y^{+}$range for all data sets. For the Fernholz-Vagt data, the $y^{+}$range of agreement between computed and measured was very short ( Table 9 ). Because measured values of $U_{z}^{+}$are small by definition, it is difficult to judge agreement with this relation. The constant $B_{7}$ was found to be $\sim 4.7$ and $B_{8} \sim 0.15$.

The Law-of-the-Wall search for the data presented in this study was plotted separately as all the nine Law of the Walls for this particular data set ( Fig 110). This was made to see the effect of the uncertainties in the data in judging the performance of the Law-of-the-Wall relations. The uncertainty analysis was carried out by using the previously discussed analysis used in Chapter III 
, and the uncertainties in each quantity. The data used was of the last 6 stations of the LDV data where the skin friction values at the wall were measured.

For this data set, within the simple Law-of-the-Wall relations Chandrashekhar- Swamy and Pierce-Krommenhoek relation seen to be working better than the others if all the profiles are included. The logarithmic region within $y^{+}=40$ to 300 was clearly observable. The constants A3 and B3 were read as, $A_{3}=1.514, B_{3}=8.98$.(eq.4). Second best fit belongs to the Johnston relation. This was observed when the least fitting third station profile was omitted. $A_{1}, B_{1}$ were read as $A_{1}=1.93, B_{1}=8.33$. The free-stream component of the other relations were seen to be less satisfactory. W component of Chandrashekhar-Swamy relation was seen as not working (Fig $110)$.

Amongst the relations which include the pressure correction terms, the Perry-Joubert relation (left-hand side of eq. 8) was seen as superior. Due to higher uncertainties observed, it was less superior to Pierce-Krommenhoek relation. The match of the left-hand side and right-hand side of (eq.8) was within a short range of $y^{+}$, from 20 to 70 . Due to the $\alpha y^{+}$term in (eq.10) being less than $(-1)$ in all stations except station 2, the White- Lesmann-Christoph relation profiles could not be fully plotted. Within the available points, only station 2 was observed to be matching the left and right hand sides of (eq.10) until $y^{+}=2000$, within the uncertainty bands. The computed $W^{+}$values from data and Mager's formulation for defining the same quantity in terms of $U^{+}$were seen to be not matching. The left-hand side of the Van Den Berg relation (eq.12), which is same as of the Coles relation and, the right-hand side of the same equation was seen to be not overlapping, except for station 7. For this station, an overlap region within $y^{+}=40$ to 700 with $\mathrm{B} 7=12.5$, was observed. The $W^{+}$component proposed in the last relation was seen as not working, since the both sides of the (eq.13) did not overlap for any profiles.

To compare the Law-of-the-Wall relations, the computed quantities at abscissa coordinates of 40 , 70,100 and 250 of each relation at each station, were plotted separately ( Fig 111 ). The uncertainties on the quantities were shown with bars. If the proposed relation is perfectly working, 
the data for each $y^{+}$, for all stations, should be computed as same.The W-L-C relation, since there were not enough computed values, was not included into this plot.The Van Den Berg relation was also excluded, since, it was same as the Coles relation, for the left-hand side of the $U$ component (eq. 12 ). From this plot it was seen that, the Pierce-Krommenhoek relation was superior to the others. Eventhough Perry-Joubert relation was seen as working as good as the previous relation, the uncertainty band was bigger; and the Johnston relation needed station 3 data to be excluded.

Overall, including all data sets, for the $U$ component of the velocity, it was observed that the Johnston and Perry-Joubert laws of the wall were superior to the others based on ( Fig 108, Fig 111 ). The functions which took into account the pressure gradient effect in the W-L-C, Perry-Joubert, and Van Den Berg law of the wall relations were seen to perform well for some profiles up to $y^{+}=1000$, but overall the predictions agreed with data only over short ranges of $y^{+}$. Between the Johnston and Perry-Joubert relations, Johnston's relation performs better in 6 of the 9 data sets used.

The $\mathrm{W}$ component of velocity could not be represented with any of the Laws-of-the-Wall presented. Mager's method presented in the W-L-C scheme consistently estimated higher values than the data, with up to 100 percent difference. The Van Den Berg relation resulted in much different values than the data for $y^{+}>30$, except for the Elsenaar-Boelsma's data in which the prediction was good up to $y^{+}=200$. The Chandrashekhar-Swamy relation produced very large scatter of the data except for Bissonnette-Mellor data ( Fig 103 ). If there has to be a choice made among these three methods, Mager's method might be suggested (Fig $106 \mathrm{c}$ and d). 


\subsection{TURBULENCE MODELING}

\subsection{INTRODUCTION}

This chapter describes the performance of selected turbulence models in simulating the shear stress data.The data used were from the same data sets gathered for the investigation of the existence of the Law-of-the-Wall velocity profile for three-dimensional boundary layers, except the Fernholz-Vagt data, since $\overline{v w}$ data were not available. Turbulence models selected were chosen among the models which did not necessitate solving the governing equations. This restricts the discussion to algebraic eddy viscosity models.

The comparison of the computed and experimental data was performed using two parameters. The shear stress vector in the plane parallel to the floor with components of $\overline{-U v}_{F S}$ and ${\overline{-v w_{F S}}}_{\text {can }}$ be expressed using complex numbers in the $|\tau| e^{i \alpha}$ form, where $|\tau|=\left[\overline{(-u v)_{F s}^{2}}+\overline{(-v w)_{F s}^{2}}\right]^{1 / 2}$ and $\alpha$ is the shear stress angle. The ratio of the measured and computed shear stresses presented in the complex form give two parameters: 


$$
\begin{aligned}
\frac{|\tau|_{m}}{|\tau|_{c}} & =\text { Magnitude ratio } \\
\alpha_{m}-\alpha_{c} & =\text { Direction angle difference }
\end{aligned}
$$

where $\mathrm{m}$ denotes the measured quantities and $\mathrm{c}$ stands for the computed values. If the model was able to predict the magnitude and the direction of the measured shear stress vector data perfectly, the ratio of magnitudes would be 1 , and the difference between the shear stress directions would be zero.

All the computations were carried out using the data in free-stream coordinates, and $\mathrm{U}$ and $\mathrm{W}$ in this chapter denote the mean velocity components in the $x_{F S}$ and $z_{F S}$ directions, respectively. Except at the zeroth and first stations of the present data, the shear stresses at the wall were taken from the data sets included in this study. At station 0, the wall stress was found using the Clauser 2-D Law of the Wall, and at station 1, it was computed with Johnston's 3-D Law of the Wall. The required mean flow quantities were also taken from the data, as if the solutions of the governing equations were same as the data.The necessary maximum shear stress magnitudes in the layers for the Johnson-King model at each station were taken as the maximum shear stress magnitudes which satisfy the realizability conditions. Also since the comparison is done in free-stream coordinates, the necessary experimental shear stresses which were expressed in a coordinate system other than free-stream coordinates were transformed into the free-stream coordinates using the tensor transformation in Appendix III. This was needed for the Dechow and Elsenaar-Boelsma data. 


\subsection{SELECTED ALGEBRAIC TURBULENCE}

\section{MODELS}

\subsubsection{CEBECI-SMITH MODEL}

The Cebeci-Smith model used in this study is the one described by Cebeci ( 1984 ). The model uses two different eddy-viscosity definitions, one described for the inner region and one for the outer region.

In the inner region, the eddy-viscosity is defined as

$$
\begin{aligned}
v_{t i} & =F^{2} l^{2}\left[\left(\frac{\partial U}{\partial y}\right)^{2}+\left(\frac{\partial W}{\partial y}\right)^{2}\right]^{1 / 2} \\
l & =\kappa y \\
F & =1-\exp \left(-y^{+} / A^{+}\right) \\
y^{+} & =\frac{y u_{\tau}}{v} \\
u_{\tau} & =\left(\frac{\tau_{w}}{\rho}\right)^{1 / 2} \\
\tau_{w} & =v\left[\left(\frac{\partial U}{\partial y}\right)_{w}^{2}+\left(\frac{\partial W}{\partial y}\right)_{w}^{2}\right]_{w}^{1 / 2} \\
A^{+} & =26 \quad \kappa=0.4
\end{aligned}
$$

in which $l$ is the mixing length, and $F=\left[1-\exp \left(y^{+} / A^{+}\right)\right]$is the van Driest damping function.

The outer region eddy-viscosity is given by

$$
v_{t o}=0.0168 \delta_{2}^{*} \gamma_{k} U_{e}
$$




$$
\begin{aligned}
& \delta_{2}^{*}=\left|\int_{0}^{\infty}\left(1-\frac{\left(U^{2}+W^{2}\right)^{1 / 2}}{U_{e}}\right) d y\right| \\
& \gamma_{k}=\left[1+5.5\left(\frac{y}{\delta}\right)^{6}\right]^{-1}
\end{aligned}
$$

where $\gamma_{k}$ is Klebanoff's intermittency correction, and $\delta_{i}^{*}$ is the displacement thickness. The boundary layer thickness $\delta$ is defined as the point in the layer where $\left(U^{2}+W^{2}\right) / U_{e}^{2}$ is 0.99 . By using a smoothing function, the eddy-viscosity distribution in the layer can be defined as:

$$
v_{t}=v_{t o}\left[1-\exp \left(-v_{t i} / v_{t o}\right)\right]
$$

and the shear stresses are found by using:

$$
-\overline{u v}_{F S}=v_{t} \frac{\partial U}{\partial y},-\overline{v w}_{F S}=v_{t} \frac{\partial W}{\partial y}
$$

\subsubsection{ROTTA'S MODEL}

The anisotropic eddy-viscosity model used is based on work by Rotta ( 1979 ). An analysis of the pressure strain terms in the governing equations for the stresses led Rotta to an anisotropic eddy-viscosity model ( Rotta, 1977 ). The model uses anisotropy constant $\mathrm{T}$ defined as

$$
T=\frac{\left(v_{T}\right)_{\text {transverse }}}{\left(v_{T}\right)_{\text {streamwise }}}
$$

the ratio of the transverse eddy-viscosity to the streamwise eddy-viscosity in local free-stream coordinates.

By assuming $T$ constant in the layer, $v_{T}$ in the free-stream coordinates can be computed as 


$$
v_{T}=F^{2} l^{2}\left[\left(\frac{\partial U}{\partial y}\right)^{2}+\left(\frac{\partial W}{\partial y}\right)^{2}+\frac{(T-1)\left(W \frac{\partial U}{\partial y}-U \frac{\partial W}{\partial y}\right)^{2}}{U^{2}+W^{2}}\right]
$$

and the stresses are related to the mean flow gradients with

$$
\begin{aligned}
v_{t} & =v_{t o}\left[1-\exp \left(-v_{T} / v_{t o}\right)\right] \\
-\overline{w v}_{F S} & =v_{t}\left(a_{x x} \frac{\partial U}{\partial y}+a_{x z} \frac{\partial W}{\partial y}\right) \\
-\overline{v w}_{F S} & =v_{t}\left(a_{x z} \frac{\partial U}{\partial y}+a_{z z} \frac{\partial W}{\partial y}\right)
\end{aligned}
$$

where

$$
a_{x x}=\frac{U^{2}+T W^{2}}{U^{2}+W^{2}} \quad, a_{z z}=\frac{W^{2}+T U^{2}}{U^{2}+W^{2}} \quad, a_{x z}=(1-T) \frac{U W}{U^{2}+W^{2}} \quad, \quad l=\kappa y
$$

In this study, $v_{T}$ defined by Rotta was used as the inner layer eddy-viscosity and the outer layer eddy-viscosity was kept the same as the Cebeci-Smith model. For the pressure-driven flow data, three different anisotropy constants, $\mathrm{T}=0.3, \mathrm{~T}=0.5$ and $\mathrm{T}=0.7$, were tested. For the shear-driven data, since the use of the constant less than 1 increased the difference between the measured and computed shear stress magnitude, $T=1.2$ and $T=1.5$ were also applied.

\subsubsection{PATEL'S MODEL}

The third model selected is the one equation (k) model of Wolfshtein ( 1969 ) as used by Chen and Patel ( 1988 ) in the $k-\varepsilon$ turbulence model with the fully elliptic Reynolds-averaged Navier-Stokes equations to compute the flow characteristics in the boundary layer or wake of axisymmetric bodies. The eddy-viscosity in this model is defined as: 


$$
\begin{aligned}
v_{t} & =c_{\mu} \sqrt{k} l_{\mu} \\
l_{\mu} & =c_{\nu} \nu\left[1-\exp \left(-R_{y} / A_{\mu}\right)\right] \\
c_{l} & =\kappa c_{\mu}^{-3 / 4} \\
R e & =U_{r e f} L / v=\text { Reynolds number } \\
R_{y} & =\operatorname{Re} \sqrt{k} y=\text { Turbulent Reynolds number } \\
L & =\text { Body length }, A_{\mu}=70, c_{\mu}=0.09, \kappa=0.4, k=T K E=\frac{\frac{u^{2}}{v^{2}}+\frac{w^{2}}{2}}{2}
\end{aligned}
$$

and the stresses in cartesian coordinates could be computed using ( Stern, Yoo and Patel, 1988)

$$
\begin{aligned}
\overline{u_{i} u_{j}} & =-2 v_{T} S_{i j}+2 k \delta_{i j} / 3 \\
S_{i j} & =\frac{1}{2}\left(U_{i, j}+U_{j, i}\right) \\
\delta & =1 \text { if } i=j \\
& =0 \text { if } i \neq j
\end{aligned}
$$

After neglecting the derivatives other than those with respect to $\mathrm{y}$, the stresses in this study are computed using

$$
\overline{w_{F S}}=-v_{t} \frac{\partial U}{\partial y} \quad, \quad \overline{w_{F S}}=-v_{t} \frac{\partial W}{\partial y}
$$

The validity of the equations for the turbulence model as given was defined to be restricted to the viscous sublayer, buffer layer, and a part of the fully turbulent layer. Therefore, the comparison with the data is only meaningful below $y^{+}=150$ ( Patel and Chen, 1987 ). Since the model length used in some of the experiments did not exist, to be consistent within the analysis carried out, the body length $\mathrm{L}$ was kept as $1 \mathrm{~m}$ for all the data sets. 


\subsubsection{JOHNSON-KING MODEL}

Eddy viscosity model introduced by Johnson and King ( 1984 ) for 2-D flows subject to strong pressure gradients and separation was extended to 3-D flows by Abid ( 1988 ).

Instead of using the wall skin friction as the Cebeci-Smith model does, the model utilizes the maximum shear stress in the layer to define the eddy viscosities and the Van Driest damping function which is effective near the wall. The inner layer eddy viscosity, which has the same form as Cebeci-Smith model, differs due to the use of the maximum shear stress in the layer. The model is defined as follows:

$$
\begin{aligned}
v_{t i} & =F^{2} l\left(\frac{\tau_{M}}{\rho}\right)^{1 / 2} \\
F & =1-\exp \left(-y \frac{\left(\tau_{M} / \rho\right)^{1 / 2}}{v A^{+}}\right) \\
l & =\kappa y \\
\frac{\tau_{M}}{\rho} & =\left(\overline{u v}^{2}+\overline{v w}^{2}\right)_{\max }^{1 / 2} \\
A^{+} & =15
\end{aligned}
$$

The outer eddy viscosity was also modified to take into account the effect of the maximum shear stress in the outer layer. Outer layer eddy viscosity is defined as:

$$
v_{t o}=\sigma(0.0168) \gamma_{k} \int_{0}^{\infty}\left(V_{e}-V\right) d y
$$

where $\sigma$ is found when the relation

$$
\left(\frac{\tau_{M}}{\rho}\right)=v_{t}\left[\left(\frac{\partial U}{\partial y}\right)^{2}+\left(\frac{\partial W}{\partial y}\right)^{2}\right]_{\max }^{1 / 2}
$$


is satisfied at the location in the layer where the shear stress is maximum. Once $\sigma$ is found the shear stresses are found using

$$
\overline{w v}=-v_{t} \frac{\partial U}{\partial y} \quad, \quad \overline{v w}=-v_{t} \frac{\partial W}{\partial y}
$$

where

$$
v_{t}=v_{t o}\left(1-\exp \left(-v_{t i} / v_{t o}\right)\right)
$$

Even though the maximum shear stress magnitude was found using an ordinary differential equation derived from the T.K.E. equation, which is valid along a path where the shear stress is maximum, in this study it was assumed that this equation could exactly compute the maximum shear stress. The location of it in the layer which would be found once the governing equations are solved was also assumed to be found accurately. Once these assumptions are made, the constant multiplier $\sigma$ in the $v_{t o}$ equation was found by Newton iteration and by using the experimental $\tau_{M}$ and mean flow gradients. For the Bissonnette-Mellor low and high Reynolds number data and at first and seventh stations of the present data, $\sigma$ could not be computed, since the iteration did not converge. At these stations $\sigma$ was kept as 1 .

The necessary mean flow gradients to calculate computed shear stresses for each flow were found by the same parabola fitting program used before.

\subsection{RESULTS AND DISCUSSION}

The comparison of the computed and measured stress magnitudes and angles for the present data are presented using $\log (y / t)$ as the abscissa of the plots, to be in accordance with the presentations of the other flow variables. However, to distinguish the different regions in the layers different 
symbols corresponding to inner, logarithmic and outer regions were used. Also the uncertainties in the variables were found using the uncertainties in each experimental shear-stress and assuming that the computed shear-stresses did not induce any uncertainty. These uncertainties are plotted as bars at each point. The uncertainty of the magnitude ratio for this flow was also used to eliminate the data points which had uncertainties too high to be acceptable. The data presented are the ones which have uncertainties less than \pm 1 and which satisfy the realizability conditions ( Figs $112-117)$.

The Cebeci-Smith model at the zeroth station of the present data set seems to underpredict the magnitude of the computed shear stresses ( Fig 112 ). Since this station closely resembles a 2-D flow and since the model was developed using available 2-D data, the ratio of stresses should be 1 . These high shear stresses observed are attributed to the uncertainties, and the ratio of magnitudes is 1 within the limits of the uncertainty in most of the log and outer regions. The direction difference is zero at this station within the uncertainty limits. Even though station 1 plots show scatter, the magnitude ratio was close to one and stress direction difference was again seen to be zero within the uncertainty bands. Station 2 profiles show higher $\left|\tau_{m}\right|$ within the inner region and part of the $\log$ region. Although including the uncertainty bands the magnitude ratio is still 1.0 , the values were seen to be $\simeq 1.2$ in the part of the log and outer regions. The direction difference at this station was seen to be decreasing down to $0^{\circ}$ at the log region starting from a value of $\simeq 60^{\circ}$ in the near wall region. At station 3 even though the end of the log region and beginning of the outer region magnitude ratio values are close to 1 including the uncertainty bands; overall, a decrease was observed. Even though the uncertainty in the angle difference is increased, the quantity is close to zero in the whole layer. Station 4 magnitude ratios resemble station 3 values. The inner layer and part of the outer layer ratios were seen to be less than 1 , however, most of the log region values were 1 within the uncertainty bands. Angle difference at this station reaches to $100^{\circ}$ near the wall which decreases down to zero in the log region. The near wall values of the magnitude ratio at station 5 are seen to be gradually increasing from a value of $\simeq 0.2$ at the beginning of log region, which was also observed at station 4 . The end of the log region and outer region values were seen 
to be 1.0 within the uncertainty bands. The high angle difference observed at station 4 is seen to be reduced to $\simeq 60^{\circ}$ near the wall by station 5 . At station 6 the decrease of magnitude ratio near the wall which started at station 3 is most visible. The low, near wall values reach a maximum at $y / t \simeq 0.05$ with a value of $\simeq 1.65$. The outer region values were seen to be $\simeq 1$. 1 . The angle difference was seen to form a peak at $y / t \simeq 0.015$ at a value of $\simeq 60^{\circ}$ and a lower peak at $y / t \simeq 0.08$ at $-25^{\circ}$. Station 7 values show the same characteristics of sixth station. While the log region values are close to $\simeq 0.9$, outer region values of the magnitude ratio are around 1.1 . The angle difference at this station was reduced, and while the difference in the inner and $\log$ regions were $\simeq 20^{\circ}$, outer region values were $\simeq-20^{\circ}$. Overall it was observed that the Cebeci-Smith model resulted in lower magnitude ratios near the wall and higher magnitude ratios in the outer region, and the shear stress direction difference was most noticable in the near wall and beginning of log regions.

Rotta's model with $\mathrm{T}=0.3$ seems to be mostly effective in raising appreciably the magnitude ratios in the $\log$ region, in the range $y / t \simeq 0.01-0.1$, (Fig 113 ). This effect is seen with the first station, however the effect at this station was small. At station 2 , the log region values were seen to be shifted up as much as 0.1 , but the effect was reduced at the outer region. This is due to the use of $v_{T}$ only in the inner region. Very near wall values were also less affected than the log region values. At station 3 , the shift at $y / t \simeq 0.03$ was $\simeq 0.4$, which gave a magnitude ratio of 1.5 at this location. In the log layer, reduction of the computed shear stress magnitude results in values of more than 1. The stress vector direction difference at these two stations was also seen to be bigger than zero in the $\log$ and outer regions, opposite to values observed for the Cebeci-Smith model including the uncertainties. The increment in the magnitude ratios for stations 4 and 5 was similar to the previous two stations. While the maximum shift in the magnitude ratio at station 4 was $\simeq 0.5$, it was $\simeq 0.7$ at station 5. Even though the magnitude ratio for the outer region of station 4 was $\simeq 1.0$, at both stations while the near wall ratios were less than 1, the logarithmic regions had ratios on the order of $\simeq 1.4-1.8$. The outer region values of station 5 were reduced to the ratio of 1 , but were higher than Cebeci-Smith model results. At station 4, while the very near wall direction difference was $\simeq 100^{\circ}$, it was seen to be $\simeq 30^{\circ}$ in the log and outer regions. At station 5 , the difference was seen to be 
reduced to $\simeq 25^{\circ}$ from $\simeq 50^{\circ}$ of near wall difference. The Cebeci-Smith model at these two stations seemed to be predicting the stress vector direction in the log and outer regions. In the upper part of the log region and the lower part of outer region, the magnitude ratio increase at the next two stations was also accompanied with a direction difference increase. While the increment at $y / t \simeq 0.03$ was $\simeq 0.7$ for station 6 , it was $\simeq 0.4$ at $\mathrm{y} / \mathrm{t} \simeq 0.1$ for station 7 . Station 6 magnitude difference was seen to be $\simeq 25^{\circ}$ for the log and outer regions, and station 7 values were seen to be close to zero in the whole layer.

Using different anisotropy constants, $T=0.5$ and 0.7 , to compute $v_{T}$, results in the magnitude ratios and direction differences which are in between the results found using Cebeci-Smith $(T=1)$ and Rotta's models with $\mathrm{T}=0.3$ ( Fig 114 and 115 ). For this data set, it was observed that using an anisotropic eddy-viscosity amplified the existing differences of the measured and computed shear stress magnitude and directions with the Cebeci-Smith model.

Discussion on Patel's model predictions are restricted until the beginning of logarithmic region since the equations used were for this region. Quantities were plotted for $y^{+}$values less than 250 . In all the stations, Patel's model was seen to underpredict the magnitude ratios very near the wall, similar to the Cebeci-Smith model.

For the zeroth station, in the log region, while the magnitude ratio was $\simeq 1.0$, the stress vector direction difference was seen to be zero within the uncertainty bands ( Fig 116). At station 1, the ratio seems to be underpredicted, and even though the direction difference seems to be zero in the uncertainty bands, actual values were $\simeq 15^{\circ}$ till $\mathrm{y} / \mathrm{t} \simeq 0.03$ and were seen to $\mathrm{be} \simeq-25^{\circ}$ at the last three points. The magnitude ratio for the second station was again seen to be 1.0 within the uncertainty limits, and the direction difference except near the wall below $y^{+}<40$ is seen to be zero. The ratio of magnitudes above $y / t \simeq 0.03$ for the $3 \mathrm{rd}$ station was $\simeq 0.7-0.8$, and, in the same region, the two vectors were aligned. Station 4 values in the near wall and $\log$ regions were scattered between $0.5-0.8$ with an average of $\simeq 0.7$ in the uncertainty band. While at the first two points the angle difference was $\simeq 40^{\circ}$, the next point in near wall region had $\simeq 100^{\circ}$ of difference. This difference reduced to 
zero in the $\log$ region. The magnitude ratio at station 5 was again close to 1.0 in the log region, but the direction difference was seen to be $\simeq 50^{\circ}$ near the wall. The differences at the last 3 points were again zero. The gradual reduction in the ratio observed in the next 2 profiles was accompanied by less difference in the directions. While the ratio was $\simeq 0.8$ at station 6 , it was $\simeq 0.6$ at station 7 . The angle difference at station 6 peaked at $\mathrm{y} / \mathrm{t} \simeq 0.02$ at a value of $50^{\circ}$. Station 7 values for the direction difference was seen to be constant at $\simeq 15^{\circ}$. In comparison to Cebeci-Smith or Rotta models, Patel's model seems to underpredict the magnitude ratio in general, and the direction difference was similar to the Cebeci-Smith model results.

Since, the maximum shear stress for the Johnson-King model was used to find the $\sigma$ parameter in the outer eddy-viscosity definition such that the shear stress computed would be same as the experimental value, one point in each of the magnitude ratio profiles is assured to be 1.0 . In the application of the Johnson-King model, this maximum value is obtained from a solution of a partial differential equation. As mentioned before, in this study it was taken from the data as if the solution of this equation were same as the data.

At station 0 , even though the very near wall and most of the outer region magnitude ratios are less than 1.0 , the $\log$ region values were seen to be $\simeq 1.0$, and the direction difference at the same station was zero in the most of the layer ( Fig 117 ). At station 1, the scatter of the data resulted in divergence of the iteration procedure, so $\sigma$ was taken as 1.0. Data show that, within the uncertainty bands, the magnitude ratio in the log region is close to 1.0 . The direction difference was $\simeq 10^{\circ}$ in the near wall and part of the $\log$ region and was $\simeq 15^{\circ}$ in the outer region. Within the uncertainty bands $\log$ region magnitude ratios of station 2 are $\simeq 1.0$. Near wall and outer regions show underestimated ratios. The $\log$ region and outer region directions of the two stress vectors were the same, which was reached after a gradual decrease starting from a value of $\simeq 100^{\circ}$ near the wall. Magnitude ratios of station 3 show the same hill shaped distribution as station 2, with a peak in the $\log$ region at $\simeq 1.0$ with underestimated values in the near wall and outer regions. Also, the direction difference is seen to be zero within the uncertainty bands. For station 4 , the values are scattered within $0.7-1.0$, closer to 0.9 in the average. The high direction difference on the order of $\simeq 100^{\circ}$ near 
the wall decreases to zero in the logarithmic region. The same type of development was also observed for station 5; high near wall values of direction differences were seen to be reduced to zero in the $\log$ region at $y / t \simeq 0.02$. The magnitude ratios in the $\log$ region values of this station were predicted correctly as 1.0 within the uncertainty bands. At station 6 the near wall values as seen in most of the profiles were lower than 1.0 ; starting with $y / t \simeq 0.03$ the ratios were close to being 1.0 in the uncertainty bands. The difference in the directions of the computed and experimental stress vector parallel to the wall were seen to be reaching to a peak at $y / t \simeq 0.02$ at a value of $50^{\circ}$. Further in the layer, the difference was gradually reduced and was zero within the bands in the log and outer regions. At station 7, $\sigma$ was taken as 1.0. Starting from very near the wall, a gradual increase of the magnitude ratios in the layer were seen. This direction difference, which was seen to be $\simeq 15^{\circ}$ for the near wall part of the log region, was lower than zero in the outer region.

Overall, the Cebeci-Smith model and the modification to it by using Rotta's anisotropy constant were seen to overpredict the magnitude ratios and Patel's model was seen to underpredict. Even though the Johnson-King model inherently includes the maximum stress to find the stress distribution in the layers, it was observed that it underpredicted the magnitude ratios within the near wall and outer regions. None of the models were able to predict the stress direction perfectly, and except Rotta's model, which overpredicts the direction in most of the stations, they all seemed to work equally well.

The same type of analysis were carried out for the other data sets and the results are presented as $y^{+}$vs. the magnitude ratios and $y^{+}$vs. the angle difference for each data set in one figure.

Estimated shear stress magnitudes for the Elsenaar-Boelsma flow with the Cebeci-Smith model decrease proceeding downstream ( Fig 118 ). While the first station values were on the order of 1.6, by station 5 the ratios were seen to be scattered around 1.0. At station 10 , the ratio was $\simeq 0.6$. Mostly the data for this set above $y^{+}=2000$ were too highly scattered to be able to judge on the performance of the models. The difference of the computed and experimental data sets were seen to be above zero approximately $10^{\circ}$ starting with the fourth station values. Tenth station values 
were seen to be negative in $y^{+}=300$ to 600 . Use of an anisotropy constant $T=0.3$ seems to be effective starting with station 6 ( Fig 118 ). The magnitude ratio for stations 7 and 8 within $y^{+}=500-2000$ were seen to be $\simeq 0.7-0.8$ with a shift of $\simeq 0.1$ with respect to the Cebeci-Smith model. For station 9 and 10 , the increase in the ratio was 0.6 and 1.0 , respectively, in the $y^{+}=500-1000$ range. The stress vector direction difference was seen to be in the negative side for all the stations, and the difference is seen to be increasing proceeding downstream. The next two applications of the Rotta model with $T=0.5$ and $T=0.7$ give better results in terms of both the magnitude ratio and the direction difference ( Fig 118 ). The $\mathrm{T}=0.7$ case was seen to be able to predict the stress vector direction correctly except at station 10 . Even though use of $T=0.5$ results in lower magnitude ratios for station 10 and ratios on the order of 1 for the other stations, both $T=0.5$ and $T=0.7$ anisotropy constant were not able to resolve the decrease in the magnitude ratio proceeding downstream.

The Johnson-King model magnitude ratio results are seen to be scattered within 0.4 to 1.3 . Even though ratios of stations $5,6,7,8$ within $y^{+}=300-900$ are close to 1.0 , station 4 ratios are seen to be scattered within 0.8-1.2 range. Station 7 values are $\simeq 0.8$, and station 10 values are within 0.4-0.6 range. Direction differences are within $0^{\circ}-15^{\circ}$ range for all stations ( Fig 118 ).

Patel's model at the points where it could be used are seen to be scattered within $0.8-1.2$ except station 10 values which are $\simeq 0.6$. The direction difference is also seen within the same range of $0^{\circ}-15^{\circ}$

First station magnitude ratios for the Dechow flow are observed to be predicted correctly with the Cebeci-Smith model ( Fig 119 ). However, a further increase up to 1.2 by station 3, and then a decrease down to 0.6 by station 7 is also observed. The direction difference for this flow is seen much higher than zero, especially below $y^{+}=400$ and for stations 5,6 and 7. Although other station values of the direction difference are still higher than zero, they were below $10^{\circ}$.

Application of Rotta's model with $T=0.3$ shows its effect starting with station 4 ( Fig 119 ). The next three station ratios increased by up to an amount of 0.2 . While the ratio at $y^{+}=100$ ranges 
from 1 to 1.6 , at $y^{+}=300$, it is seen in between 0.7 to 1.2 . The angle difference is seen close to zero for all stations except below $y^{+}=80$. As seen before, the effect of using $T=0.5$ and $T=0.7$ resulted in ratios and angle differences between Cebeci-Smith and Rotta $T=0.3$ models ( Fig 119 ).

The Johnson-King model results in a decrease of the magnitudes within the first four stations in the layer towards the outer region ( Fig 119 ). The fifth station values are seen close to 1.0 ; the sixth and seventh station values were seen to be increasing in the layers. While the direction difference is within $\simeq 10^{\circ}$ for the first four stations, the next three station values showed higher misalignments, reaching up to $\simeq 75^{\circ}$ at station 7 .

Patel's model is seen to predict low magnitude ratios for all the stations with similar direction differences observed for Johnson-King model ( Fig 119 ). While the ratio for station 3 is $\simeq 0.9$, it is $\simeq 0.5$ by station 7 .

Magnitude ratios calculated using Muller's data set with the Cebeci-Smith model are seen to be changing from 0.6 to 1.5 for station $\mathrm{A} 11$ ( Fig 120 ). For stations $\mathrm{B} 21$ and $\mathrm{E} 53$, the magnitude ratio profiles are seen to be changing in the whole layers, more than fluctuating around a value. Last four station values of the magnitude ratios are seen to be in the range of 0.6 to 0.8 . Use of Rotta's model with $T=0.3$ does not result in considerable change for any of the station values except for station F65 where an increase of $\simeq 0.2$ in the whole layer is observed ( Fig 120). The angle differences computed with the Cebeci-Smith model are close to zero for all stations above $y^{+}=200$. Rotta's model, however, resulted in differences within $-25^{\circ}$ and $10^{\circ}$.

The Johnson-King model predicts the magnitude ratios and the stress angles for this flow in between $y^{+}=400-800$, except for station B21 where the values increase towards the edge of the layer ( Fig 120). However, below $y^{+}=400$ the ratios are scattered within 0.7-1.1 range.

Patel's model results in magnitude ratios of $\simeq 0.6$ for the A11,B21,D42 and F65 stations. The third and fifth station values were seen to be 0.8 and 1.0, respectively ( Fig 120). The stress angle 
difference except stations E53 and F65 were seen to be zero. For these stations the differences were $\simeq-10^{\circ}$ and $\simeq 10^{\circ}$, respectively.

Anderson's data set with the Cebeci-Smith model show a decrease in the calculated ratios proceeding downstream above $y^{+}=200$. ( Fig 121 ). While the ratio is $\simeq 1.0-1.1$ for his first station, the ratio drops down to 0.5 by station 5 . The direction differences increase for the first four stations from $\simeq 0^{\circ}$ to $\simeq 40^{\circ}$. At station 5 , the values are in this range. However, use of Rotta's model with $\mathrm{T}=0.3$ results in $\simeq 0^{\circ}$ differences ( Fig 121 ). The magnitude ratios also range between $0.8-1.1$ except for station 5, where all the values of the profile are higher than 1.2. Even though use of $T=0.5$ or $T=0.7$ reduces this station's ratios down to $0.8-1.1$ range, the angle differences were increased ( Fig 121 ).

Below $y^{+}=100$, the Johnson-King model for this flow overpredicts the magnitude ratios. Except at station 5 , the ratios in the region $y^{+}=100-300$ are scattered within a range of $0.9 \cdot 1.1$. Above this value, while ratios of station $2,3,4$ are close to 1.0 , first station values decrease in the layer towards the edge. Station 5 values show a lower peak at $y^{+}=250$ with a value of 0.5 . The stress angle differences increase until station 4 up to a value of $\simeq 40^{\circ}$. At station 5 , the difference below $y^{+}=200$ is observed to be $\simeq 10^{\circ}$. Magnitude ratios computed using Patel's model, except at station 1 , show continuous decrease till $y^{+}=250$. At station 1, values were in 0.8-0.9 range ( Fig 121 ).

For the shear-driven flows application of the models were seen to be less satisfactory than the pressure-driven flows. For Lohmann's flow while the 2nd station magnitude ratios were close to 1.0 , the downstream station values increase to $\simeq 3$ at station 9 (Fig 122 ). Differences in the directions of the stress vectors for stations 2 and 4 peak between $y^{+}=500-1000$ are on the order of $25^{\circ}$ and $-25^{\circ}$, respectively.

Use of Rotta's model with $\mathrm{T}$ less than 1.0 not only increased the magnitude ratios but also increased the direction differences. Therefore, $T=1.2$ and 1.5 were also used. Even though reduced magnitude ratios are observed, the station 2 values increase semi-logarithmically from a value of 0.4 to 1.5 in 
the layer, and station 9 values except below $y^{+}=200$ are higher than 1 . The station 2 values are close to 1.0 with $\mathrm{T}=1.5$ ( Fig 122 ).

The Johnson-King model predictions for the shear stress magnitude of Lohmann flow are correct below $y^{+}=250$. Above this point in the layers, the angle difference is similar to the Cebeci-Smith model ( Fig 122 ).

Patel's model for this data set underpredicts the magnitude ratios, especially for station 2 . The ratios seen at this station were $\simeq 0.4$. The direction differences are within $\simeq 0^{\circ}$ to $10^{\circ}$ ( Fig 122 ).

The same kind of flow structure is also observed for the low and high Reynolds number flows of Bissonnette-Mellor data. The scale of the magnitude plots were adjusted high enough to present the values. For all the four stations above $y^{+}=40$, magnitude ratios values were higher than 2.0 . The direction difference was also seen to be above $100^{\circ}$ for all the stations. The same as for Lohmann's data, the use of anisotropy constants below 1.0 results in very high magnitude ratios ranging up to 20 ( Fig 123 and 124). The angle difference was also seen to be increased. The values close to zero for Rotta's model with $T=0.3$ below $y^{+}=100$ are due to angle differences being more than $180^{\circ}$. Even though calculations using $T=1.5$ give some values close to 1.0 near the wall above $y^{+}=100$ in the layers, most of the magnitude ratios are above 2.0. Angle differences are on the order of $100^{\circ}$ or higher in most part of the layers.

The Cebeci-Smith model for the high Re number data result in ratios above 1.0 but below 4.0 ( Fig 124 ). For this case also, the direction differences were high, between $60^{\circ}-100^{\circ}$. Although Rotta's model with $\mathrm{T}=0.3$ gives better direction differences on the order of $25^{\circ}-75^{\circ}$, the magnitude ratios reach up to 12.0 . Rotta's model with $\mathrm{T}=1.5$ results in magnitude ratios still above 1.0 for most of the stations, mostly on the order of 1.5 to 2.25 . Direction differences are the same as Cebeci-Smith model ( Fig 124 ). 
Below $y^{+}=40$ for the low Re number case, the Johnson-King model was seen to be satisfactory in predicting the magnitude ratios. Above this $y^{+}$value, ratios were seen to be very higher than 1 ( Fig 123 ).

Patel's model predictions in the range of $y^{+}=60-250$ were seen to be scattered within $1.0-1.5$ range, with direction differences similar to Cebeci-Smith model predictions (Fig 123 and 124 ).

For the high Re number case of the same data set, ratios found using Johnson-King model are close to 1.0 ( Fig 124 ). Even though the values above this height are not as high as seen in the low $\operatorname{Re}$ number case, they still are scattered between 1.0-3.5 (Fig 123 ). Directions of the stresses predicted were off by $60^{\circ}-100^{\circ}$.

The range of the computed magnitude ratios and angle differences at $y^{+}=200$ for the flows studies may be found at Table 10 .

In all cases, the Cebeci-Smith model overpredicts for the almost 2-D stations but decreases in the magnitude ratio proceeding downstream in the individual flows. The angle difference is in most of the profiles in the positive side. For the shear-driven flows the ratios change between 0.5 to 10.0 and the angle differences are very high, especially for Bissonnette-Mellor flow.

Rotta's model with anisotropy constant $T=0.3$ for the pressure-driven data predicts the angle differences in a wider band, even though results in magnitude ratios closer to 1.0 and with lower scatter of the data. The effect was mostly seen at the stations where the velocity gradients were higher. This resulted in overestimated magnitude ratios for the most downstream stations such as in Olcmen, Elsenaar-Boelsma and Anderson flows. The constants $T=0.5$ and $T=0.7$ result in magnitude ratios and angle differences between the predicted values of the Cebeci-Smith model and $T=1.0$. The effect of the anisotropy constant $T$ less than 1.0 on the shear-driven flow magnified the difference in the measured and computed stresses, which resulted in overestimated magnitude 
ratios and angle differences. Even though $T=1.5$ for this type of flows is seen to be reducing the observed magnitude ratios, the ratios were still higher than 1.0 at most of the stations.

Patel's model for the pressure-driven flow, in the same $y^{+}$range was observed to predict magnitude ratios lower than the Cebeci-Smith model values. The angle differences are the same as the Cebeci-Smith model. For the shear-driven flow, the magnitude ratios calculated with this model are closer to 1.0 than any other model examined.

The Johnson-King model in the form used in this study mostly underpredicts the magnitude ratios in the almost 2-D flow stations of the pressure-driven flow data, especially in the outer region. For the stations where 3-D flow is developed, the scatter of the magnitude ratios are around 1.0 and in a narrower band than the Cebeci-Smith or any other model. For the shear-driven data except at the two most downstream stations of Lohmann's data, the stresses are overpredicted. Also, as mentioned before, $\sigma$ for the Bissonnette- Mellor data was kept as 1.0, since the equation to find the $\sigma$ did not converge. The angle differences obtained with the Johnson-King model were not different than Cebeci-Smith model.

In conclusion, none of the models predict the shear stress data well. Table 10 shows the range of the angle differences and the magnitude ratios calculated by using different models, for different flows at $y^{+}=200$. If there must be a choice made among these models, it may be suggested basing on ( Table 10 ) that, for the pressure-driven flows the Johnson-King model and for the shear-driven cases Patel's model could be used. 


\subsection{CONCLUSIONS}

A three dimensional turbulent boundary layer generated by a $3: 2$ elliptical nose, NACA 0020 tail cylinder protruding from a flat plate was experimentally studied. The experiments were conducted in a low speed wind tunnel at a nominal reference velocity of $27 \mathrm{~m} / \mathrm{sec}$. In the test section, the Reynolds number of the flow based on the momentum thickness at 0.75 chord upstream of the body on the centerline of the tunnel was $\simeq 5936$. Mean velocity and stress measurements were carried out by using hot-wire anemometry and laser-Doppler-velocimetry techniques. Data presented also include the static pressure measurements on the plate surrounding the wing and on the wing and the skin friction magnitude and direction on the wall. The incoming boundary layer was studied using the hot-wire technique. With the available data as input, the flow field can be computed and therefore another benchmark case is thus presented.

The existence of differences between the quantities measured with the hot-wire and LDV techniques, especially for the $u^{\prime}$ fluctuating velocity component near the wall and in the regions corresponding to the logarithmic regions of the $U / U_{\text {ref }}$ profiles on the order of $10 \%$, necessitated that the data be validated. For this purpose, LDV measurements were repeated by using the Burst Spectrum Analyzer and the same optical systems used in the measurements done with the Swept Spectrum Analyzers. The existence of the difference between some measured quantities, especially 
of the normal stresses with the LDV and the hot-wire techniques was established. The difference is attributed to the effect of the wall on the wire which needs to be studied further.

The data were further used to investigate the flow characteristics. The anisotropy constant $\mathrm{N}$, which is the ratio of the eddy viscosities in the $\mathrm{z}$ and $\mathrm{x}$ directions, is not unity as is frequently used in turbulence closure models. Although in the normal stress coordinates, $\mathrm{N}$ is seen to be close to 1.0 , it varies through the boundary layer and from station to station. Therefore, the concept of isotropy or even the constancy of the ratio in a preferred direction is questionable.

The structural parameter $A_{1}$, as used by Bradshaw ( 1967 ) to relate the shear stresses to the TKE of the flow, is not a constant of 0.15 in the whole layer. While in the outer region $A_{1}$ is close to 0.15 including the uncertainty band, it is less than 0.15 near the wall with no specific variations in the profiles.

The comparison of shear stress and flow gradient angles magnitudes revealed that the shear stress angle lags behind the flow gradient angle, especially in the inner region. In the outer region, especially at the stations where the mean flow field had higher gradients, the lag was more distinguishable. This also shows that the anisotropy of the near wall turbulence is greater than in the outer regions.

The $u_{r}$, friction velocity could not be observed as the velocity scale of the turbulence structure since, neither the fluctuating velocity nor the shear stress profiles could be correlated by normalizing with this velocity.

While the mixing lengths computed from the data near the wall follow the suggested variation of $0.41 \mathrm{y}$, while they were different constants at each station's outer region.

The TKE profiles show that in the successive locations the TKE of the flow does not change significantly. This suggests that the flow studied is close to an equilibrium flow in which the production equals the dissipation at each station, and convection is negligible. 
Both the TKE and stress production terms are seen to be significant below the logarithmic regions of the $U / U_{\text {ref }}$ profiles.

The existence of a Law of the Wall for 3-D pressure and shear driven turbulent boundary layers was also studied. For the axial component of the flows, among the 9 models chosen to be tested with 9 different data sets Johnston's model was seen to be superior, since the scatter of the 6 data sets in the log regions in this model's coordinate system was less than the other models' scatter. For the lateral component of the flows, even though at some locations the Law of the Wall of Mager was predicts $100 \%$ larger than the data, it is seen to be working qualitatively better than the other wall laws.

Among the 4 eddy-viscosity models tested, compared to the other models the Johnson-King model was seen to predict the shear stress magnitudes and directions closer to the pressure-driven flow data. For the shear-driven data Patel's model was seen to be superior. Even though the comparison of the Algebraic eddy-viscosity models show that some models are working better than the others this conclusion in terms of the turbulence modeling would be misguiding; since none of the models are able to predict the data perfectly. The modeling of turbulence, as mentioned in the Introduction chapter, necessitates many terms to be measured. Data sets including the triple correlations of the fluctuating velocities, simultaneous fluctuating velocity gradients seem to be the first step towards better understanding the nature of turbulence. 


\section{REFERENCES}

Abid, R., "Extension of the Johnson-King Turbulence Model to 3-D Flows", AIAA Paper-88-0223, AIAA 26th Aerospace Sciences Meeting, Jan 11-14, 1988, Reno, Nevada.

Abid, R., "An Anisotropic Eddy Viscosity for 3-D Separated Boundary Layer Flows", SAE Technical Paper Series 881544, 400 Commonwealth Drive, Warrendale, PA, 15096-0001.

Abid, R. and Schmitt, R., "Critical Examination of Turbulence Models for a Separated Three-Dimensional Turbulent Boundary Layer", Rech. Aerosp., No 1984-6.

Ahn, S., "Unsteady Features of Turbulent Boundary Layers", M.S. Thesis, Dept. of Aerospace and Ocean Eng., VPI\&SU, 1986.

Ailinger, K., " Measurements of Surface Shear Stresses under a Three- Dimensional Turbulent Boundary Layer using Oil-Film Laser Interferometry" , M.S. Thesis, Dept. of Aerospace and Ocean Eng., VPI\&SU, 1990.

Anderson, S.D., and Eaton, J.K., " An Experimental Investigation of Pressure-Driven Three-Dimensional Turbulent Boundary Layers",Report MD-49 June 1987a,Thermosciences Divison,Department of Mechanical Eng., Stanford, CA, 96305.

Anderson, S.D., and Eaton, J.K., "Experimental Study of a Pressure-Driven Three-Dimensional Turbulent Boundary Layer", AIAA Journal, Vol. 25, No 8, August 1987b, pp. 1086-1092.

Arnal, D., Cousteix, J., "Numerical Study of Corner Flows", IUTAM Symposium Berlin/Germany 1982, Editors H.H. Fernholz and E. Krause, "Three-Dimensional Turbulent Boundary Layers", Springer-Verlag, Berlin, Heidelberg, New-York, pp. 343-352.

Baldwin, B.S., and Lomax, H., "Thin Layer Approximation and Algebraic Model for Separated Flows", AlAA-Paper-78-257, 1978.

Bernard, P.S., "Limitations of the Near-Wall k-epsilon Turbulence Model", AlAA Journal, Vol. 24, No 4, April 1986.

Bertin, J.J., and Smith, M.L., Aerodynamics for Engineers, First edition, 1979, Prentice Hall, Englewood Cliffs, New Jersey, 07632, pp. 63-68. 
Bissonnette, L.R., and Mellor, G.L., "Experiments on the Behavior of an Axisymmetric Turbulent Boundary Layer with a Sudden Circumferential Strain", J. Fluid Mech., 1974, Vol. 63, Part 2, pp. 369-413.

Bradshaw, P., Turbulence, Topics in Applied Physics, Volume 12, Second edition, Springer Verlag, Berlin, Heidelberg, New York, 1978, pp. 1-44.

Bradshaw,P., Cebeci,T. and Whitelaw,J., Engineering Calculation Methods for Turbulent Flow, Academic Press, New York, 1981.

Bradshaw, P., Ferriss, D.H., and Atwell, N.P., "Calculation of Boundary Layer Development Using the Energy Equation", J. Fluid Mech., 1967, Vol. 28, Part 3, pp. 593-616.

Bradshaw, P., "Calculation of Three-Dimensional Turbulent Boundary Layers", J. Fluid Mech., 1972, Vol. 46, Part 3, pp. 417-445.

Bradshaw, P., "Physics and Modelling of Three-Dimensional Boundary Layers", AGARD-Report No:741, 1987, --Computation of Three-Dimensional Boundary Layers Including Separation--7 Rue Ancelle 92200, Neuilly sur Seine, France.

Cebeci, T., and Smith, A.M.O., Analysis of Turbulent Boundary Layers, Academic Press, New York, 1974.

Cebeci, T., Kaups, K. and Ramsey, J.A., " A General Method for Calculating Three-Dimensional Compressible Laminar and Turbulent Boundary Layers on Arbitrary Wings", NASA CR-2777, Jan 1977, NASA Washington, DC, 20546.

Cebeci, T., "Calculation of Three-Dimensional Boundary Layers 1. Swept Infinite Cylinders and Small Cross Flow", AIAA Journal, Vol. 12, No 6, June 1974.

Cebeci, T., "Problems and Opportunities with Three-Dimensional Boundary Layers", AGARD-Report, No 719, 1984, 7 Rue Ancelle 92200, Neuilly sur Seine, France.

Chandrashekhar, N. and Swamy, N.V.C., "Wall Shear Stress Interference for Three-Dimensional Turbulent Boundary Layer Velocity Profiles", Trans. of ASME, Journal of Fluids Eng. ,97, December 1975, pp. 550-557.

Chen, H.C., and Patel, V.C., "Near-Wall Turbulence Models for Complex Flows Including Separation", AIAA Journal, Vol. 26, No 6, June 1988, pp. 641-648.

Clauser, F.H., "The Turbulent Boundary Layer", Advances in Applied Mechanics, Vol. IV, Academic Press, New York, 1956.

Coles, D., "The Law-of-the-Wake in the Turbulent Boundary Layer", J. Fluid Mech., July 1956, Vol. 1, Part 2, pp. 191-226.

Cousteix, J., "Three-Dimensional Boundary Layers. Introduction to Calculation Methods", AGARD-FDP-VKI Special Course 14-18 April 1986.

Cousteix, J., "Three-Dimensional and Unsteady Boundary Layer Computations", Annual Review of Fluid Mechanics, 1986, pp. 173-196.

Cousteix, J., "Three-Dimensional Boundary Layers, Inroduction to Calculation Methods", AGARD-Report No:741, 1987, --Computation of Three-Dimensional Boundary Layers Including Separation--7 Rue Ancelle 92200, Neuilly sur Seine, France. 
Cousteix, J., "Integral Method and Turbulence Models Applied to Three-Dimensional Boundary Layers", IUTAM Symposium Berlin/Germany 1982, Editors H.H. Fernholz and E. Krause, "Three-Dimensional Turbulent Boundary Layers", Springer-Verlag, Berlin, Heidelberg, New-York, pp. 286-297.

Daly, B.J., and Harlow, H.F., "Transport Equations in Turbulence", The Physics of Fluids, Vol. 13, No 11, November 1970, pp. 2634-2649.

Dechow, R., and Felsch, K.O., "Measurements of the Mean Velocity and of the Reynolds Stress Tensor in a Three-Dimensional Turbulent Boundary Layer Induced by a Cylinder Standing on a Flat Wall", Symposium on Turbulent Shear Flows, pp. 9.11-9.20 April 18-20, 1977a, University Park, PA.

Dechow, R., "Mittlere Geschwindigkeit und Reynoldsscher Spannungstensor in der dreidimensionale turbulenten Wandgrenzschicht von einen Stehenden Zylinder", Mitteilungen des Instituts fur Stromungsmechanik und Stromungsmoschinen, Universitat(TH) Karlsruhe, Heft 21 März $1977 b$.

Devenport, W.J., and Simpson, R.L., "Some Time Dependent Features of Turbulent Appendage-Body Juncture Flows", 16th Symposium on Naval Hydrodynamics, July 14-18, 1986, Berkley, CA.

Devenport, W.J. and Simpson, R.L., "An Experimental Investigation of the Flow Past an Idealized Wing-Body Junction: Preliminary Data Report", Dept. of Aerospace and Ocean Eng., VPI\&SU, Dec. 1987.

Devenport, W.J. and Simpson, R.L., "Turbulence Structure near the Nose of a Wing-Body Junction", AIAA-87-1310, AIAA 19th Fluid Dynamics, Plasma Dynamics and Lasers Conference, June 8-10, 1987a)/Honolulu, Hawaii.

Dickinson, S.C., "An Experimental Investigation of Appandage-Flat Plate Junction Flow Volume I: Description", DTNSRDC-86/051, December 1986, David W. Taylor Naval Ship Research and Development Center, Bethesda, MD 20084-5000.

Driver, D.M., Hebbar, S.K., "Experimental Study of a Three-Dimensional, Shear Driven, Turbulent Boundary Layer Using a Three-Dimensional Laser-Doppler Velocimeter", AIAA-85-1610,NASA Ames Research Center, Moffat Field, CA, 94305.

Durst, F., Melling, A., Whitelaw, J.H., Principles and Practice of Laser-Doppler Anemometry , Academic Press, Second Edition, 1981.

East, L.F., and Hoxey, R.P., "Low-Speed Three-Dimensional Turbulent Boundary Layer Data", Part 1 and Part 2, Reports and Memoranda, No:3653, March 1969.

Echols, W.H., and Young, J.A., "Studies of Portable Air-Operated Aerosol Generators", NRL Report 5929, 1963.

Elsenaar, A., and Boelsma, S.H., "Measurements of the Reynolds Stress Tensor in a Three-Dimensional Turbulent Boundary Layer Under Infinite Swept Wing Conditions", NLR TR 7409521.

Fernholz, H.H., and Vagt, J.D., "Turbulence Measurements in an Adverse-Pressure Gradient Three-Dimensional Turbulent Boundary Layer Along a Circular Cylinder", J. Fluid Mech., 1981, Vol. 111, pp.233-269. 
Fernholz, H.H., and Vagt, B., Dziomba, B. and Dengel, P., "Messungen in einen dreidimensionalen turbulenten Wandgrenzschicht an einer gekrummten Wand: Tabellarische Darstellung ber MeBwerte", Hermann-Fottinger-Institut fur Thermound Fluiddynamik, Technische Universitat Berlin, Institutibericht Nr. 01/78.

Fleming, J., "An Experimental Study of a Turbulent Wing-Body Junction and Wake Flow", M.S. Thesis, Dept. of Aerospace and Ocean Eng., VPI \& SU, 1990

Frederick, D., and Chang, T.S. "Continuum Mechanics", Scientific Publishers, Inc., Cambridge, 1972, pp.11

George, W.K., and Lumley, J.L., "The Laser-Doppler Velocimeter and Its Application to the Measurement of Turbulence", J. Fluid Mech., 1973, Vol. 60, Part 2, pp. 321-362.

Gorski, J.J., Govindan, T.R., Lakshminarayana, B., "Computation of Three-Dimensional Turbulent Shear Flows in Comers", AIAA Journal, Vol. 23, No 5, May 1985, pp. 685-692.

Gruschwitz, E., "Turbulent Reinbungsschichten mit Seckundarstromung", Ingenieur-Archiv., 4, 1935 , pp. 355-365.

Hanjalic, K., and Launder B.E., "A Reynolds Stress Model of Turbulence and Its Application to Thin Shear Layers", J. Fluid Mech., 52, 1972, pp. 609.

Holman, J.P., Experimental Methods for Engineers, McGraw-Hill Book Company, 1984, Fourth Edition, pp. 57.

Hong, S.K., and Murthy, S.N.B., "Effective Velocity of Transport in Curved Wall Boundary Layers", AIAA Journal, Vol. 24, No 3, March 1986.

Hornung, H.G., and Joubert, P.N., "The Mean Velocity Profile in Three-Dimensional Turbulent Boundary Layers", J. Fluid Mech., Vol. 15, Part 3, 1963, pp. 368-384.

Horstman, C.C., and Hung, C.M., "Computation of Three-Dimensional Turbulent Separated Flow at Supersonic Speeds", AlAA-Paper-79-0002, 1979.

Humphreys, D.A., "Viscous Flow- Its Analysis and Computation", FFA Report 142, Stockholm, 1986.

Humphreys, D.A., and Lindhout, J.P.F., "Calculation Methods for Three-Dimensional Turbulent Boundary Layers", Progress in Aerospace Sciences, Vol. 25, 1988, pp. 107-129.

Johnson, D.A., and King, L.S., "A New Turbulence Closure Model for Boundary Layer Flows with Strong Adverse Pressure Gradients and Separation", AIAA Paper-84-0175, AIAA 22nd Aerospace Sciences Meeting, Jan 9-12, 1984, Reno, Nevada.

Johnston, J.P., "On the Three-Dimensional Turbulent Boundary Layer Generated by Secondary Flow" Journal of Basic Eng., Trans. of the ASME, March 1960, pp. 233-248.

Johnston, J.P., "Three-Dimensional Turbulent Boundary Layer", ScD Thesis, M.I.T., Gas Turbine Laboratory Report No 39, May 1957.

Jones, W.P., and Launder, B.E., "The Prediction of Laminarization with a Two-Equation Model of Turbulence", International Journal of Heat and Mass Transfer, Vol. 15, 1972, pp. 301-314. 
Jones, W.P., and Launder, B.E., "The Calculation of Low-Reynolds Number Phenomena with a Two-Equation of Turbulence", International Journal of Heat and Mass Transfer, Vol. 16, 1973, pp. 1119-1130.

Kirtley, K., and Lakshminarayana, B., "Computation of Internal Incompressible Separated Flows Using a Space Marching Technique", AlAA-Paper-85-1624, 1985.

Klebanoff, P.S., "Characteristics of Turbulence in a Boundary Layer with Zero Pressure Gradient", NACA Rept. 1247, 1955. nd Three-Dimensional Incompressible Turbulent Boundary Layer Equations", Trans. of ASME, Journal of Fluids Eng., Vol. 95, September 1973, pp. 445-458.

Kliensiek, W.F. and Pierce, F.J., "Simultaneous Later Skewing in a Three-Dimensional Turbulent Boundary Layer", Trans. of ASME, Journal of Fluids Eng., Vol. 92, March 1970, pp. 83-90.

Kline, S.J., and McClintock, F.A., "Describing Uncertainties in Single-Sample Experiments", Mech. Eng., Jan. 1953, pp. 3.

Kline, S.J., Cantwell, B., and Lilley, G.M. (eds.), "Complex Turbulent Flows: Comparison of Computation and Experiment, Vol II, Taxonomies, Reporters Summaries, Evaluation and Conclusions", Stanford University Press, Stanford, CA, 1982 a).

Kline, S.J., Cantwell, B., and Lilley, G.M. (eds.), "Complex Turbulent Flows: Comparison of Computation and Experiment, Vol III, "Comparison of Computation with Experiment, and Computors' Summary Reports", Stanford University Press, Stanford, CA, 1982 b).

Kolmogorov, A.N., "Equations of Turbulent Motion of an Incompressible Fluid", Izv. Akad. Nauk. SSr Seria Fizicheska Vi., No 1-2, 1942, pp. 56-58, English Translation, Mechanical Engineering Dept., Imperial College, London, England, Rept. ON/6, 1968.

Kuethe, A.M., McKee, P.G., and Curry, W.H., "Measurements in the Boundary Layer of a Yawed Wing", NACA TN 1946, September 1949.

Kussoy, M.I., Horstman, C.C., and Viegas, J.R., "An Experimental and Numerical Investigation of a 3-D Shock Separated Turbulent Boundary Layer", AIAA Journal, Vol. 18, Dec. 1980, pp. $1477-1484$.

Lakshminarayana, B., "Three-Dimensional Turbulent Boundary Layers", VKI for Fluid Dynamics Lecture Series, 1983-03, Feb 28-March 4, 1983.

Lakshminarayana, B., "Turbulence Modeling for Complex Shear Flows", AIAA Journal, Dec 1986, pp. 1900-1917.

Launder, B.E., and Spalding, D.B., Mathematical models of Turbulence, Academic Press, New York, 1972.

Launder, B.E., "A Generalized Algebraic Stress Transport Hypothesis", AIAA Journal, Vol. 20, No 3, March 1982.

Launder, B.E., Reece, G.J., and Rodi, W., "Progress in the Development of a Reynolds Stress Turbulence Closure", J. Fluid Mech., 1975, Vol. 68, Part 3, pp. 537-566.

Launder, B.E., Morse, A., Rodi, W., and Spalding, D.B., "The Prediction of Free Shear Flows-A Comparison of the Performance of Six Turbulence Models", Imperial College of Science and Technology, Dept. of Mech. Eng. Exhibition Road, London, S.W.7, Paper prepared for NASA conference on Free Shear Flows, Langley Field, Hampton, Virginia, July 20-21, 1972. 
Lemmerman, L.A., and Atta, E.H., "A Comparison of Existing Three-Dimensional Boundary Layer Calculation Methods", AIAA-Paper-80-0133.

Lohmann, R.P., "The Response of a Developed Turbulent Boundary Layer to Local Transverse Surface Motion", Journal of Fluids Eng., Transactions of the ASME, Sept 1976, pp. 354-363.

Lumley, J.L., "Turbulence Modeling", Journal of Applied Mechanics, December 1983, Vol. 50, pp. 1097-1103.

Mager, A., "Generalization of Boundary Layer Momentum Integral Equations to Three-Dimensional Flows, Including Those of a Rotating Disk", NACA Technical Note No $2310,1951$.

McMahon, H.,Hubbart, J., Kubendran, L., "Mean Velocities and Reynolds Stresses in a Juncture Flow", NASA Contractor Report 3605, 1982.

Marvin, J.G., "Turbulence Modeling for Computational Aerodynamics", AIAA Journal, Vol. 21, July 1983, pp. 941.

Merati, P., McMahon, H.M., Yoo, K.M., "Experimental Modeling of a Turbulent Flow in the Junction and Wake of an Appendage Flat Plate", 1st National Fluid Dynamics Congr., Cincinatti, OH, July 24, 1988.

Miller, J.A., "A Simple Linearized Hot-wire Anemometer", Journal of Fluids Eng., Trans. of $A S M E, 1976,98$, pp. 550- .

Monson, D.J., and Higuchi, H., "Skin Friction Measurements by a Dual-Laser-Beam interferometer Technique", AIAA Journal, Vol. 19, No 6, June 1981, pp. 739-744.

Müller, U.R., "Comparison of Three-Dimensional Turbulent Boundary Layer Calaulations with Experiment", IUTAM Symposium Berlin/Germany 1982a, Editors H.H. Fernholz and E. Krause, "Three-Dimensional Turbulent Boundary Layers", Springer-Verlag, Berlin, Heidelberg, New-York, pp. 309-352.

Müller, U.R., "Measurement of the Reynolds Stresses and the Mean Flow field in a Three-Dimensional Pressure-Driven Boundary Layer", J. Fluid Mech., 1982b, Vol. 119, pp. 121-153.

Patel, V.C., and Chen, H.C., "Turbulent Wake of a Flat Plate", AIAA Journal, August 1987, Vol. 25, No 8, pp. 1078-1085.

Perry, A.E., and Joubert, P.N., "A Three-Dimensional Turbulent Boundary Layer", J. Fluid Mech., 1965, Vol. 22, Part 2, pp. 285-304.

Pierce, F.J., and McAllister, J.E., "Measurements in a Pressure-Driven and a Shear-Driven Three-Dimensional Turbulent Boundary Layer", IUTAM Symposium Berlin/Germany 1982, Editors H.H. Fernholz and E. Krause, "Three-Dimensional Turbulent Boundary Layers", Springer-Verlag, Berlin, Heidelberg, New-York, pp. 45-54.

Pierce, F.J. and Krommenhoek, D.H., "Wall Shear Stress Diagnostics in 3-D Turbulent Boundary Layers", Interim Technical Report No 2, ARO-D Project 6858E, VPI\&SU, 1968.

Pierce, F.J., McAllister, J.E. and Tennant, M.H., "Near-Wall Similarity in Three-Dimensional Turbulent Boundary Layers, Part I: Model Review, Part II: Pressure-Driven Flow Results", Proceedings AIAA/ASME Joint Fluids Conf., June 7-11, 1982, pp. 85-103, ASME publication. 
Pierce, F.J., and East, J.L., "Near Wall Collateral Flow in Three-Dimensional Turbulent Boundary Layers", AIAA Journal, Vol. 10, No 3, March 1972, pp. 334-336.

Prahlad, T.S., "Wall Similarity in Three-Dimensional Turbulent Boundary Layers", AlAA Journal, Vol.6, No.9, September 1968, pp 1772-1774.

Prahlad, T.S., "Mean Velocity Profiles in Three-Dimensional Incompressible Turbulent Boundary Layers", AIAA Journal, Vol. 11, No 3, March 1973.

Prandtl, L., "Uber ein neues Formelsystem fur die ausgebildete Turbulenz", Nachrichten Akademie der Wissenschaften, Göttingen, Math-Phys. Klasse, 1945, P. 6.

Reynolds, W.C., "Computation of Turbulent Flows", Annual Review of Fluid Mechanics, Vol. 8, 1976, pp.183.

Rhyming, I.L., Fannelop, T.K., "A Three-Dimensional Law-ot-the-Wall Including Skewness and Roughness Effects", IUTAM Symposium Berlin/Germany 1982, Editors H.H. Fernholz and E. Krause, "Three-Dimensional Turbulent Boundary Layers", Springer-Verlag, Berlin, Heidelberg, New-York, pp. 324-342.

Rodi, W., "Examples of Turbulence Models for Incompressible Flows", AIAA Journal, Vol. 20 , NO 7, July, 1982, pp. 872-879.

Rodi, W., Turbulence Models and Their Application in Hydraulics, Book publication of the International Association for Hydraulic Research, Delft, the Netherlands, 1980.

Rotta, J.C., "On the Effect of the Pressure Strain Correlations on the Three-Dimensional Turbulent Boundary Layers", Turbulent Shear Flows, July 2-4, 1979, Imperial College, London.

Rotta, J.C., "A Family of Turbulence Models for Three-Dimensional Thin Shear Layers", Turbulent Shear Flows 1, April 18-20 1977, University Park, Pennsylvania.

Rotta, J.C., A Family of Turbulence Models for Three-Dimensional Boundary Layers, Turbulent Shear Flows, Vol. II, Springer-Verlag, New York, 1979.

Rotta, J.C., "Turbulent Boundary Layers in Incompressible Flow", Progress in Aeronautical Sciences, Vol. 2, Boundary Layer Problems, 1962, Edited by, Ferri, A., Kuchemann, D., Sterne, L.H.G., Pergamon Press, The MacMillan Company, New York.

Schetz, J.A., Foundations of Boundary Layer Theory for Momentum, Heat, and Mass Transfer, 1984, Prentice-Hall, Inc., Englewood Cliffs, N.J. 07632. pp.139-219

Schumann, U., "Realizability of Reynolds-Stress Turbulence Models", Physics of Fluids, Vol. 20, No 5, May 1977, pp.721-725.

Shabaka, I.M.M.A., "Turbulent Flow in an Idealized Wing-Body JUnction ", Ph.D. Dissertation, Imperial College of Science and Technology, London, 1979.

Shabaka, I.M.M.A., and Bradshaw, P., "Turbulent Flow Measurements in an Idealized Wing-Body Junction", AIAA Journal, 19, 1981, pp. 131-132.

Simpson, R.L., and Barr, P.W., "Velocity Measurements in a Separating Turbulent Boundary Layer Using Sampling Spectrum Analysis", Proc. Second International Workshop on Laser Velocimetry, II, pp. 15-43, Stevenson, W.H., and Thompson, H.D., editors. 
Simpson, R.L., and Barr, P.W., "Laser Doppler Velocimeter Signal Processing Using Sampling Spectrum Analysis", Rev. Sci. Inst., 1975, 46, pp. 835-837.

Simpson, R.L., and Chew, Y.T., "Measurements in Steady and Unsteady Separated Turbulent Boundary Layers", Laser Velocimetry and Particle Sizing, Thompson, H.D., and Stevenson, W.H., editors, NY, Hemisphere, 1979, pp. 179-196.

Simpson, R.L., Heizer, K.W., and, Nasburg, R.E., "Performance Characteristics of a Simple Linearized Hot-Wire Anemometer", Journal of Fluids Eng., Trans. of ASME, Vol. 101, 1979, PP. 381-

Smith, P.D., "The Numerical Computation of Three-Dimensional Boundary Layers", IUTAM Symposium Berlin/Germany 1982, Editors H.H. Fernholz and E. Krause, "Three-Dimensional Turbulent Boundary Layers", Springer-Verlag, Berlin, Heidelberg, New-York, pp. 205-285.

Stern, F., Yoo, S.Y., Patel, V.C., "Interactive and Large-Domain Solutions of Higher-Order Viscous-Flow Equations", AIAA Journal, September 1988, Vol. 26, No 9, pp. 1052-1060.

Sutton, P. "private communication", 1985

Suvaganam, "Evaluation of Low Reynolds Number Turbulence Models for Attached and Separated Flows", AIAA-Paper-85-0375, 1985.

Tennekes, H., and Lumley, J.L., A First Course in Turbulence, The MIT Press, Cambridge,Massachusetts, and London, England, First Printing 1972, Eleventh Printing 1987, pp. 63.

Van Den Berg, B., "A Three-Dimensional Law-of-the-Wall for Turbulent Shear Flows", J. Fluid Mech., 1975, Vol. 70, Part 1, pp. 149-160.

Van Den Berg, B., "Some Notes on Three-Dimensional Turbulent Boundary Layer Data and Turbulence Modelling", IUTAM Symposium Berlin/Germany 1982, Editors H.H. Fernholz and E. Krause, "Three-Dimensional Turbulent Boundary Layers", Springer-Verlag, Berlin, Heidelberg, New-York.

Van Den Berg, B., A. Elsenaar, J.P.F. Lindhout and P. Wesseling, "Measurements in an Incompressible Three-Dimensional Turbulent Boundary Layer, Under Infinite Swept-Wing Conditions, and Comparison with Theory", J. Fluid Mech., 1975, Vol. 70, Part 1, pp. 127-148.

Van Den Berg, B., "Three-Dimensional Shear Layer Experiments and Their Use as Test Cases for Calculation Methods", AGARD-Report No:741,1987, .-Computation of Three-Dimensional Boundary Layers Including Separation--7 Rue Ancelle 92200, Neuilly sur Seine, France.

Van Den Berg, B., and Elsenaar, A., "Measurements in a Three-Dimensional Incompressible Turbulent Boundary Layer in an Adverse Pressure Gradient Under Infinite Swept Wing Conditions", 1972, NLR TR 72092 U.

Van Den Berg, B., Humphreys, D.A., Krause, E., and Lindhout, J.P.F., " Three-Dimensional Turbulent Boundary Layer Calculations and Experiments", Vueweg, Braunschweig, 1988.

Vagt, J.D., "Experimental Techniques in Three-Dimensional Turbulent Boundary Layers",IUTAM Symposium Berlin/Germany 1982, Editors H.H. Fernholz and E. Krause, "Three-Dimensional Turbulent Boundary Layers", Springer-Verlag, Berlin, Heidelberg, New-York, pp. 19-43. 
Wheeler, A.J., and Johnston, J.P., "Three-Dimensional Turbulent Boundary-Layers, an Assesment of Prediction Methods", Report MD-30, July 1971, Thermosciences Divison, Department of Mechanical Eng., Stanford Univ., Stanford, California.

White, F.M., Lessmann, R.C., Christoph, G.H., "A Three-Dimensional Integral Method for Calculating Incompressible Turbulent Skin Friction", Journal of Fluids Eng., Vol. 97, Dec 1975 , pp. 550-557.

Wilcox, D.C., "A Complete Model of Turbulence Revisited", AlAA-Paper-84-0176, AIAA 22nd Aerospace Sciences Meeting, Jan 9-12, 1984, Reno, Nevada.

Wilcox, D.C., Rubesin, M.W., "Progress in Turbulence Modeling for Complex Flowfields Including the Effect of Compressibility", NASA TP 1517, 1980.

Visbal, M., and Knight, D., "The Baldwin-Lomax Turbulence Model for Two-Dimensional Shock-Wave/Boundary-Layer Interactions", AIAA Journal, Vol. 22, July 1984, pp.921.

Wolfshtein, M., "The Velocity and Temperature Distribution in One-Dumensional Flow with Turbulence Augmentation and Pressure Gradient", International Journal of Heat and Mass Transfer, Vol. 12, March 1969, pp. 301-318. 


\section{TABLES}


Table 1. LEFT-HAND-SIDE HOT-WIRE LOCATIONS.

\begin{tabular}{|c|c|c|c|c|c|c|}
\hline $\begin{array}{l}\text { Station } \\
\text { Number }\end{array}$ & $\begin{array}{l}\text { File } \\
\text { Name }\end{array}$ & $\begin{array}{l}X \\
\text { (inches) }\end{array}$ & $\begin{array}{l}Z \\
\text { (inches) }\end{array}$ & $\begin{array}{l}U_{\text {ref }} \\
\mathrm{m} / \mathrm{sec}\end{array}$ & $\begin{array}{l}\beta_{F S} \\
\text { (degree) }\end{array}$ & $\begin{array}{l}\beta_{N S} \\
\text { (degree) }\end{array}$ \\
\hline 18 & LHS18 & -9.477 & 1.079 & 27.4 & 1.17 & 0. \\
\hline 17 & LHS17 & -6.478 & 1.125 & 27.49 & -0.69 & 0.97 \\
\hline 16 & LHS16 & -4.487 & 1.316 & 27.54 & 1.648 & 6.1 \\
\hline 15 & LHS 15 & -3.496 & 1.449 & 27.46 & 2.189 & 8.52 \\
\hline 14 & LHS 14 & -2.759 & 1.586 & 27.51 & 2.144 & 11.66 \\
\hline 13 & LHS 13 & -2.287 & 1.752 & 27.47 & 5.504 & 21.5 \\
\hline 12 & LHS 12 & -1.804 & 1.88 & 27.46 & 6.53 & 14.84 \\
\hline 11 & LHS 11 & -1.328 & 2.035 & 27.53 & 9.01 & 18 \\
\hline 10 & LHS 10 & -0.904 & 2.326 & 27.47 & 10.95 & 32.07 \\
\hline 9 & LHS9 & -0.472 & 2.578 & 27.44 & 12.05 & 30.22 \\
\hline 8 & LHS8 & 0.194 & 2.724 & 27.44 & 13.87 & 30.86 \\
\hline 7 & LHS7 & 0.258 & 2.939 & 27.46 & 10.47 & 25.33 \\
\hline 6 & LHS6 & 0.726 & 3.117 & 27.5 & 9.48 & 20.82 \\
\hline 5 & LHS5 & 1.193 & 3.295 & 27.58 & 9.15 & 20.82 \\
\hline 4 & LHS4 & 1.674 & 3.432 & 27.53 & 7.34 & 15.89 \\
\hline 3 & LHS3 & 2.165 & 3.527 & 27.5 & 5.03 & 10.92 \\
\hline 2 & LHS2 & 2.66 & 3.6 & 27.49 & 5.03 & 8.39 \\
\hline 1 & LHS1 & 3.655 & 3.678 & 27.62 & 4.3 & 4.48 \\
\hline
\end{tabular}




\begin{tabular}{|l|l|l|l|l|l|l|}
\hline Table & 2. & 0.75 \\
LiJCATIONS.
\end{tabular}




\begin{tabular}{|c|c|c|c|c|c|c|c|c|}
\hline $\begin{array}{l}\text { Station } \\
\text { Number }\end{array}$ & $\begin{array}{l}\text { File } \\
\text { Name }\end{array}$ & $\begin{array}{l}X \\
\text { (inches) }\end{array}$ & $\begin{array}{l}Z \\
\text { (inches) }\end{array}$ & $\begin{array}{l}U_{\text {ref }} \\
\mathrm{m} / \mathrm{sec}\end{array}$ & $\begin{array}{l}\beta_{F S} \\
\text { (degree) }\end{array}$ & $\begin{array}{l}\beta_{N S} \\
\text { (degree) }\end{array}$ & $\begin{array}{l}\beta_{w} \\
\text { (degree) }\end{array}$ & $\begin{array}{c}u_{4} \\
\mathrm{~m} / \mathrm{sec}\end{array}$ \\
\hline 0 & LDV0 & -4.487 & -1.316 & 27.57 & -1.684 & -6.1 & -3.816 & 1.152 \\
\hline 1 & LDV1 & -3.496 & -1.449 & 27.44 & -2.288 & -8.52 & -17.712 & 0.864 \\
\hline 2 & LDV2 & -2.287 & -1.752 & 27.40 & -5.576 & -21.5 & -21.724 & 0.865 \\
\hline 3 & LDV3 & -1.328 & -2.035 & 27.60 & -8.774 & -18 & -39.823 & 0.957 \\
\hline 4 & LDV4 & -0.472 & -2.578 & 27.62 & -10.163 & -30.22 & -26.337 & 1.105 \\
\hline 5 & LDV5 & 0.258 & -2.939 & 27.59 & -9.392 & -25.326 & -9.808 & 1.154 \\
\hline 6 & LDV6 & 1.193 & -3.295 & 27.25 & -6.509 & -20.82 & -4.692 & 1.162 \\
\hline 7 & LDV7 & 2.165 & -3.527 & 27.29 & -3.753 & -10.92 & -1.048 & 1.203 \\
\hline
\end{tabular}


Table 4. RIGHT-HAND-SIDE HOT-WIRE LOCATIONS.

\begin{tabular}{|l|l|l|l|l|l|l|}
\hline $\begin{array}{l}\text { Station } \\
\text { Number }\end{array}$ & \multicolumn{1}{l}{$\begin{array}{l}\text { File } \\
\text { Name }\end{array}$} & \multicolumn{1}{l}{$\begin{array}{l}\mathrm{X} \\
\text { (inches) }\end{array}$} & \multicolumn{2}{l}{$\begin{array}{l}\text { (inches) } \\
\text { (in/sec }\end{array}$} & $\begin{array}{l}\boldsymbol{U}_{\text {ref }} \\
\text { (degree) }\end{array}$ & $\begin{array}{l}\beta_{\text {NS }} \\
\text { (degree) }\end{array}$ \\
\hline 1 & RHS1 & -3.496 & -1.449 & 26.99 & -3.098 & -8.52 \\
\hline 2 & RHS2 & -2.287 & -1.752 & 26.99 & -3.858 & -21.5 \\
\hline 3 & RHS3 & -1.328 & -2.035 & 27.00 & -6.745 & -18 \\
\hline 4 & RHS4 & -0.472 & -2.578 & 26.99 & -9.249 & -30.22 \\
\hline 6 & RHS6 & 1.193 & -3.295 & 26.97 & -5.421 & -20.82 \\
\hline 7 & RHS7 & 2.165 & -3.527 & 27.06 & -4.615 & -10.92 \\
\hline
\end{tabular}




\begin{tabular}{|l|l|l|l|l|l|l|}
\hline Table 5. & AVERAGED NOMINAL VALUES OF THE \\
LNCERTAINTIES ÄT \\
DIFFERENT REGIONS OF THE BOUNDARY LAYER. \\
\hline Region \\
\hline Near wall & 0.063 & 0.007 & 0.033 & 0.063 & 0.013 & 0.027 \\
\hline Logarithmic & 0.023 & 0.015 & 0.018 & 0.004 & 0.004 & 0.004 \\
\hline Outer & 0.035 & 0.011 & 0.051 & 0.005 & 0.003 & 0.005 \\
\hline & $\overline{\boldsymbol{w}} / \boldsymbol{U}_{\text {ref }}^{2}$ & $\overline{\boldsymbol{u w}} / U_{\text {ref }}^{2}$ & $\overline{\bar{w}} / U_{\text {ref }}^{2}$ & & & \\
\hline Near wall & 0.004 & 0.004 & 0.002 & & & \\
\hline Logarithmic & 0.0004 & 0.0004 & 0.0003 & & & \\
\hline Outer & 0.00005 & 0.0002 & 0.0002 & & & \\
\hline
\end{tabular}




\begin{tabular}{|c|c|c|c|c|c|}
\hline \multirow{2}{*}{$\begin{array}{l}\text { Table } 6 . \\
\text { Station } \\
\text { Number }\end{array}$} & \multicolumn{5}{|c|}{$\begin{array}{l}\text { SEVERAL THICKNESSES COMPUTED } \\
\text { THE LDV DATA IN } \\
\text { FREE-STREAM COORDINATES. }\end{array}$} \\
\hline & $\begin{array}{l}\delta \\
(\mathrm{cm}) \\
\end{array}$ & $\begin{array}{l}\delta_{1} \\
(\mathrm{~mm})\end{array}$ & $\begin{array}{l}\delta_{2} \\
(\mathrm{~mm})\end{array}$ & $\begin{array}{l}\delta_{3} \\
(\mathrm{~mm}) \\
\end{array}$ & $\begin{array}{l}\delta_{4} \\
(\mathrm{~mm})\end{array}$ \\
\hline 0 & 3.921 & 6.521 & 6.518 & 4.627 & 0.107 \\
\hline 1 & 3.899 & 6.676 & 6.66 & 4.683 & 0.82 \\
\hline 2 & 3.996 & 6.626 & 6.57 & 4.628 & 1.183 \\
\hline 3 & 3.424 & 5.941 & 5.802 & 4.184 & 1.732 \\
\hline 4 & 3.885 & 5.323 & 5.165 & 3.984 & 1.802 \\
\hline 5 & 4.1457 & 4.981 & 4.846 & 3.877 & 1.691 \\
\hline 6 & 3.864 & 4.76 & 4.663 & 3.778 & 1.668 \\
\hline 7 & 3.757 & 4.512 & 4.46 & 3.592 & 0.787 \\
\hline
\end{tabular}

$\delta=$ Boundary Layer Thickness

$\delta_{1}=\int_{0}^{\delta}\left(1-\frac{U}{U_{e}}\right) d y=$ Displacement Thickness

$\delta_{2}=\int_{0}^{\delta}\left(1-\frac{\sqrt{U^{2}+W^{2}}}{U_{e}}\right) d y=$ Displacement Thickness

$\delta_{3}=\int_{0}^{\delta}\left(1-\frac{U}{U_{e}}\right) \frac{U}{U_{e}} d y=$ Momentum Thickness

$\delta_{4}=\int_{0}^{\delta}\left(-\frac{W}{U_{e}}\right) d y=$ Lateral Displacement Thickness 
Table 7. SUMMARY OF EXPERIMENTAL DATA SETS.

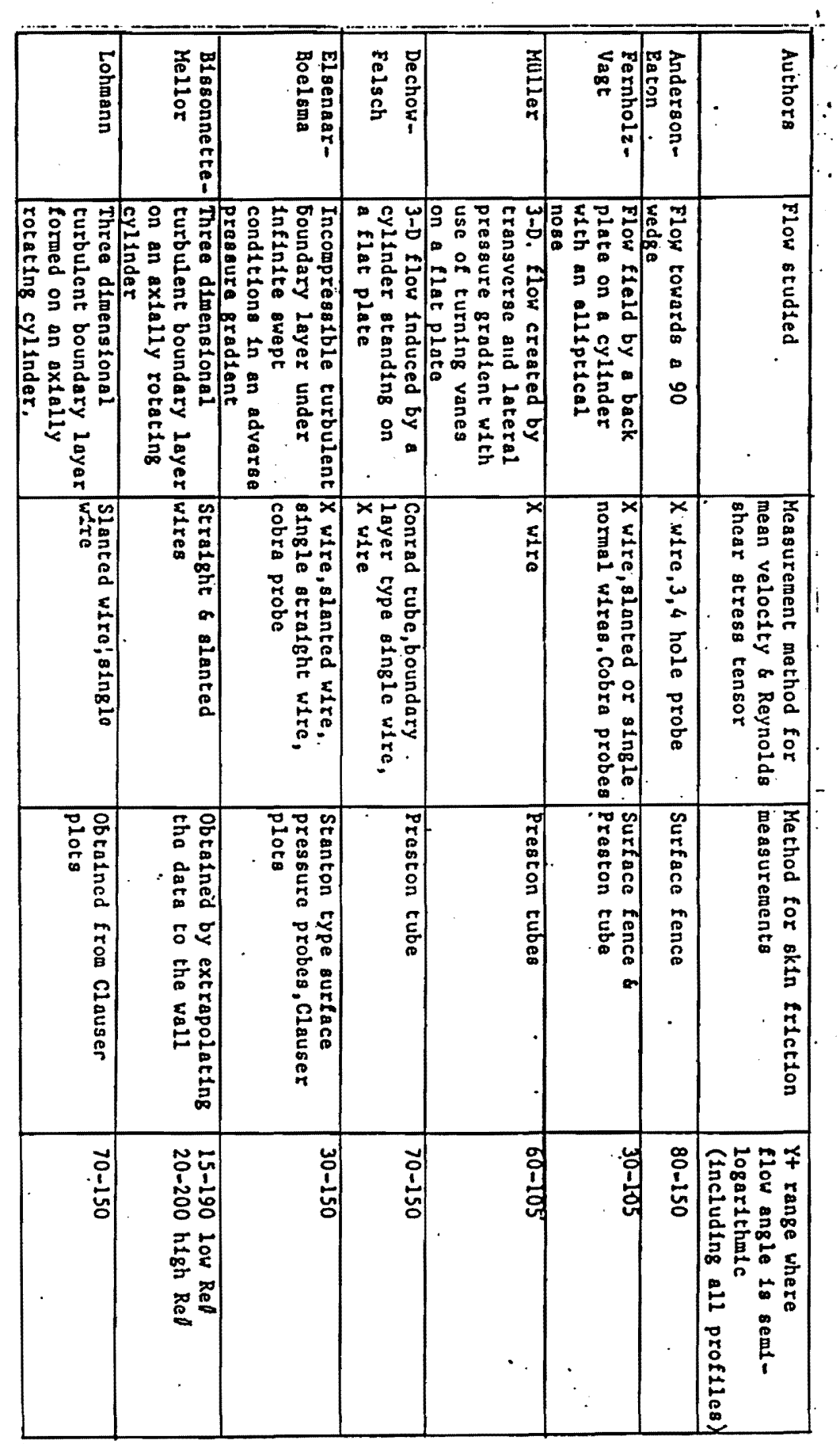




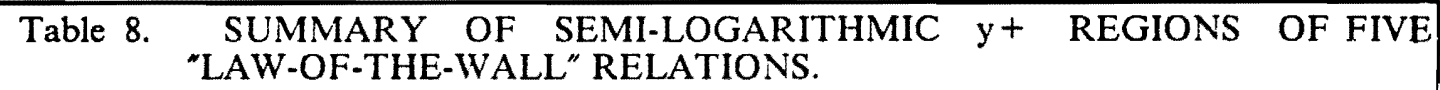

\begin{tabular}{|l|l|l|l|l|l|}
\hline $\begin{array}{l}\text { Flows /Models } \\
\text { studied }\end{array}$ & Coles & Johnston & \multicolumn{1}{l}{$\begin{array}{l}\text { Hornung- } \\
\text { Joubert }\end{array}$} & \multicolumn{1}{l|}{$\begin{array}{l}\text { Pierce- } \\
\text { Krommenhoek }\end{array}$} & \multicolumn{1}{l|}{$\begin{array}{l}\text { Chandrashekhar- } \\
\text { Swamy }\end{array}$} \\
\hline $\begin{array}{l}\text { Elsenaar- } \\
\text { Boelsma }\end{array}$ & $45-400$ & $60-250$ & $30-450$ & $35-250$ & $35-250$ \\
\hline Muller & $30-250$ & $30-200$ & $30-250$ & $20-200$ & $20-200$ \\
\hline $\begin{array}{l}\text { Fernholz- } \\
\text { Vagt }\end{array}$ & $15-150$ & $15-100$ & $15-100$ & $15-100$ & $15-100$ \\
\hline $\begin{array}{l}\text { Dechow- } \\
\text { Felsch }\end{array}$ & $20-200$ & $20-150$ & $20-150$ & $20-150$ & $20-150$ \\
\hline Anderson & $60-200$ & $60-200$ & $60-200$ & $60-200$ & $60-200$ \\
\hline $\begin{array}{l}\text { Bissonnette- } \\
\text { Mellor Low Re }\end{array}$ & $20-200$ & $45-250$ & $30-200$ & $20-200$ & $20-200$ \\
\hline $\begin{array}{l}\text { Bissonnette- } \\
\text { Mellor High Re }\end{array}$ & - & $20-250$ & - & $25-250$ & $25-250$ \\
\hline Lohmann & - & $60-250$ & - & $40-150$ & $40-150$ \\
\hline Olcmen & $40-500$ & $40-200$ & $40-400$ & $40-250$ & $40-250$ \\
\hline
\end{tabular}


Table 9. OVERLAP $y+$ RANGES FOR THE LEFT-HAND SIDE OF THE PROPOSED MODELS FOR PRESSUREDRIVEN DATA SETS FOR THE AXIAL COMPONENT.

\begin{tabular}{|l|l|l|l|}
\hline $\begin{array}{l}\text { Flows /Models } \\
\text { studied }\end{array}$ & \multicolumn{1}{l}{$\begin{array}{l}\text { Perry- } \\
\text { Joubert }\end{array}$} & $30-200$ & $30-200$ \\
\hline $\begin{array}{l}\text { Elsenaar- } \\
\text { Boelsma }\end{array}$ & $40-500$ & $4-60$ & $4-40$ \\
\hline Muller & $6-150$ & $15-30$ & $15-30$ \\
\hline $\begin{array}{l}\text { Fernholz- } \\
\text { Vagt }\end{array}$ & $10-100$ & $7-60$ & $7-20$ \\
\hline $\begin{array}{l}\text { Dechow- } \\
\text { Felsch }\end{array}$ & $7-20$ & $60-100$ & $60-80$ \\
\hline Anderson & $60-100$ & $20-40$ & $30-40$ \\
\hline Olcmen & $20-100$ & & \\
\hline
\end{tabular}




\begin{tabular}{|c|c|c|c|c|}
\hline \multicolumn{5}{|c|}{$\begin{array}{l}\text { Table 10a. THE RANGES AND ABSOLUTE RANGES OF ANGLE DIFFERENCES } \\
\left(\alpha_{m}-\alpha_{c}\right) \text { in degrees CALCULATED WITH SEVERAL TURBULENCE } \\
\text { MODELS AT } y+=200 .\end{array}$} \\
\hline $\begin{array}{l}\text { Flows /Models } \\
\text { studied }\end{array}$ & $\begin{array}{l}\text { Cebeci- } \\
\text { Smith }\end{array}$ & $\begin{array}{l}\text { Rotta } \\
0.3\end{array}$ & $\begin{array}{l}\text { Rotta } \\
0.5\end{array}$ & $\begin{array}{l}\text { Rotta } \\
0.7\end{array}$ \\
\hline $\begin{array}{l}\text { Elsenaar- } \\
\text { Boelsma }\end{array}$ & $\begin{array}{l}0^{\circ} \rightarrow 12.8^{\circ} \\
12.8^{\circ}\end{array}$ & $\begin{array}{l}-6.4^{\circ} \rightarrow 3.2^{\circ} \\
9.6^{\circ}\end{array}$ & ${ }^{-3.2^{\circ} \rightarrow 6.4^{\circ}}$ & $\begin{array}{l}0^{\circ} \rightarrow 6.4^{\circ} \\
6.4^{\circ}\end{array}$ \\
\hline $\begin{array}{l}\text { Dechow- } \\
\text { Felsch }\end{array}$ & $\begin{array}{l}6.4^{\circ} \rightarrow 31.9^{\circ} \\
31.9^{\circ}\end{array}$ & $\begin{array}{l}-9.6^{\circ} \rightarrow 3.2^{\circ} \\
12.8^{\circ}\end{array}$ & $\begin{array}{l}0^{\circ} \rightarrow 6.4^{\circ} \\
6.4^{\circ}\end{array}$ & $\begin{array}{l}6.4^{\circ} \rightarrow 22.3^{\circ} \\
22.3^{\circ}\end{array}$ \\
\hline Muller & $\begin{array}{l}-3.2^{\circ} \rightarrow 3.2^{\circ} \\
6.4^{\circ}\end{array}$ & $\begin{array}{l}-31.9^{\circ} \rightarrow 3.2^{\circ} \\
35.1^{\circ}\end{array}$ & $\begin{array}{l}12.8^{\circ} \rightarrow 3.2^{\circ} \\
16.0^{\circ}\end{array}$ & $\begin{array}{l}-9.6^{\circ} \rightarrow 3.2^{\circ} \\
12.8^{\circ}\end{array}$ \\
\hline Anderson & $\begin{array}{l}6.4^{\circ} \rightarrow 31.9^{\circ} \\
31.9^{\circ}\end{array}$ & $\begin{array}{l}-6.4^{\circ} \rightarrow 3.2^{\circ} \\
9.6^{\circ}\end{array}$ & $\begin{array}{l}0^{\circ} \rightarrow 16.0^{\circ} \\
16.0^{\circ}\end{array}$ & $\begin{array}{l}0^{\circ} \rightarrow 25.5^{\circ} \\
25.5^{\circ}\end{array}$ \\
\hline Olcmen & $\begin{array}{l}-31.9^{\circ} \rightarrow 25.5^{\circ} \\
57.4^{\circ}\end{array}$ & $\begin{array}{l}-25.5^{\circ} \rightarrow 35.1^{\circ} \\
60.6^{\circ}\end{array}$ & $\begin{array}{l}-28.7^{\circ} \rightarrow 22.3^{\circ} \\
51.1^{\circ}\end{array}$ & $-31.9^{\circ} \rightarrow 12.8^{\circ}$ \\
\hline Lohmann & $\begin{array}{l}-3.2^{\circ} \rightarrow 6.4^{\circ} \\
9.6^{\circ}\end{array}$ & $\begin{array}{l}-31.9^{\circ} \rightarrow 16.0^{\circ} \\
47.9^{\circ}\end{array}$ & $\begin{array}{l}-12.8^{\circ} \rightarrow 9.6^{\circ} \\
22.3^{\circ}\end{array}$ & $\begin{array}{l}-6.4^{\circ} \rightarrow 9.6^{\circ} \\
16.0^{\circ}\end{array}$ \\
\hline $\begin{array}{l}\text { Bissonnette- } \\
\text { Mellor low } \mathrm{Re}\end{array}$ & $\begin{array}{l}83.0^{\circ} \rightarrow 102.1^{\circ} \\
102.1^{\circ}\end{array}$ & $\begin{array}{l}57.5^{\circ} \rightarrow 95.7^{\circ} \\
95.7^{\circ}\end{array}$ & $\begin{array}{l}67.0^{\circ} \rightarrow 102.1^{\circ} \\
102.1^{\circ}\end{array}$ & $\begin{array}{l}76.6^{\circ} \rightarrow 98.9^{\circ} \\
98.9^{\circ}\end{array}$ \\
\hline $\begin{array}{l}\text { Bissonnette- } \\
\text { Mellor high } \mathrm{Re}\end{array}$ & $\begin{array}{l}57.5^{\circ} \rightarrow 70.2^{\circ} \\
70.2^{\circ}\end{array}$ & $\begin{array}{l}31.9^{\circ} \rightarrow 41.5^{\circ} \\
41.5^{\circ}\end{array}$ & $\begin{array}{l}38.3^{\circ} \rightarrow 51.1^{\circ} \\
51.1^{\circ}\end{array}$ & $\begin{array}{l}57.5^{\circ} \rightarrow 73.4^{\circ} \\
73.4^{\circ}\end{array}$ \\
\hline $\begin{array}{l}\text { Flows /Models } \\
\text { studied }\end{array}$ & $\begin{array}{l}\text { Rotta } \\
1.2\end{array}$ & $\begin{array}{l}\text { Rotta } \\
1.5\end{array}$ & $\begin{array}{l}\text { Jolınson- } \\
\text { King }\end{array}$ & Patel \\
\hline $\begin{array}{l}\text { Elsenaar- } \\
\text { Boelsma }\end{array}$ & - & - & $\begin{array}{l}3.2^{\circ} \rightarrow 12.8^{\circ} \\
12.8^{\circ}\end{array}$ & $\begin{array}{l}0^{\circ} \rightarrow 12.8^{\circ} \\
12.8^{\circ}\end{array}$ \\
\hline $\begin{array}{l}\text { Dechow- } \\
\text { Felsch }\end{array}$ & $\begin{array}{l}- \\
-\end{array}$ & - & $\begin{array}{l}6.4^{\circ} \rightarrow 31.9^{\circ} \\
31.9^{\circ}\end{array}$ & $\begin{array}{l}3.2^{\circ} \rightarrow 28.7^{\circ} \\
28.7^{\circ}\end{array}$ \\
\hline Muller & - & - & $\begin{array}{l}-6.4^{\circ} \rightarrow 3.2^{\circ} \\
9.6^{\circ}\end{array}$ & $\begin{array}{l}-9.6^{\circ} \rightarrow 3.2^{\circ} \\
12.8^{\circ}\end{array}$ \\
\hline Anderson & - & $\overline{-}$ & $\begin{array}{l}6.4^{\circ} \rightarrow 35.1^{\circ} \\
35.1^{\circ}\end{array}$ & $\begin{array}{l}6.4^{\circ} \rightarrow 35.1^{\circ} \\
35.1^{\circ}\end{array}$ \\
\hline Olcmen & - & $\overline{-}$ & $\begin{array}{l}-35.1^{\circ} \rightarrow 25.5^{\circ} \\
60.6^{\circ}\end{array}$ & $\begin{array}{l}-35.1^{\circ} \rightarrow 0^{\circ} \\
35.1^{\circ}\end{array}$ \\
\hline Lohmann & $\begin{array}{l}-3.2^{\circ} \rightarrow 6.4^{\circ} \\
9.6^{\circ}\end{array}$ & $\begin{array}{l}-3.2^{\circ} \rightarrow 6.4^{\circ} \\
9.6^{\circ}\end{array}$ & $\begin{array}{l}-3.2^{\circ} \rightarrow 6.4^{\circ} \\
9.6^{\circ}\end{array}$ & $\begin{array}{l}-3.2^{\circ} \rightarrow 6.4^{\circ} \\
9.6^{\circ}\end{array}$ \\
\hline $\begin{array}{l}\text { Bissonnette- } \\
\text { Mellor low } \mathrm{Re}\end{array}$ & $\begin{array}{l}83.0^{\circ} \rightarrow 102.1^{\circ} \\
102.1^{\circ}\end{array}$ & $\begin{array}{l}83.0^{\circ} \rightarrow 102.1^{\circ} \\
102.1^{\circ}\end{array}$ & $\begin{array}{l}76.6^{\circ} \rightarrow 102.1^{\circ} \\
102.1^{\circ}\end{array}$ & $\begin{array}{l}79.8^{\circ} \rightarrow 102.1^{\circ} \\
102.1^{\circ}\end{array}$ \\
\hline $\begin{array}{l}\text { Bissonnette- } \\
\text { Mellor high Re }\end{array}$ & $\begin{array}{l}63.8^{\circ} \rightarrow 73.4^{\circ} \\
73.4^{\circ}\end{array}$ & $\begin{array}{l}38.3^{\circ} \rightarrow 51.1^{\circ} \\
51.1^{\circ}\end{array}$ & $\begin{array}{l}54.3^{\circ} \rightarrow 63.8^{\circ} \\
63.8^{\circ}\end{array}$ & $\begin{array}{l}54.3^{\circ} \rightarrow 67.0^{\circ} \\
67.0^{\circ}\end{array}$ \\
\hline
\end{tabular}


Table 10b. THE RANGES AND ABSOLUTE RANGES OF MAGNITUDE RATIOS ( $\left.\tau_{m} / \tau_{c}\right)$ CALCULATED WITH SEVERAL TURBULENCE MODELS AT $\mathrm{y}+=200$.

\begin{tabular}{|c|c|c|c|c|}
\hline $\begin{array}{l}\text { Flows /Models } \\
\text { studied }\end{array}$ & $\begin{array}{l}\text { Cebeci- } \\
\text { Smith } \\
\end{array}$ & $\begin{array}{l}\text { Rotta } \\
0.3 \\
\end{array}$ & $\begin{array}{l}\text { Rotta } \\
0.5\end{array}$ & $\begin{array}{l}\text { Rotta } \\
0.7 \\
\end{array}$ \\
\hline $\begin{array}{l}\text { Elsenaar- } \\
\text { Bolesma }\end{array}$ & $\begin{array}{l}-0.38 \rightarrow 0.81 \\
1.19\end{array}$ & $\begin{array}{l}-0.34 \rightarrow 1.02 \\
1.36\end{array}$ & $\begin{array}{l}-0.3 \rightarrow 0.98 \\
1.28\end{array}$ & $\begin{array}{l}-0.34 \rightarrow 0.89 \\
1.23\end{array}$ \\
\hline $\begin{array}{l}\text { Dechow- } \\
\text { Felsch }\end{array}$ & $\begin{array}{l}-0.47 \rightarrow 0.34 \\
0.81\end{array}$ & $\begin{array}{l}-0.26 \rightarrow 0.3 \\
0.56\end{array}$ & 0.6 & 0.72 \\
\hline Muller & $\begin{array}{l}-0.38 \rightarrow 0.02 \\
0.4\end{array}$ & $\begin{array}{l}-0.4 \rightarrow 0.21 \\
0.61\end{array}$ & $\begin{array}{l}-0.36 \rightarrow 0.15 \\
0.51\end{array}$ & $\begin{array}{l}-0.38 \rightarrow 0.13 \\
0.51\end{array}$ \\
\hline Anderson & $\begin{array}{l}-0.34 \rightarrow 0.17 \\
0.51\end{array}$ & $\begin{array}{l}-0.26 \rightarrow 0.64 \\
0.9\end{array}$ & $\begin{array}{l}-0.34 \rightarrow 0.47 \\
0.81\end{array}$ & $\begin{array}{l}-0.36 \rightarrow 0.19 \\
0.55\end{array}$ \\
\hline Olcmen & $\begin{array}{l}-0.32 \rightarrow 0.4 \\
0.72\end{array}$ & $\begin{array}{l}-0.3 \rightarrow 0.85 \\
1.15\end{array}$ & $\begin{array}{l}-0.3 \rightarrow 0.64 \\
0.94\end{array}$ & $\begin{array}{l}-0.3 \rightarrow 0.34 \\
0.64\end{array}$ \\
\hline Lohmann & $\begin{array}{l}-0.51 \rightarrow 0.51 \\
1.02\end{array}$ & $\begin{array}{l}0.51 \rightarrow 6.47 \\
6.47 \\
\end{array}$ & $\begin{array}{l}0.17 \rightarrow 3.15 \\
3.15 \\
\end{array}$ & $\begin{array}{l}-0.17 \rightarrow 1.53 \\
1.7\end{array}$ \\
\hline $\begin{array}{l}\text { Bissonnette- } \\
\text { Mellor low Re }\end{array}$ & $\begin{array}{l}1.91 \rightarrow 4.47 \\
4.47 \\
\end{array}$ & $\begin{array}{l}11.06 \rightarrow 19.15 \\
19.15\end{array}$ & $\begin{array}{l}5.74 \rightarrow 12.98 \\
12.98 \\
\end{array}$ & $\begin{array}{l}3.4 \rightarrow 8.51 \\
8.51 \\
\end{array}$ \\
\hline $\begin{array}{l}\text { Bissonnette- } \\
\text { Mellor high Re }\end{array}$ & $\begin{array}{l}0.51 \rightarrow 2.17 \\
2.17 \\
\end{array}$ & $\begin{array}{l}1.53 \rightarrow 4.34 \\
4.34 \\
\end{array}$ & $\begin{array}{l}1.28 \rightarrow 3.57 \\
3.57\end{array}$ & $\begin{array}{l}0.77 \rightarrow 2.43 \\
2.43\end{array}$ \\
\hline $\begin{array}{l}\text { Flows /Models } \\
\text { studied }\end{array}$ & $\begin{array}{l}\text { Rotta } \\
1.2\end{array}$ & $\begin{array}{l}\text { Rotta } \\
1.5\end{array}$ & $\begin{array}{l}\text { Johnson- } \\
\text { King }\end{array}$ & Patel \\
\hline $\begin{array}{l}\text { Elsenaar- } \\
\text { Bolesma }\end{array}$ & - & - & $\begin{array}{l}-0.55 \rightarrow 0.38 \\
0.93\end{array}$ & $\begin{array}{l}-0.43 \rightarrow 0.06 \\
0.49\end{array}$ \\
\hline $\begin{array}{l}\text { Dechow- } \\
\text { Felsch }\end{array}$ & $\overline{-}$ & - & $\begin{array}{l}-0.21 \rightarrow 0.13 \\
0.34\end{array}$ & $\begin{array}{l}-0.64 \rightarrow-0.17 \\
0.64\end{array}$ \\
\hline Muller & $\overline{-}$ & - & $\begin{array}{l}0^{-0.3 \rightarrow 0.02} \\
0.32\end{array}$ & $\begin{array}{l}-0.43 \rightarrow-0.21 \\
0.43\end{array}$ \\
\hline Anderson & - & - & $\begin{array}{l}-0.32 \rightarrow 0.09 \\
0.41\end{array}$ & $\begin{array}{l}-0.7 \rightarrow-0.26 \\
0.7\end{array}$ \\
\hline Olcmen & $\overline{-}$ & $\overline{-}$ & $\begin{array}{l}-0.28 \rightarrow 0.21 \\
0.49\end{array}$ & $\begin{array}{l}-0.43 \rightarrow 0 \\
0.43\end{array}$ \\
\hline Lohmann & $\begin{array}{l}-1.19 \rightarrow 0.68 \\
1.87\end{array}$ & $\begin{array}{l}-1.36 \rightarrow-0.17 \\
1.36\end{array}$ & $\begin{array}{l}0.09 \rightarrow 0.17 \\
0.17\end{array}$ & $\begin{array}{l}-2.55 \rightarrow-0.94 \\
2.55\end{array}$ \\
\hline $\begin{array}{l}\text { Bissonnette- } \\
\text { Mellor low } \operatorname{Re}\end{array}$ & $\begin{array}{l}2.34 \rightarrow 5.96 \\
5.96\end{array}$ & $\begin{array}{l}1.49 \rightarrow 4.68 \\
4.68 \\
\end{array}$ & $\begin{array}{l}1.7 \rightarrow 3.4 \\
3.4\end{array}$ & $\begin{array}{l}0.85 \rightarrow 6.81 \\
6.81 \\
\end{array}$ \\
\hline $\begin{array}{l}\text { Bissonnette- } \\
\text { Mellor high } \mathrm{Re}\end{array}$ & $\begin{array}{l}0.13 \rightarrow 1.53 \\
1.53\end{array}$ & $\begin{array}{l}1.28 \rightarrow 3.83 \\
3.83\end{array}$ & $\begin{array}{l}2.04 \rightarrow 3.83 \\
3.83\end{array}$ & $\begin{array}{l}-1.02 \rightarrow 2.81 \\
3.83\end{array}$ \\
\hline
\end{tabular}




\section{FIGURES}




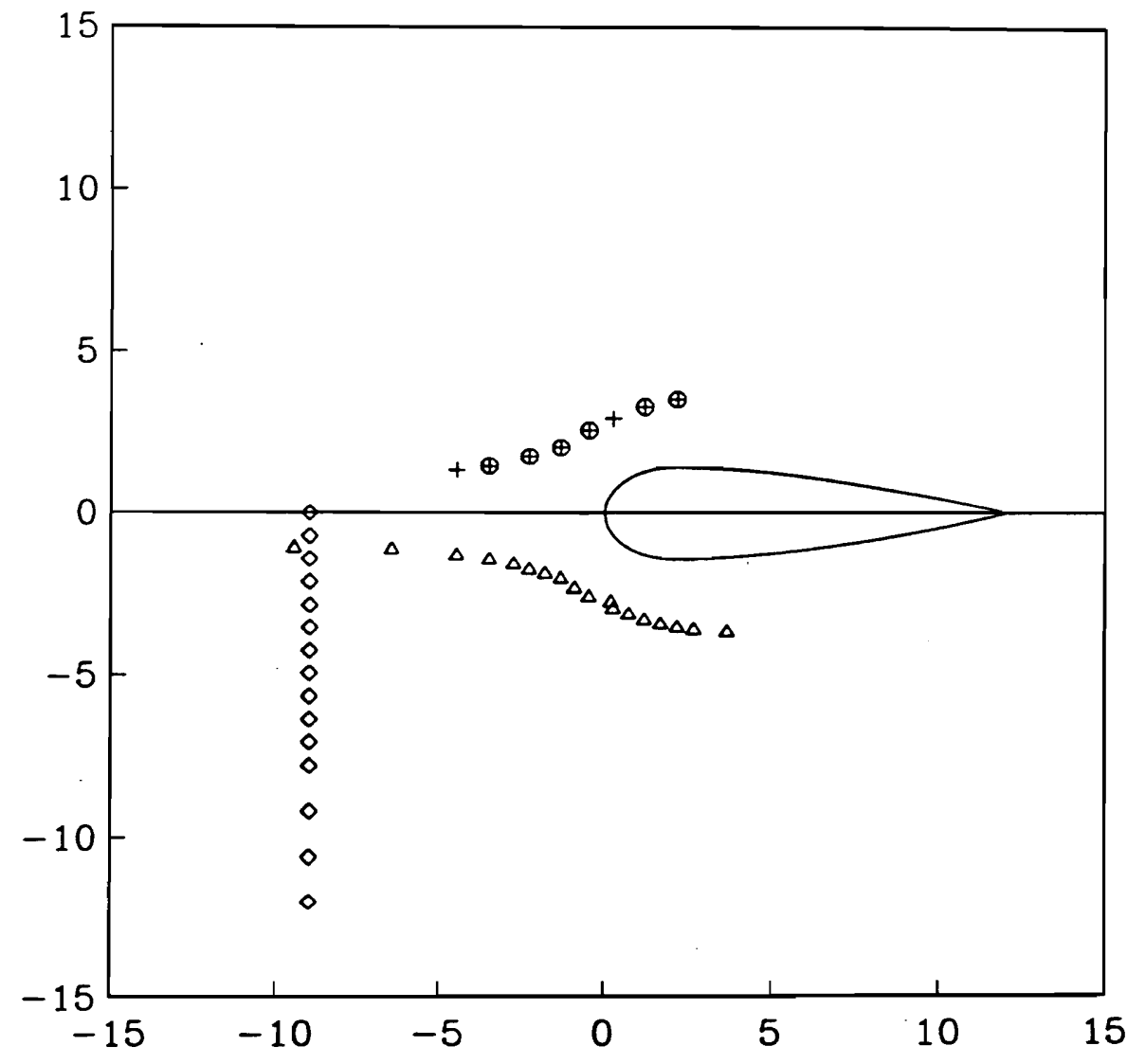

Figure 1. Mean velocity, normal and shear stress measurement locations.: The scale of the plot is in inches. Left-hand side hot-wire locations, right-hand side hot-wire locations, .75 chord upstream hot-wire locations, LDV locations. 


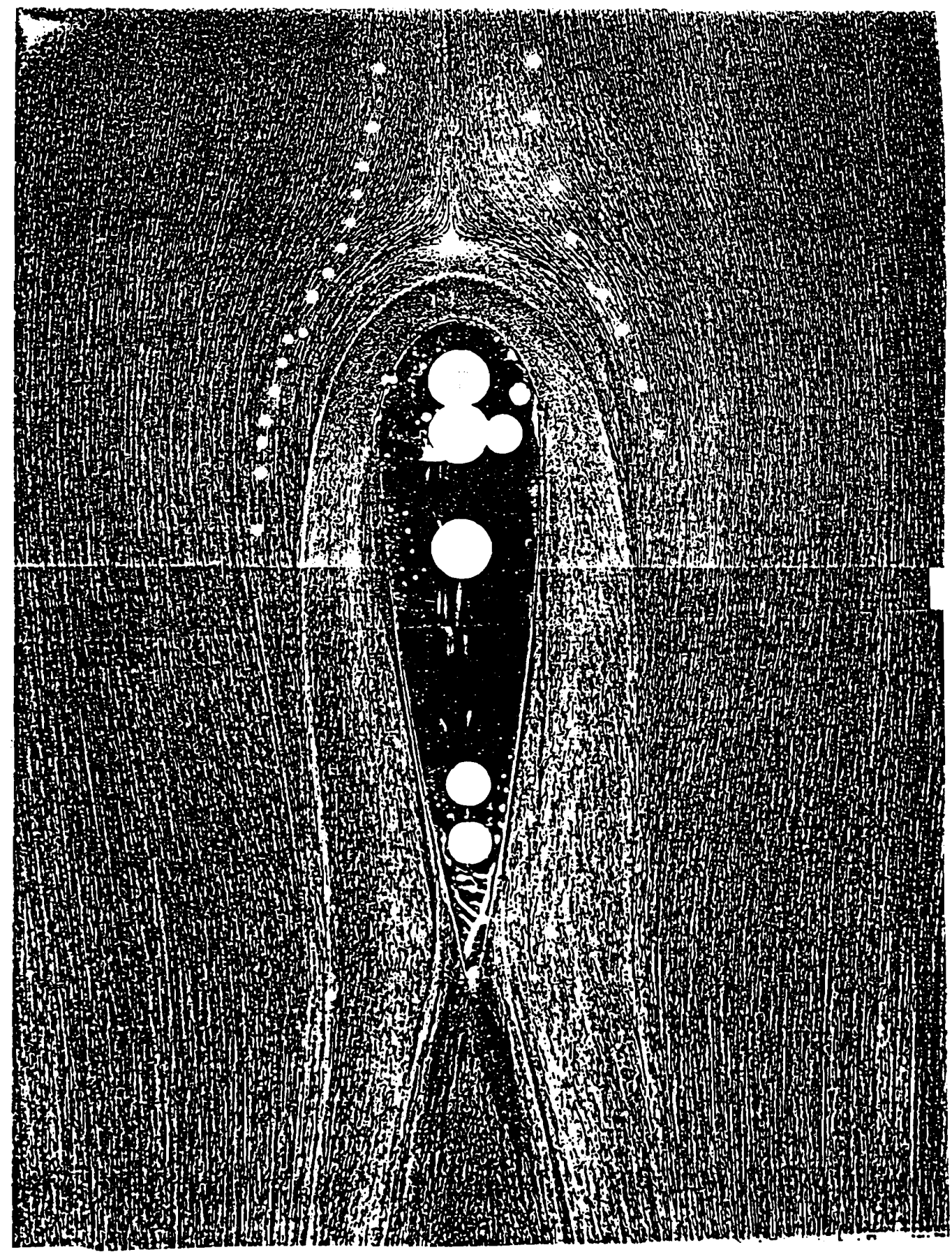

Figure 2a. Oil flow visualization picture taken on the test wall surrounding the wing, at 27 $\mathrm{m} / \mathrm{sec}$ nominal reference velocity.: The dots on both sides denote the velocity and stress measurement locations. 


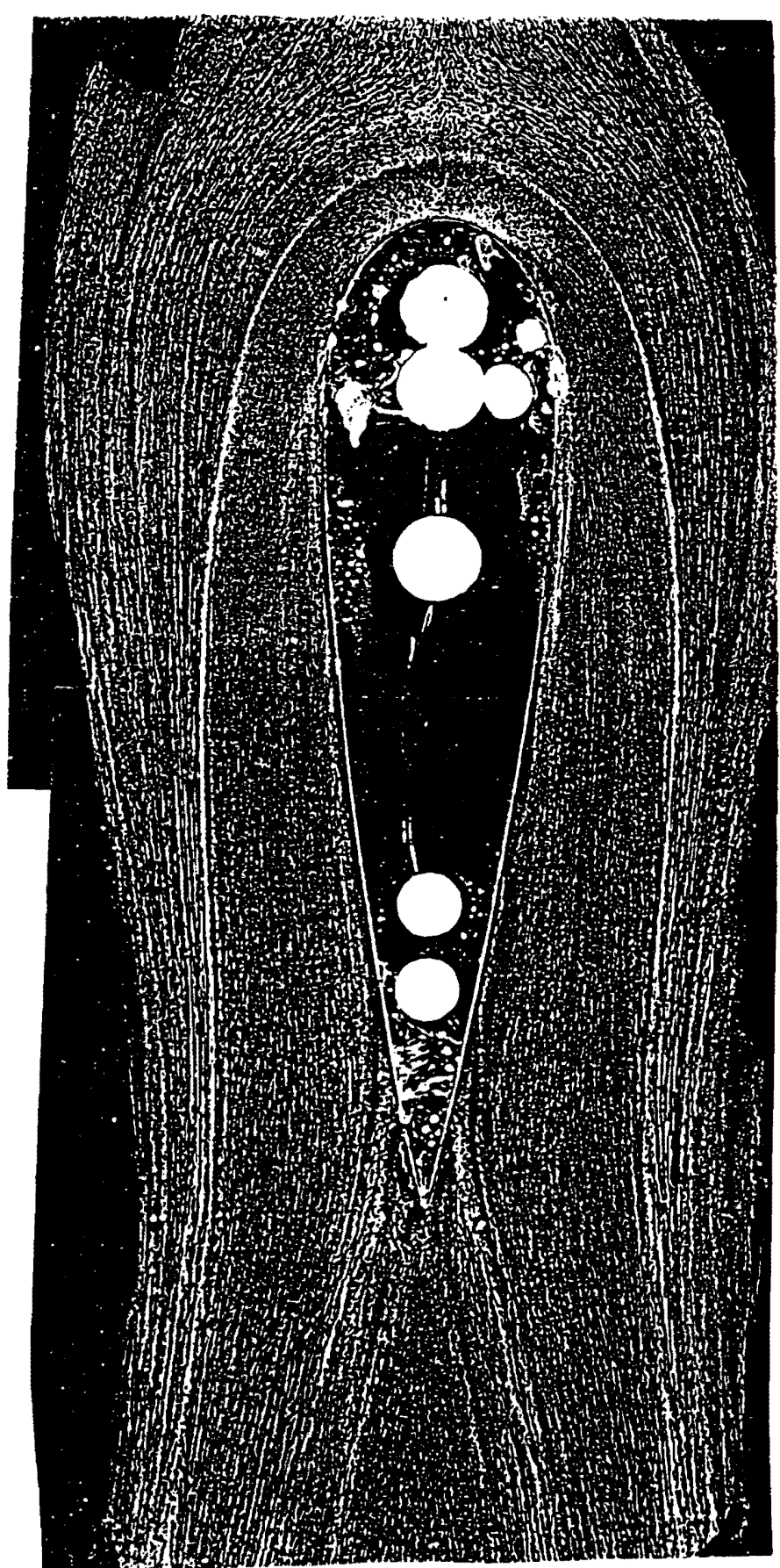

Figure $2 \mathrm{~b}$. Oil flow visualization picture taken on the test wall surrounding the wing, at 27 $\mathrm{m} / \mathrm{sec}$ nominal reference velocity. 


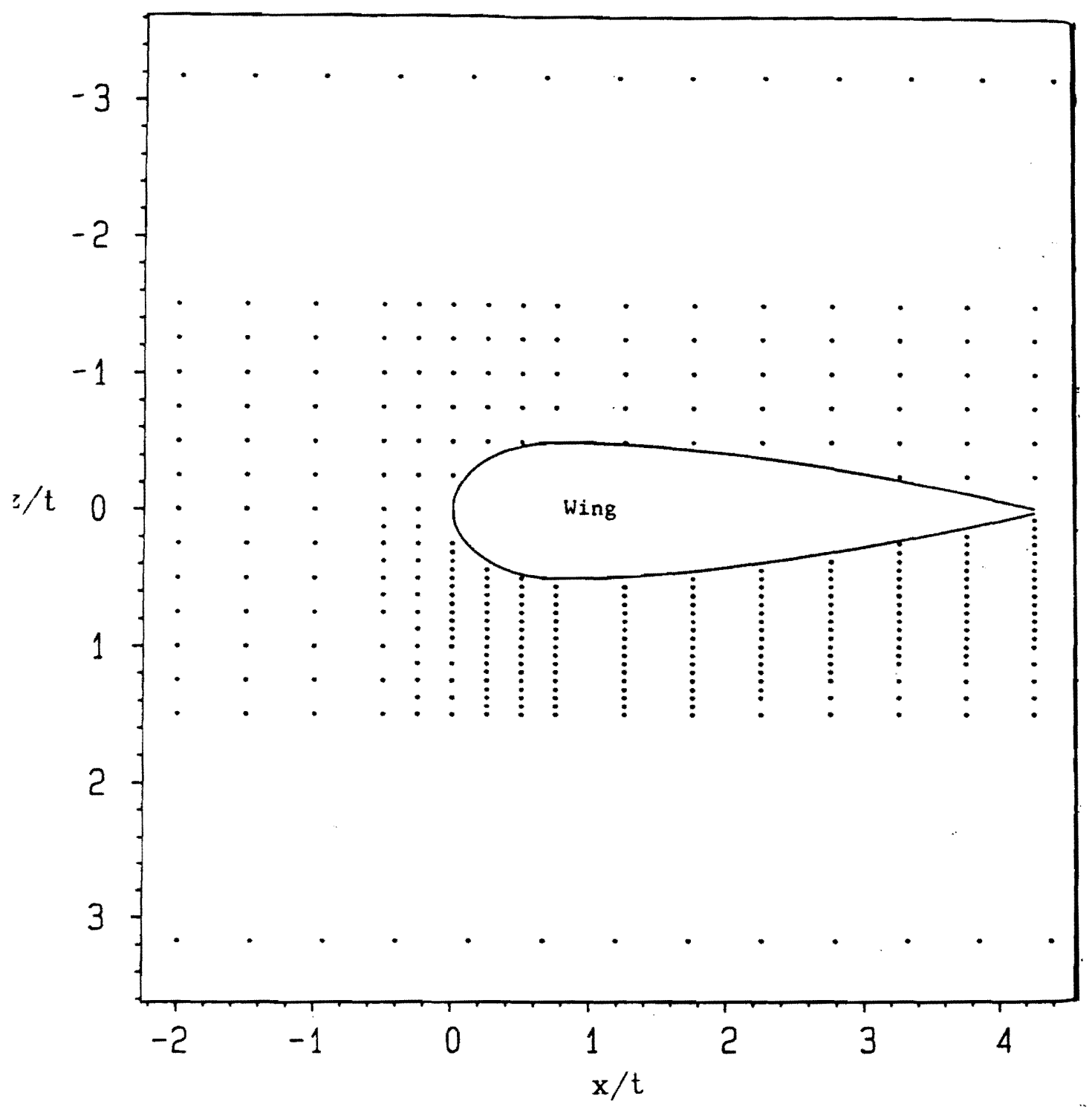

Figure 3a. Positions of pressure tappings on the test wall surrounding wing. 

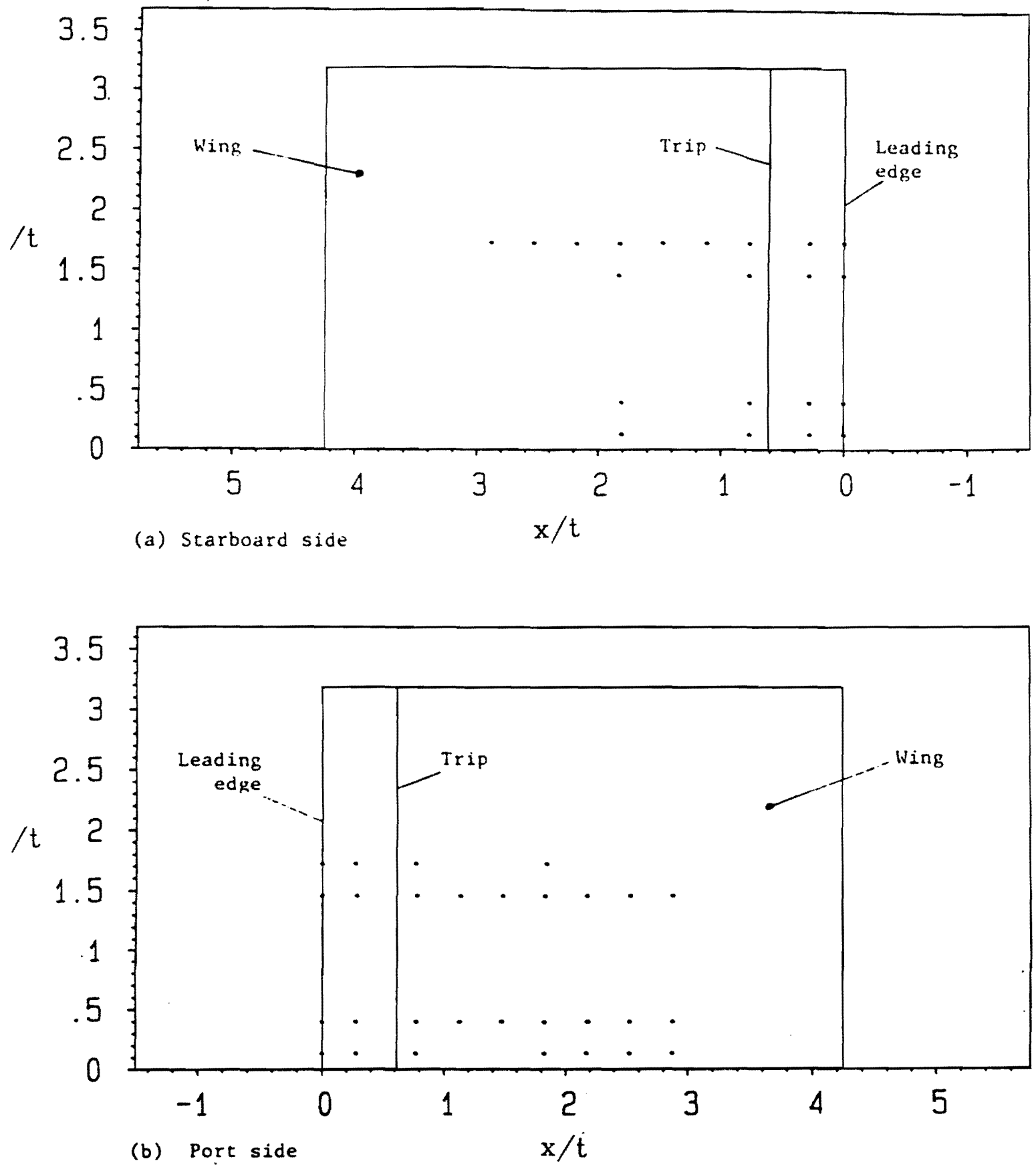

Figure $3 \mathrm{~b}$. Positions of pressure tappings on the wing surface projected on to an XY plane. 


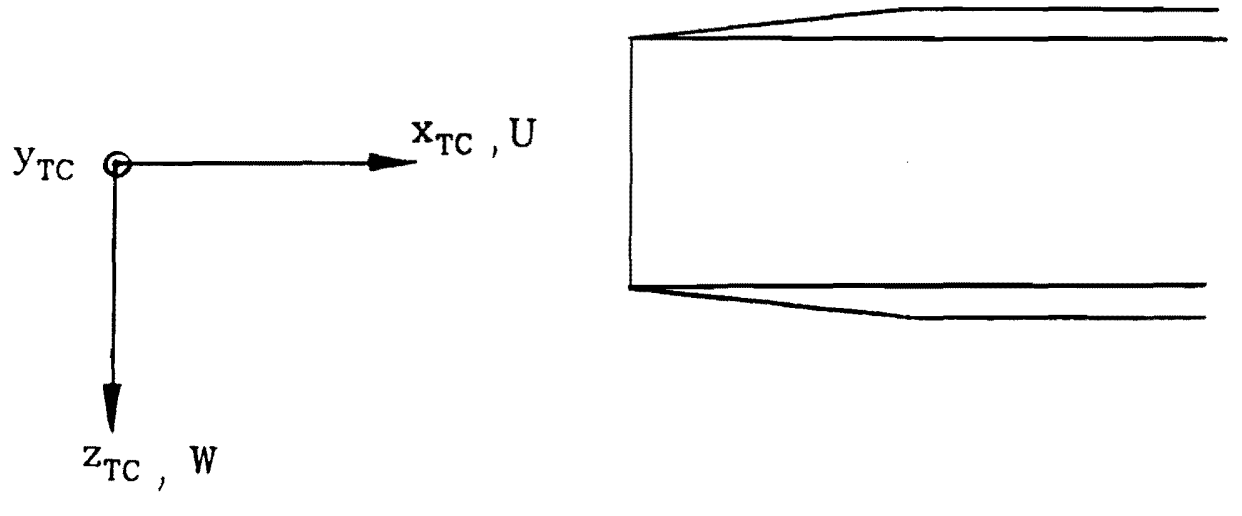

Figure 4. Hot-wire probe, in the tunnel. 


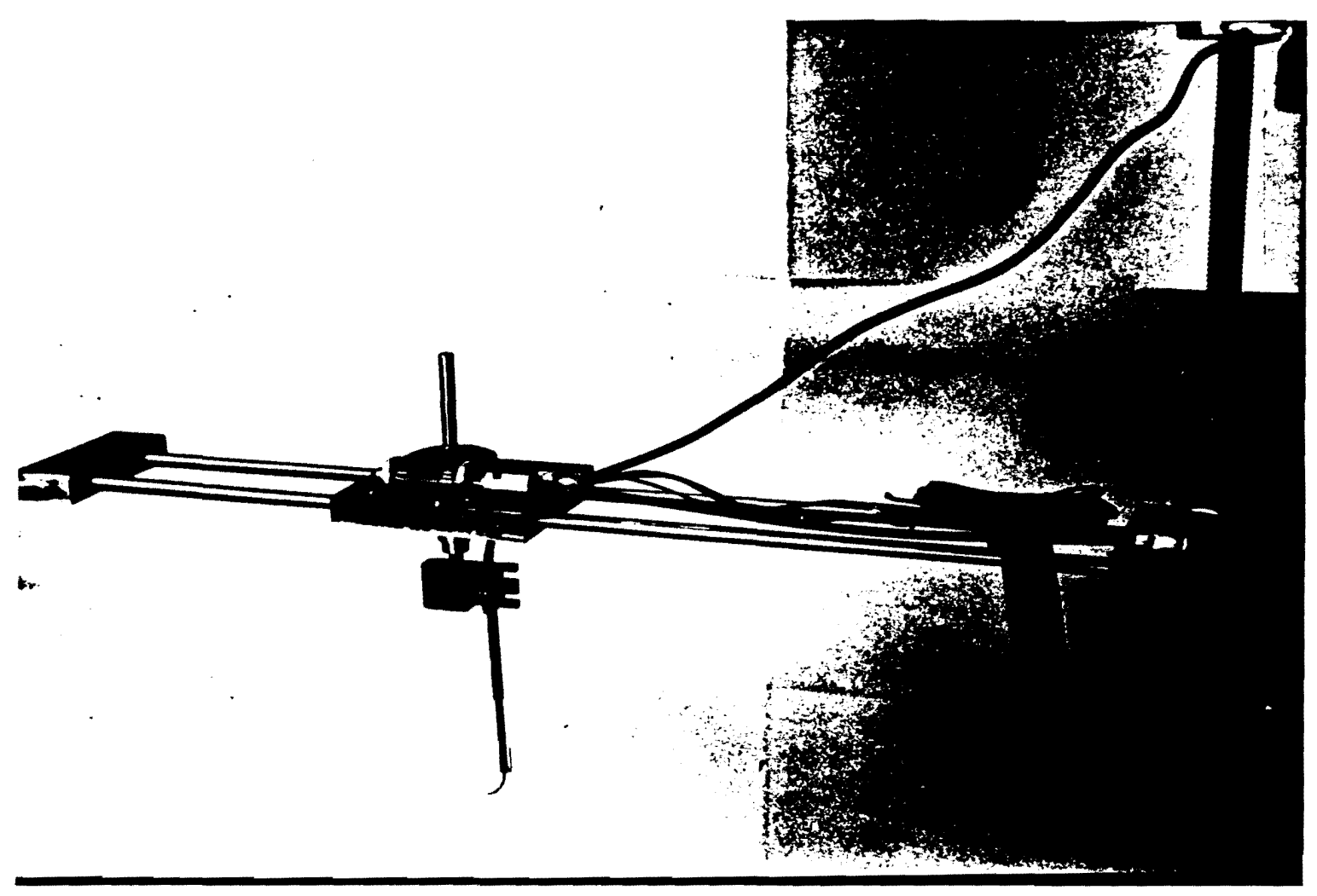

Figure 5. Probe holder designed to reach to measurement locations. 


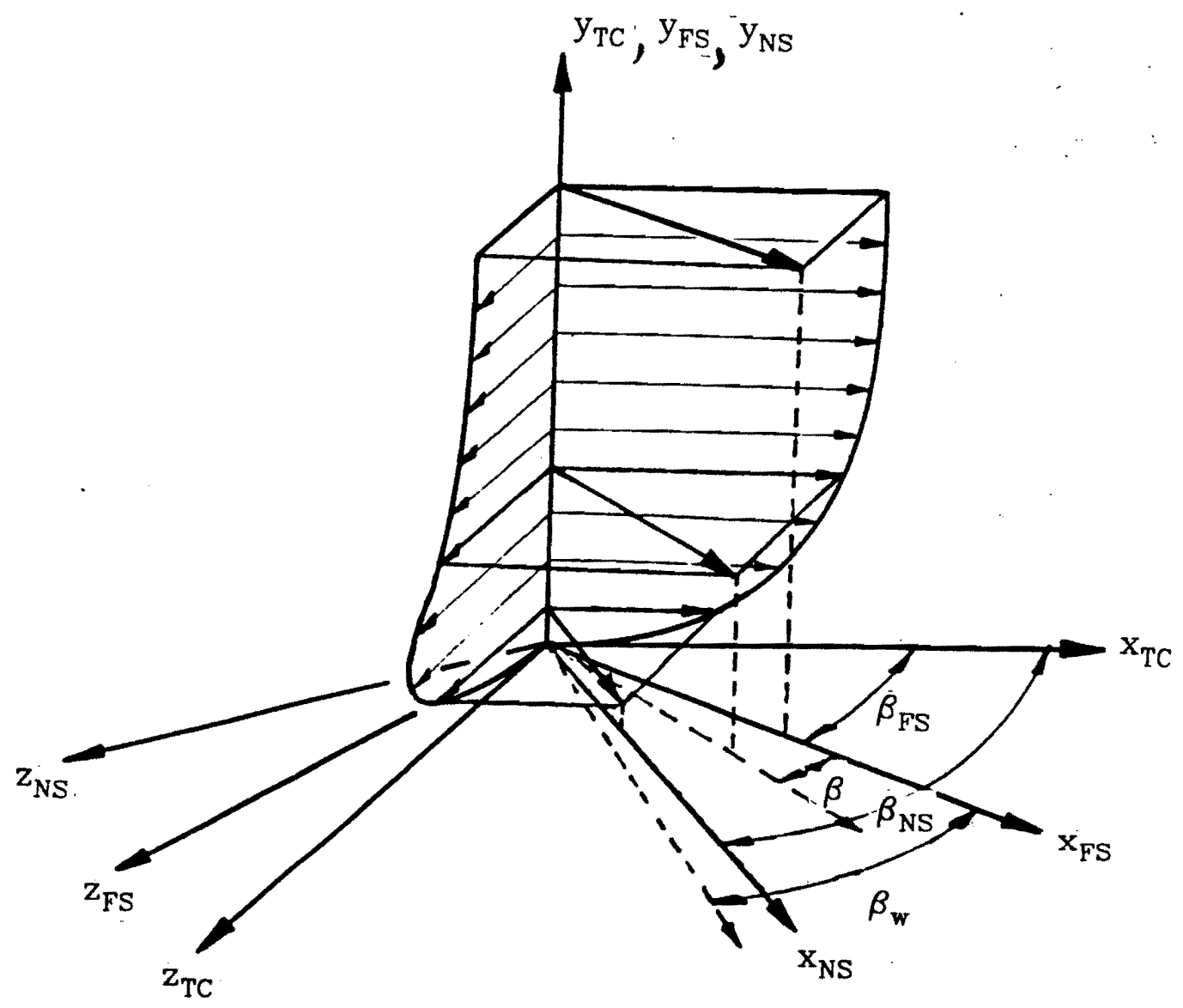

Figure 6. Definition of the angles and the coordinate axes used in the present study.: $x_{T C}, y_{T C}, z_{T C}=$ Tunnel coordinates, $x_{F S}, y_{F S}, z_{F S}=$ Free-stream coordinates, $x_{N S}, y_{N S}, z_{N S}=$ Maximum normal stress coordinates. 


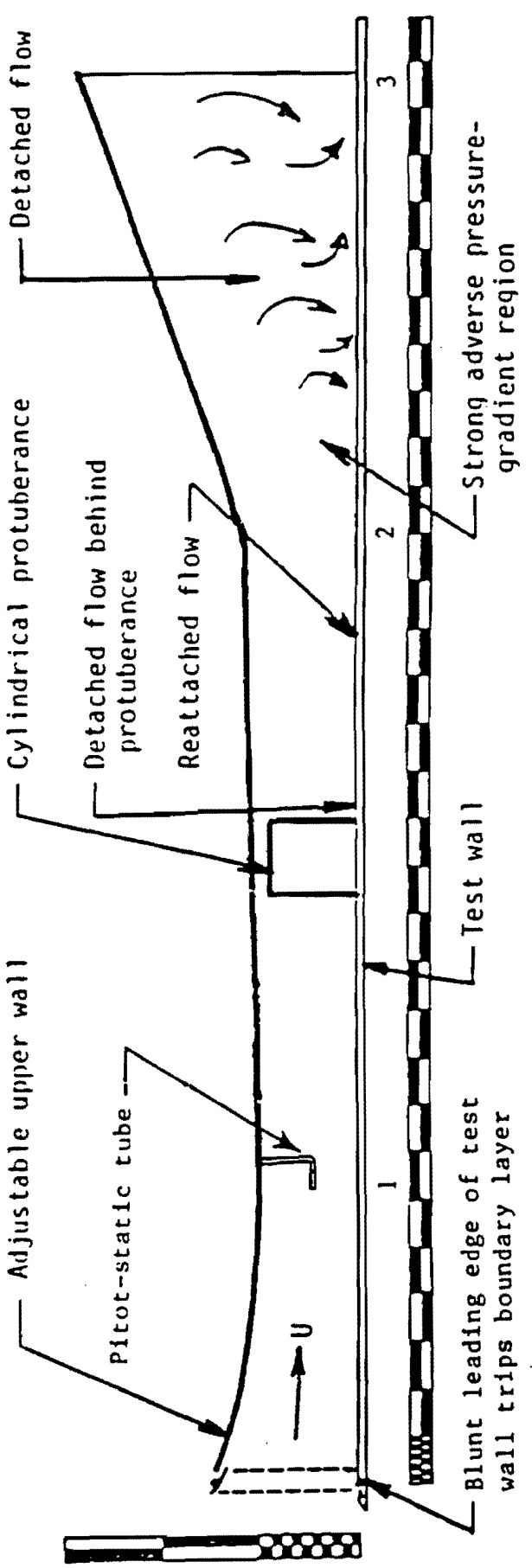

Figure 7. Sideview of the wind-tunnel test section for the wing-body junction experiments.: Major divisions on scales $=10$ inches. 


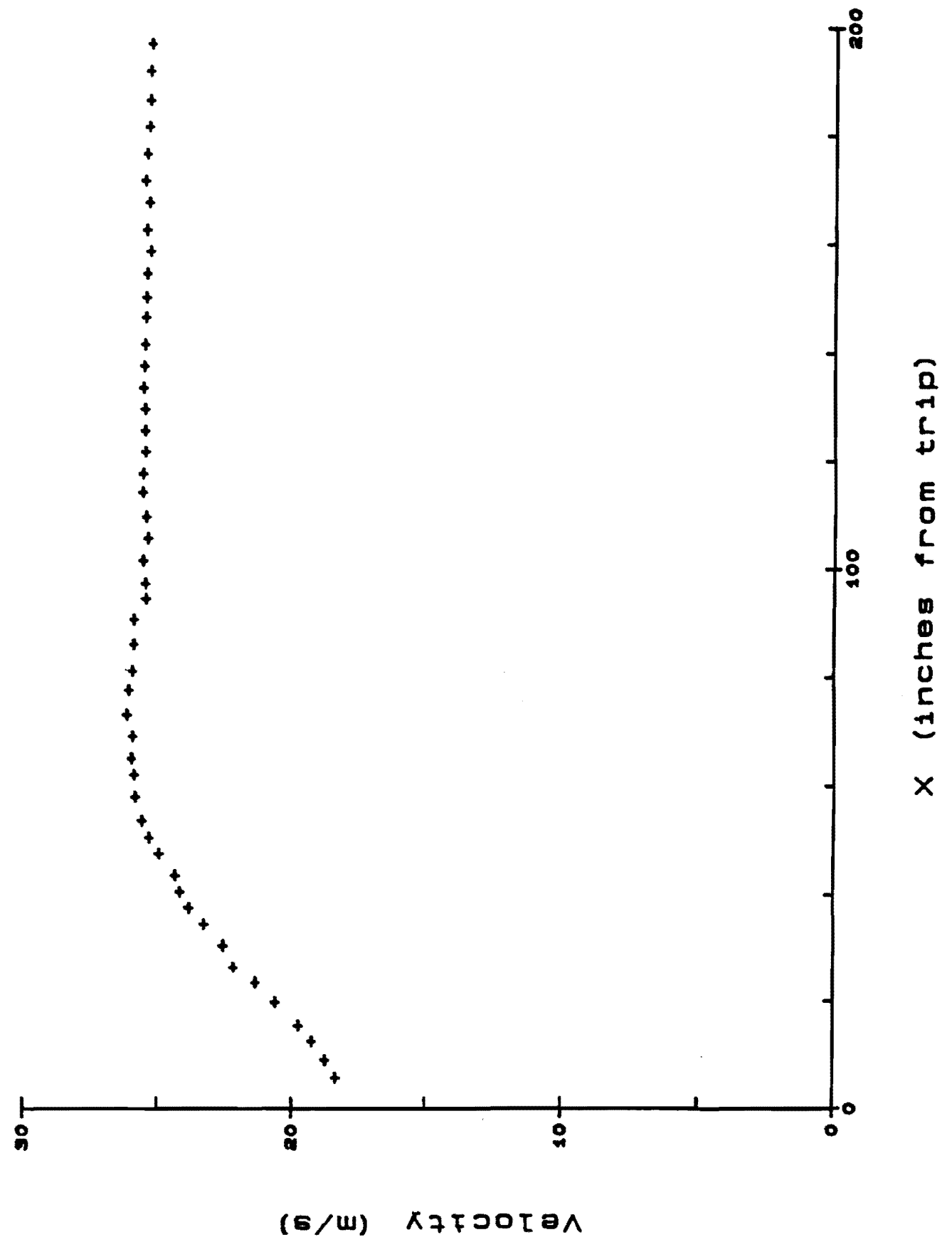

Figure 8. Free-stream velocity distribution along the axis of the tunnel test section at 27 $\mathrm{m} / \mathrm{sec}$ reference velocity 


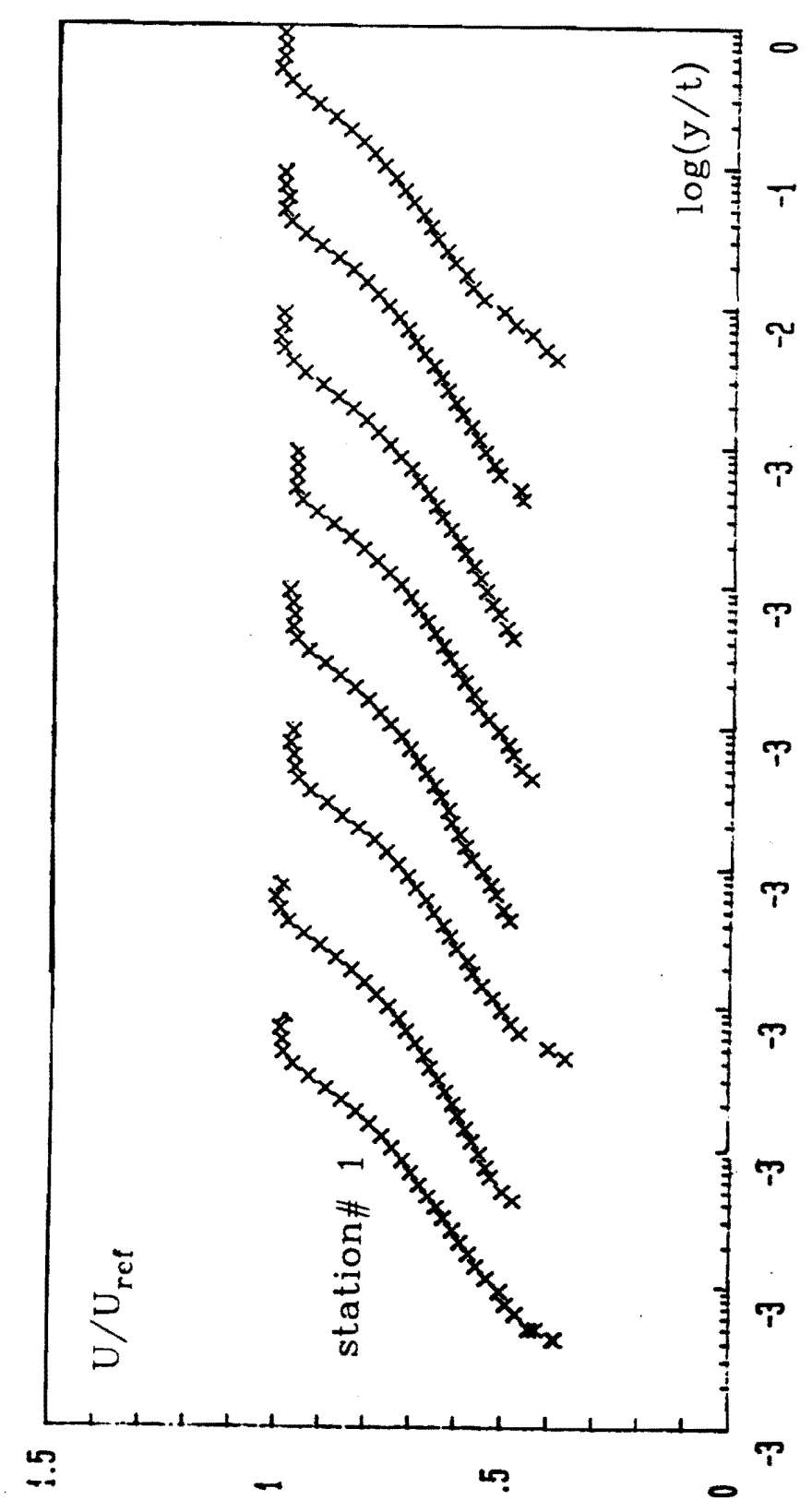

Figure 9a. U velocity component measured with the hot-wire technique at .75 chord length ( chord $=12$ inches upstream of the body expressed in tunnel coordinates. ): The height in the profiles is nondimensionalized with $t=2.824$ inches maximum body thickness. Profiles from left to right correspond to the stations proceeding in $+z_{T C}$ direction. Note the shifted scale of the absissa. ( see also fig 4 and table 2 ) 


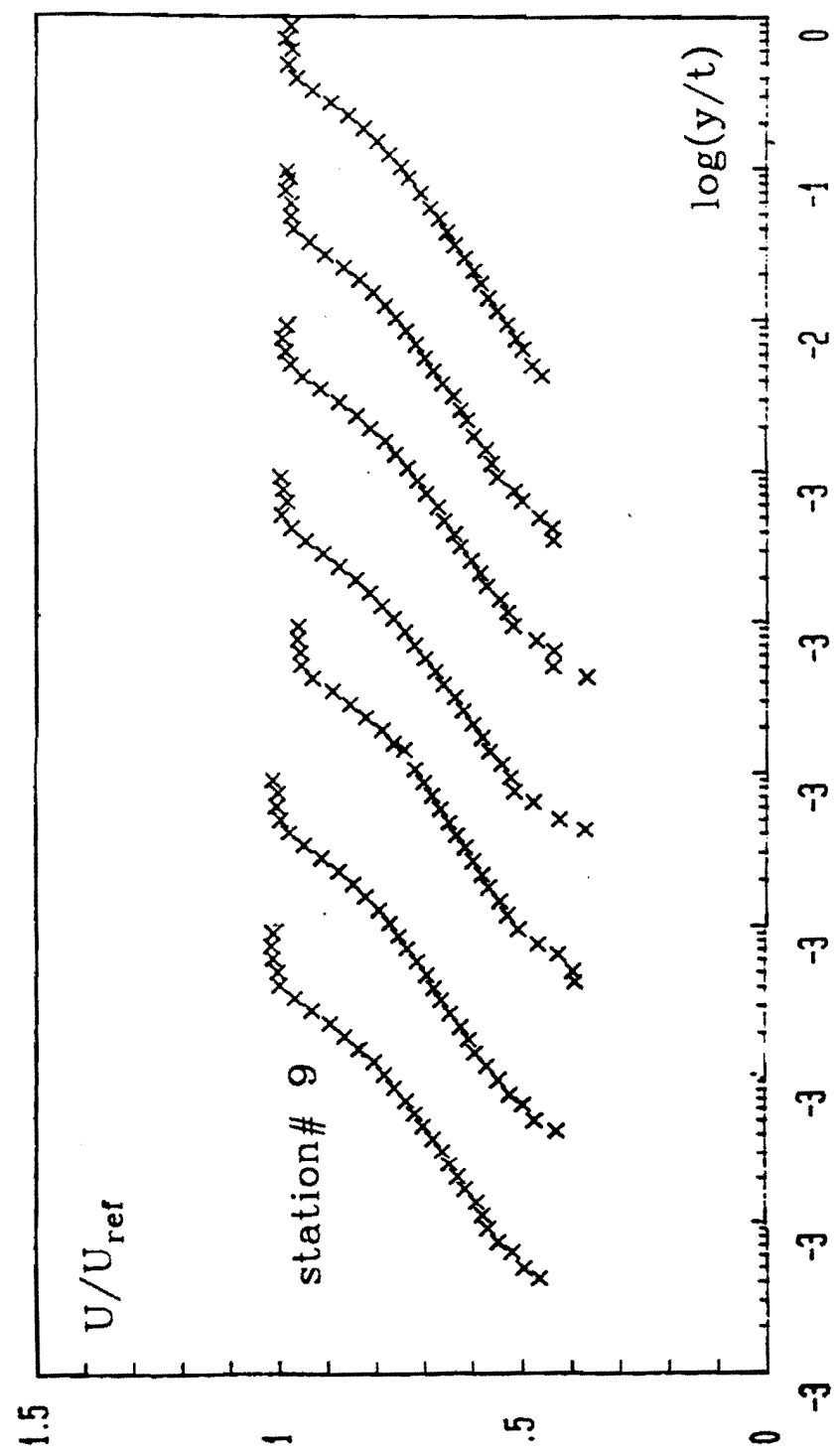

Figure $9 \mathrm{~b}$. U velocity component measured with the hot-wire technique at .75 chord length upstream of the body continued 


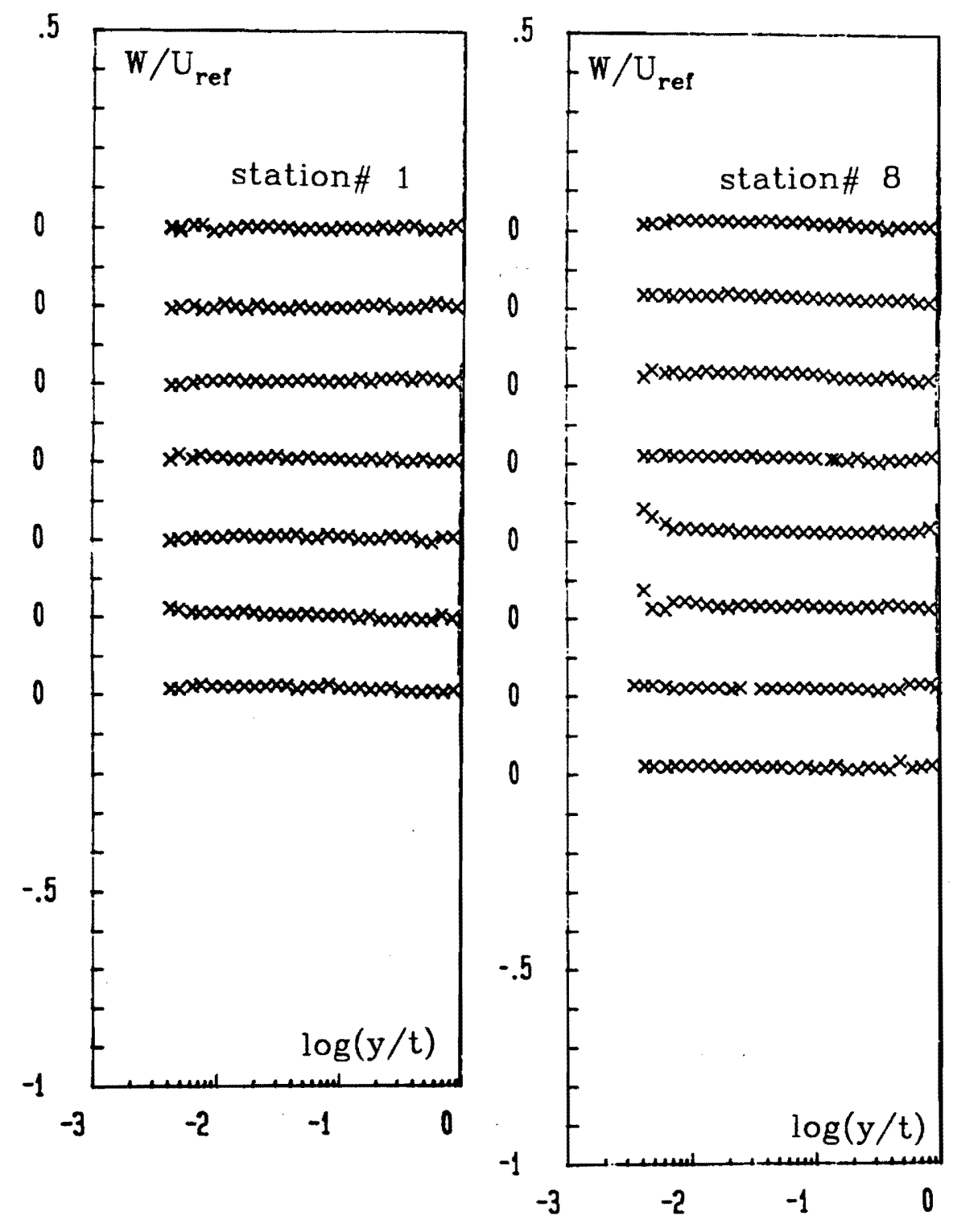

Figure 9c. W velocity component measured with the hot-wire technique at .75 chord length ( chord $=12$ inches upstream of the body expressed in tunnel coordinates.: The height in the profiles is nondimensionalized with $t=2.824$ inches maximum body thickness. Profiles from top to bottom starting with the left-hand side figure correspond to the stations proceeding in $+z_{T C}$ direction. Note the shifted scale of the ordinate. ( see also fig 4 and table 2 ) 


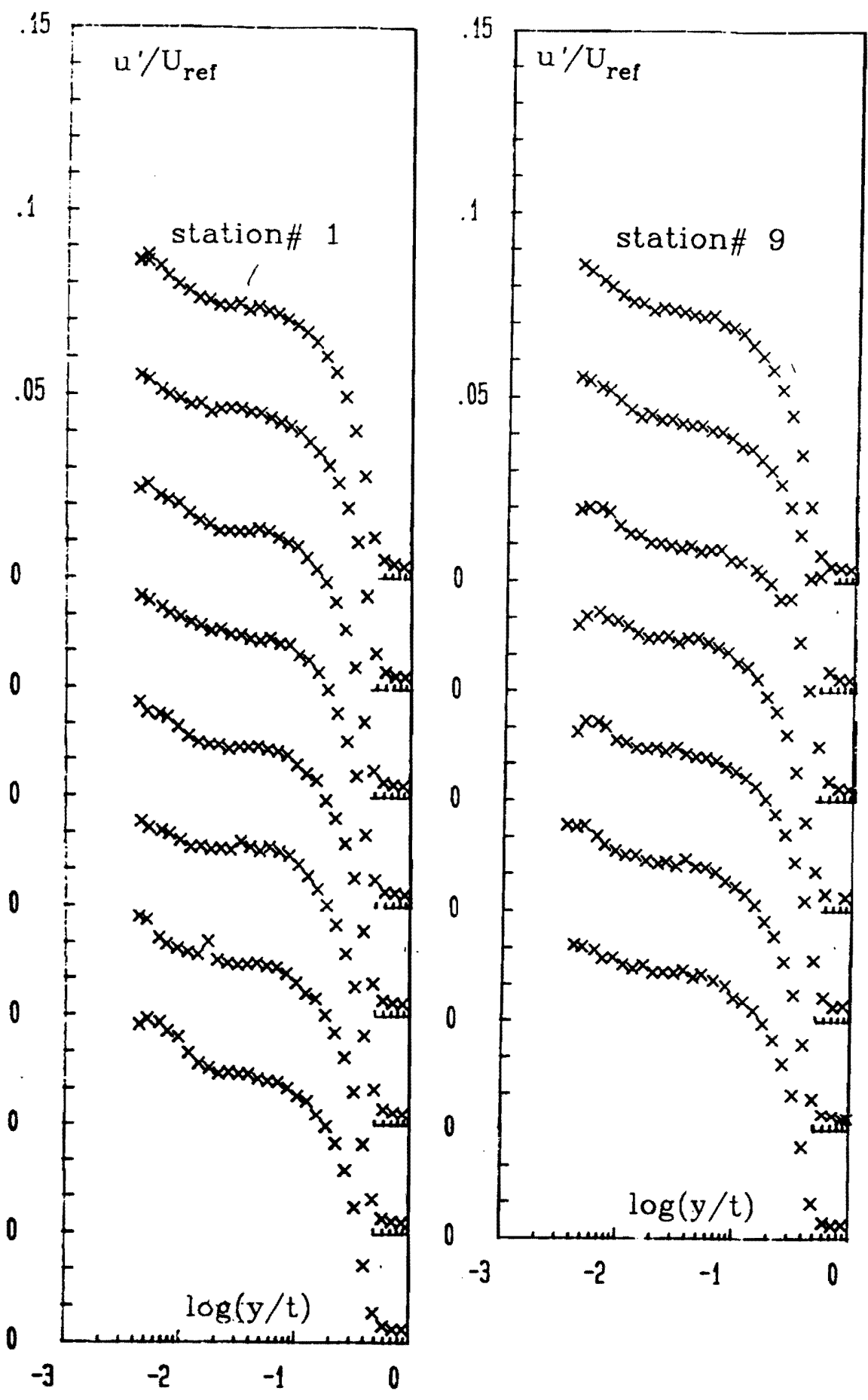

Figure 9d. $u^{\prime}$ fluctuation velocity component measured with the hot-wire technique at .75 chord length ( chord $=12$ inches upstream of the body expressed in tunnel coordinates.: The height in the profiles is nondimensionalized with $t=2.824$ inches maximum thickness. Profiles from top to bottom starting with the left-hand side figure correspond to the stations proceeding in $+z_{T C}$ direction. Note the shifted scale of the ordinate. (see also fig 4 and table 2 ) 


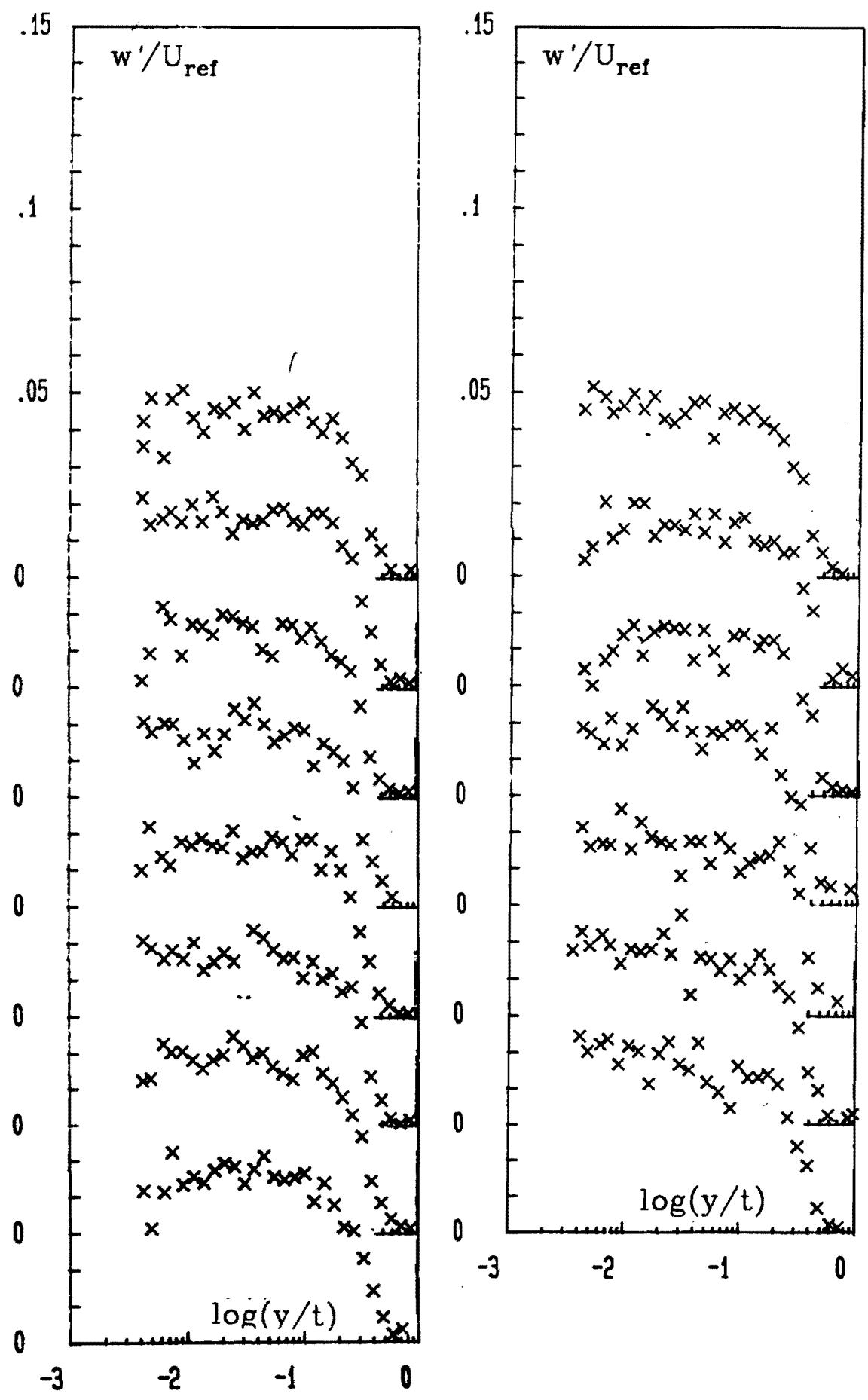

Figure 9e. $\quad w^{\prime}$ fluctuation velocity component measured with the hot-wire technique at .75 chord length ( chord $=12$ inches upstream of the body expressed in tunnel coordinates.: The height in the profiles is nondimensionalized with $t=2.824$ inches maximum thickness. Profiles from top to bottom starting with the left-hand side figure correspond to the stations proceeding in $+z_{T C}$ direction. Note the shifted scale of the ordinate. ( see also fig 4 and table 2 ) 


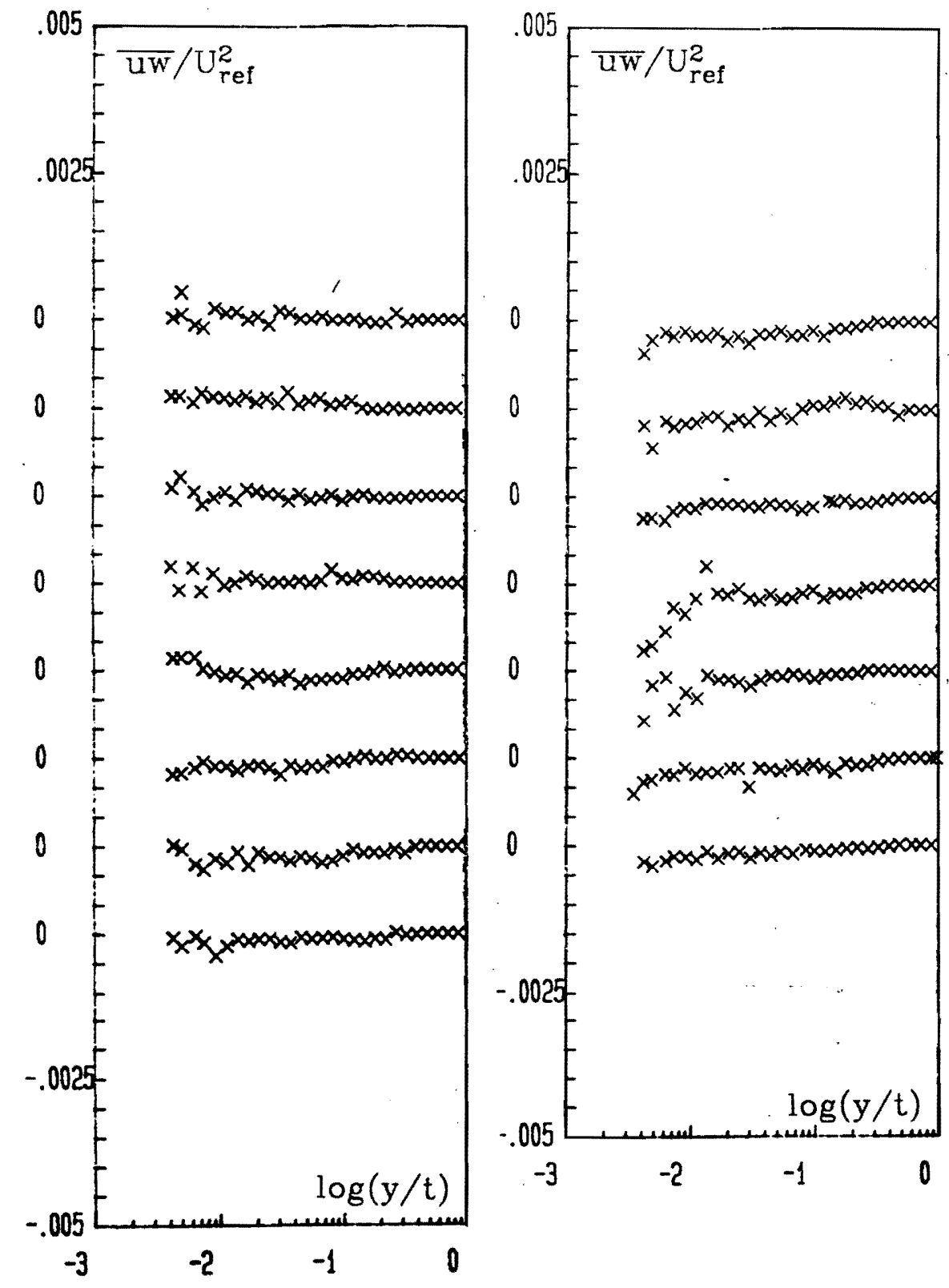

Figure 9f. $-\overline{\mathrm{uw}}$ shear stress component measured with the hot-wire technique at .75 chord length ( chord $=12$ inches thickness.: The height in the profiles is nondimensionalized with $t=2.824$ inches maximum thickness. Profiles from top to bottom starting with the left-hand side figure correspond to the stations proceeding in $+z_{T C}$ direction. Note the shifted scale of the ordinate. ( see also fig 4 and table 2 ) 


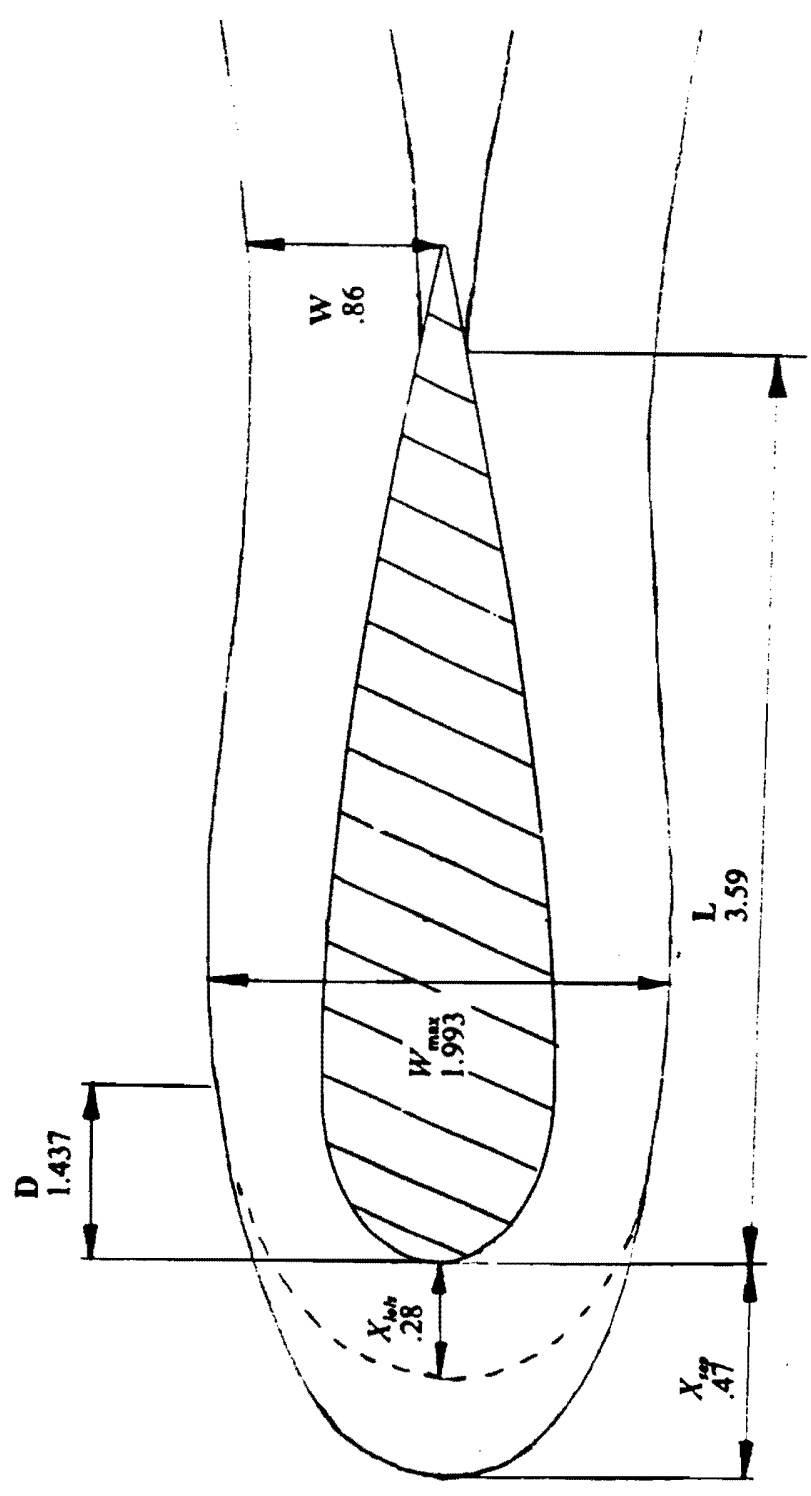

Figure 10. Characteristic dimensions visualized with the oil flow technique.: The characteristic dimensions are nondimensionalized with the maximum body thickness $\mathrm{t}=2.824$ inches. 


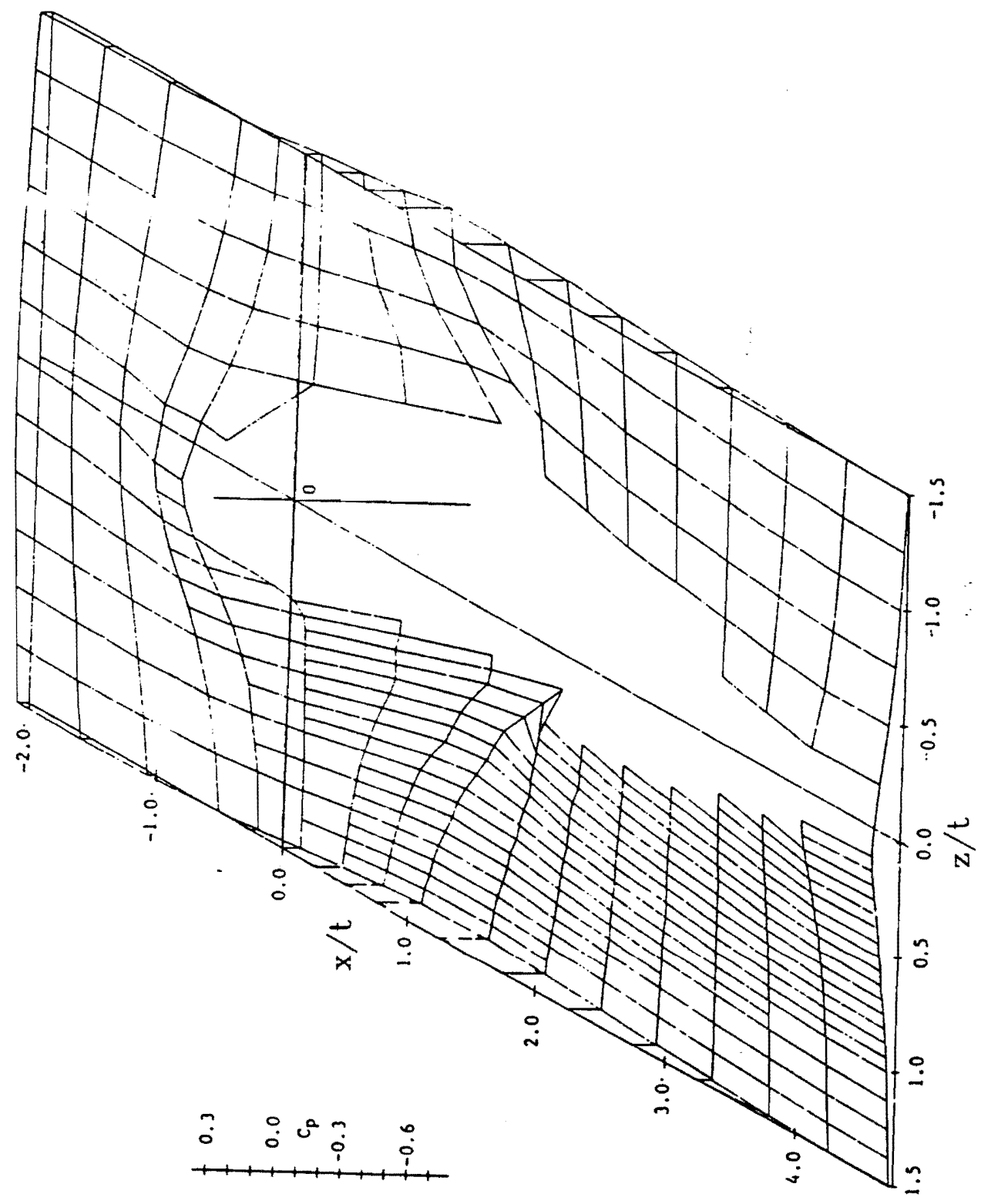

Figure 11. Distribution of time-mean static pressure on the test wall surrounding the wing at Reynolds number based on momentum thickness at 0.75 upstream of the body on the centerline of the tunnel of 5936. 


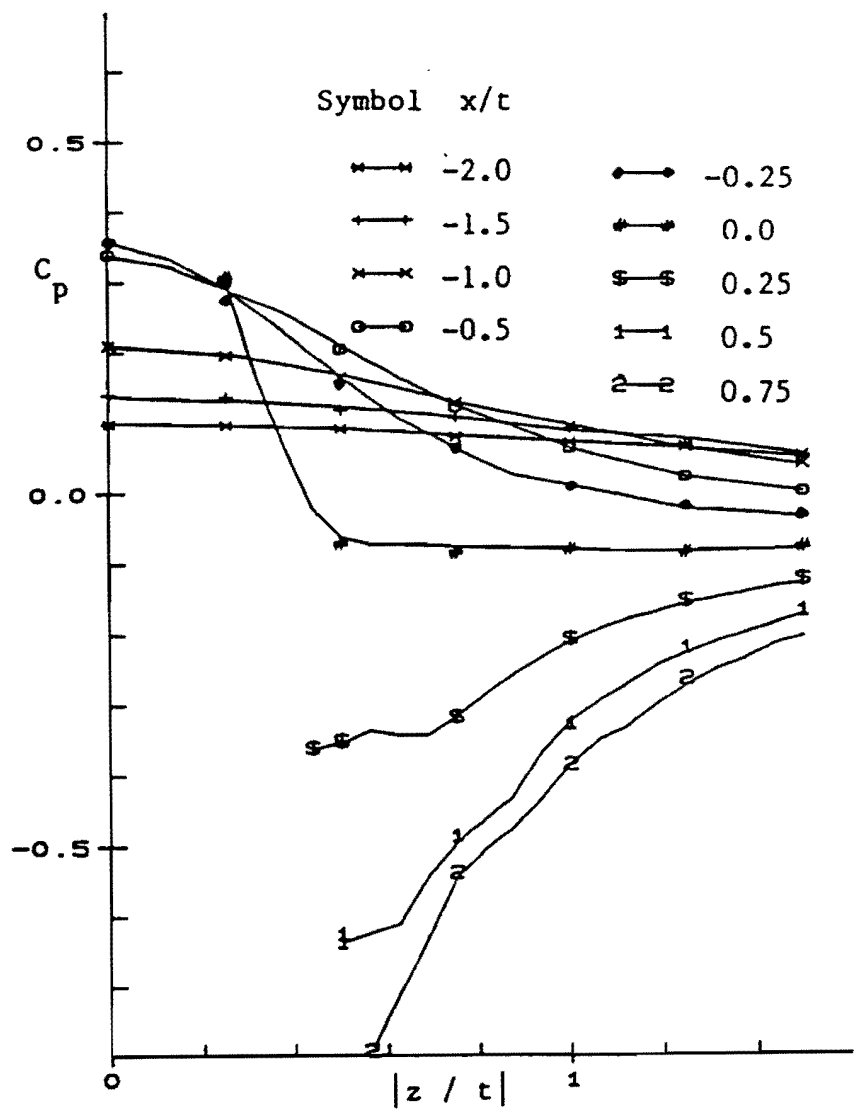

Figure 12a. Distribution of time-mean static pressure on the wall surrounding the wing at Reynolds number based on momentum thickness of 5936.: Lines indicate $+z_{T C}$, points indicate $-z_{T C}$. 


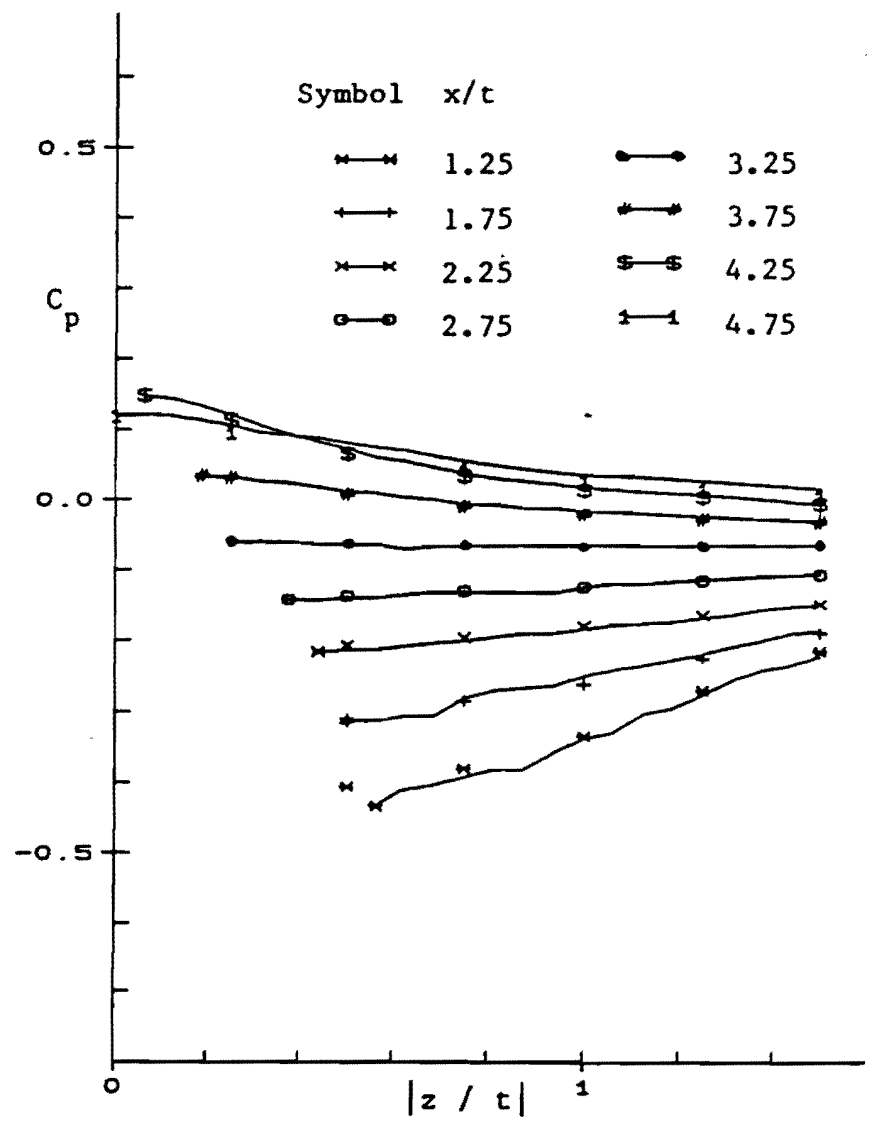

Figure 12b. Distribution of time-mean static pressure on the wall surrounding the wing at Reynolds number based on momentum thickness of 5936: Lines indicate $+z_{T C}$, points indicate $-z_{T C}$. 


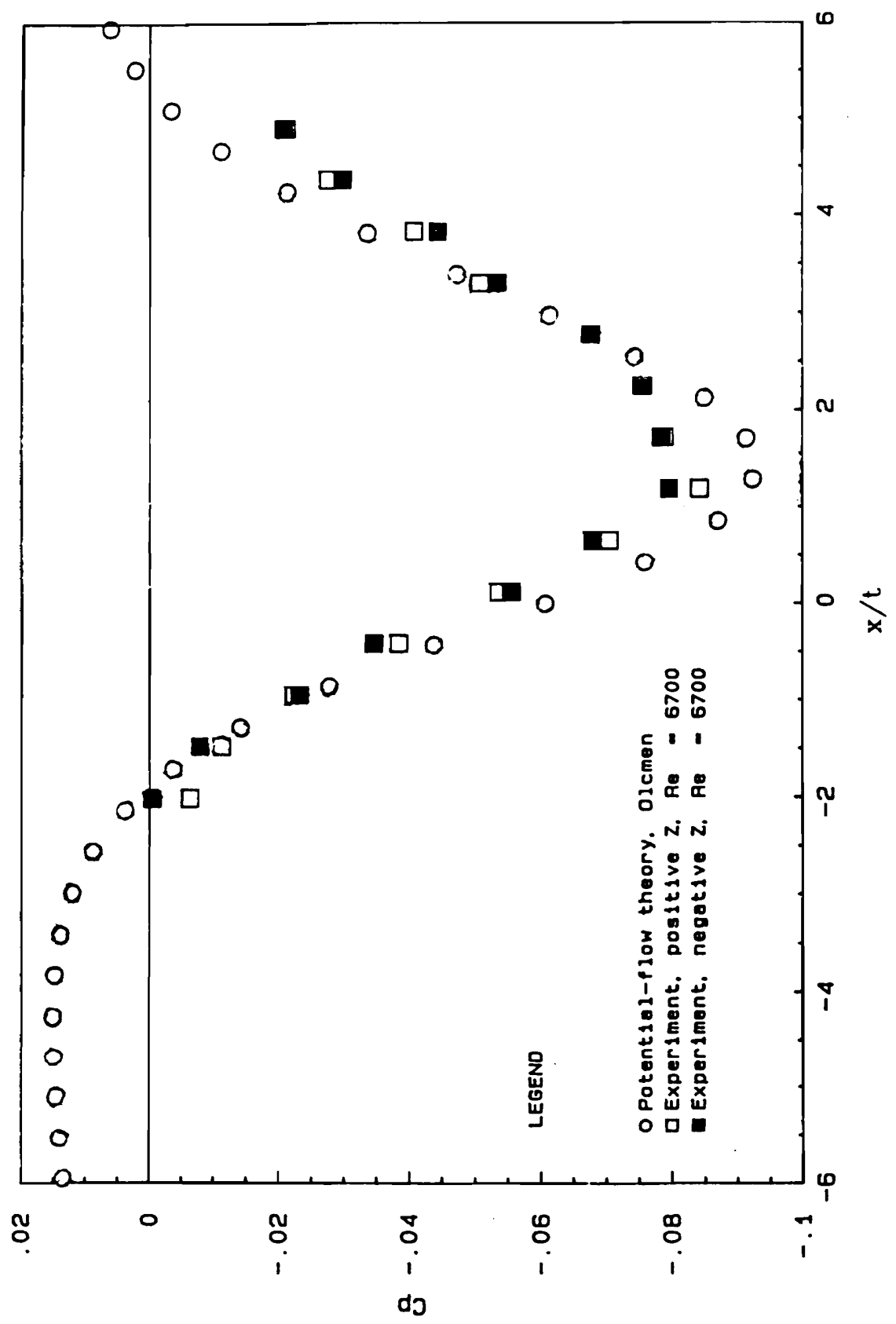

Figure 13. Comparison of surface pressure distributions in tunnel coordinates at $z / t=+-3.187$ 


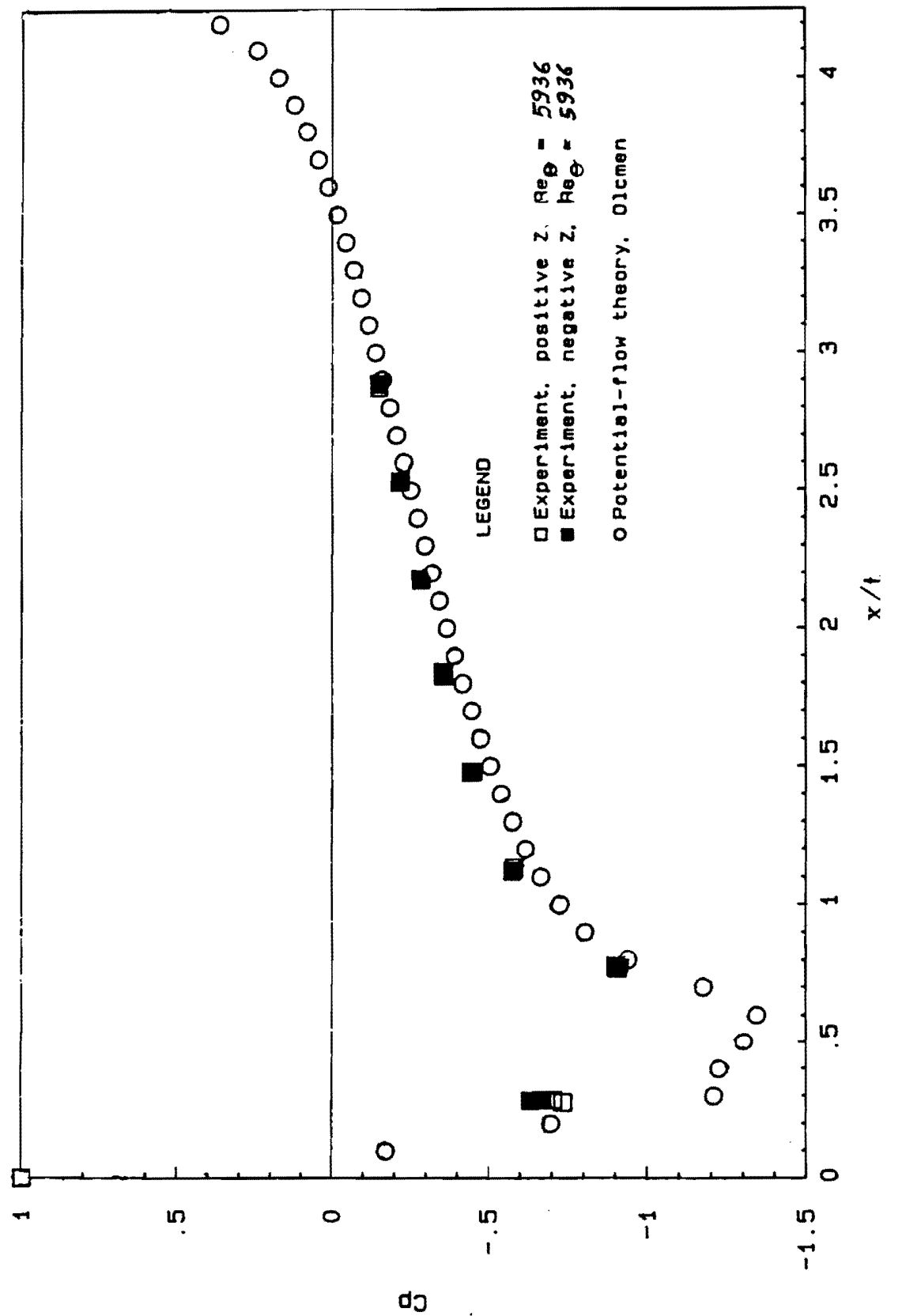

Figure 14. Comparison of surface pressure distributions on wing. 


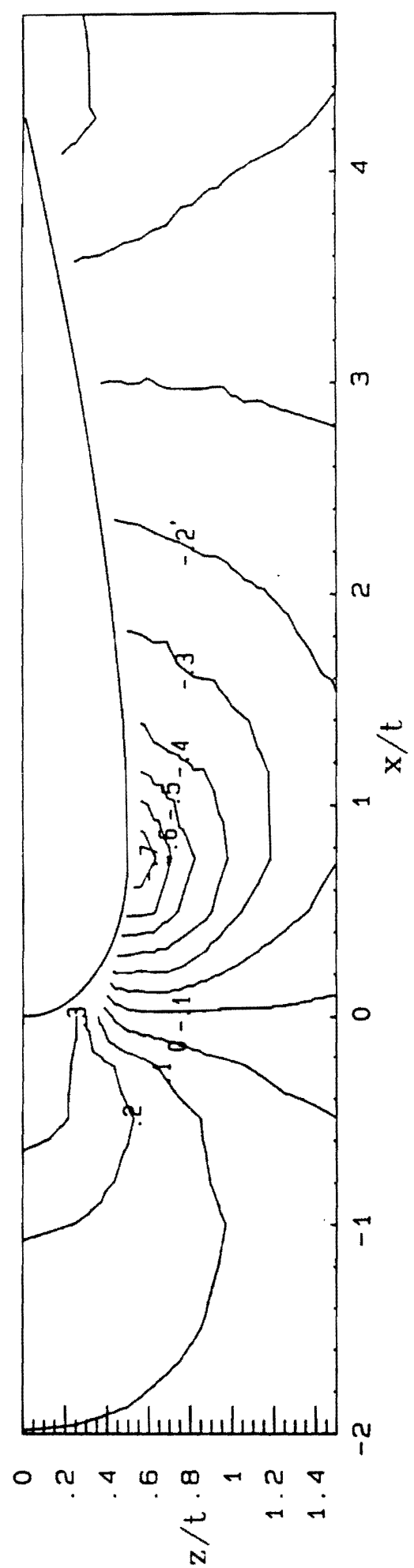

Figure 15a. Contours of measured mean static pressure on the test wall. 


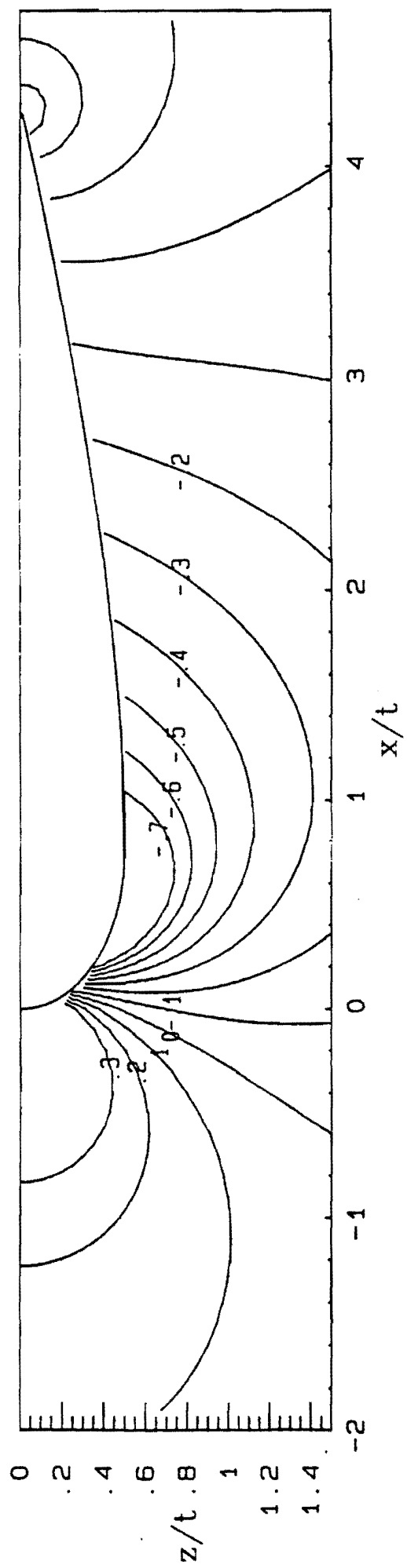

Figure 15b. Contours of computed mean static pressure on the test wall:: Two-dimensional inviscid calculation of Olcmen. 

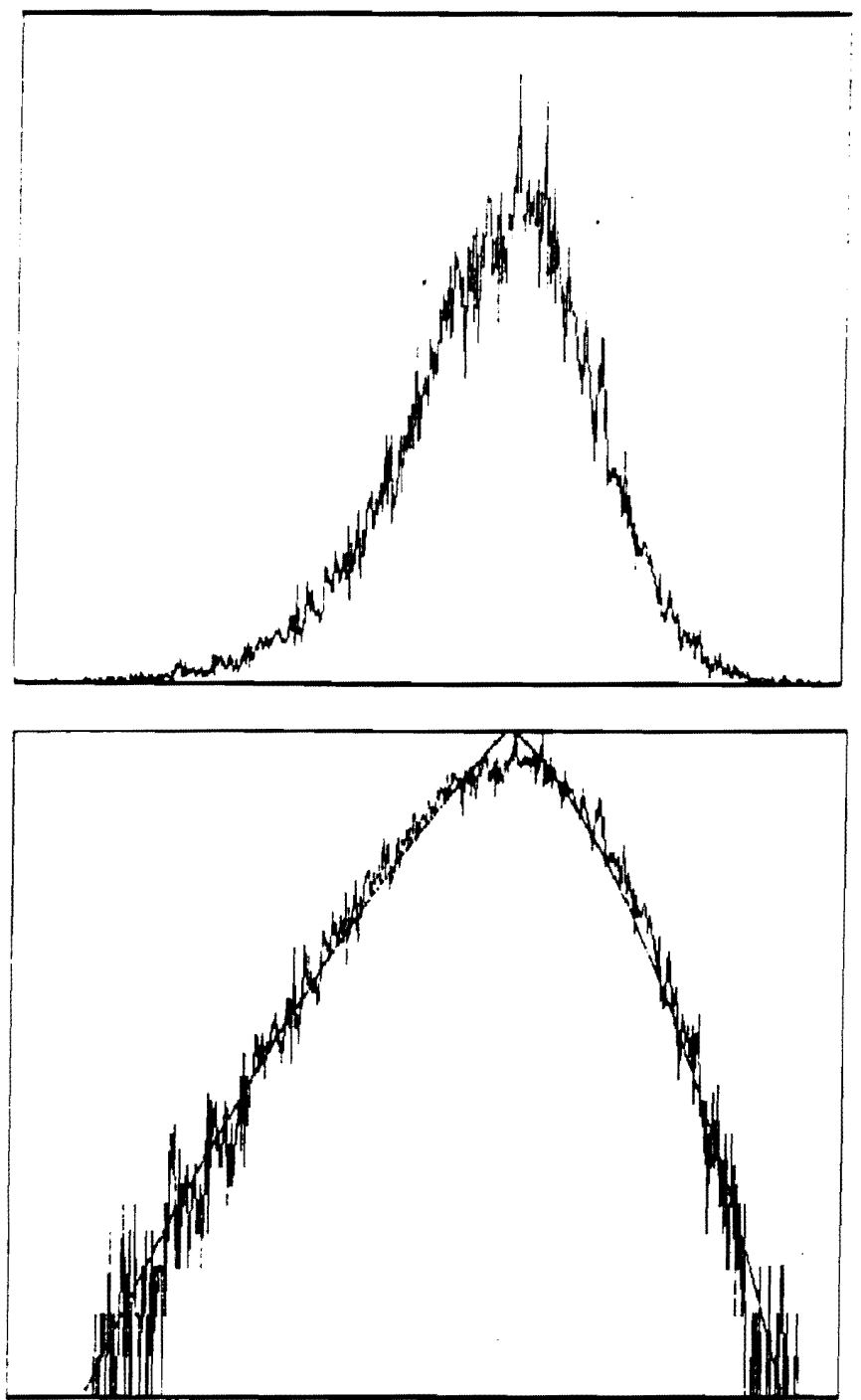

Figure 16a. Scheamatic figure of the histogram noise level determining routine.: top:The histogram with the noise.bottom:Parabola fitting to both sides of the peak of the logarithm of the histogram values. 

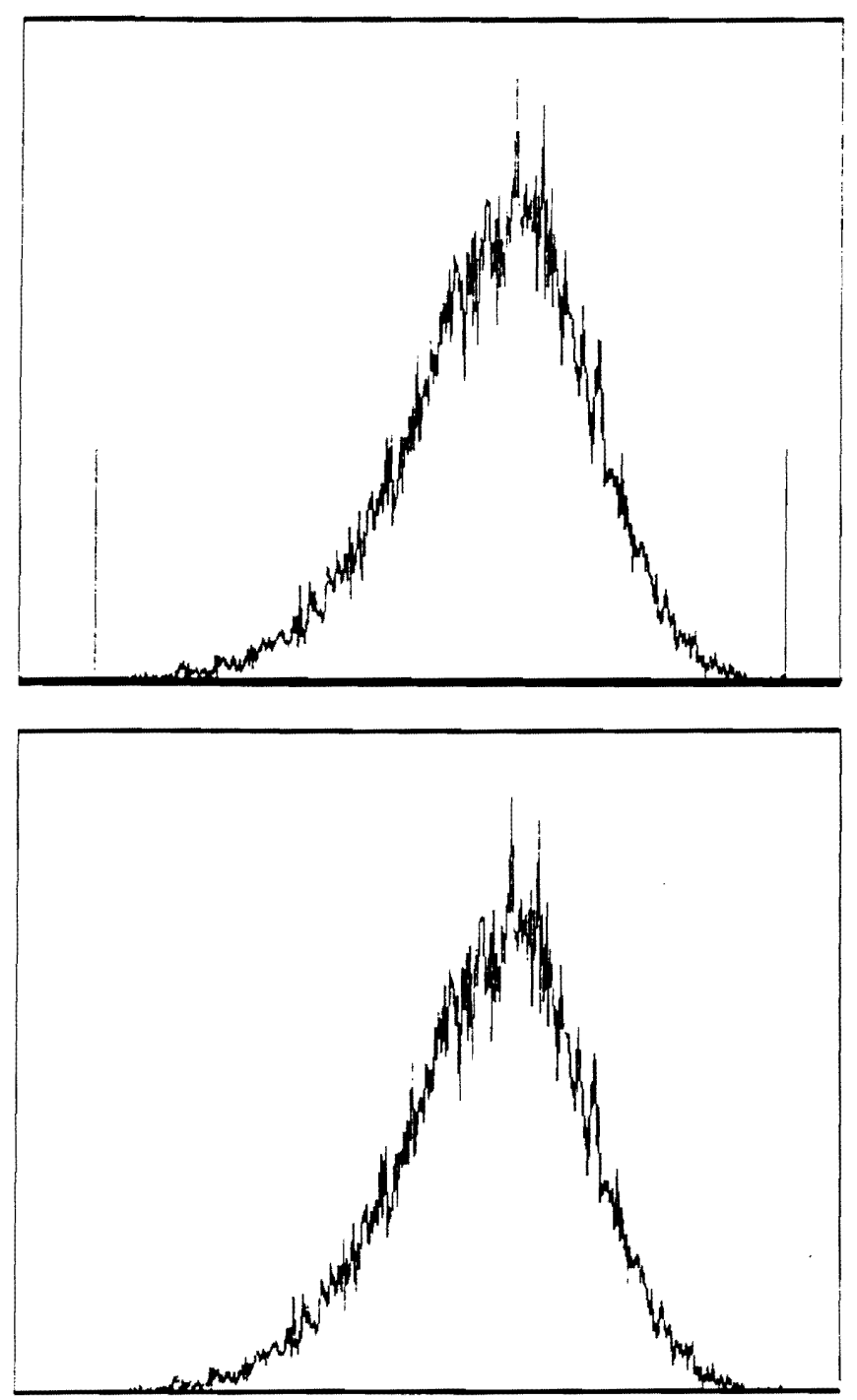

Figure 16b. Scheamatic figure of the histogram noise level determining routine.: top:The noise level of the histogram..bottom:The histogranafter the noise is removed. 

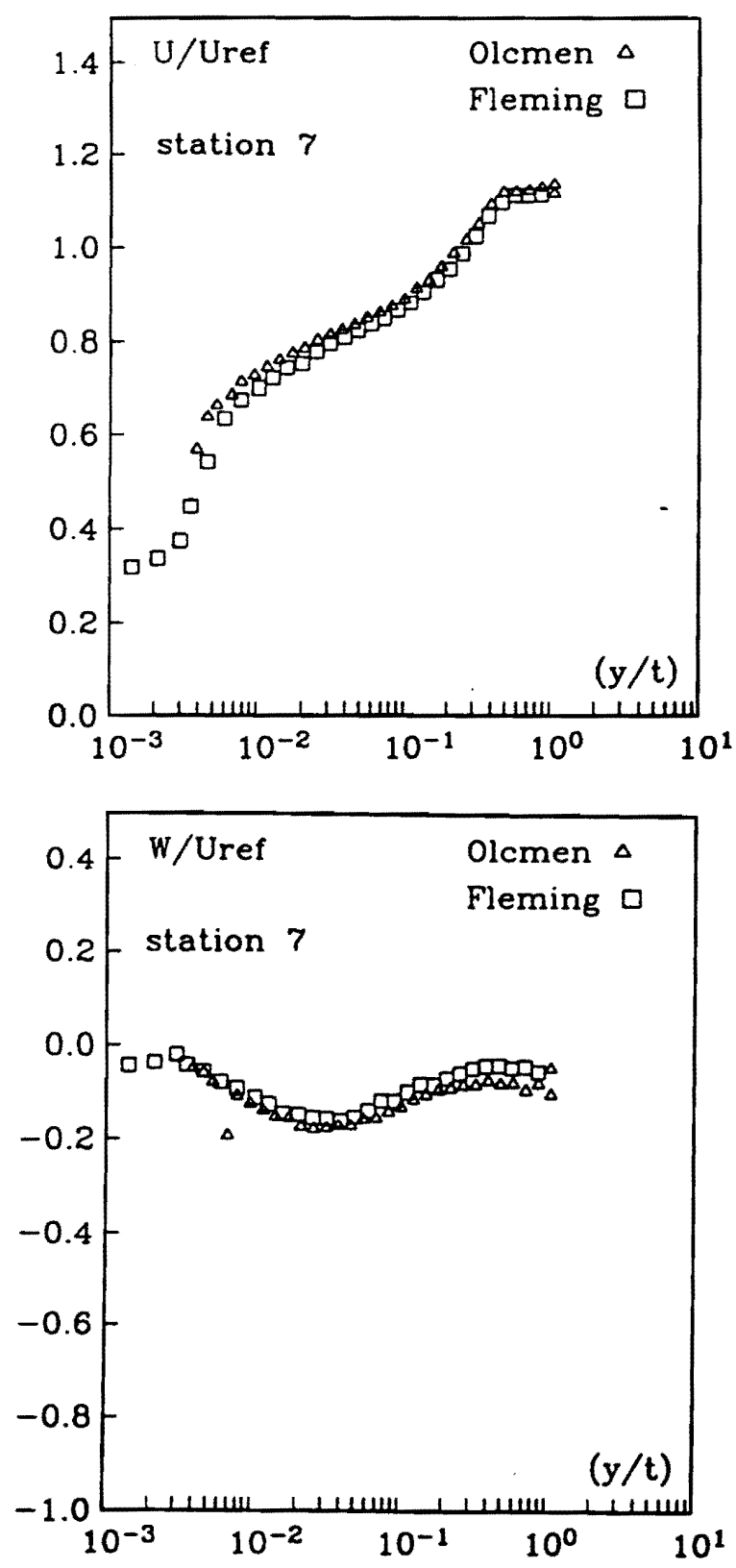

Figure 16b. Comparison of $U$ and $W$ mean velocity components measured with hot-wire technique using two different set of equipments and reduction programs.: At station 7 on the right-hand side of the wing. 

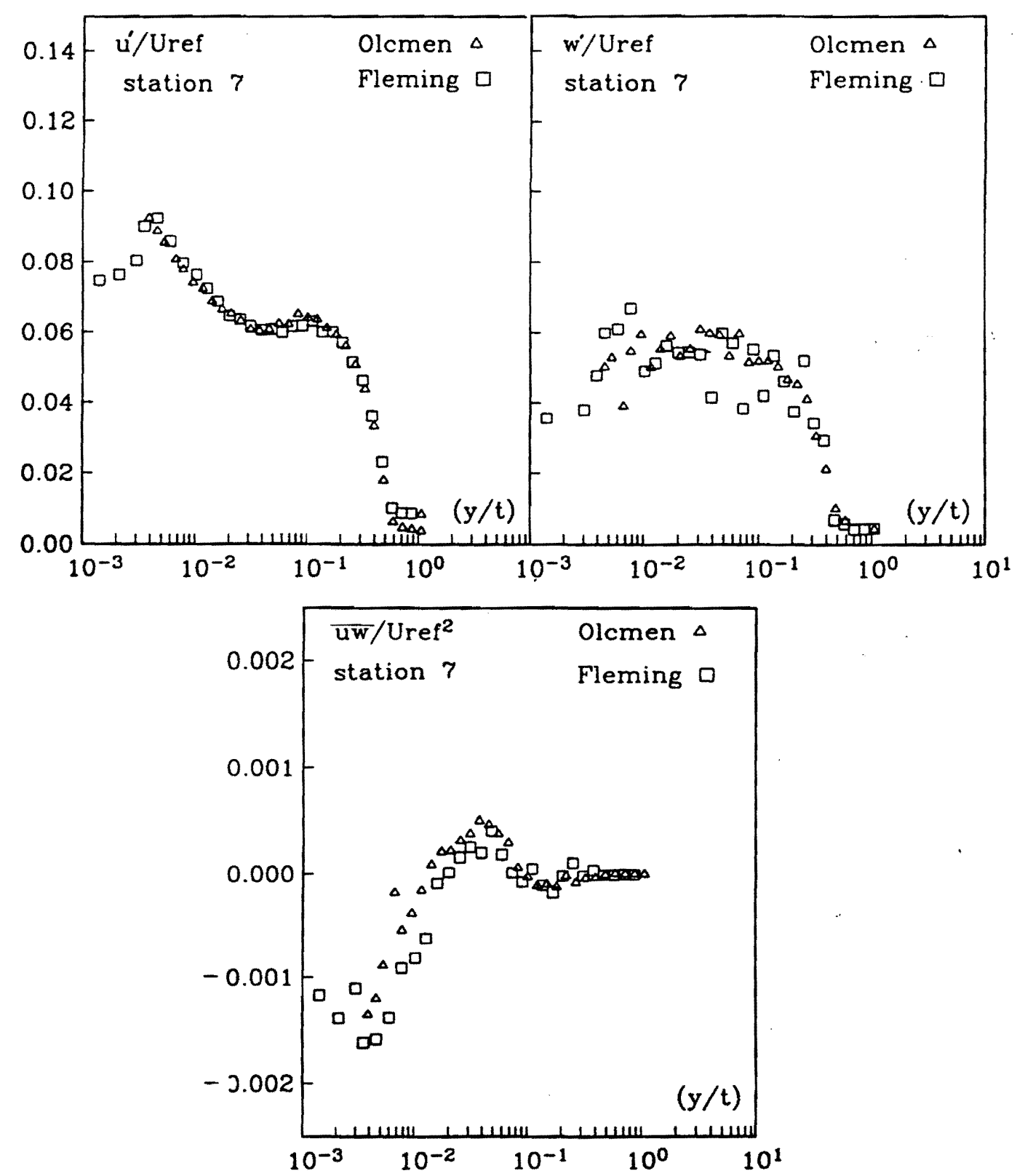

Figure 17b. Comparison of $u^{\prime}$ and $w^{\prime}$ fluctuation velocity and-uw shear stress components measured with hot-wire technique using two different set of equipments and reduction programs: At station 7 on the right-hand side of the wing. 


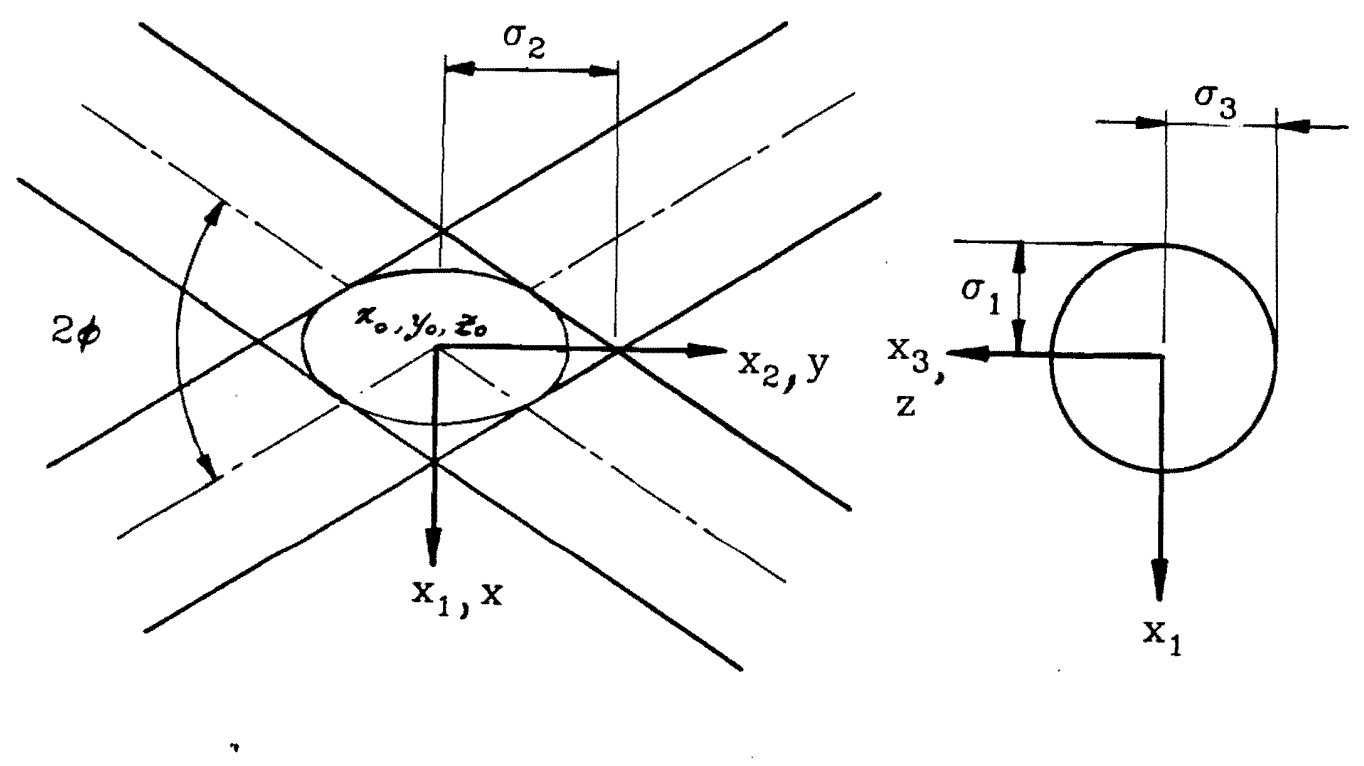

Figure 18. Probe volume formed by two intersecting laser beams. 


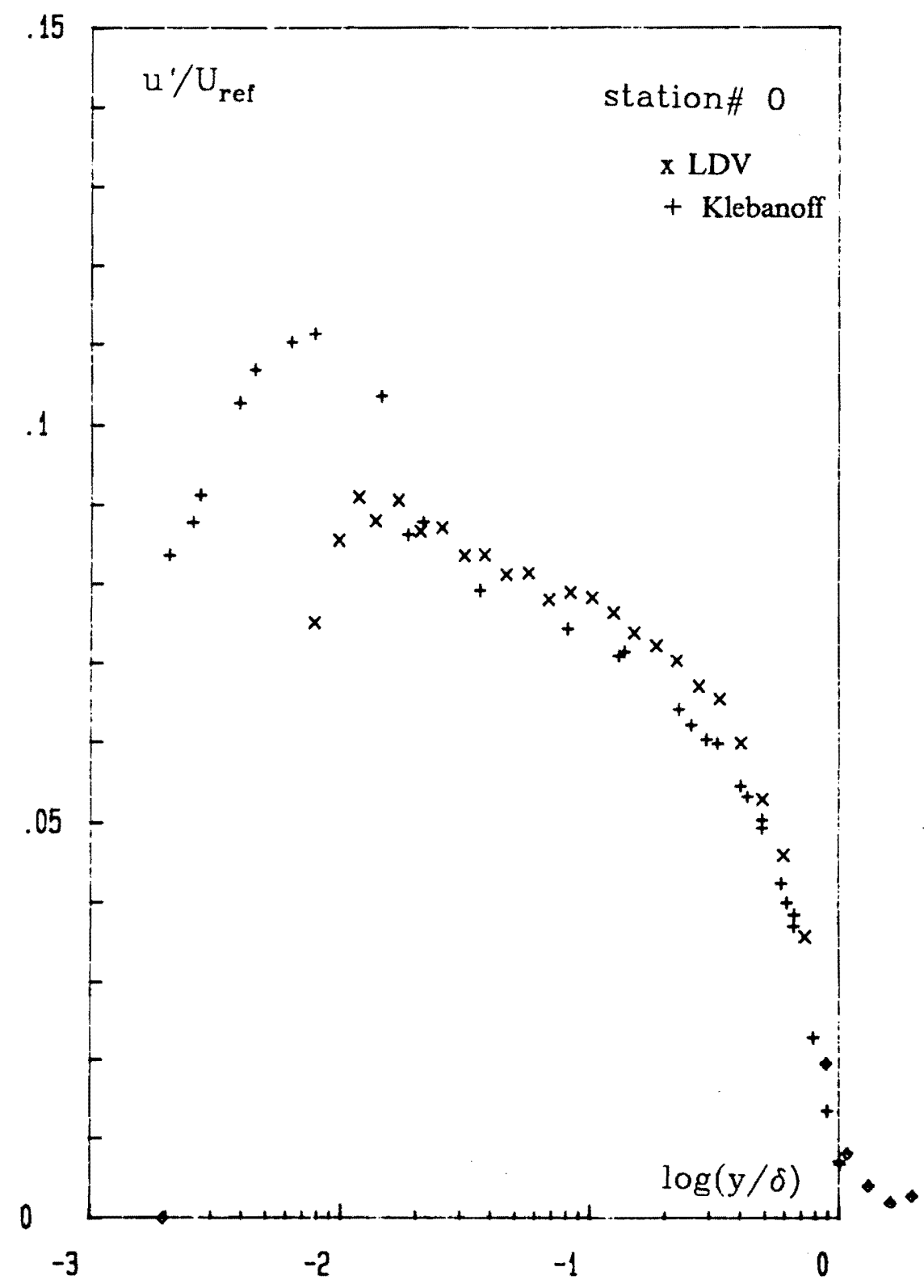

Figure 19. Comparison of station 0 u'fluctuation velocity LDV profile with Klebanoff's two-dimensional turbulent boundary layer data.: X LDV data, + Klebanoff's data. 

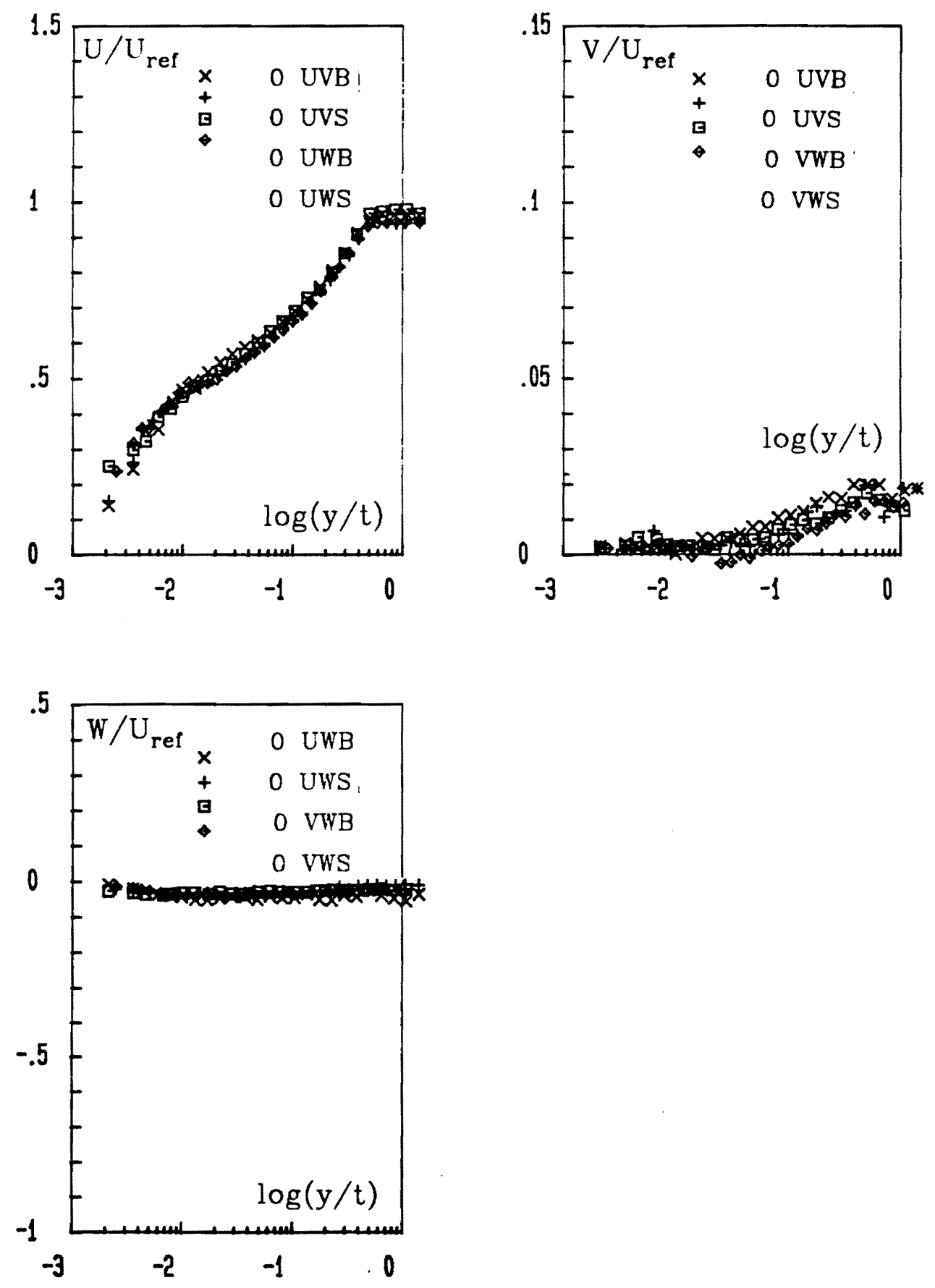

Figure 20a. U,V,W mean velocity components measured with two different LDV techniques presented in tunnel coordinates.: UV, UW and VW show the LDV beam system used to obtain the data. Extensions $B$ and $S$ denote the measurements taken with Burst Spectrum Analyzer and fast sampling rate swept-spectrum analyzer,respectively. Station numbers are also shown in the figures. 

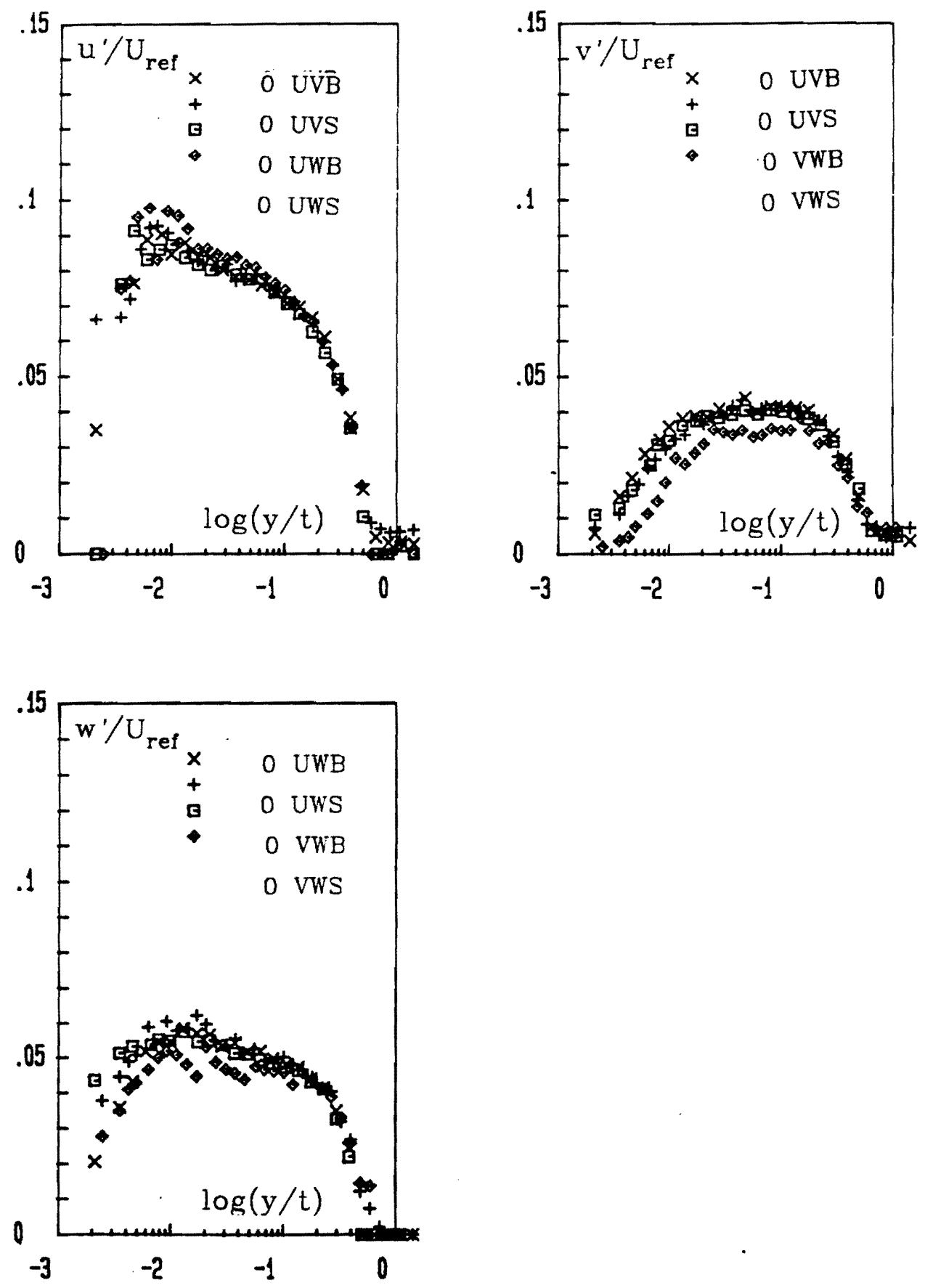

Figure 20b. $u^{\prime}, v^{\prime}, w^{\prime}$ fluctuation velocity components measured with two different LDV techniques presented in tunnel coordinates.: UV, UW and VW show the LDV beam system used to obtain the data. Extensions $B$ and $S$ denote the measurements taken with Burst Spectrum Analyzer and fast sampling rate swept-spectrum analyzer,respectively. Station numbers are also shown in the figures. 

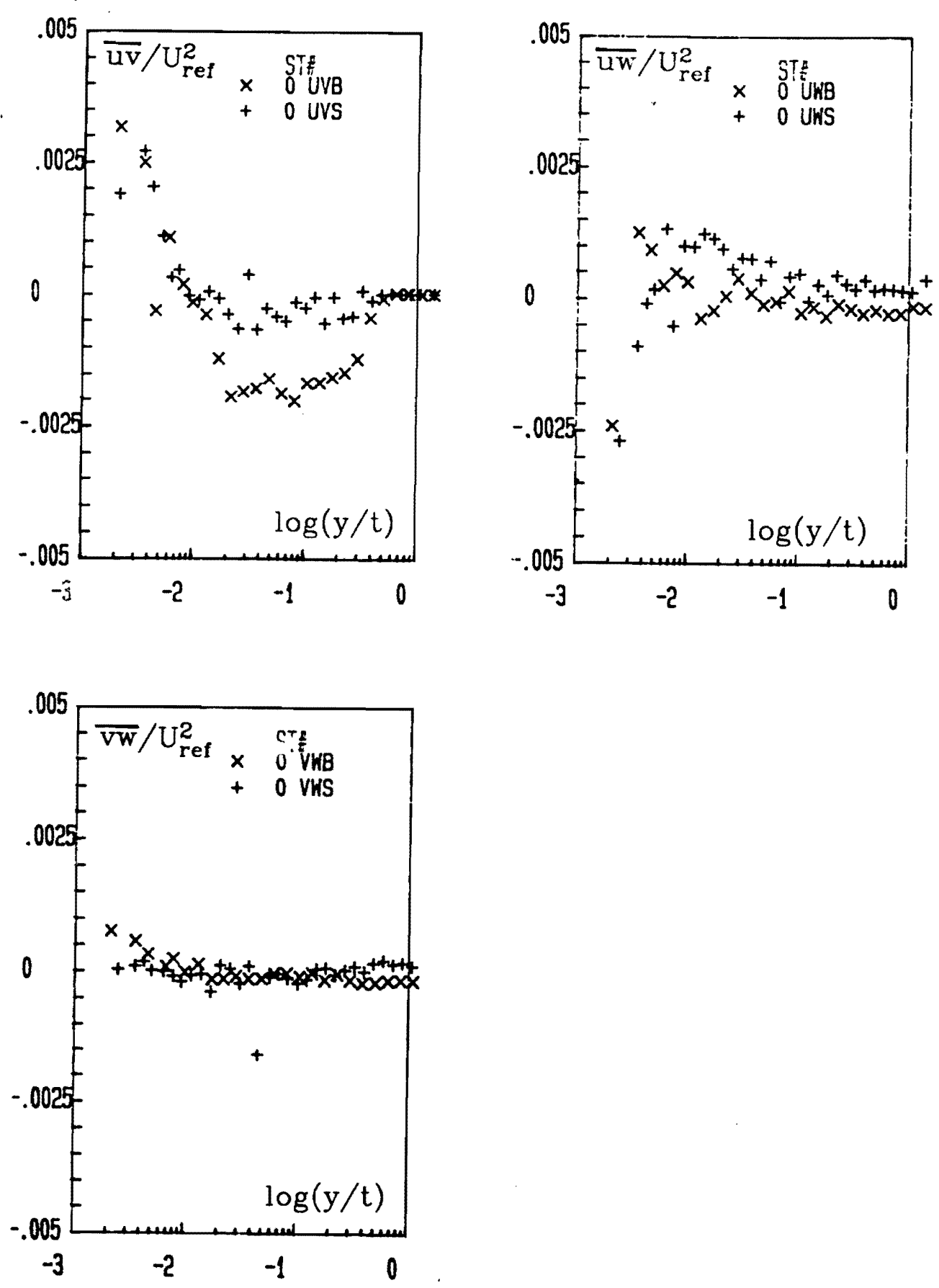

Figure 20c. $-\overline{\mathrm{uv}}, \overline{\mathrm{uw}}, \overline{\mathrm{vw}}$ shear stress components measured with two different LDV techniques presented in tunnel coordinates.: UV, UW and VW show the LDV beam system used to obtain the data. Extensions $B$ and $S$ denote the measurements taken with Burst Spectrum Analyzer and fast sampling rate swept-spectrum analyzer,respectively. Station numbers are also shown in the figures. 

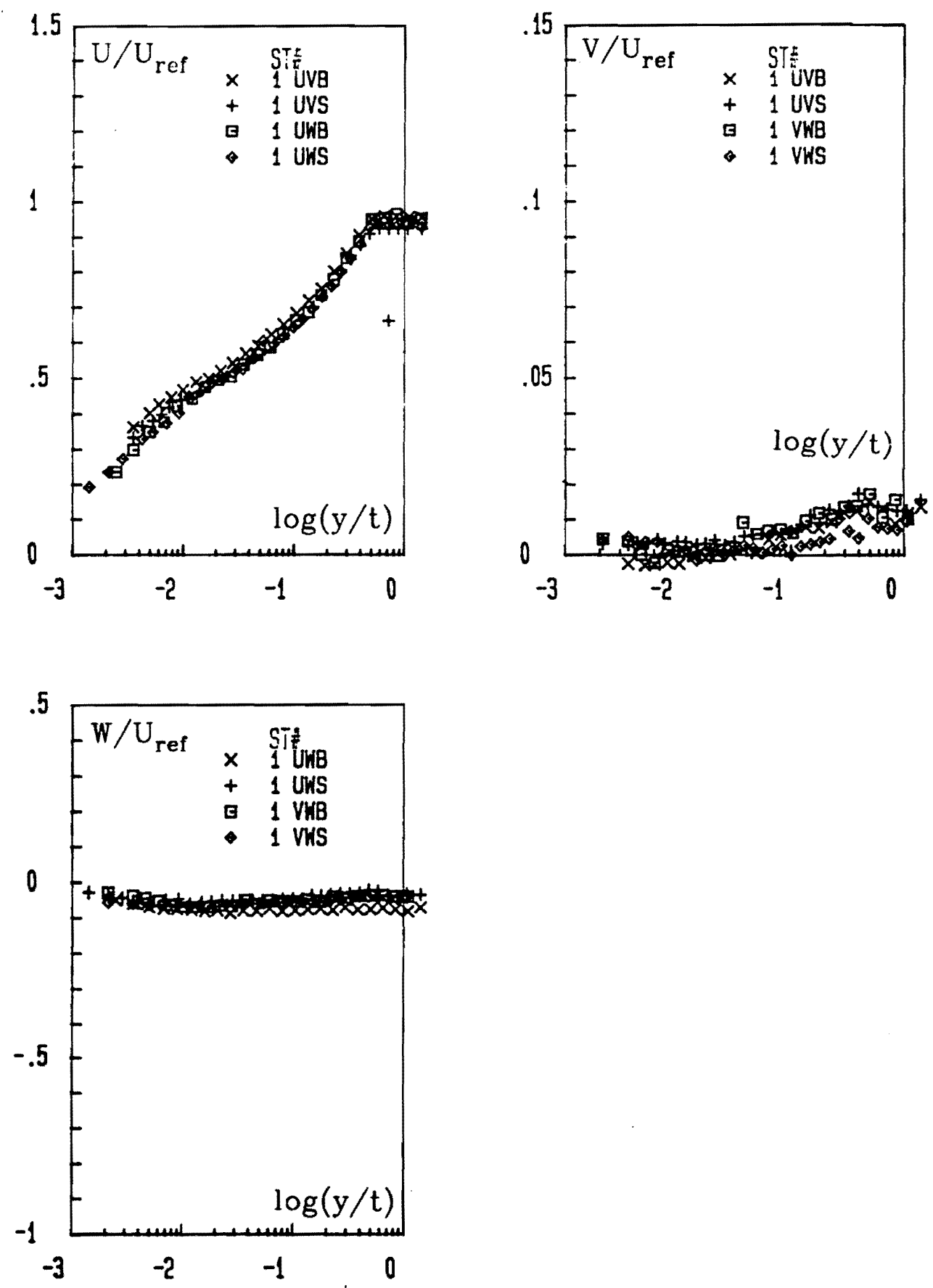

Figure 20d. U,V,W mean velocity components measured with two different LDV techniques presented in tunnel coordinates.: UV, UW and VW show the LDV beam system used to obtain the data. Extensions B and $S$ denote the measurements taken with Burst Spectrum Analyzer and fast sampling rate swept-spectrum analyzer,respectively. Station numbers are also shown in the figures. 

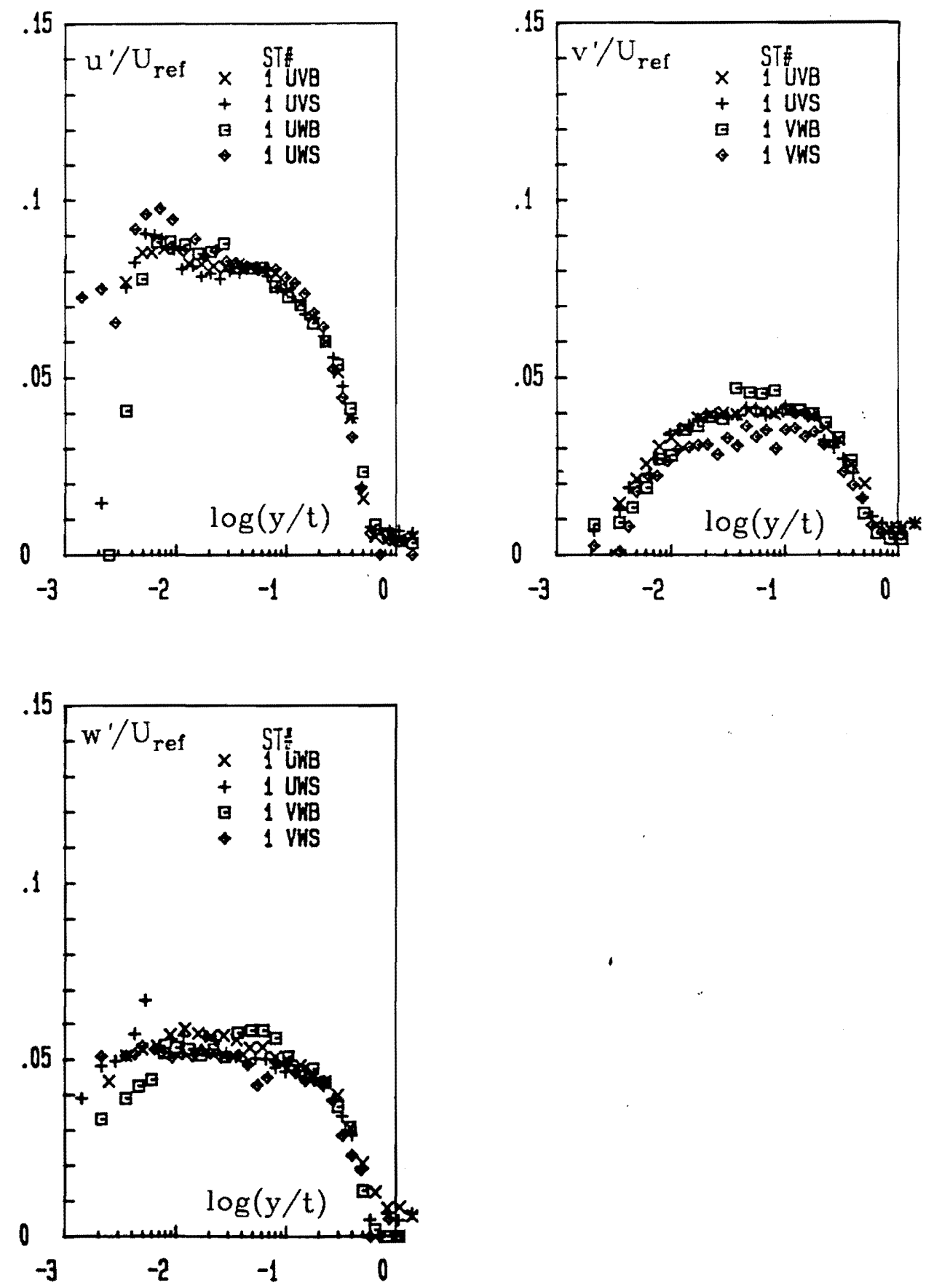

Figure 20e. $u^{\prime}, v^{\prime}, w^{\prime}$ fluctuation velocity components measured with two different LDV techniques presented in tunnel coordinates.: UV, UW and VW show the LDV beam system used to obtain the data. Extensions $B$ and $S$ denote the measurements taken with Burst Spectrum Analyzer and fast sampling rate swept-spectrum analyzer, respectively. Station numbers are also shown in the figures. 

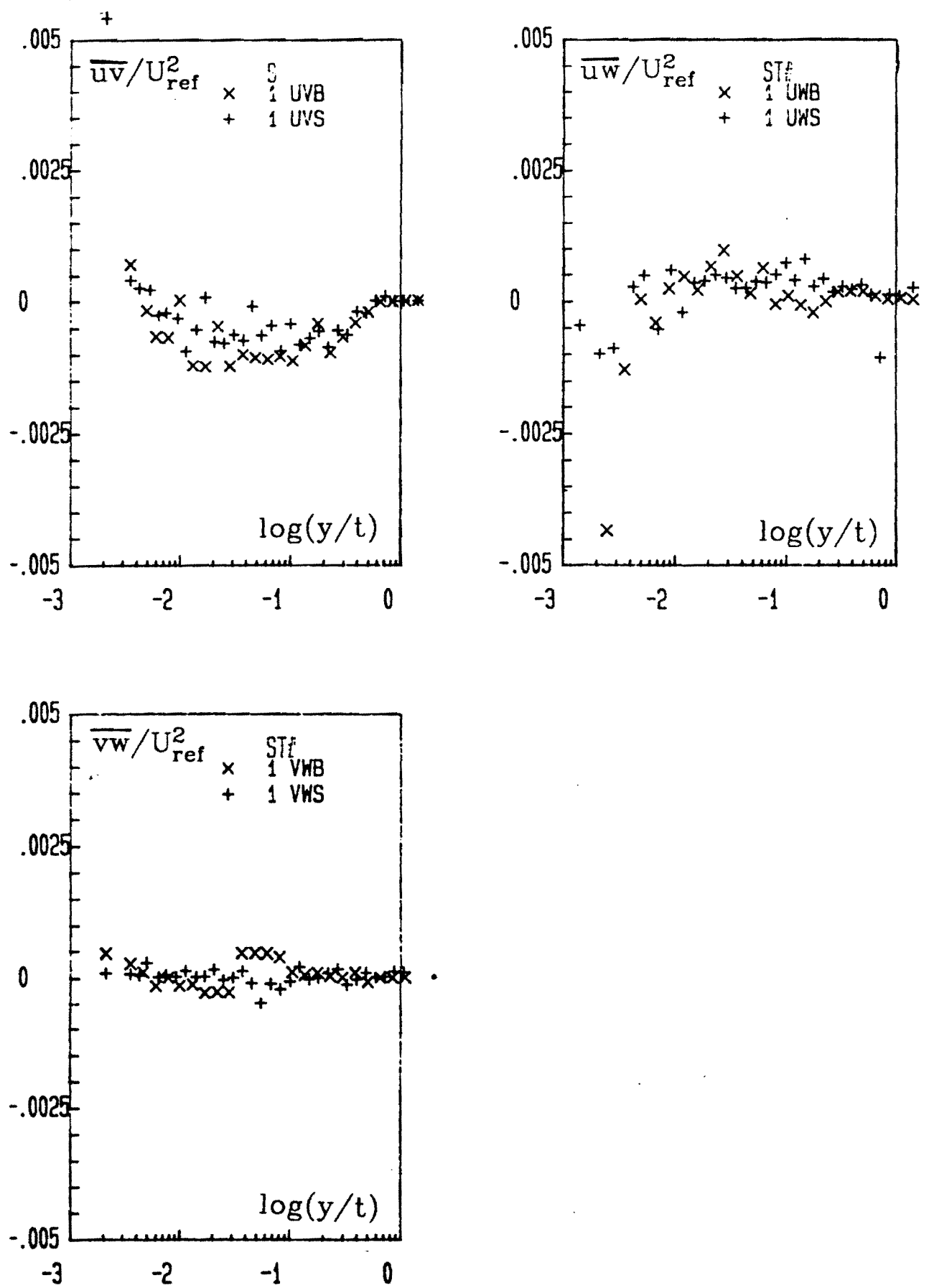

Figure 20f. $-\overline{\mathrm{UV}},-\overline{\mathrm{UW}}, \overline{\mathrm{WW}}$ shear stress components measured with two different LDV techniques presented in tunnel coordinates.: UV, UW and VW show the LDV beam system used to obtain the data. Extensions $B$ and $S$ denote the measurements taken with Burst Spectrum Analyzer and fast sampling rate swept-spectrum analyzer,respectively. Station numbers are also shown in the figures. 

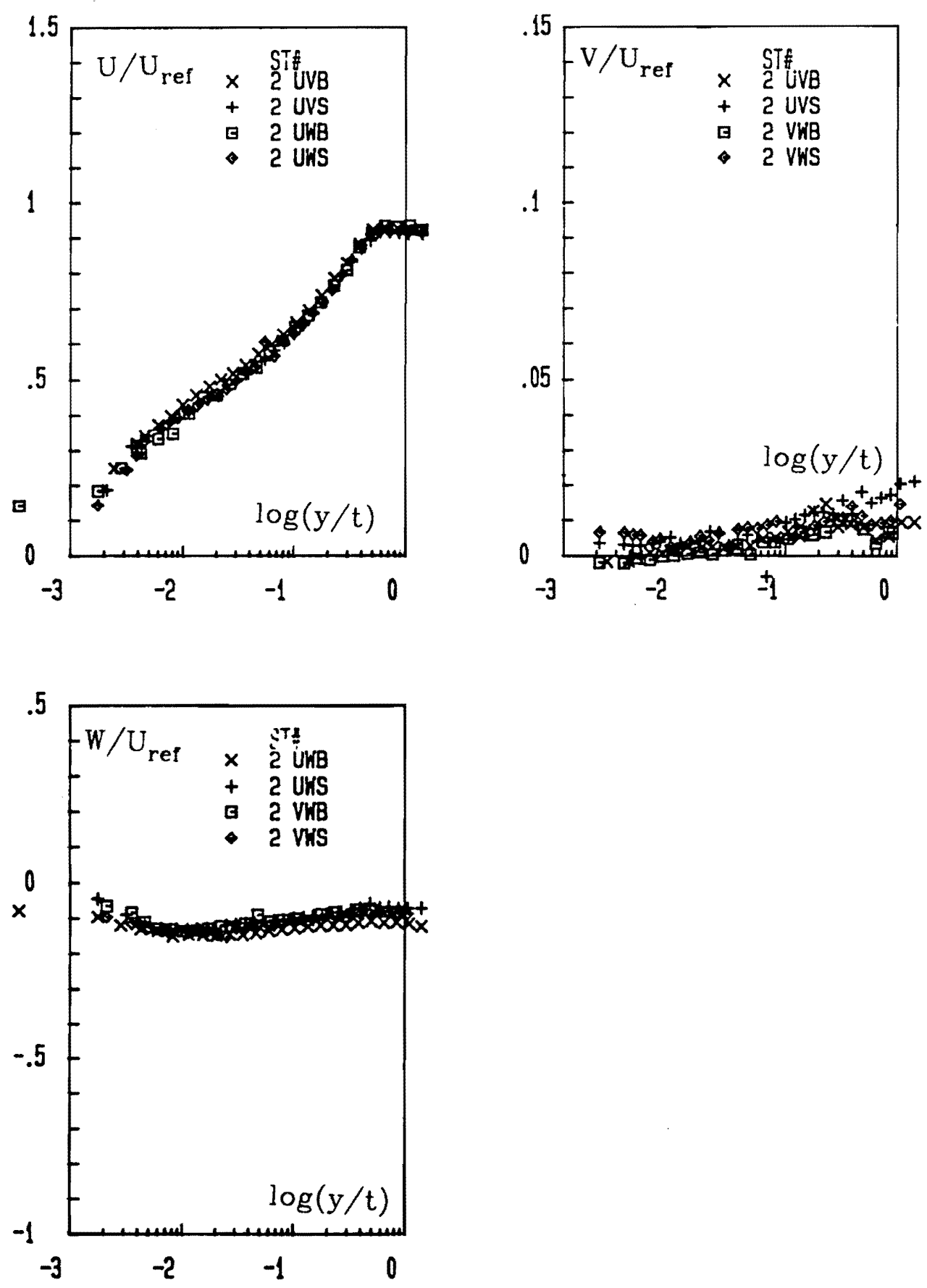

Figure 20g. U,V,W mean velocity components measured with two different LDV techniques presented in tunnel coordinates.: UV, UW and VW show the LDV beam system used to obtain the data. Extensions $B$ and $S$ denote the measurements taken with Burst Spectrum Analyzer and fast sampling rate swept-spectrum analyzer,respectively. Station numbers are also shown in the figures. 

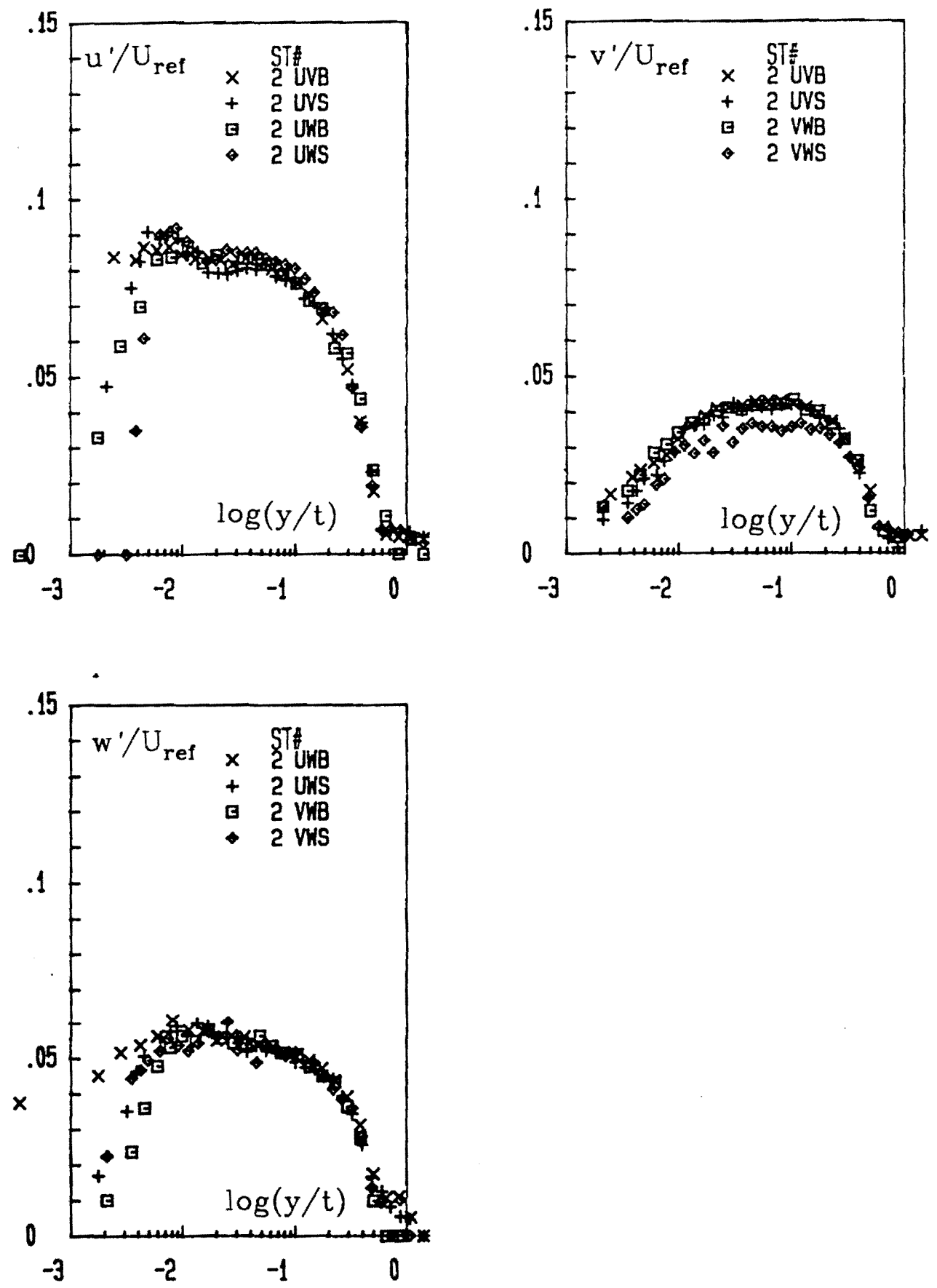

Figure 20h. $u^{\prime}, v^{\prime}, w^{\prime}$ fluctuation velocity components measured with two different LDV techniques presented in tunnel coordinates.: UV, UW and VW show the LDV beam system used to obtain the data. Extensions $B$ and $S$ denote the measurements taken with Burst Spectrum Analyzer and fast sampling rate swept-spectrum analyzer, respectively. Station numbers are also shown in the figures. 

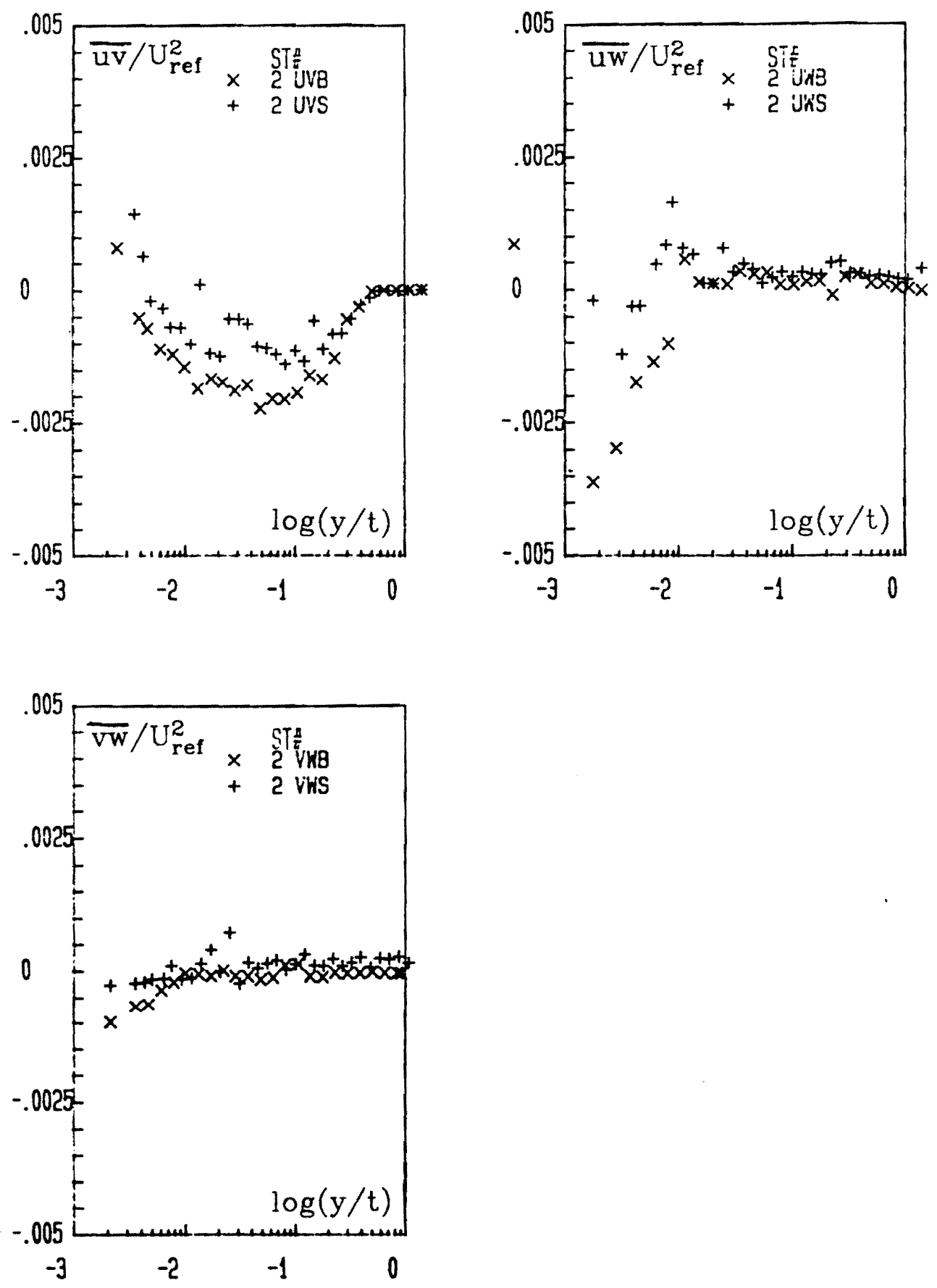

Figure 20i. $-\overline{u v}, \overline{u w},-\overline{v w}$ shear stress components measured with two different LDV techniques presented in tunnel coordinates.: UV, UW and VW show the LDV beam system used to obtain the data. Extensions $B$ and $S$ denote the measurements taken with Burst Spectrum Analyzer and fast sampling rate swept-spectrum analyzer,respectively. Station numbers are also shown in the figures. 

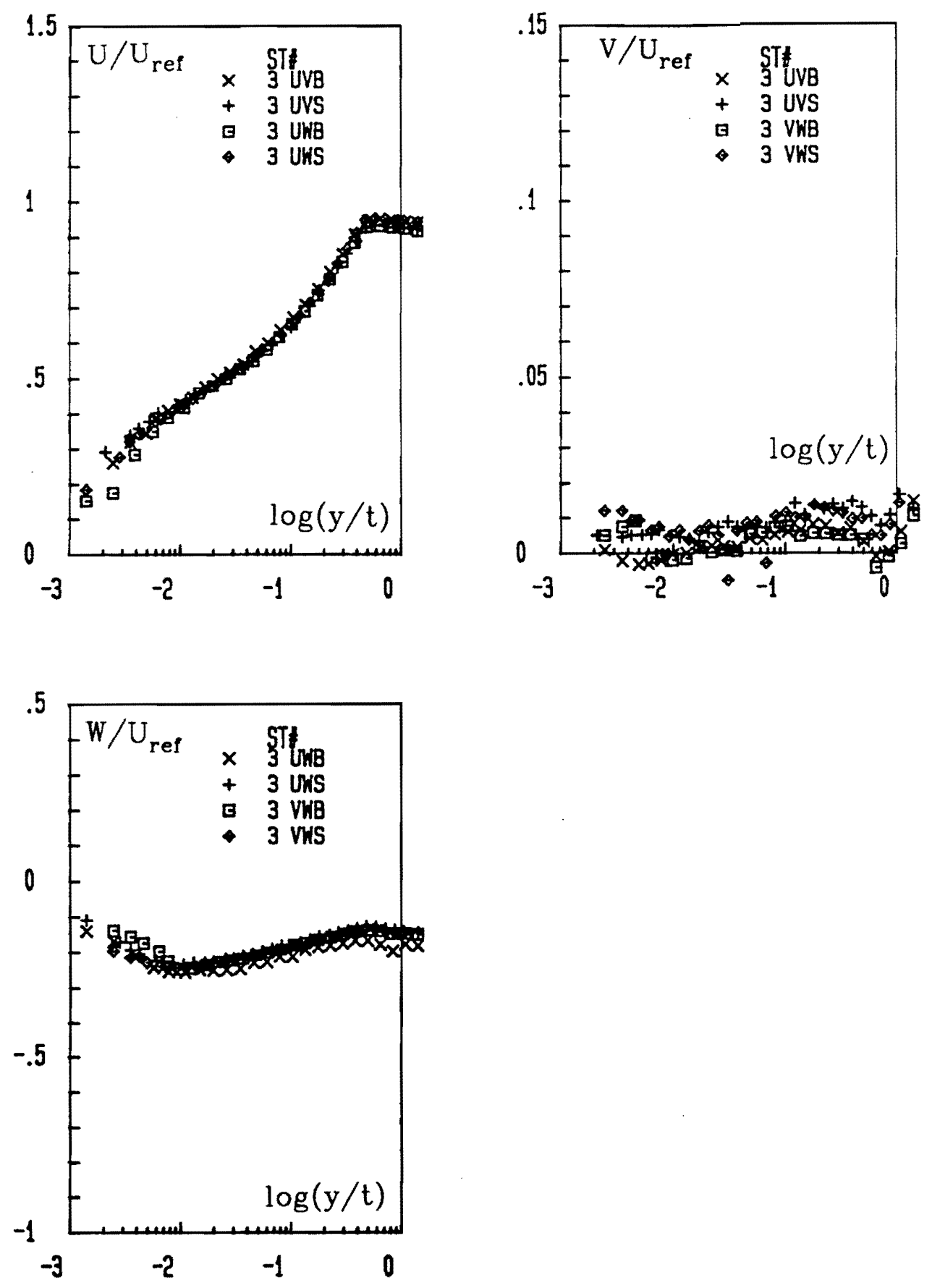

Figure $20 \mathrm{j}$. U,V,W mean velocity components measured with two different LDV techniques presented in tunnel coordinates.: UV, UW and VW show the LDV beam system used to obtain the data. Extensions B and $S$ denote the measurements taken with Burst Spectrum Analyzer and fast sampling rate swept-spectrum analyzer,respectively. Station numbers are also shown in the figures. 

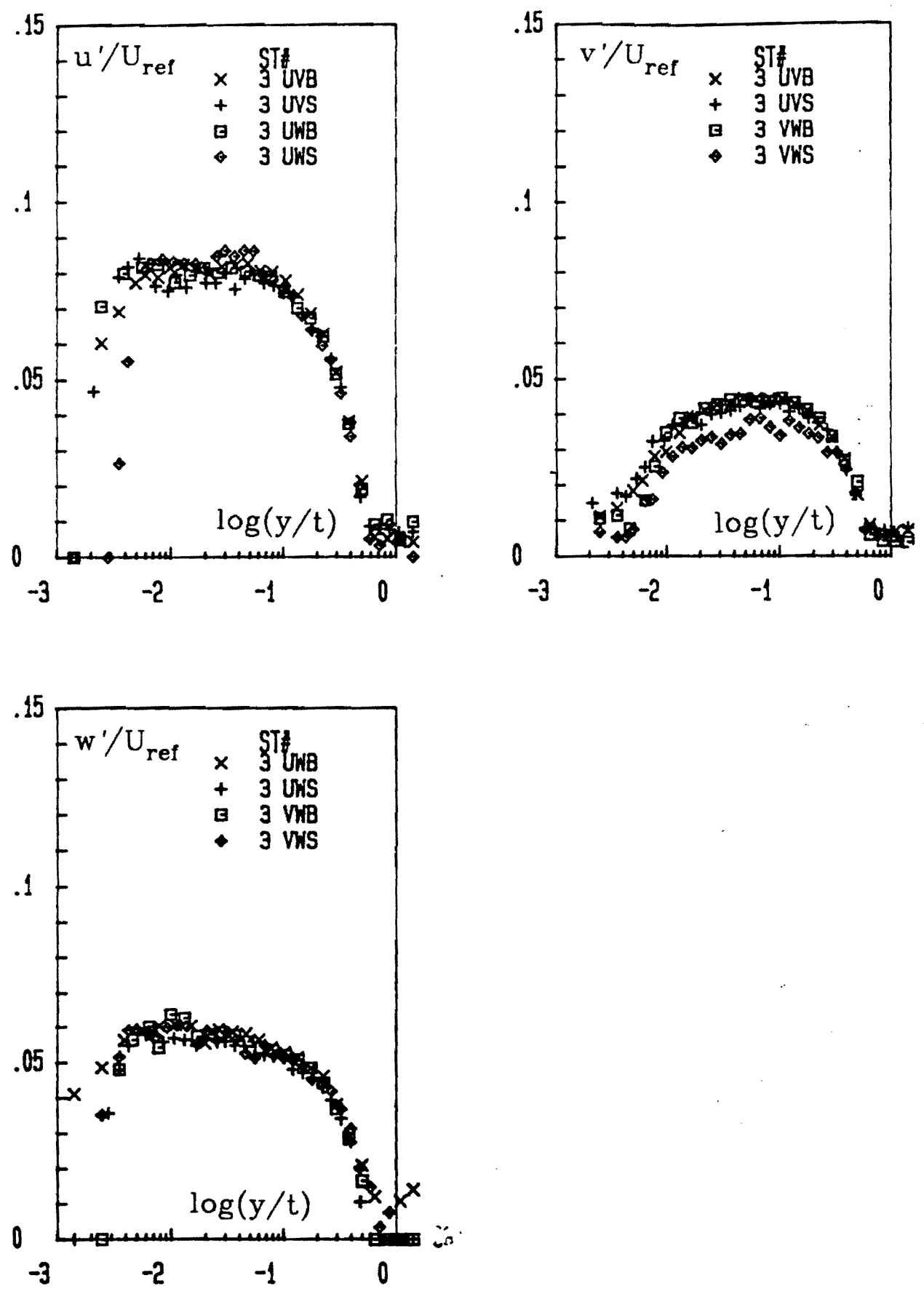

Figure 20k. $u^{\prime}, v^{\prime}, w^{\prime}$ fluctuation velocity components measured with two different LDV techniques presented in tunnel coordinates.: UV, UW and VW show the LDV beam system used to obtain the data. Extensions $B$ and $S$ denote the measurements taken with Burst Spectrum Analyzer and fast sampling rate swept-spectrum analyzer,respectively. Station numbers are also shown in the figures. 

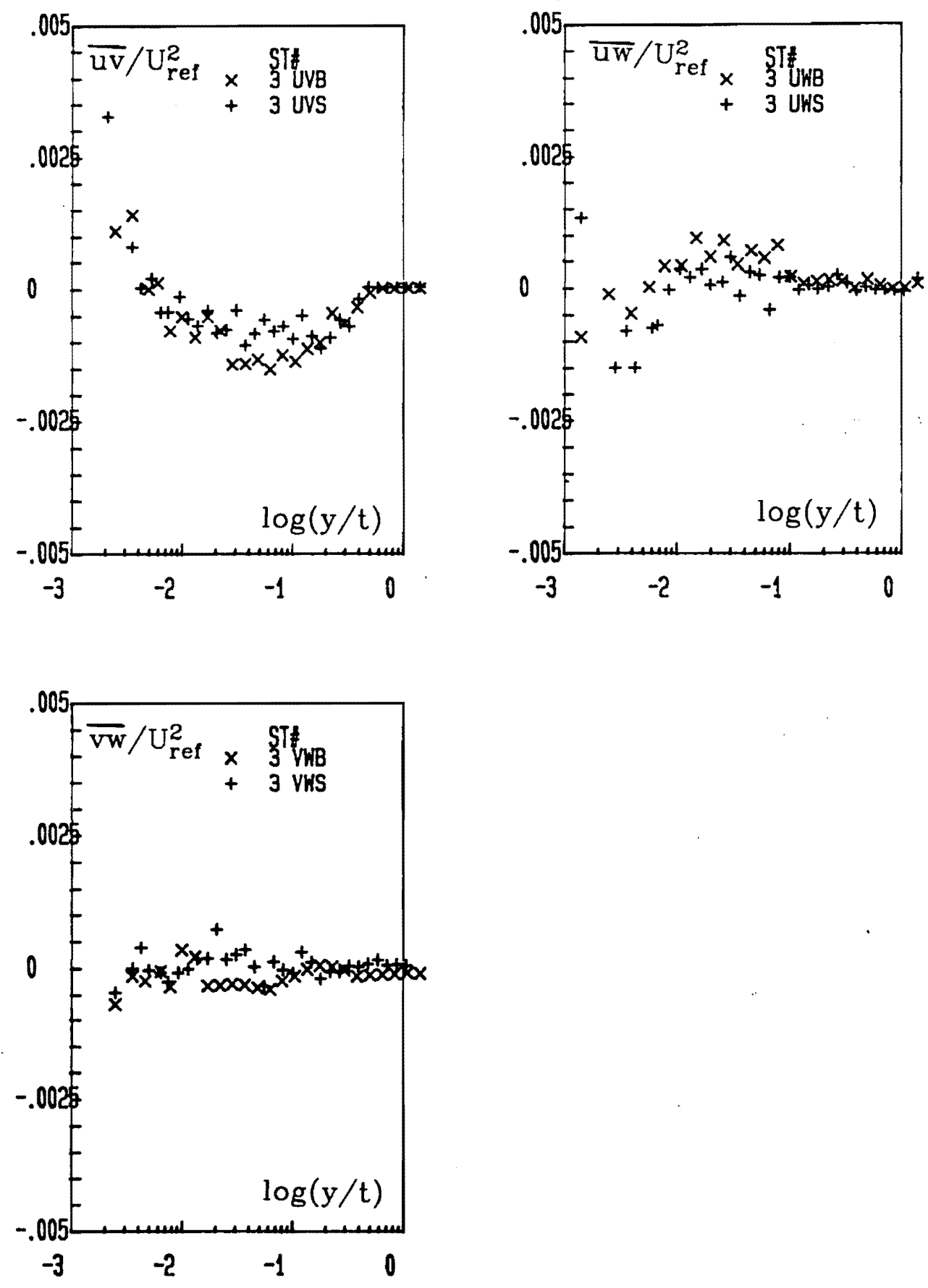

Figure 201. $-\overline{u v},-\overline{u w},-\overline{v w}$ shear stress components measured with two different LDV techniques presented in tunnel coordinates.: UV, UW and VW show the LDV beam system used to obtain the data. Extensions $B$ and $S$ denote the measurements taken with Burst Spectrum Analyzer and fast sampling rate swept-spectrum analyzer,respectively. Station numbers are also shown in the figures. 

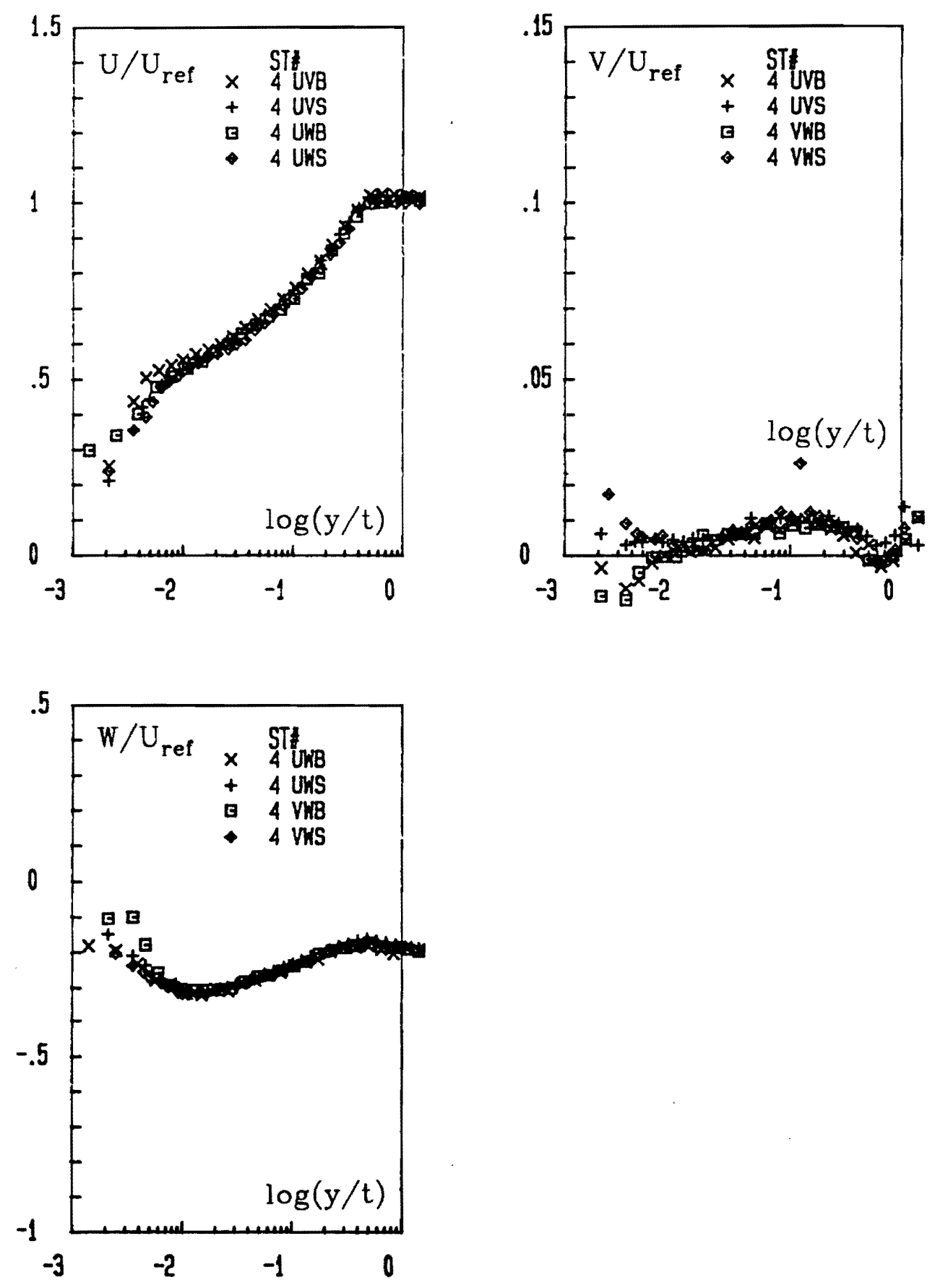

Figure $20 \mathrm{~m}$. U,V,W mean velocity components measured with two different LDV techniques presented in tunnel coordinates.: UV, UW and VW show the LDV beam system used to obtain the data. Extensions $B$ and $S$ denote the measurements taken with Burst Spectrum Analyzer and fast sampling rate swept-spectrum analyzer,respectively. Station numbers are also shown in the figures. 

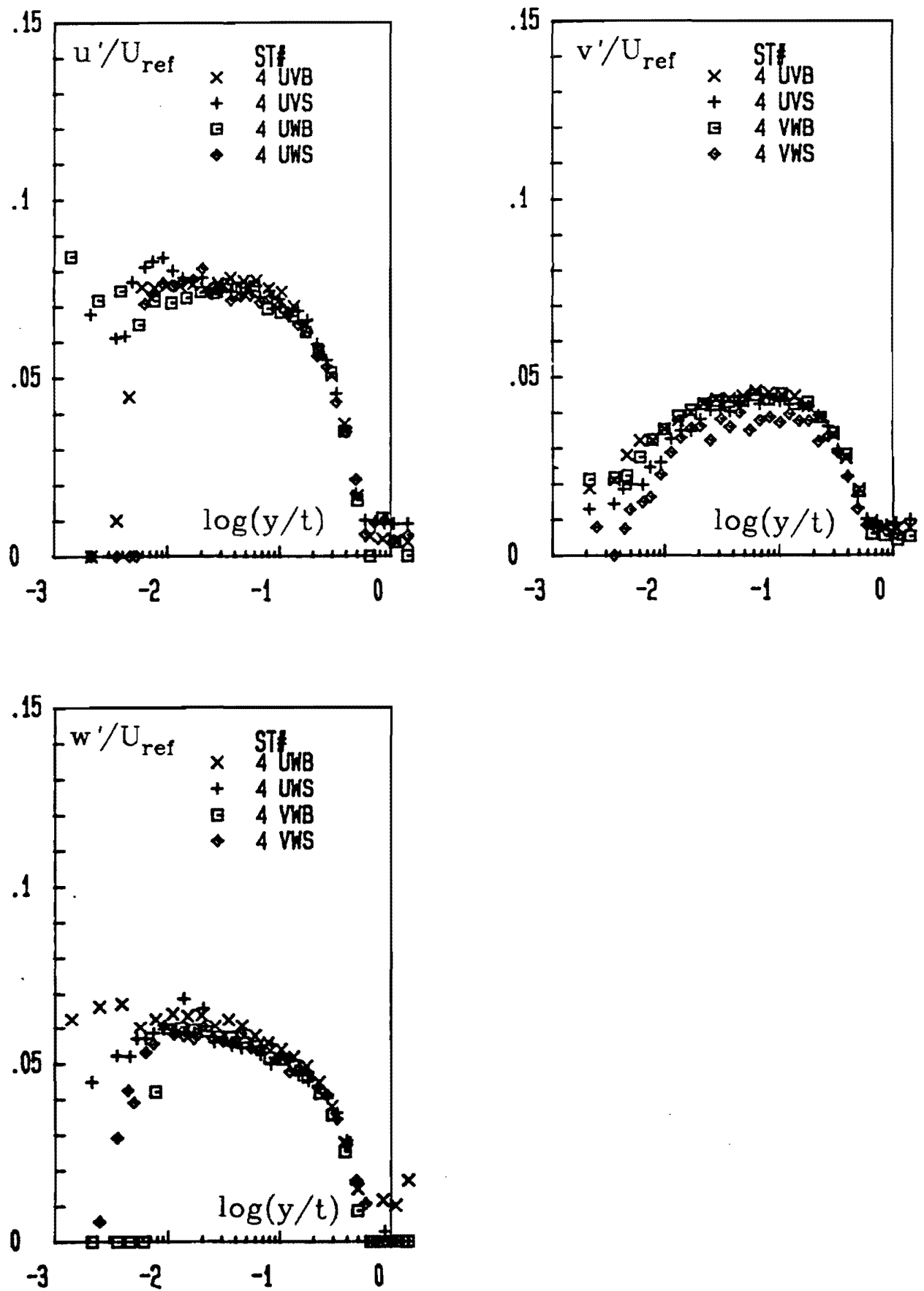

Figure 20n. $u^{\prime}, v^{\prime}, w^{\prime}$ fluctuation velocity components measured with two different LDV techniques presented in tunnel coordinates.: UV, UW and VW show the LDV beam system used to obtain the data. Extensions $B$ and $S$ denote the measurements taken with Burst Spectrum Analyzer and fast sampling rate swept-spectrum analyzer,respectively. Station numbers are also shown in the figures. 

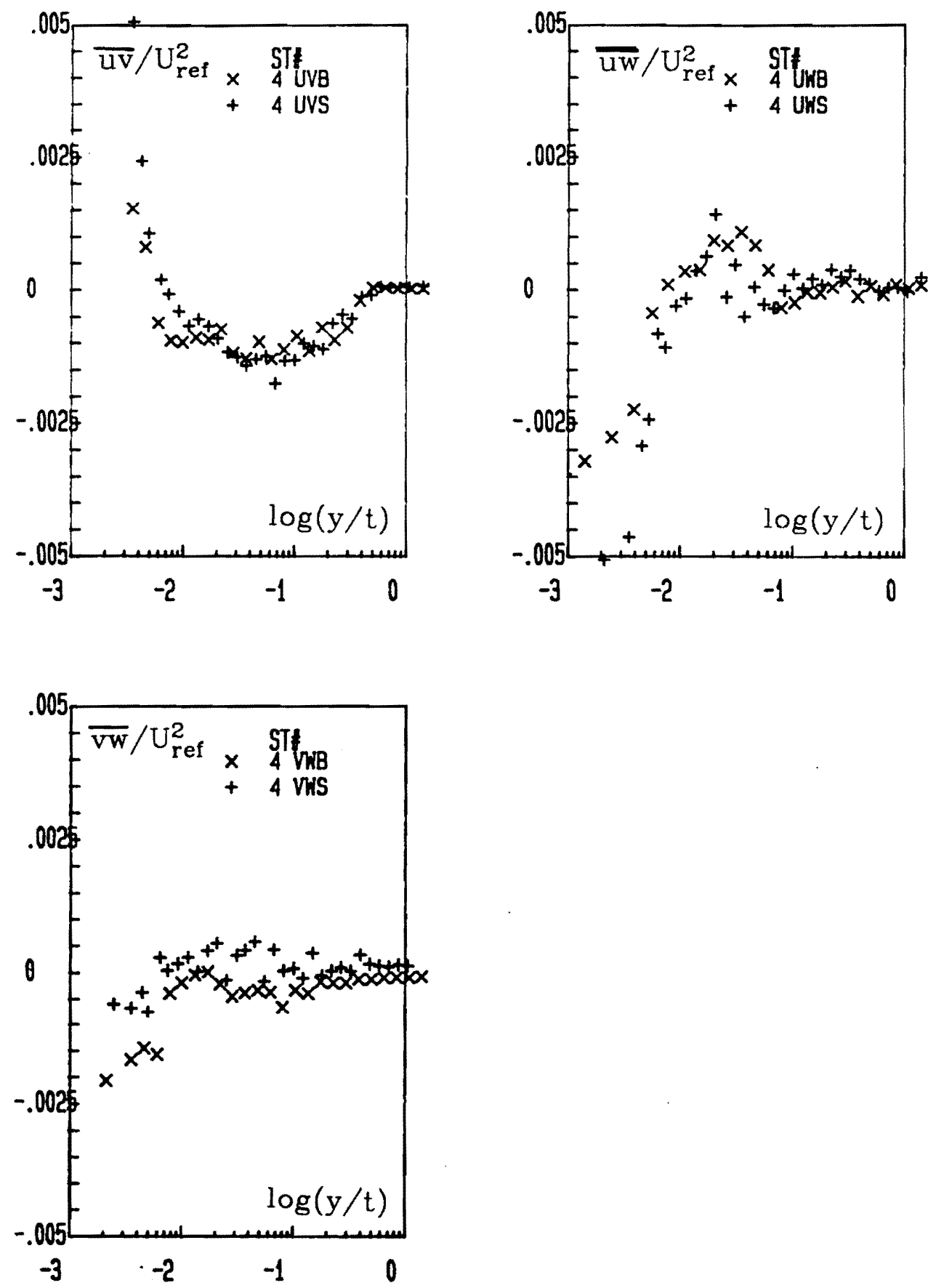

Figure 20o. $-\overline{\mathrm{uv}},-\overline{\mathrm{uw}},-\overline{\mathrm{vw}}$ shear stress components measured with two different LDV techniques presented in tunnel coordinates.: UV, UW and VW show the LDV beam system used to obtain the data. Extensions $B$ and $S$ denote the measurements taken with Burst Spectrum Analyzer and fast sampling rate swept-spectrum analyzer,respectively. Station numbers are also shown in the figures. 

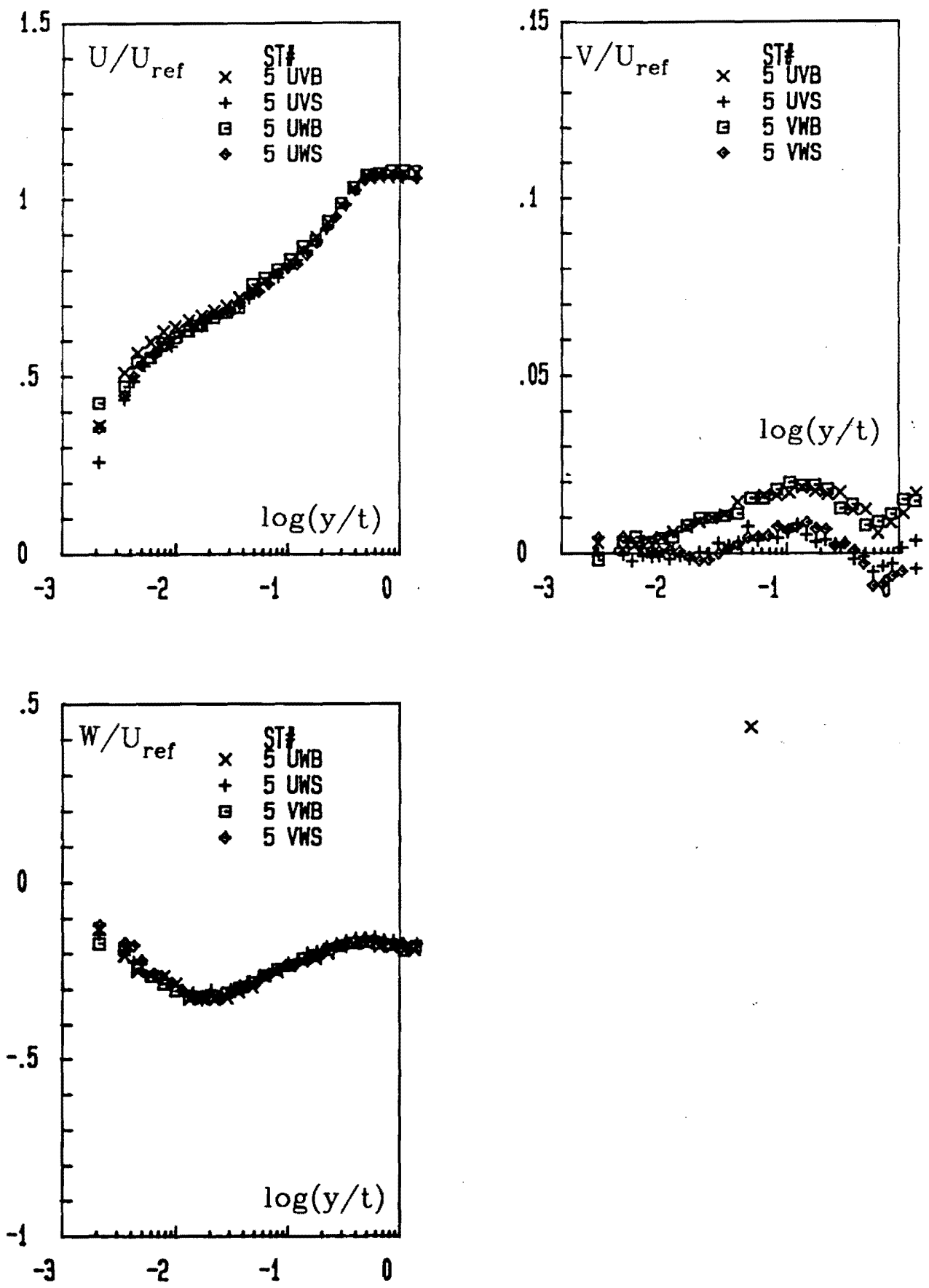

$x$

Figure 20p. U,V,W mean velocity components measured with two different LDV techniques presented in tunnel coordinates.: UV, UW and VW show the LDV beam system used to obtain the data. Extensions $B$ and $S$ denote the measurements taken with Burst Spectrum Analyzer and fast sampling rate swept-spectrum analyzer,respectively. Station numbers are also shown in the figures. 

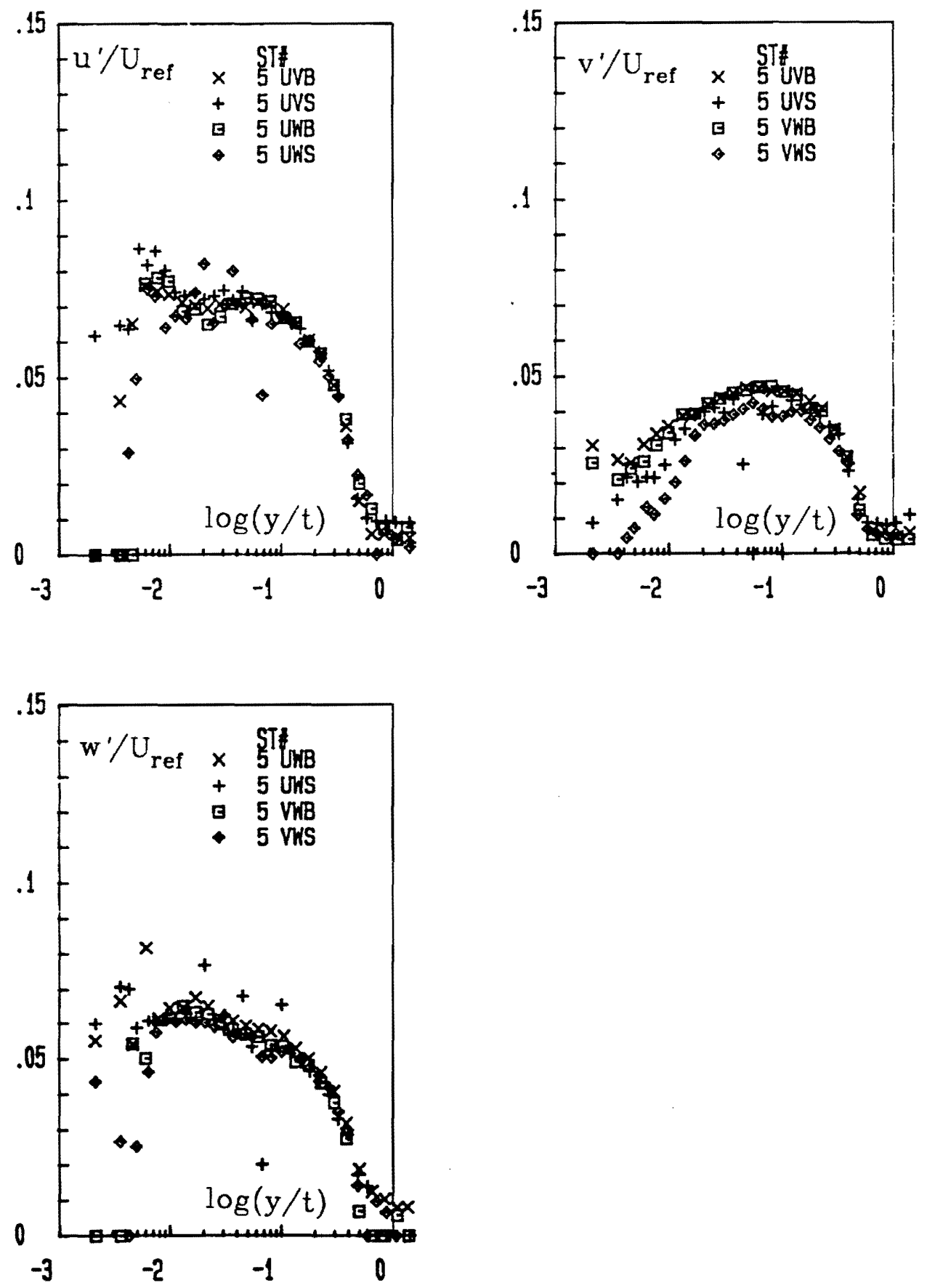

Figure 20q. $u^{\prime}, v^{\prime}, w^{\prime}$ fluctuation velocity components measured with two different LDV techniques presented in tunnel coordinates.: UV, UW and VW show the LDV beam system used to obtain the data. Extensions $B$ and $S$ denote the measurements taken with Burst Spectrum Analyzer and fast sampling rate swept-spectrum analyzer,respectively. Station numbers are also shown in the figures. 

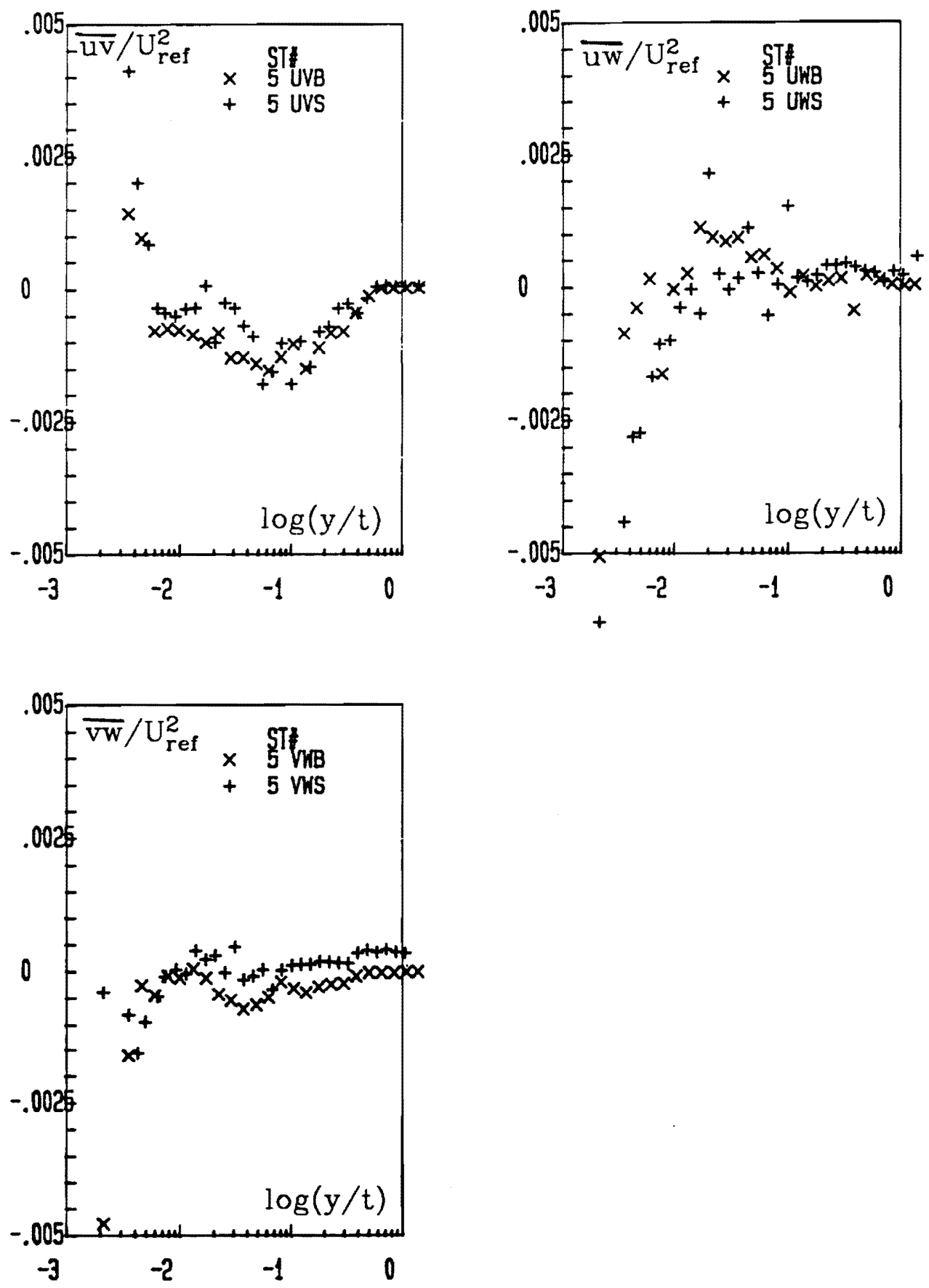

Figure 20r. $-\overline{u v},-\overline{u w},-\overline{v w}$ shear stress components measured with two different LDV techniques presented in tunnel coordinates.: UV, UW and VW show the LDV beam system used to obtain the data. Extensions $B$ and $S$ denote the measurements taken with Burst Spectrum Analyzer and fast sampling rate swept-spectrum analyzer,respectively. Station numbers are also shown in the figures. 

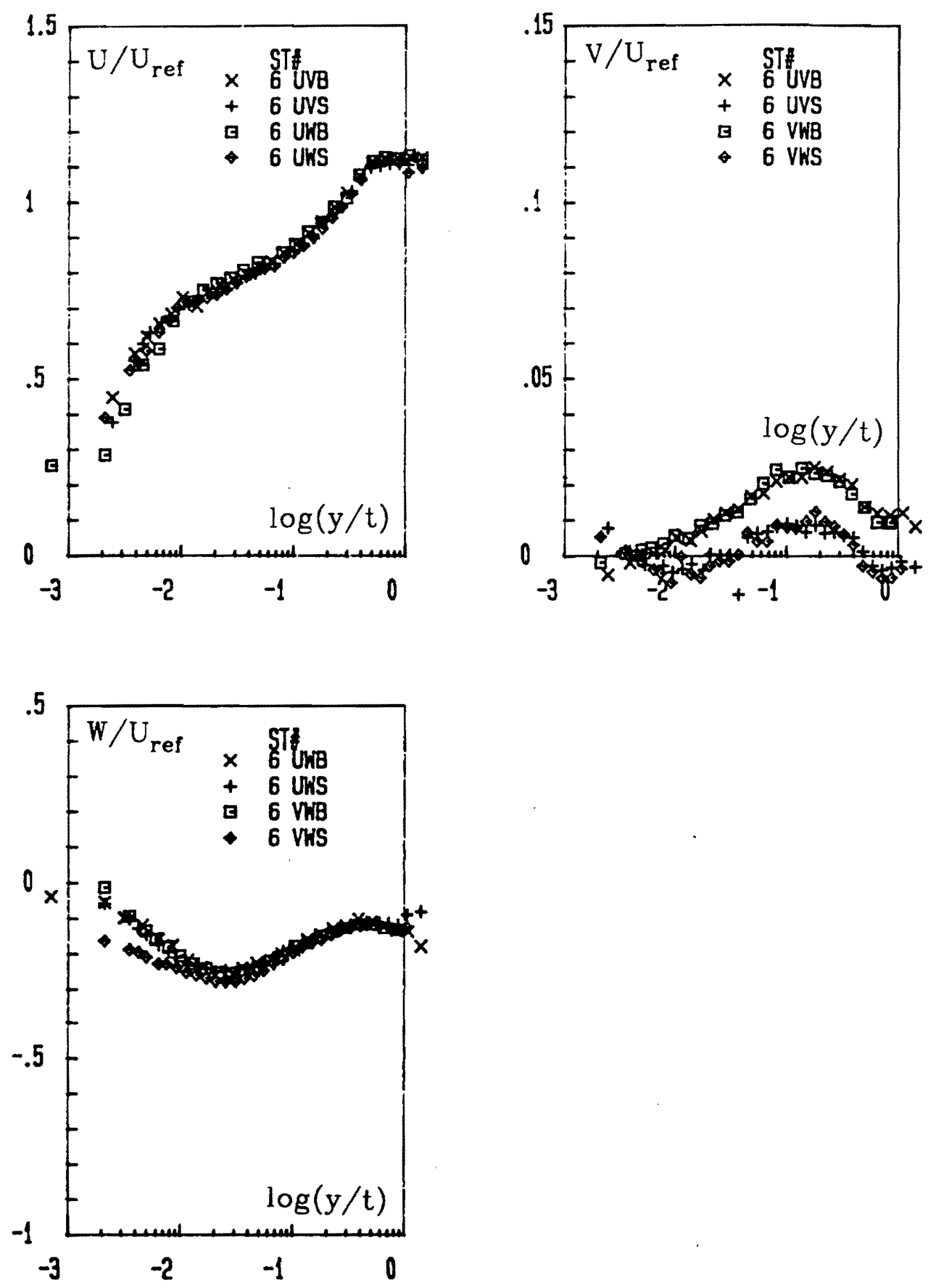

Figure 20s. U,V,W mean velocity components measured with two different LDV techniques presented in tunnel coordinates.: UV, UW and VW show the LDV beam system used to obtain the data. Extensions $B$ and $S$ denote the measurements taken with Burst Spectrum Analyzer and fast sampling rate swept-spectrum analyzer,respectively. Station numbers are also shown in the figures. 

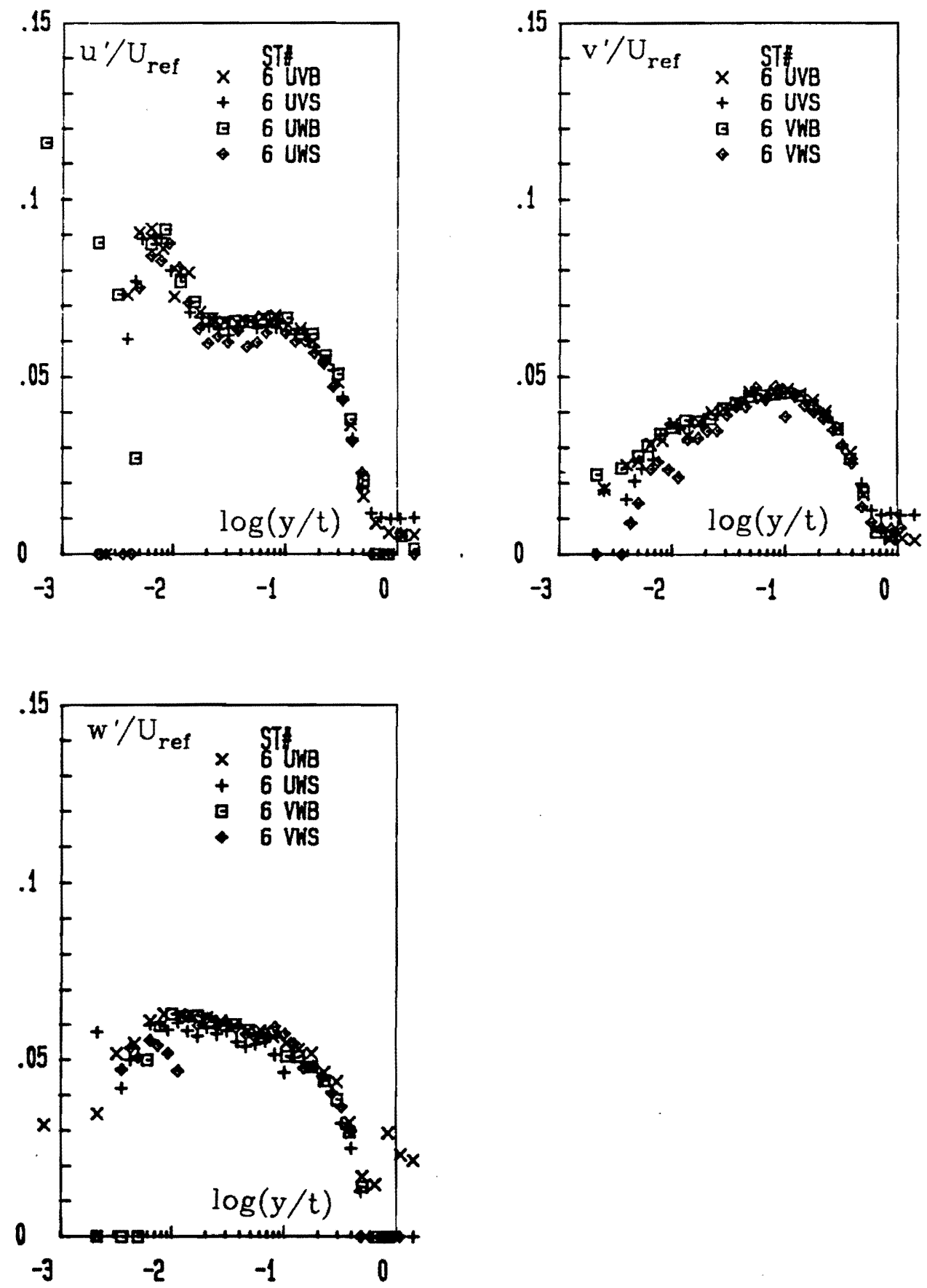

Figure 20t. $u^{\prime}, v^{\prime}, w^{\prime}$ fluctuation velocity components measured with two different LDV techniques presented in tunnel coordinates.: UV, UW and VW show the LDV beam system used to obtain the data. Extensions $B$ and $S$ denote the measurements taken with Burst Spectrum Analyzer and fast sampling rate swept-spectrum analyzer,respectively. Station numbers are also shown in the figures. 

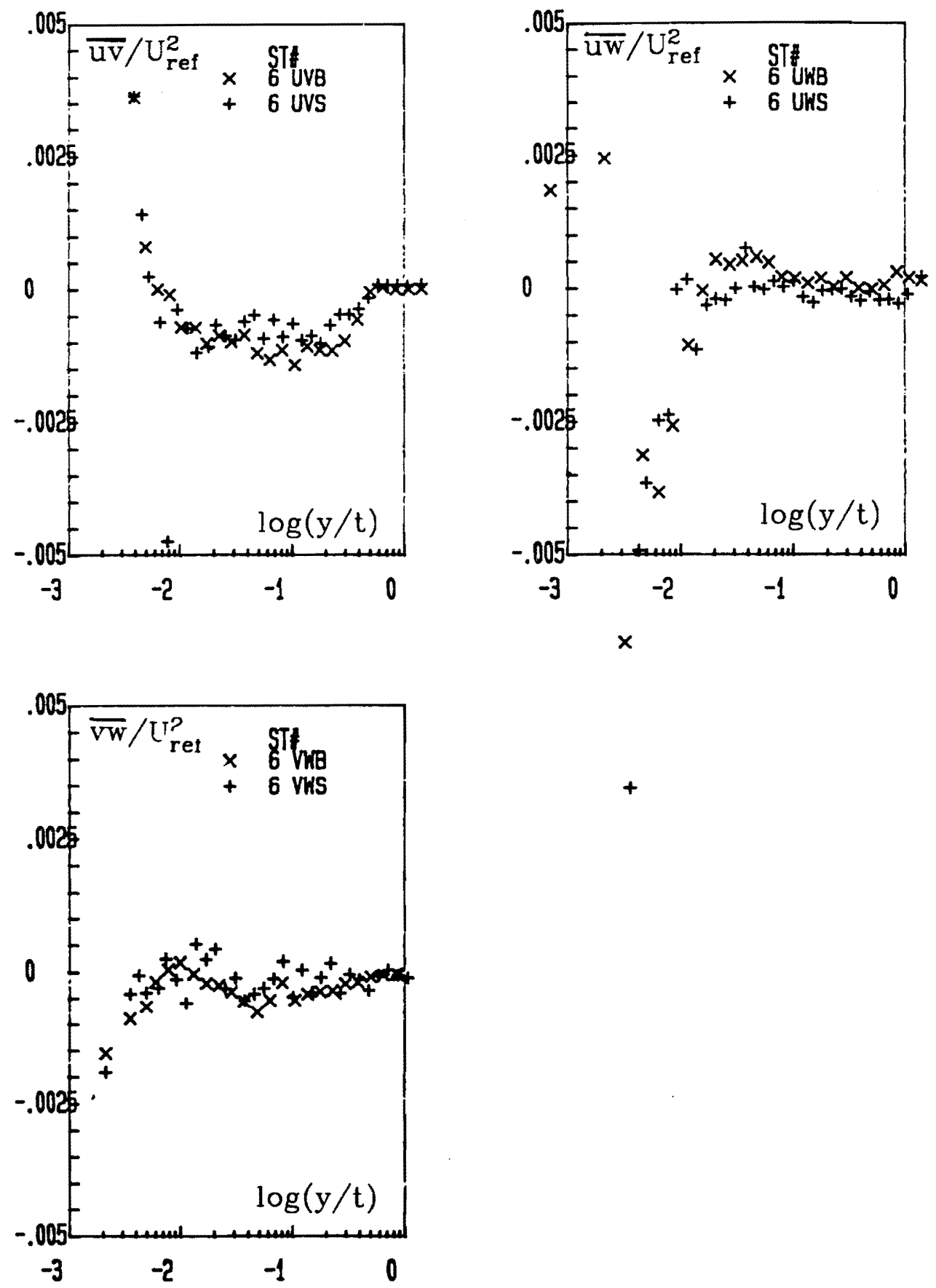

Figure 20u. $-\overline{u v},-\overline{u w},-\overline{v w}$ shear stress components measured with two different LDV techniques presented in tunnel coordinates.: UV, UW and VW show the LDV beam system used to obtain the data. Extensions $B$ and $S$ denote the measurements taken with Burst Spectrum Analyzer and fast sampling rate swept-spectrum analyzer,respectively. Station numbers are also shown in the figures. 

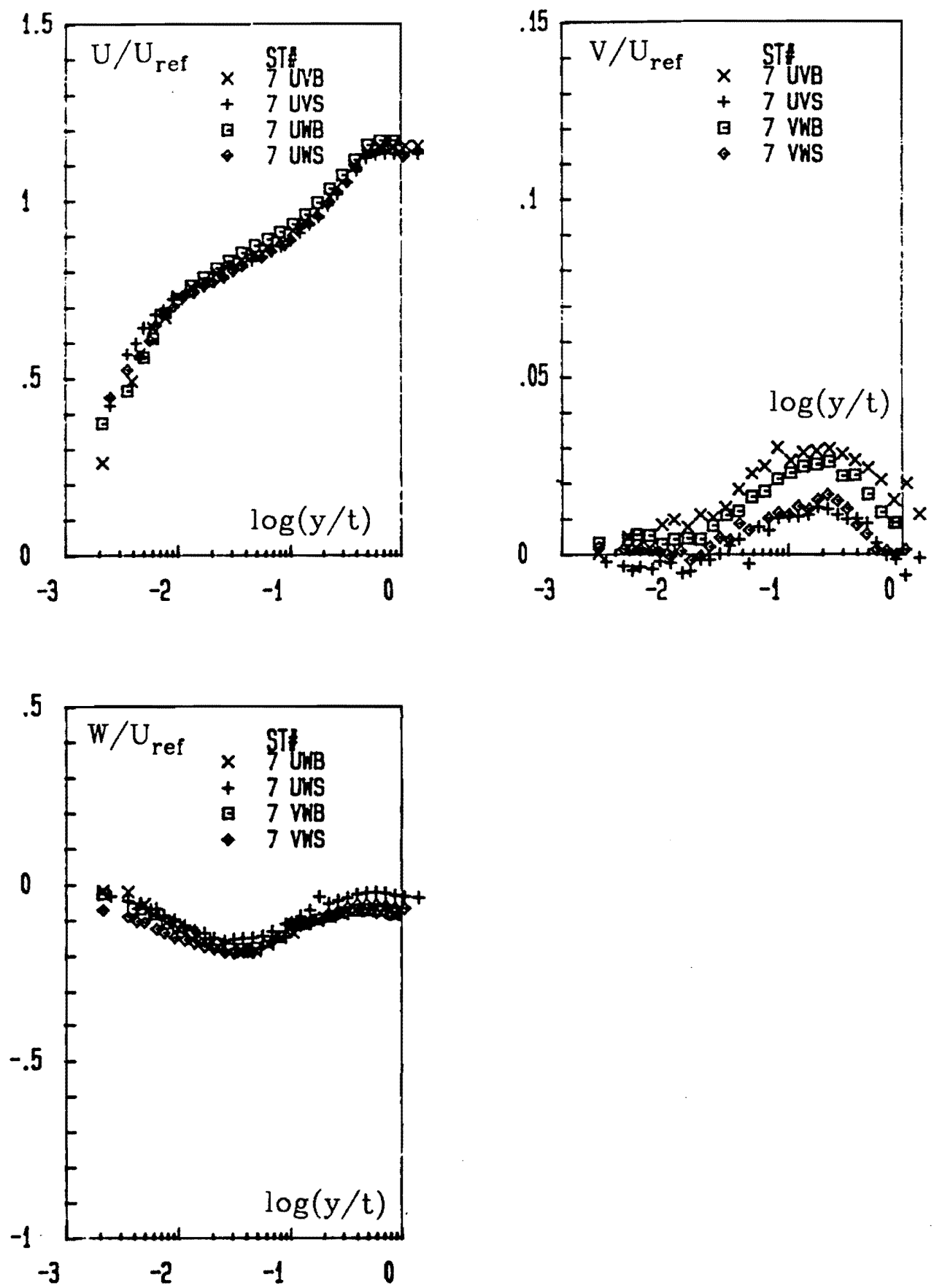

Figure 20w. U,V,W mean velocity components measured with two different LDV techniques presented in tunnel coordinates.: UV, UW and VW show the LDV beam system used to obtain the data. Extensions B and S denote the measurements taken with Burst Spectrum Analyzer and fast sampling rate swept-spectrum analyzer,respectively. Station numbers are also shown in the figures. 

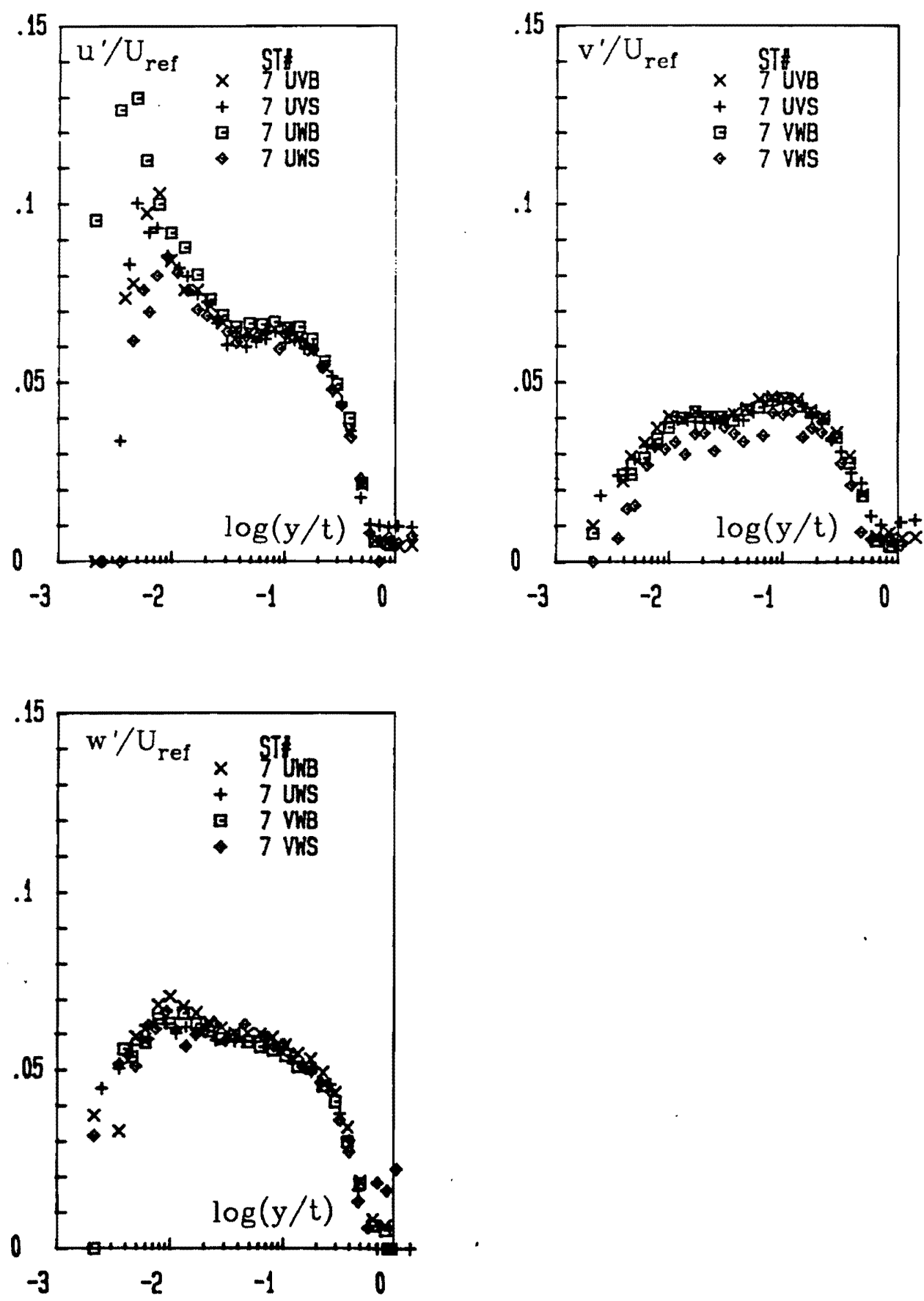

Figure 20x. $u^{\prime}, v^{\prime}, w^{\prime}$ fluctuation velocity components measured with two different LDV techniques presented in tunnel coordinates.: UV, UW and VW show the LDV beam system used to obtain the data. Extensions $B$ and $S$ denote the measurements taken with Burst Spectrum Analyzer and fast sampling rate swept-spectrum analyzer,respectively. Station numbers are also shown in the figures. 

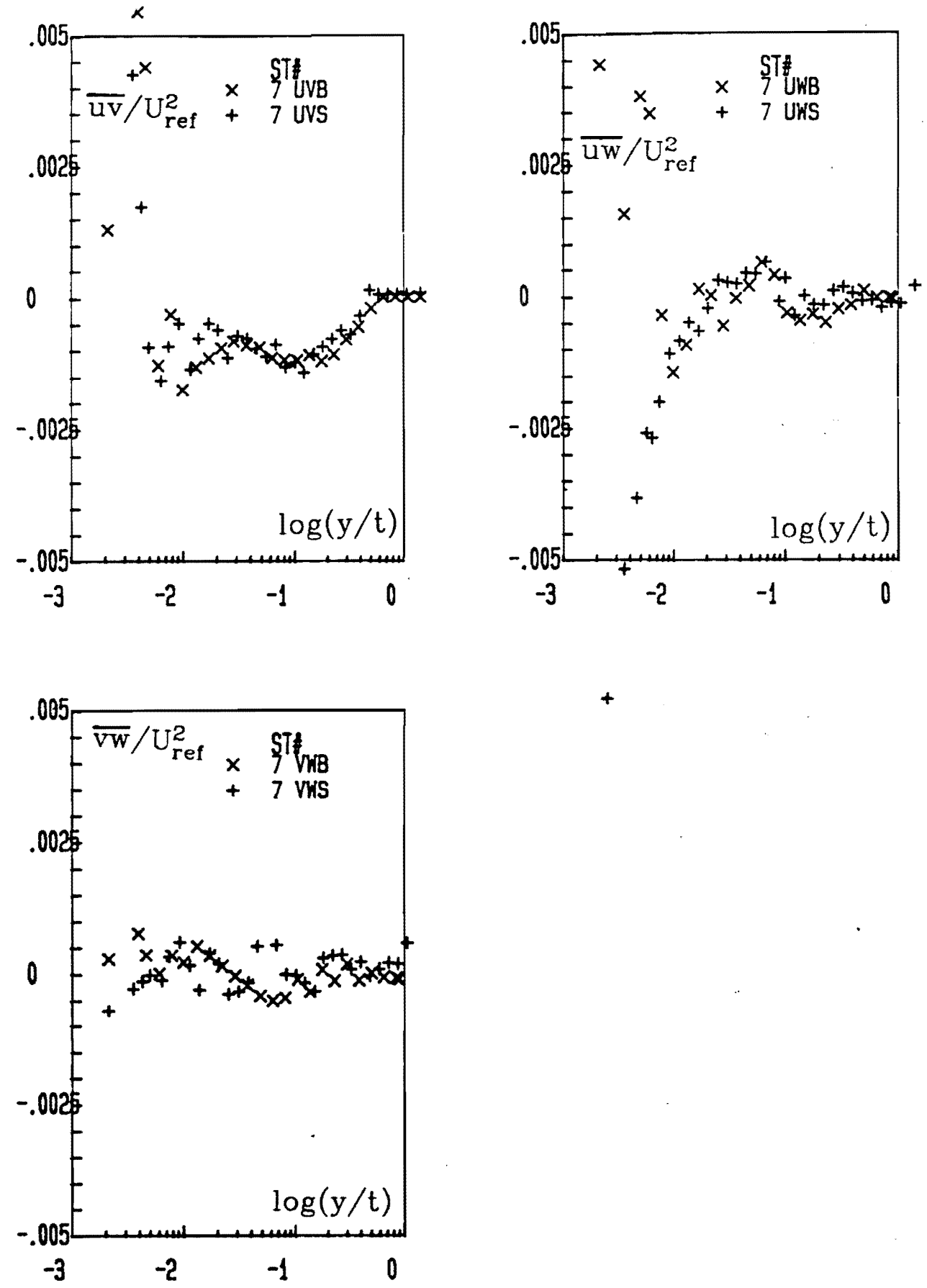

Figure 20y. $-\overline{\mathrm{uV}},-\overline{\mathrm{uw}},-\overline{\mathrm{vw}}$ shear stress components measured with two different LDV techniques presented in tunnel coordinates.: UV, UW and VW show the LDV beam system used to obtain the data. Extensions $B$ and $S$ denote the measurements taken with Burst Spectrum Analyzer and fast sampling rate swept-spectrum analyzer,respectively. Station numbers are also shown in the figures. 


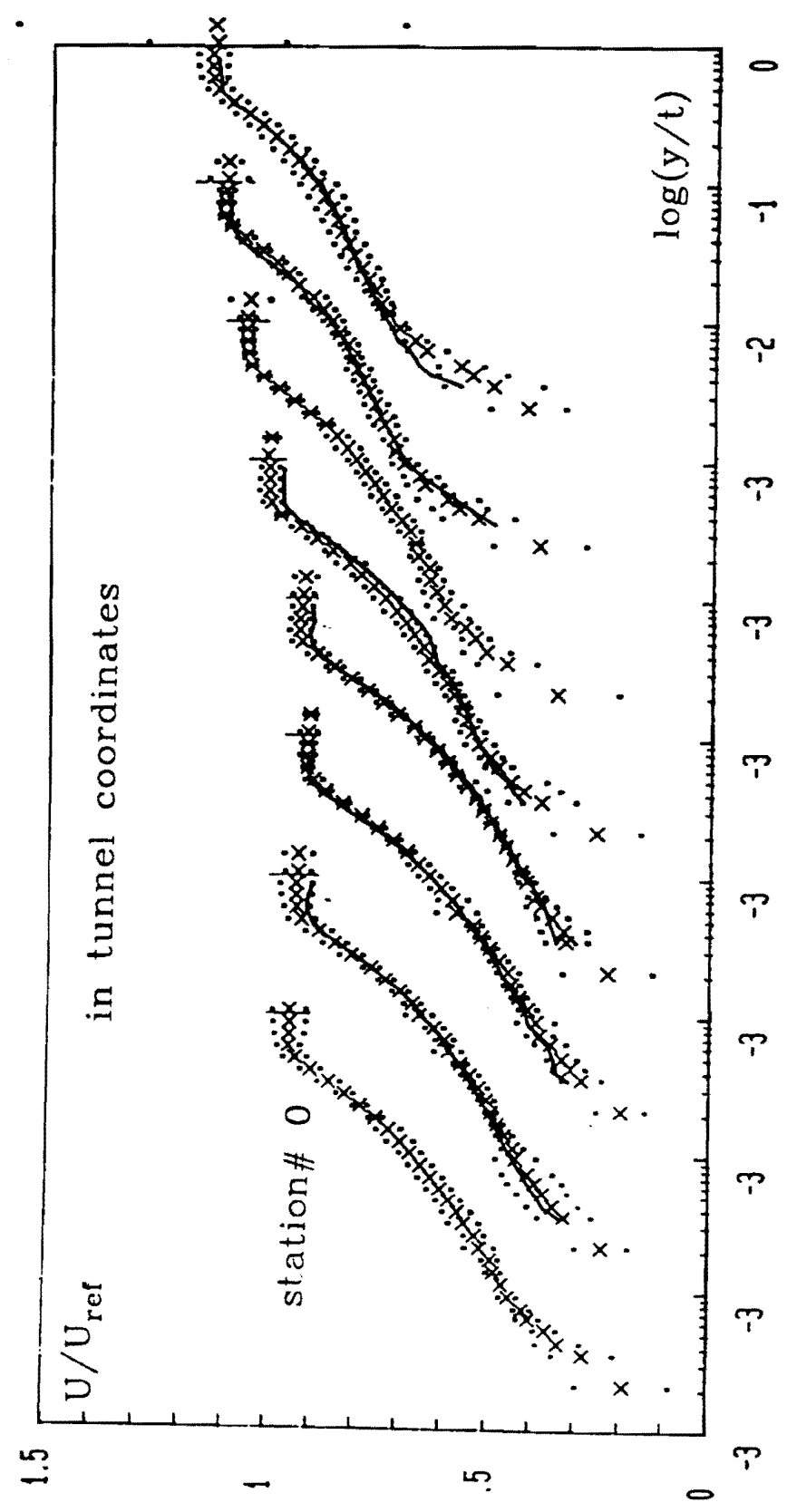

Figure 21. U component of the mean velocity vector measured with LDV technique presented in tunnel coordinates.: The stations are numbered from left to right starting with 0 . Large symbols denote the data, small symbols denote the uncertainty band. Lines are used to show the hot-wire profiles taken at the same locations. Note the shifted scale of the absissa. ( See also fig 4, and table 3 ) 

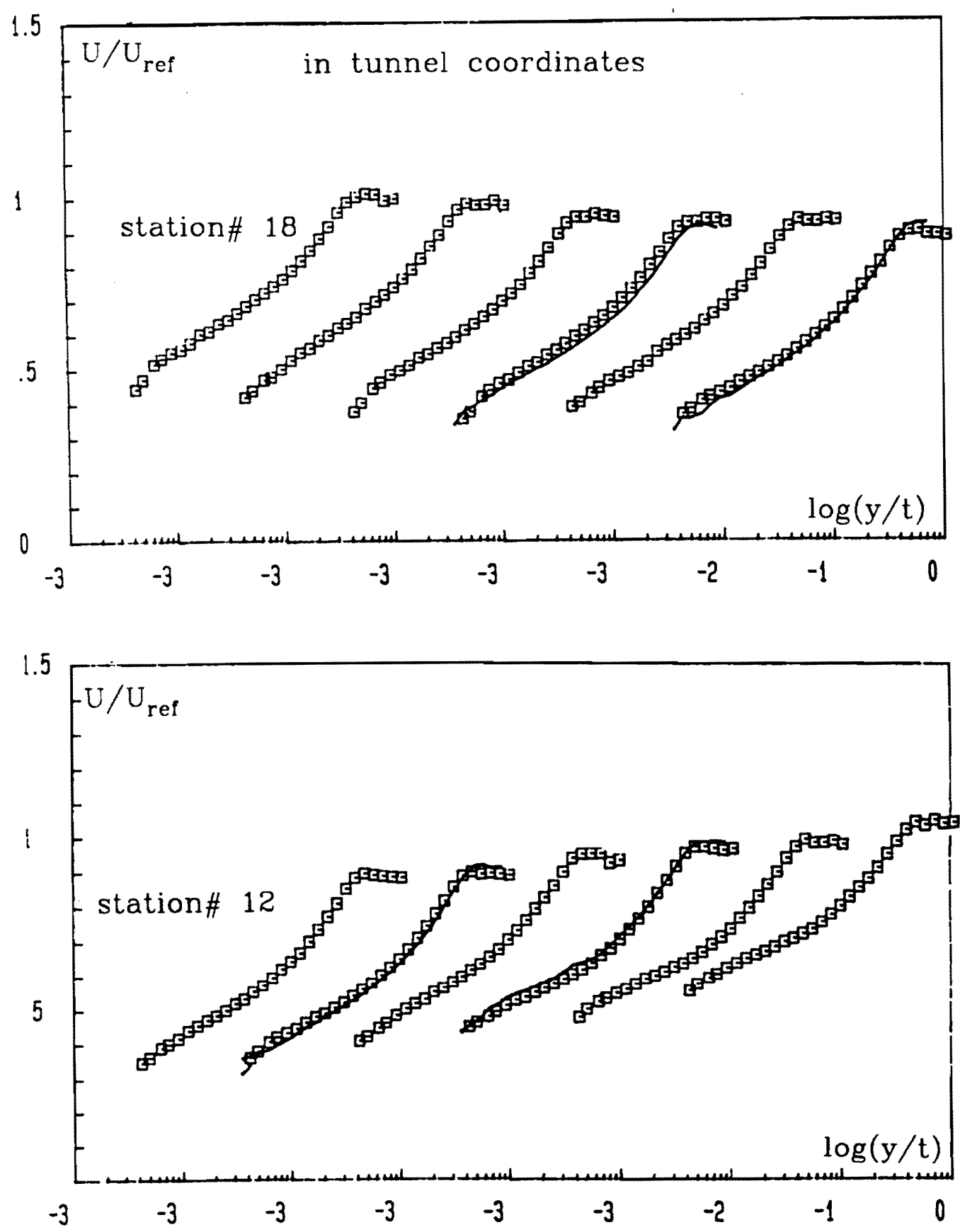

Figure 22a. U component of the mean velocity vector measured with hot-wire technique on the left-hand side of the wing presented in tunnel coordinates.: In the figures the stations are numbered from left to right starting with station 18 and station 12 , respectively. Lines are used to show the hot-wire profiles taken at the symmetric locations. Note the shifted scale of the absissa. ( See also fig 4 , and table 1) 


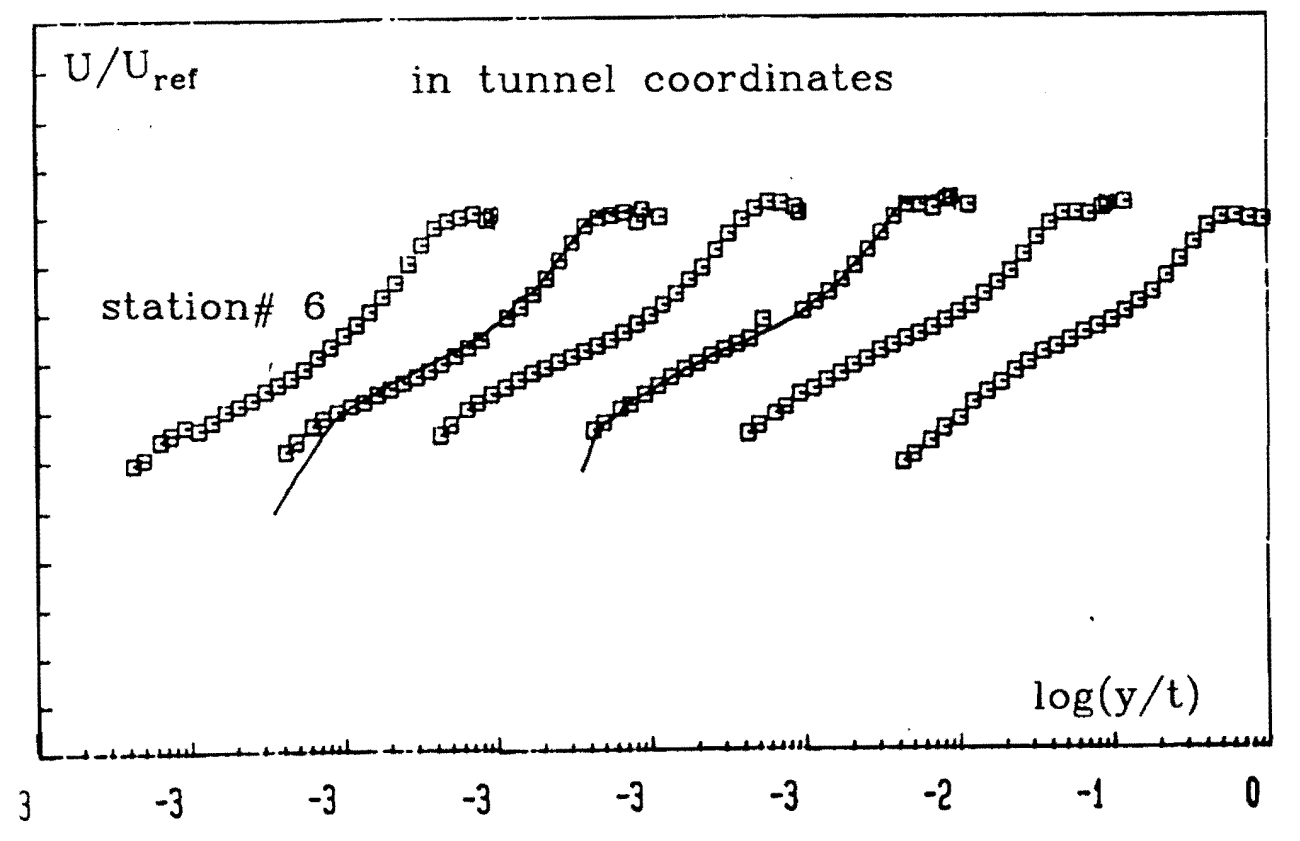

Figure 22b. U component of the mean velocity vector measured with hot-wire technique on the left-hand side of the wing presented in tunnel coordinates.: In the figure the stations are numbered from left to right starting with station 6 . Lines are used to show the hot-wire profiles taken at the symmetric locations. Note the shifted scale of the absissa. ( See also fig 4 , and table 1 ) 


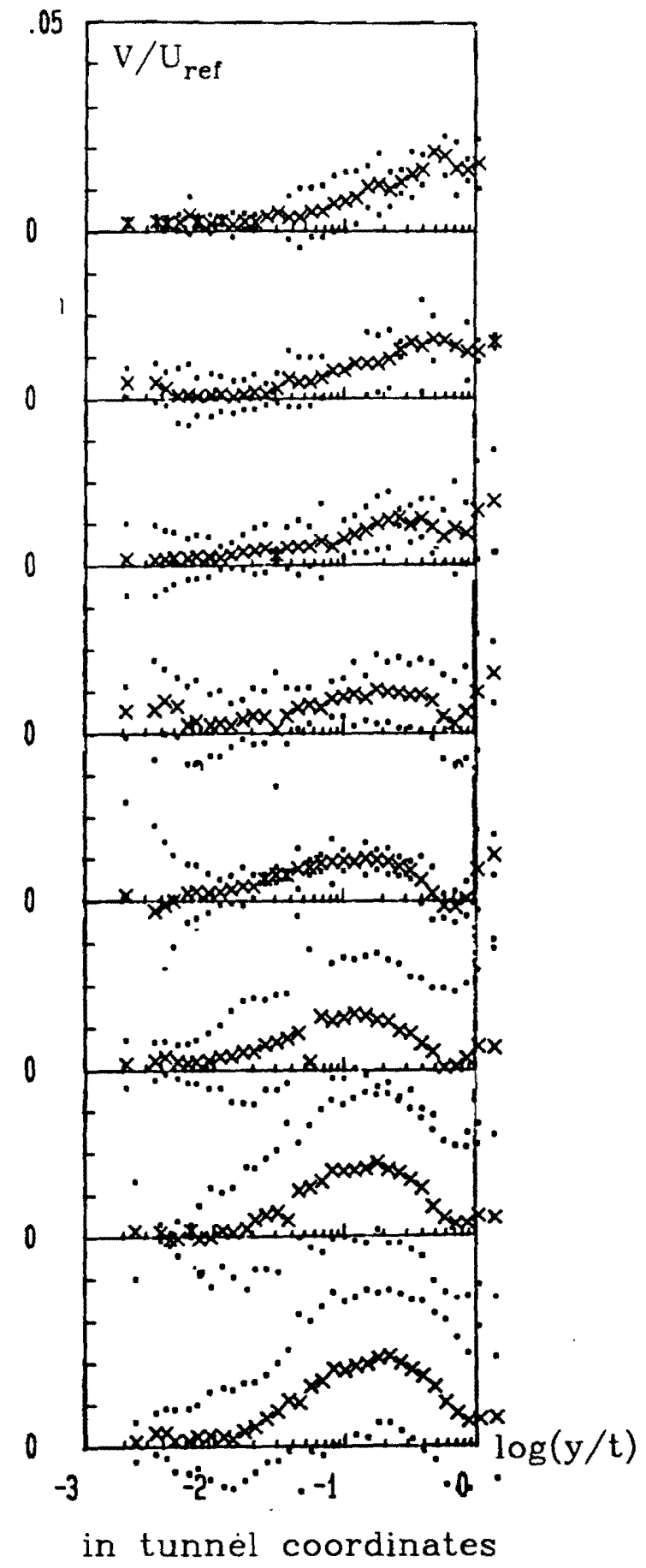

Figure 23. $\mathrm{V}$ component of the mean velocity vector measured with LDV technique presented in tunnel coordinates.: The stations are numbered from top to bottom starting with 0 . Large symbols denote the data, small symbols denote the uncertainty band. 


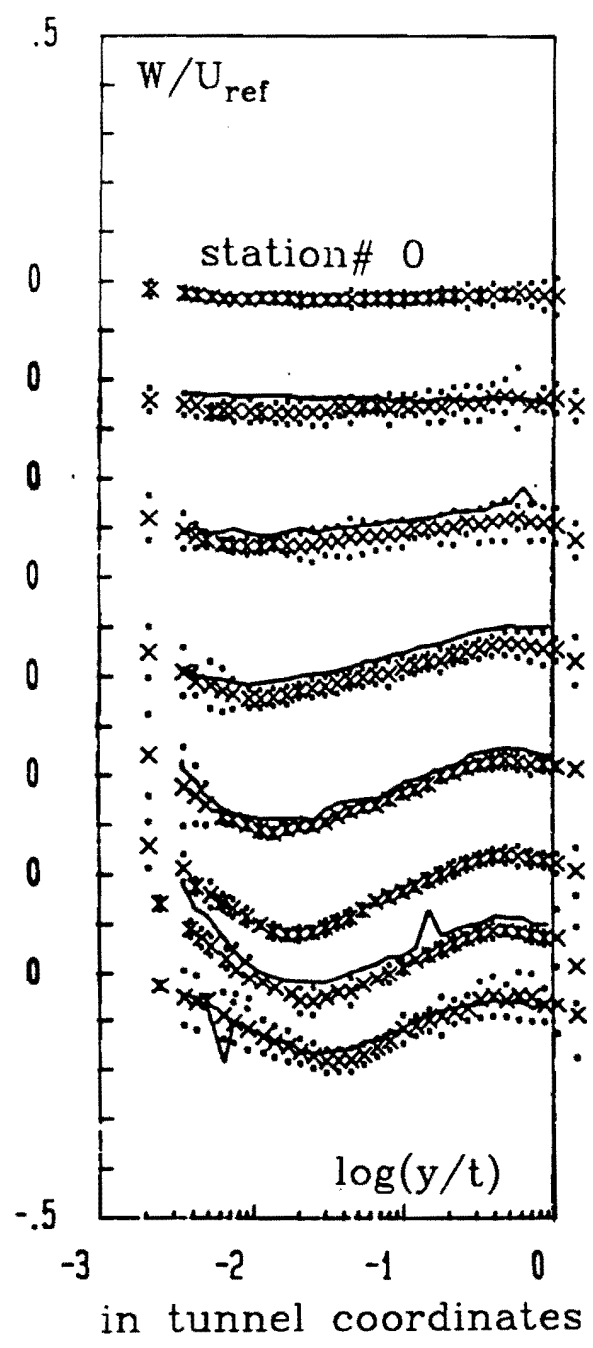

Figure 24. W component of the mean velocity vector measured with LDV technique presented in tunnel coordinates.: The stations are numbered from top to bottom starting with 0 . Large symbols denote the data, small symbols denote the uncertainty band. Lines are used to show the hot-wire profiles taken at the same locations. Note the shifted scale of the ordinate. (See also fig 4 , and table 3 ) 


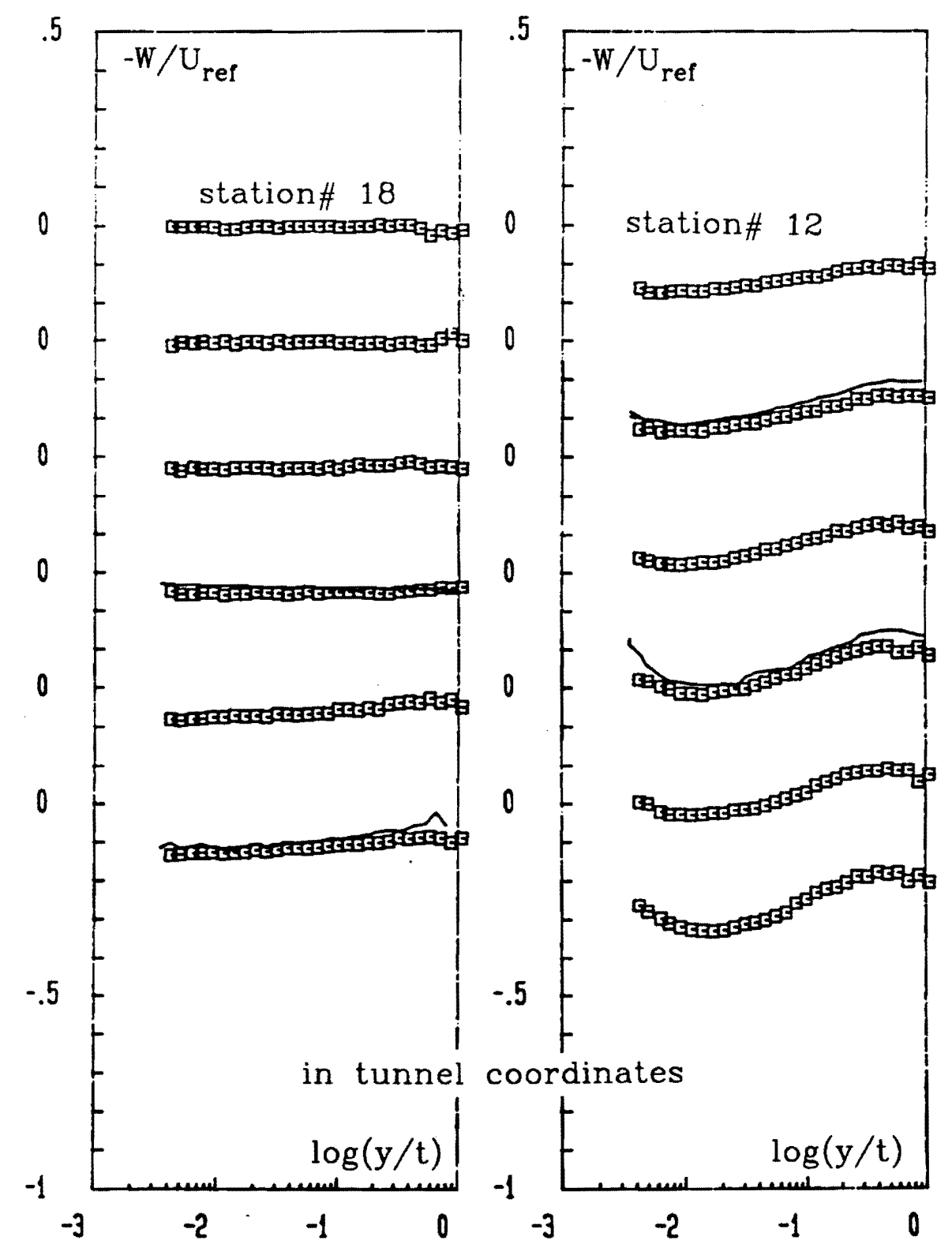

Figure 25a. W component of the mean velocity vector measured with hot-wire technique on the left-hand side of the wing presented in tunnel coordinates.: In the figures the stations are numbered from top to bottom starting with station 18 and station 12, respectively. Lines are used to show the hot-wire profiles taken at the symmetric locations. Note the shifted scale of the ordinate and the minus sign of the velocity. ( See also fig 4, and table 1 ) 


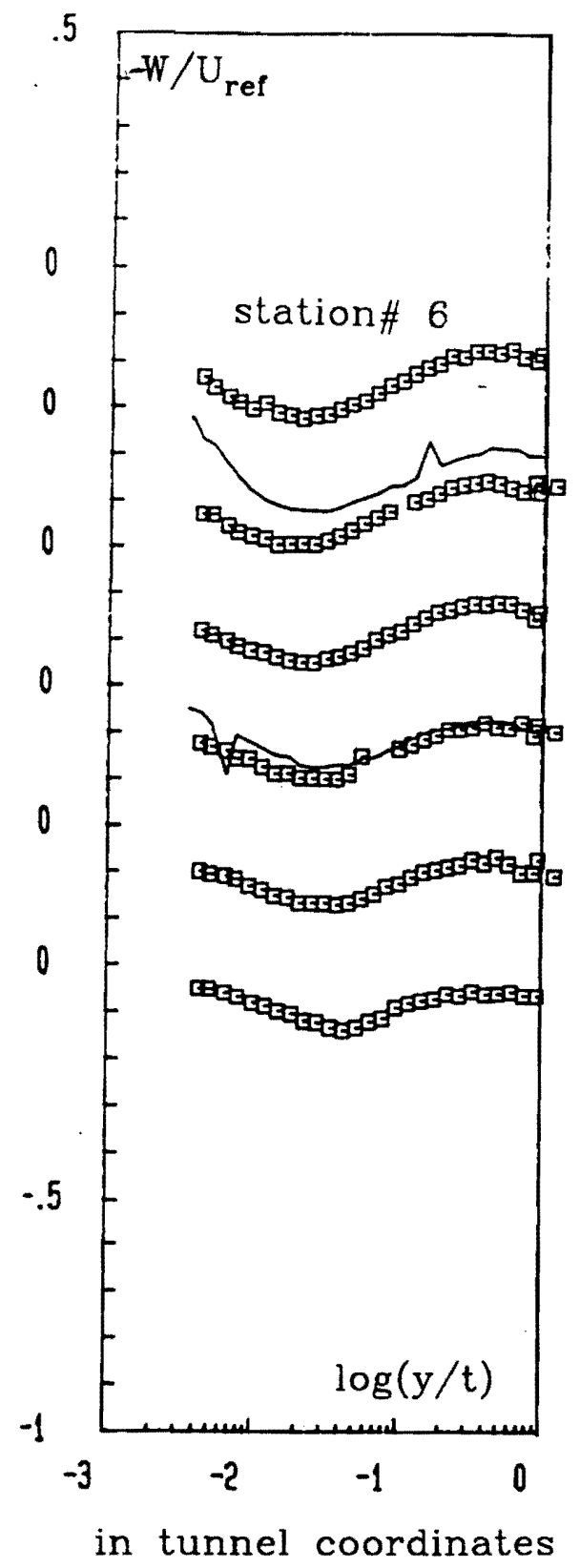

Figure 25b. W component of the mean velocity vector measured with hot-wire technique on the left-hand side of the wing presented in tunnel coordinates.: In the figure the stations are numbered from top to bottom starting with station 6 . Lines are used to show the hot-wire profiles taken at the symmetric locations. Note the shifted scale of the ordinate and the minus sign of the velocity. ( See also fig 4, and table 1) 


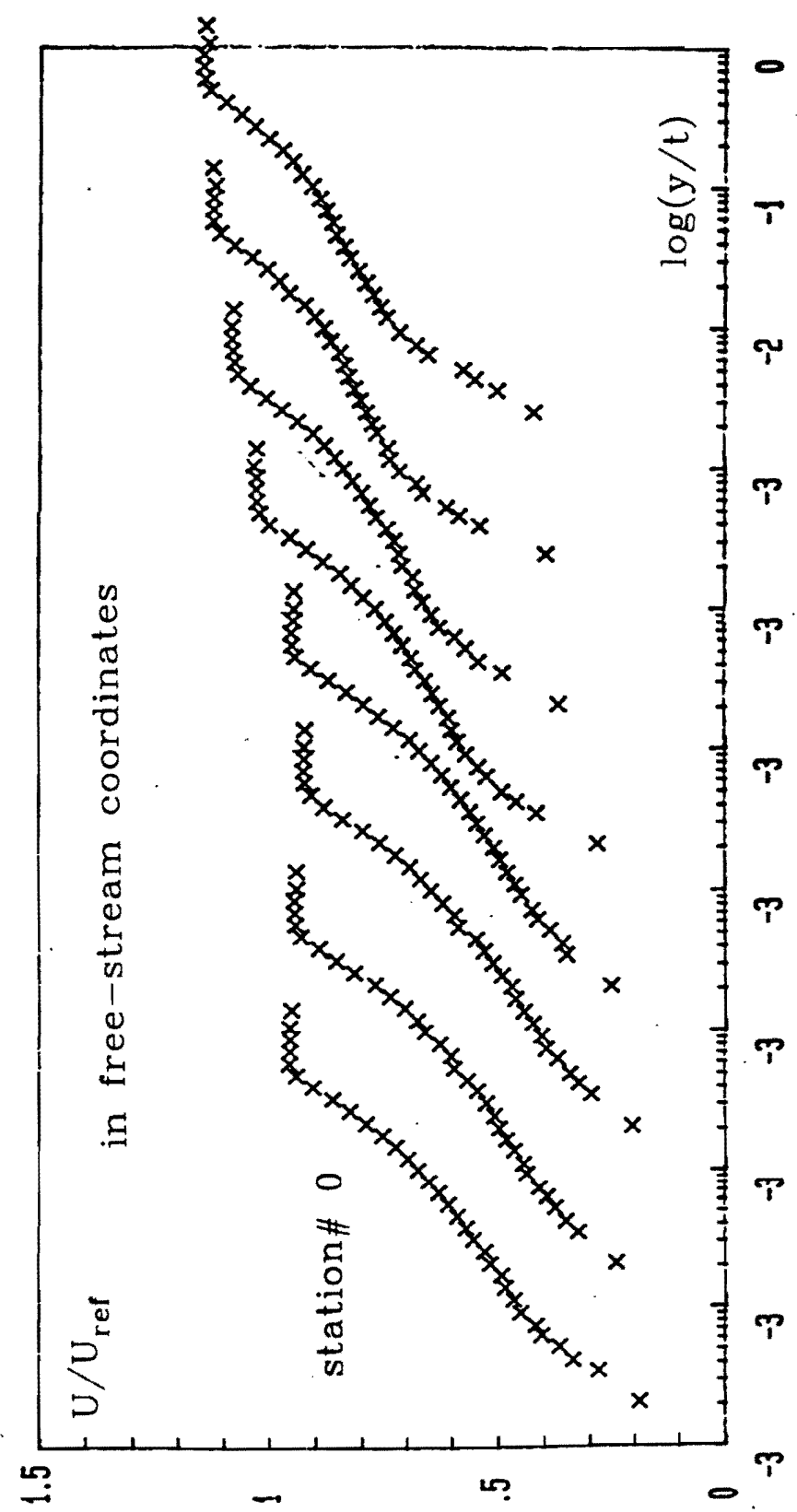

Figure 26. U component of the mean velocity vector measured with LDV technique presented in free-stream coordinates.: The stations are numbered from left to right starting with 0 . Note the shifted scale of the absissa. (See also fig 4, and table 3 ) 

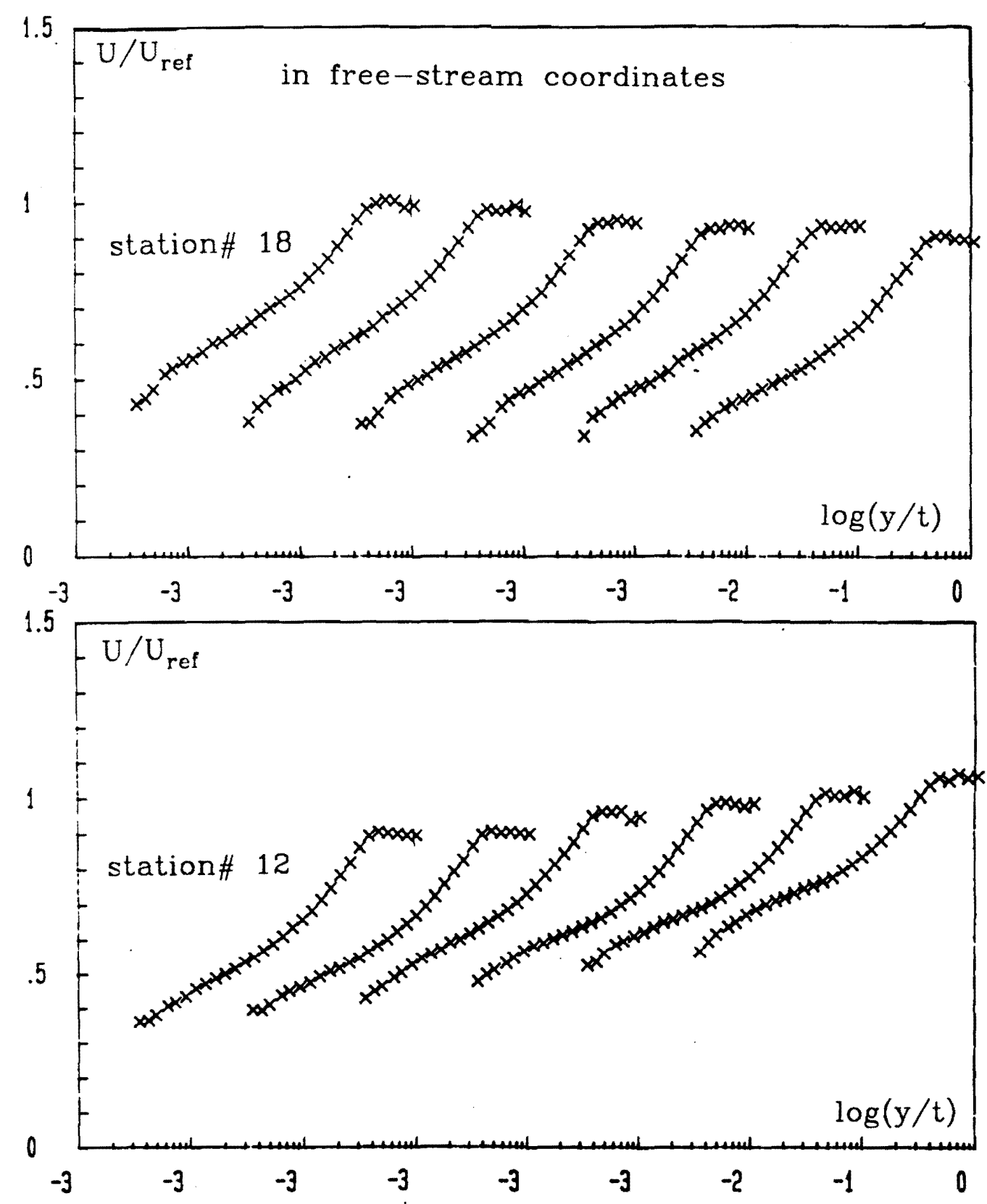

Figure 27a. U component of the mean velocity vector measured with hot-wire technique on the left-hand side of the wing presented in free-stream coordinates.: In the figures the stations are numbered from left to right starting with station 18 and station 12 , respectively. Note the shifted scale of the absissa. ( See also fig 4 , and table 1 ) 


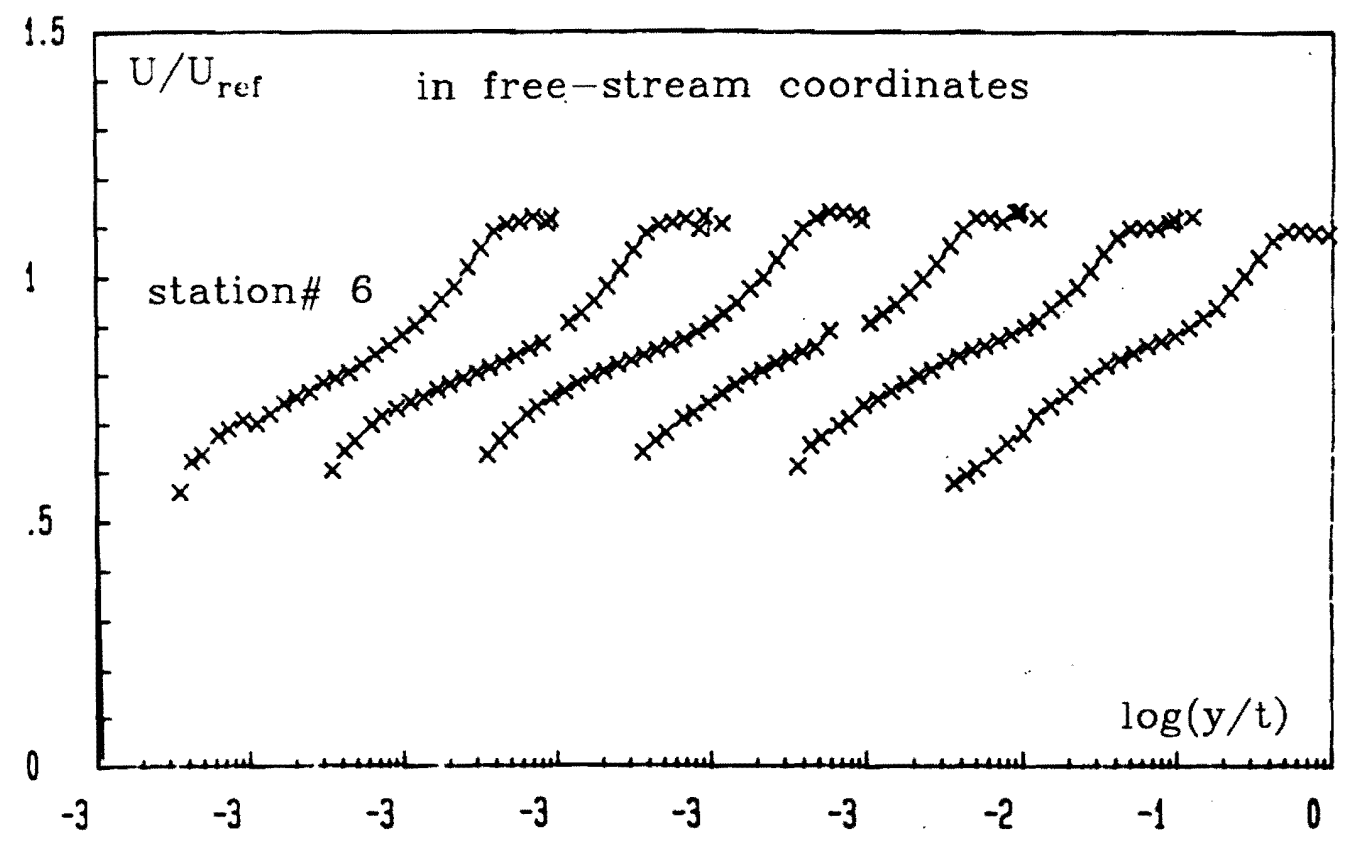

Figure 27b. $U$ component of the mean velocity vector measured with hot-wire technique on the left-hand side of the wing presented in free-stream coordinates.: In the figure the stations are numbered from left to right starting with station 6 . Note the shifted scale of the absissa. (See also fig 4, and table 1 ) 


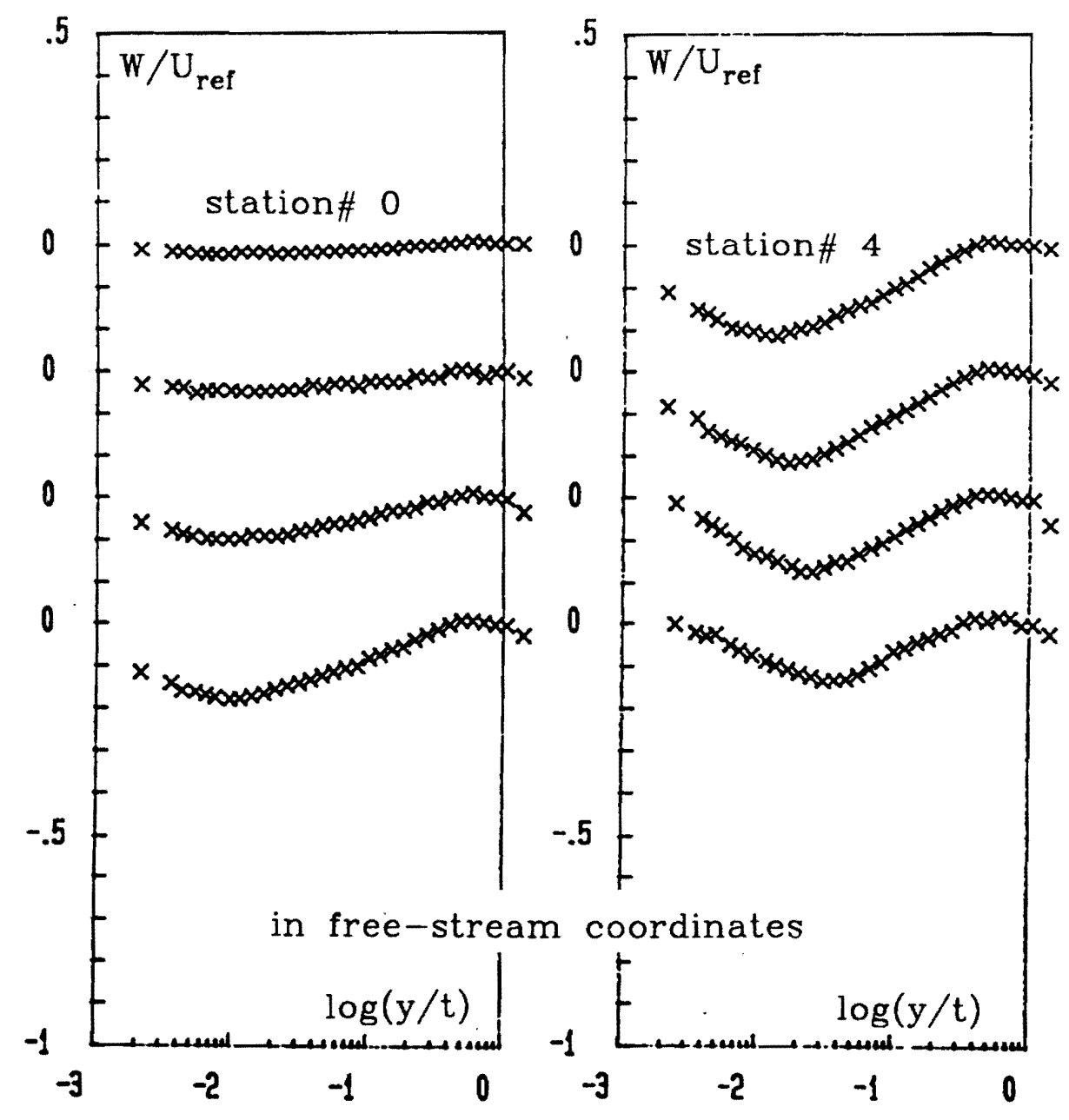

Figure 28. W component of the mean velocity vector measured with LDV technique presented in free-stream coordinates.: In the figures the stations are numbered from top to bottom starting with station 0 and station 4 , respectively. Note the shifted scale of the ordinate. (See also fig 4 , and table 3 ) 


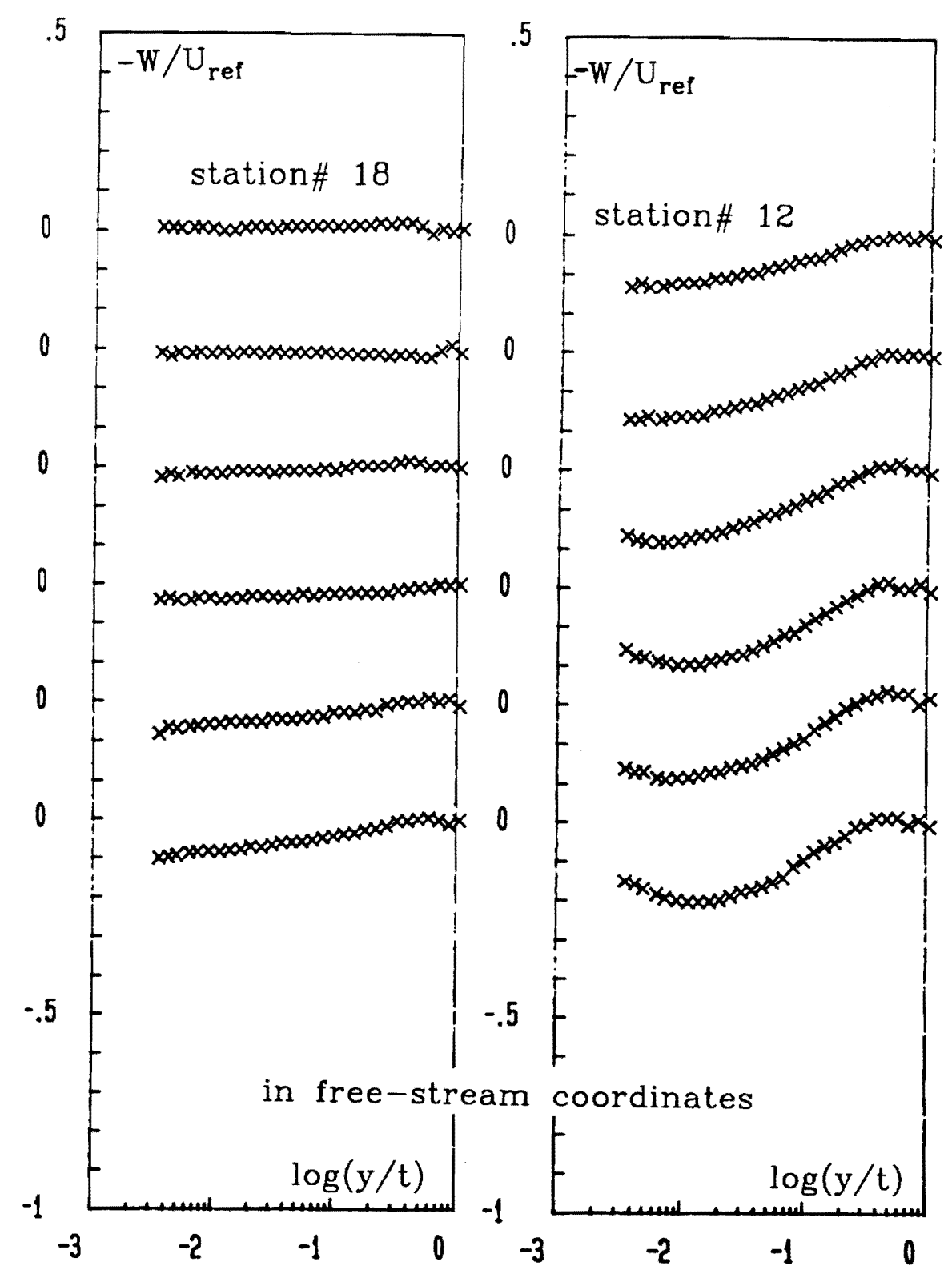

Figure 29a. W component of the mean velocity vector measured with hot-wire technique on the left-hand side of the wing presented in free-stream coordinates.: In the figures the stations are numbered from top to bottom starting with station 18 and station 12 , respectively. Note the shifted scale of the ordinate and the minus sign of the velocity. ( See also fig 4 , and table 1 ) 


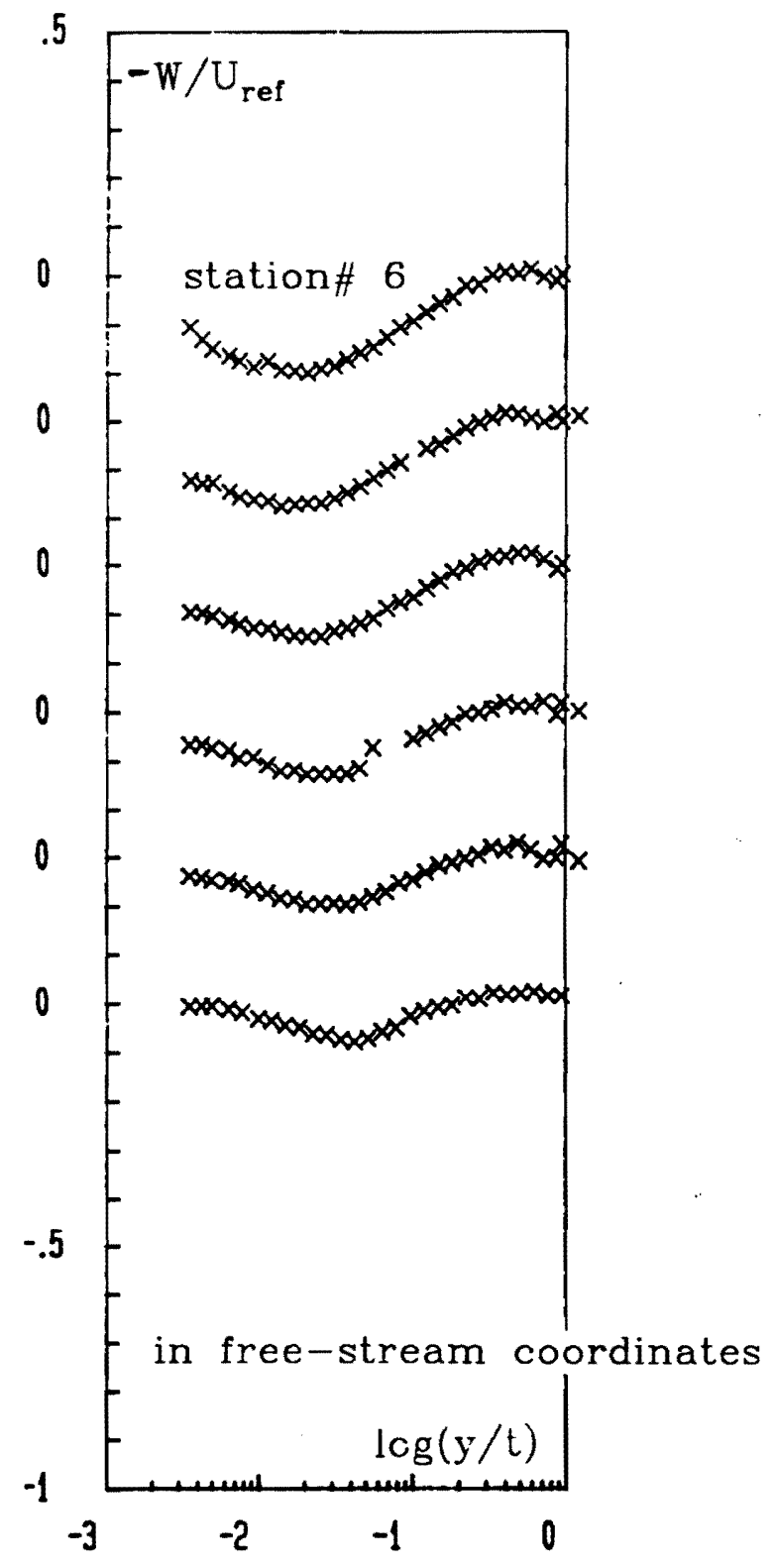

Figure 29b. W component of the mean velocity vector measured with hot-wire technique on the left-hand side of the wing presented in free-stream coordinates.: In the figure the stations are numbered from top to bottom starting with station 6 . Note the shifted scale of the ordinate and the minus sign of the velocity. (See also fig 4, and table 1 ) 


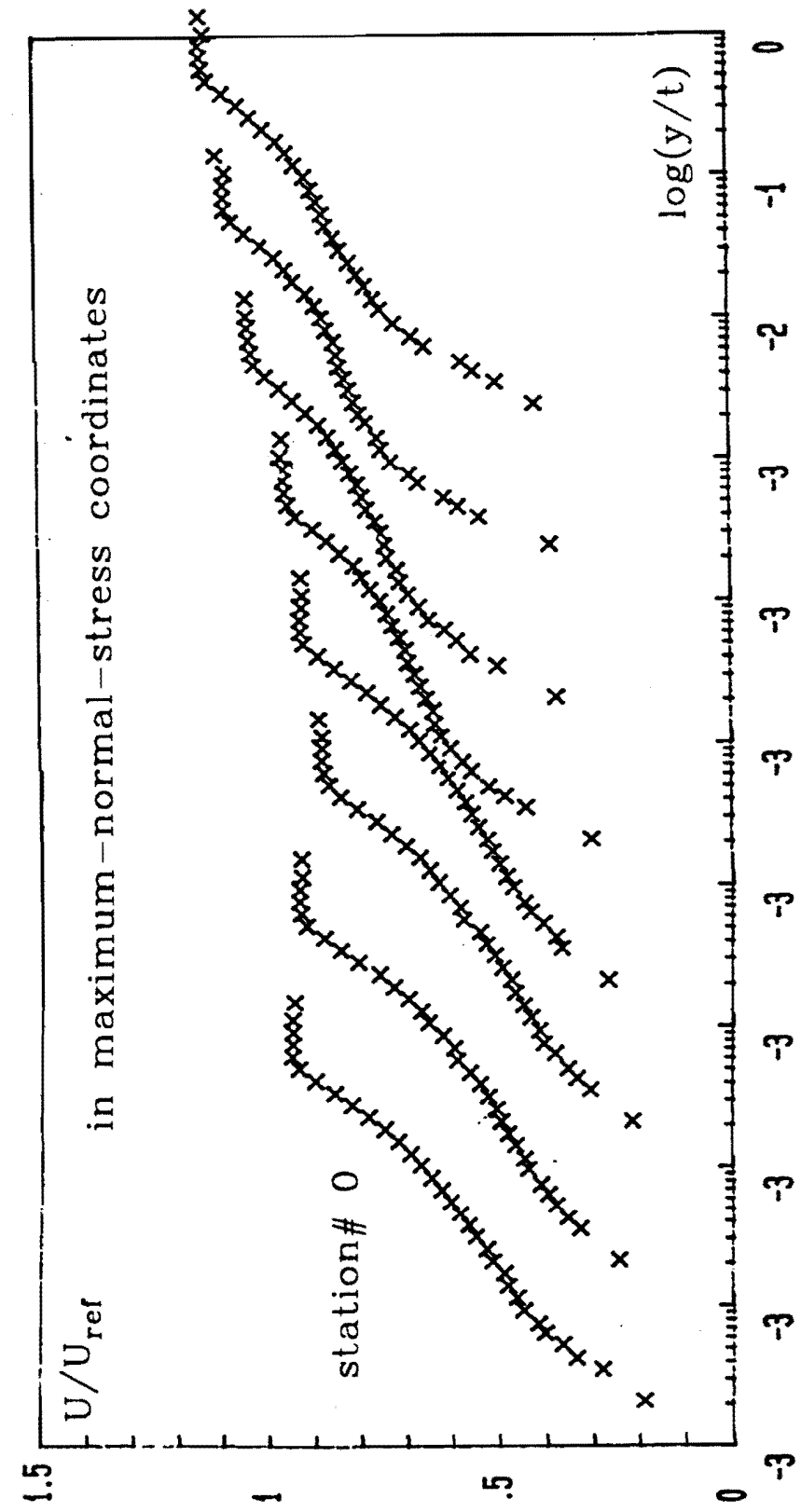

Figure 30. $U$ component of the mean velocity vector measured with LDV technique presented in maximum normal stress coordinates.: The stations are numbered from left to right starting with 0 . Note the shifted scale of the absissa. ( See also fig 4 , and table 3 ) 

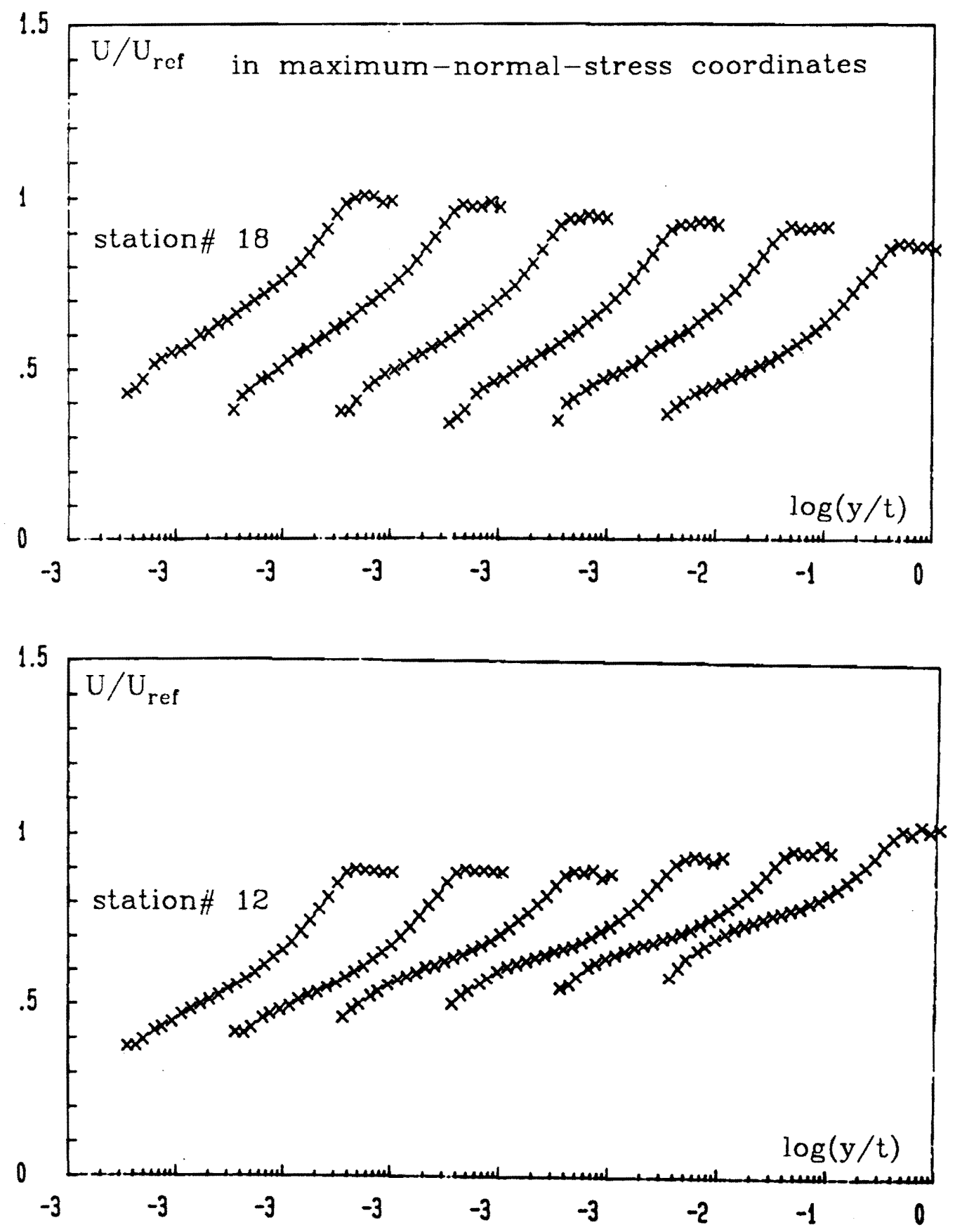

Figure 31a. $U$ component of the mean velocity vector measured with hot-wire technique on the left-hand side of the wing presented in normal stress coordinates.: In the figures the stations are numbered from left to right starting with station 18 and station 12, respectively. Note the shifted scale of the absissa. ( See also fig 4 , and table 1) 


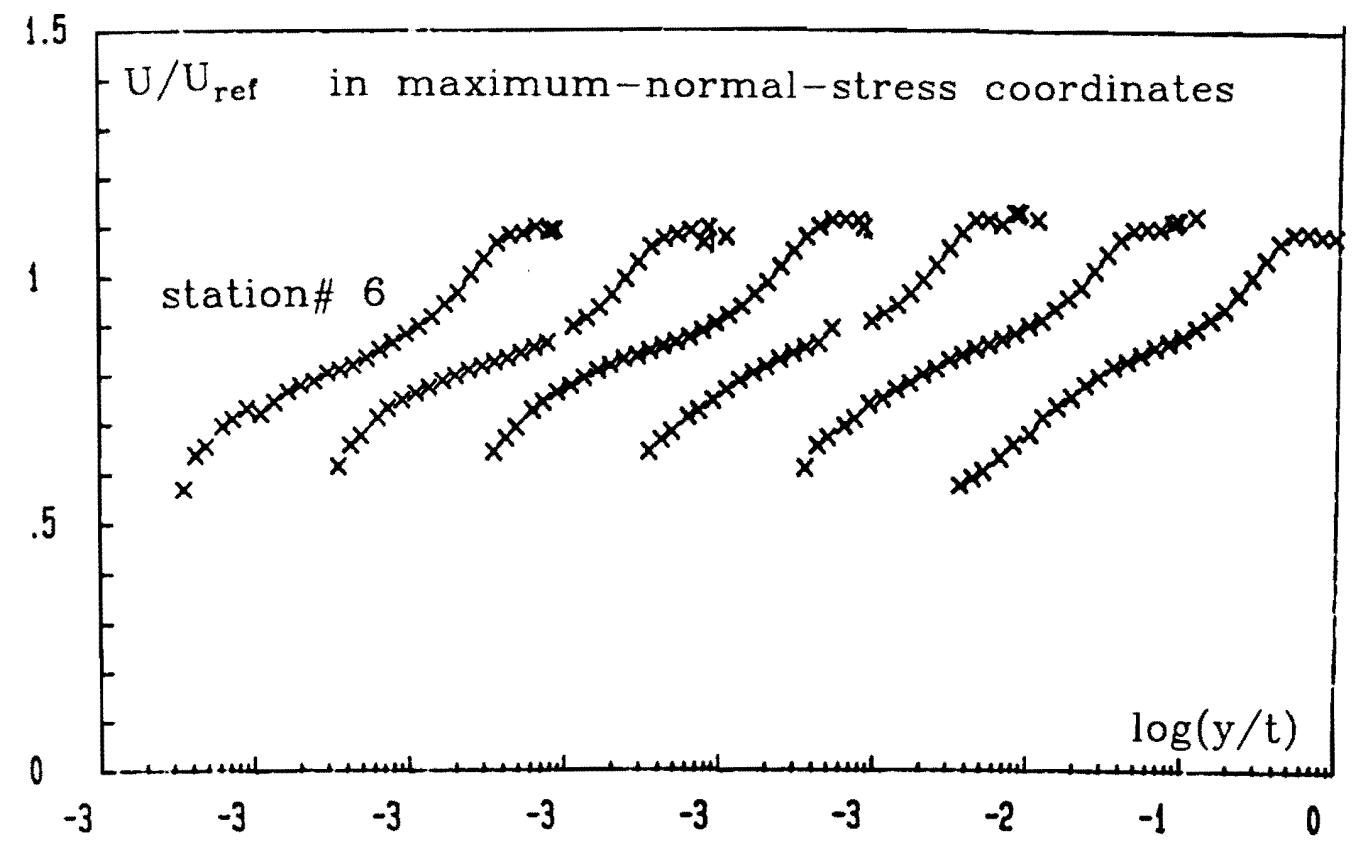

Figure 31b. U component of the mean velocity vector measured with hot-wire technique on the left-hand side of the wing presented in normal stress coordinates.: In the figure the stations are numbered from left to right starting with station 6 . Note the shifted scale of the absissa. ( See also fig 4, and table 1 ) 


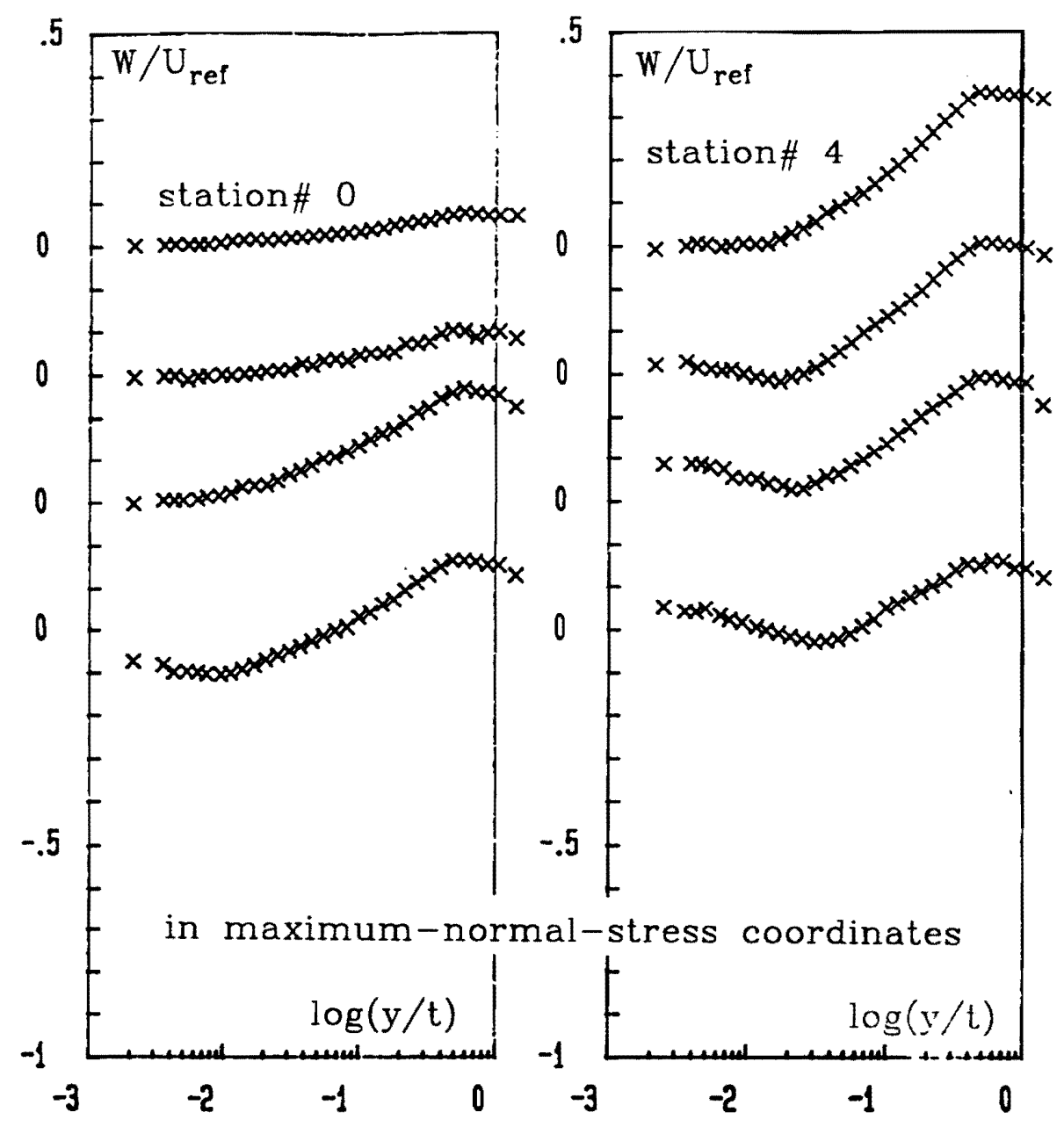

Figure 32. $\mathrm{W}$ component of the mean velocity vector measured with LDV technique presented in maximum normal stress coordinates.: In the figures the stations are numbered from top to bottom starting with station 0 and station 4 , respectively. Note the shifted scale of the ordinate. (See also fig 4, and table 3 ) 


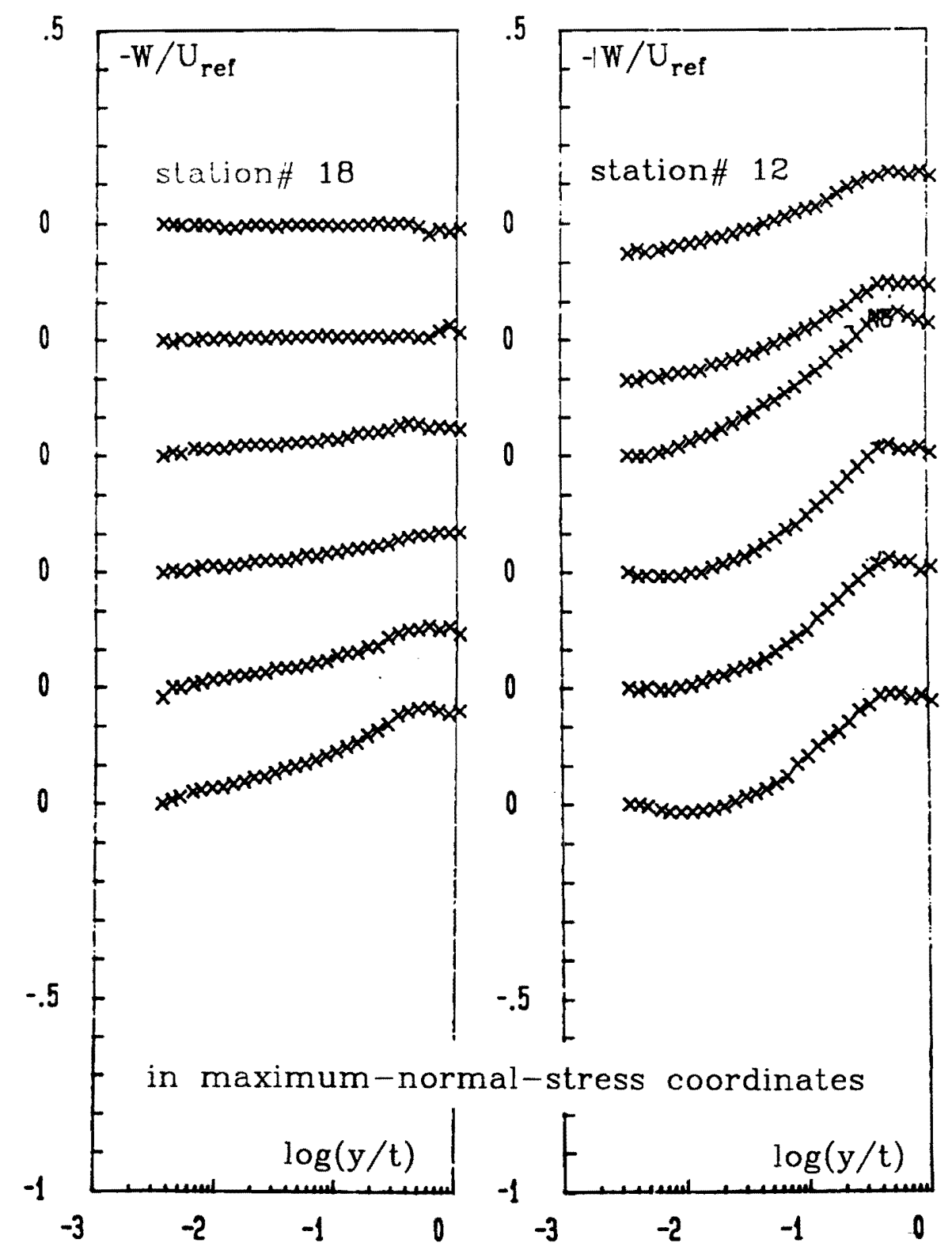

Figure 33a. W component of the mean velocity vector measured with hot-wire technique on the left-hand side of the wing presented in normal stress coordinates.: In the figures the stations are numbered from top to bottom starting with station 18 and station 12 , respectively. Note the shifted scale of the ordinate and the minus sign of the velocity. ( See also fig 4 , and table 1 ) 


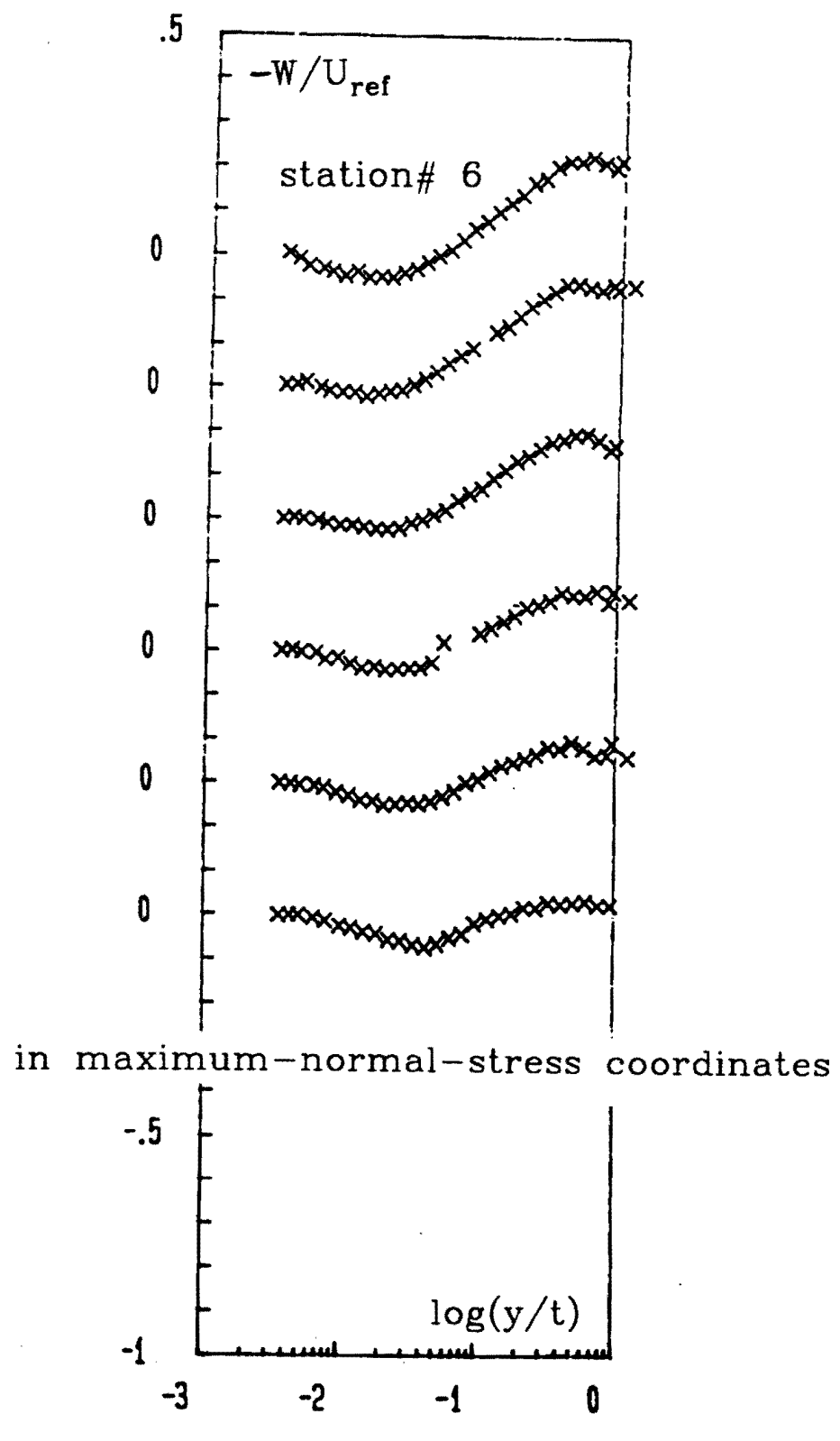

Figure 33b. W component of the mean velocity vector measured with hot-wire technique on the left-hand side of the wing presented in normal stress coordinates.: In the figure the stations are numbered from top to bottom starting with station 6 . Note the shifted scale of the ordinate and the minus sign of the velocity. (See also fig 4 , and table 1 ) 

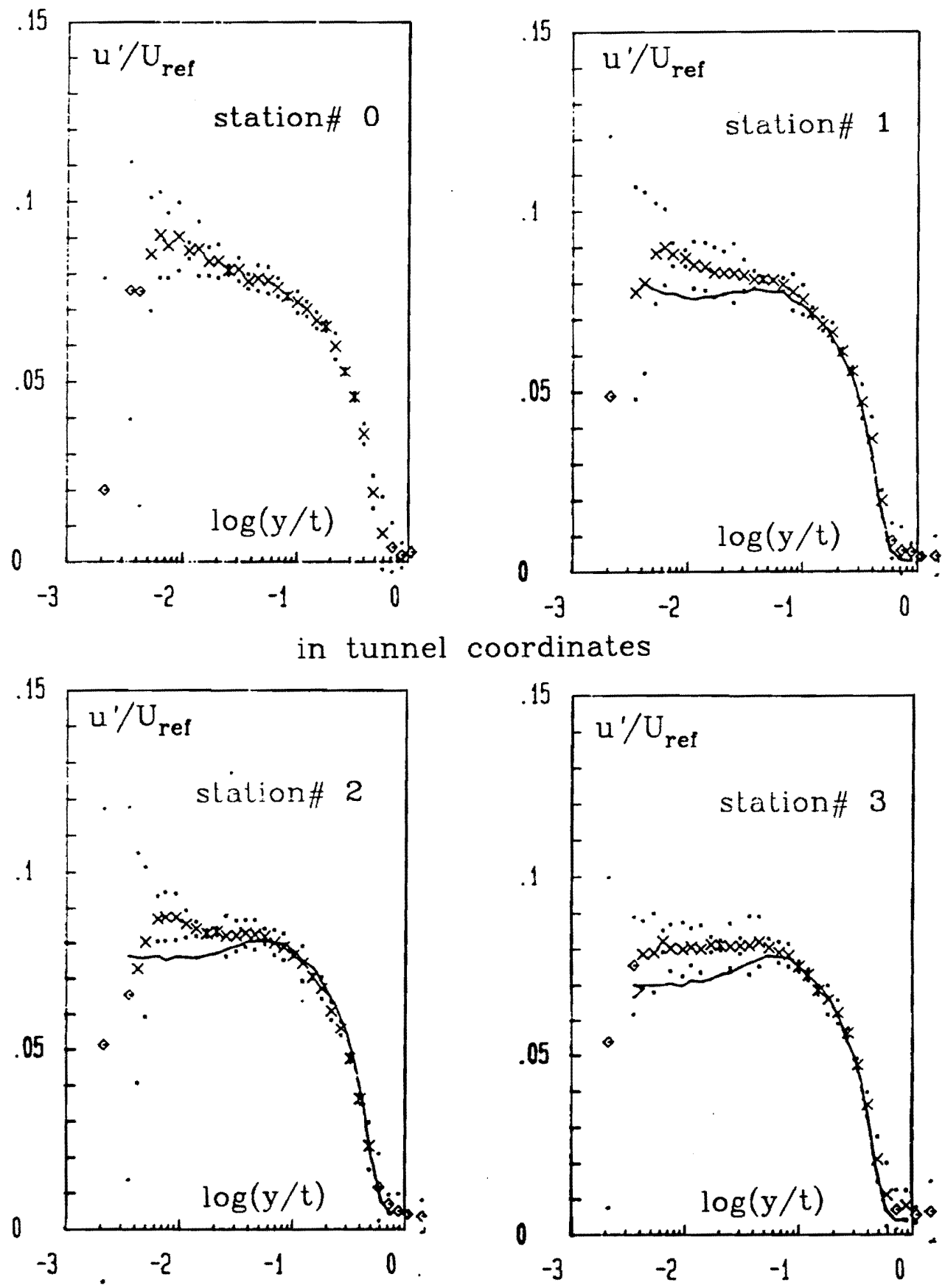

Figure 34a. $u^{\prime}$, fluctuating velocity component measured with LDV technique presented in tunnel coordinates.: The station numbers are shown in the figures. Large symbols denote the data, small symbols denote the uncertainty band. Lines are used to show the hot-wire profiles taken at the same locations. Different symbols, very near the wall and near the layer edge show the points where the realizability conditions are not satisfied. ( See also fig 4, and table 3 ) 

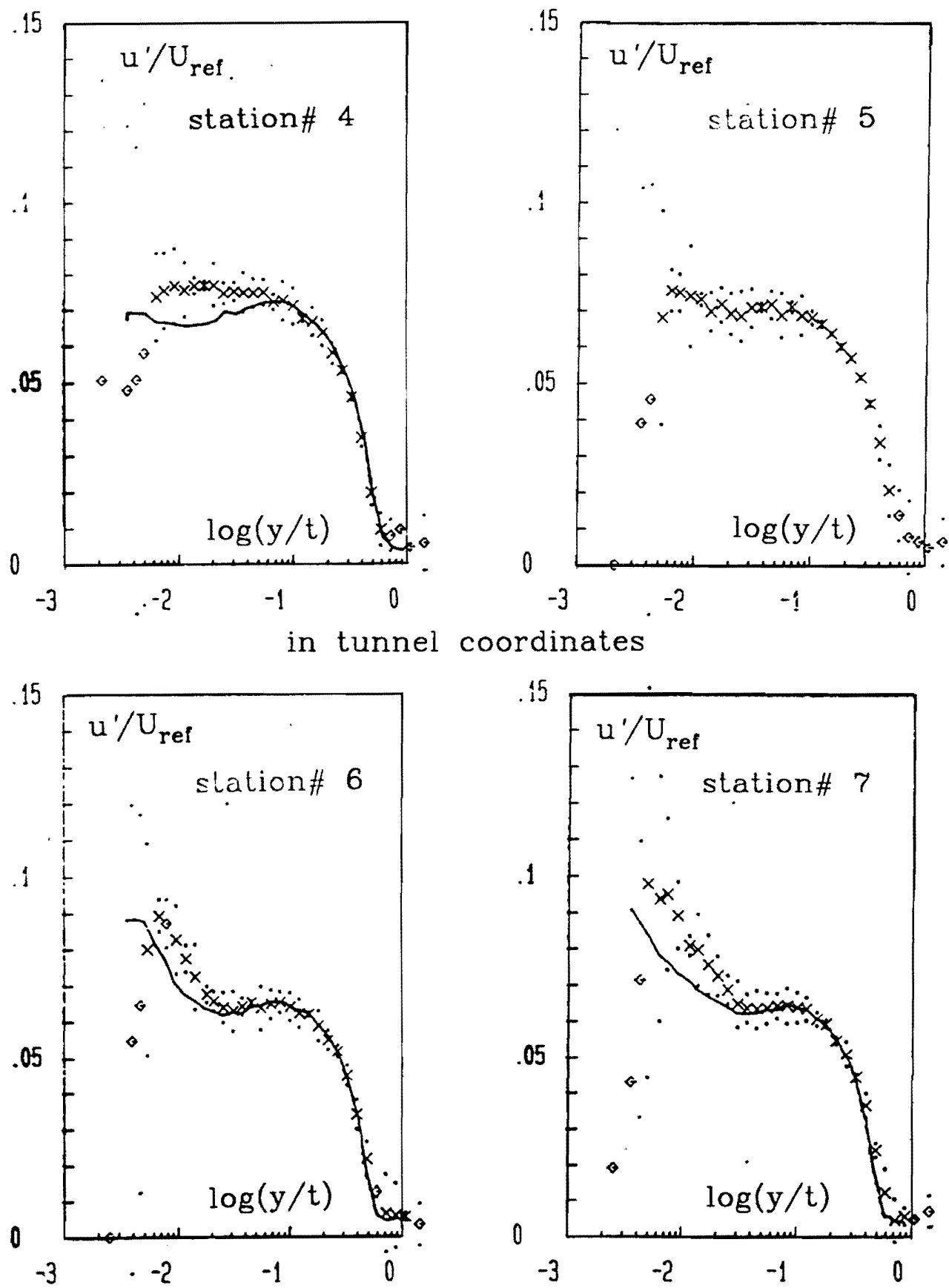

Figure 34b. $u^{\prime}$, fluctuating velocity component measured with LDV technique presented in tunnel coordinates.: The station numbers are shown in the figures. Large symbols denote the data, small symbols denote the uncertainty band. Lines are used to show the hot-wire profiles taken at the same locations. Different symbols, very near the wall and near the layer edge show the points where the realizability conditions are not satisfied. (See also fig 4 , and table 3 ) 
in tunnel coordinates

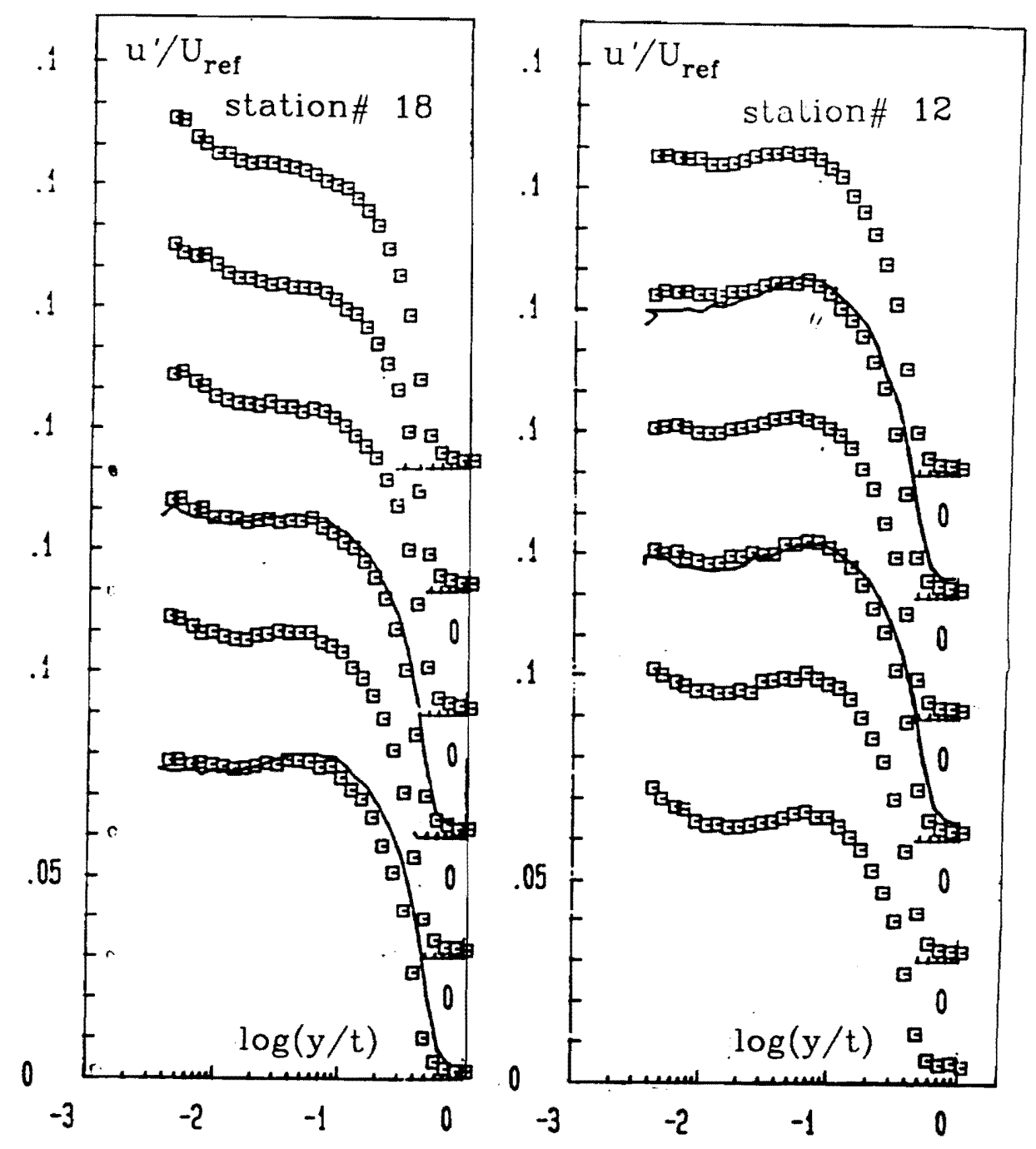

Figure 35a. $\mathbf{u}^{\prime}$, fluctuating velocity component measured with hot-wire technique on the left-hand side of the wing presented in tunnel coordinates.: In the figures the stations are numbered from top to bottom starting with station 18 and station 12 , respectively. Lines are used to show the hot-wire profiles taken at the symmetric locations. Note the shifted scale of the ordinate. (See also fig 4 , and table 1) 


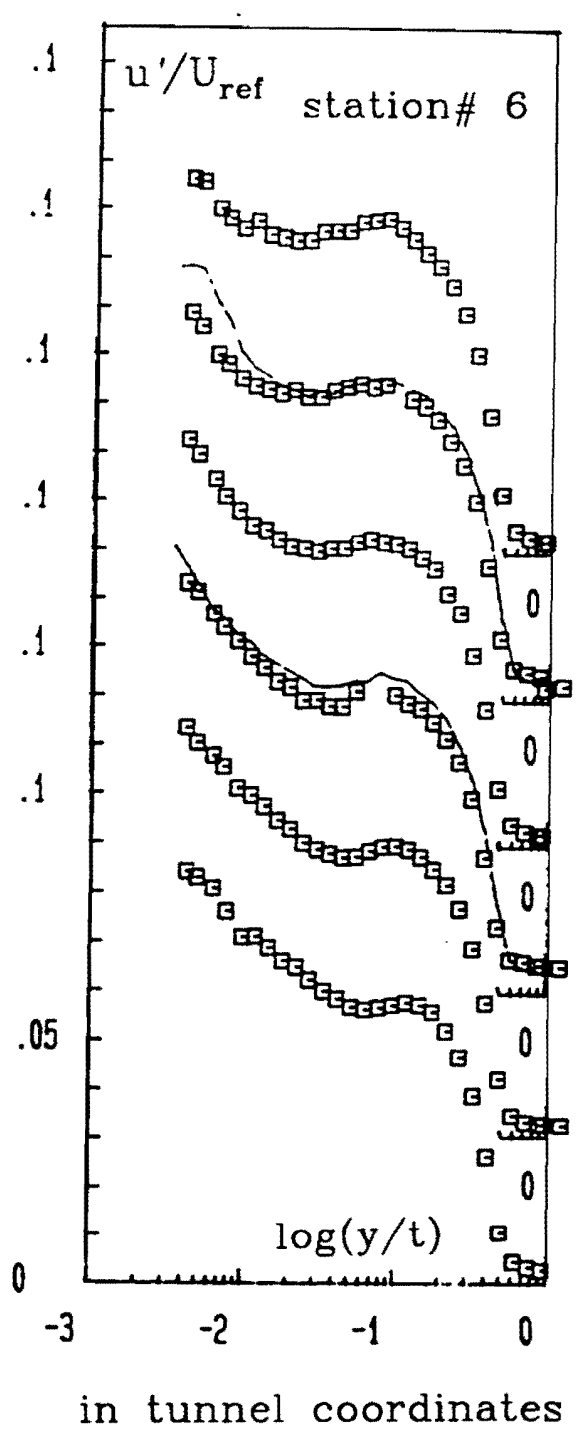

Figure 35b. $u^{\prime}$, fluctuating velocity component measured with hot-wire technique on the left-hand side of the wing presented in tunnel coordinates.: In the figure the stations are numbered from top to bottom starting with station 6 . Lines are used to show the hot-wire profiles taken at the symmetric locations. Note the shifted scale of the ordinate. ( See also fig 4 , and table 1 ) 

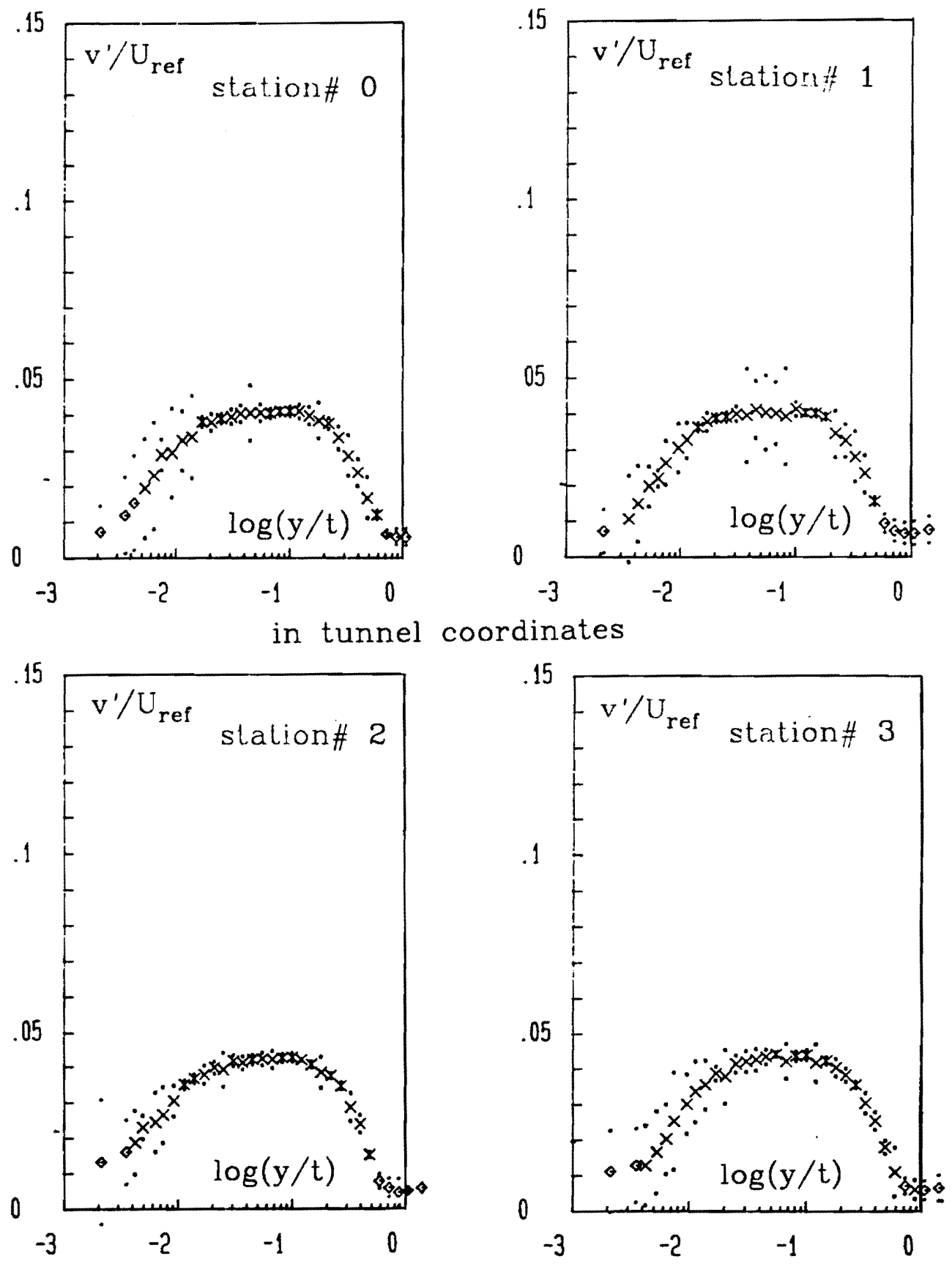

Figure 36a. $\quad v^{\prime}$, fluctuating velocity component measured with LDV technique presented in tunnel coordinates.: The station numbers are shown in the figures. Large symbols denote the data, small symbols denote the uncertainty band. Different symbols, very near the wall and near the layer edge show the points where the realizability conditions are not satisfied. 

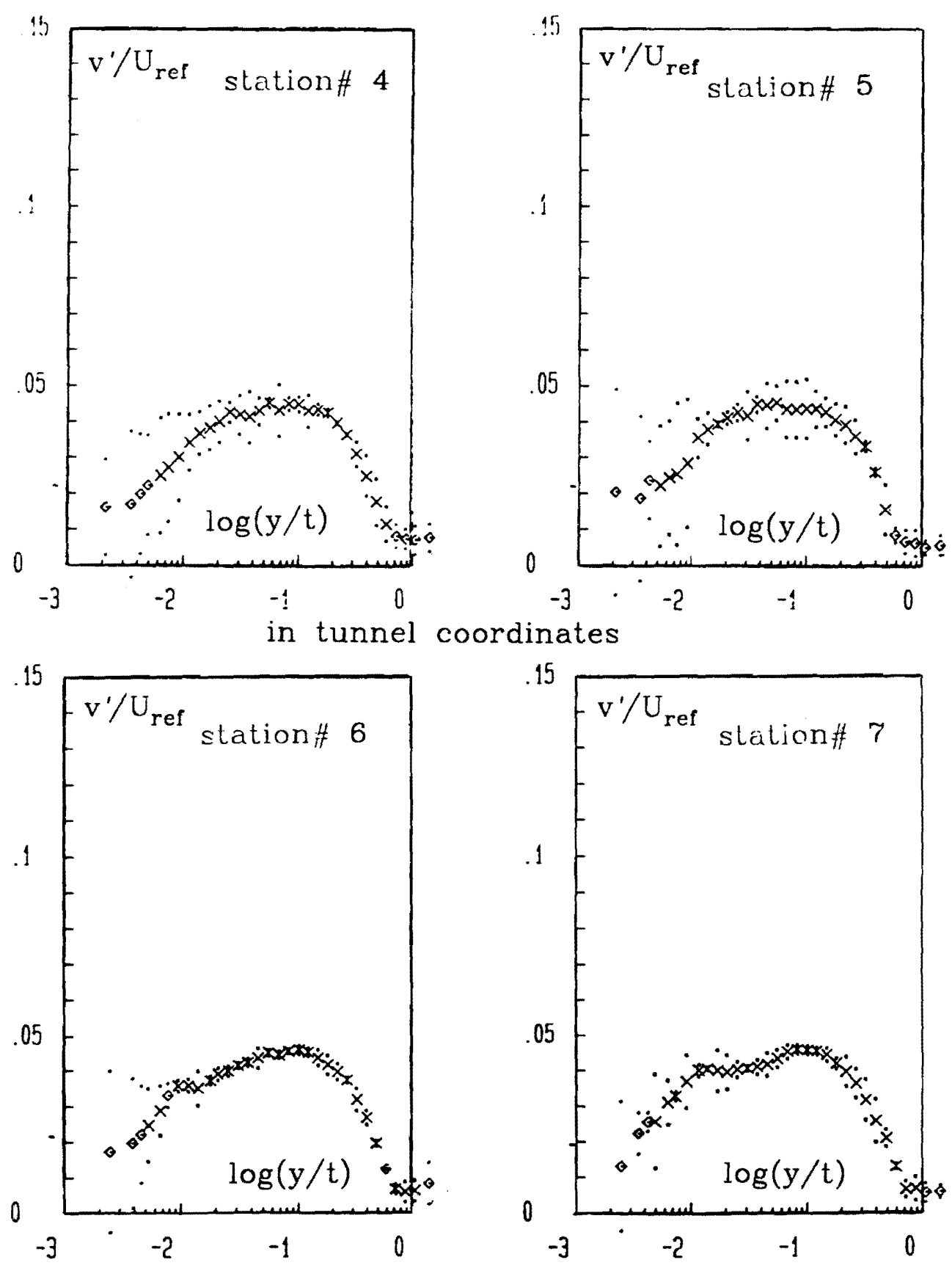

Figure 36b. $\quad v^{\prime}$, fluctuating velocity component measured with LDV technique presented in tunnel coordinates.: The station numbers are shown in the figures. Large symbols denote the data, small symbols denote the uncertainty band. Different symbols, very near the wall and near the layer edge show the points where the realizability conditions are not satisfied. 

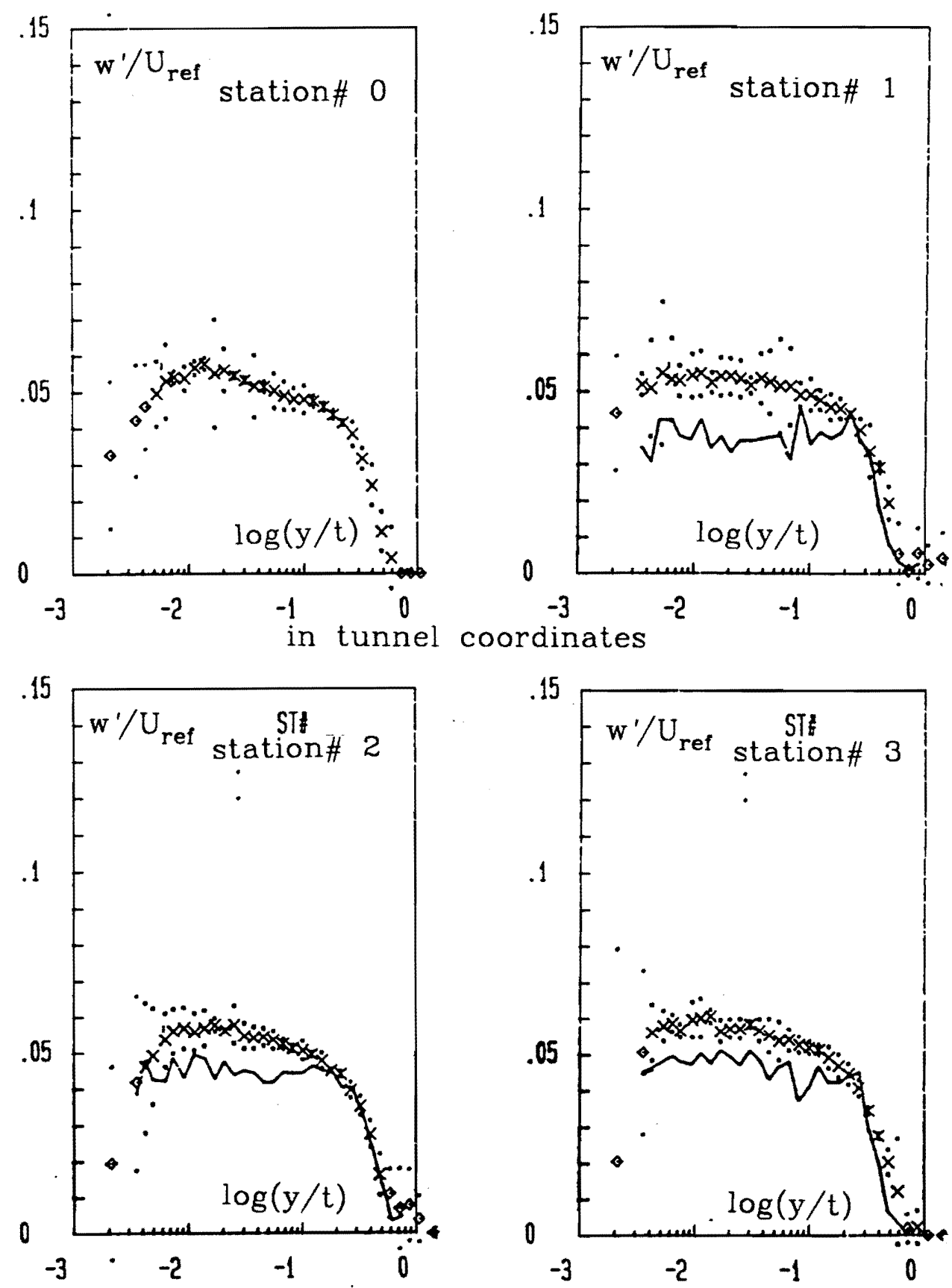

Figure 37a. w' fluctuating velocity component measured with LDV technique presented in tunnel coordinates.: The station numbers are shown in the figures. Large symbols denote the data, small symbols denote the uncertainty band. Lines are used to show the hot-wire profiles taken at the same locations. Different symbols, very near the wall and near the layer edge show the points where the realizability conditions are not satisfied. (See also fig 4 , and table 3 ) 

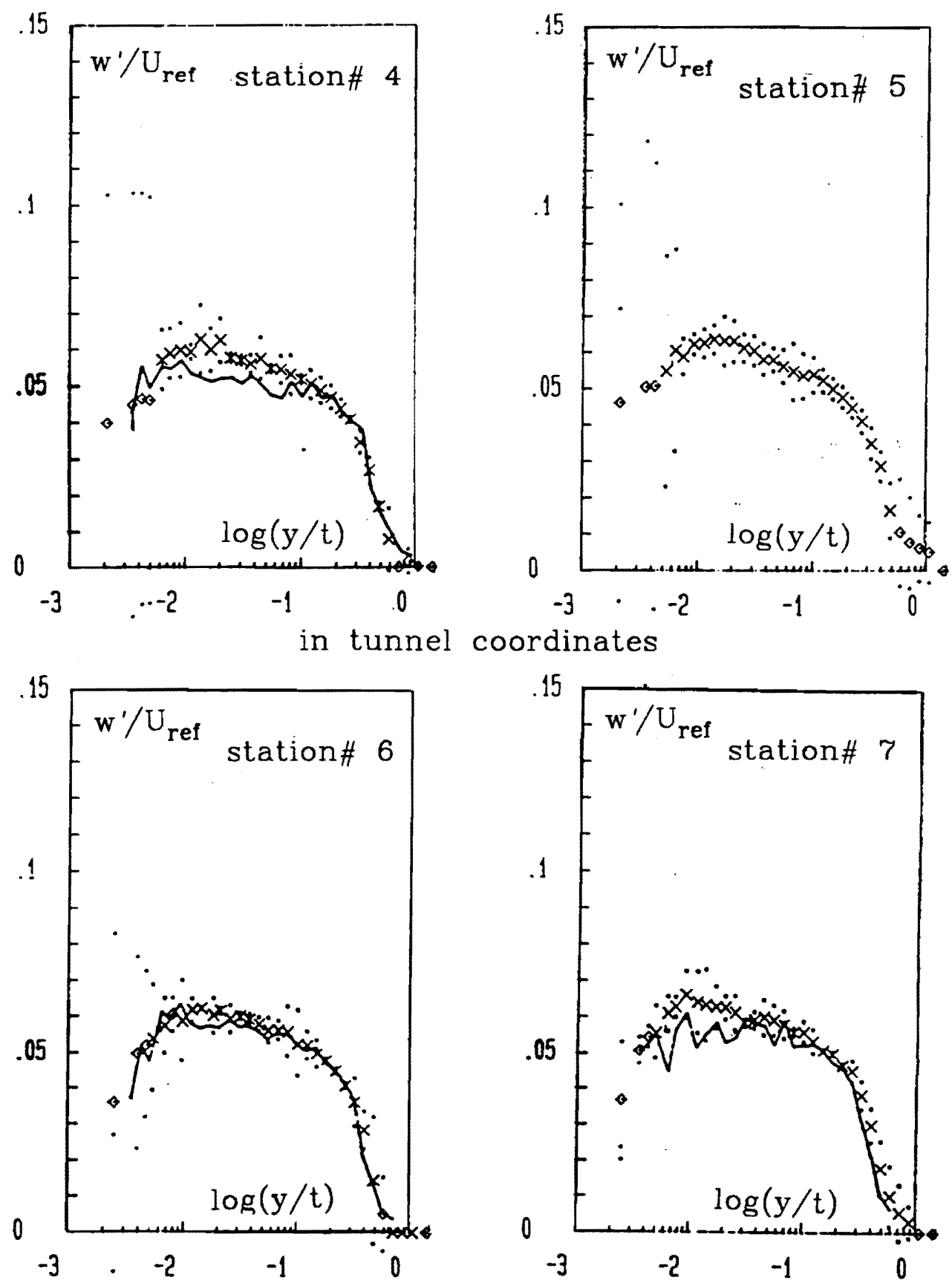

Figure 37b. w' fluctuating velocity component measured with LDV technique presented in tunnel coordinates.: The station numbers are shown in the figures. Large symbols denote the data, small symbols denote the uncertainty band. Lines are used to show the hot-wire profiles taken at the same locations. Different symbols, very near the wall and near the layer edge show the points where the realizability conditions are not satisfied. ( See also fig 4, and table 3 ) 


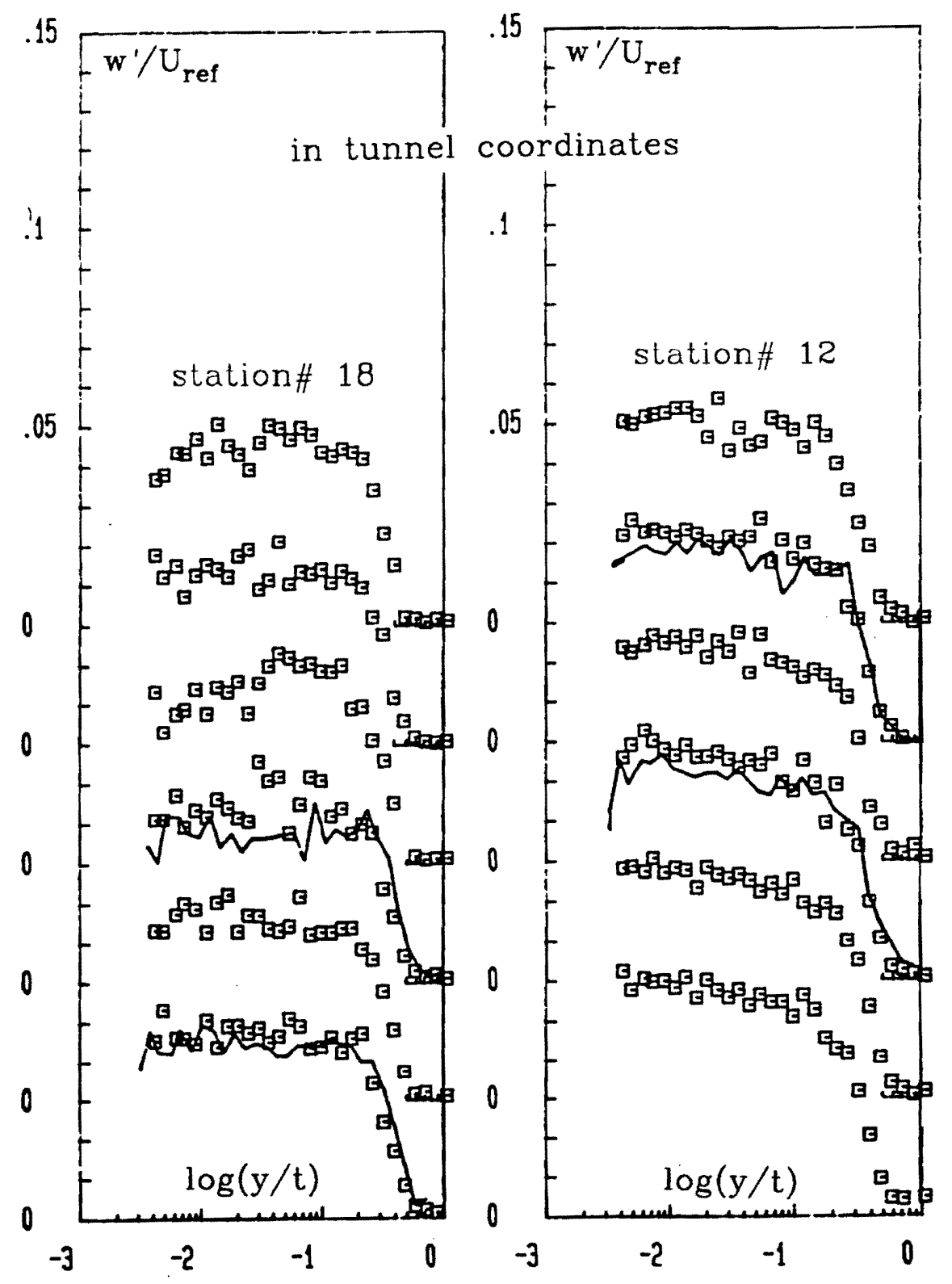

Figure 38a. w', fluctuating velocity component measured with hot-wire technique on the left-hand side of the wing presented in tunnel coordinates.: In the figures the stations are numbered from top to bottom starting with station 18 and station 12 , respectively. Lines are used to show the hot-wire profiles taken at the symmetric locations. Note the shifted scale of the ordinate. ( See also fig 4 , and table 1) 


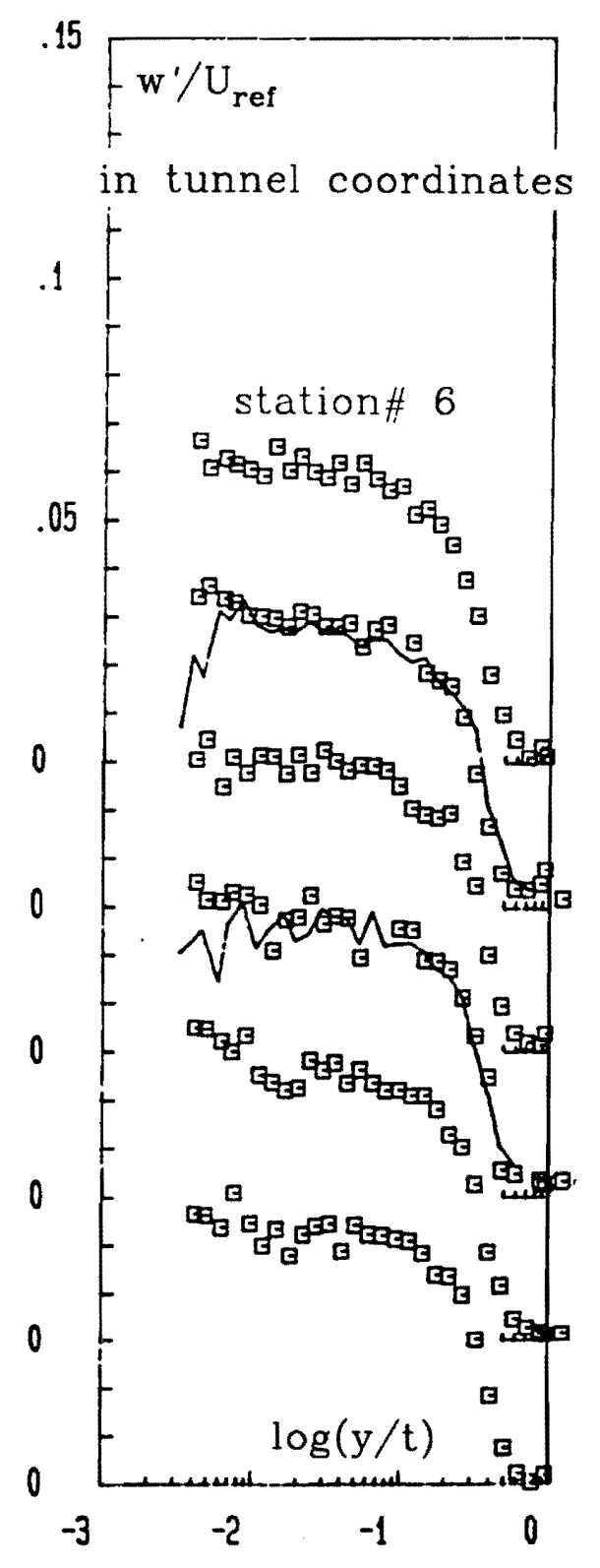

Figure 38b. w', fluctuating velocity component measured with hot-wire technique on the left-hand side of the wing presented in tunnel coordinates.: In the figure the stations are numbered from top to bottom starting with station 6 . Lines are used to show the hot-wire profiles taken at the symmetric locations. Note the shifted scale of the ordinate. (See also fig 4 , and table 1 ) 


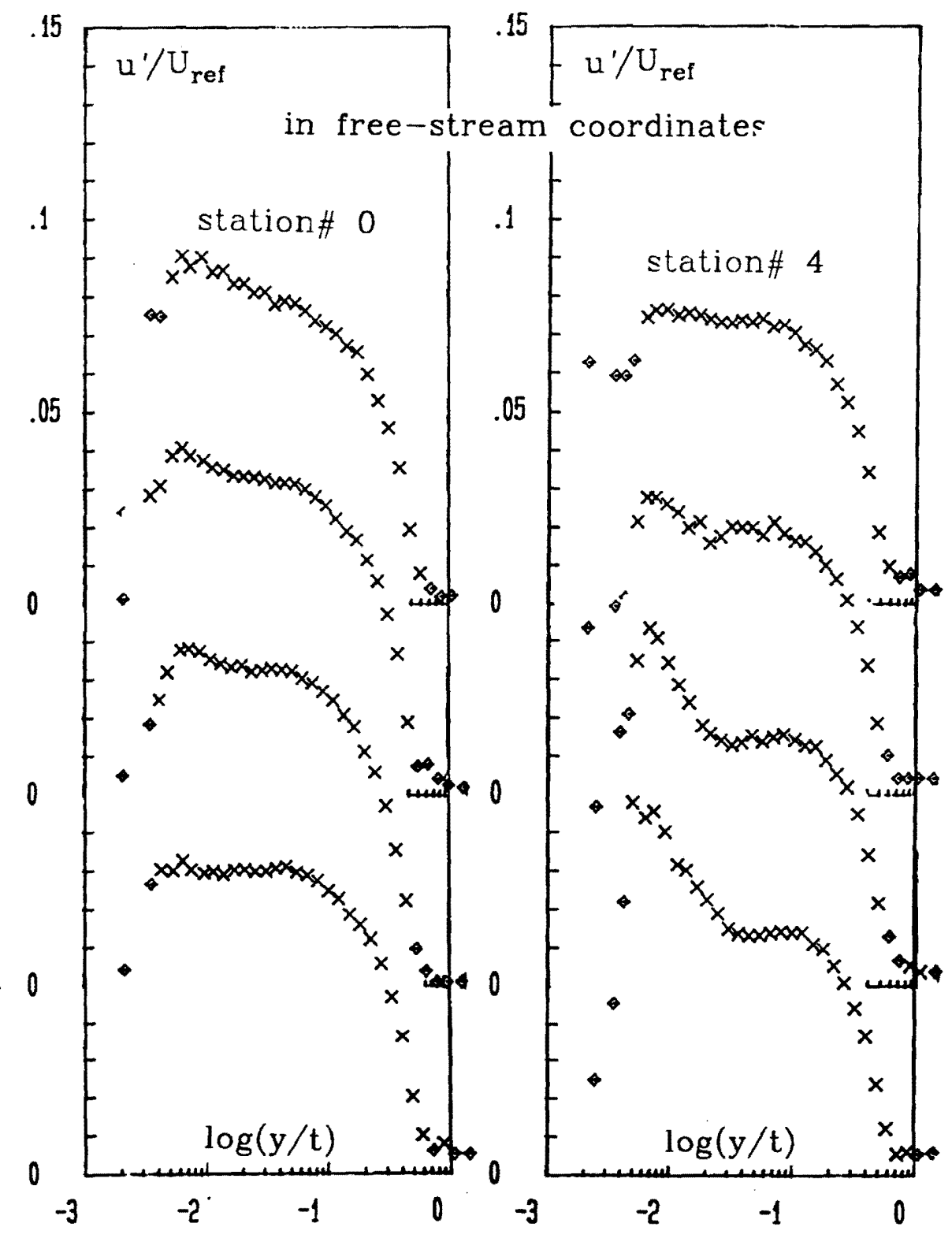

Figure 39. $\mathbf{u}^{\prime}$, fluctuating velocity component measured with LDV technique presented in free-stream coordinates.: In the figures the stations are numbered from top to bottom starting with station 0 and station 4 , respectively. Different symbols, very near the wall and near the layer edge show the points where the realizability conditions are not satisfied. (See also fig 4 , and table 3 ) 


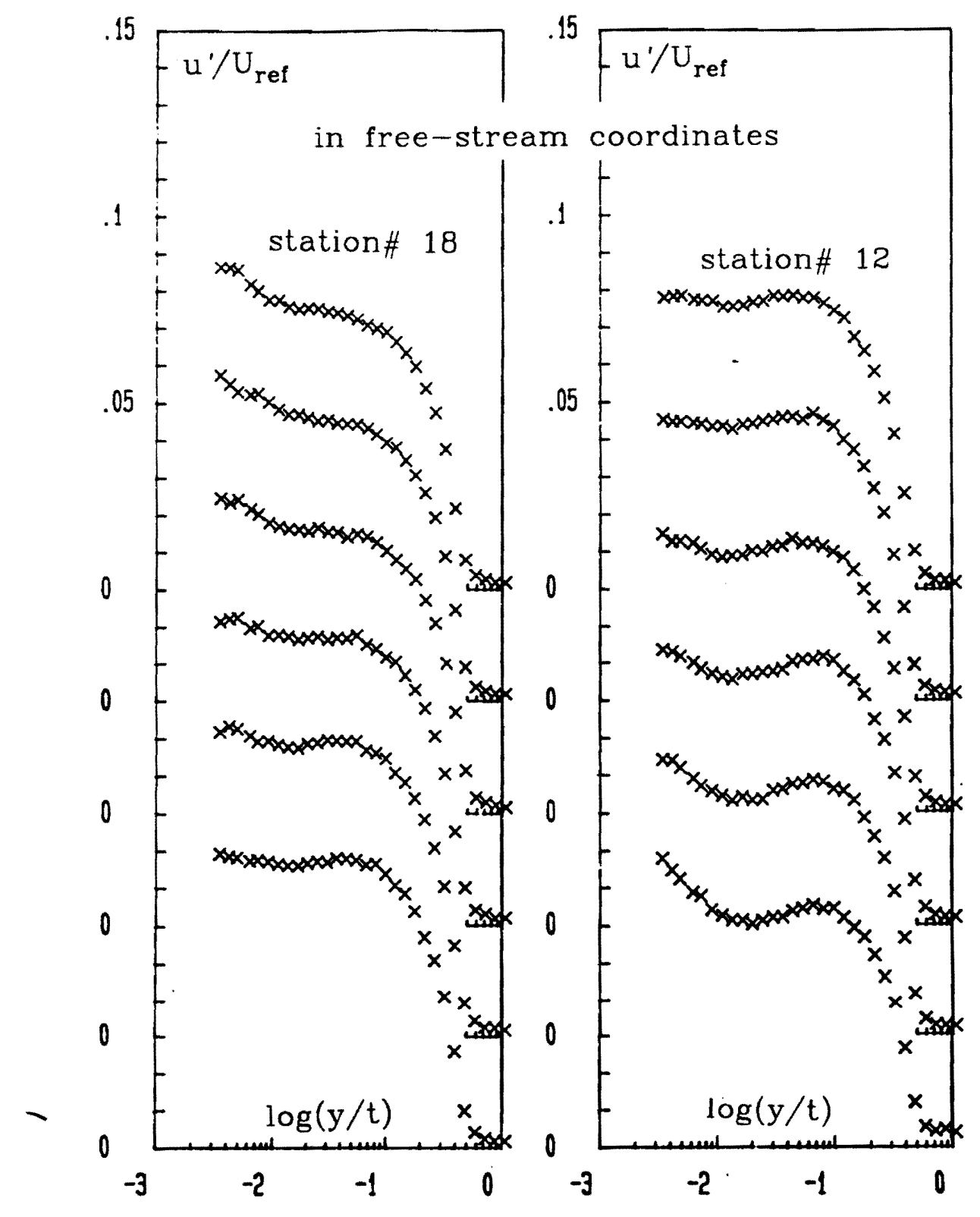

Figure 40a. $u^{\prime}$, fluctuating velocity component measured with hot-wire technique on the left-hand side of the wing presented in free-stream coordinates.: In the figures the stations are numbered from top to bottom starting with station 18 and station 12, respectively. Note the shifted scale of the ordinate. ( See also fig 4, and table 1 ) 


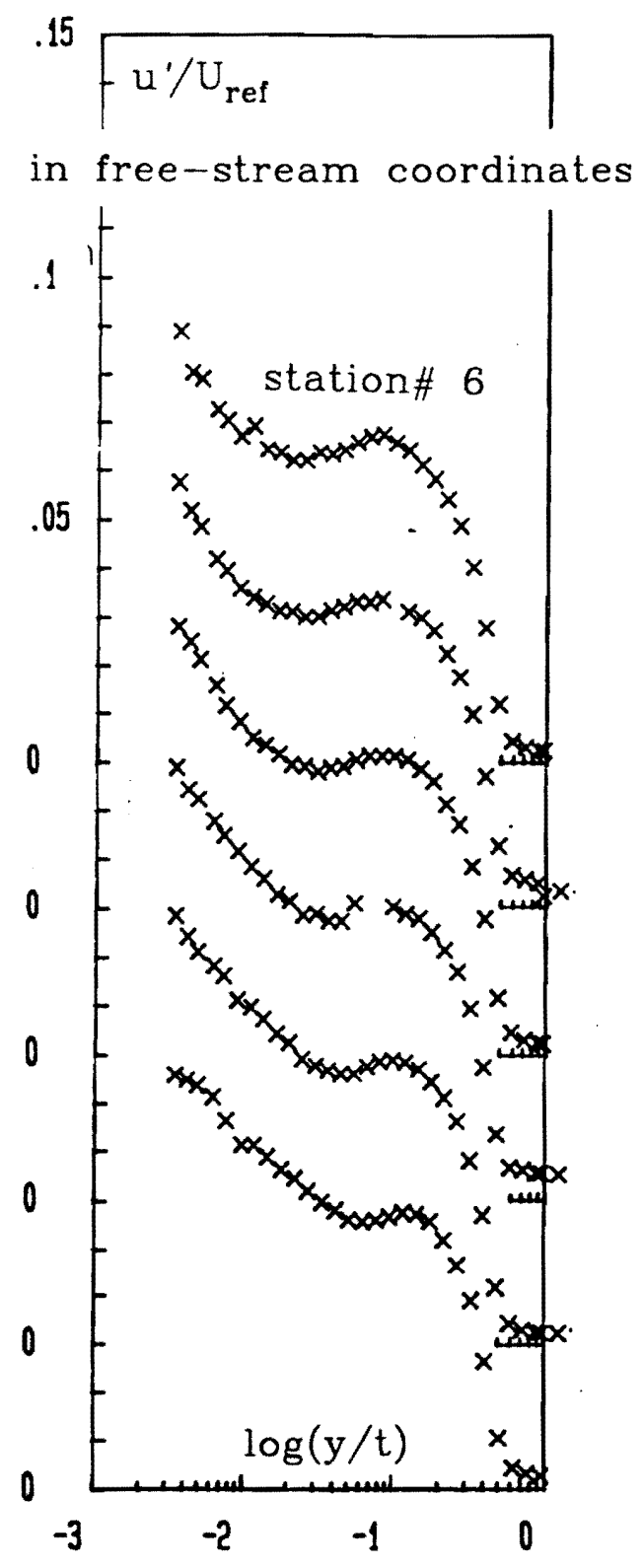

Figure 40b. $\mathrm{u}^{\prime}$, fluctuating velocity component measured with hot-wire technique on the left-hand side of the wing presented in free-stream coordinates.: In the figure the stations are numbered from top to bottom starting with station 6 . Note the shifted scale of the ordinate. (See also fig 4 , and table 1 ) 


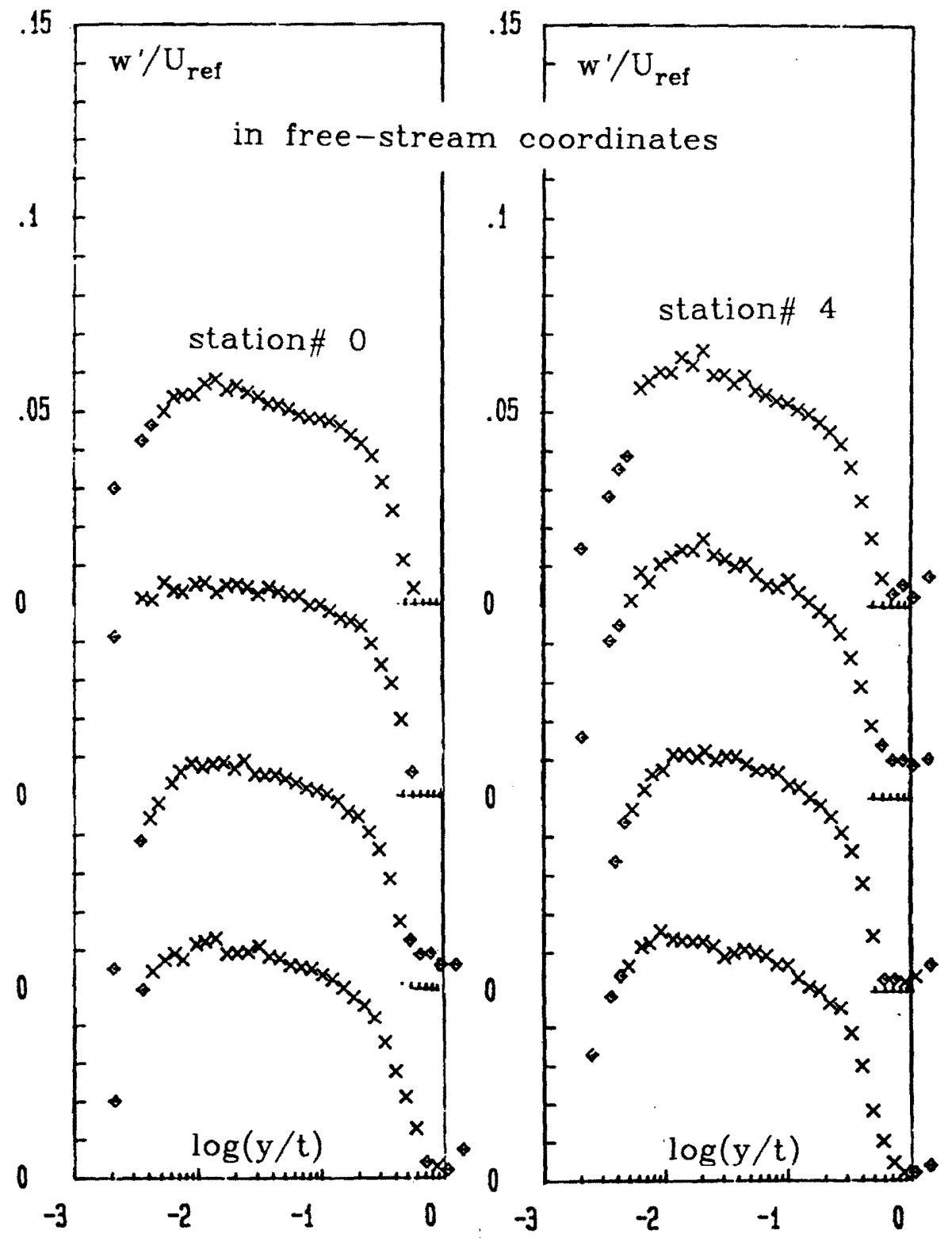

Figure 41. w' fluctuating velocity component measured with LDV technique presented in free-stream coordinates.: In the figures the stations are numbered from top to bottom starting with station 0 and station 4 , respectively. Different symbols, very near the wall and near the layer edge show the points where the realizability conditions are not satisfied. (See also fig 4 , and table 3 ) 


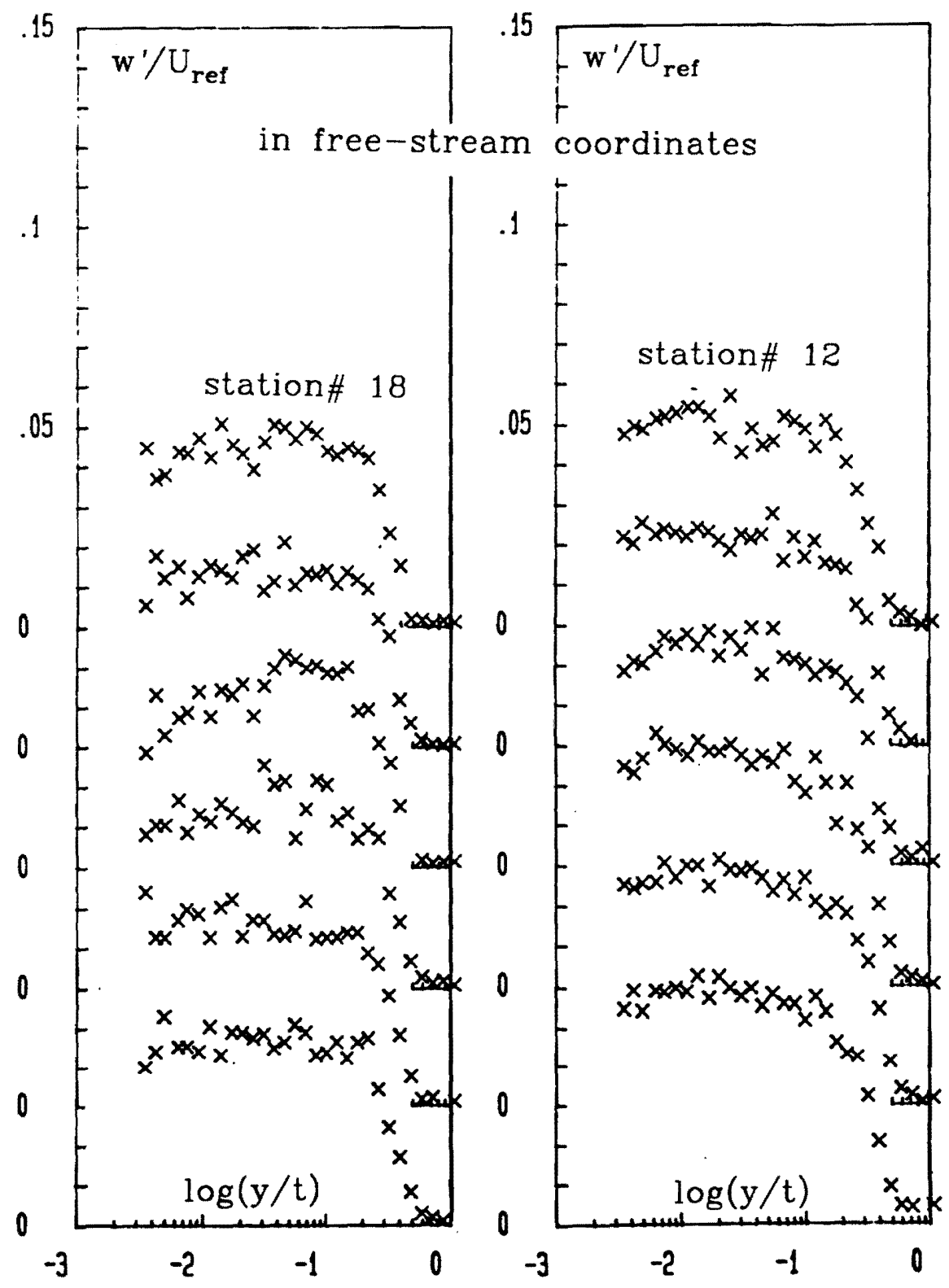

Figure 4la. w', fluctuating velocity component measured with hot-wire technique on the left-hand side of the wing presented in free-stream coordinates.: In the figures the stations are numbered from top to bottom starting with station 18 and station 12 , respectively. Note the shifted scale of the ordinate. ( See also fig 4, and table 1) 


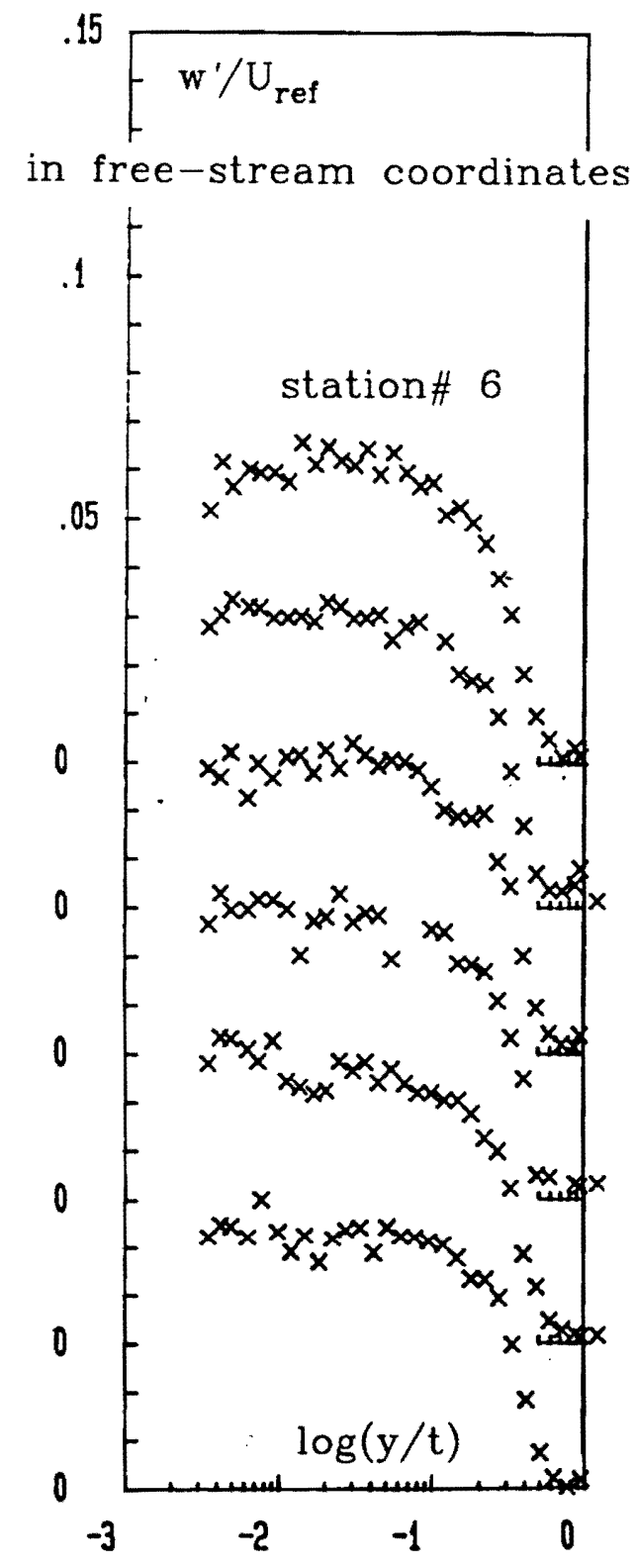

Figure 4lb. w', fluctuating velocity component measured with hot-wire technique on the left-hand side of the wing presented in free-stream coordinates.: In the figure the stations are numbered from top to bottom starting with station 6 . Note the shifted scale of the ordinate. (See also fig 4 , and table 1 ) 


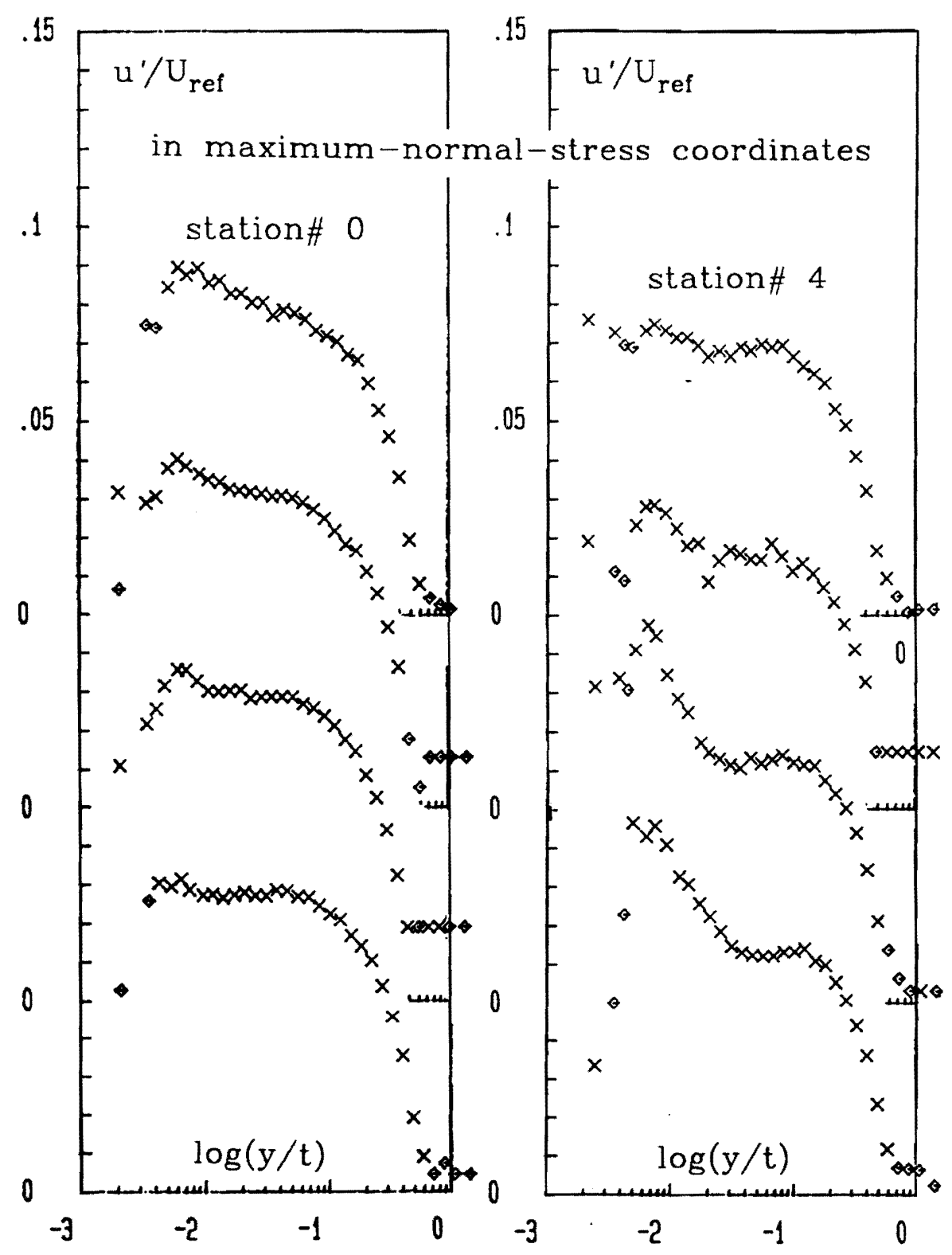

Figure 43. $\mathbf{u}^{\prime}$, fluctuating velocity component measured with LDV technique presented in maximum normal stress coordinates.: In the figures the stations are numbered from top to bottom starting with station 0 and station 4, respectively. Different symbols, very near the wall and near the layer edge show the points where the realizability conditions are not satisfied. ( See also fig 4 , and table 3 ) 


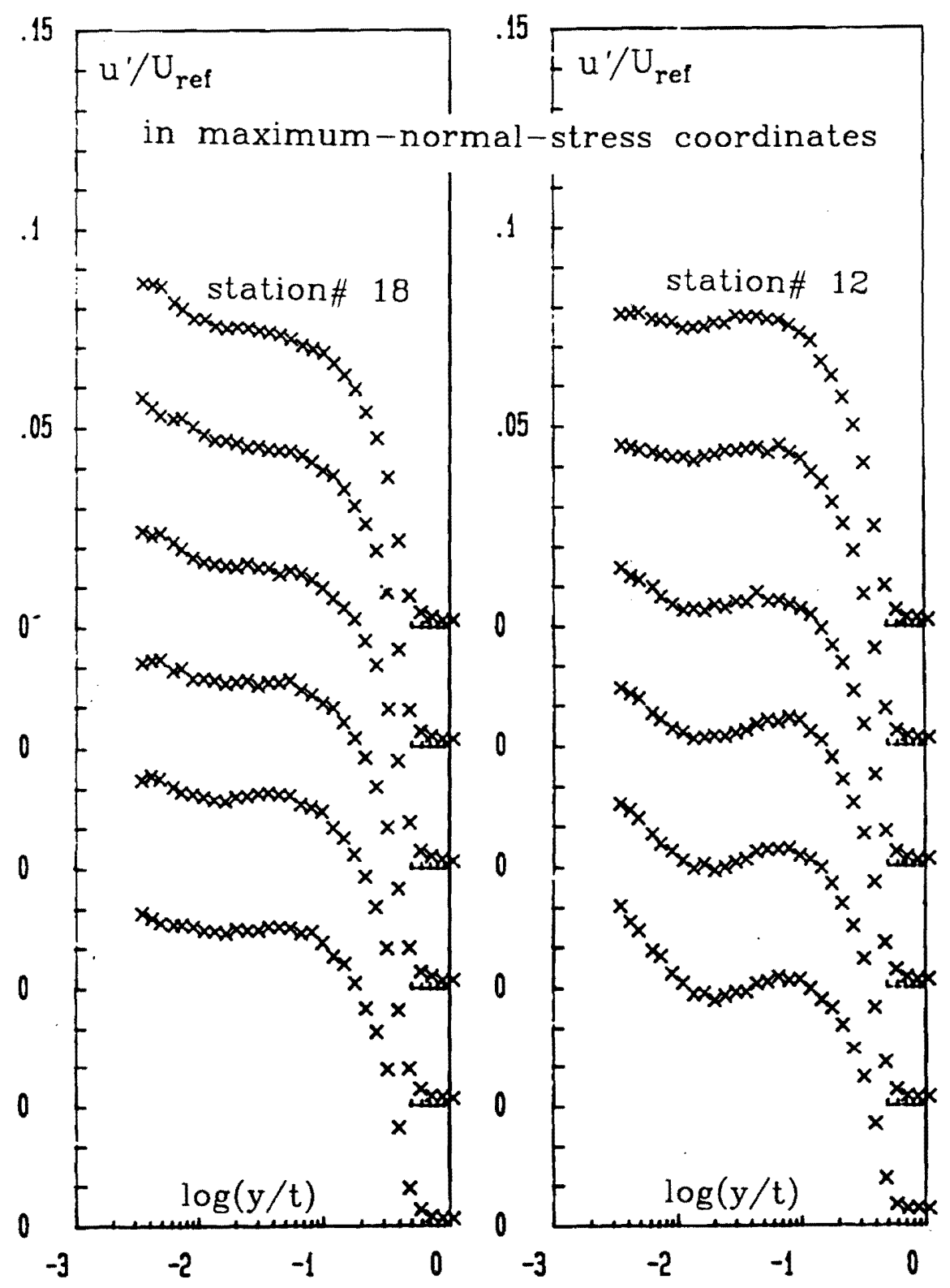

Figure 44a. $u^{\prime}$, fluctuating velocity component measured with hot-wire technique on the left-hand side of the wing presented in normal stress coordinates.: In the figures the stations are numbered from top to bottom starting with station 18 and station 12, respectively. Note the shifted scale of the ordinate. (See also fig 4, and table 1 ) 


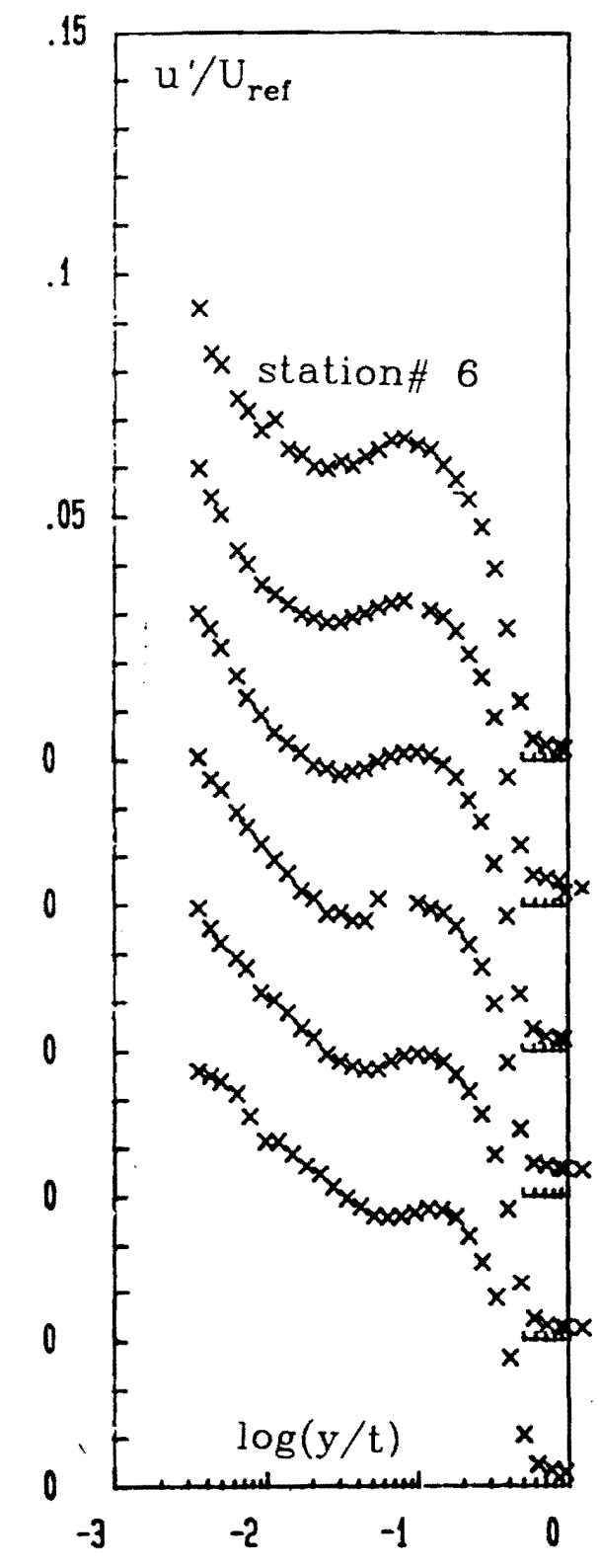

in maximum-normal-stress coordinates

Figure $44 \mathrm{~b}$. $u^{\prime}$, fluctuating velocity component measured with hot-wire technique on the left-hand side of the wing presented in normal stress coordinates.: In the figure the stations are numbered from top to bottom starting with station 6 . Note the shifted scale of the ordinate. (See also fig 4 , and table 1 ) 


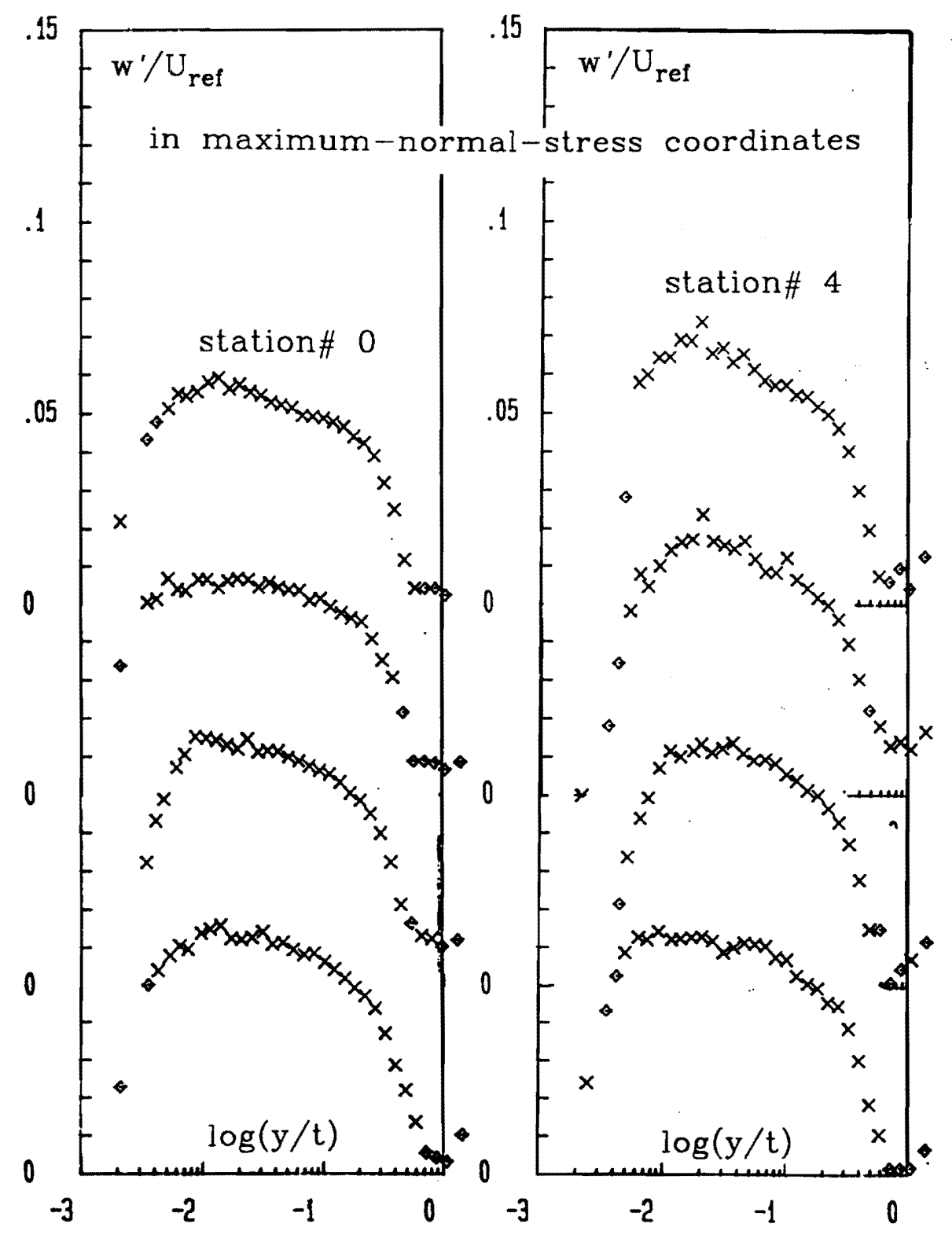

Figure 45. w' fluctuating velocity component measured with LDV technique presented in maximum normal stress coordinates.: In the figures the stations are numbered from top to bottom starting with station 0 and station 4 , respectively. Different symbols, very near the wall and near the layer edge show the points where the realizability conditions are not satisfied. (See also fig 4 , and table 3 ) 


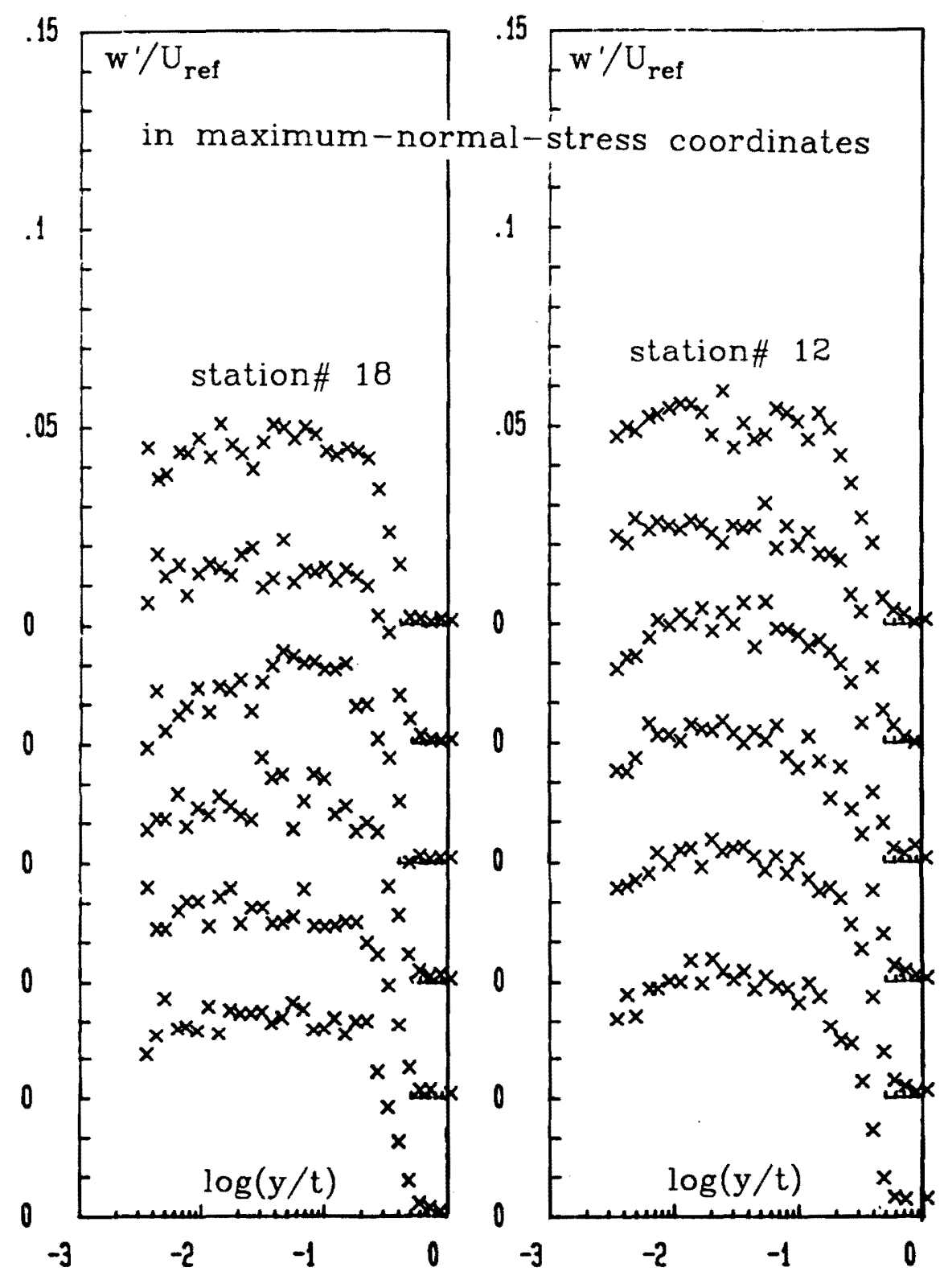

Figure 46a. w', fluctuating velocity component measured with hot-wire technique on the left-hand side of the wing presented in normal stress coordinates.: In the figures the stations are numbered from top to bottom starting with station 18 and station 12, respectively. Note the shifted scale of the ordinate. ( See also fig 4, and table 1) 


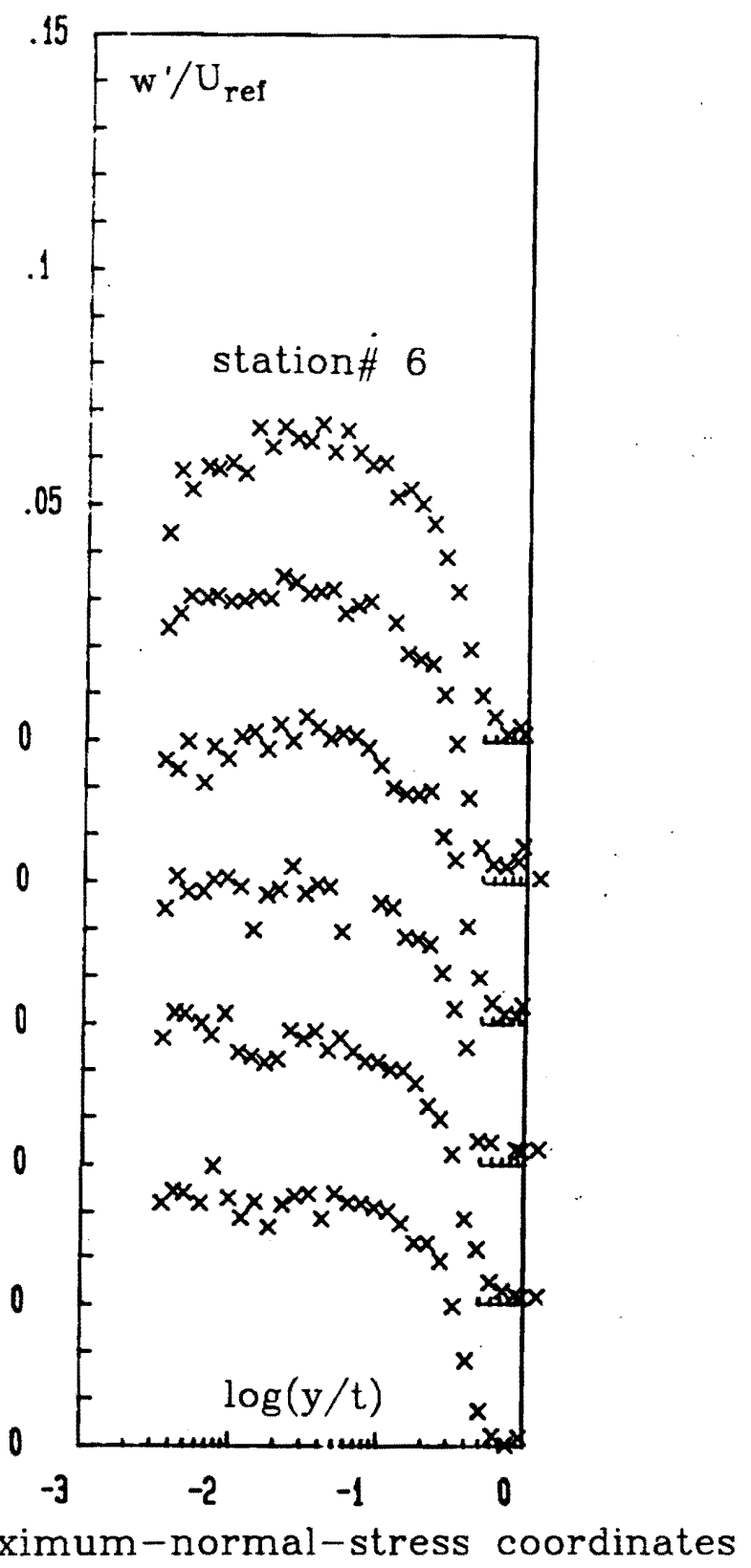

Figure 46b. w', fluctuating velocity component measured with hot-wire technique on the left-hand side of the wing presented in normal stress coordinates.: In the figure the stations are numbered from top to bottom starting with station 6 . Note the shifted scale of the ordinate. (See also fig 4 , and table 1 ) 

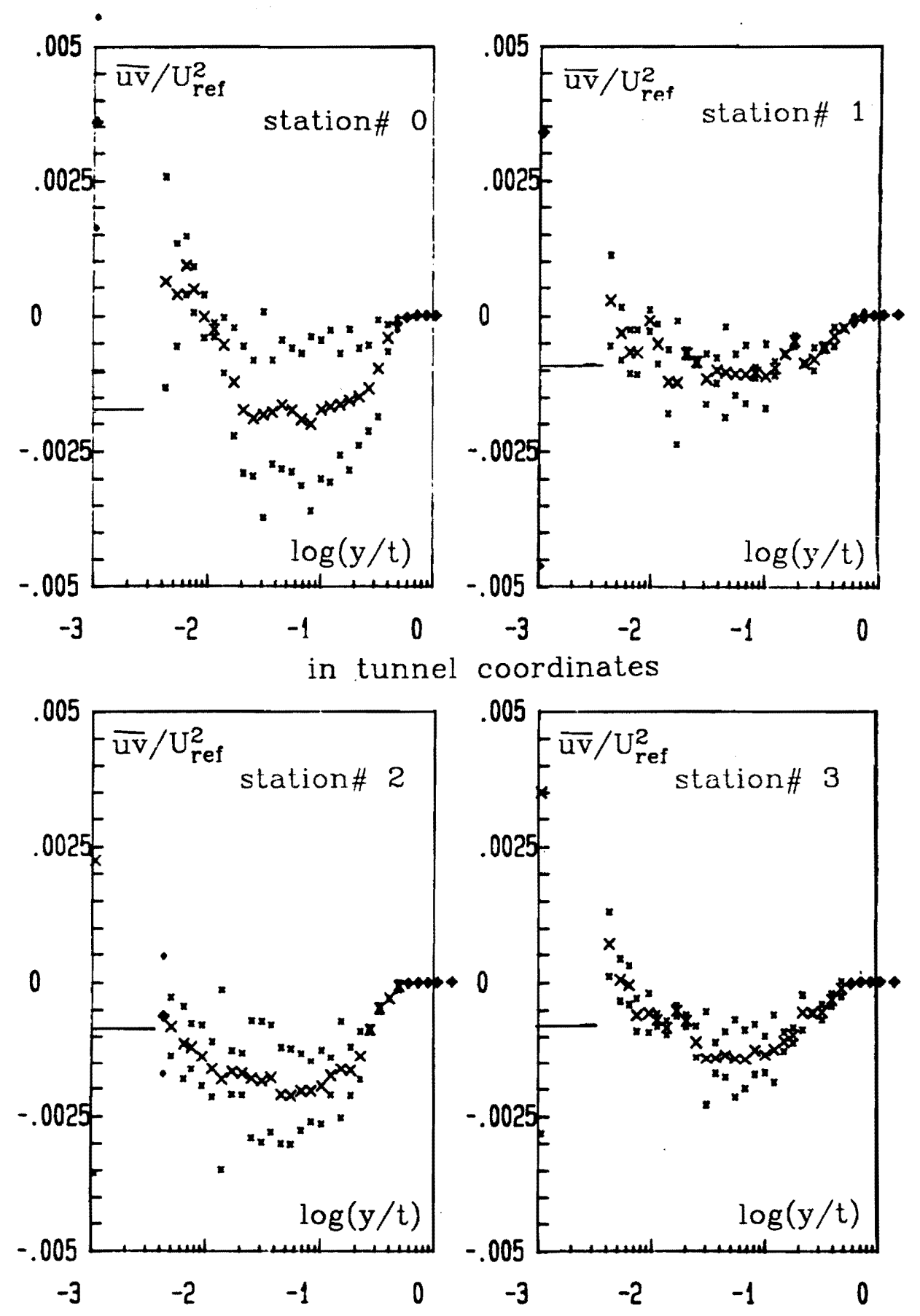

Figure 47a. $\overline{-u v}$, shear stress component measured with LDV technique presented in tunnel coordinates.: The station numbers are shown in the figures. Large symbols denote the data, small symbols denote the uncertainty band. Lines on the ordinate show the component of the shear stress on the wall. Different symbols, very near the wall and near the layer edge show the points where the realizability conditions are not satisfied. (See also fig 4, and table 3 ) 

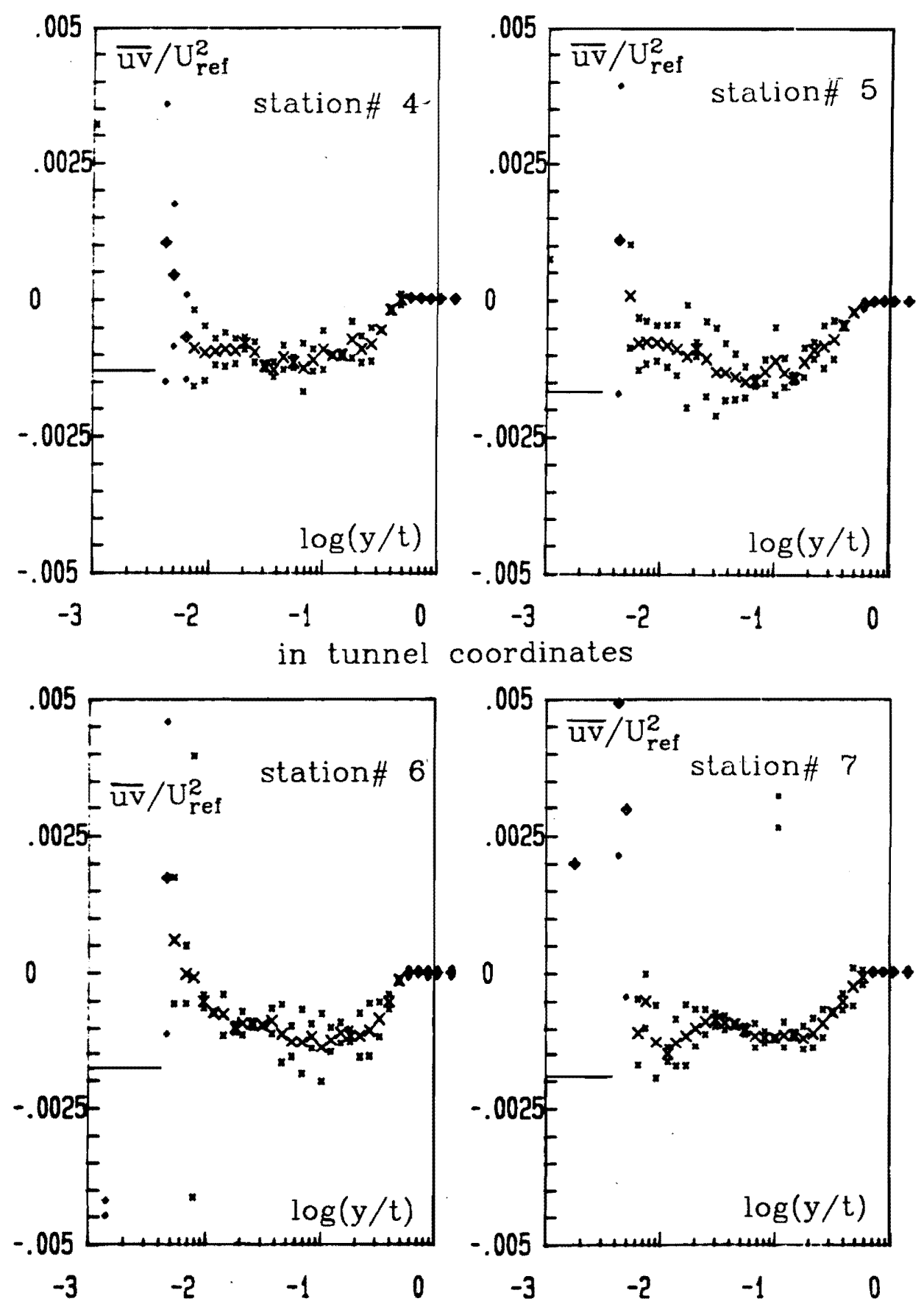

Figure 47b. -uv, shear stress component measured with LDV technique presented in tunnel coordinates.: The station numbers are shown in the figures. Large symbols denote the data, small symbols denote the uncertainty band. Lines on the ordinate show the component of the shear stress on the wall. Different symbols, very near the wall and near the layer edge show the points where the realizability conditions are not satisfied. (See also fig 4 , and table 3 ) 

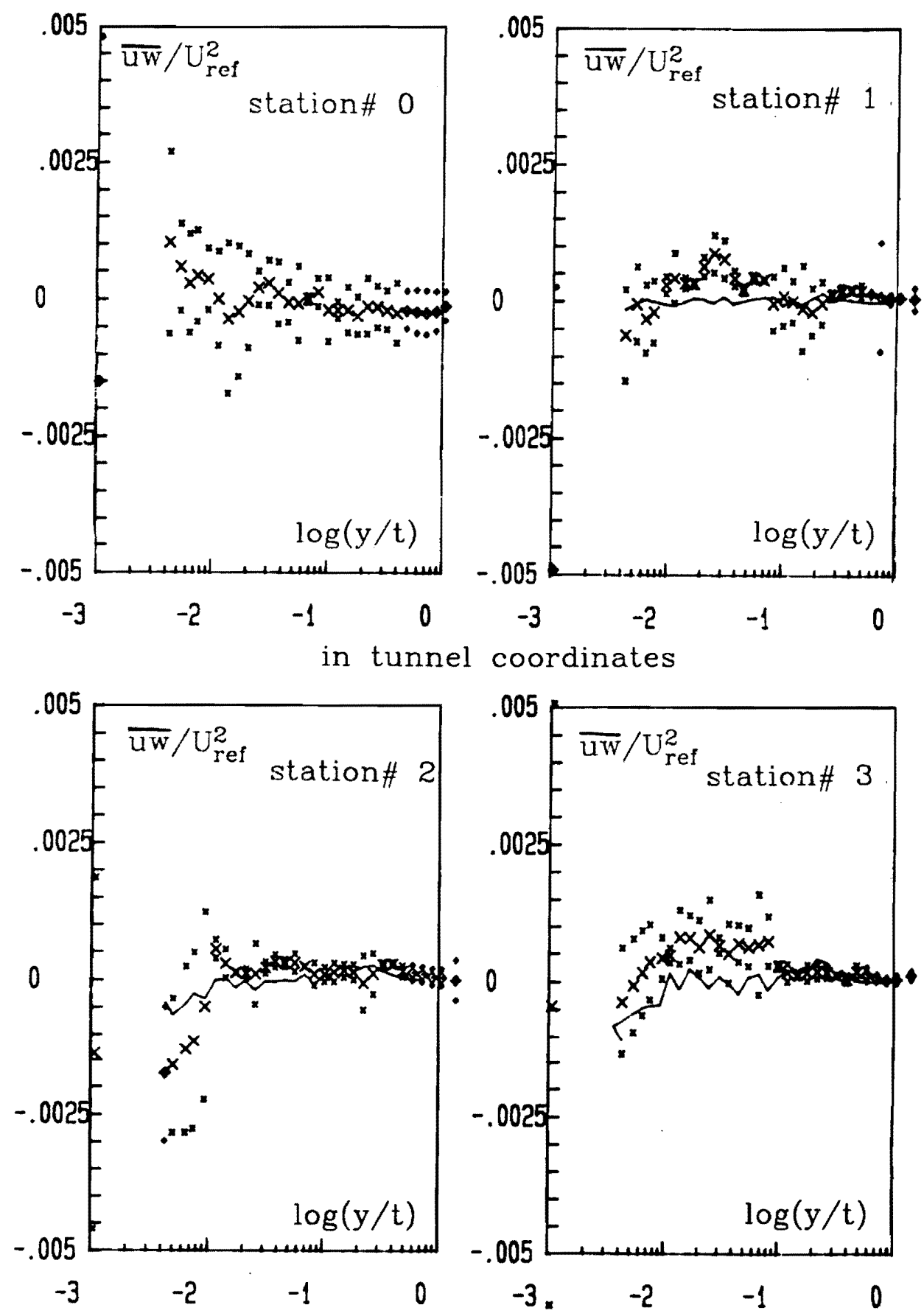

Figure 48a. $-\overline{\mathrm{uw}}$, shear stress component measured with LDV technique presented in tunnel coordinates.: The station numbers are shown in the figures. Large symbols denote the data, small symbols denote the uncertainty band. Lines are used to show the hot-wire profiles taken at the same locations. Different symbols, very near the wall and near the layer edge show the points where the realizability conditions are not satisfied. ( See also fig 4 , and table 3 ) 

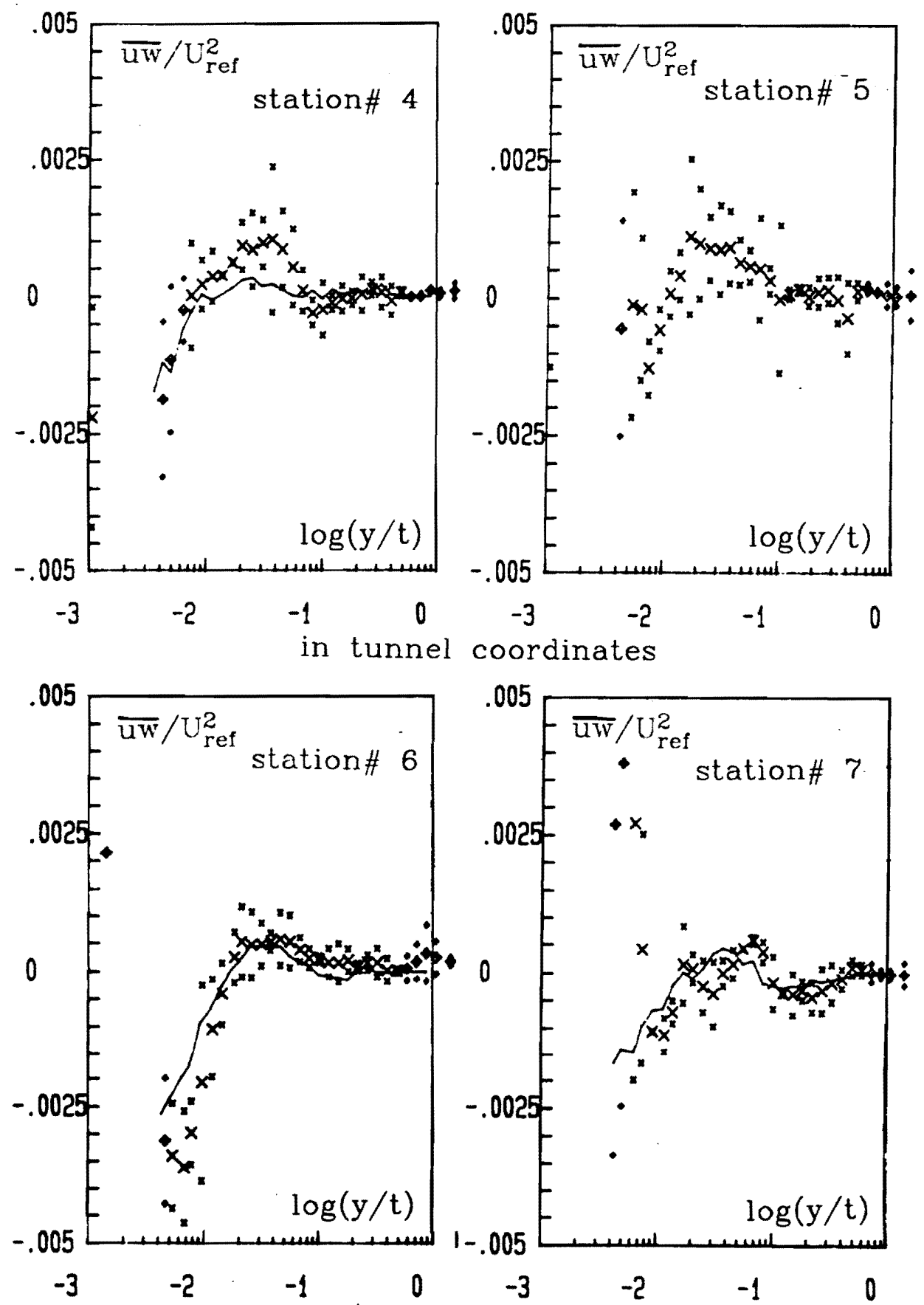

Figure 48b. $-\overline{\mathrm{uw}}$, shear stress component measured with LDV technique presented in tunnel coordinates.: The station numbers are shown in the figures. Large symbols denote the data, small symbols denote the uncertainty band. Lines are used to show the hot-wire profiles taken at the same locations. Different symbols, very near the wall and near the layer edge show the points where the realizability conditions are not satisfied. (See also fig 4 , and table 3 ) 


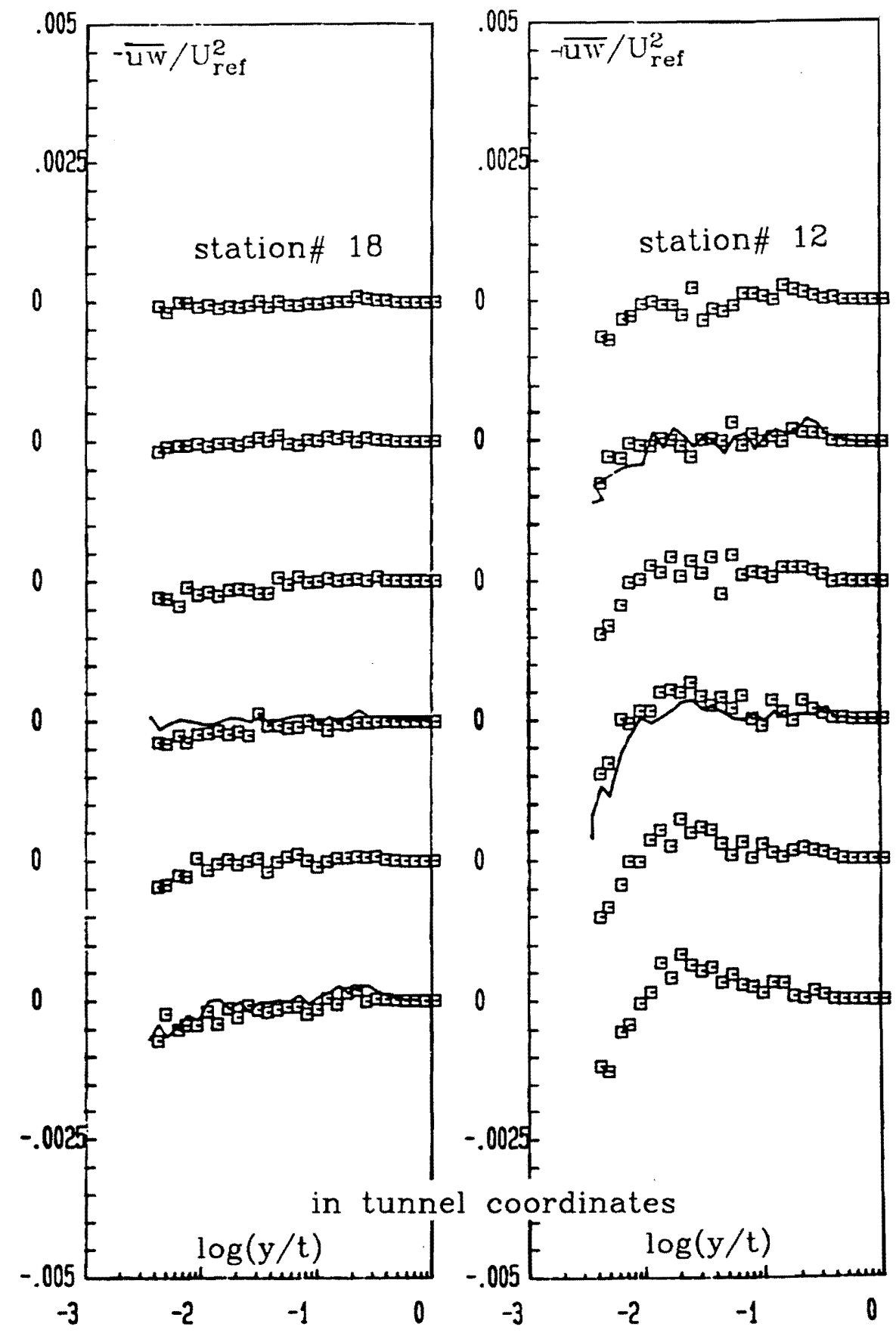

Figure 49a. $-\overline{\mathrm{uw}}$, shear stress component measured with hot-wire technique on the left-hand side of the wing presented in tunnel coordinates.: In the figures the stations are numbered from top to bottom starting with station 18 and station 12 , respectively. Lines are used to show the hot-wire profiles taken at the symmetric locations. Note the shifted scale of the ordinate and the minus sign of the stresses. ( See also fig 4, and table 1 ) 


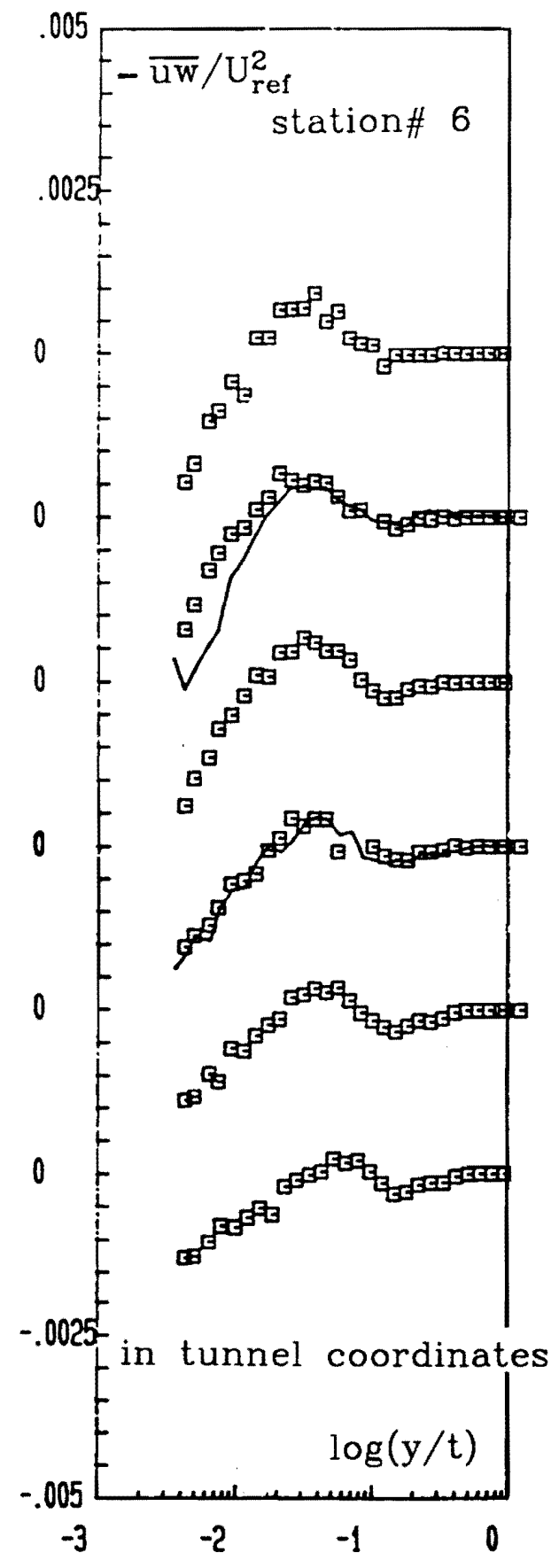

Figure $49 \mathrm{~b} . \quad-\overline{u w}$, shear stress component measured with hot-wire technique on the left-hand side of the wing presented in tunnel coordinates.:

In the figure the stations are numbered from top to bottom starting with station 6 . Lines are used to show the hot-wire profiles taken at the symmetric locations. Note the shifted scale of the ordinate and the minus sign of the stresses. (See also fig 4, and table 1) 

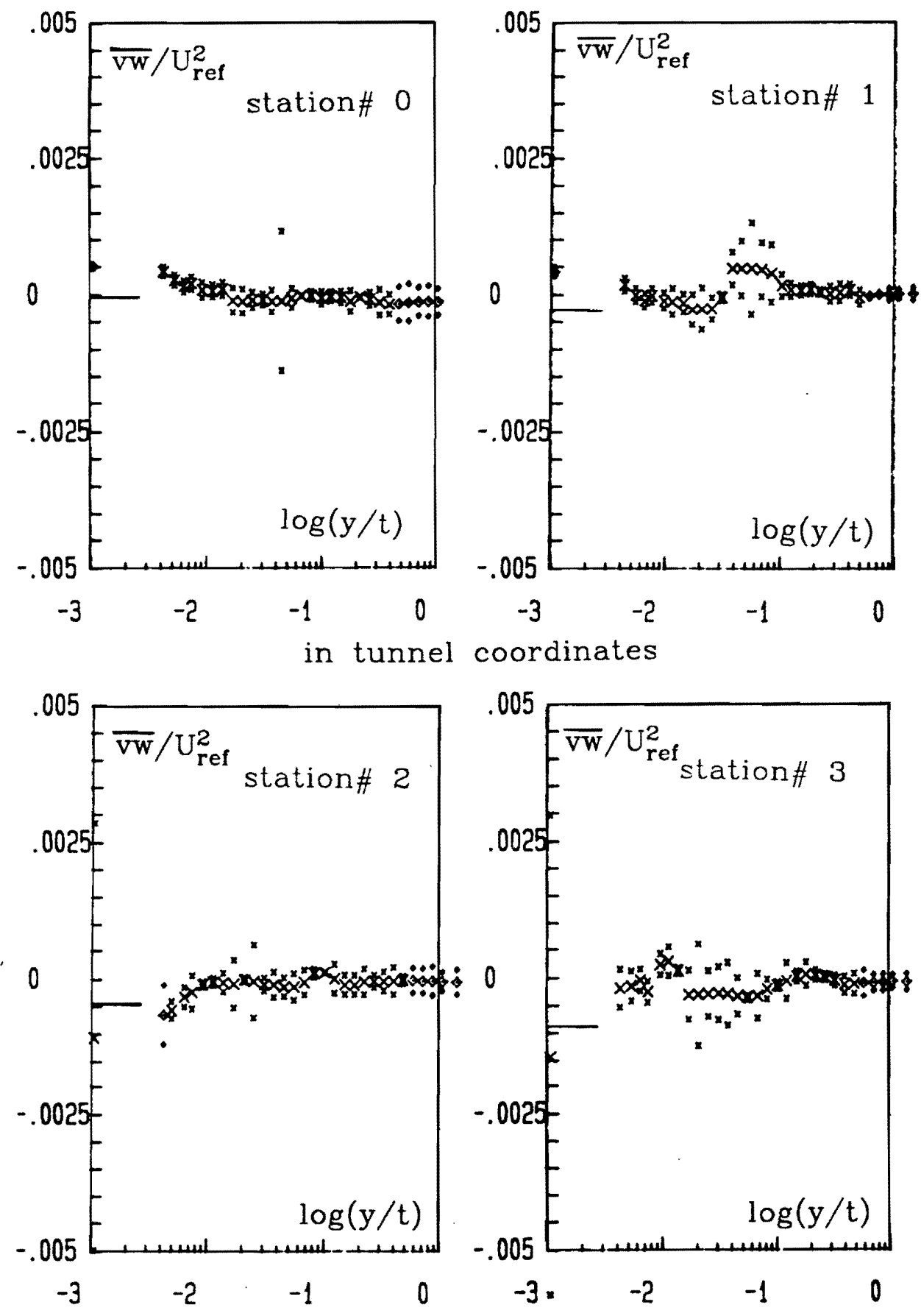

Figure 50a. $-\overline{\mathrm{vw}}$, shear stress component measured with LDV technique presented in tunnel coordinates.: The station numbers are shown in the figures. Large symbols denote the data, small symbols denote the uncertainty band. Lines on the ordinate show the component of the shear stress on the wall. Different symbols, very near the wall and near the layer edge show the points where the realizability conditions are not satisfied. 

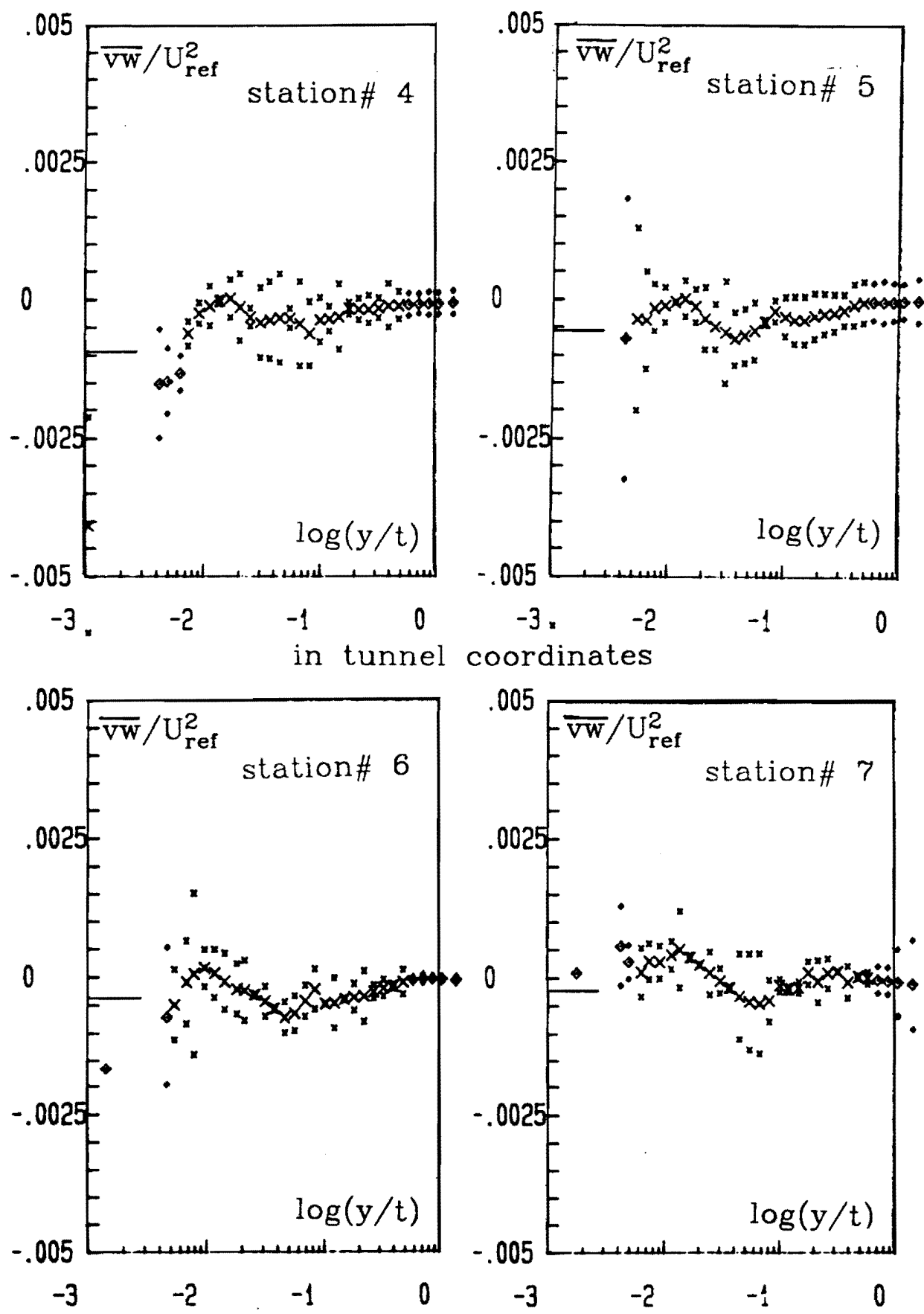

Figure 50b. $-\overline{\mathrm{vw}}$, shear stress component measured with LDV technique presented in tunnel coordinates.: The station numbers are shown in the figures. Large symbols denote the data, small symbols denote the uncertainty band. Lines on the ordinate show the component of the shear stress on the wall. Different symbols, very near the wall and near the layer edge show the points where the realizability conditions are not satisfied. 


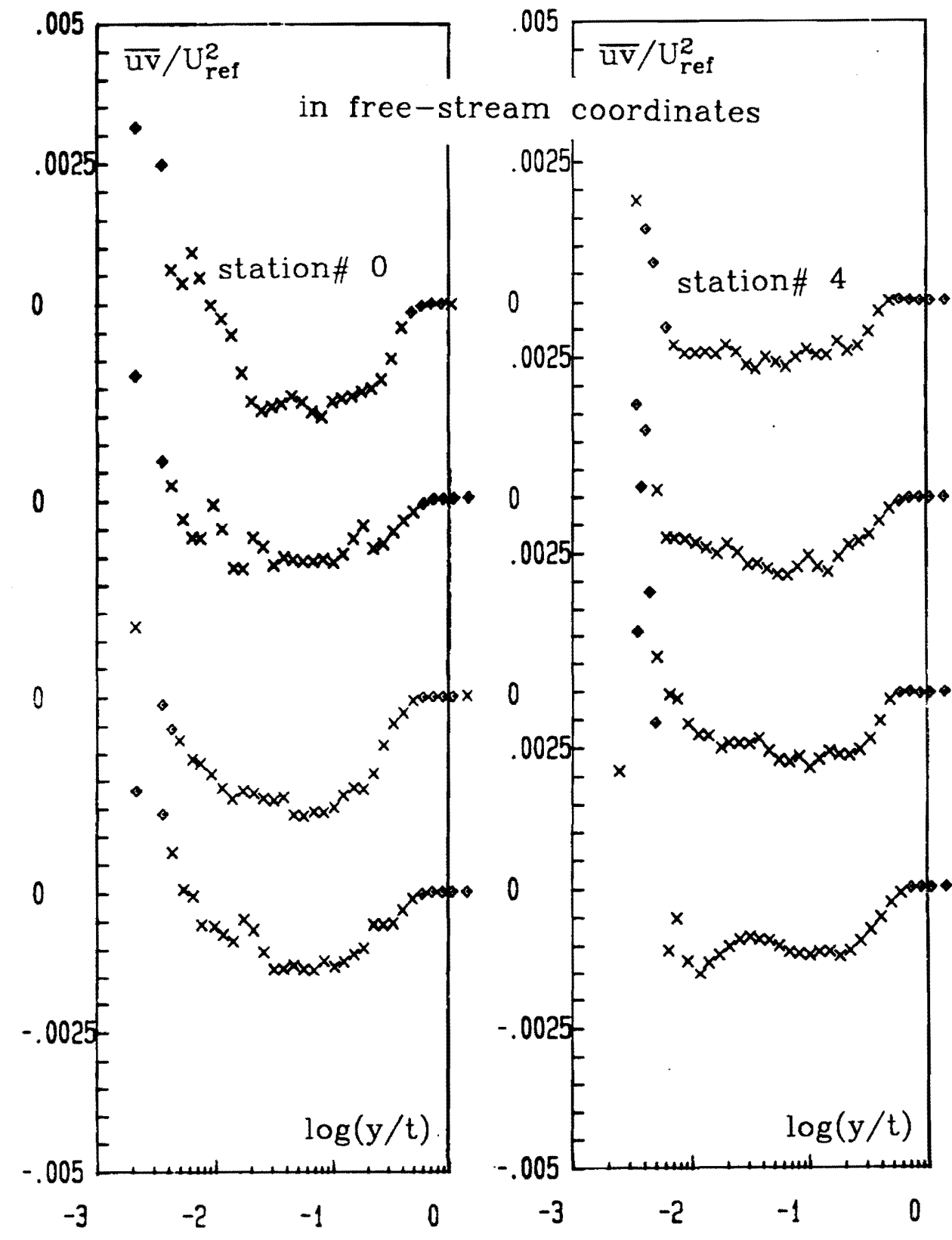

Figure 51. $-\overline{u v}$, shear stress component measured with LDV technique presented in free-stream coordinates.: In the figures the stations are numbered from top to bottom starting with station 0 and station 4 , respectively. Different symbols, very near the wall and near the layer edge show the points where the realizability conditions are not satisfied. (See also fig 4 , and table 3 ) 


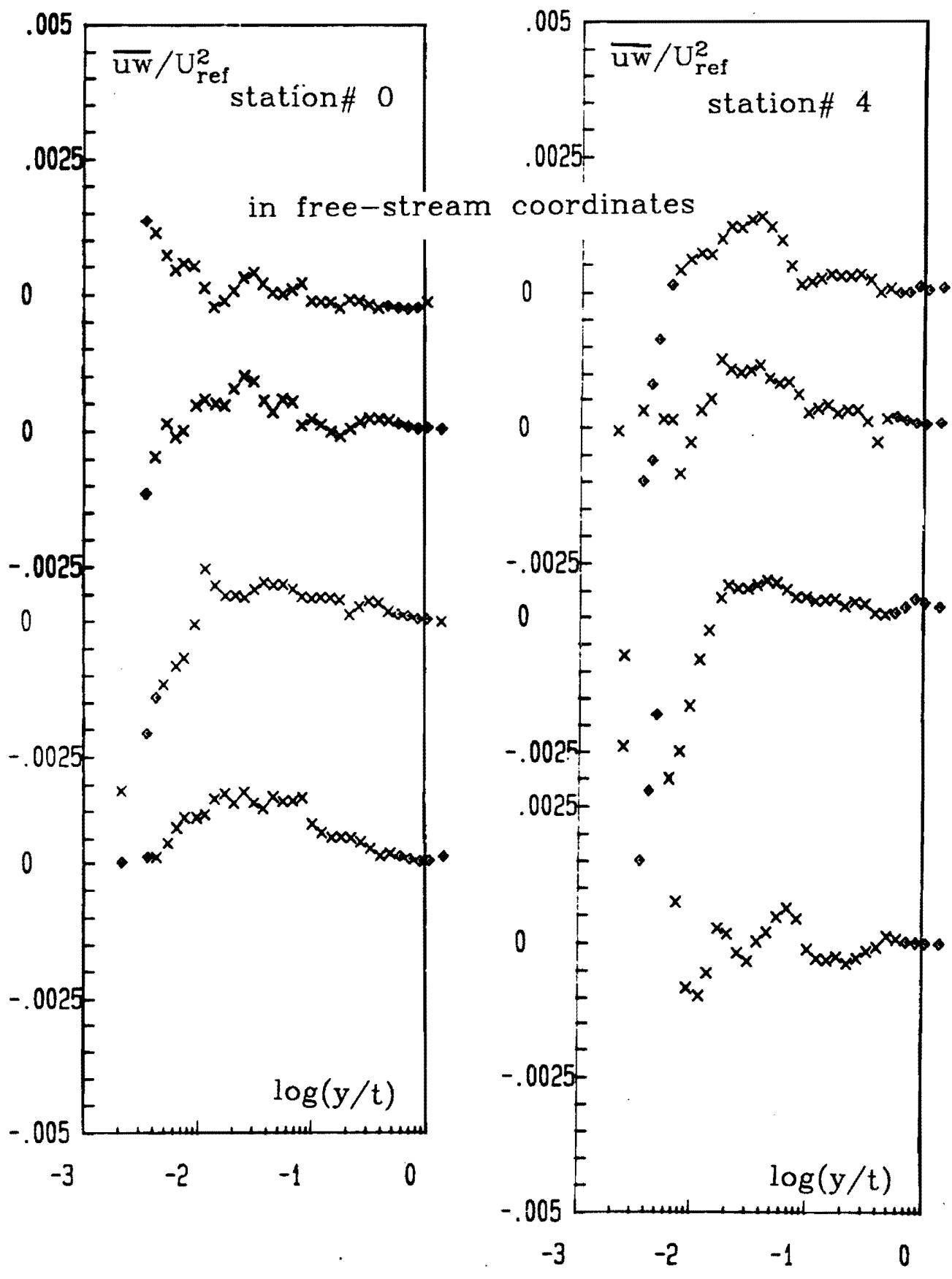

Figure 52. - -uw, shear stress component measured with LDV technique presented in free-stream coordinates.: In the figures the stations are numbered from top to bottom starting with station 0 and station 4 , respectively. Different symbols, very near the wall and near the layer edge show the points where the realizability conditions are not satisfied. (See also fig 4, and table 3 ) 


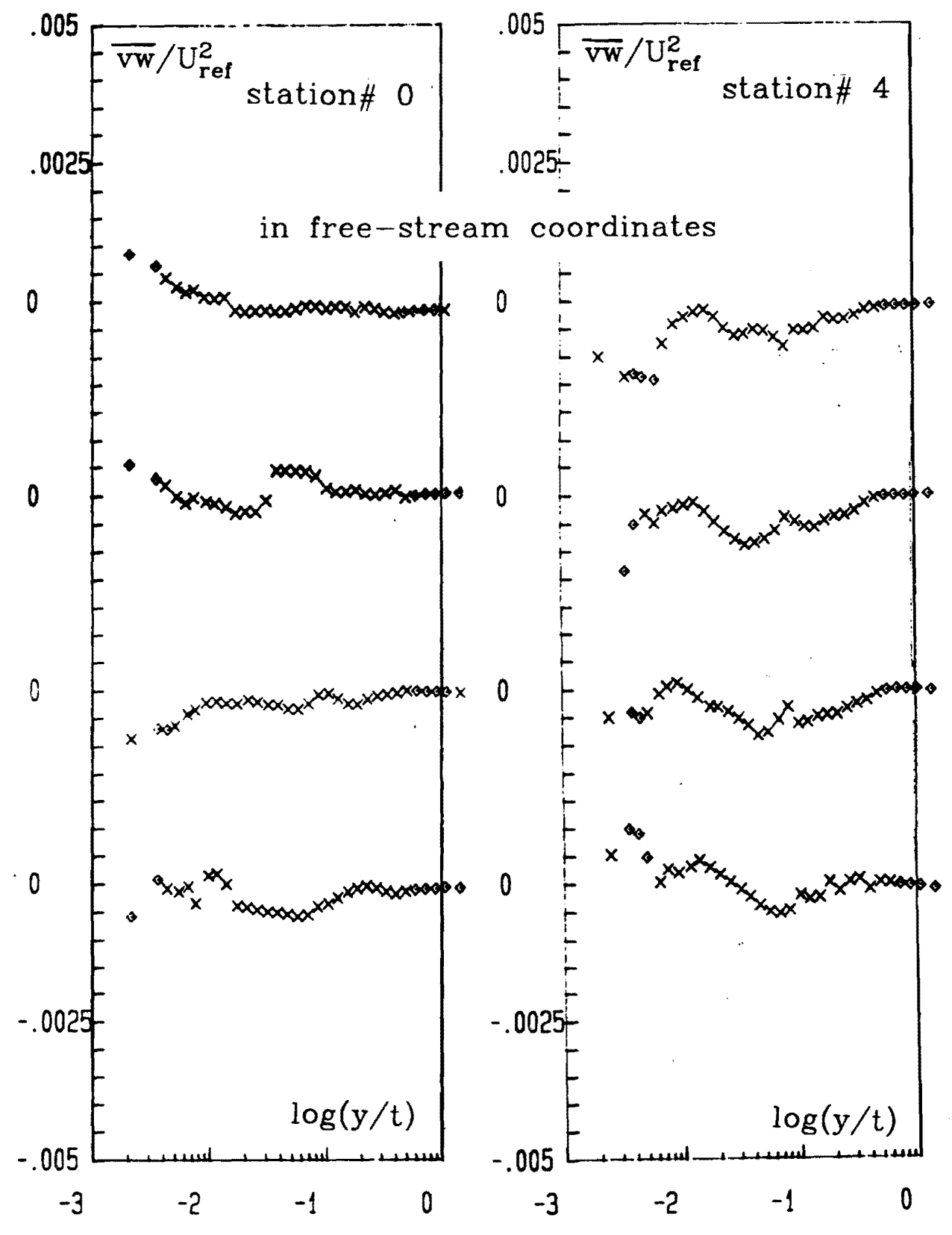

Figure 53. $-\overline{\mathrm{vw}}$, shear stress component measured with LDV technique presented in free-stream coordinates.: In the figures the stations are numbered from top to bottom starting with station 0 and station 4 , respectively. Different symbols, very near the wall and near the layer edge show the points where the realizability conditions are not satisfied. 


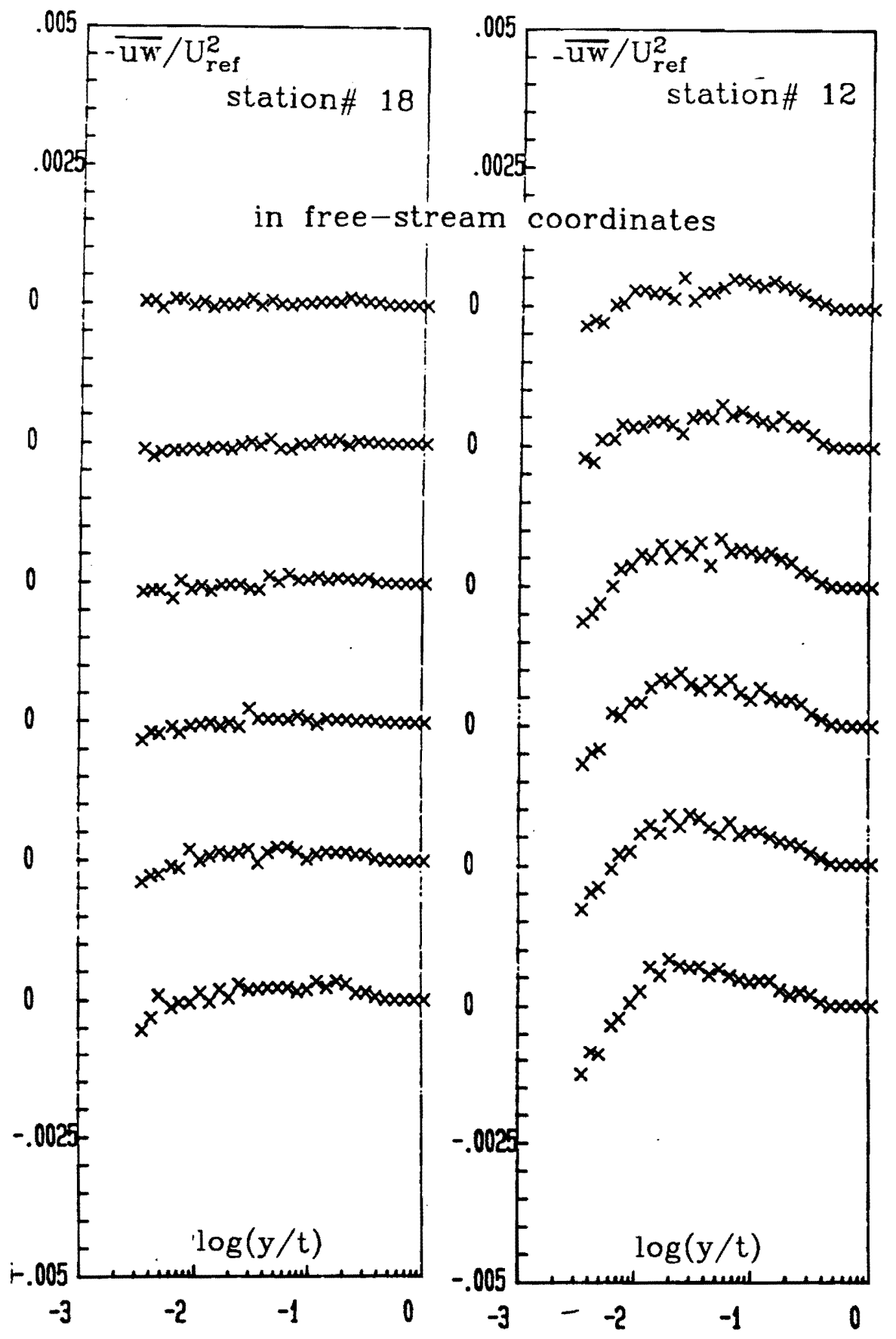

Figure 54a. $-\overline{\mathrm{uw}}$, shear stress component measured with hot-wire technique on the left-hand side of the wing presented in free-stream coordinates.: In the figures the stations are numbered from top to bottom starting with station 18 and station 12 , respectively. Note the shifted scale of the ordinate and the minus sign of the stresses. ( See also fig 4 , and table 1 ) 


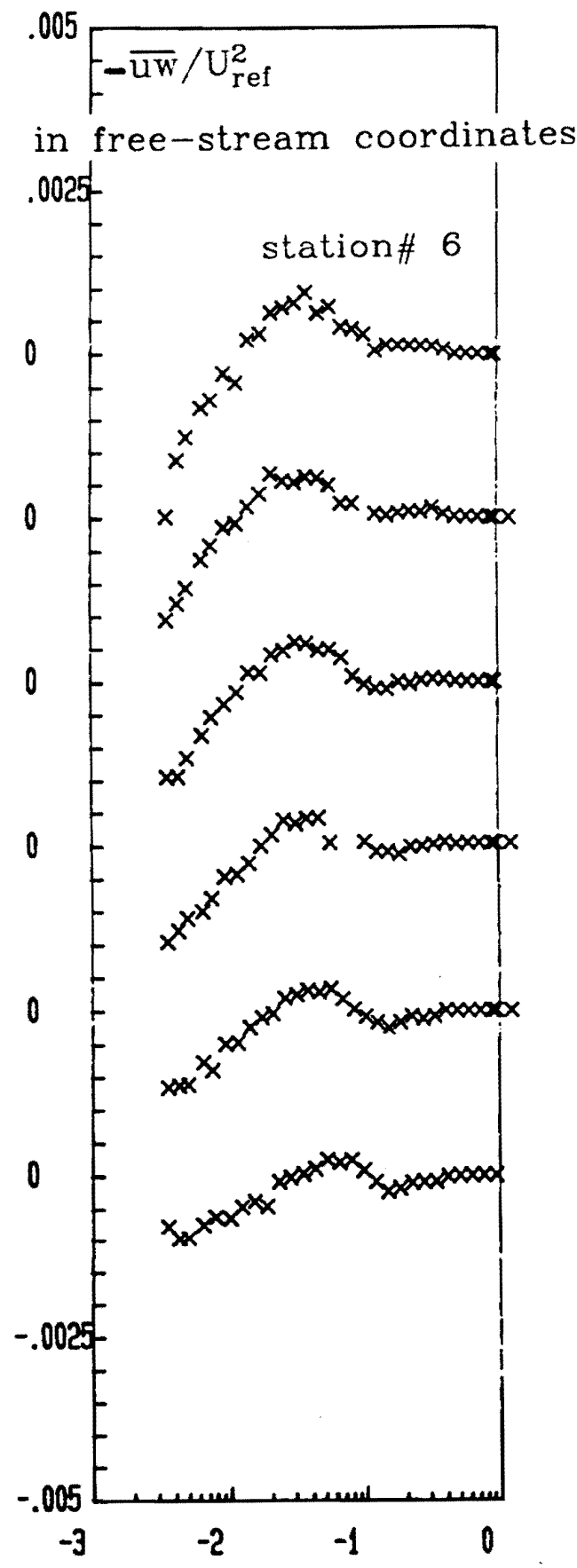

Figure 54b. $-\overline{u w}$, shear stress component measured with hot-wire technique on the left-hand side of the wing presented in free-stream coordinates.: In the figures the stations are numbered from top to bottom starting with station 6 . Note the shifted scale of the ordinate and the minus sign of the stresses. ( See also fig 4, and table 1) 


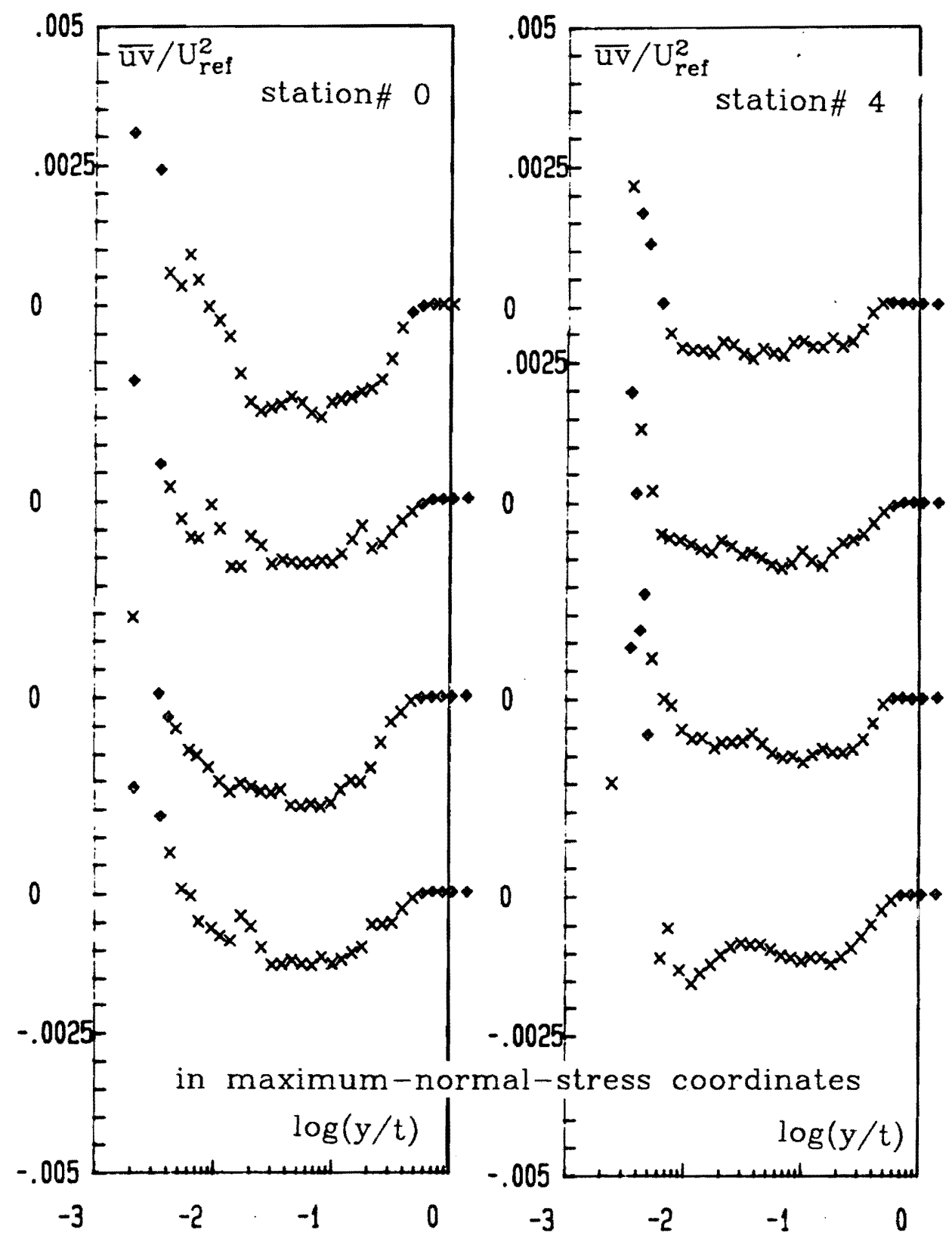

Figure 55. - $\overline{\mathrm{uv}}$, shear stress component measured with LDV technique presented in maximum normal stress coordinates.: In the figures the stations are numbered from top to bottom starting with station 0 and station 4 , respectively. Different symbols, very near the wall and near the layer edge show the points where the realizability conditions are not satisfied. (See also fig 4 , and table 3 ) 


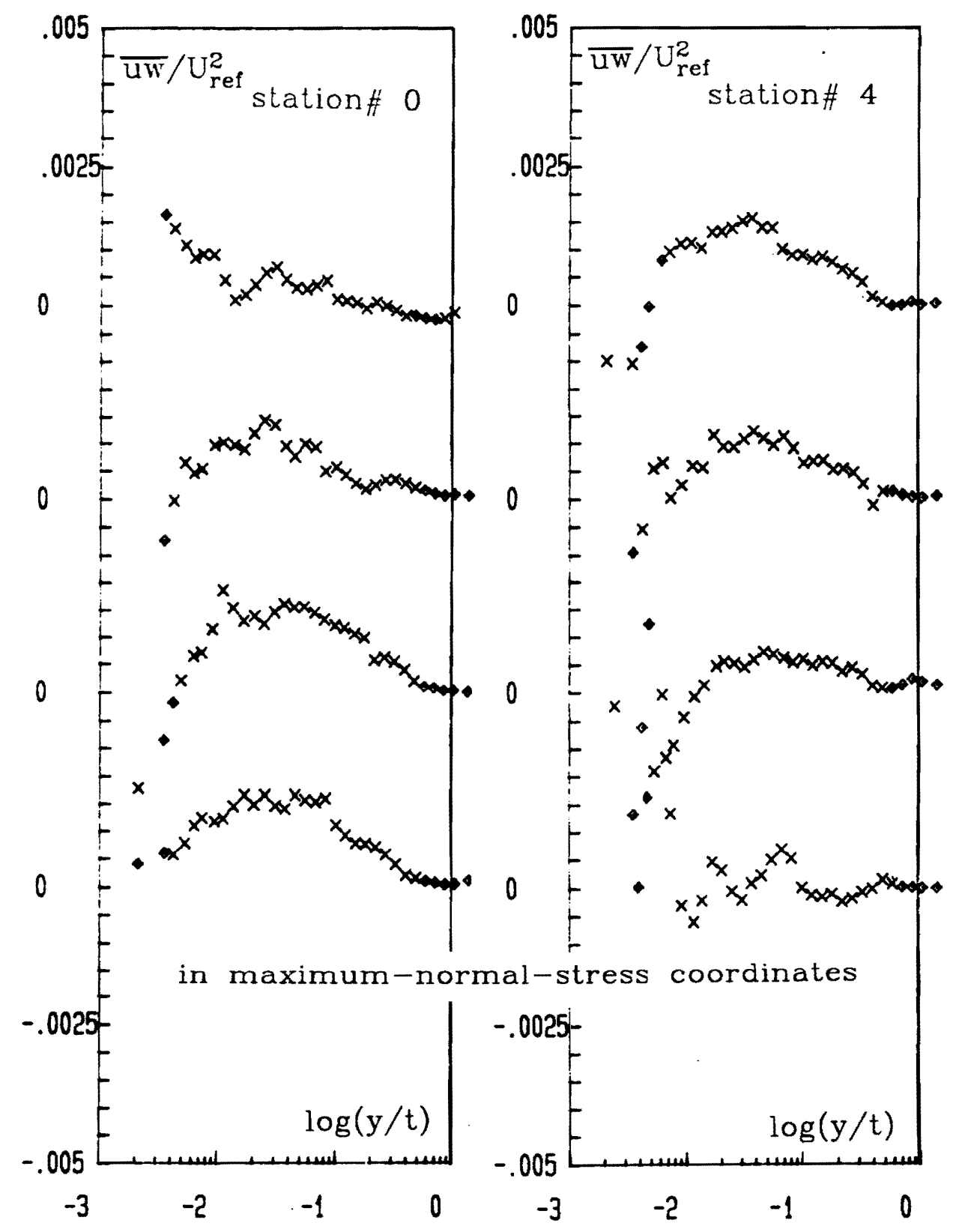

Figure 56. $-\overline{\mathrm{uw}}$, shear stress component measured with LDV technique presented in maximum normal stress coordinates.: In the figures the stations are numbered from top to bottom starting with station 0 and station 4 , respectively. Different symbols, very near the wall and near the layer edge show the points where the realizability conditions are not satisfied. ( See also fig 4 , and table 3 ) 


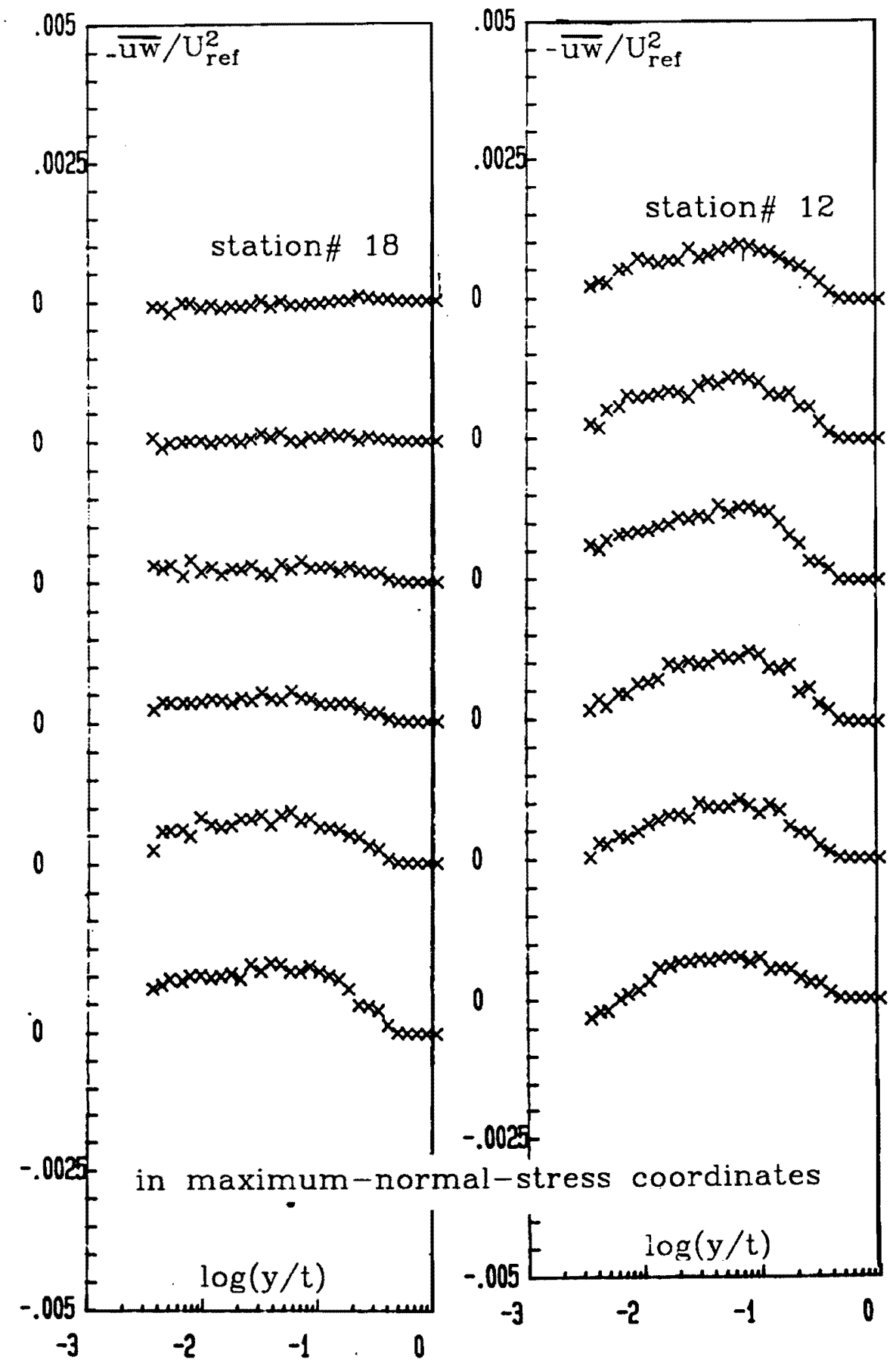

Figure 57. $-\overline{\mathrm{uw}}$, shear stress component measured with hot-wire technique on the left-hand side of the wing presented in normal stress coordinates.: In the figures the stations are numbered from top to bottom starting with station 18 and station 12 , respectively. Note the shifted scale of the ordinate and the minus sign of the stresses. ( See also fig 4 , and table 1 ) 


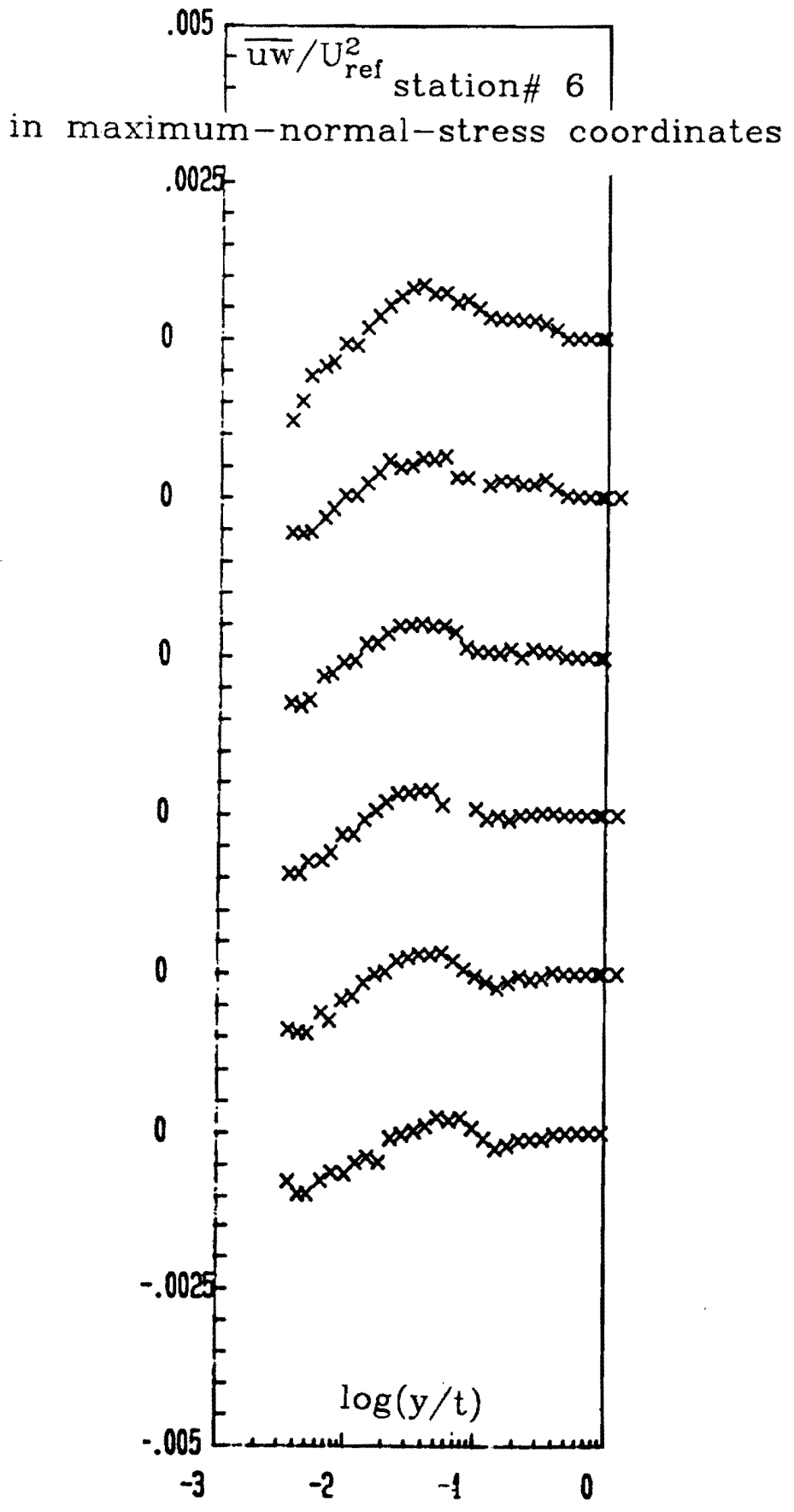

Figure 57b. $-\overline{u w}$, shear stress component measured with hot-wire technique on the left-hand side of the wing presented in normal stress coordinates.: In the figures the stations are numbered from top to bottom starting with station 6 . Note the shifted scale of the ordinate and the minus sign of the stresses. ( See also fig 4, and table 1) 


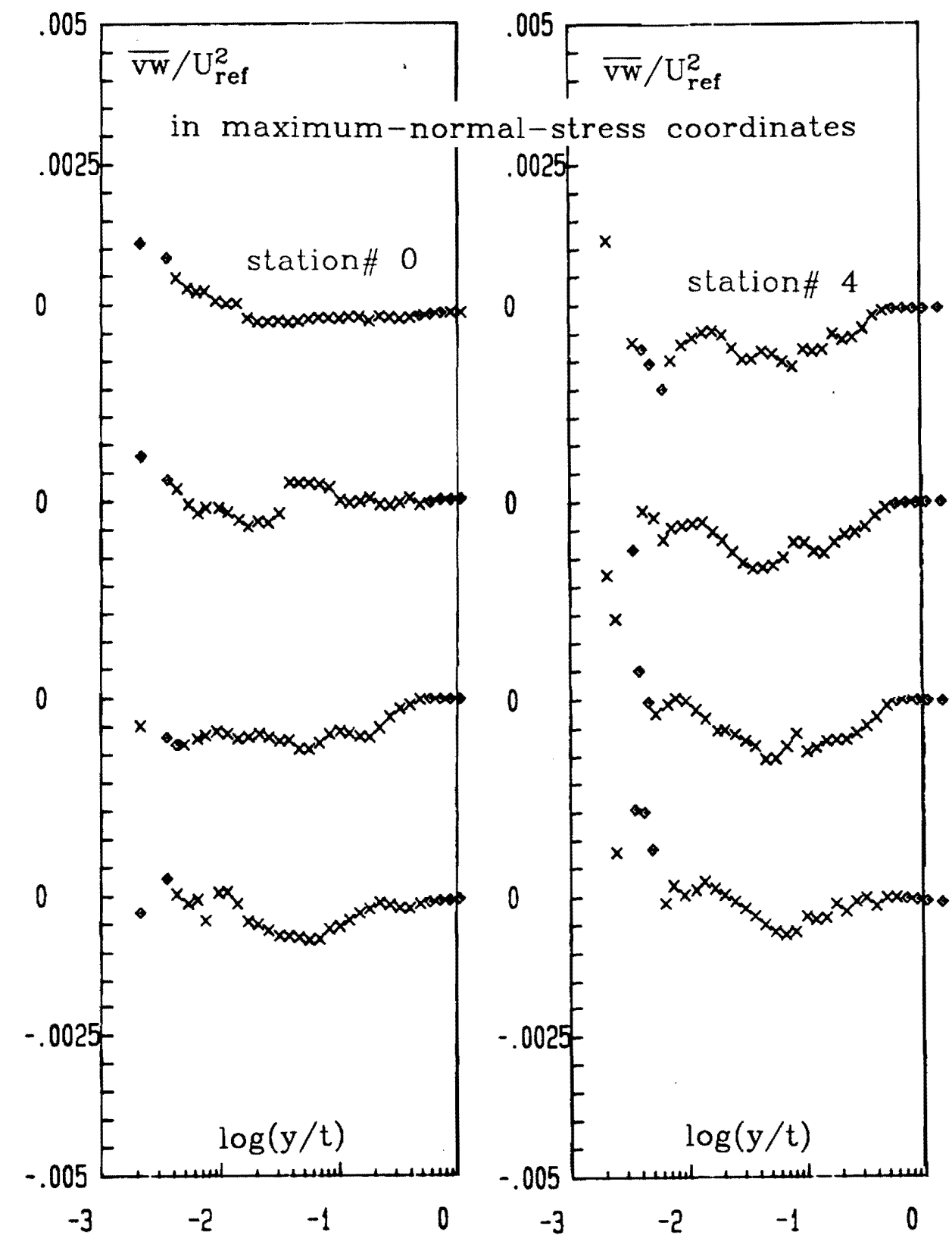

Figure 58. $-\overline{\mathrm{vw}}$, shear stress component measured with LDV technique presented in maximum normal stress coordinates.: In the figures the stations are numbered from top to bottom starting with station 0 and station 4 , respectively. Different symbols, very near the wall and near the layer edge show the points where the realizability conditions are not satisfied. 


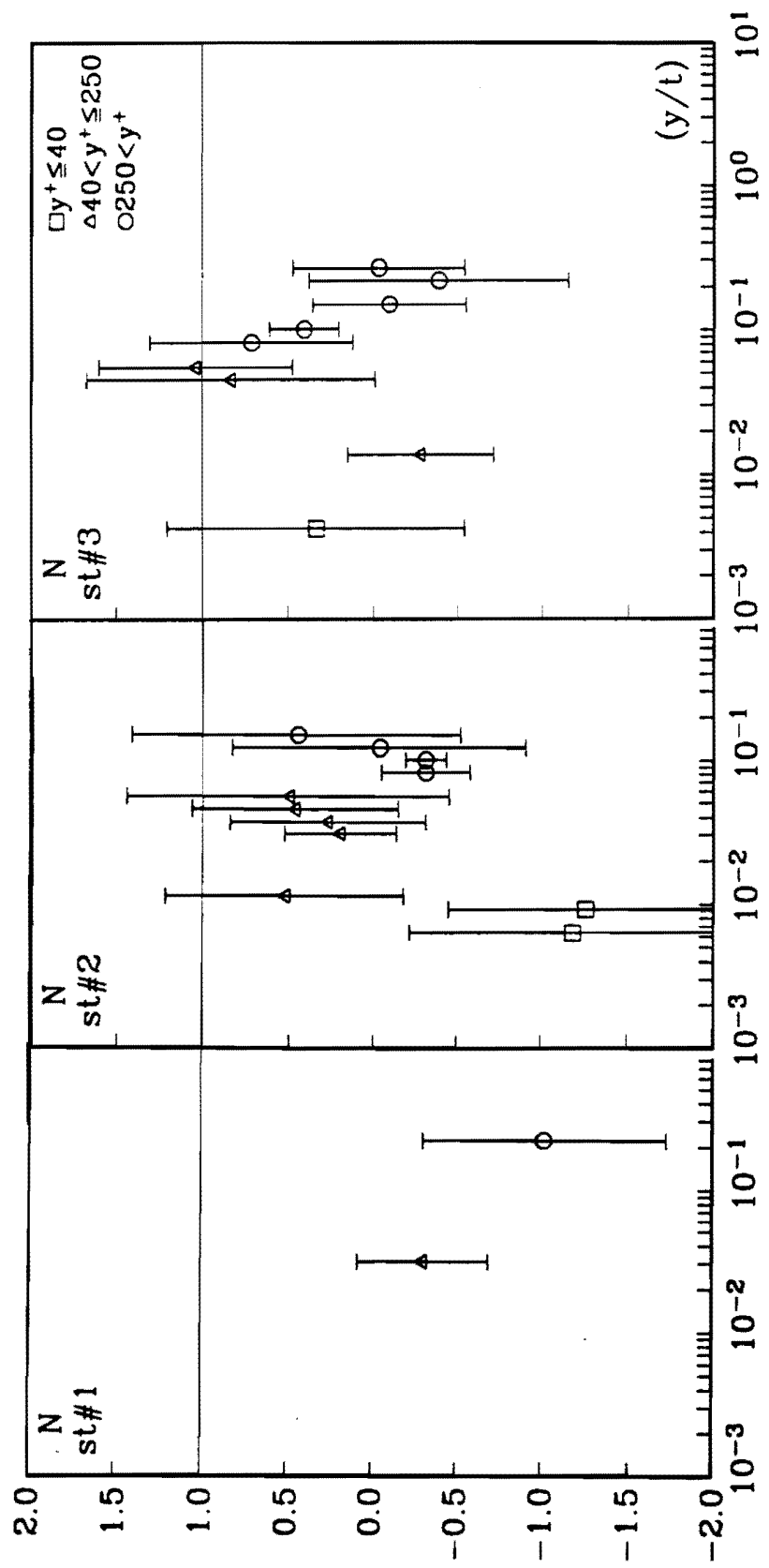

Figure 59a. N, anisotropy constant computed using LDV data in tunnel coordinates.: Stations numbers are shown in the figures. Bars denote the uncertainty bands. 


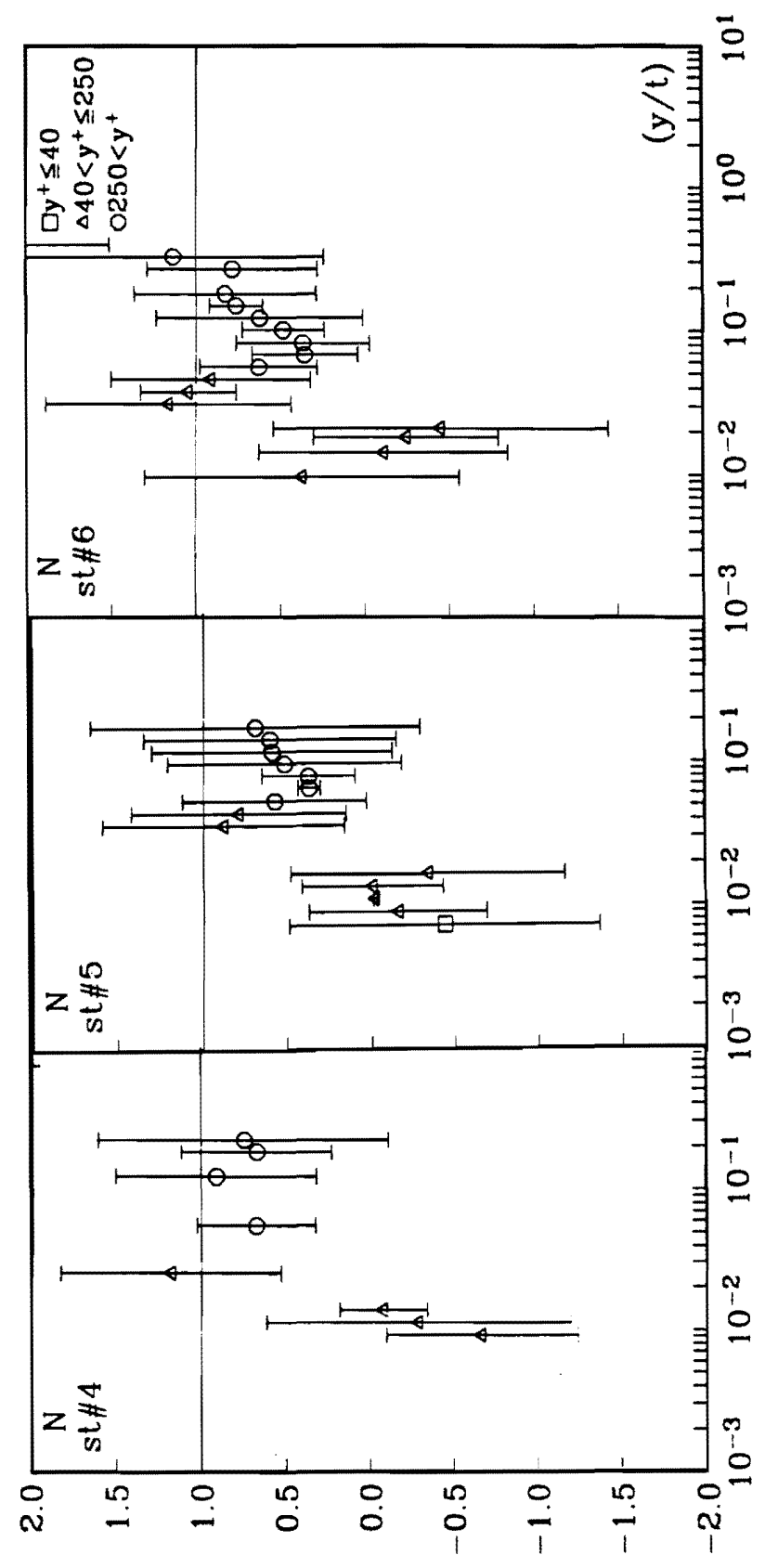

Figure 59b. $\mathrm{N}$, anisotropy constant computed using LDV data in tunnel coordinates.: Stations numbers are shown in the figures. Bars denote the uncertainty bands. 


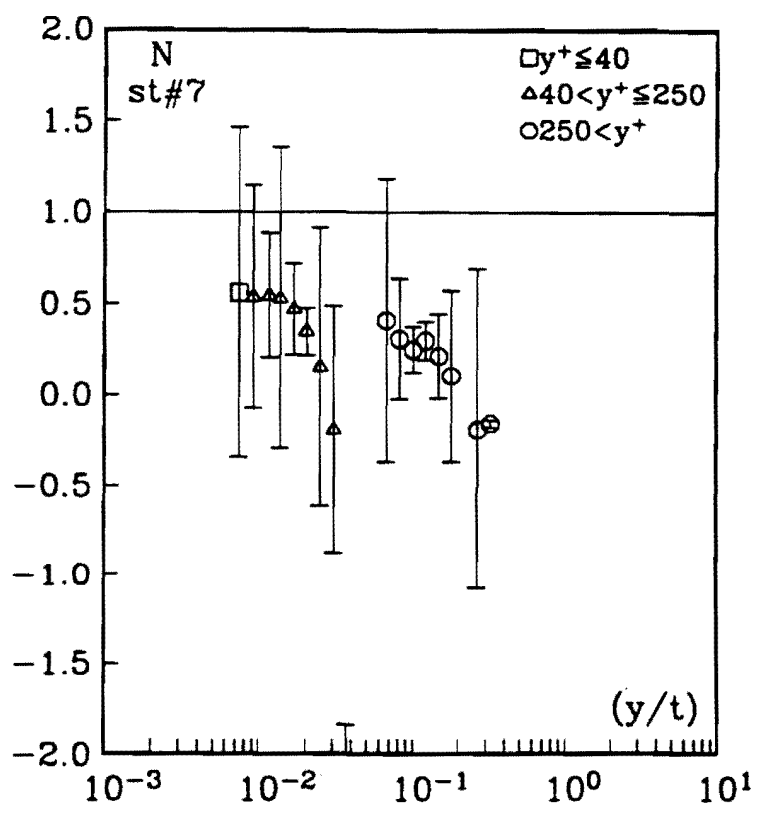

Figure 59c. N, anisotropy constant computed using LDV data in tunnel coordinates.: Stations numbers are shown in the figures. Bars denote the uncertainty bands. 


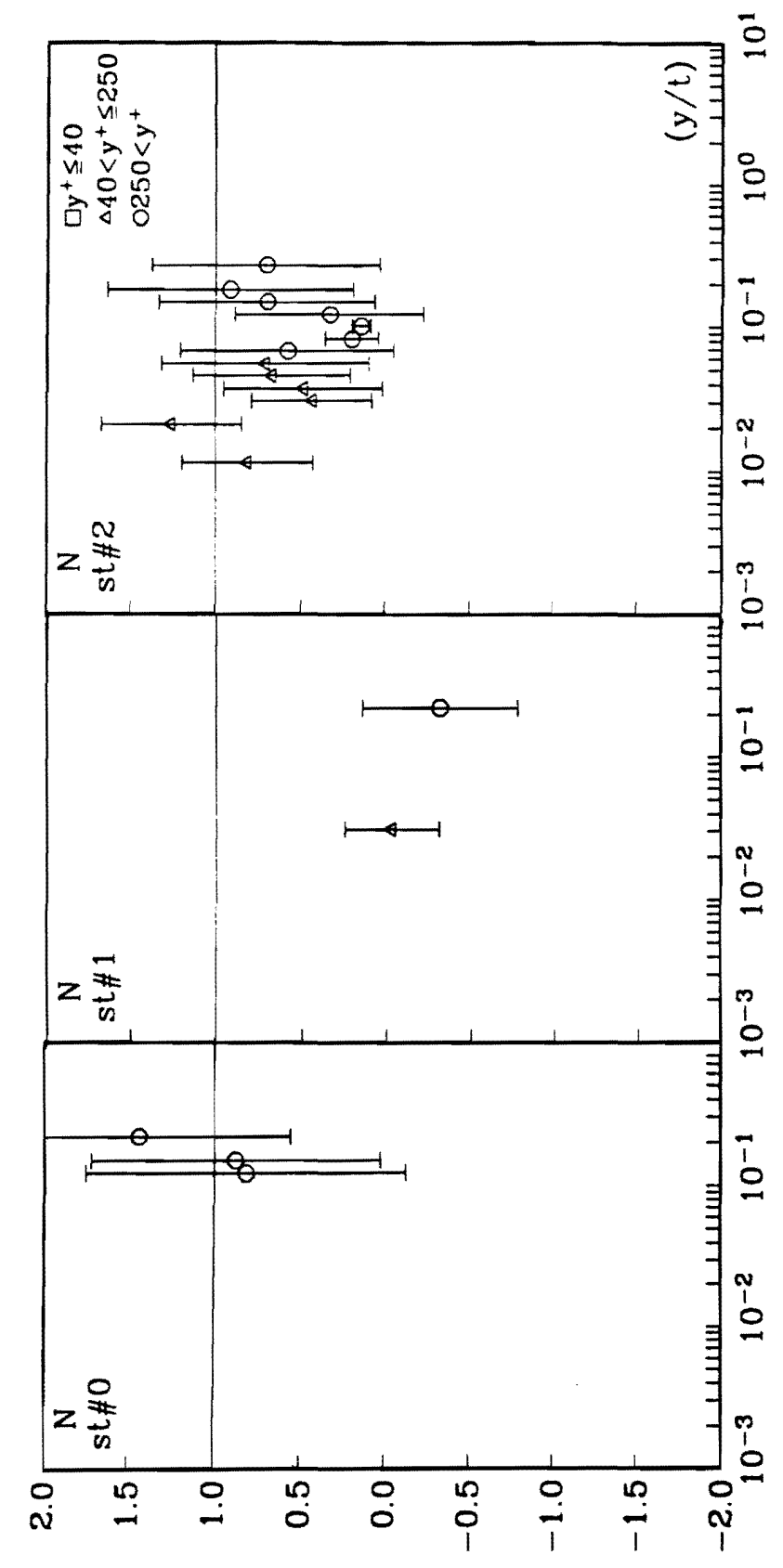

Figure 60a. $N$, anisotropy constant computed using LDV data in free-stream coordinates.: Stations numbers are shown in the figures. Bars denote the uncertainty bands. 


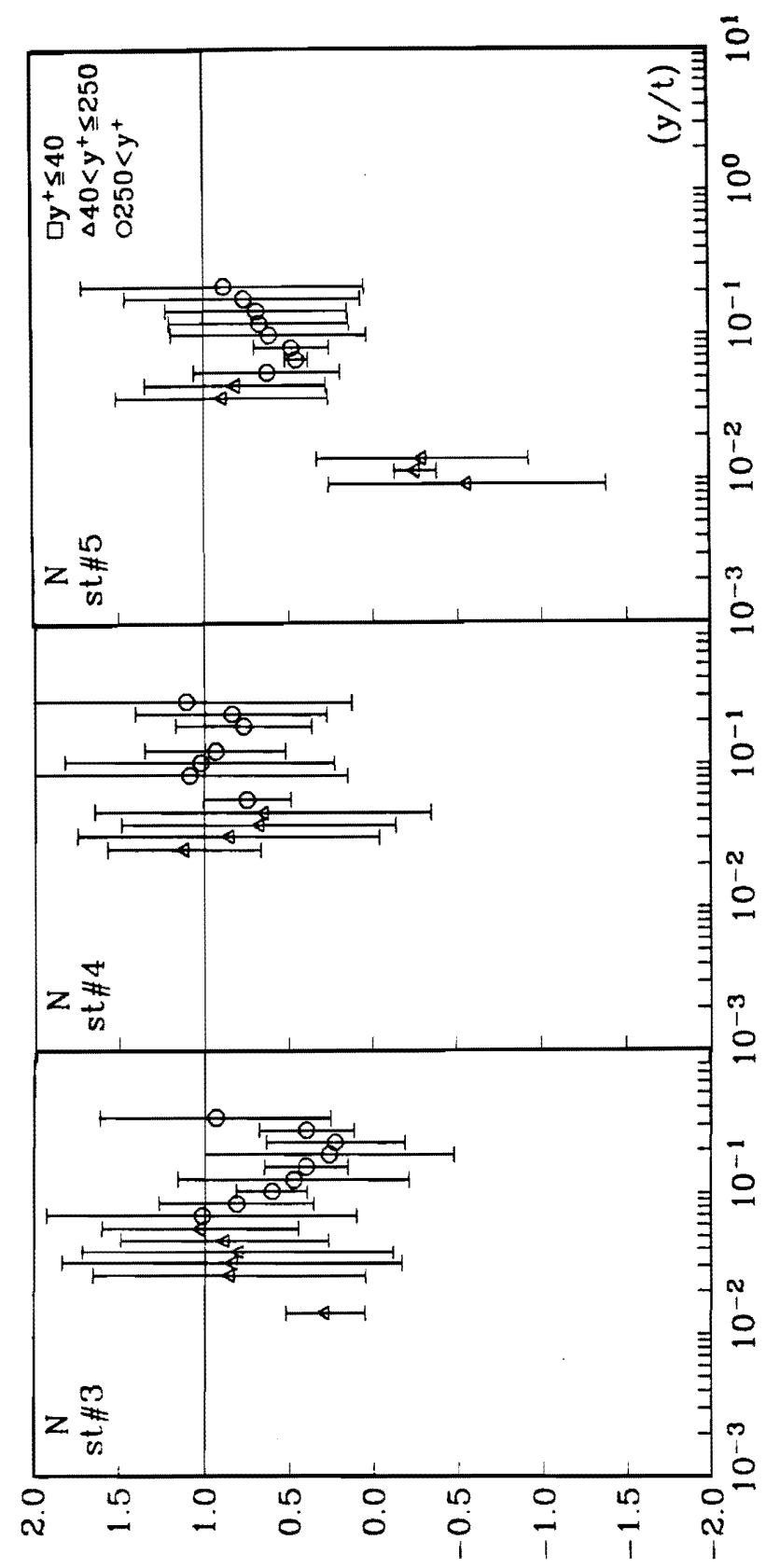

Figure 60b. N, anisotropy constant computed using LDV data in free-stream coordinates.: Stations numbers are shown in the figures. Bars denote the uncertainty bands. 


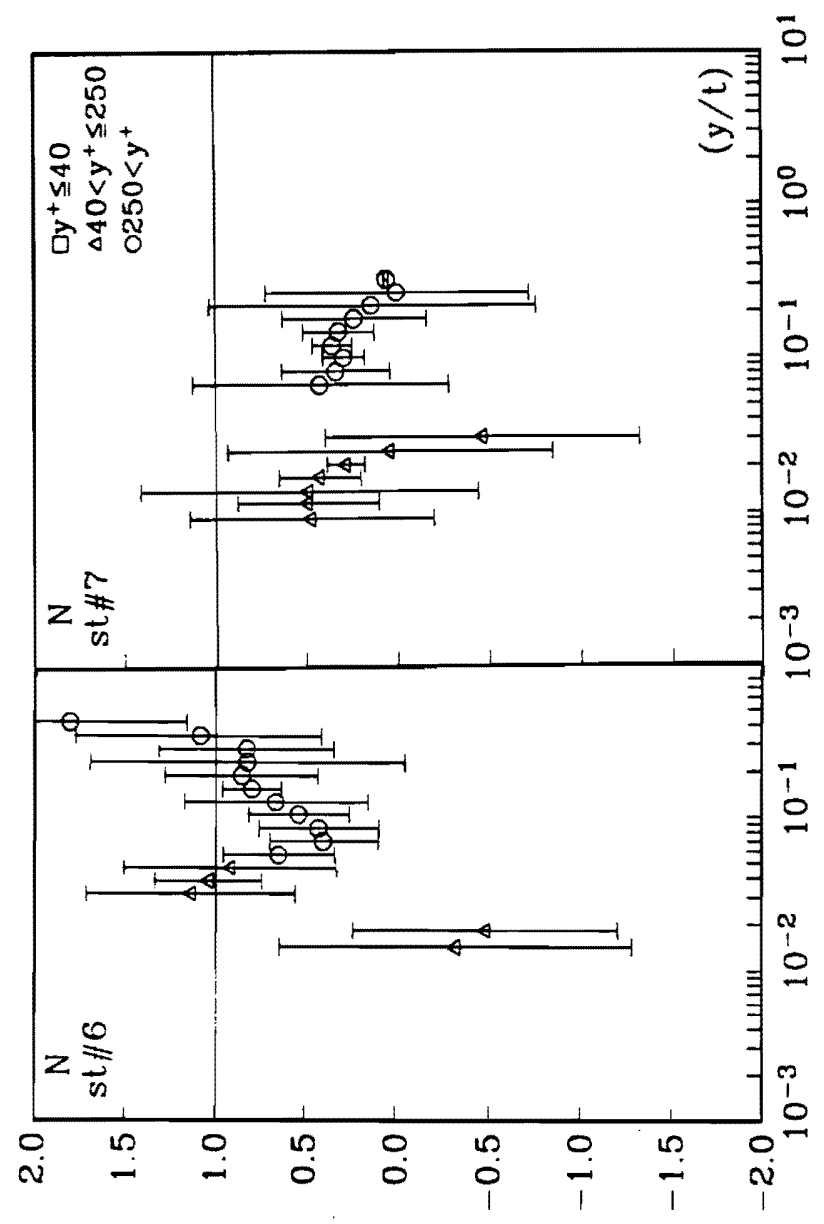

Figure $60 \mathrm{c}$. N, anisotropy constant computed using LDV data in free-stream coordinates.: Stations numbers are shown in the figures. Bars denote the uncertainty bands. 


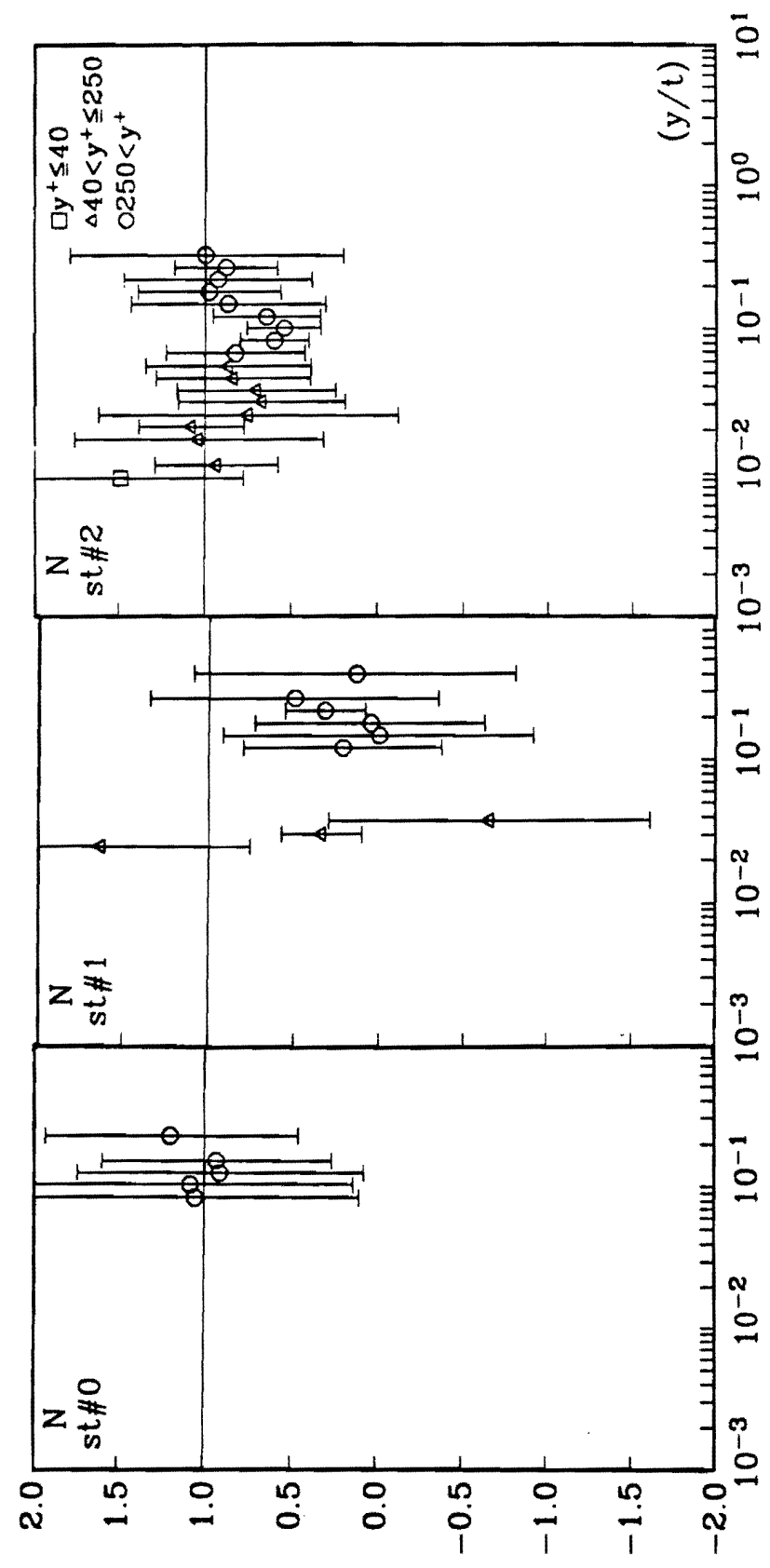

Figure 6la. N, anisotropy constant computed using LDV data in maximum normal stress coordinates.: Stations numbers are shown in the figures. Bars denote the uncertainty bands. 


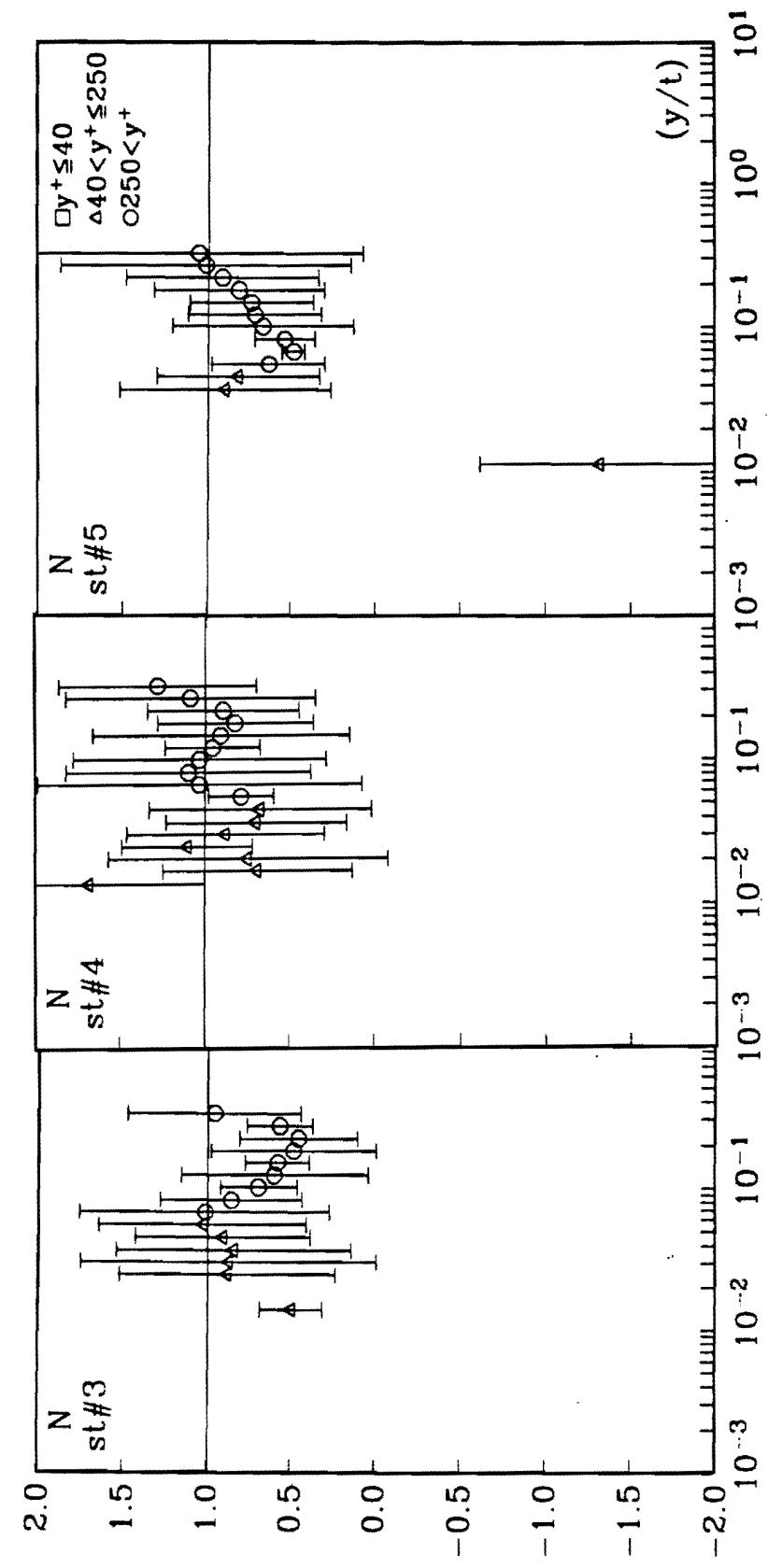

Figure 61b. $\mathrm{N}$, anisotropy constant computed using LDV data in maximum normal stress coordinates.: Stations numbers are shown in the figures. Bars denote the uncertainty bands. 


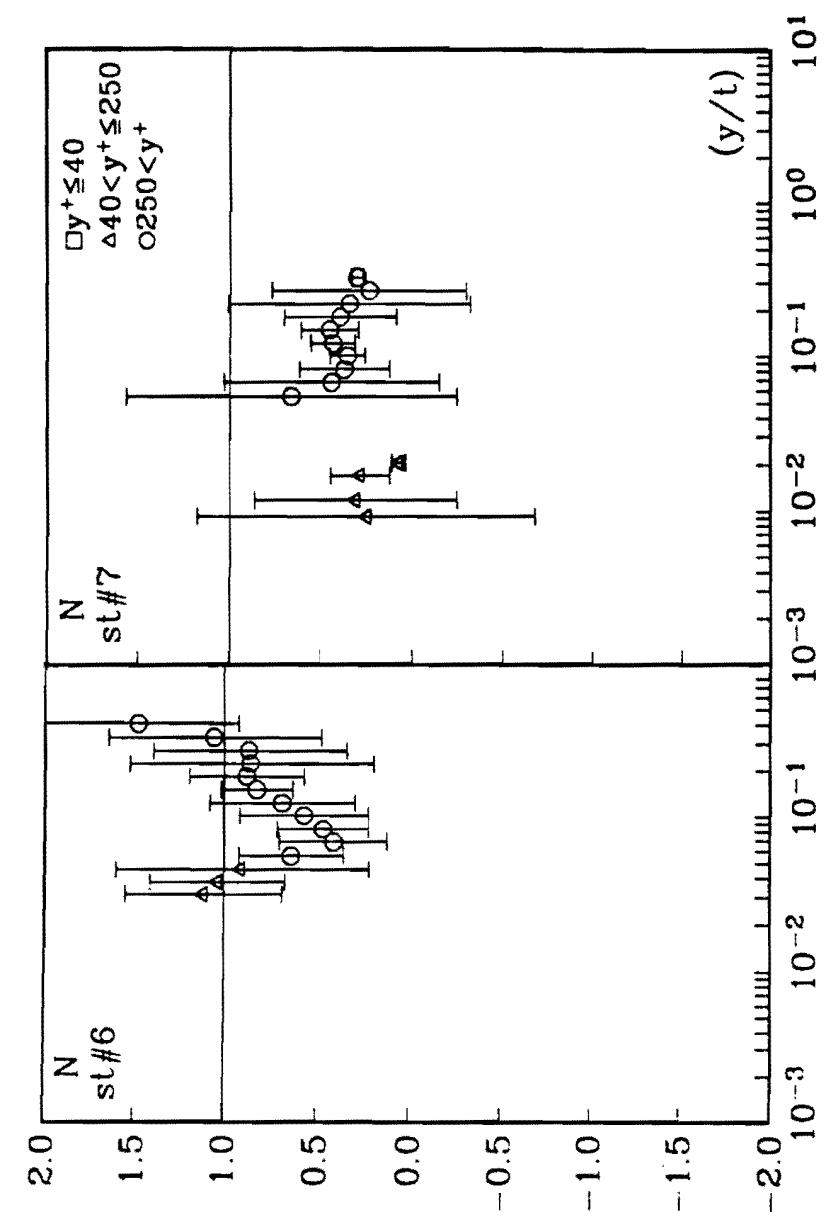

Figure 6lc. $\mathrm{N}$, anisotropy constant computed using LDV data in maximum normal stress coordinates.: Stations numbers are shown in the figures. Bars denote the uncertainty bands. 


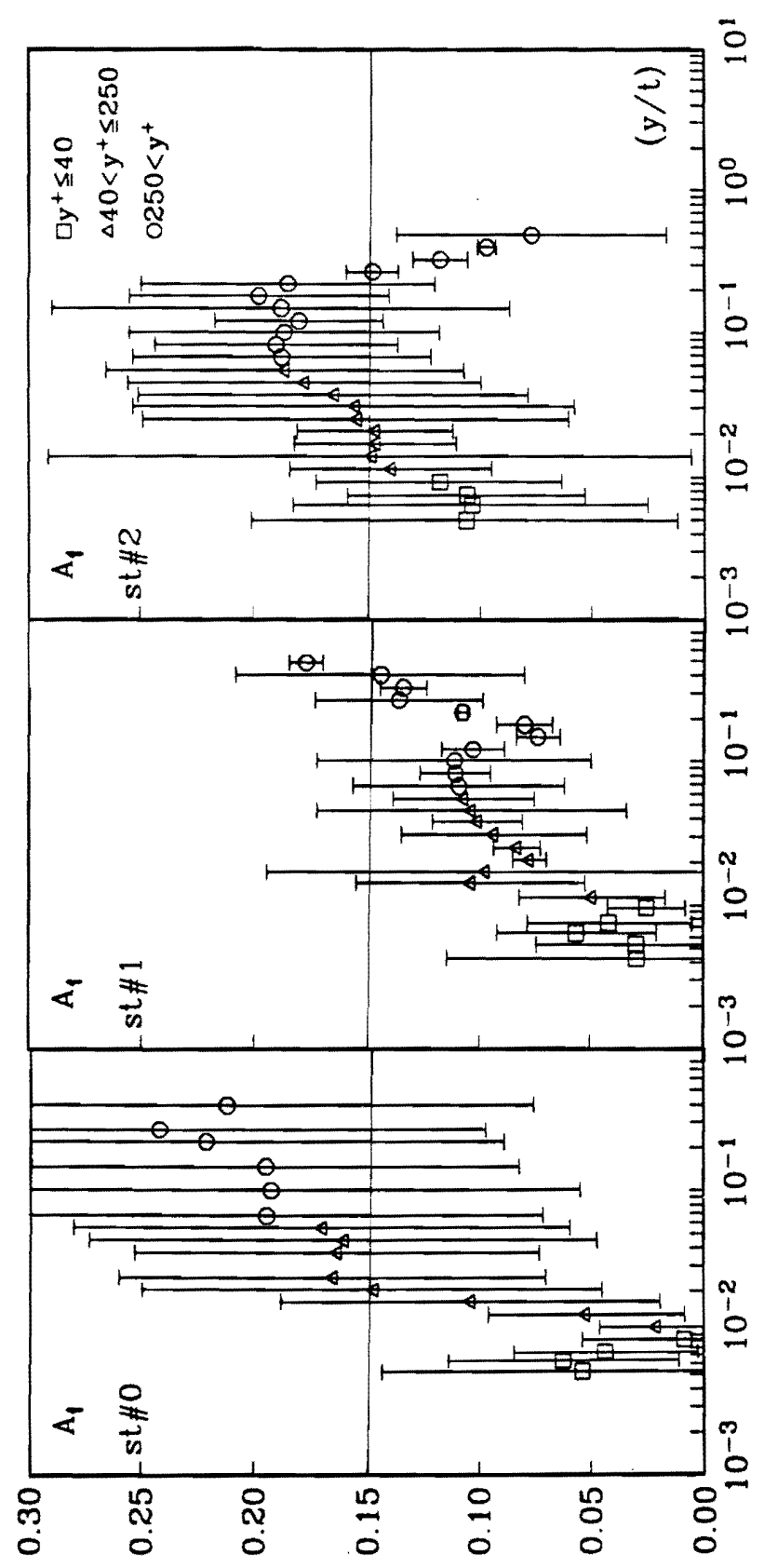

Figure 62a. A1, Townsend's structural parameter computed using LDV data.: Station numbers are shown in the figures. Bars denote the uncertainty bands. 


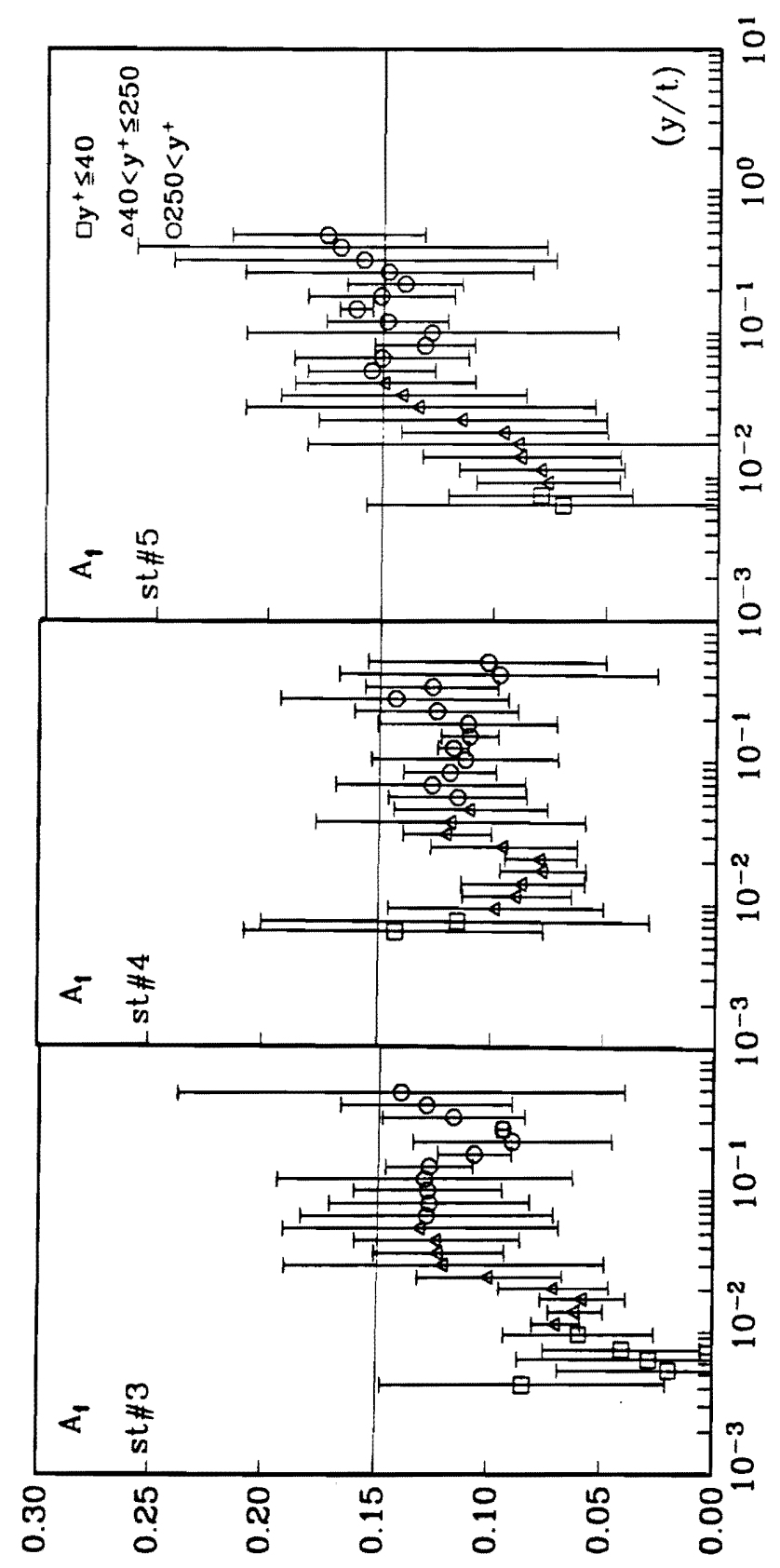

Figure 62b. A1, Townsend's structural parameter computed using LDV data.: Station numbers are shown in the figures. Bars denote the uncertainty bands. 


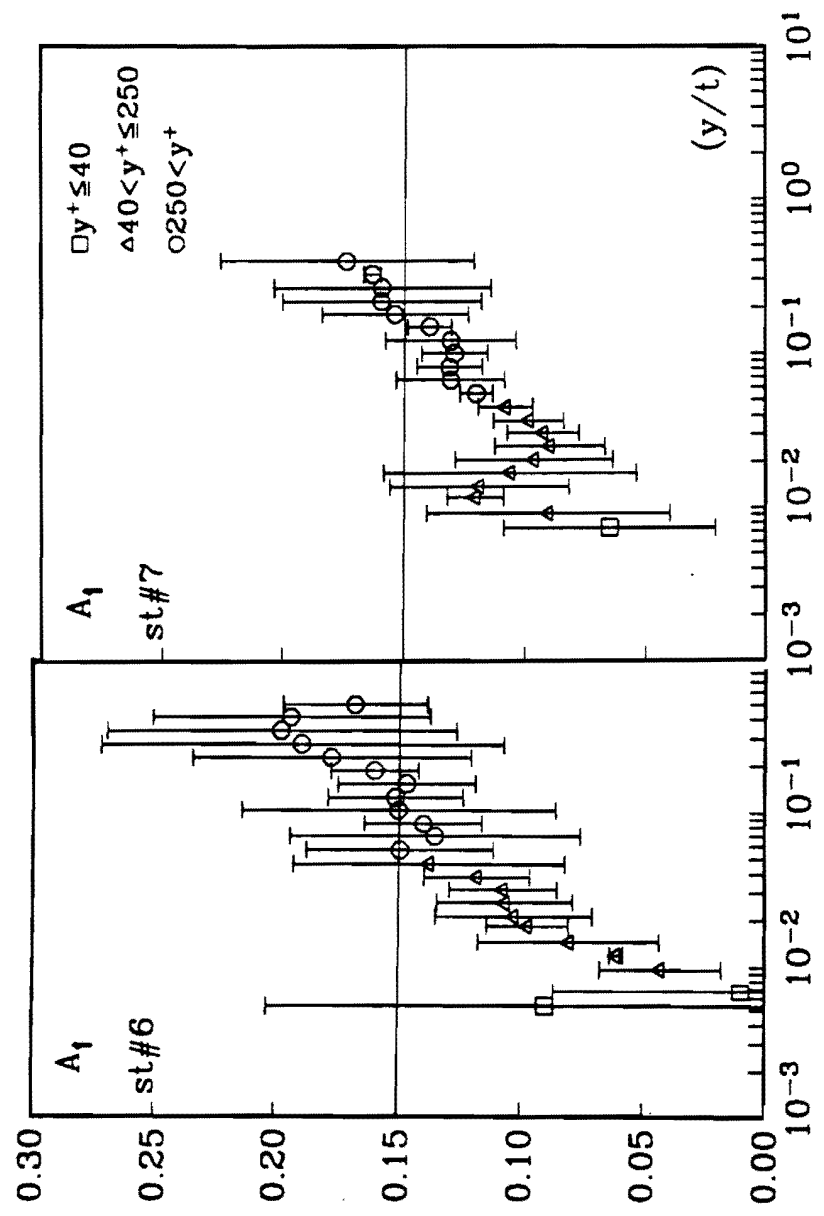

Figure 62c. A1, Townsend's structural parameter computed using LDV data.: Station numbers are shown in the figures. Bars denote the uncertainty bands. 


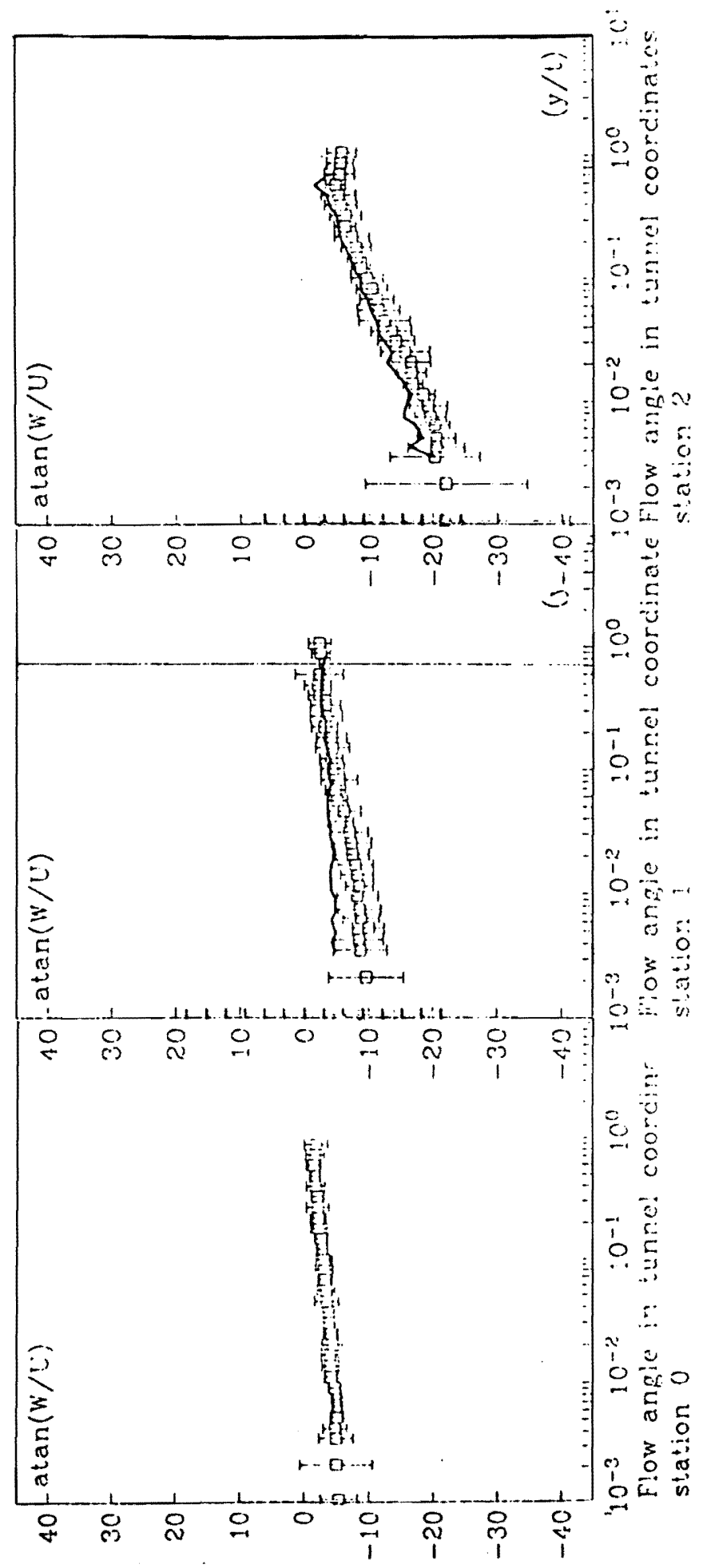

Figure 63a. Flow angle computed using LDV data in tunnel coorainates.: station numbers are shown in the figures. Lines are used to show the hot-wire profiles taken at the same locations. Bars denote the uncertainty bands. 


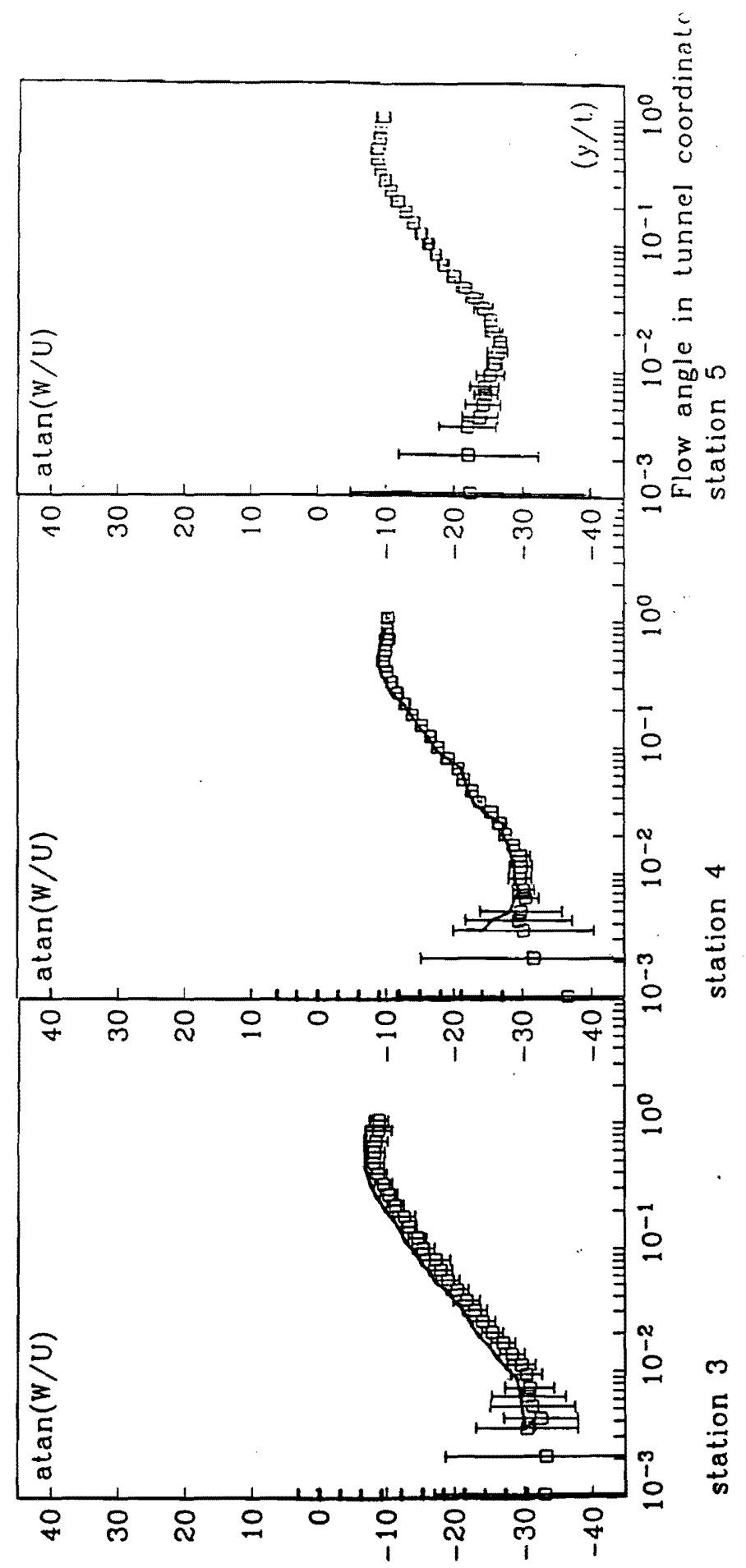

Figure 63b. Flow angle computed using LDV data in tunnel coordinates.: Station numbers are shown in the figures. Lines are used to show the hot-wire profiles taken at the same locations. Bars denote the uncertainty bands. 


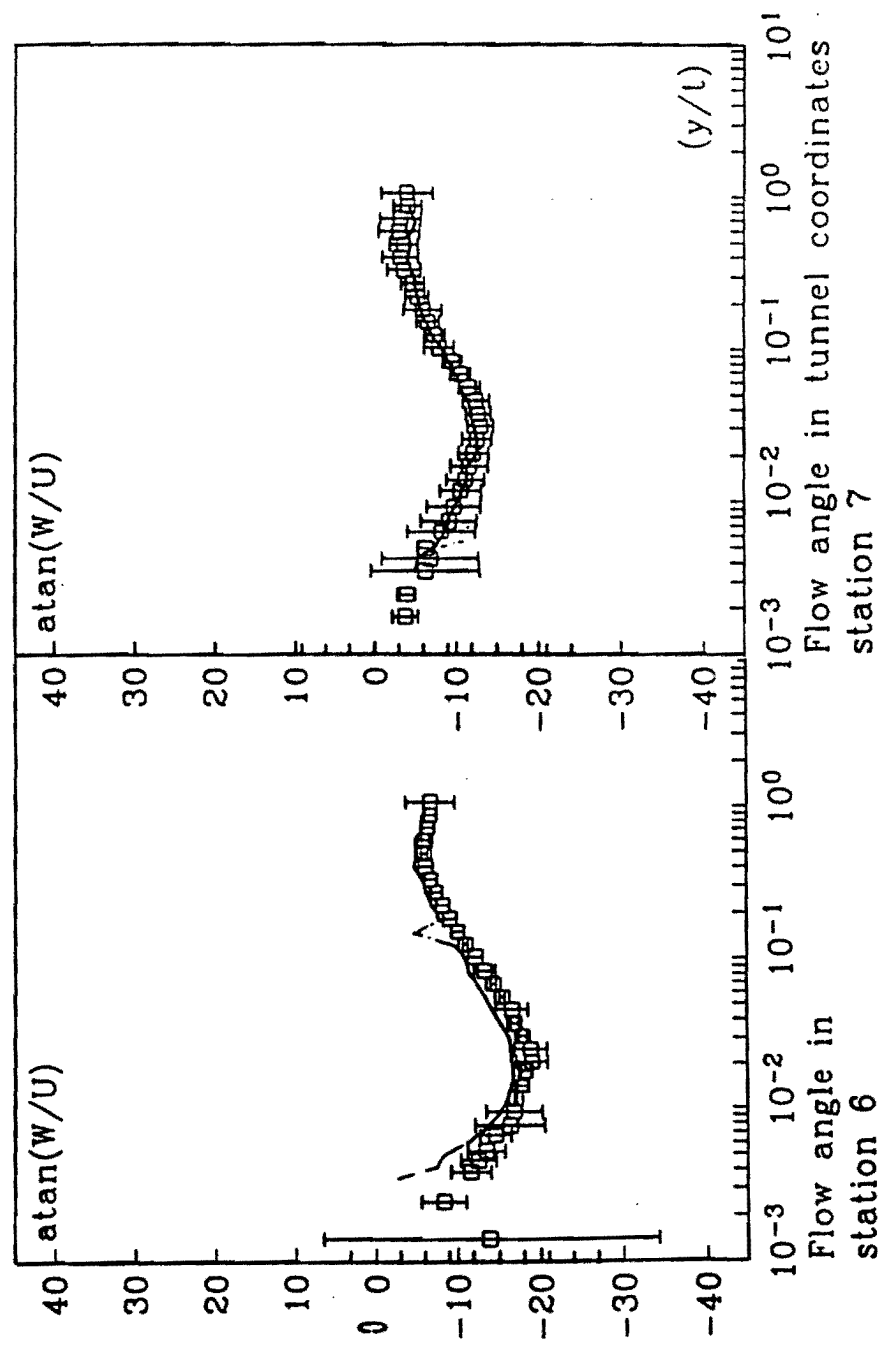

Figure 63c. Flow angle computed using LDV data in tunnel coordinates:: Station numbers are shown in the figures. Lines are used to show the hot-wire profiles taken at the same locations. Bars denote the uncertainty bands. 

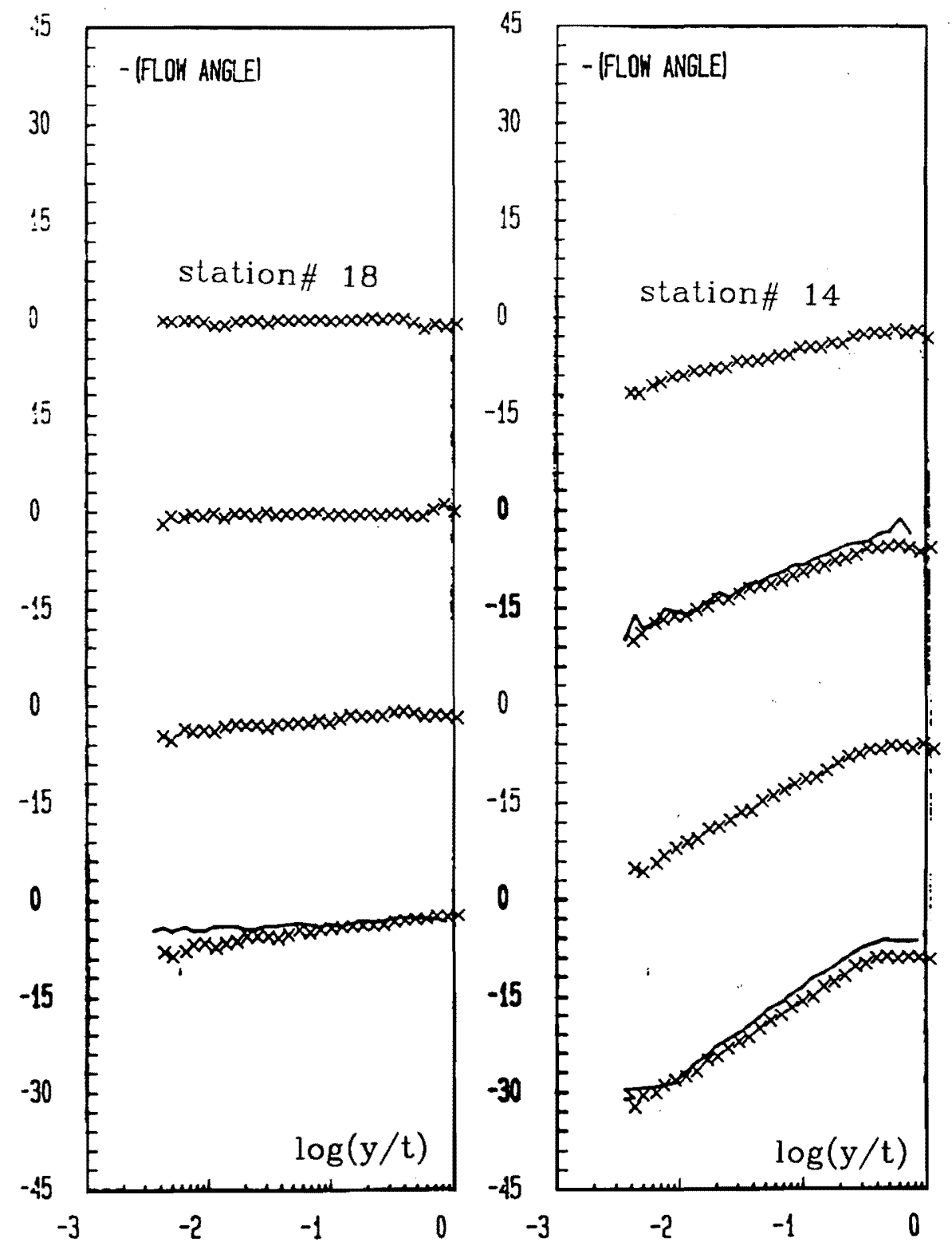

Figure 64a. Flow angle in tunnel coordinates computed using hot-wire data taken on the left-hand side of the wing.: Lines are used to show the hot-wire profiles taken at the symmetric locations. In the figures, stations are numbered from top to bottom starting with station 18 , and station 14 , respectively. Note the minus sign of the flow angle. 

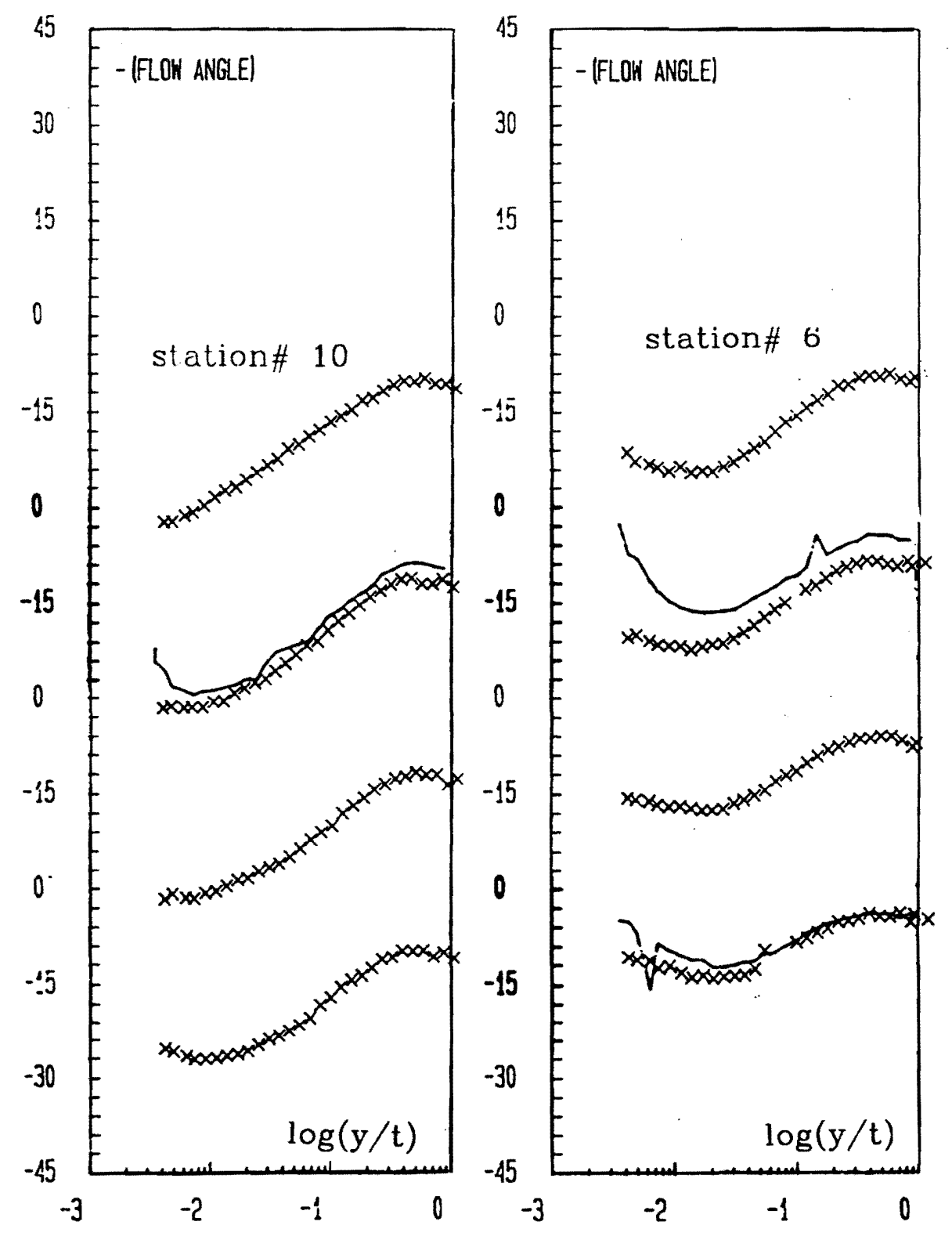

Figure 64b. Flow angle in tunnel coordinates computed using hot-wire data taken on the left-hand side of the wing.: Lines are used to show the hot-wire profiles taken at the symmetric locations. In the figures, stations are numbered from top to bottom starting with station 10 , and station 6 , respectively. Note the minus sign of the flow angle. 


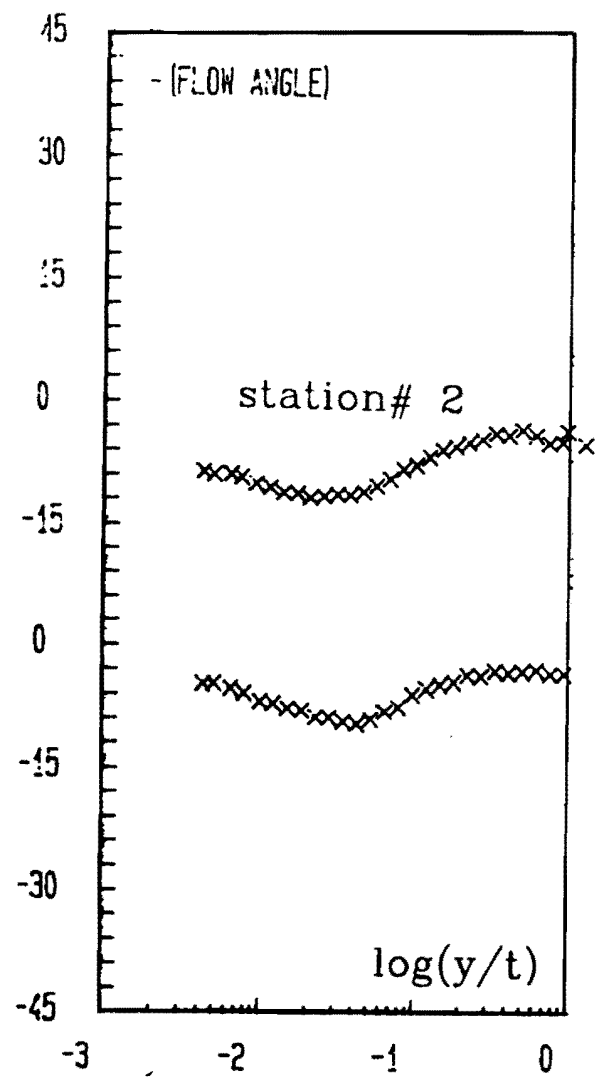

Figure 64c. Flow angle in tunnel coordinates computed using hot-wire data taken on the left-hand side of the wing.: In the figure, stations are numbered from top to bottom starting with station 2 . Note the minus sign of the flow angle. 


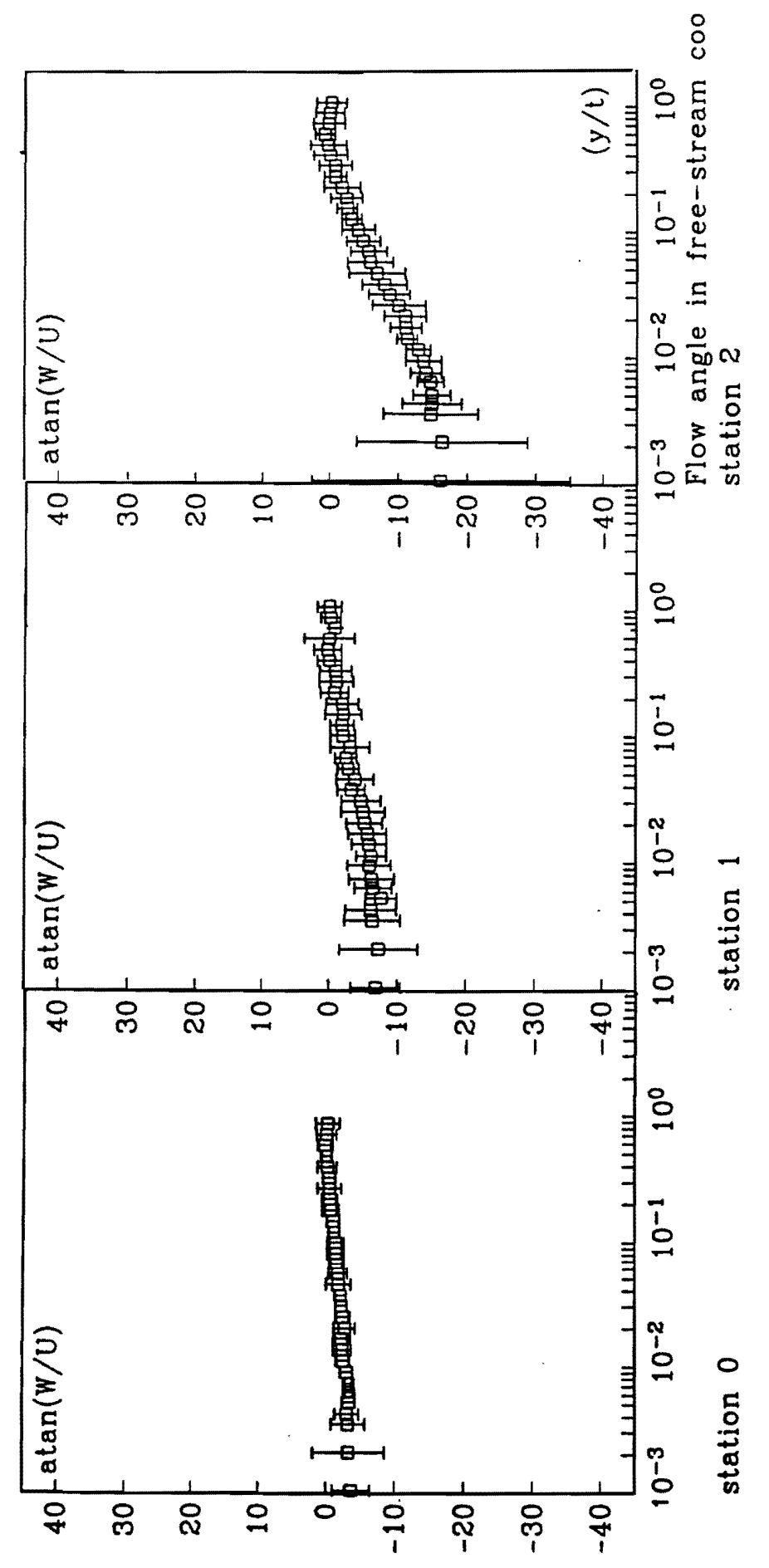

Figure 65a. Flow angle computed using LDV data in free-stream coordinates.: Station numbers are shown in the figures. Bars denote the uncertainty bands. 


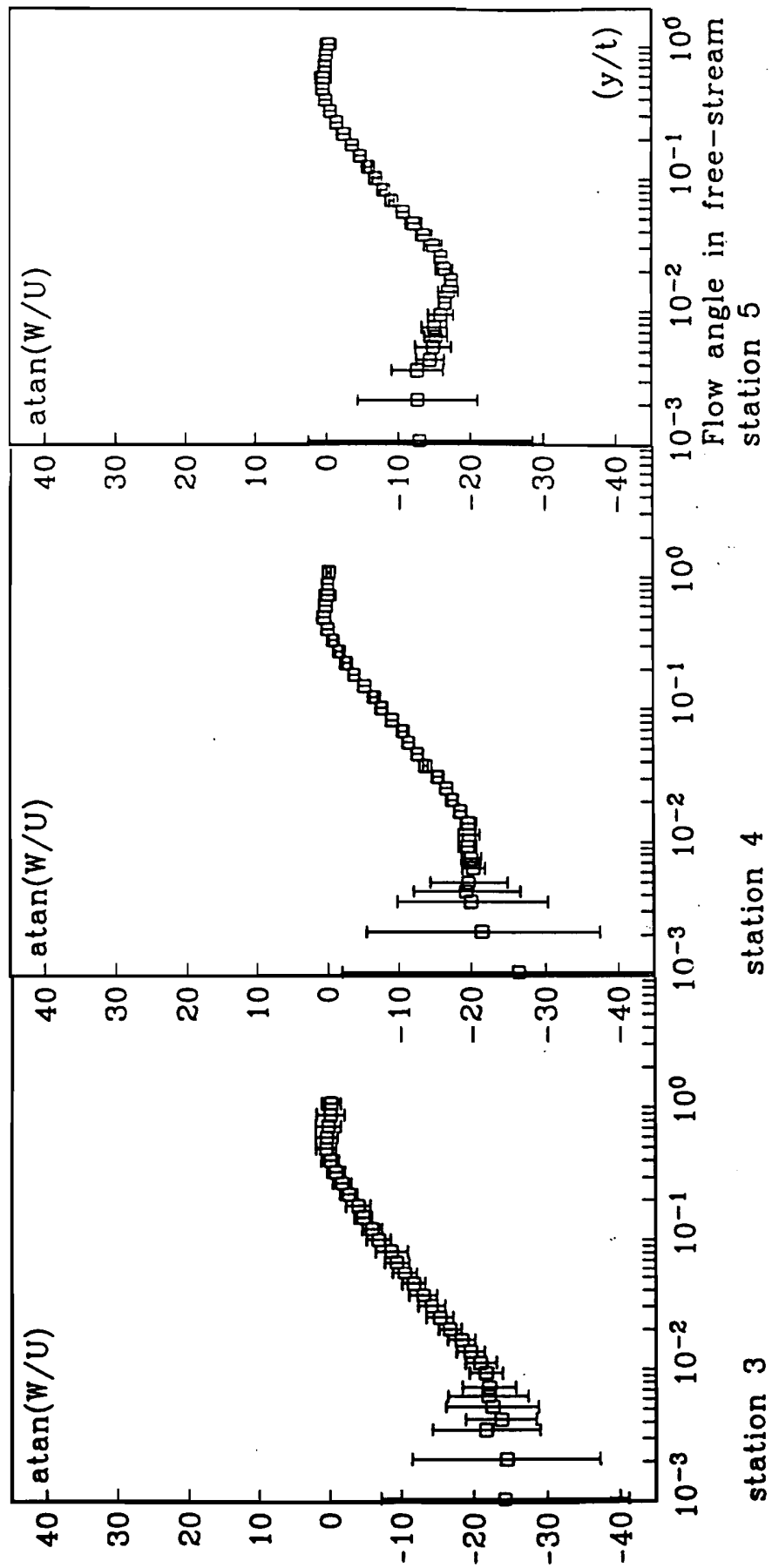

Figure 65b. Flow angle computed using LDV data in free-stream coordinates.: Station numbers are shown in the figures. Bars denote the uncertainty bands. 


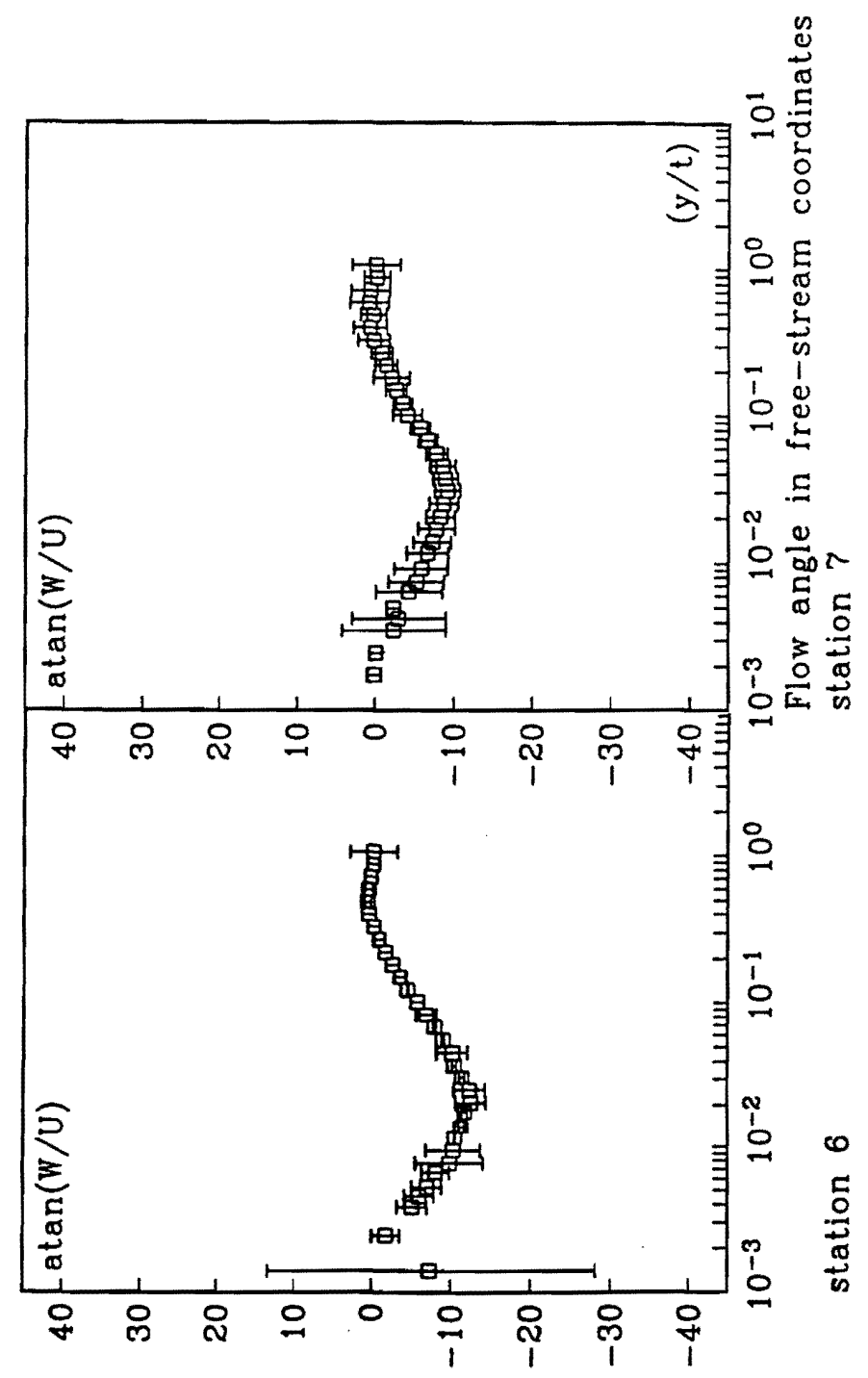

Figure 65c. Flow angle computed using LDV data in free-stream coordinates.: Station numbers are shown in the figures. Bars denote the uncertainty bands. 

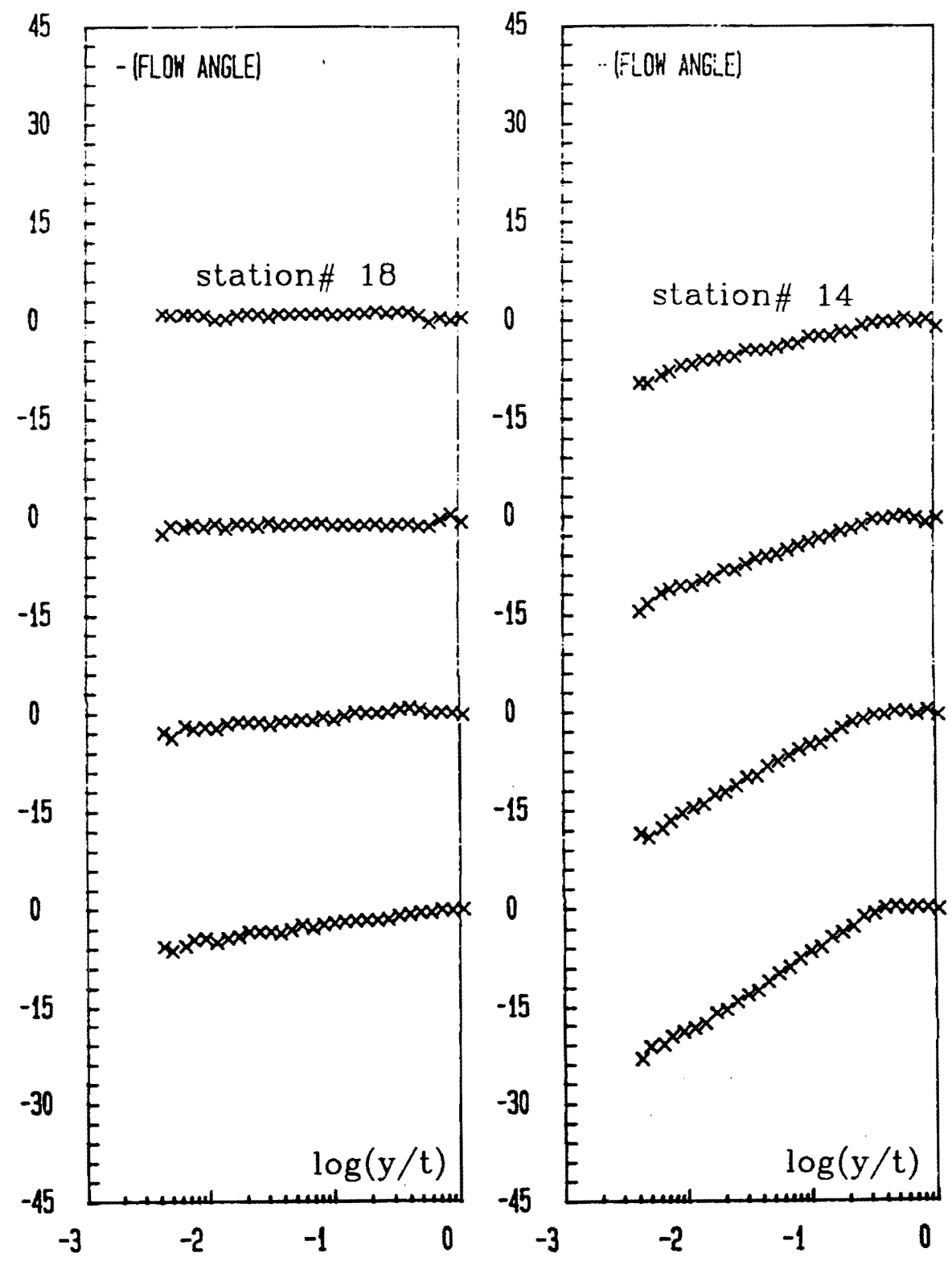

Figure 66a. Flow angle in free-stream coordinates computed using hot-wire data taken on the left-hand side of the wing.: In the figures, stations are numbered from top to bottom starting with station 18 , and station 14 , respectively. Note the minus sign of the flow angle. 


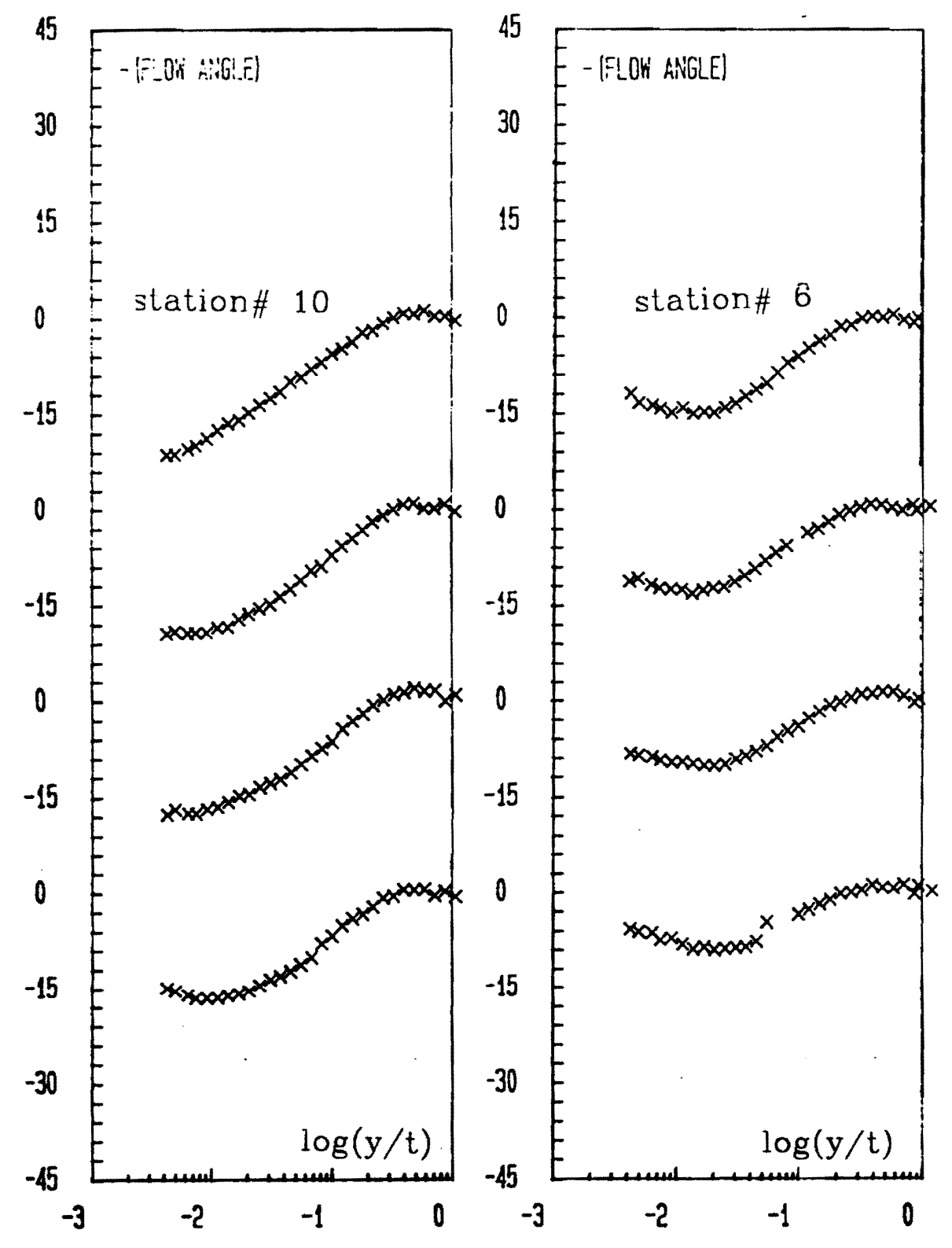

Figure 66b. Flow angle in free-stream coordinates computed using hot-wire data taken on the left-hand side of the wing.: In the figures, stations are numbered from top to bottom starting with station 10 , and station 6 , respectively. Note the minus sign of the flow angle. 


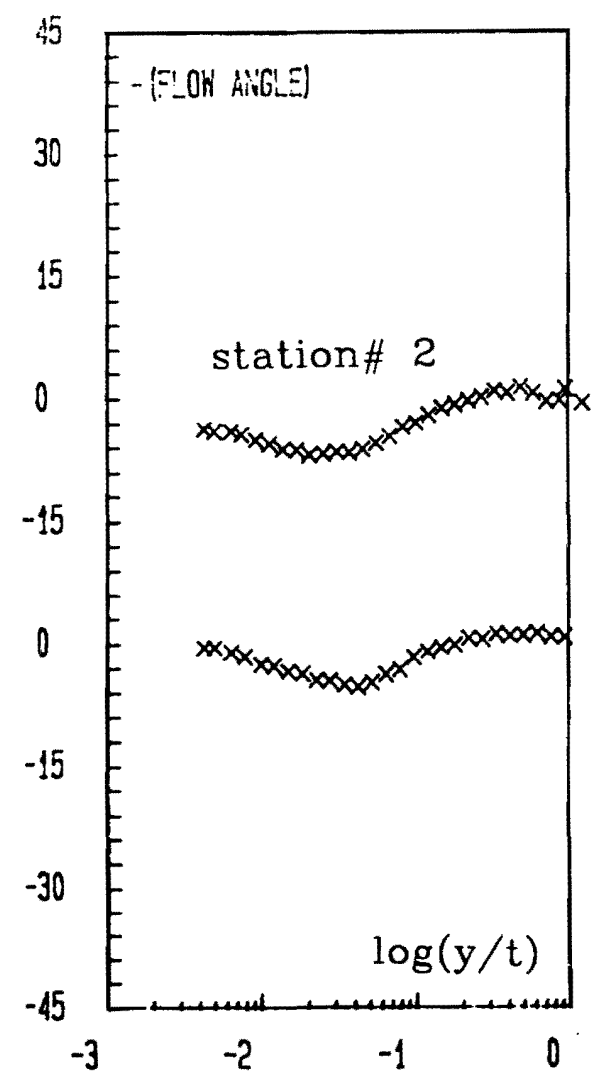

Figure 66c. Flow angle in free-stream coordinates computed using hot-wire data taken on the left-hand side of the wing.: In the figure, stations are numbered from top to bottom starting with station 2 . Note the minus sign of the flow angle. 


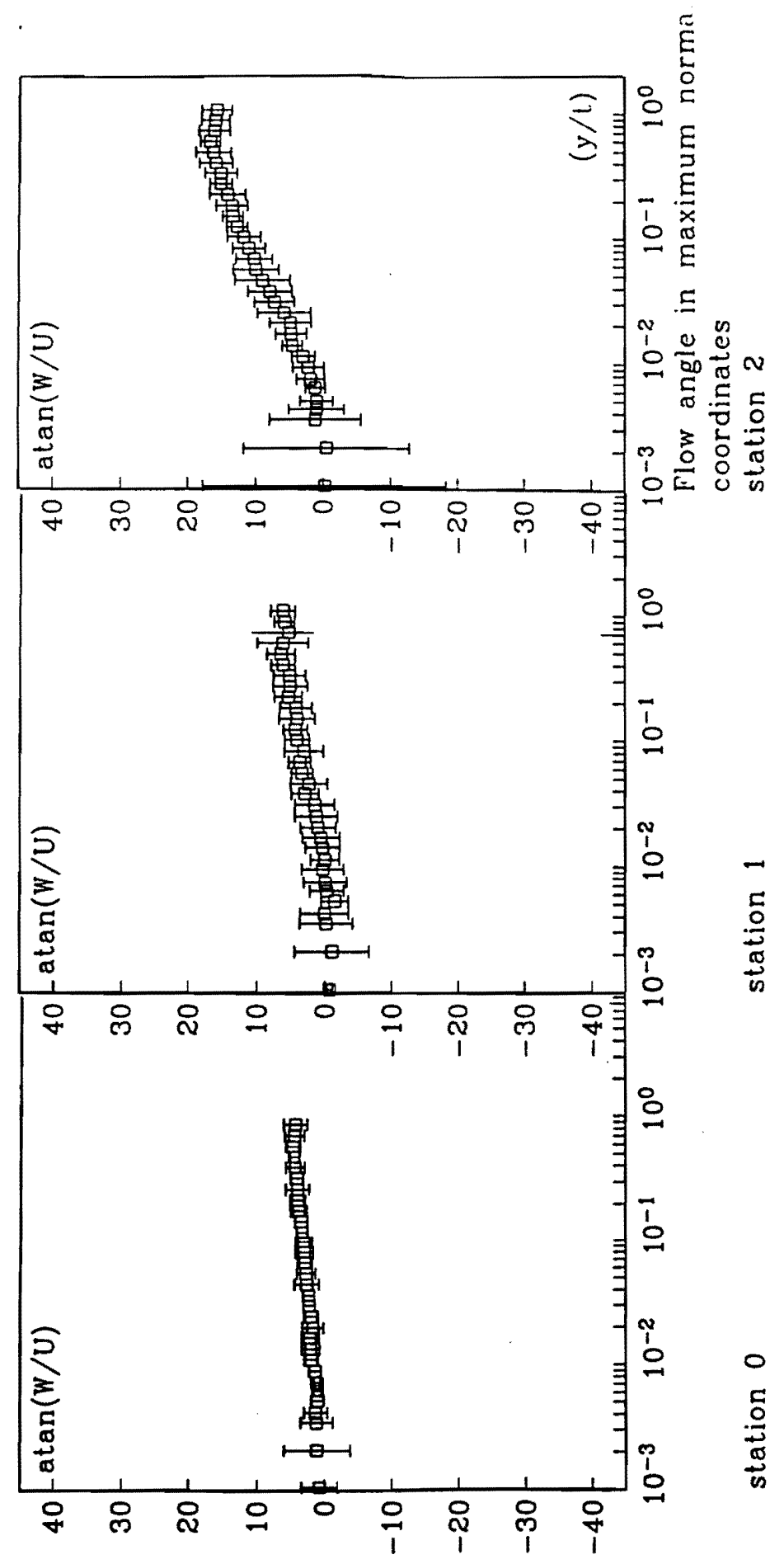

Figure 67a. Flow angle computed using LDV data in maximum normal stress coordinates.: Station numbers are shown in the figures. Bars denote the uncertainty bands. 


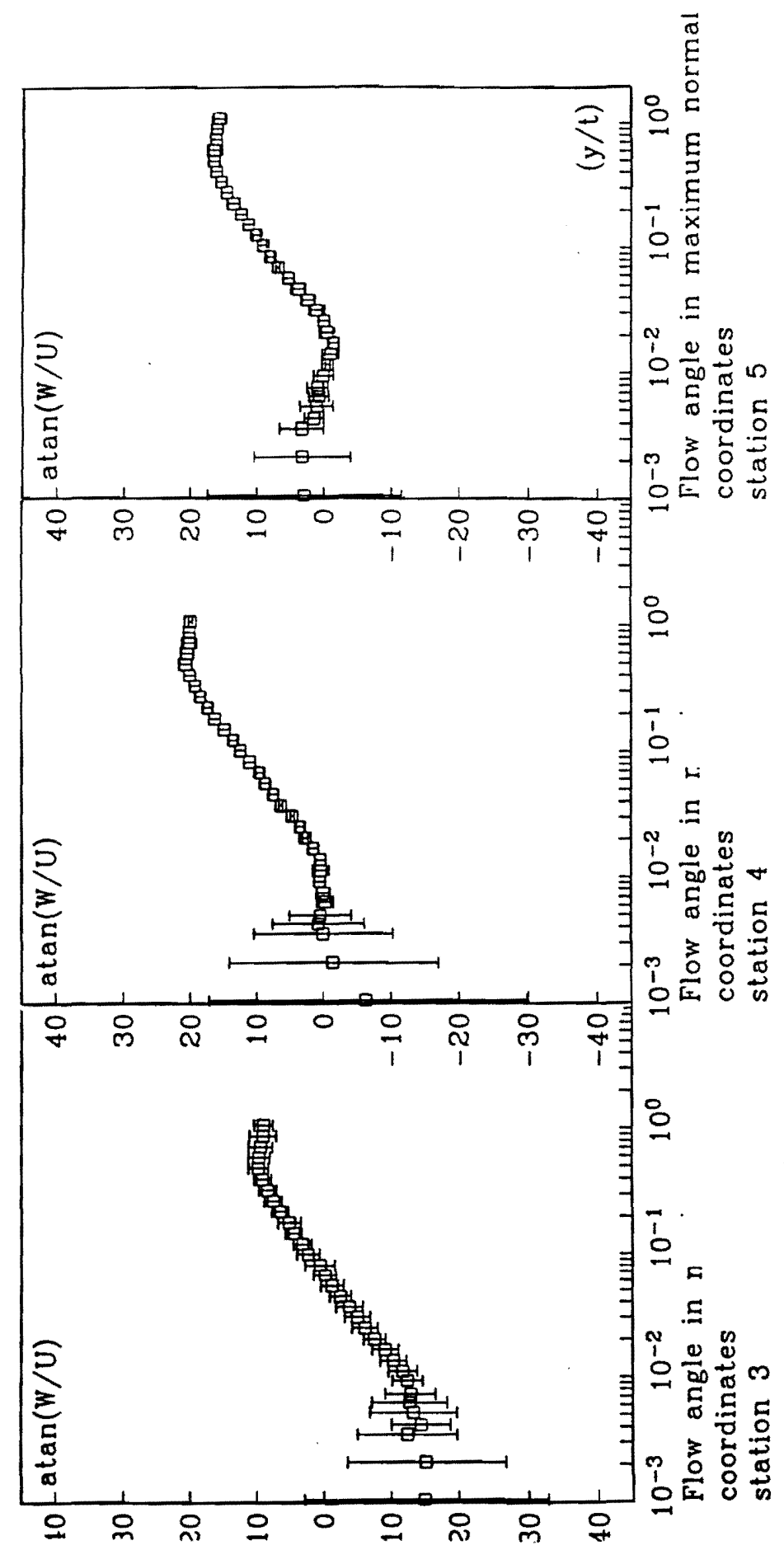

Figure 67b. Flow angle computed using LDV data in maximum normal stress coordinates.: Station numbers are shown in the figures. Bars denote the uncertainty bands. 


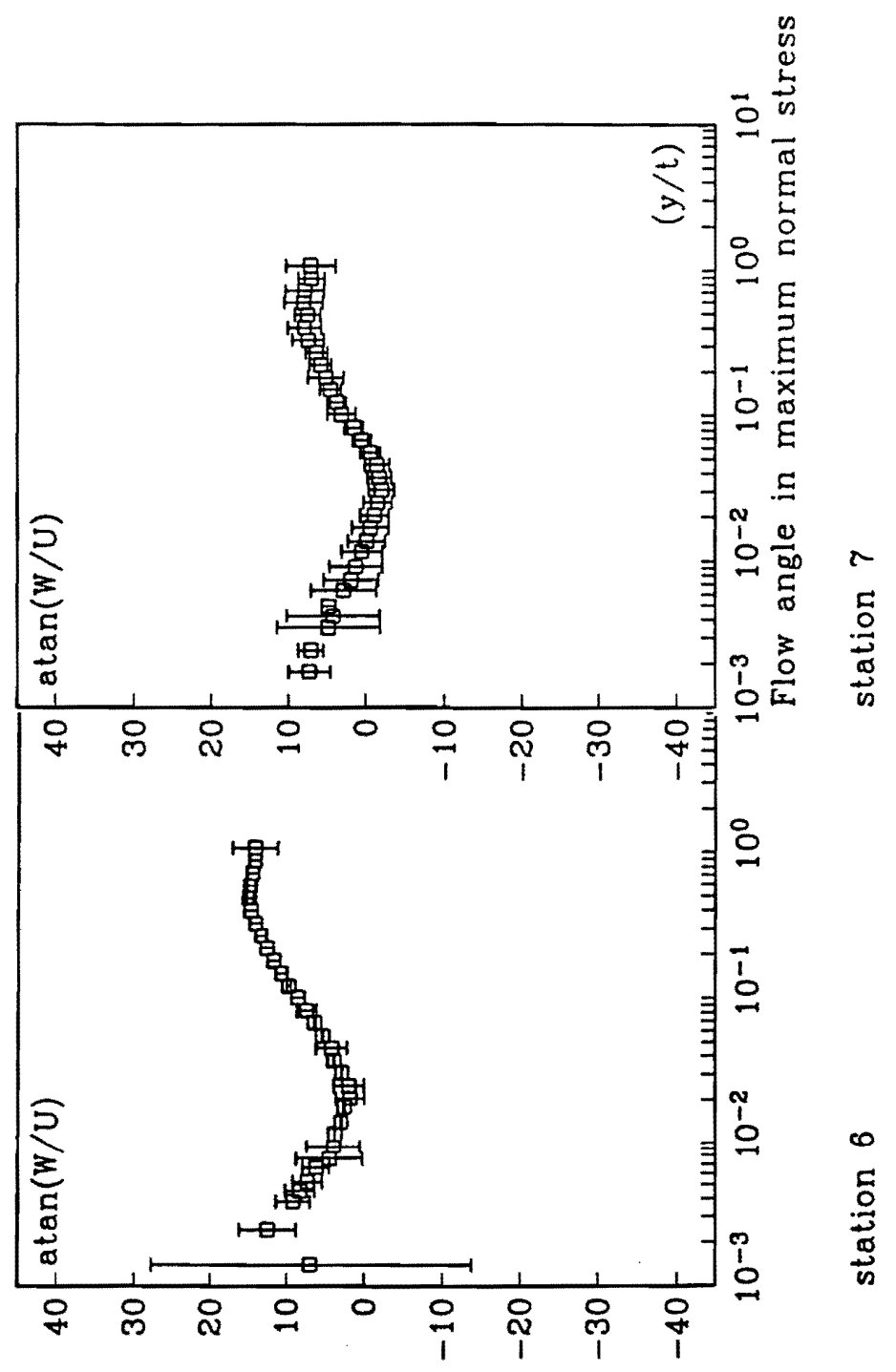

Figure 67c. Flow angle computed using LDV data in maximum normal stress coordinates.: Station numbers are shown in the figures. Bars denote the uncertainty bands. 

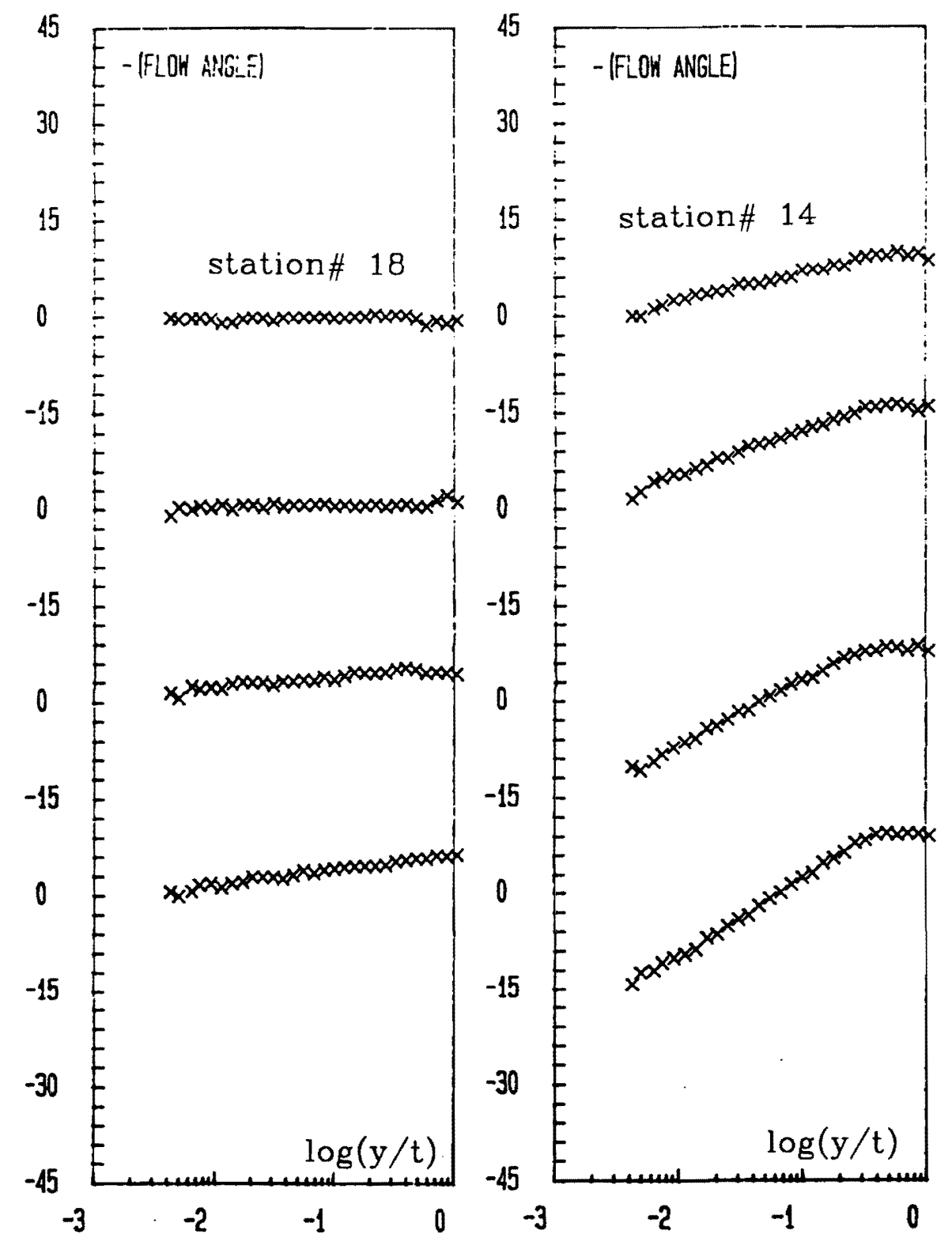

Figure 68a. Flow angle in maximum normal stress coordinates computed using hot-wire data taken on the left-hand side of the wing.: In the figures, stations are numbered from top to bottom starting with station 18, and station 14, respectively. Note the minus sign of the flow angle. 


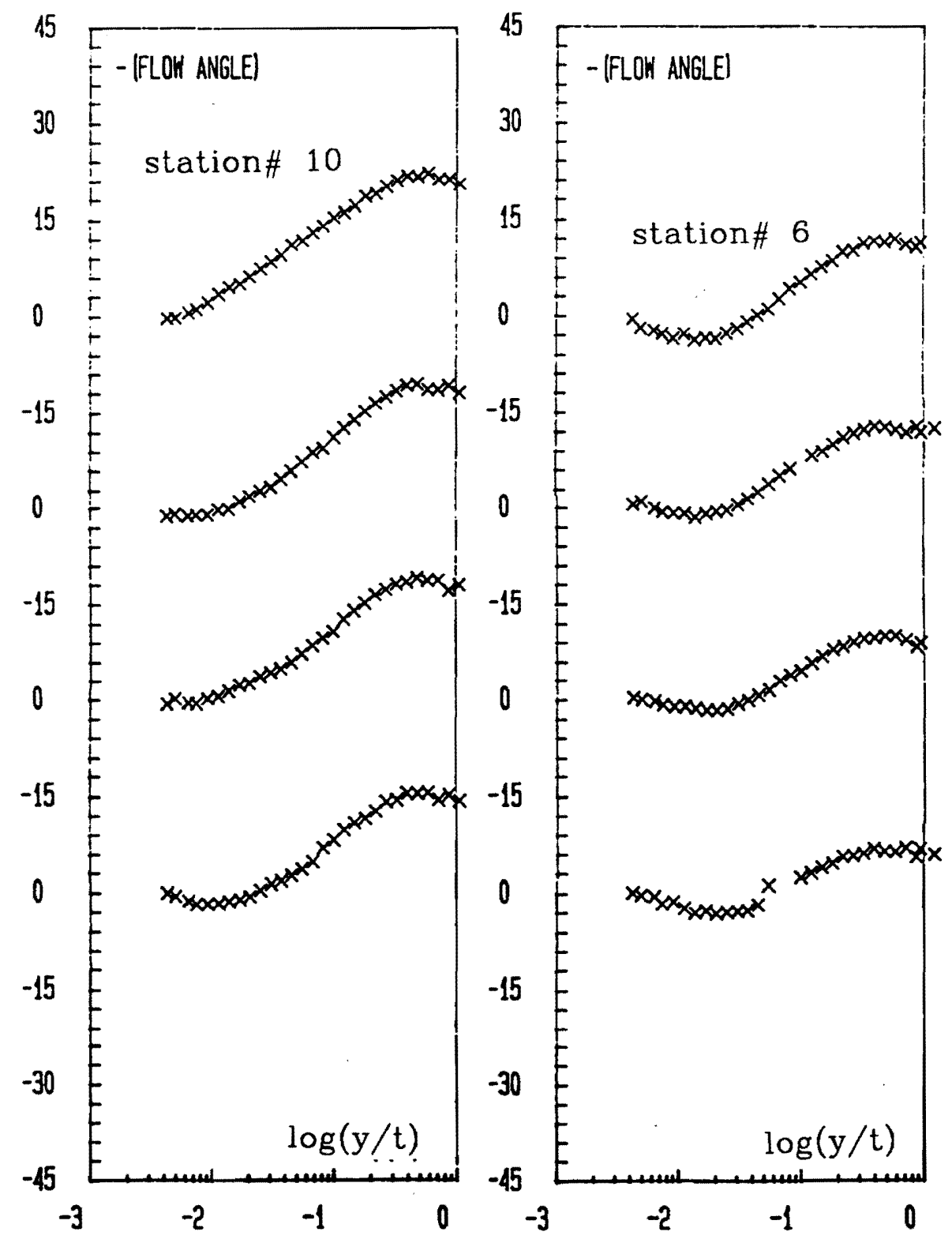

Figure 68b. Flow angle in maximum normal stress coordinates computed using hot-wire data taken on the left-hand side of the wing.: In the figures, stations are numbered from top to bottom starting with station 10 , and station 6 , respectively. Note the minus sign of the flow angle. 


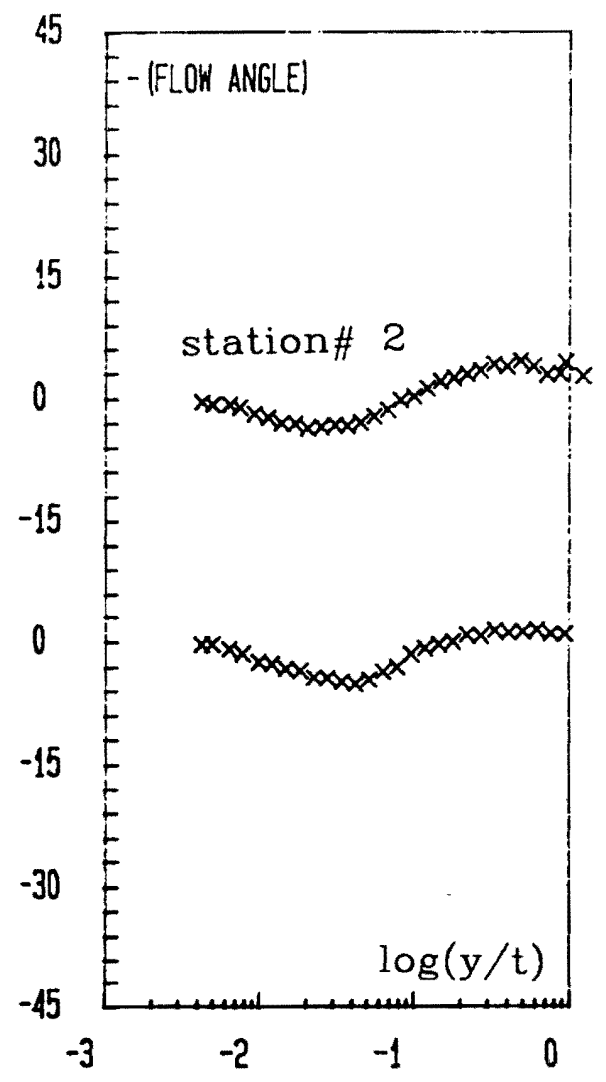

Figure 68c. Flow angle in maximum normal stress coordinates computed using hot-wire data taken on the left-hand side of the wing.: In the figure, stations are numbered from top to bottom starting with station 2 . Note the minus sign of the flow angle. 


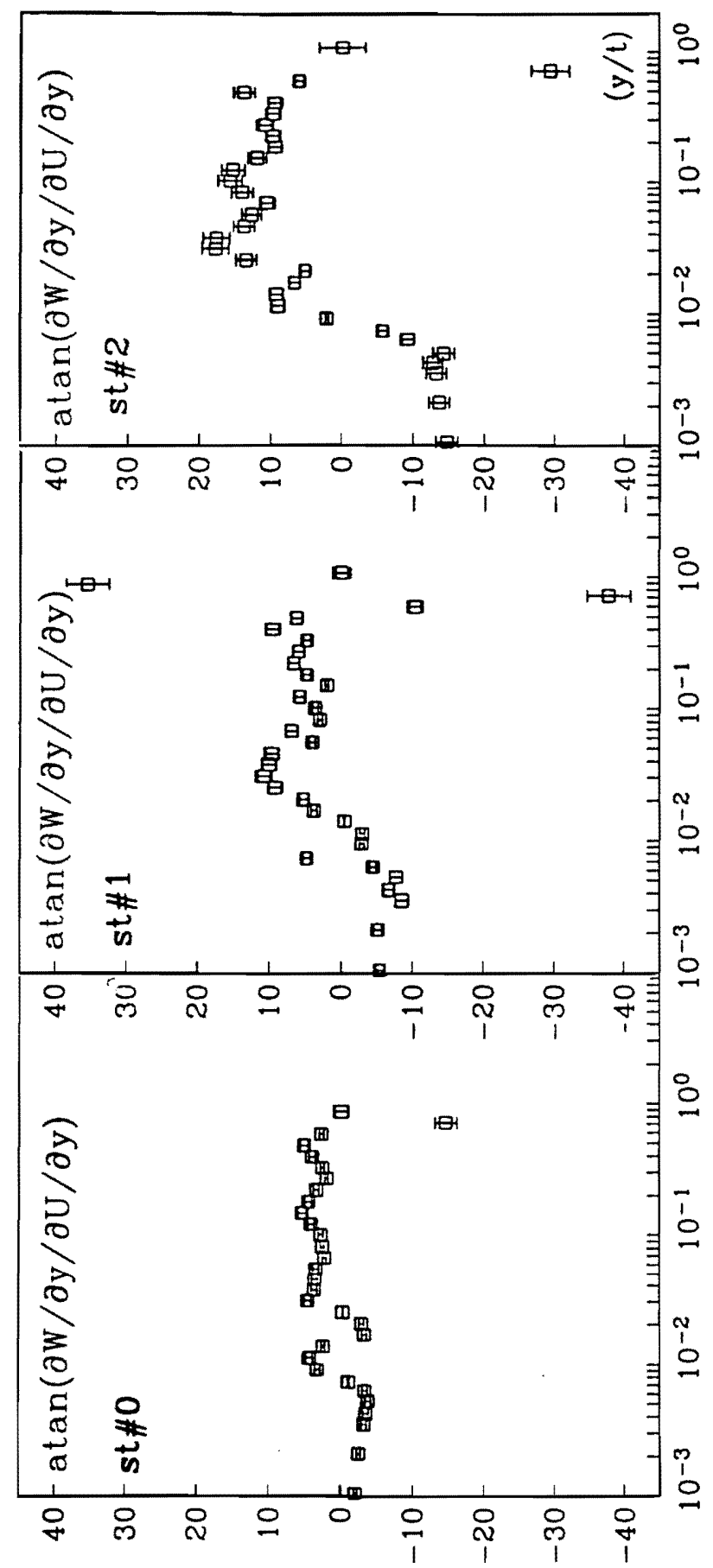

Figure 69a. Flow gradient angle computed using LDV data in free-stream coordinates.: Station numbers are shown in the figures. Bars denote the uncertainty bands. 


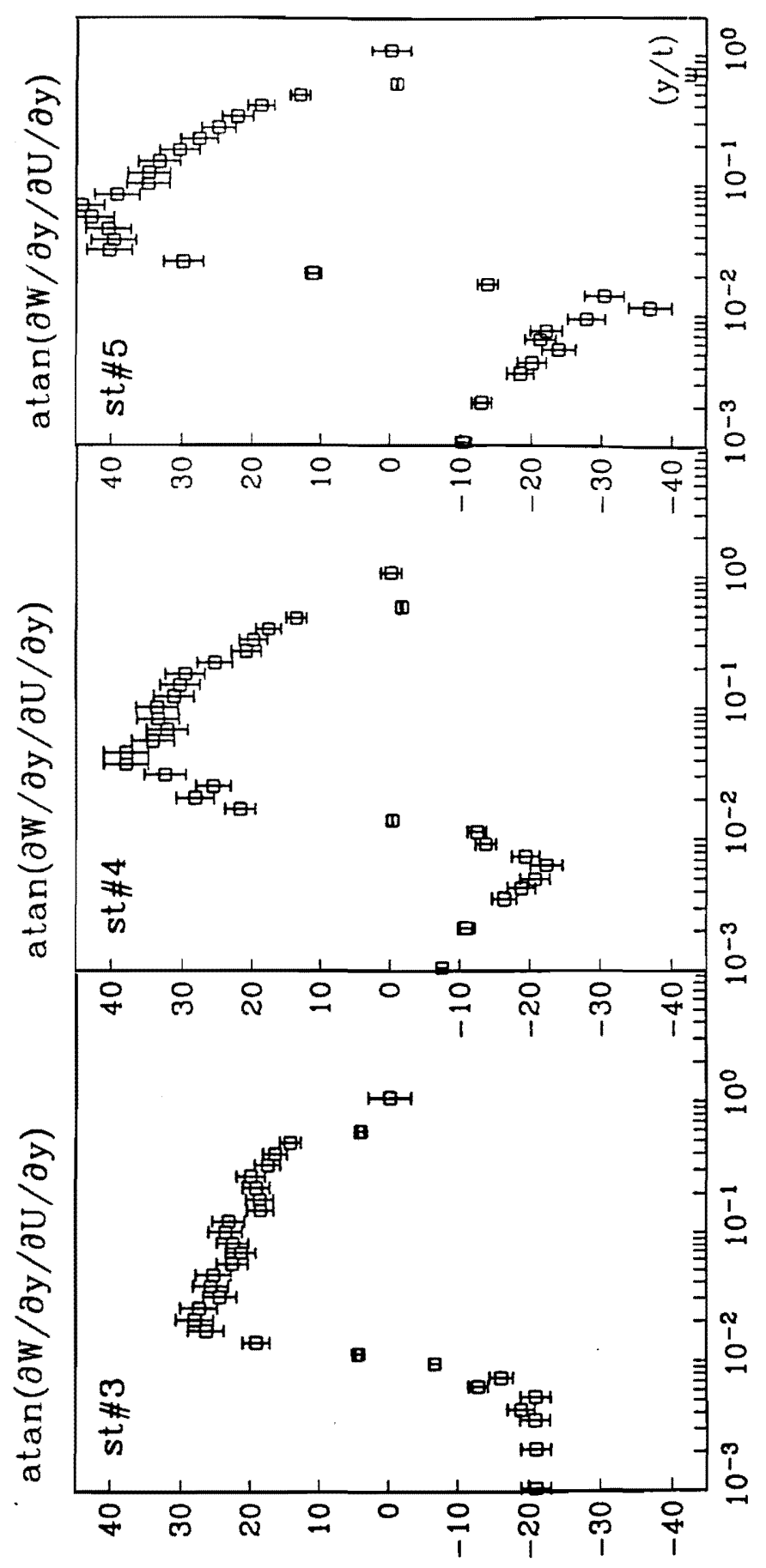

Figure 69b. Flow gradient angle computed using LDV data in free-stream coordinates.: Station numbers are shown in the figures. Bars denote the uncertainty bands. 


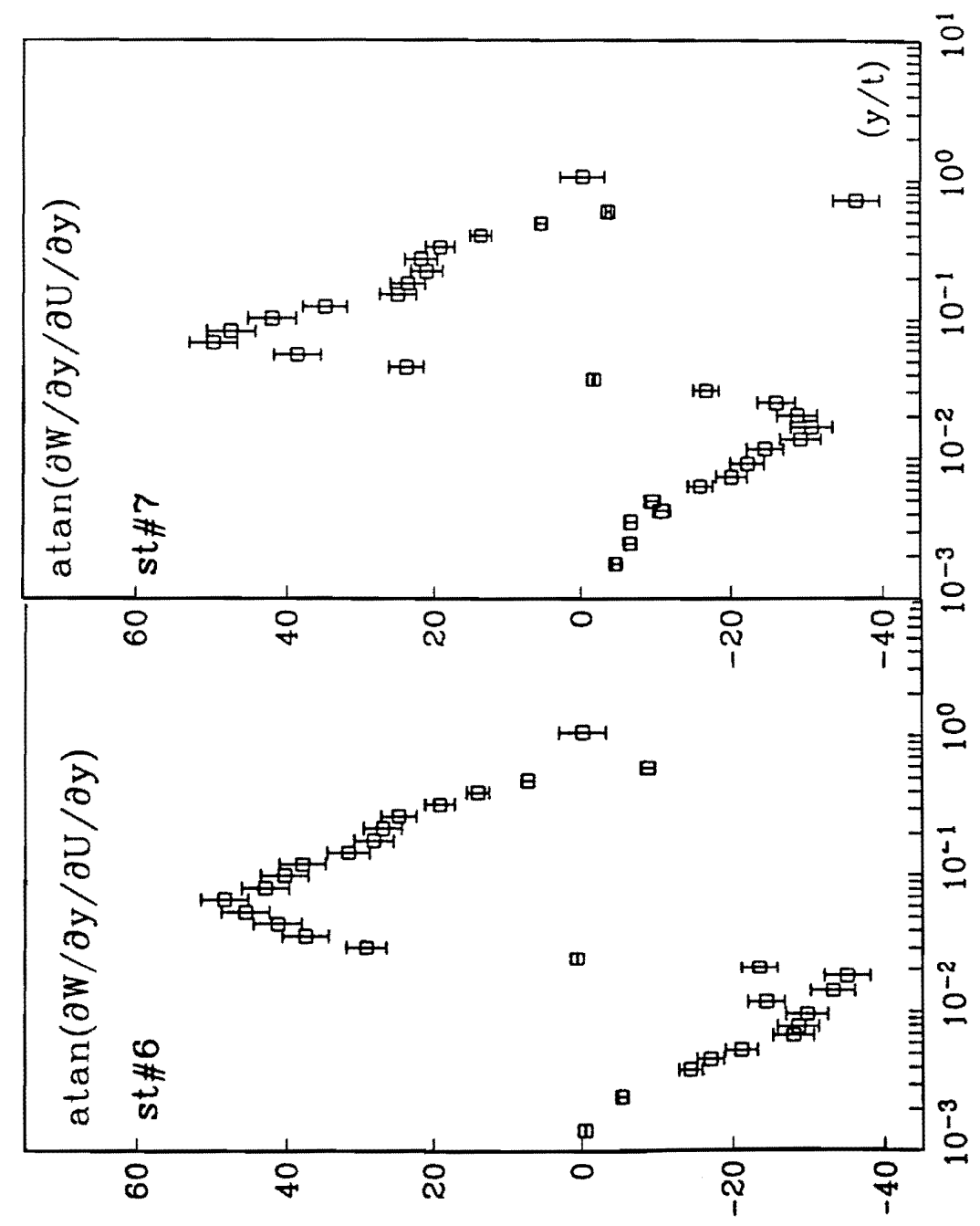

Figure 69c. Flow gradient angle computed using LDV data in free-stream coordinates.: Station numbers are shown in the figures. Bars denote the uncertainty bands. 


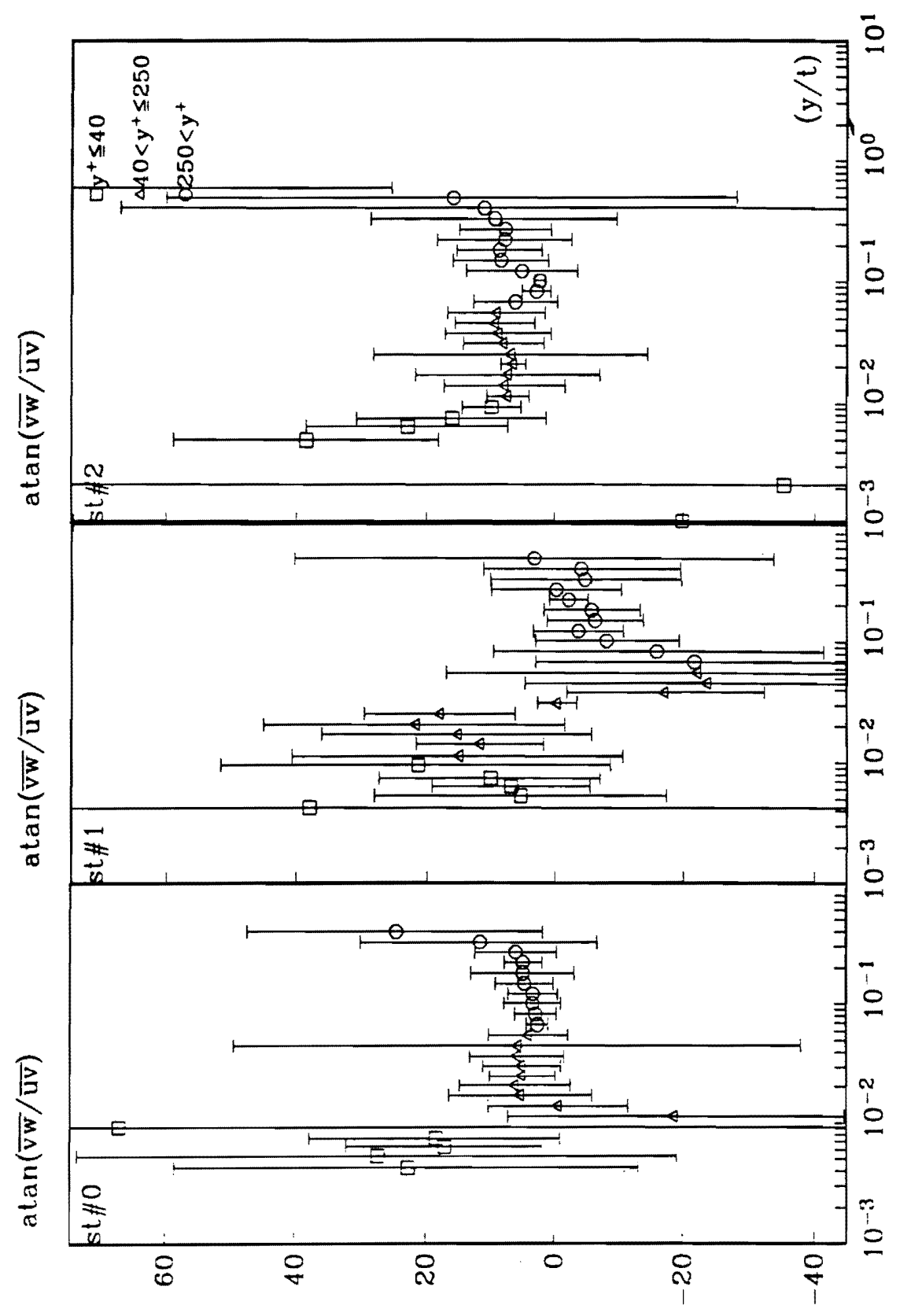

Figure 70a. Shear stress angle computed using LDV data in free-stream coordinates.: Station numbers are shown in the figures. Bars denote the uncertainty bands. 


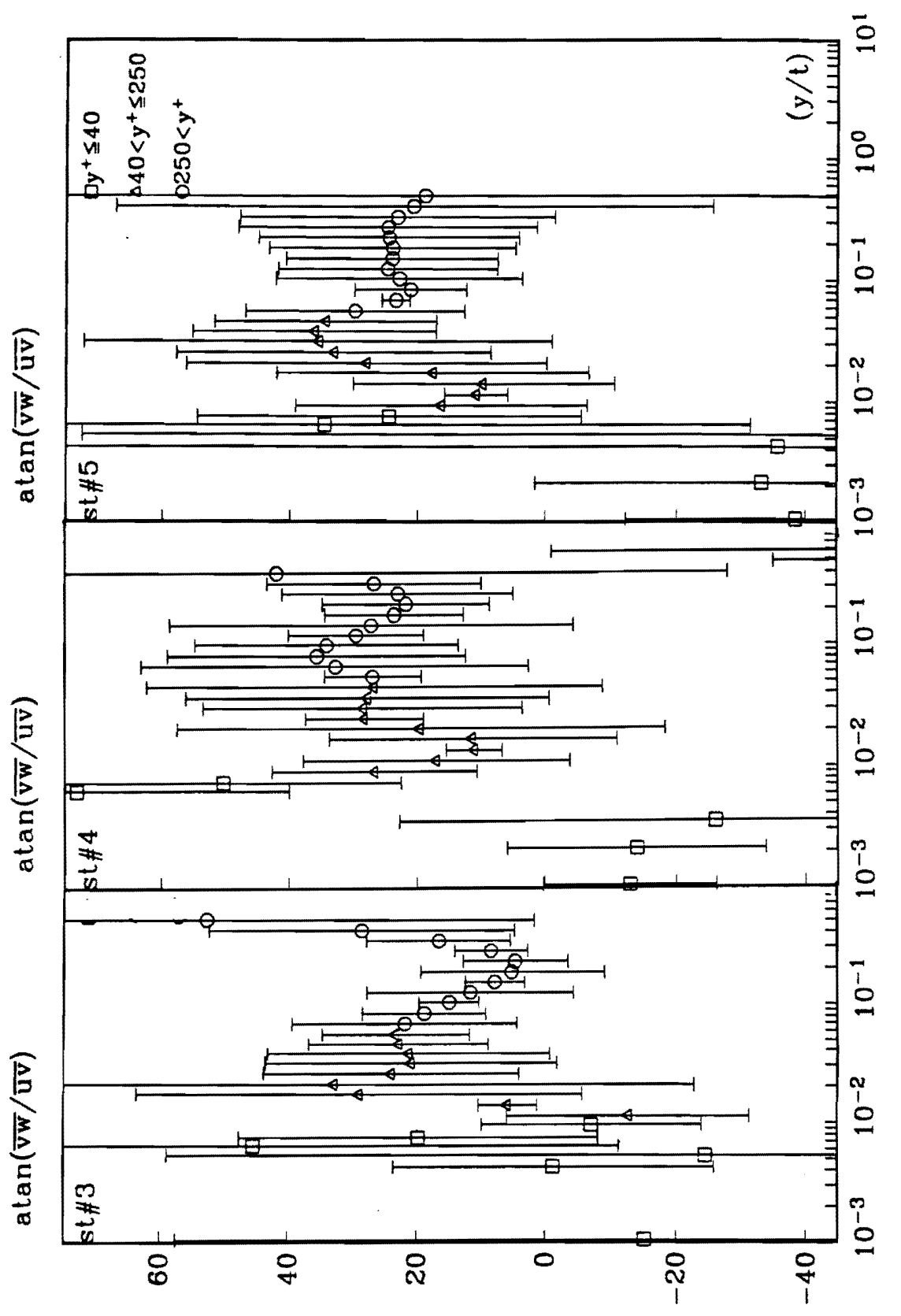

Figure 70b. Shear stress angle computed using LDV data in free-stream coordinates.: Station numbers are shown in the figures. Bars denote the uncertainty bands. 


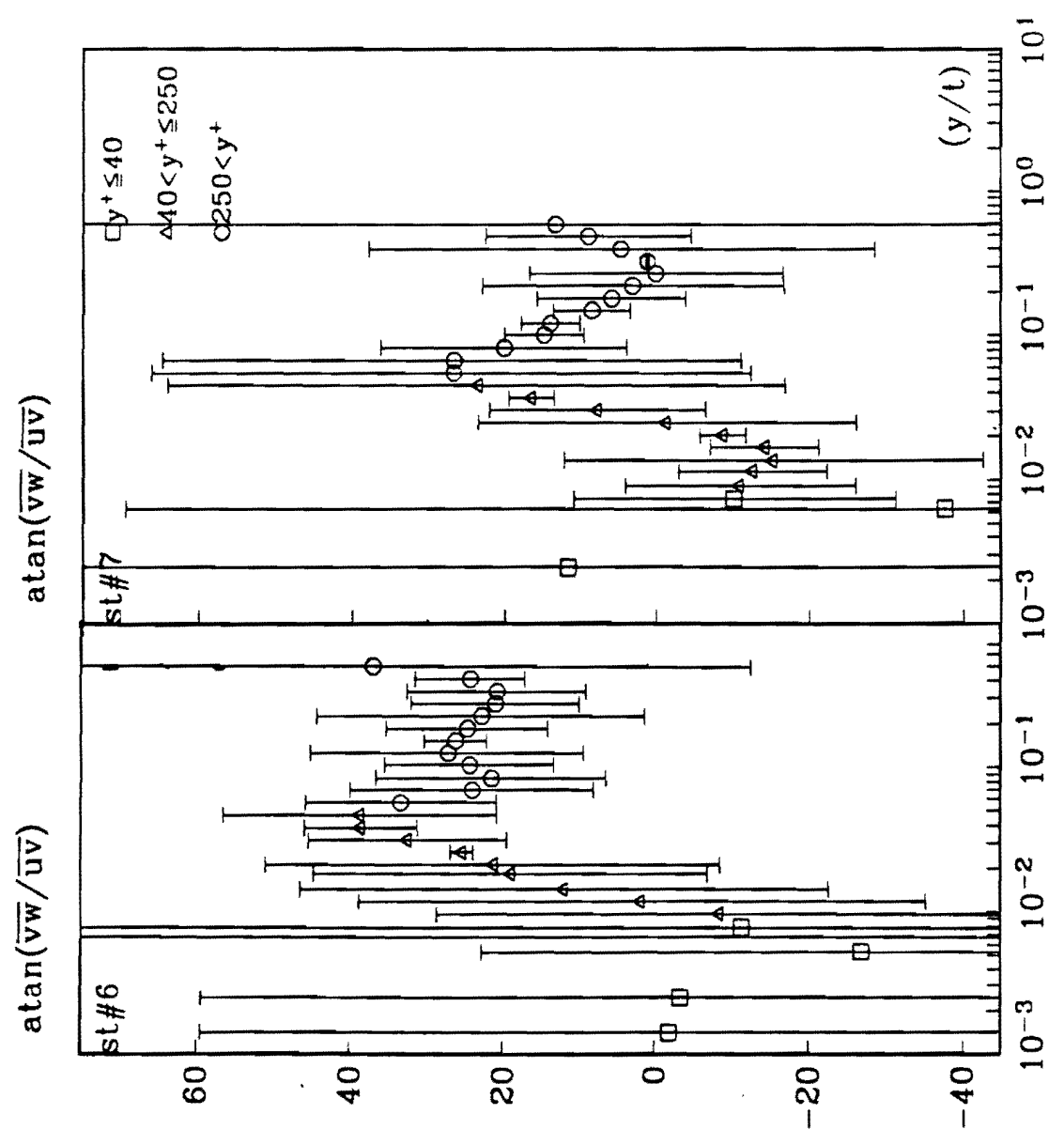

Figure 70c. Shear stress angle computed using LDV data in free-stream coordinates.: Station numbers are shown in the figures. Bars denote the uncertainty bands. 


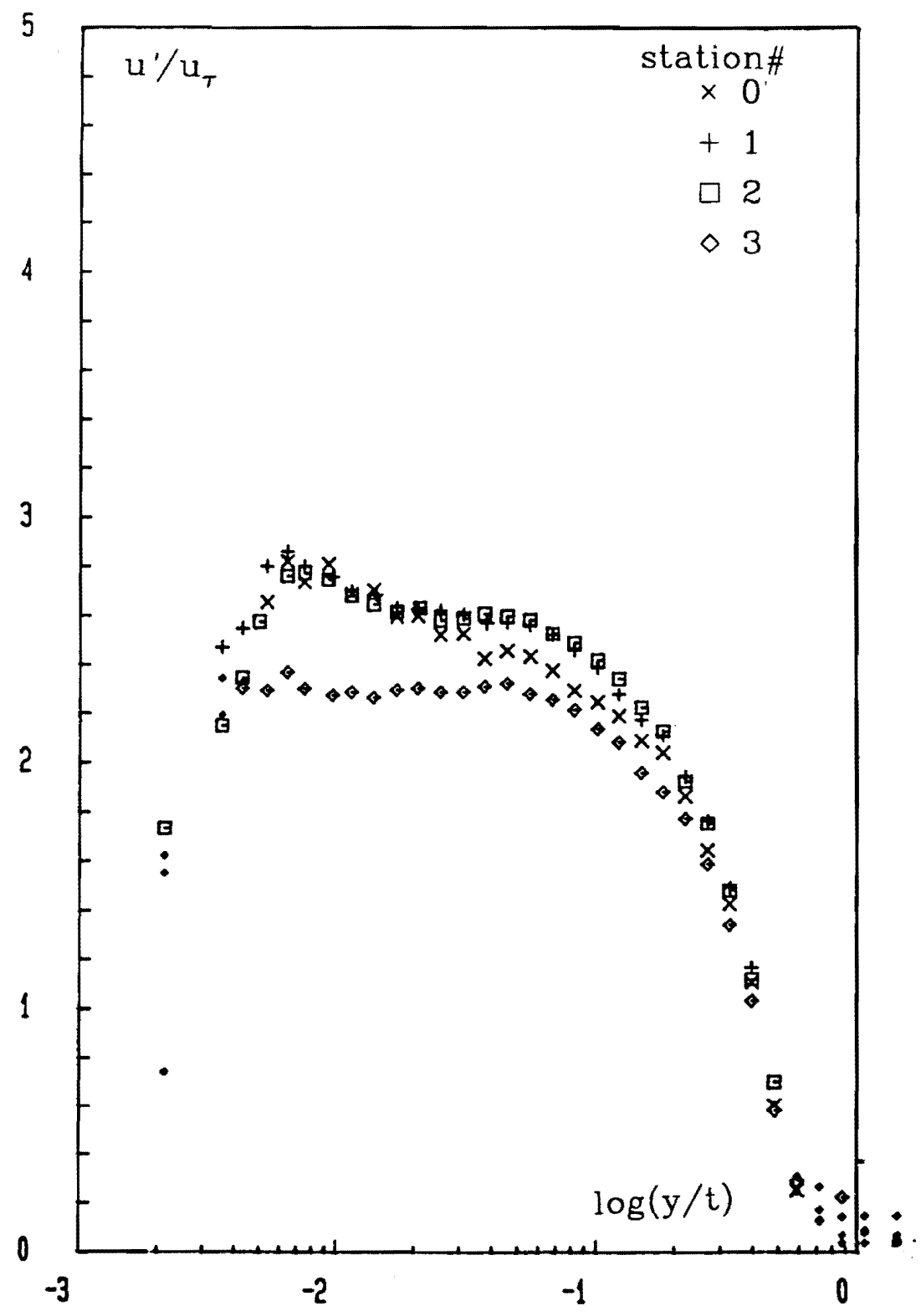

Figure 71a. u', fluctuating velocity component of LDV data in free-stream coordinates normalized with the skin friction velocity.: Station numbers are shown in the figure. 


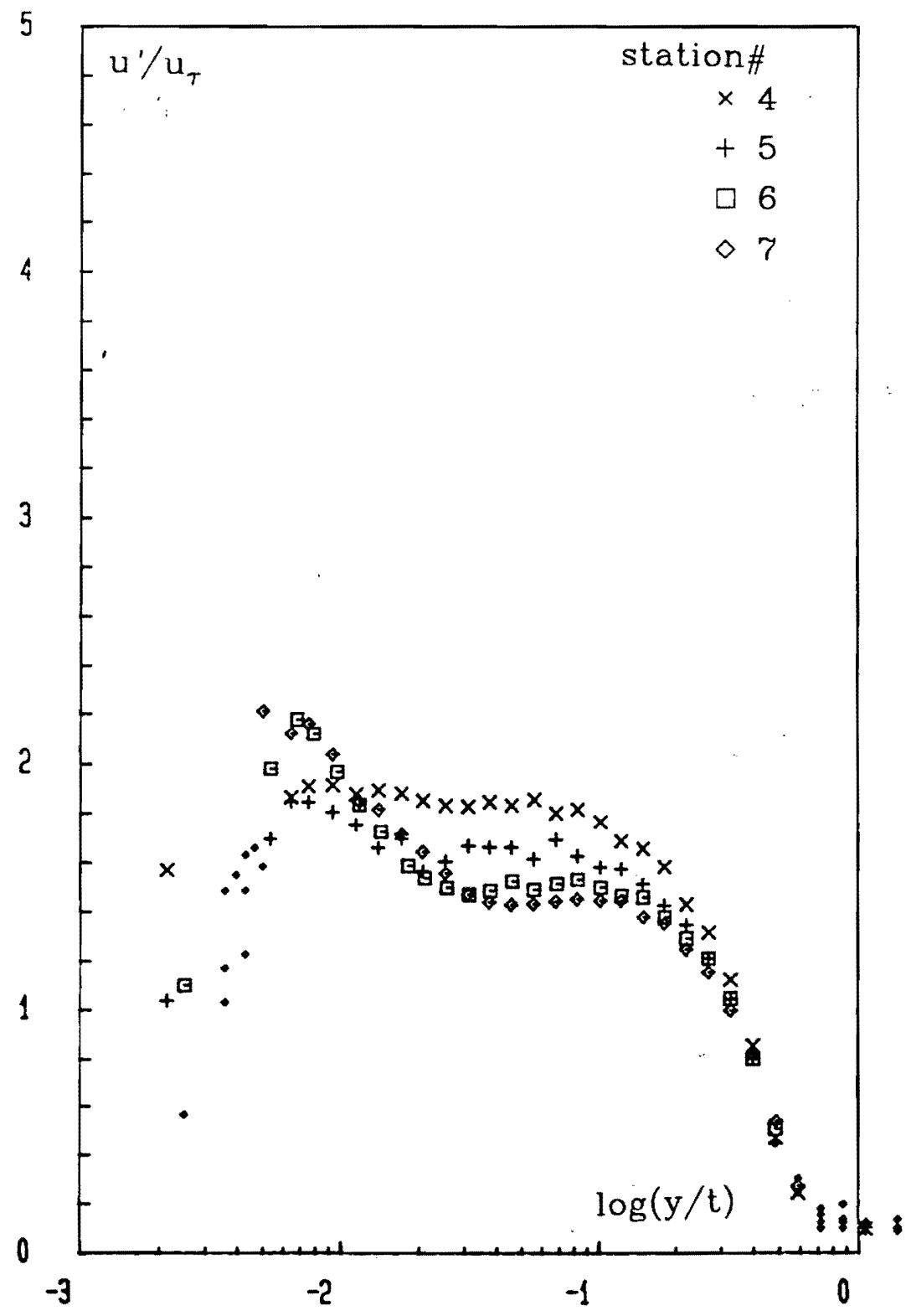

Figure 71b. $\mathrm{u}^{\prime}$, fluctuating velocity component of LDV data in free-stream coordinates normalized with the skin friction velocity.: Station numbers are shown in the figure. 


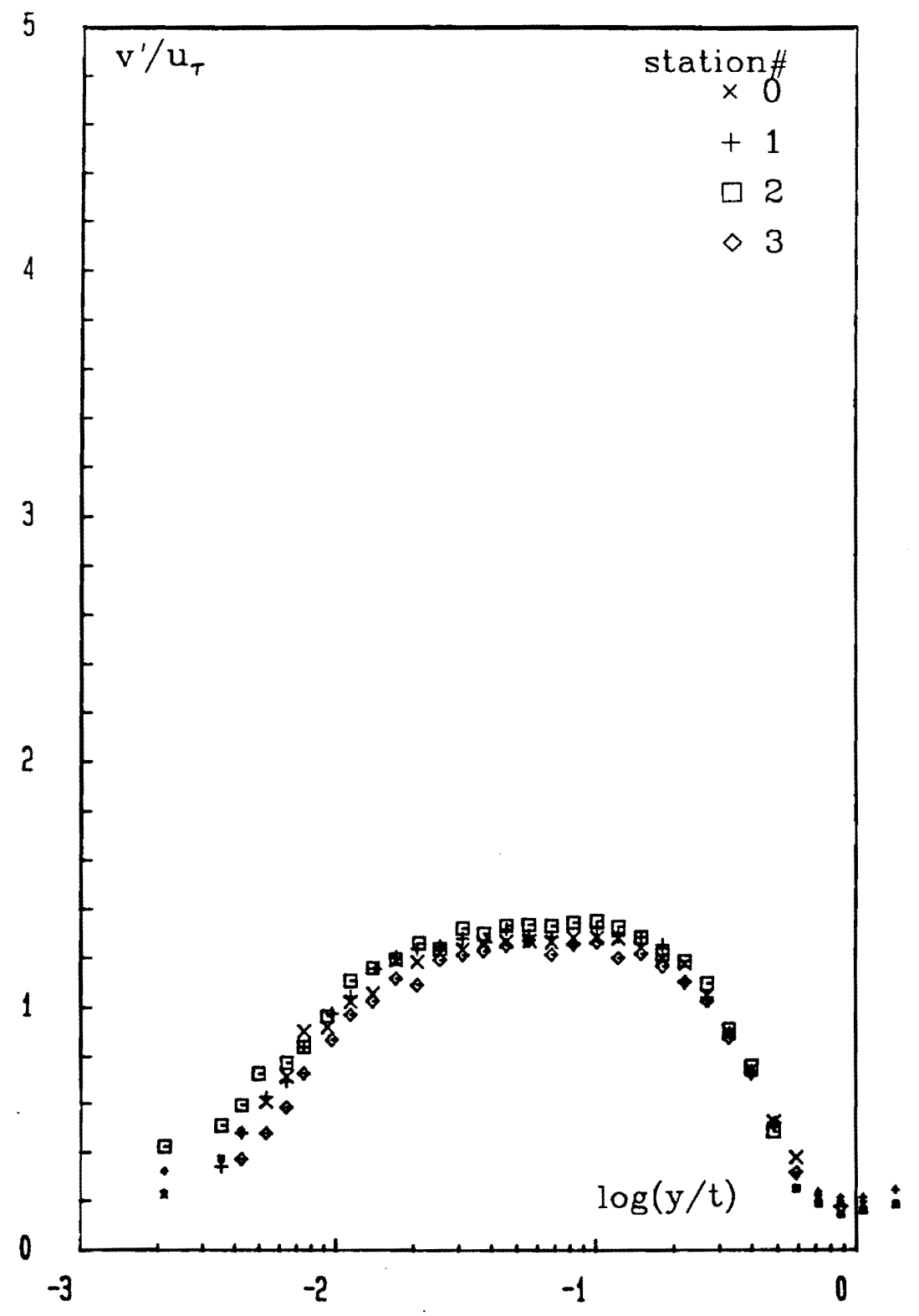

Figure 72a. $\quad \mathbf{v}^{\prime}$, fluctuating velocity component of LDV data in free-stream coordinates normalized with the skin friction velocity.: Station numbers are shown in the figure. 


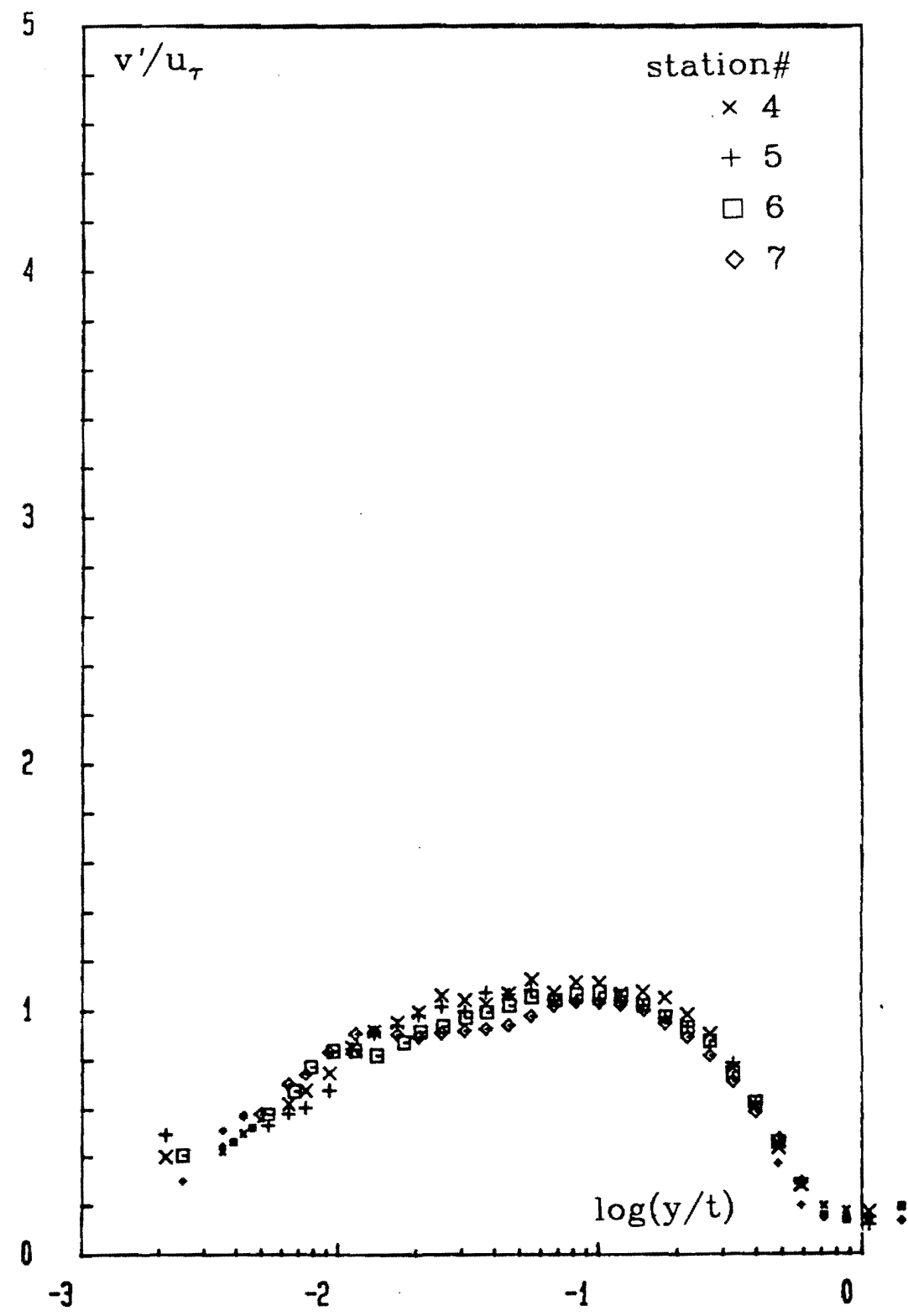

Figure 72b. $\quad v^{\prime}$, fluctuating velocity component of LDV data in free-stream coordinates normalized with the skin friction velocity.: Station numbers are shown in the figure. 


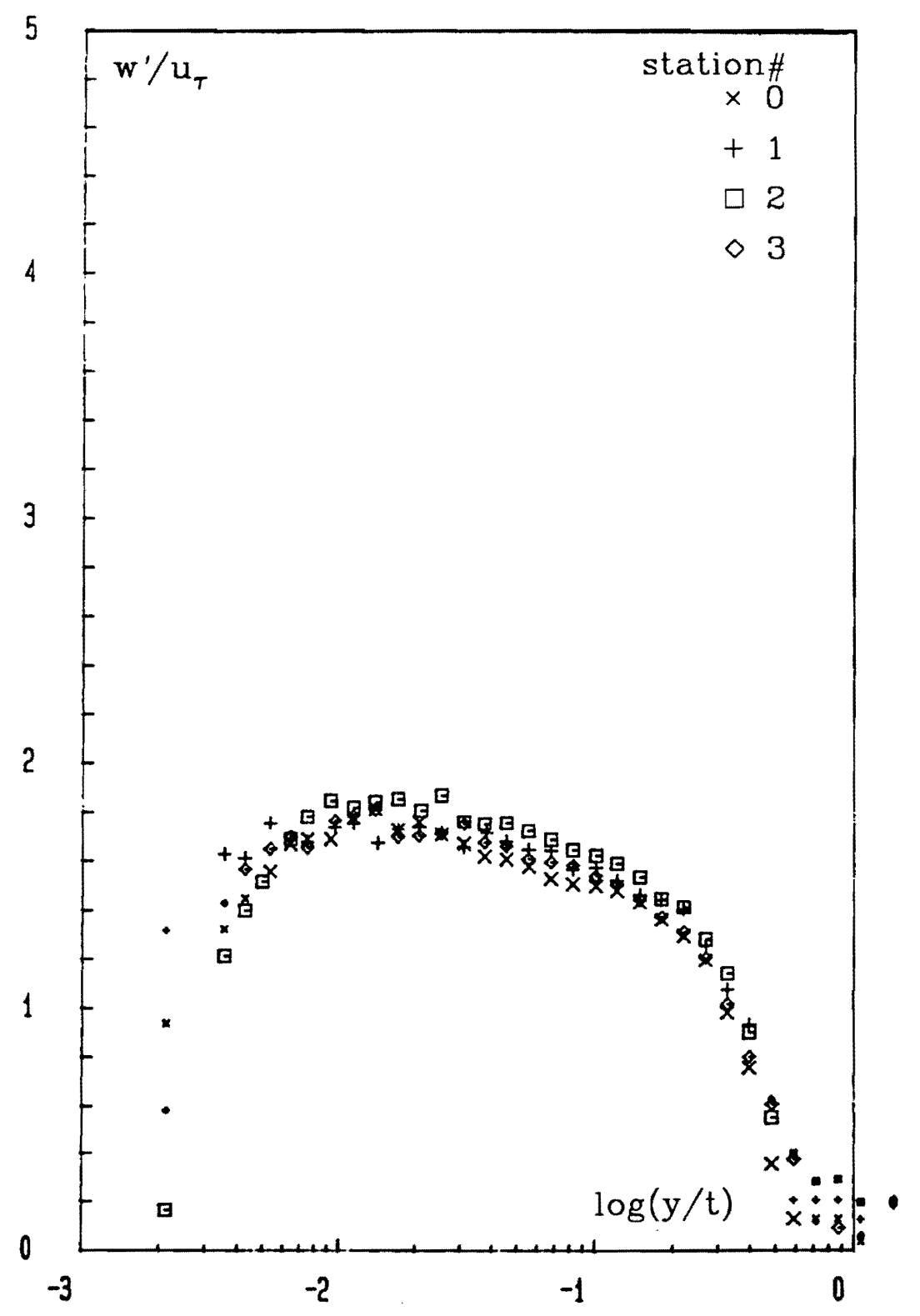

Figure 73a. $w^{\prime}$, fluctuating velocity component of LDV data in free-stream coordinates normalized with the skin friction velocity.: Station numbers are shown in the figure. 


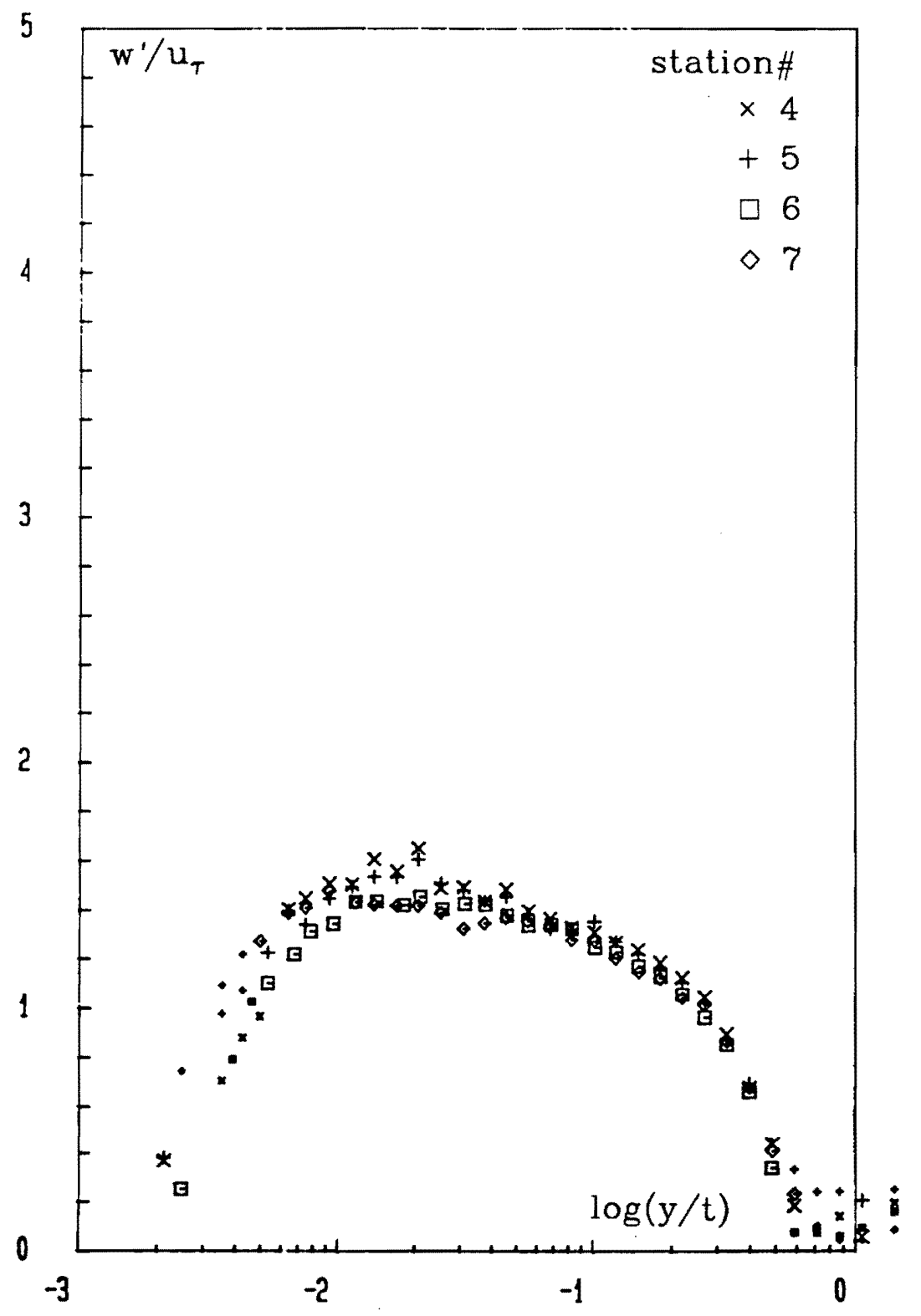

Figure 73b. $w^{\prime}$, fluctuating velocity component of LDV data in free-stream coordinates normalized with the skin friction velocity.: Station numbers are shown in the figure. 


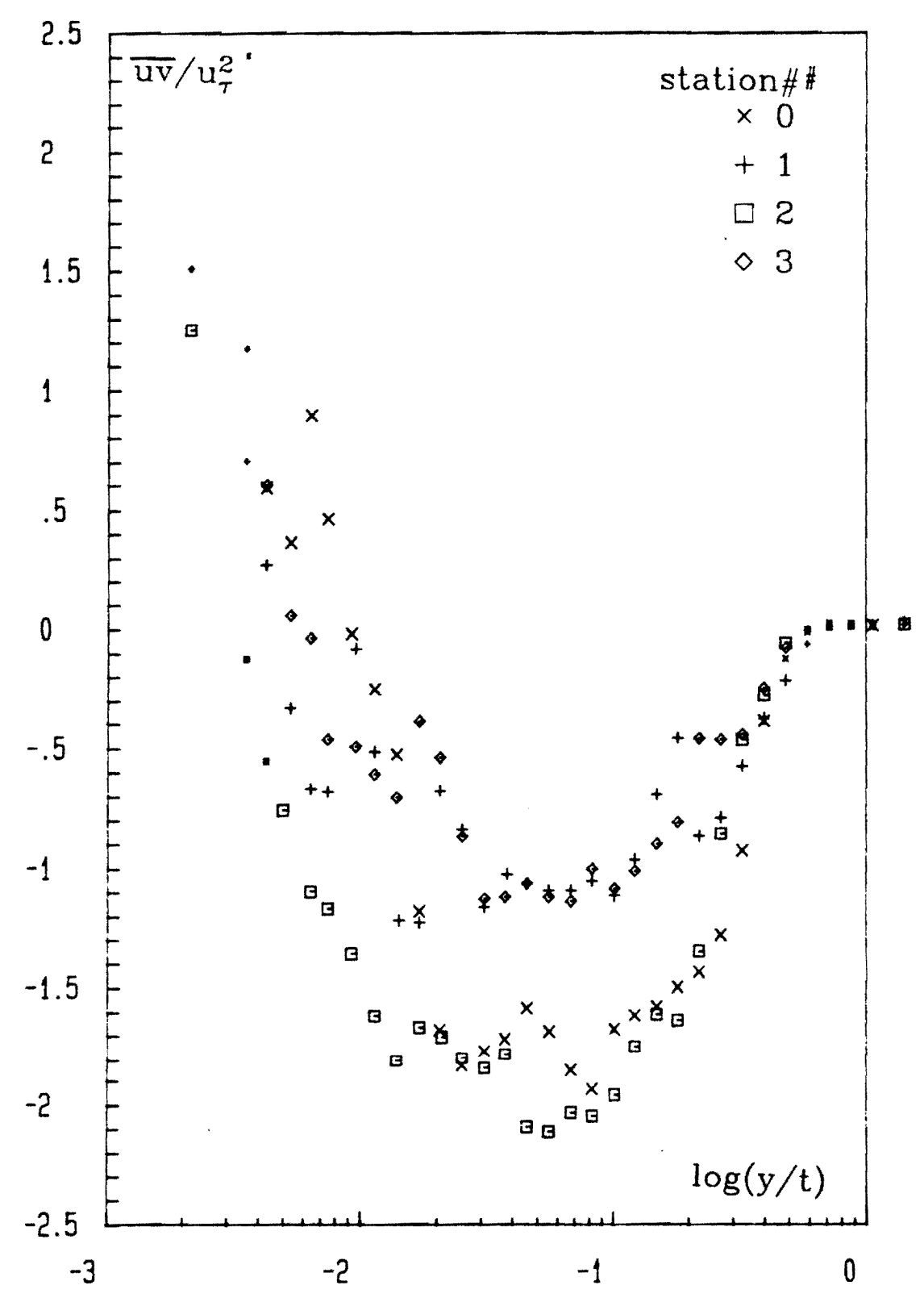
Figure $74 a .-\overline{u v}$, shear stress of LDV data in free-stream coordinates normalized with the
square of the skin friction velocity.: Station numbers are shown in the figure. 


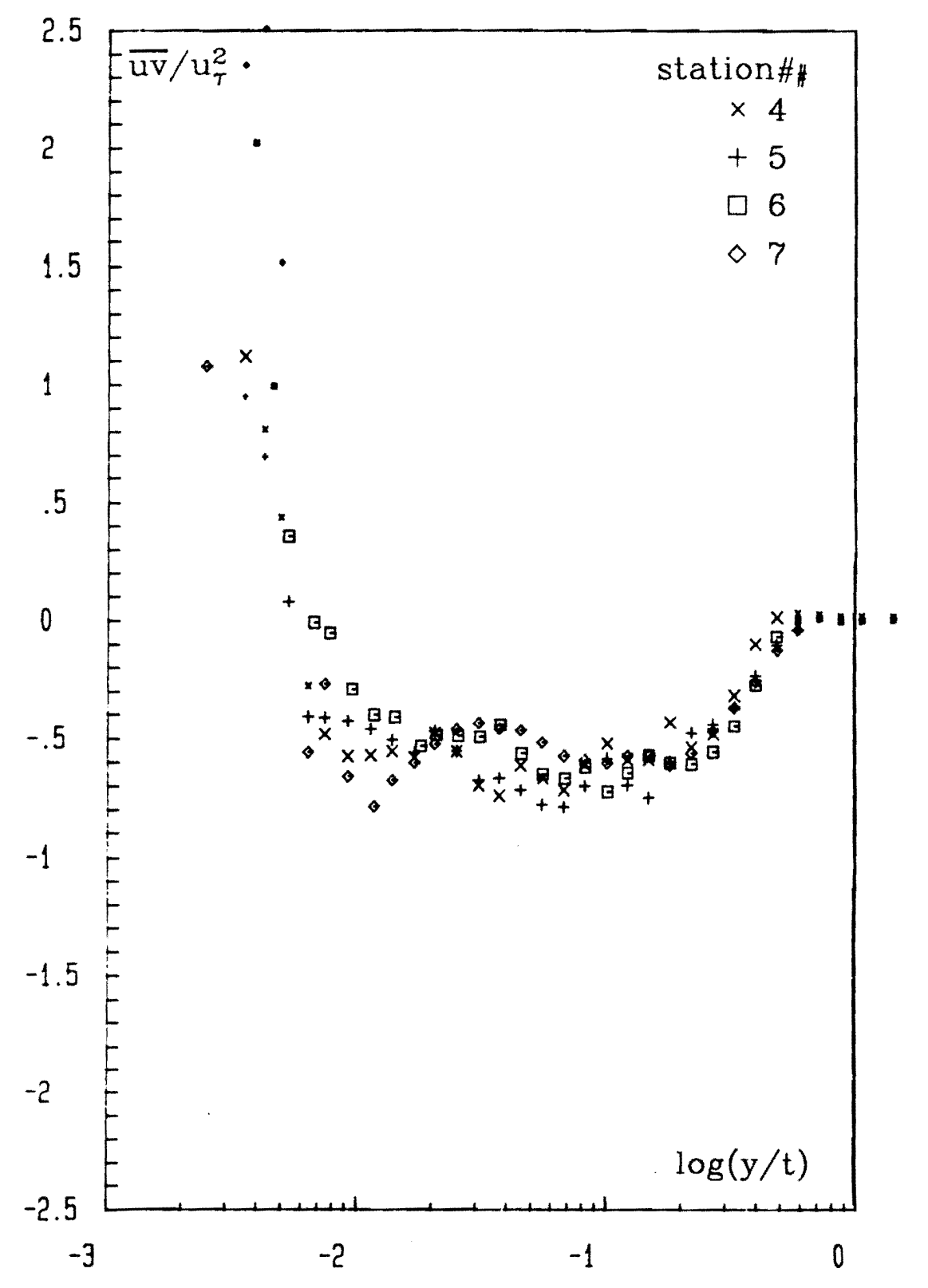

Figure $74 \mathrm{~b} .-\overline{\mathrm{uv}}$, shear stress of LDV data in free-stream coordinates normalized with the square of the skin friction velocity.: Station numbers are shown in the figure. 


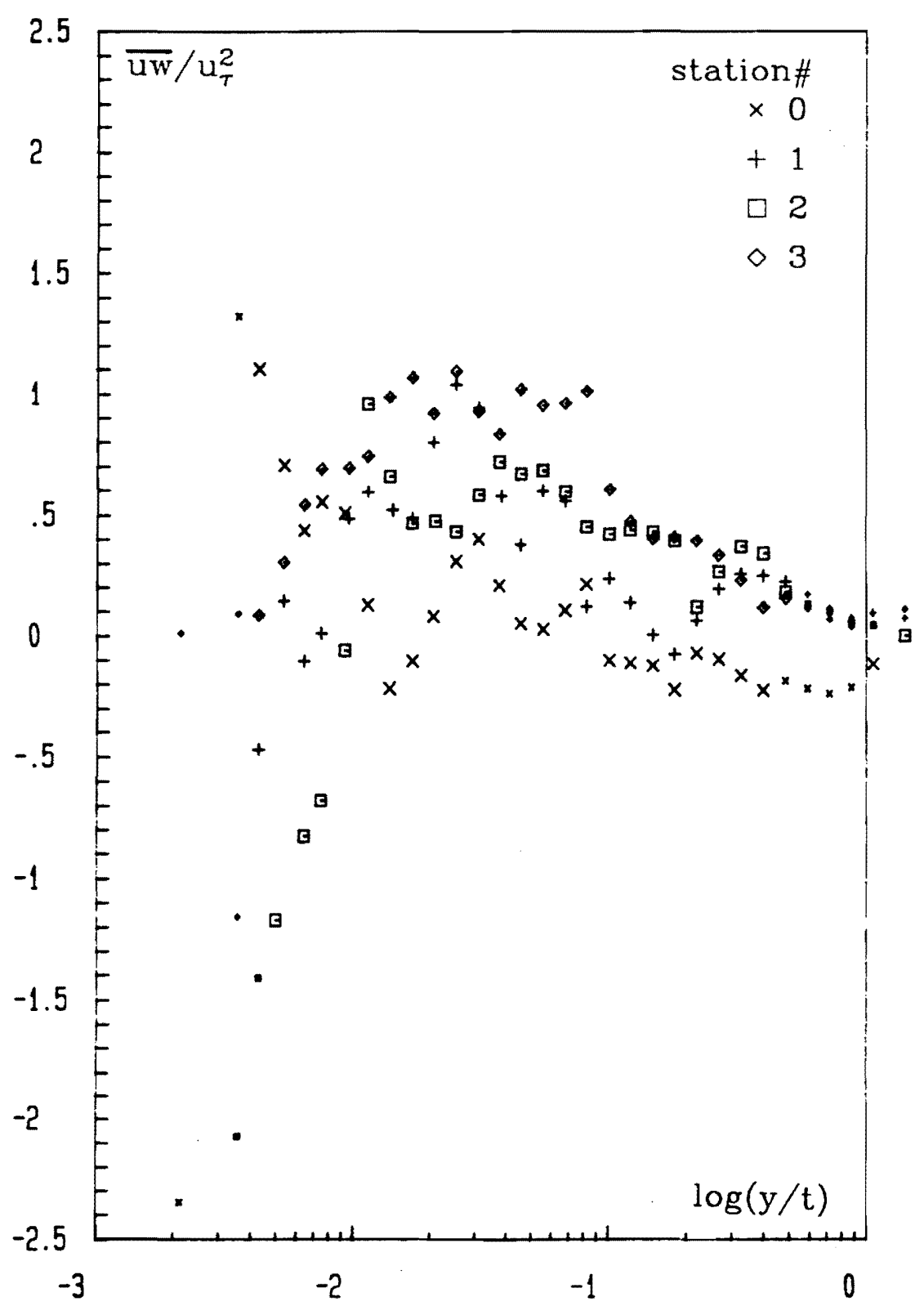

Figure 75a. - $\overline{\mathrm{uw}}$, shear stress of LDV data in free-stream coordinates normalized with the square of the skin friction velocity.: Station numbers are shown in the figure. 


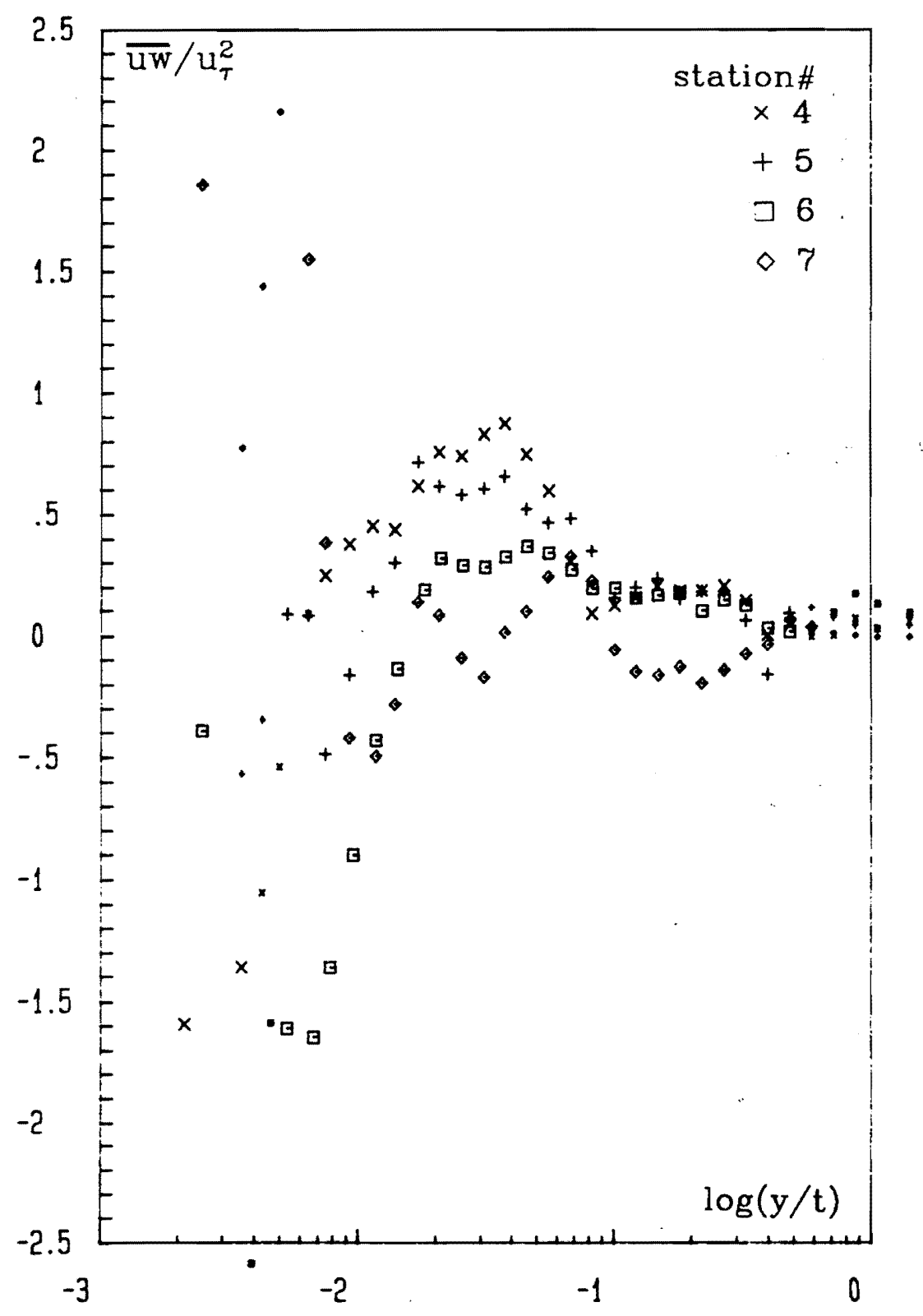

Figure 75b. $-\overline{u w}$, shear stress of LDV data in free-stream coordinates normalized with the square of the skin friction velocity.: Station numbers are shown in the figure. 


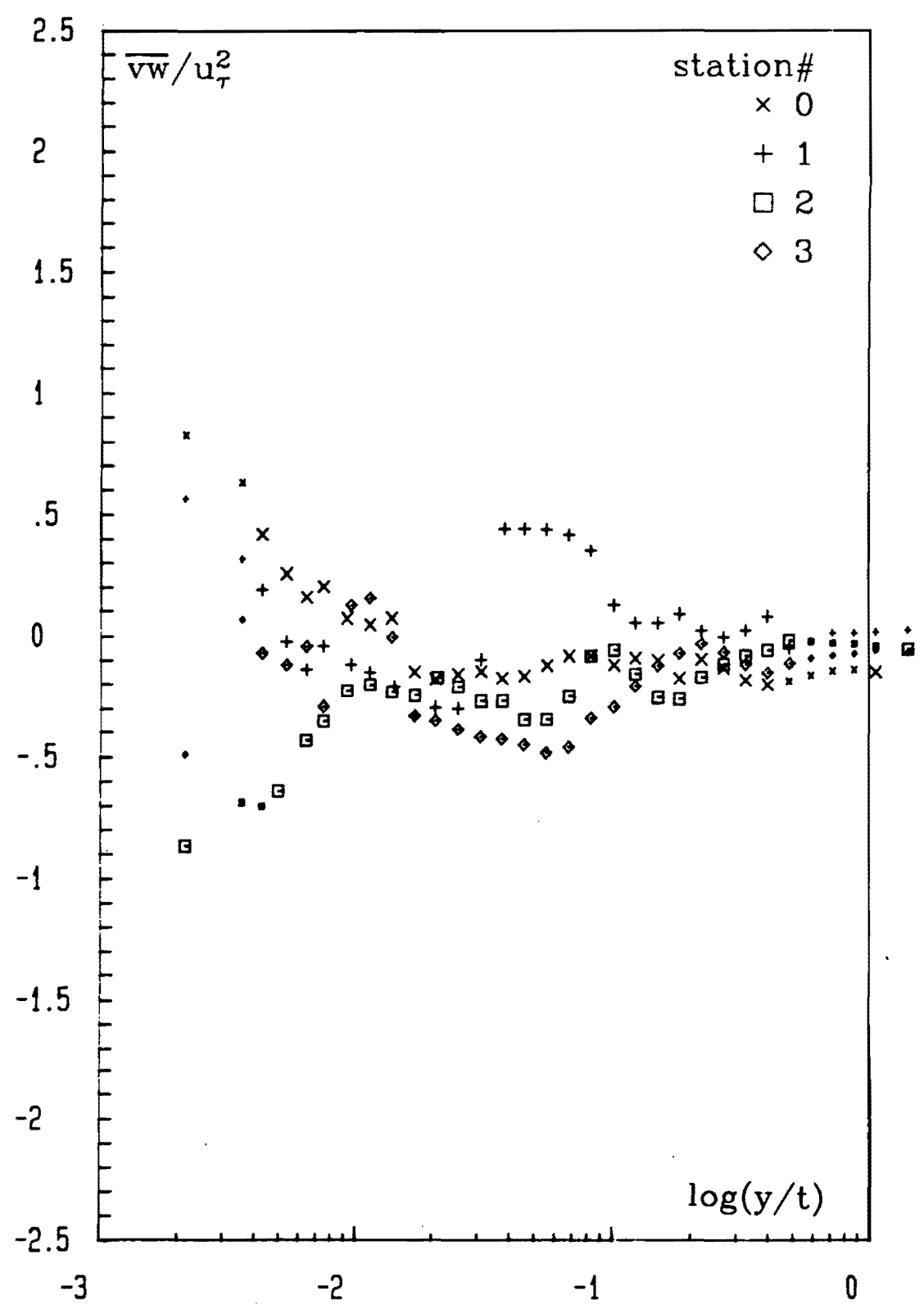

Figure 76a. $-\overline{\mathrm{ww}}$, shear stress of LDV data in free-stream coordinates normalized with the square of the skin friction velocity.: Station numbers are shown in the figure. 


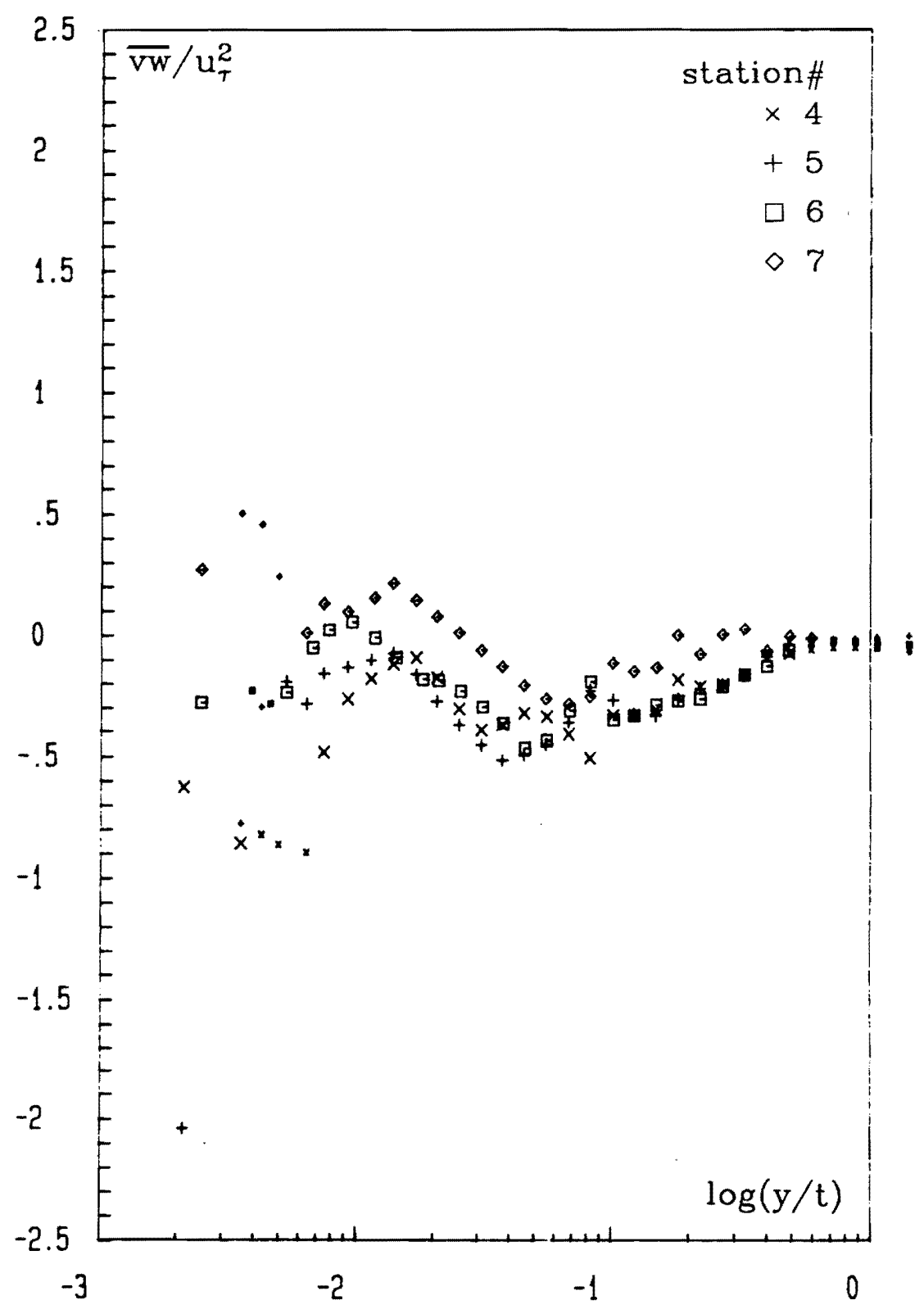

Figure 76b. $-\overline{v w}$, shear stress of LDV data in free-stream coordinates normalized with the square of the skin friction velocity.: Station numbers are shown in the figure. 


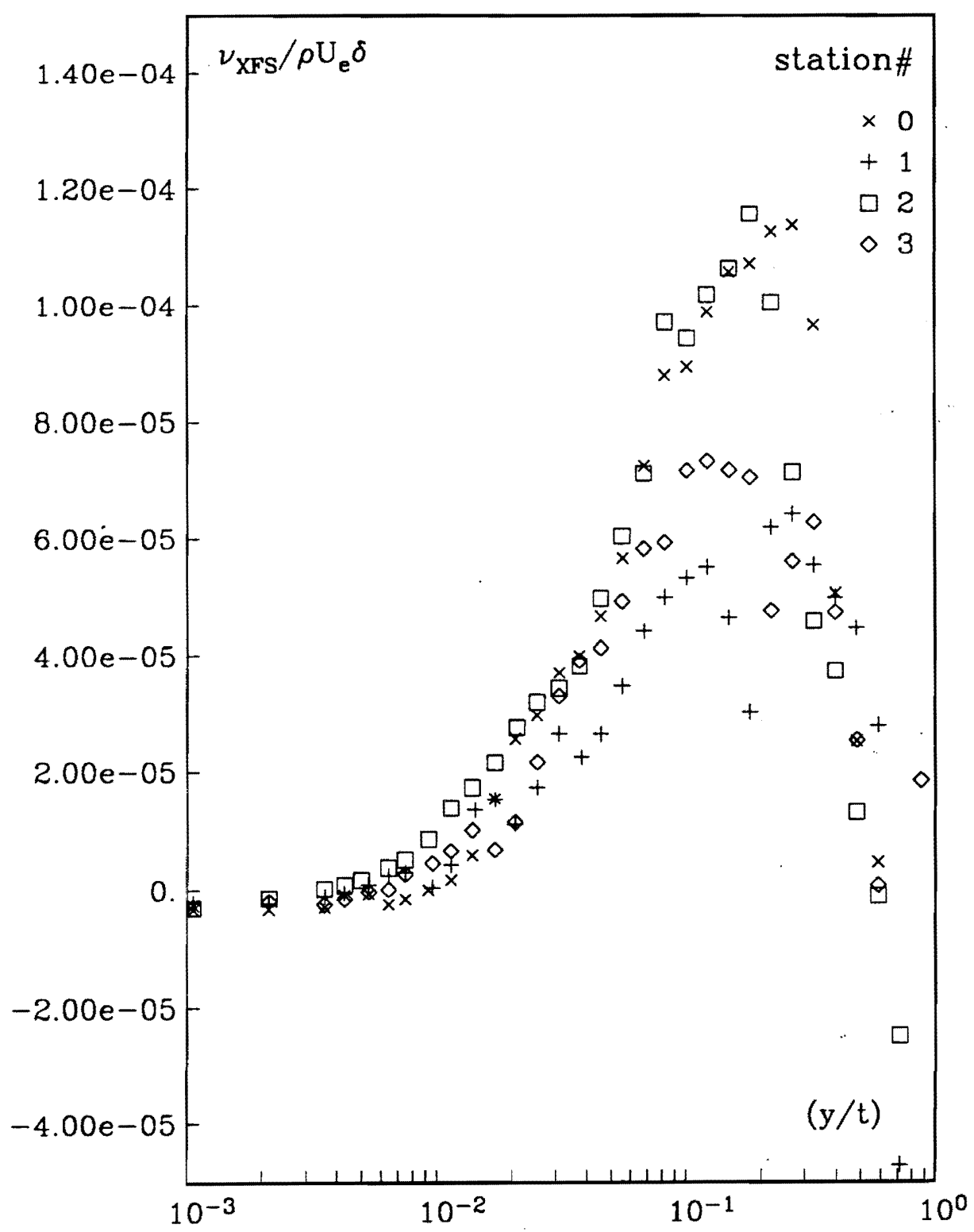

Figure 77a. $x$ eddy viscosity computed using the LDV data in free-stream coordinates.: Station numbers are shown in the figure. 


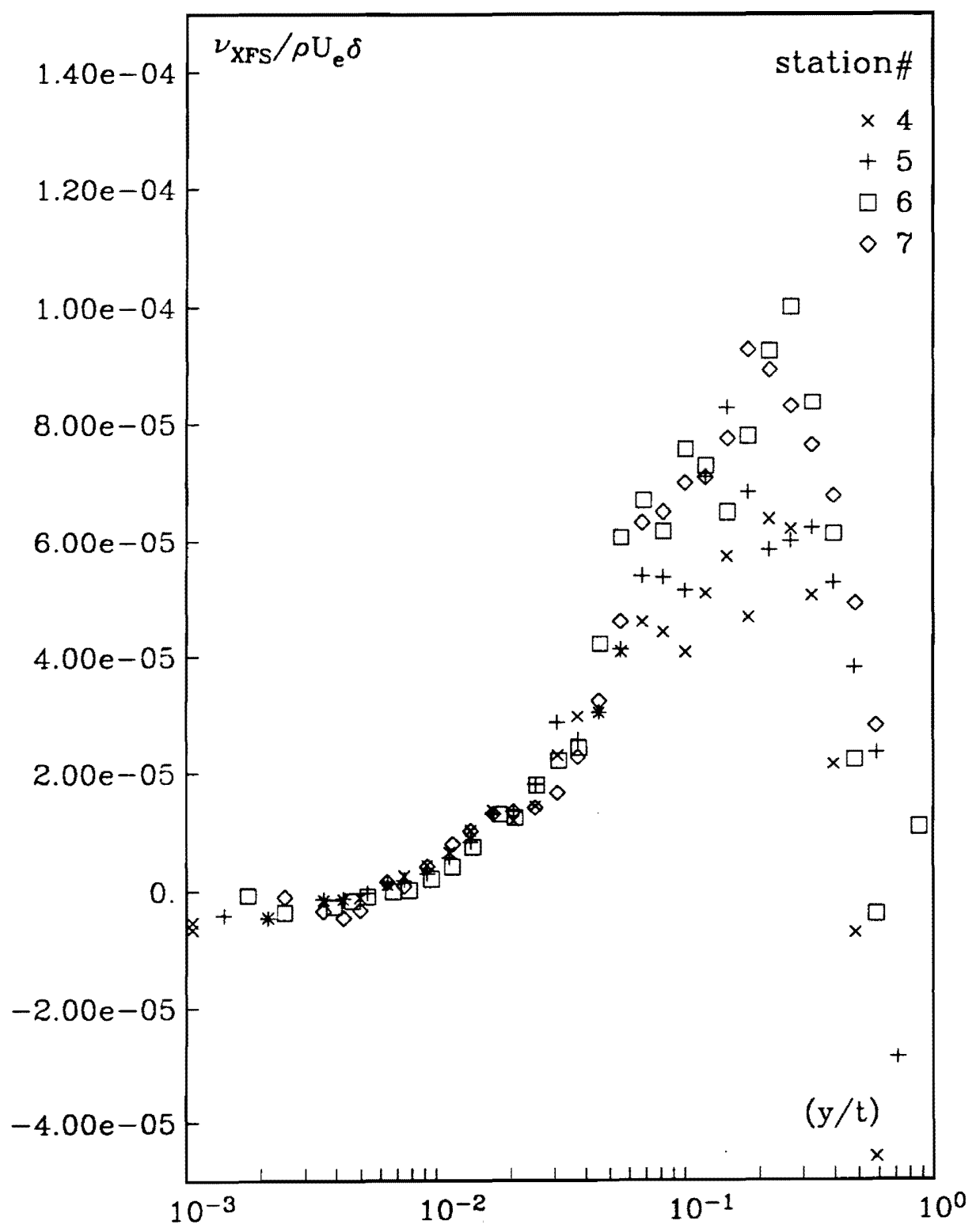

Figure 77b. $x$ eddy viscosity computed using the LDV data in free-stream coordinates.: Station numbers are shown in the figure. 


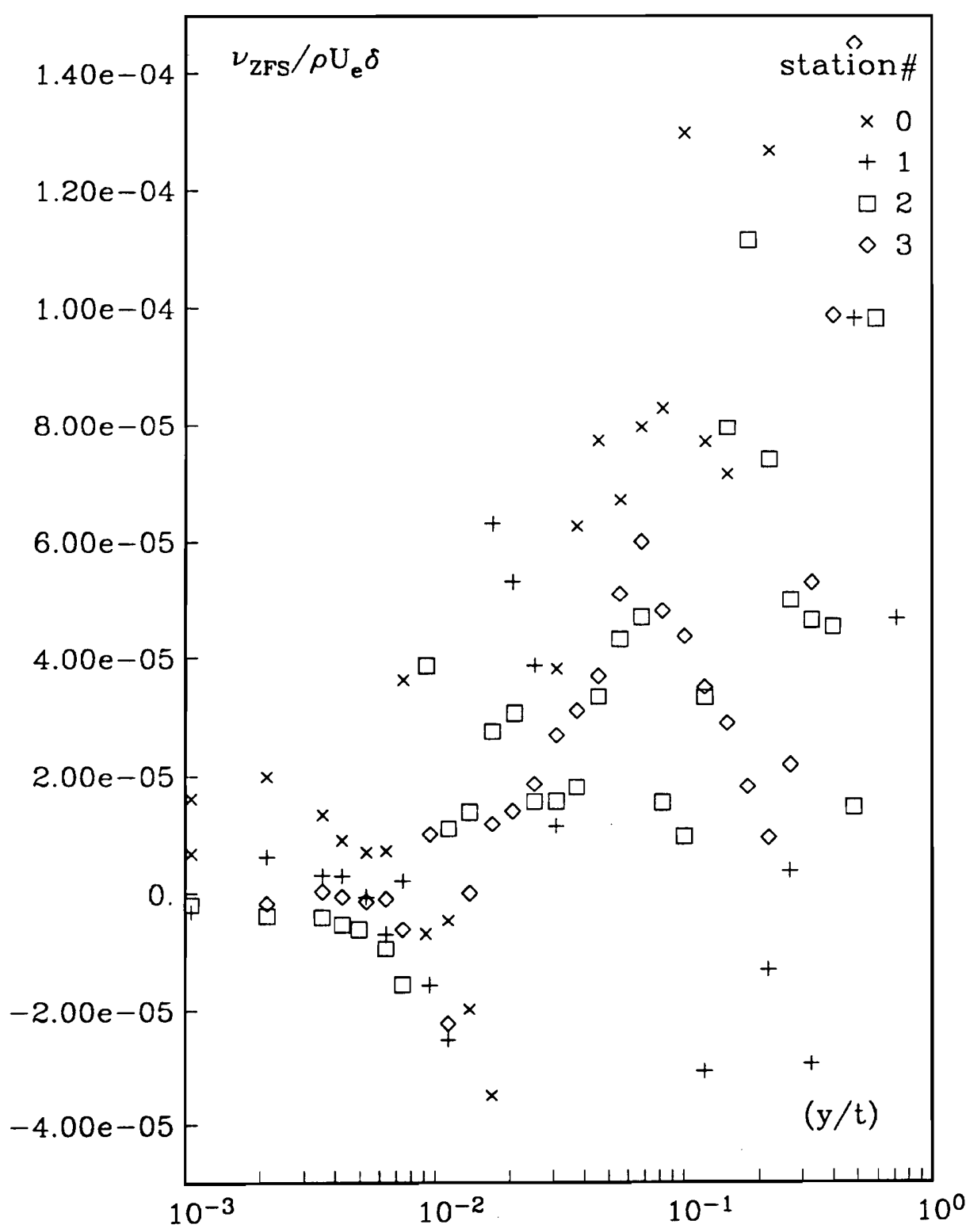

Figure 78a. $z$ eddy viscosity computed using the LDV data in free-stream coordinates.: Station numbers are shown in the figure. 


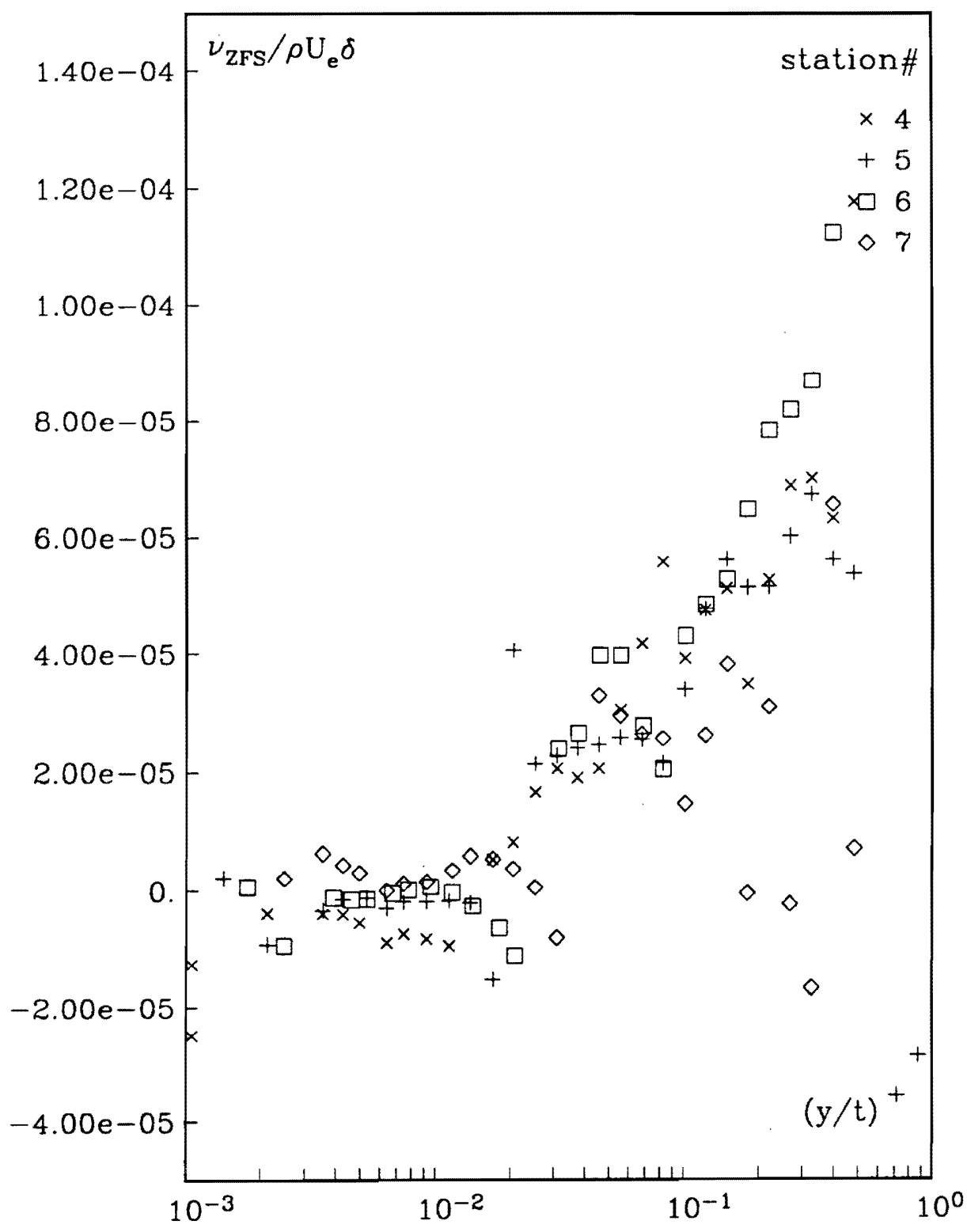

Figure 78b. $z$ eddy viscosity computed using the LDV data in free-stream coordinates.: Station numbers are shown in the figure. 


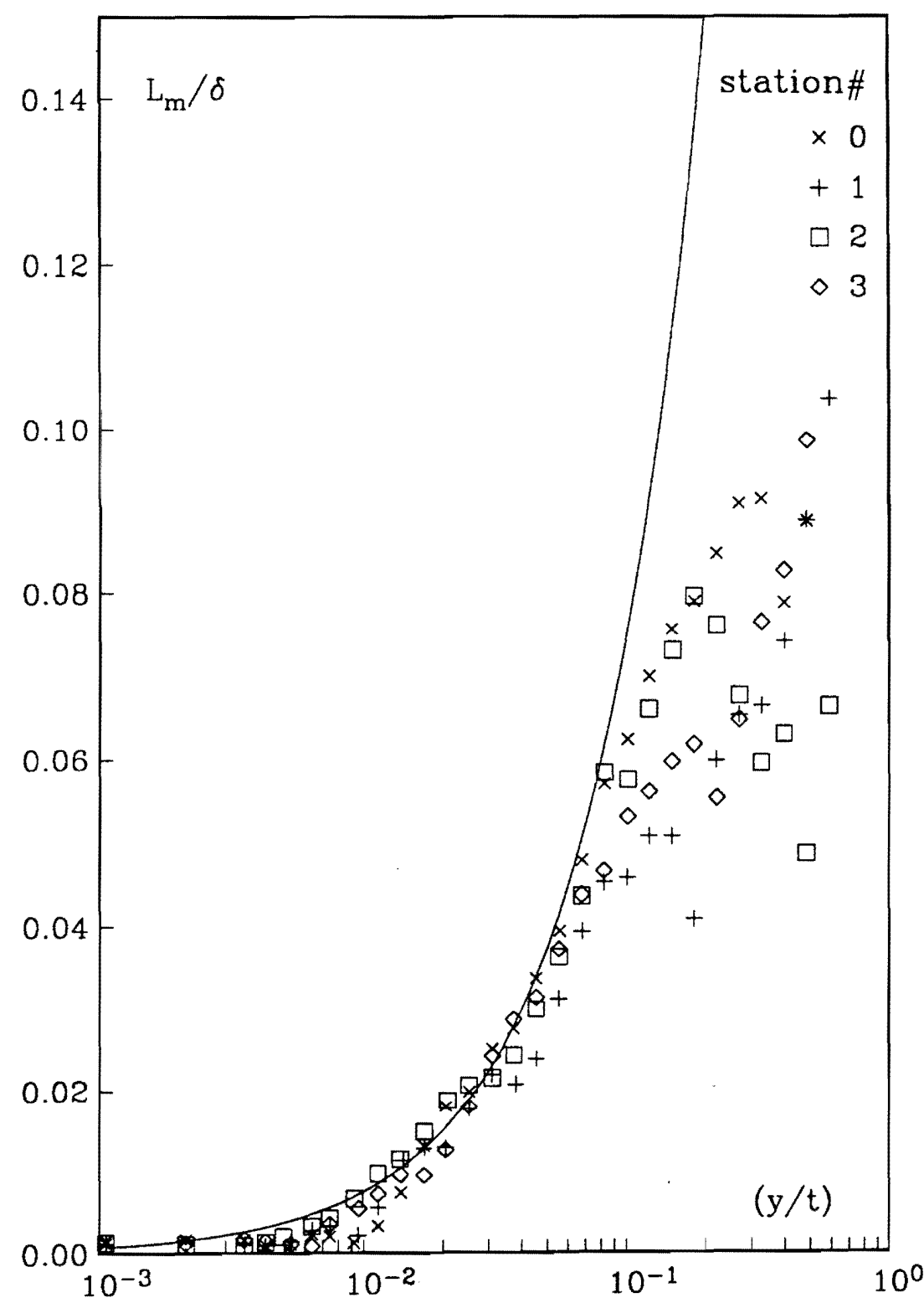

Figure 79a. Mixing length nondimensionalized with boundary layer thickness computed using LDV data.: Smooth curve denotes the $L_{m}=0.41 \mathrm{y} / \delta$ variation. Station numbers are shown in the figure. 


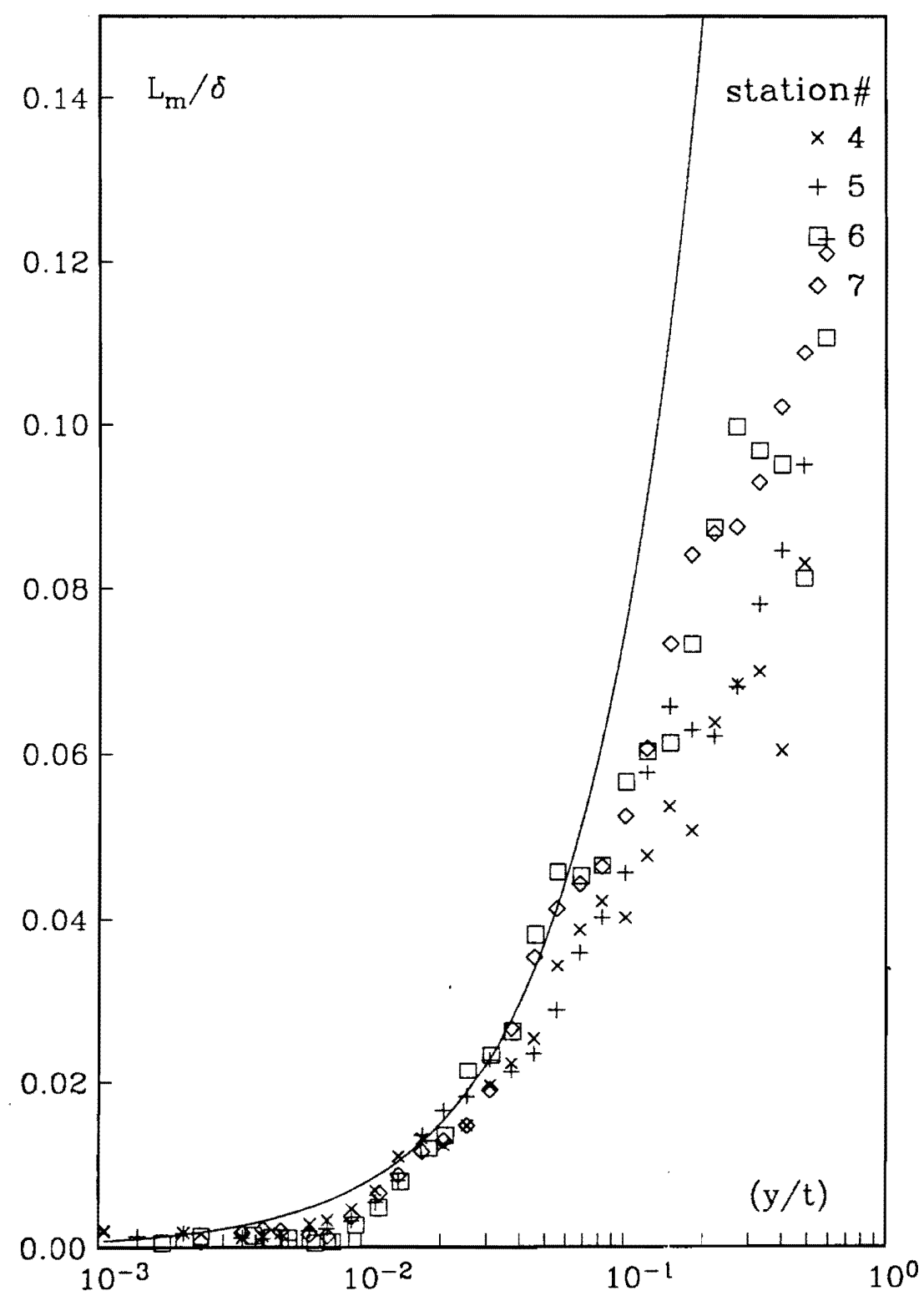

Figure 79b. Mixing length nondomensionalized with boundary layer thickness computed using LDV data.: Smooth curve denotes the $L_{m}=0.41 y / \delta$ variation. Station numbers are shown in the figure. 


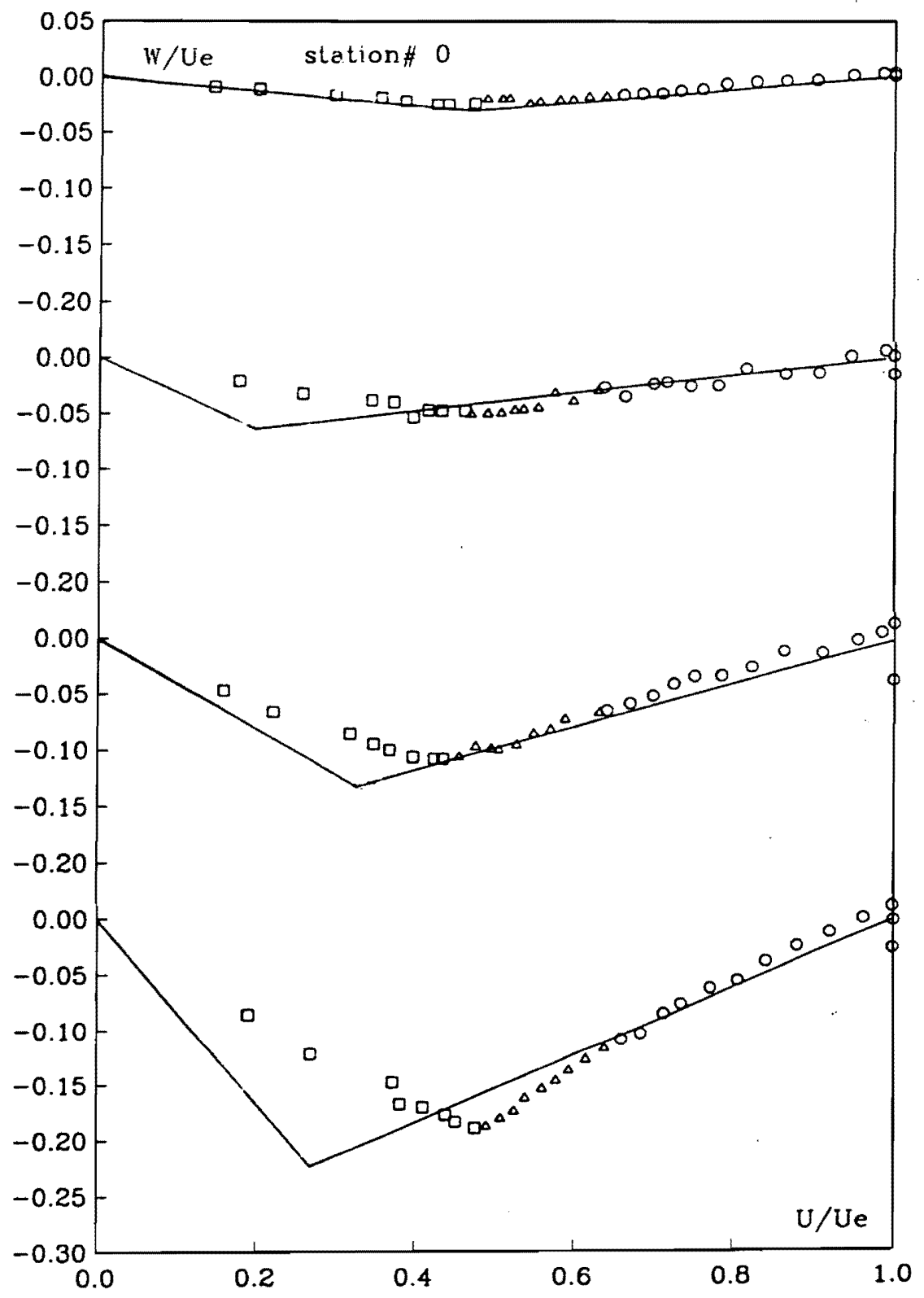

Figure $80 \mathrm{a}$. Johnston's polar plots of the present data.: The symbols show the data and the lines show the suggested relation. The sations are numbered from top to bottom starting with station 0 . 


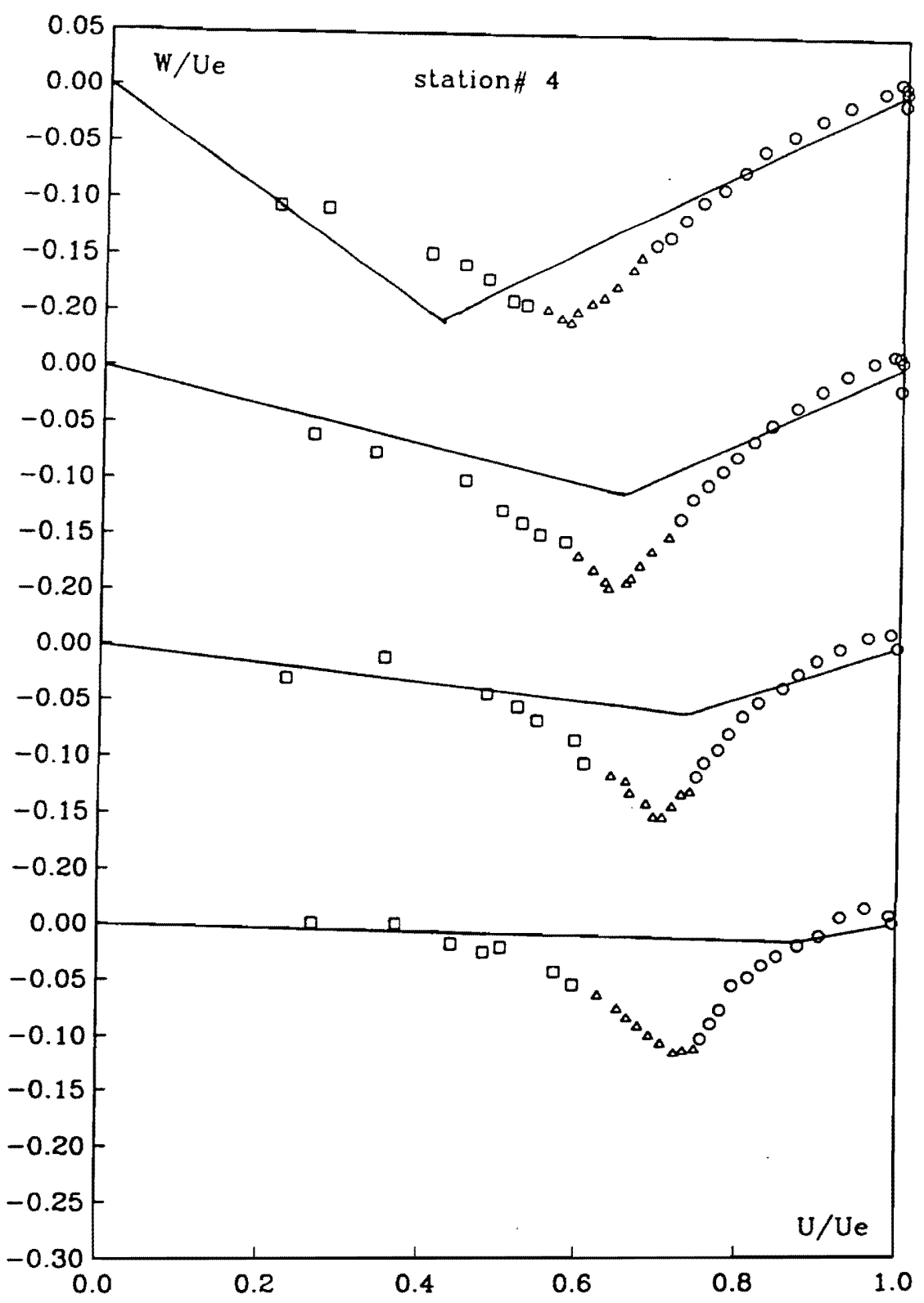

Figure 80b. Johnston's polar plots of the present data.: The symbols show the data and the lines show the suggested relation. The stations are numbered from top to bottom starting with station 4 . 


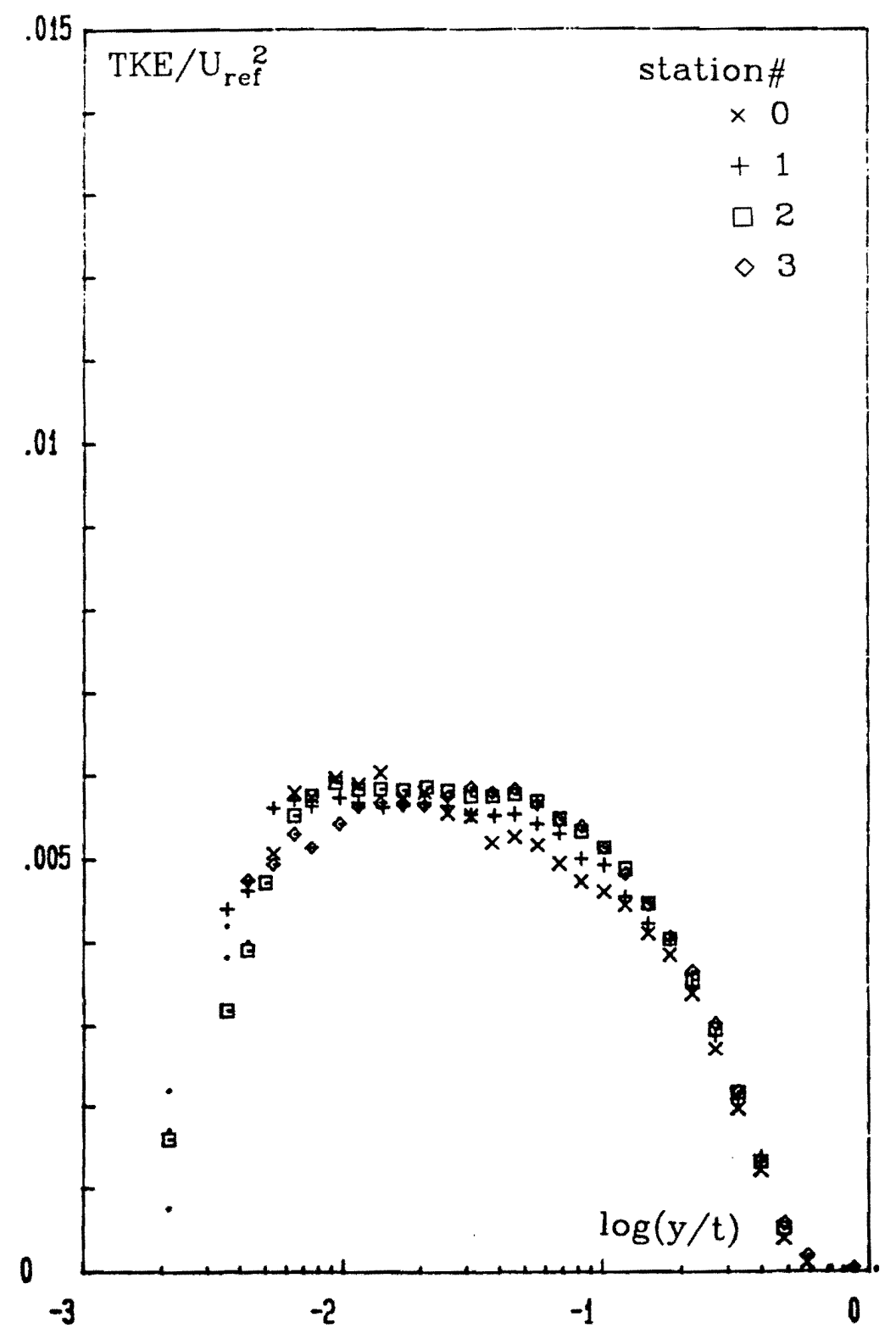

Figure 81a. Turbulent kinetic energy profiles obtained using LDV data.: Station numbers are shown in the figure. 


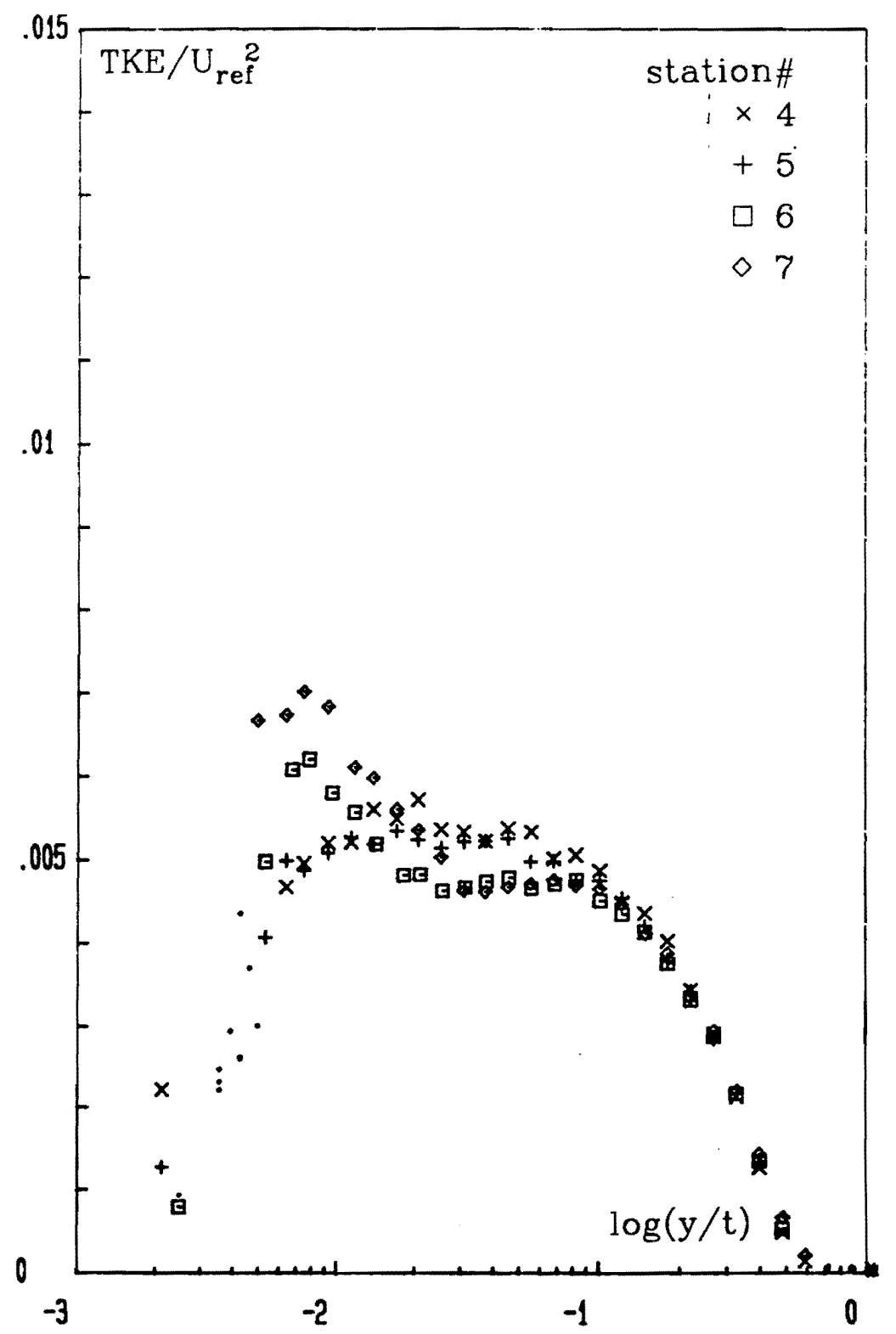

Figure $81 \mathrm{~b}$. Turbulent kinetic energy profiles obtained using LDV data.: Station numbers are shown in the figure. 


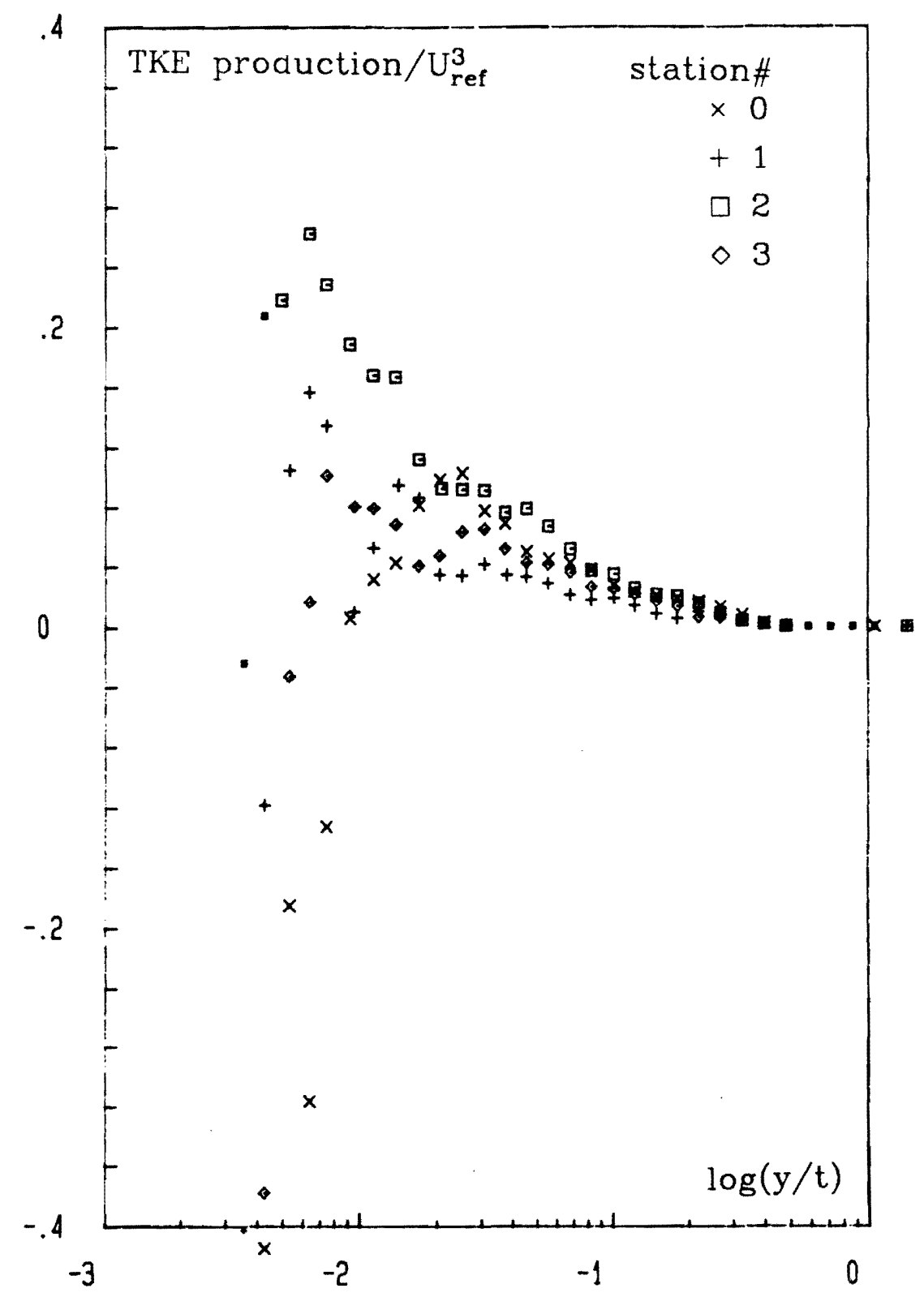

Figure 82a. Production of turbulent kinetic energy profiles obtained using LDV data in free-stream coordinates.: Station numbers are shown in the figure. 


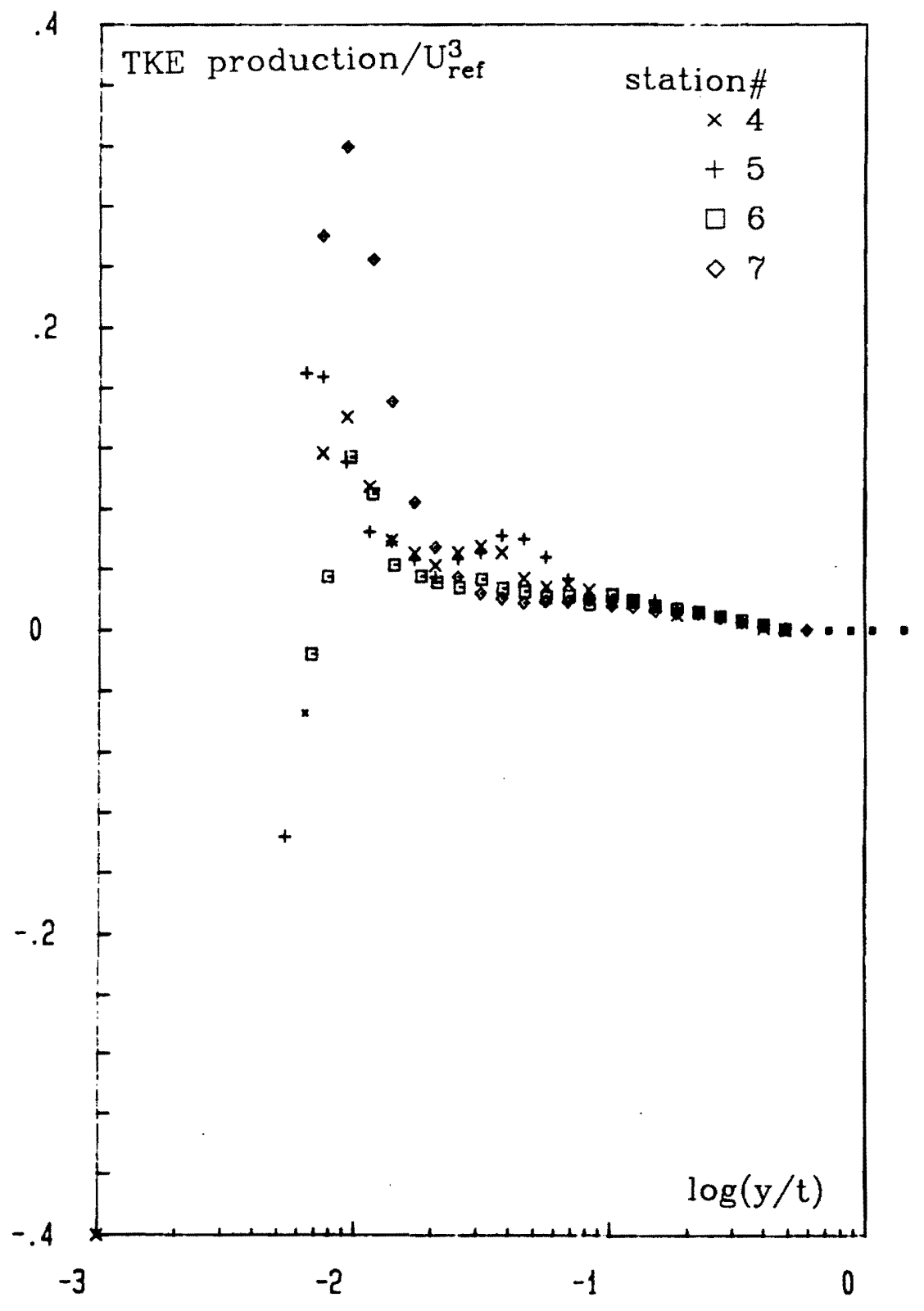

Figure 82b. Production of turbulent kinetic energy profiles obtained using LDV data in free-stream coordinates.: Station numbers are shown in the figure. 


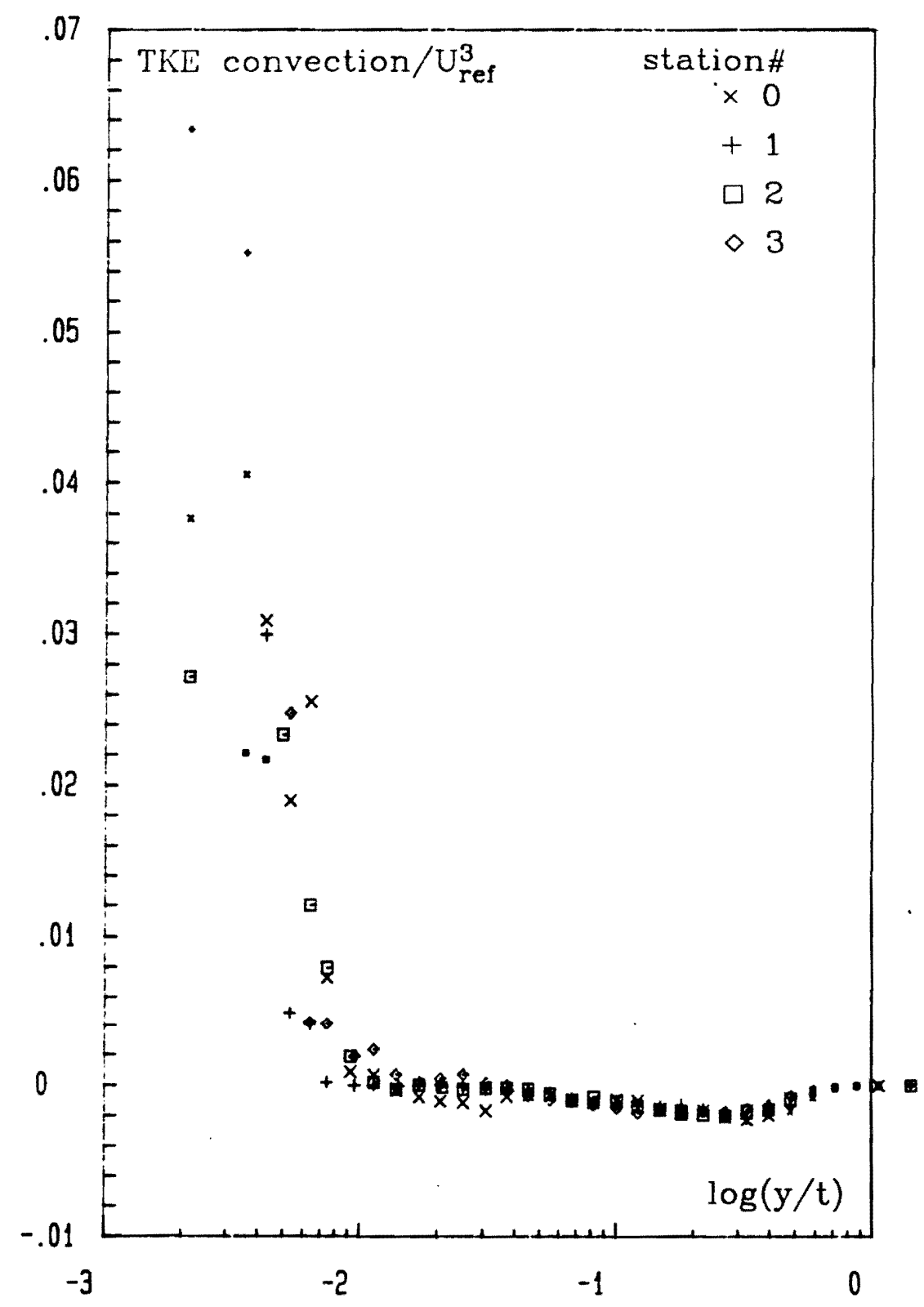

Figure 83a. Convection of turbulent kinetic energy profiles obtained using LDV data in free-stream coordinates.: Station numbers are shown in the figure. 


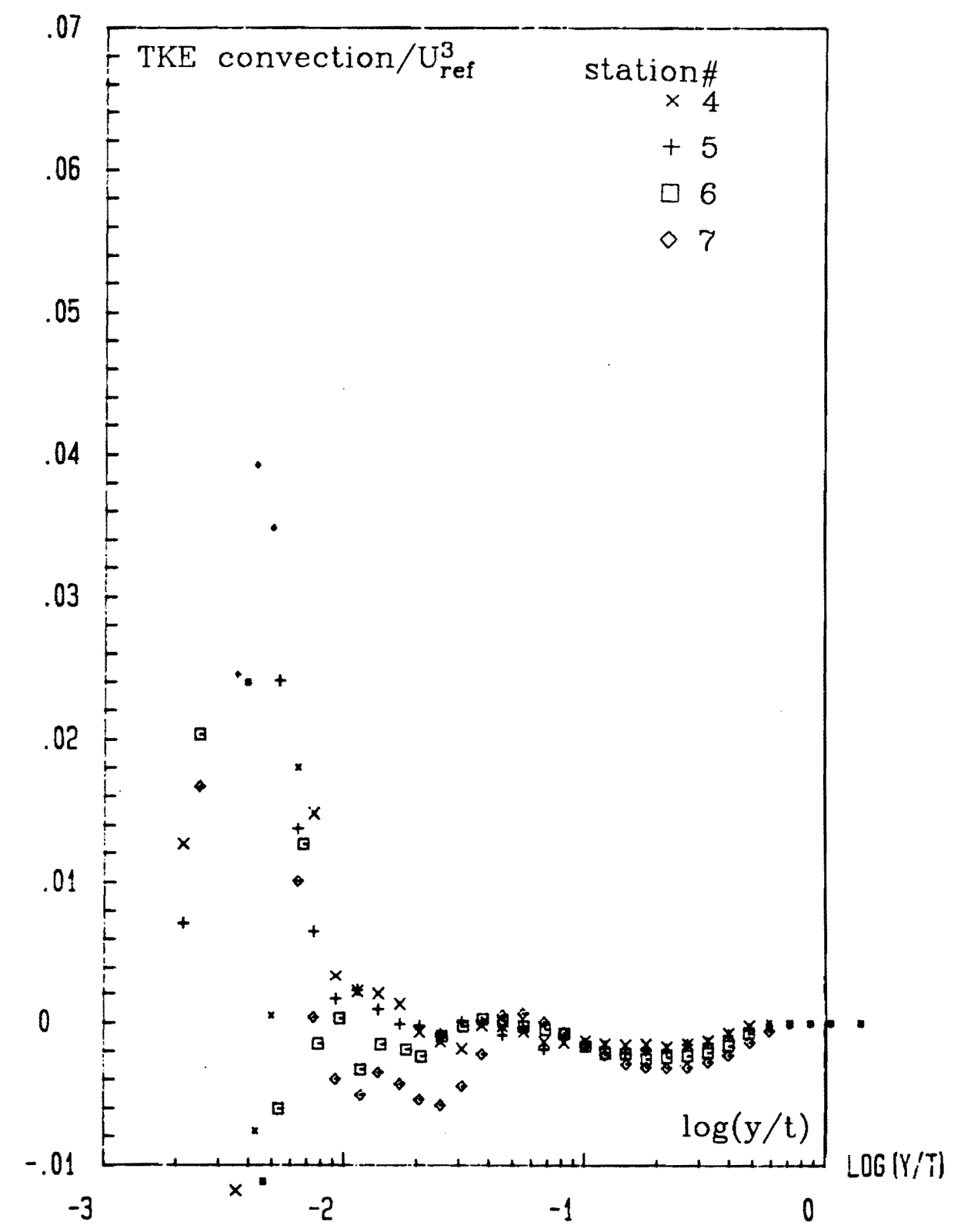

Figure 83b. Convection of turbulent kinetic energy profiles obtained using LDV data in free-stream coordinates.: Station numbers are shown in the figure. 


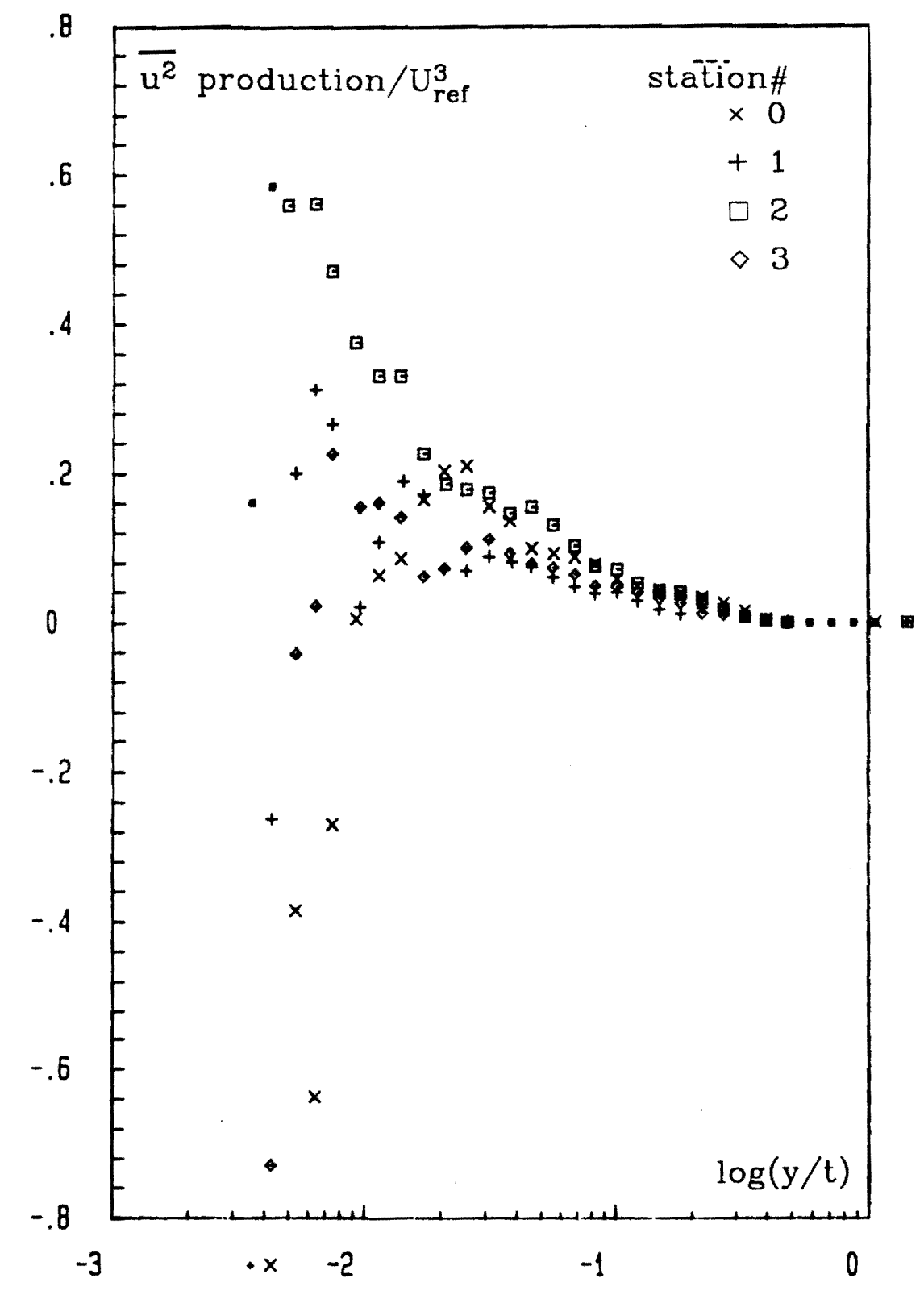

Figure 84a. Production of $\overline{\mathrm{u} 2}$ normal stress profiles computed using LDV data in free-stream coordinates.: Station numbers are shown in the figure. 


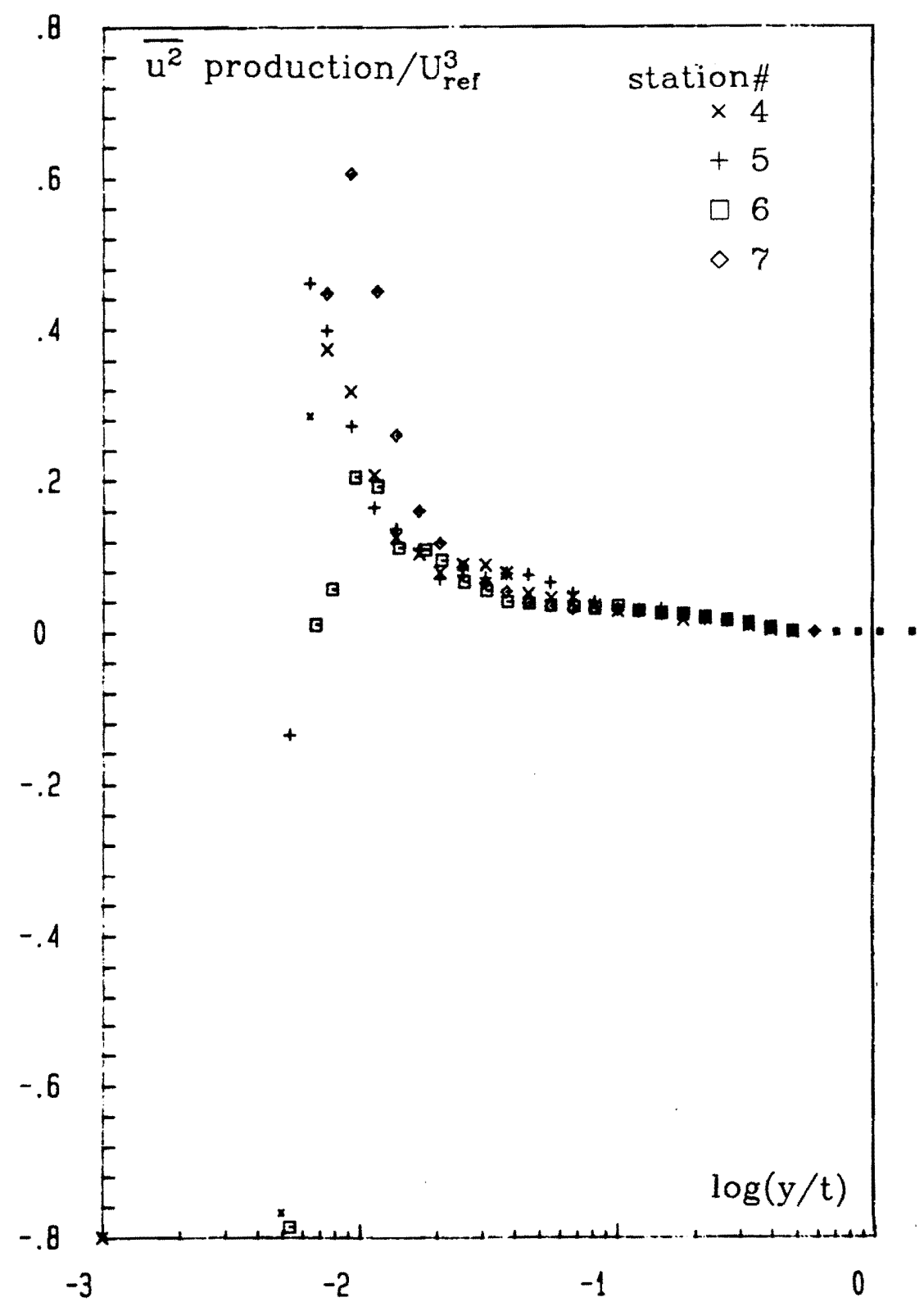

Figure $84 \mathrm{~b}$. Production of $\overline{\mathrm{u} 2}$ normal stress profiles computed using LDV data in free-stream coordinates.: Station numbers are shown in the figure. 


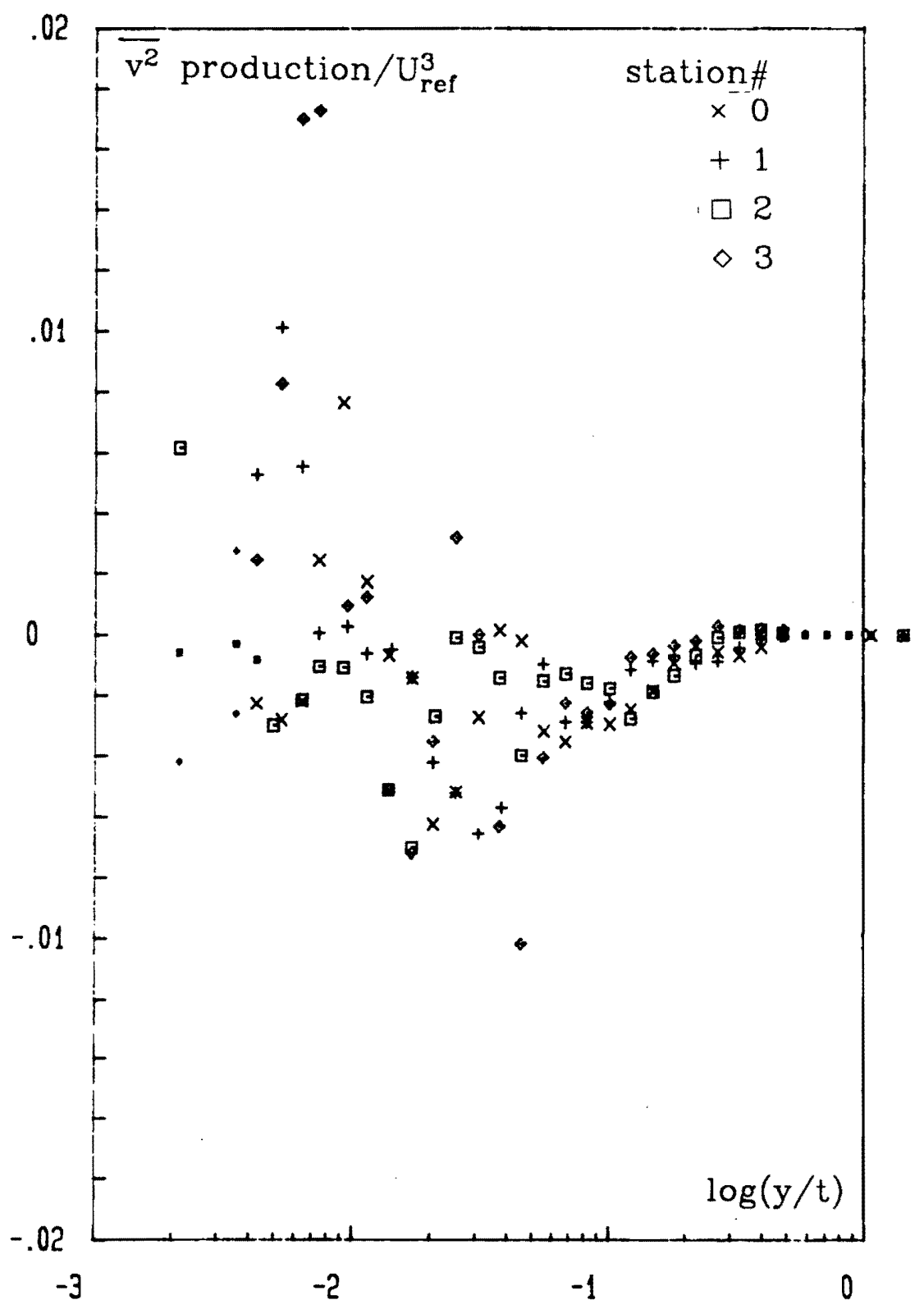

Figure $85 \mathrm{a}$. Production of $\overline{\mathrm{v} 2}$ normal stress profiles computed using LDV data in free-stream coordinates.: Station numbers are shown in the figure. 


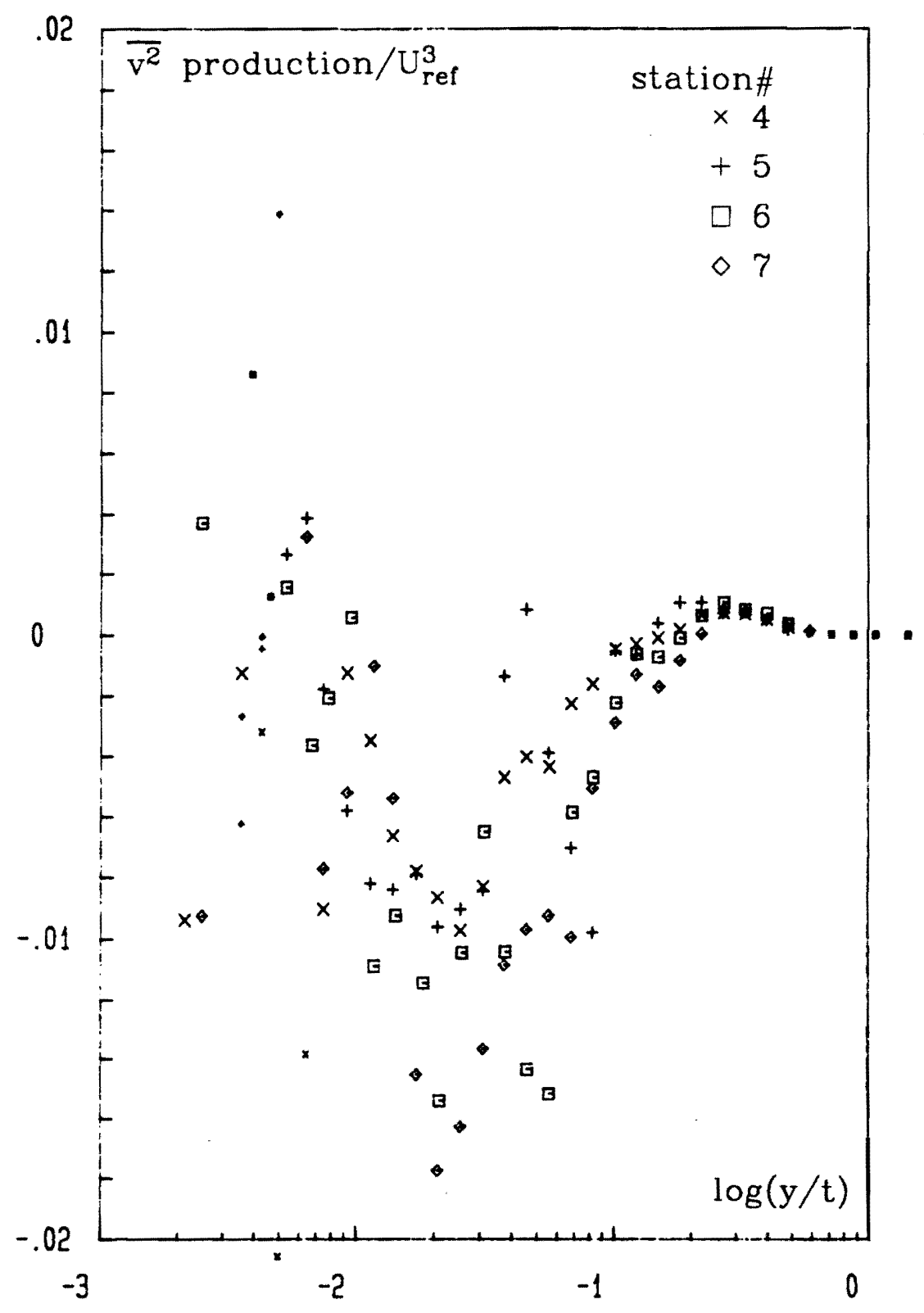

Figure $85 \mathrm{~b}$. Production of $\overline{\mathrm{v} 2}$ normal stress profiles computed using LDV data in free-stream coordinates.: Station numbers are shown in the figure. 


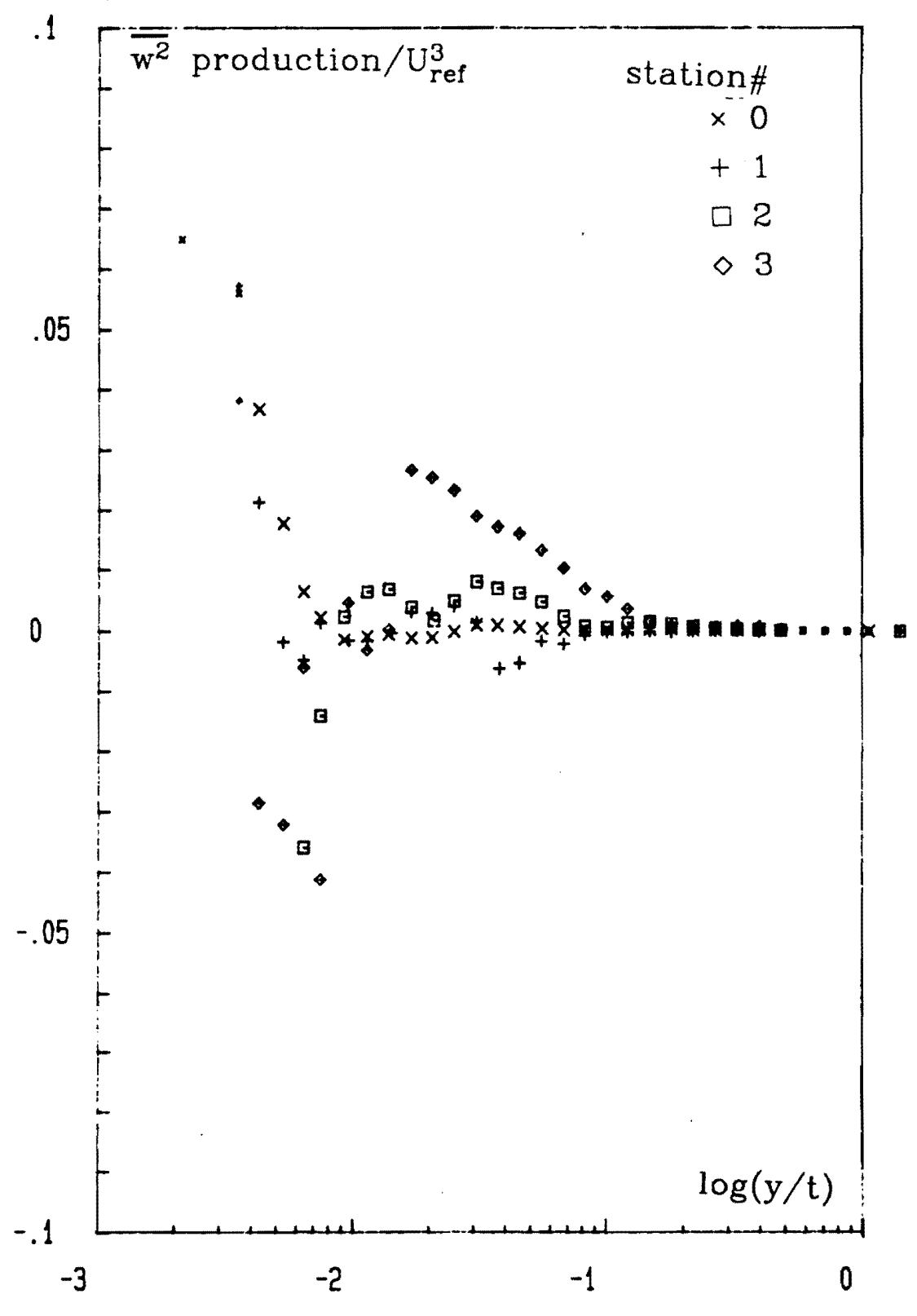

Figure 86a. Production of $\overline{w 2}$ normal stress profiles computed using LDV data in free-stream coordinates.: Station numbers are shown in the figure. 


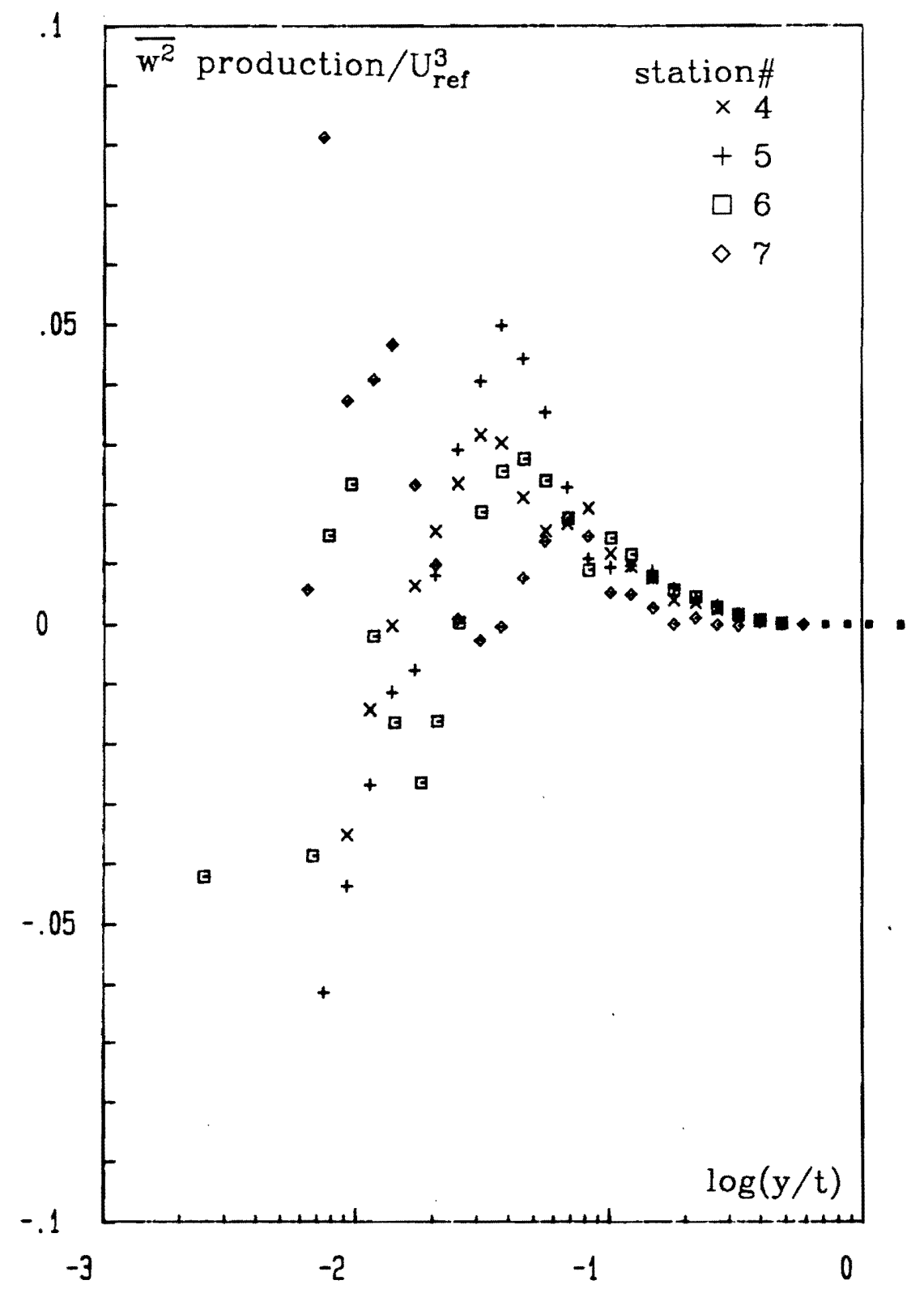

Figure $86 \mathrm{~b}$. Production of $\overline{\mathrm{w} 2}$ normal stress profiles computed using LDV data in free-stream coordinates.: Station numbers are shown in the figure. 


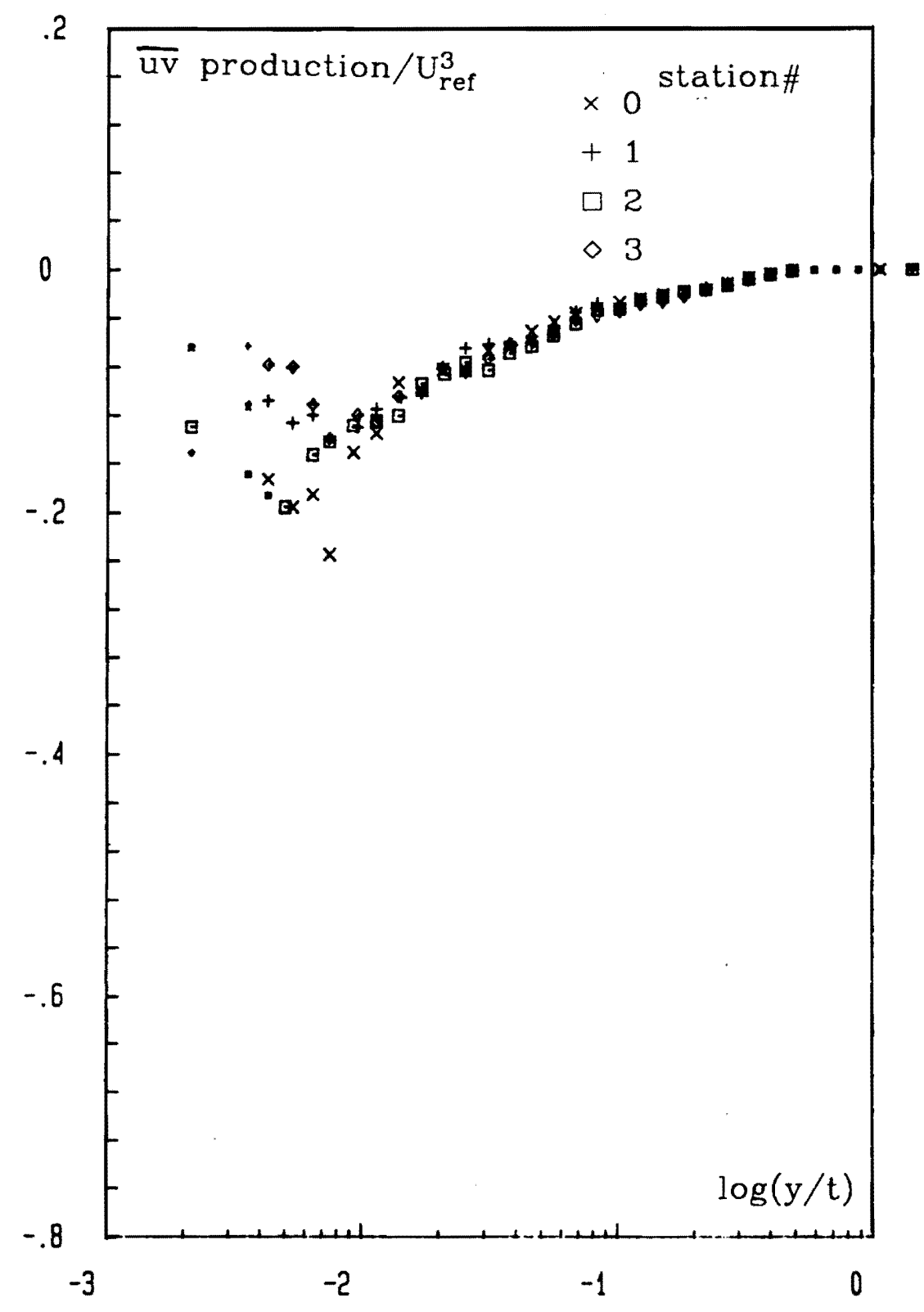

Figure 87a. Production of $-\overline{\mathrm{uv}}$ shear stress profiles computed using LDV data in free-stream coordinates.: Station numbers are shown in the figure. 


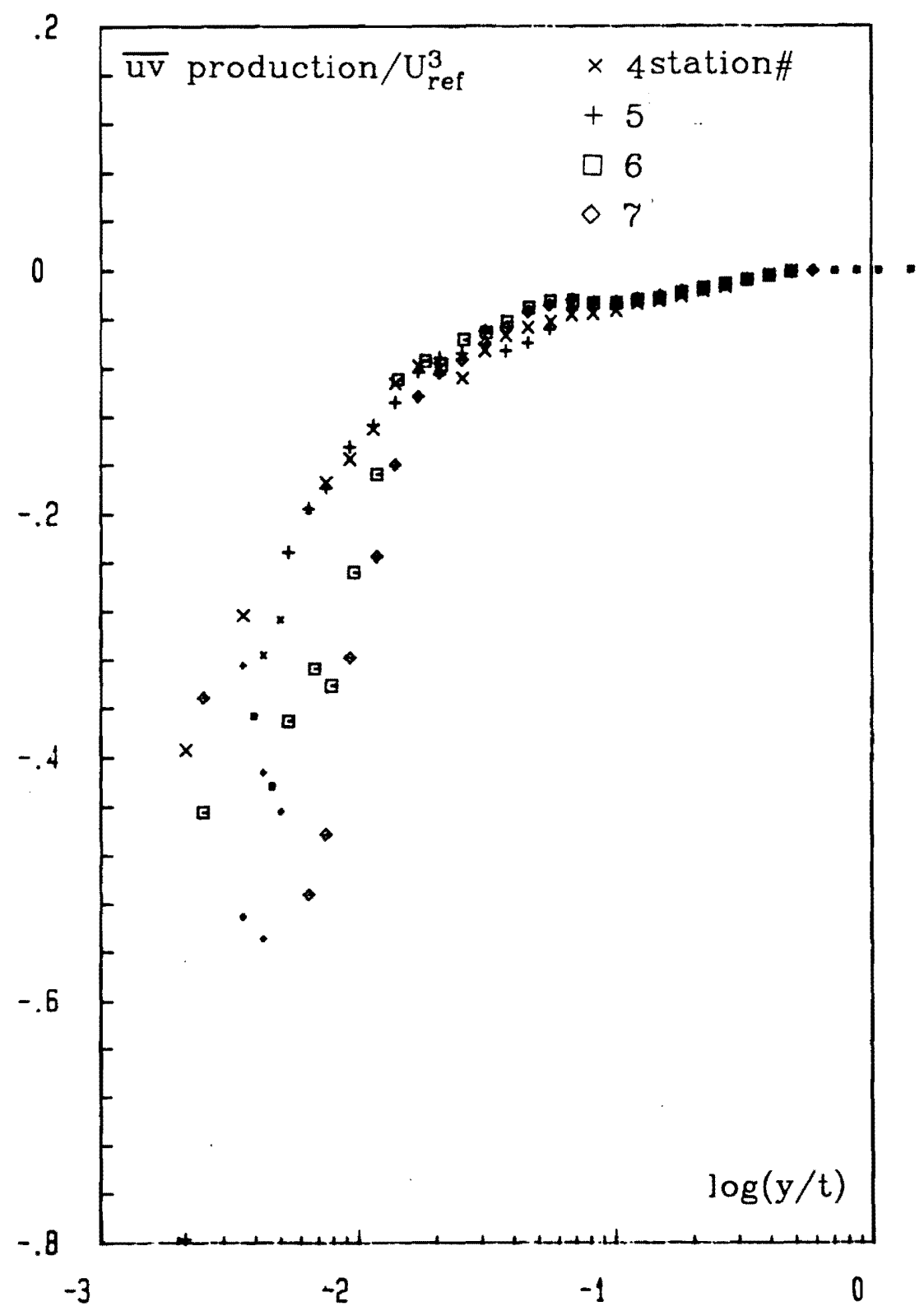

Figure 87b. Production of - $\overline{\mathrm{uV}}$ shear stress profiles computed using LDV data in free-stream coordinates.: Station numbers are shown in the figure. 


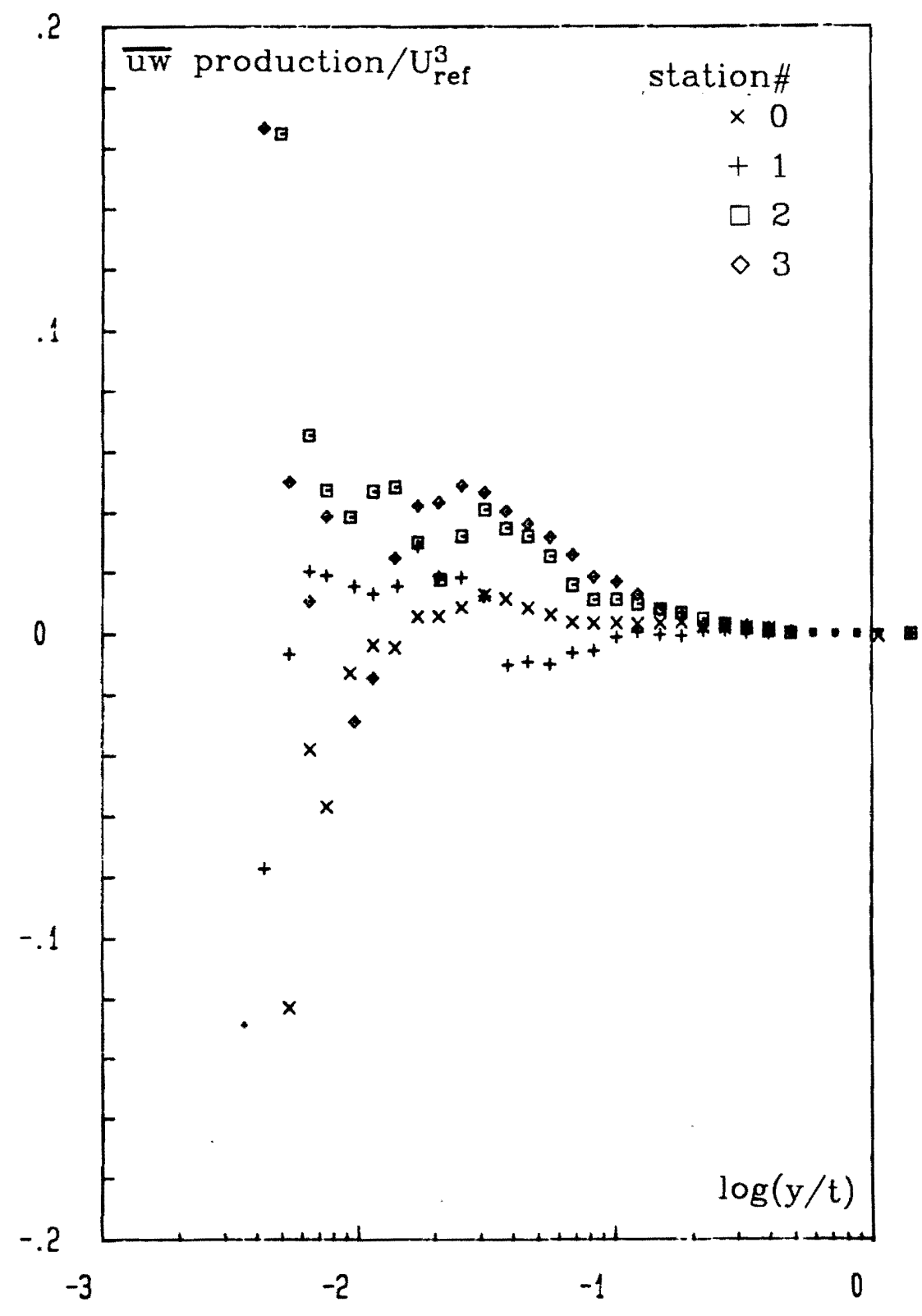

Figure 88a. Production of $-\overline{\mathrm{uW}}$ shear stress profiles computed using LDV data in free-stream coordinates.: Station numbers are shown in the figure. 


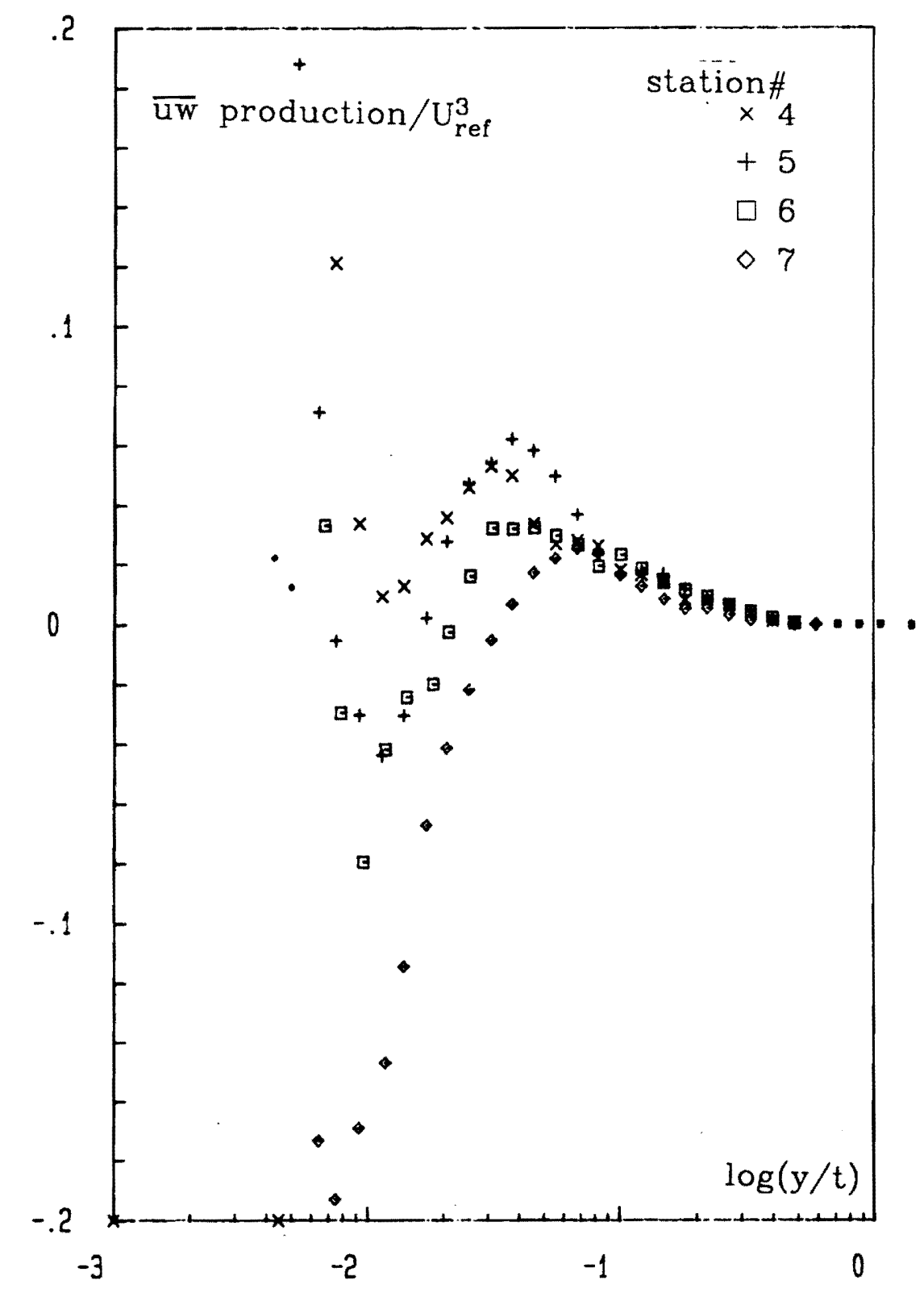

Figure $88 \mathrm{~b}$. Production of -uw shear stress profiles computed using LDV data in free-stream coordinates.: Station numbers are shown in the figure. 


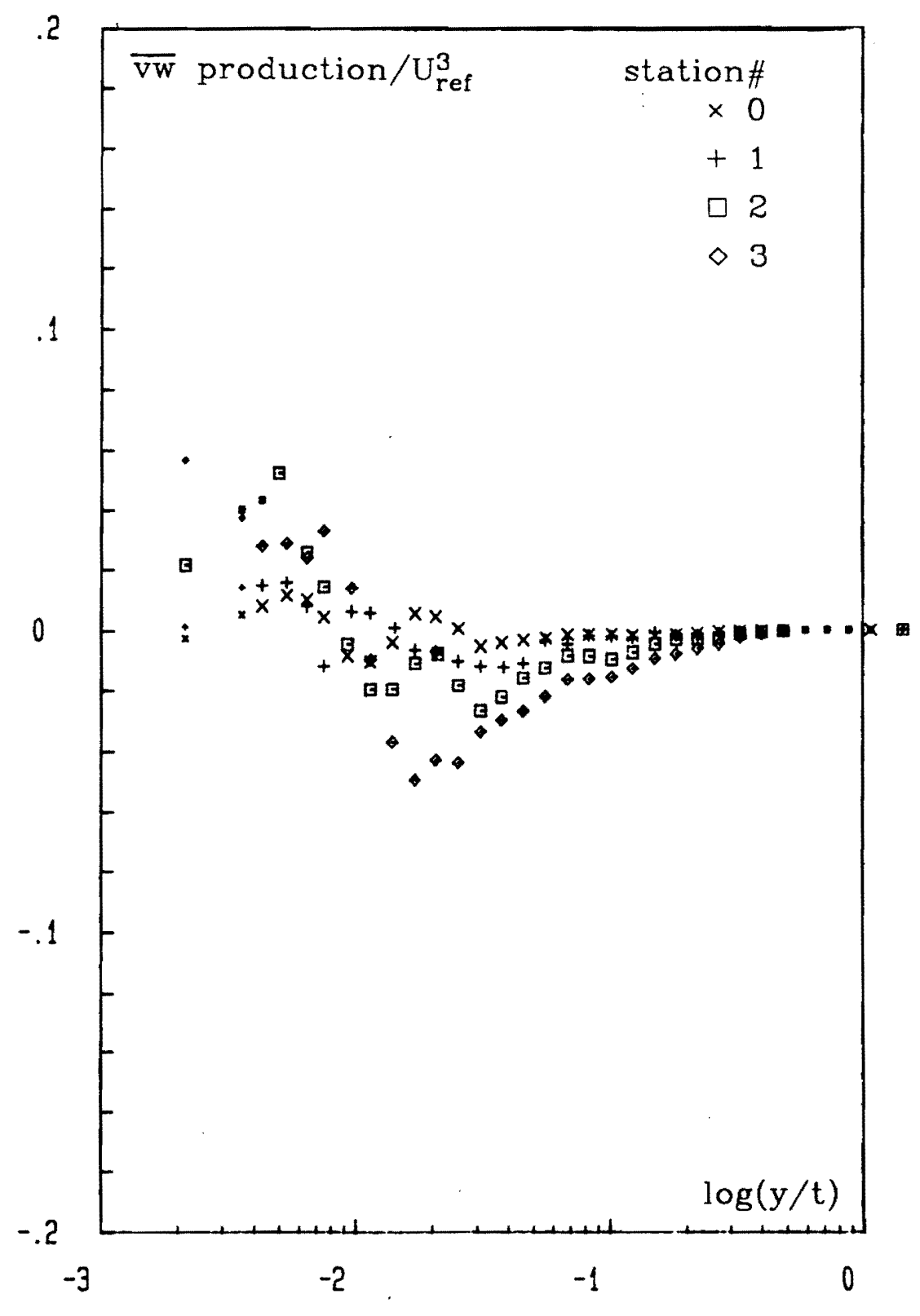

Figure 89a. Production of $-\overline{\mathrm{vw}}$ shear stress profiles computed using LDV data in free-stream coordinates.: Station numbers are shown in the figure. 


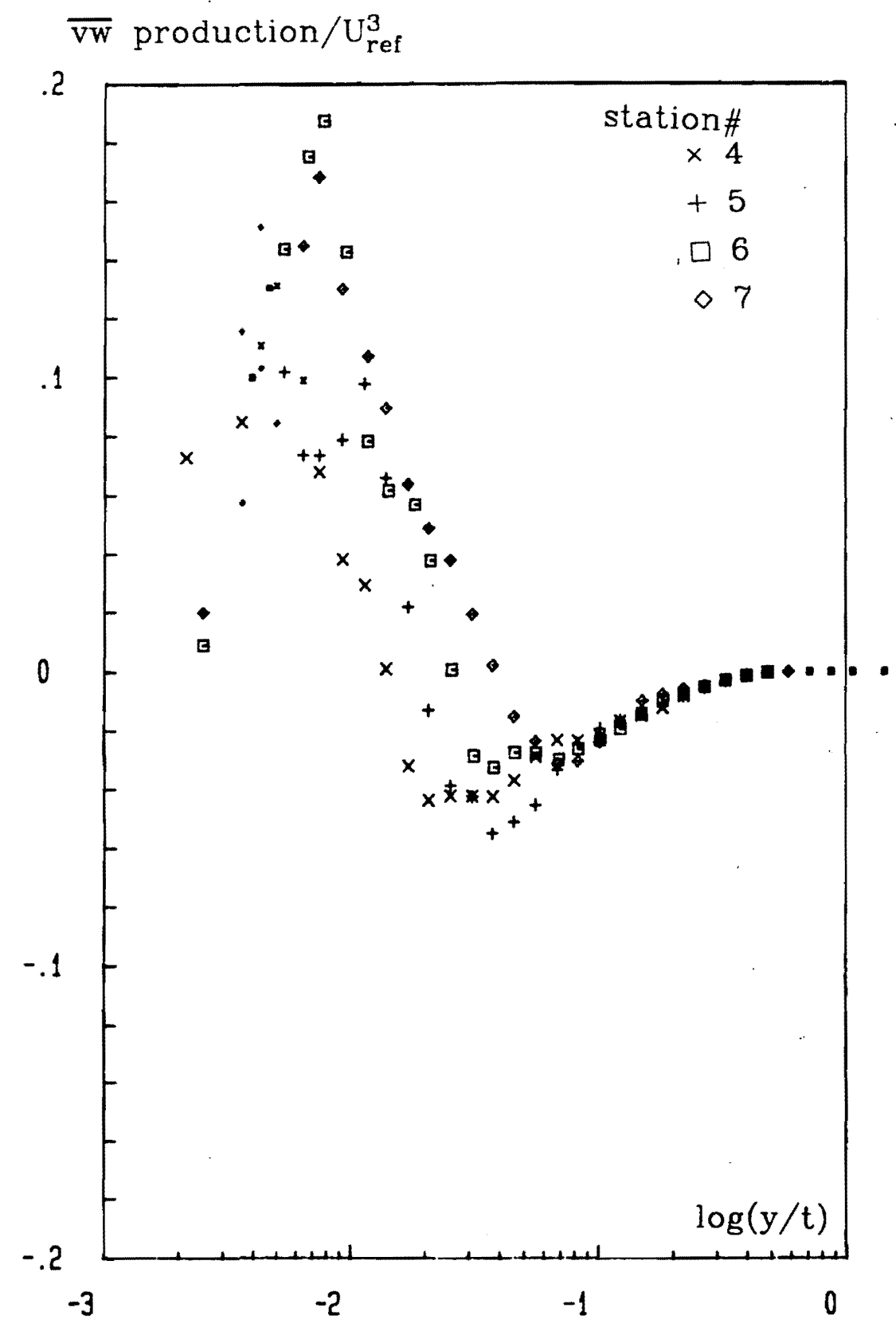

Figure $89 \mathrm{~b}$. Production of $-\overline{\mathrm{vw}}$ shear stress profiles computed using LDV data in free-stream coordinates.: Station numbers are shown in the figure. 


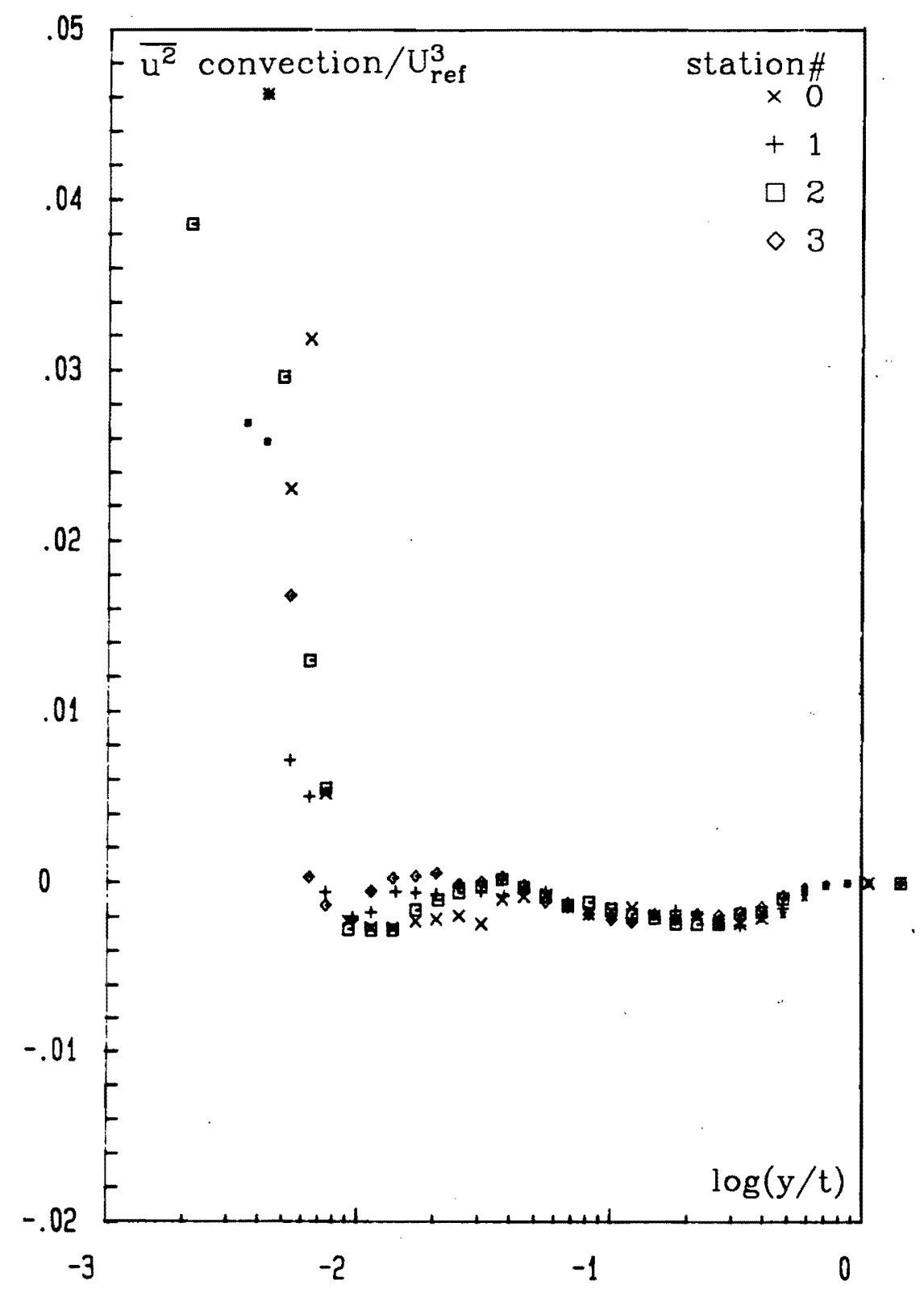

Figure 90a. Convection of $\overline{\mathrm{u} 2}$ normal stress profiles computed using LDV data in free-stream coordinates.: Station numbers are shown in the figure. 


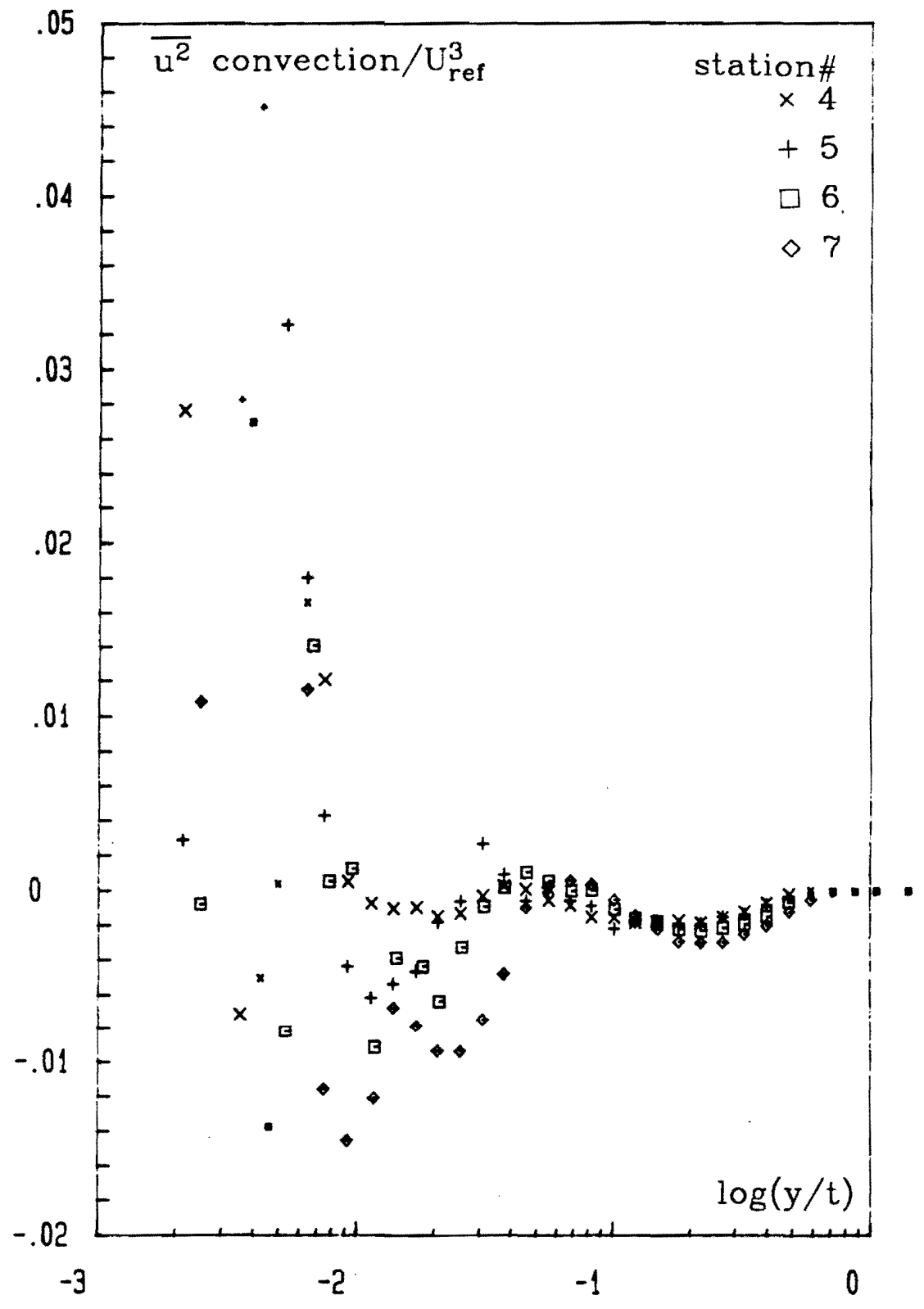

Figure 90b. Convection of $\overline{\mathrm{u} 2}$ normal stress profiles computed using LDV data in free-stream coordinates.: Station numbers are shown in the figure. 


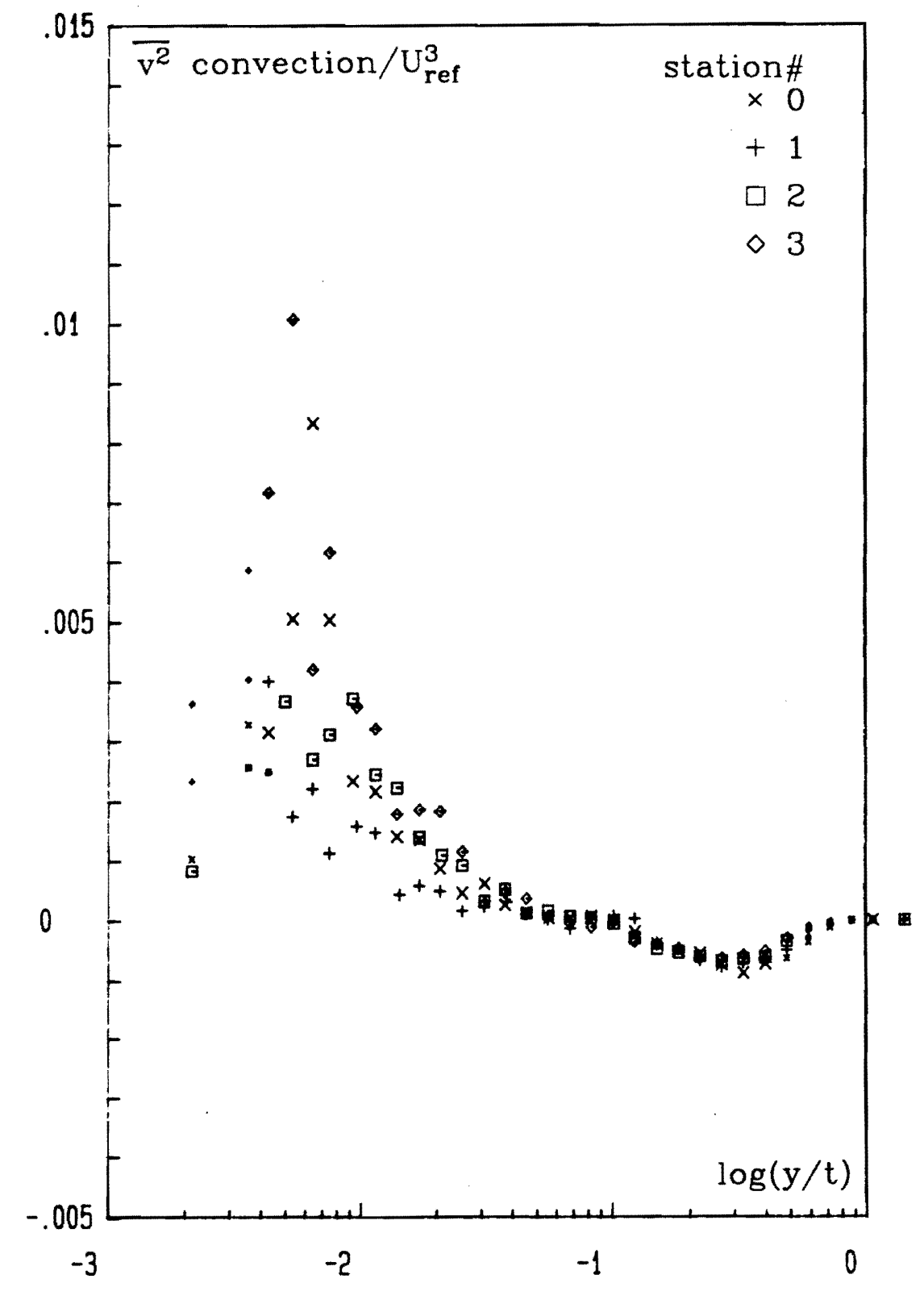

Figure 90c. Convection of $\overline{\mathrm{v} 2}$ normal stress profiles computed using LDV data in free-stream coordinates.: Station numbers are shown in the figure. 


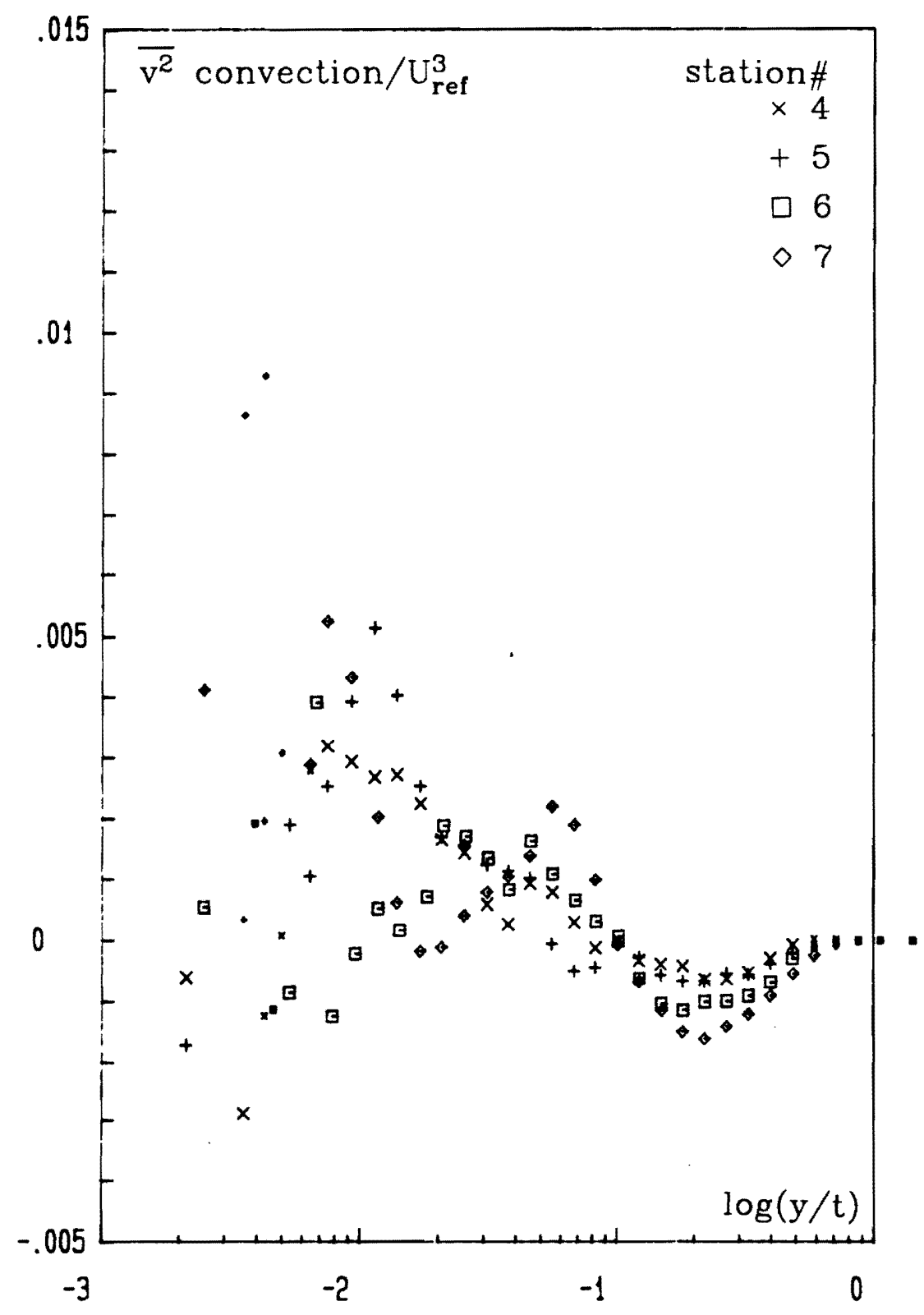

Figure 90d. Convection of $\overline{\mathrm{v} 2}$ normal stress profiles computed using LDV data in free-stream coordinates.: Station numbers are shown in the figure. 


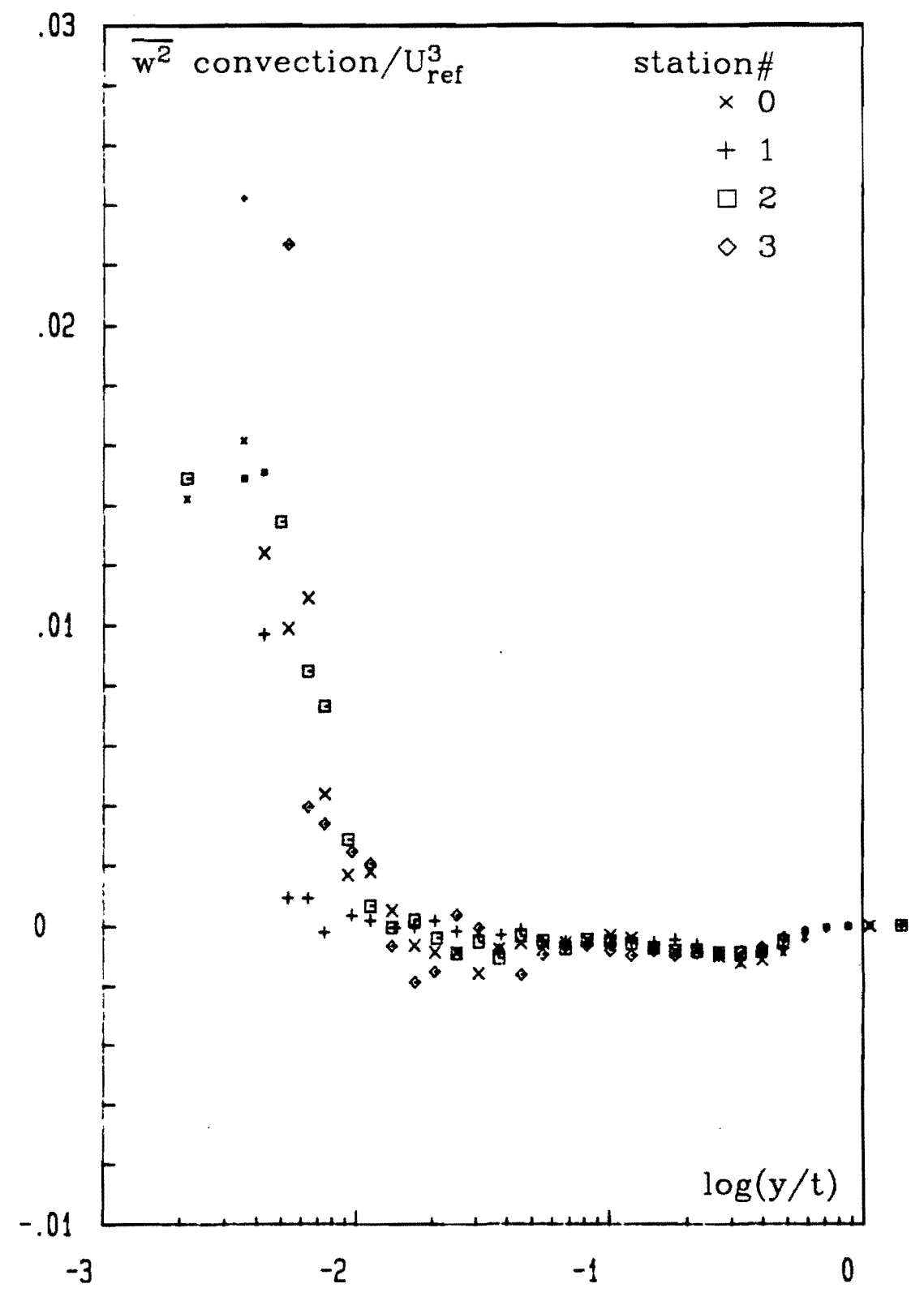

Figure 90e. Convection of $\overline{\mathrm{w} 2}$ normal stress profiles computed using LDV data in free-stream coordinates.: Station numbers are shown in the figure. 


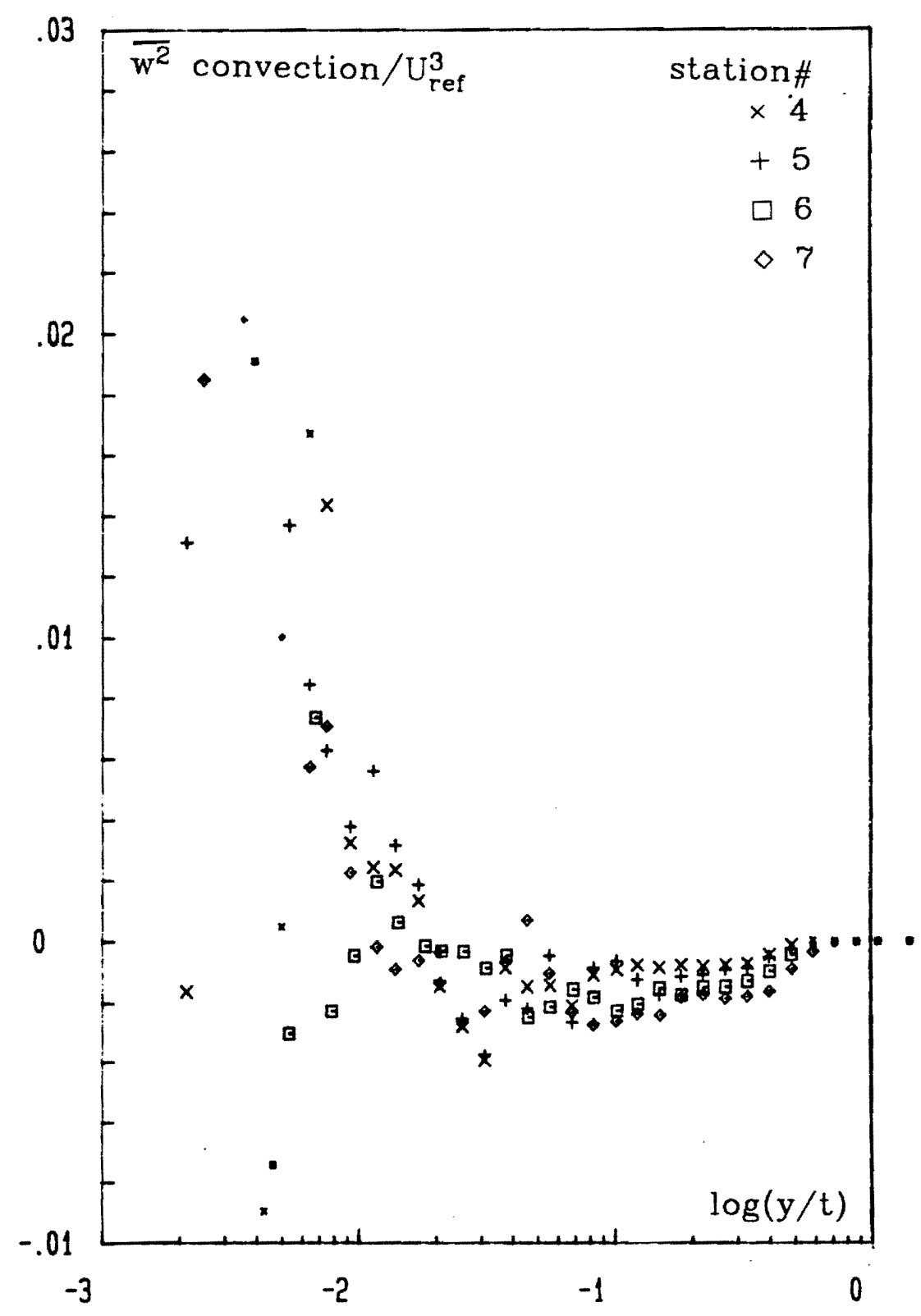

Figure 90f. Convection of $\overline{w 2}$ normal stress profiles computed using LDV data in free-stream coordinates.: Station numbers are shown in the figure. 


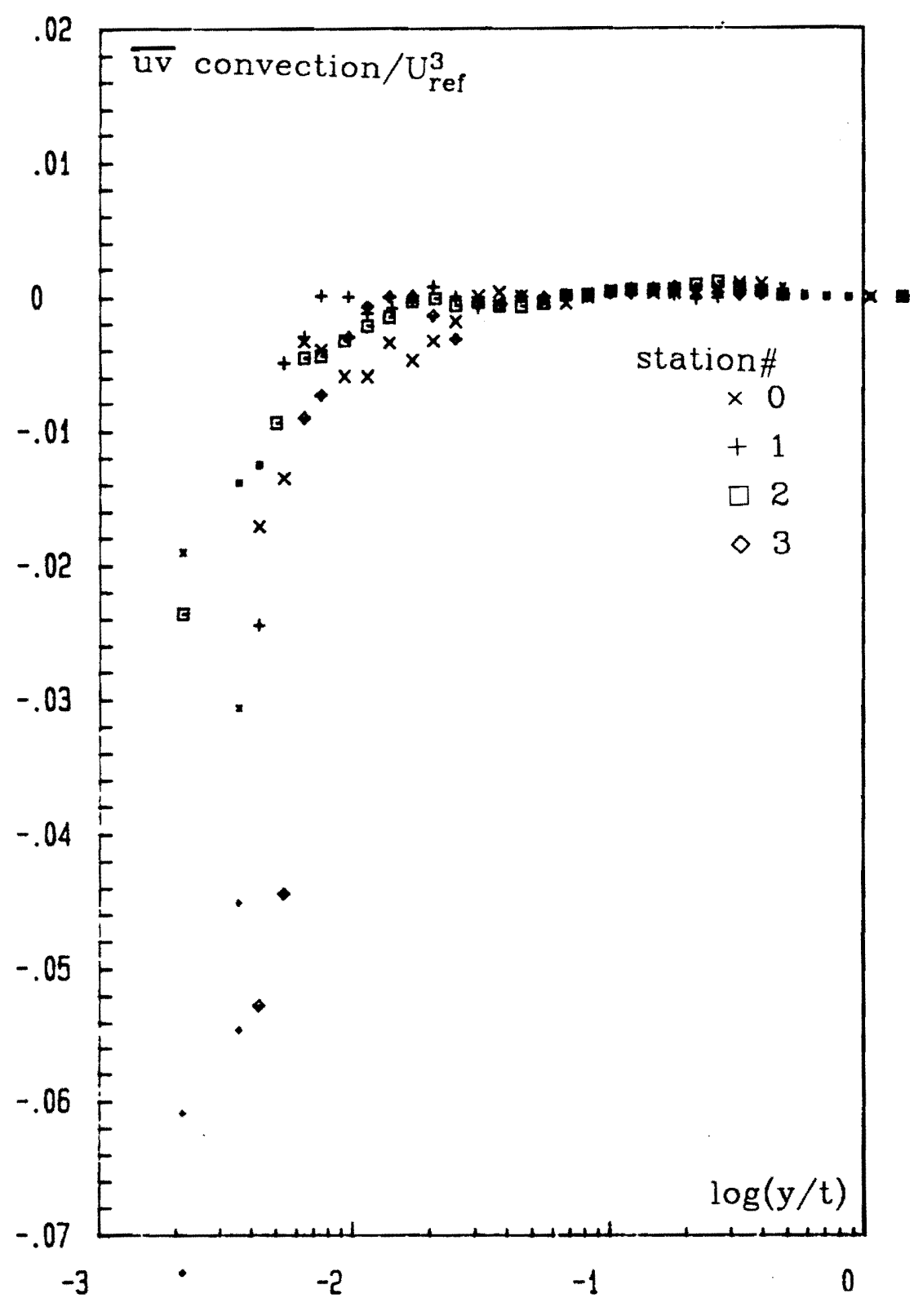

Figure $90 \mathrm{~g}$. Convection of -uv shear stress profiles computed using LDV data in free-stream coordinates.: Station numbers are shown in the figure. 


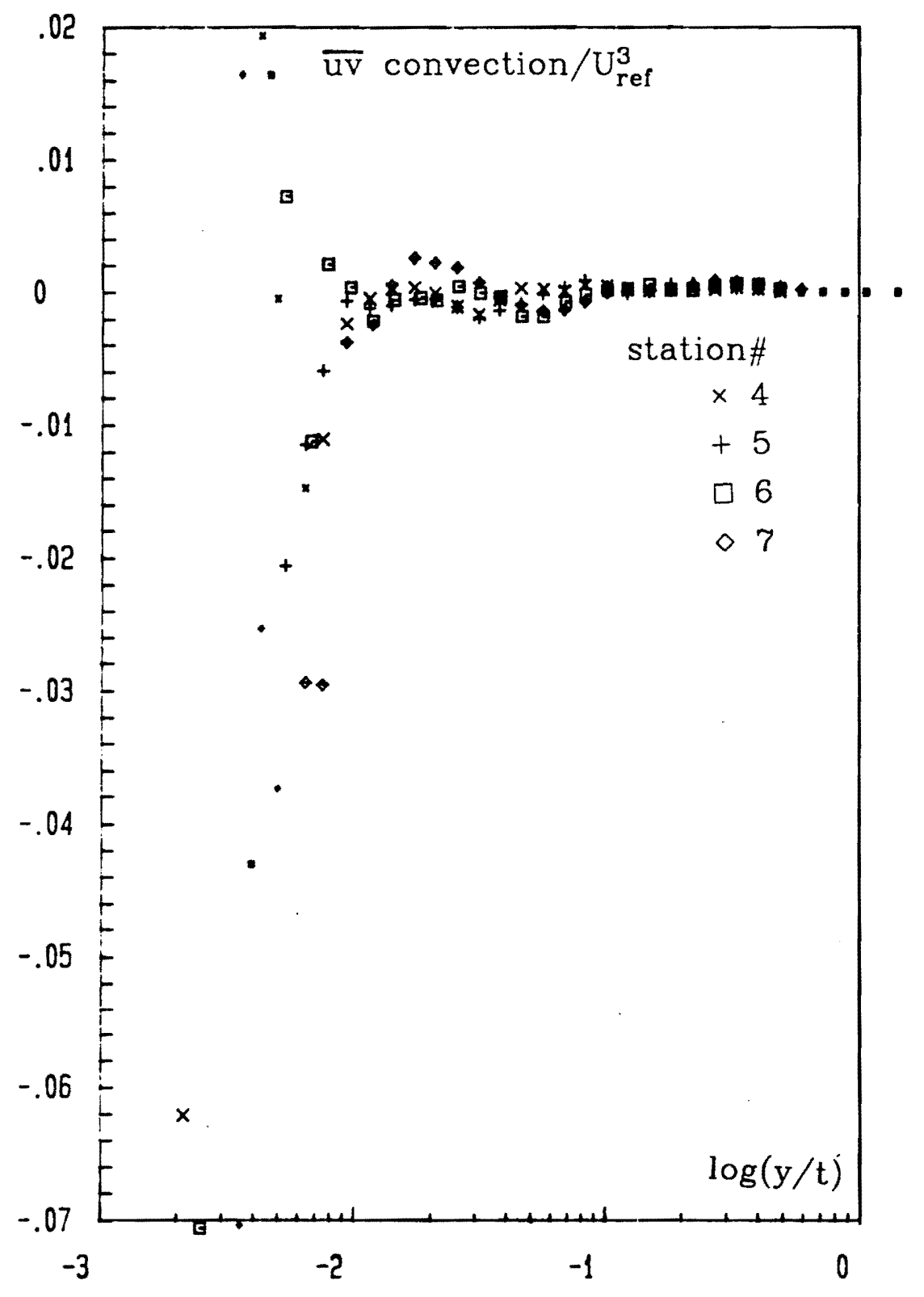

Figure 90h. Convection of $-\overline{\mathrm{uv}}$ shear stress profiles computed using LDV data in free-stream coordinates.: Station numbers are shown in the figure. 


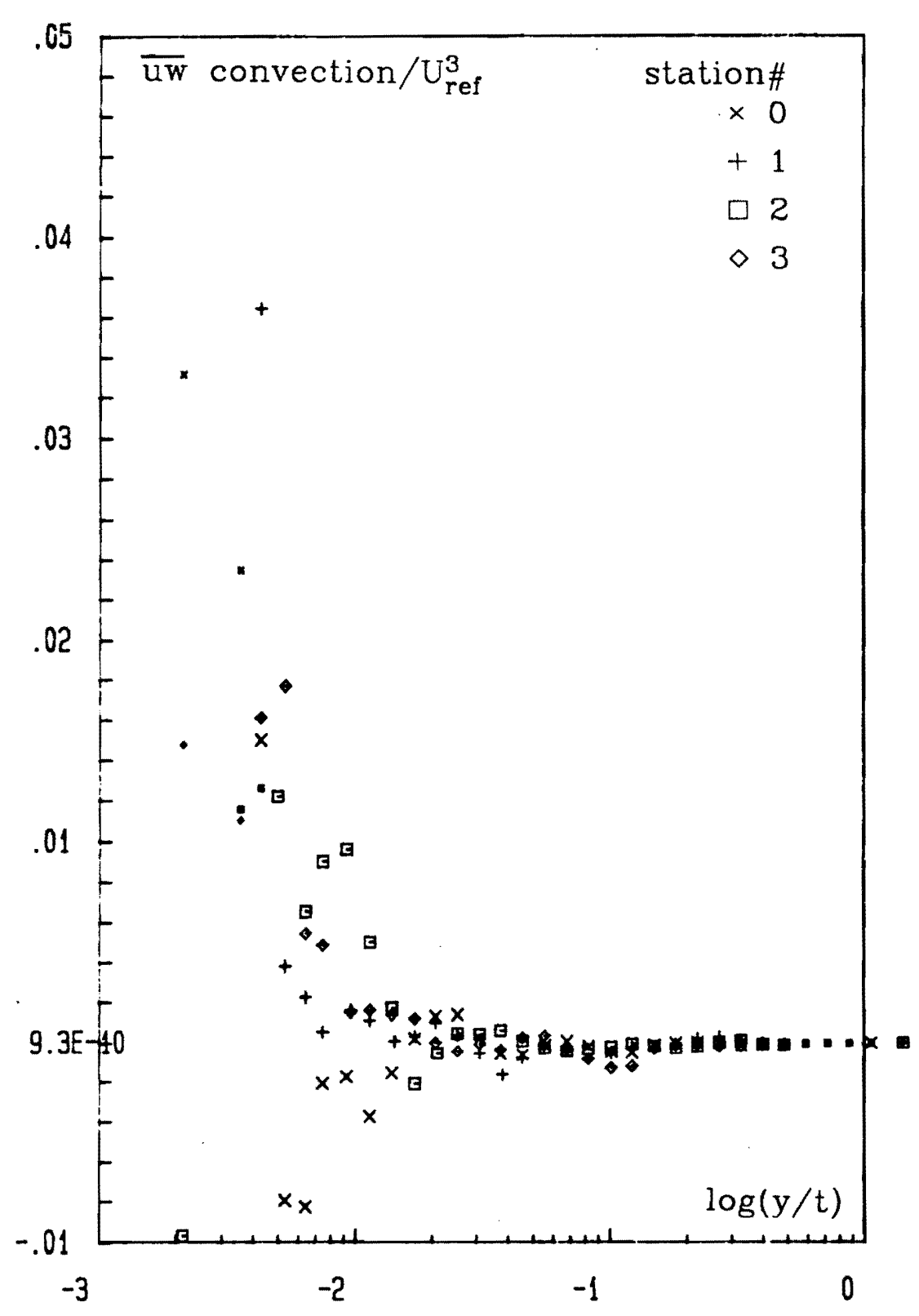

Figure 90i. Convection of - $\overline{\mathrm{uw}}$ shear stress profiles computed using LDV data in free-stream coordinates.: Station numbers are shown in the figure. 


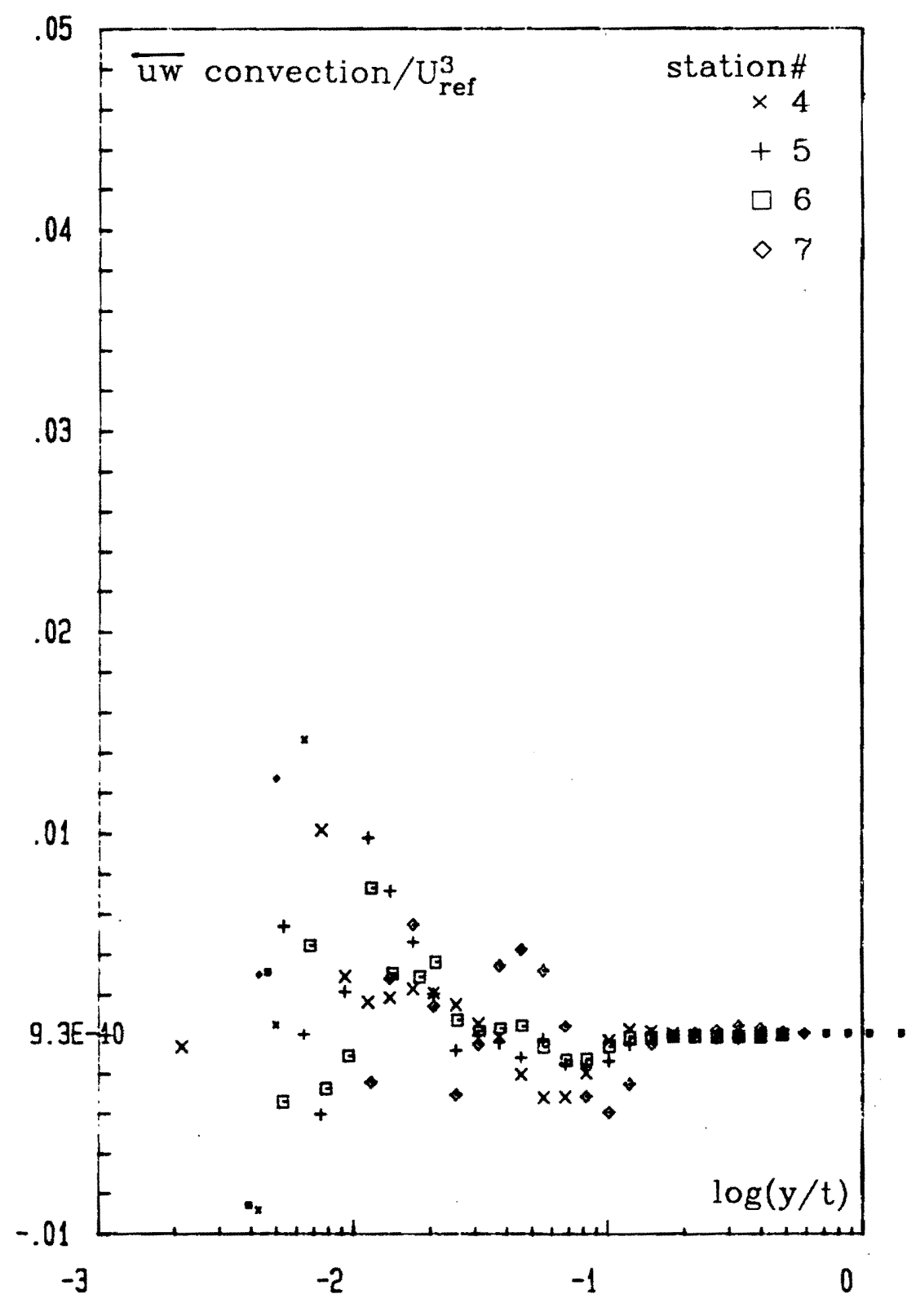

Figure $90 \mathrm{j}$. Convection of $-\overline{\mathrm{uw}}$ shear stress profiles computed using LDV data in free-stream coordinates.: Station numbers are shown in the figure. 


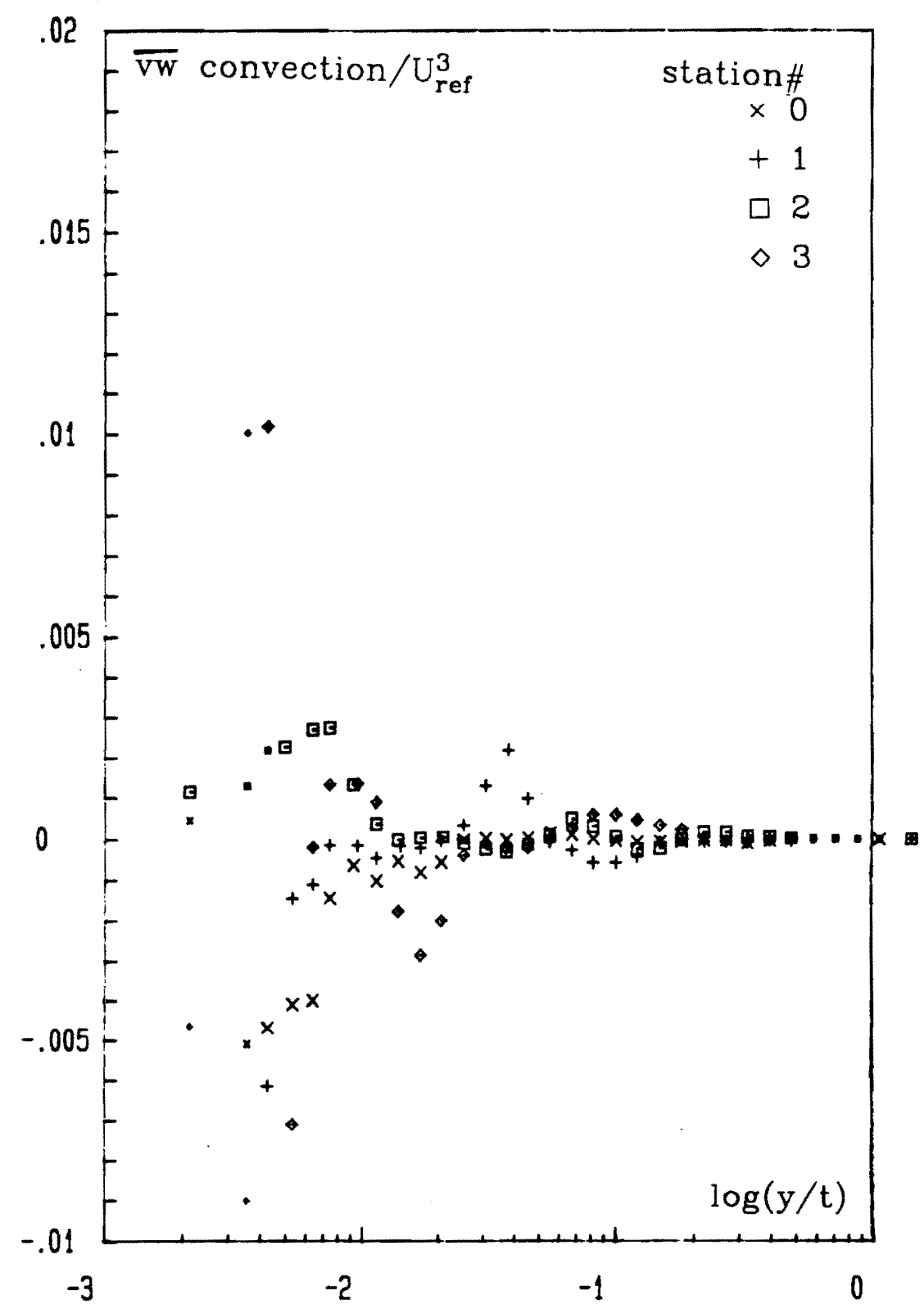

Figure $90 \mathrm{k}$. Convection of $-\overline{\mathrm{vw}}$ shear stress profiles computed using LDV data in free-stream coordinates.: Station numbers are shown in the figure. 


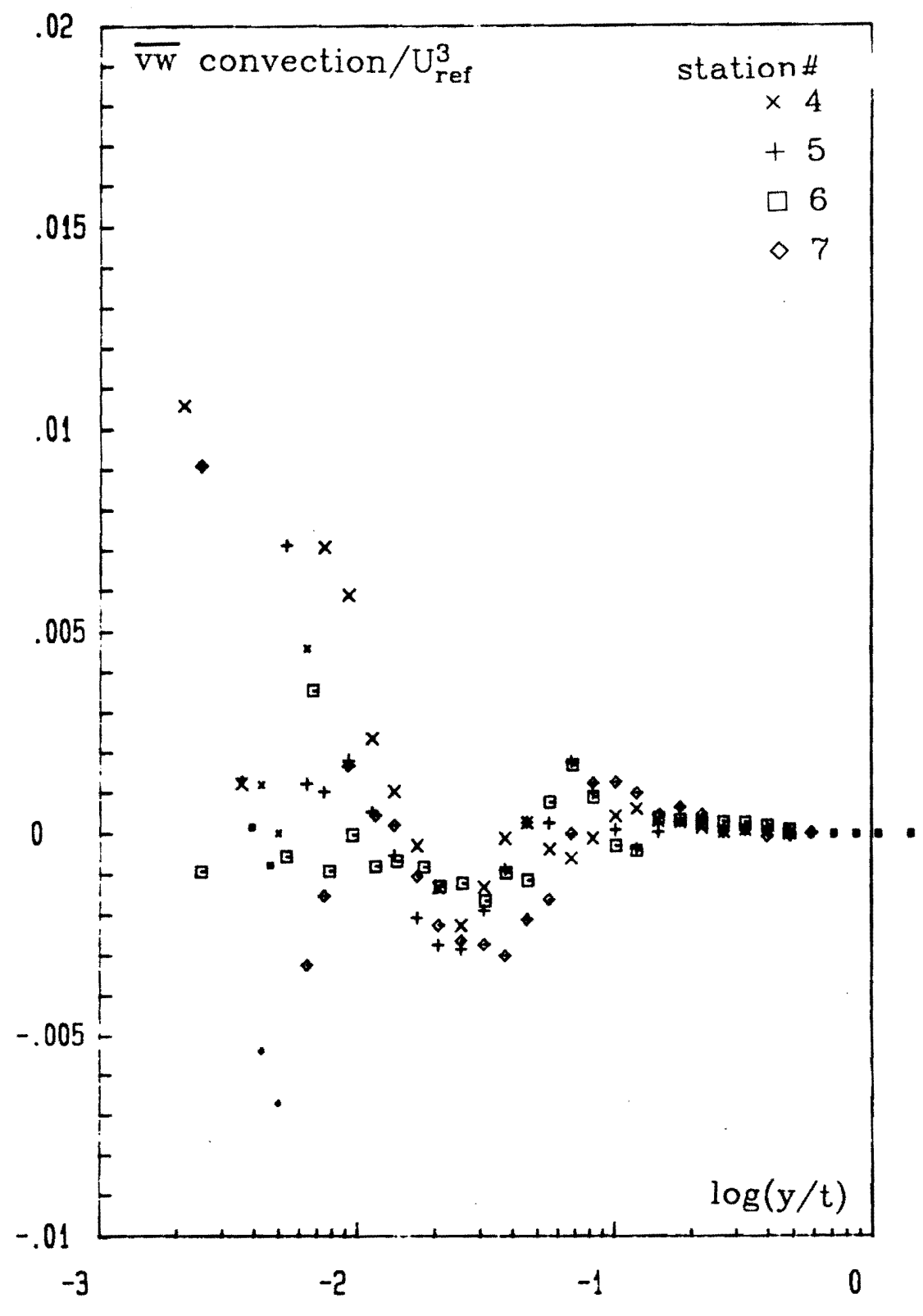

Figure 901. Convection of $-\overline{\mathrm{v} w}$ shear stress profiles computed using LDV data in free-stream coordinates.: Station numbers are shown in the figure. 


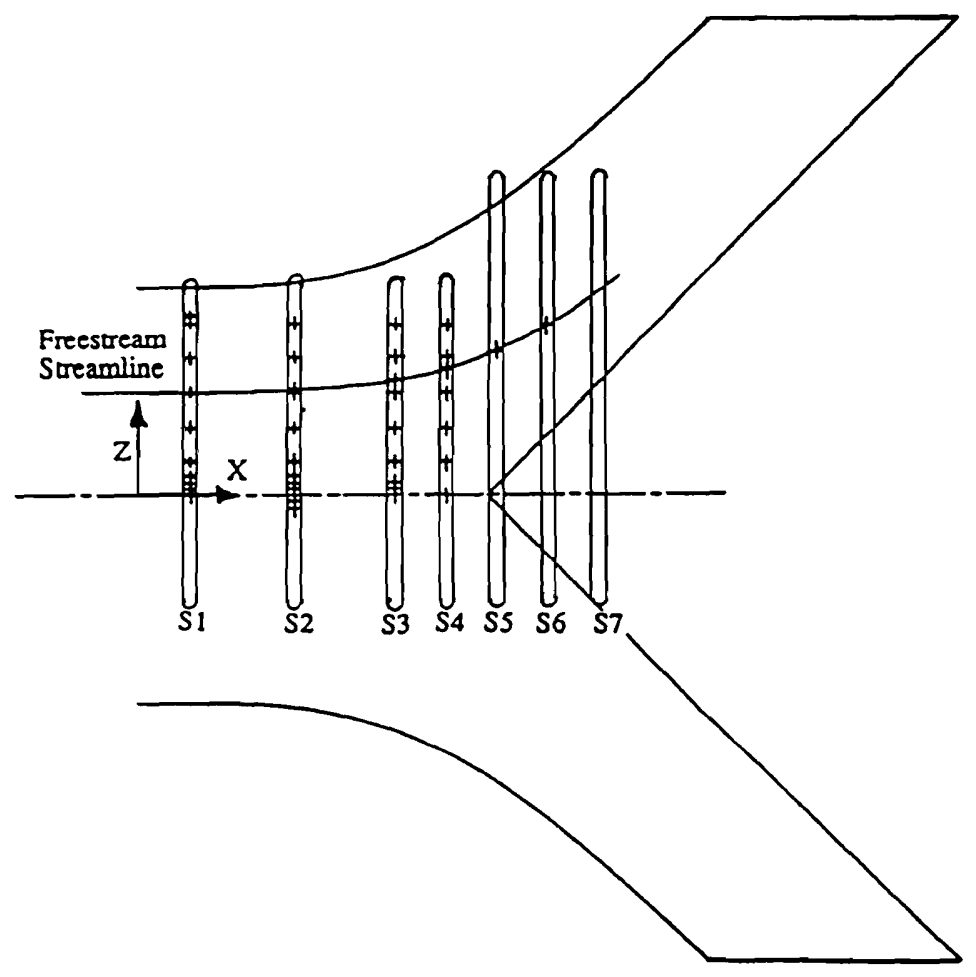

Figure 91a. Schematic figure of Anderson's experimental set up. 

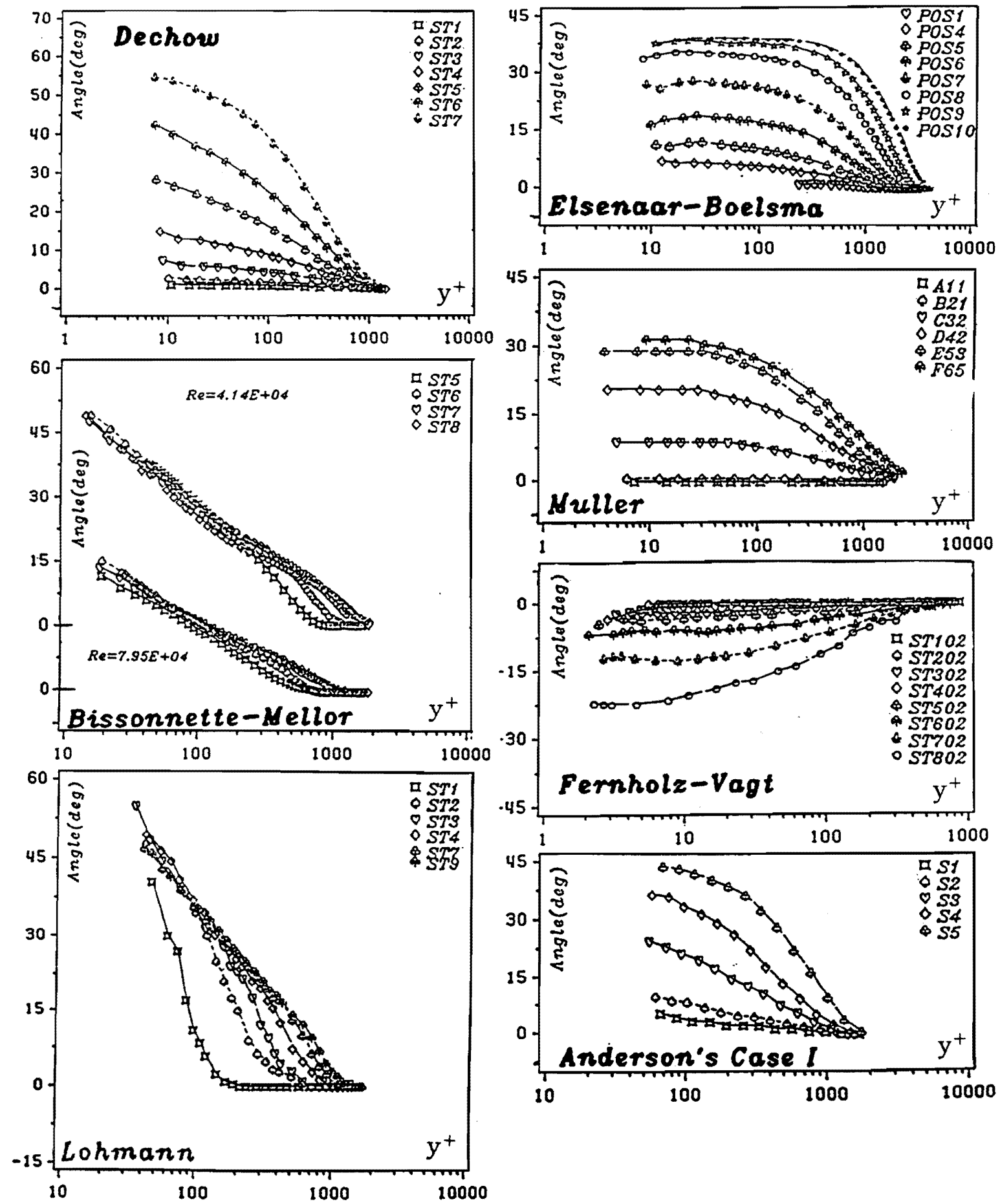

Figure 92b. Flow angles of Anderson's Case I, Fernholz-Vagt, Muller, Dechow-Felsch, Elsenaar-Boelsma, Bissonnette-Mellor, Lohmann flows. 


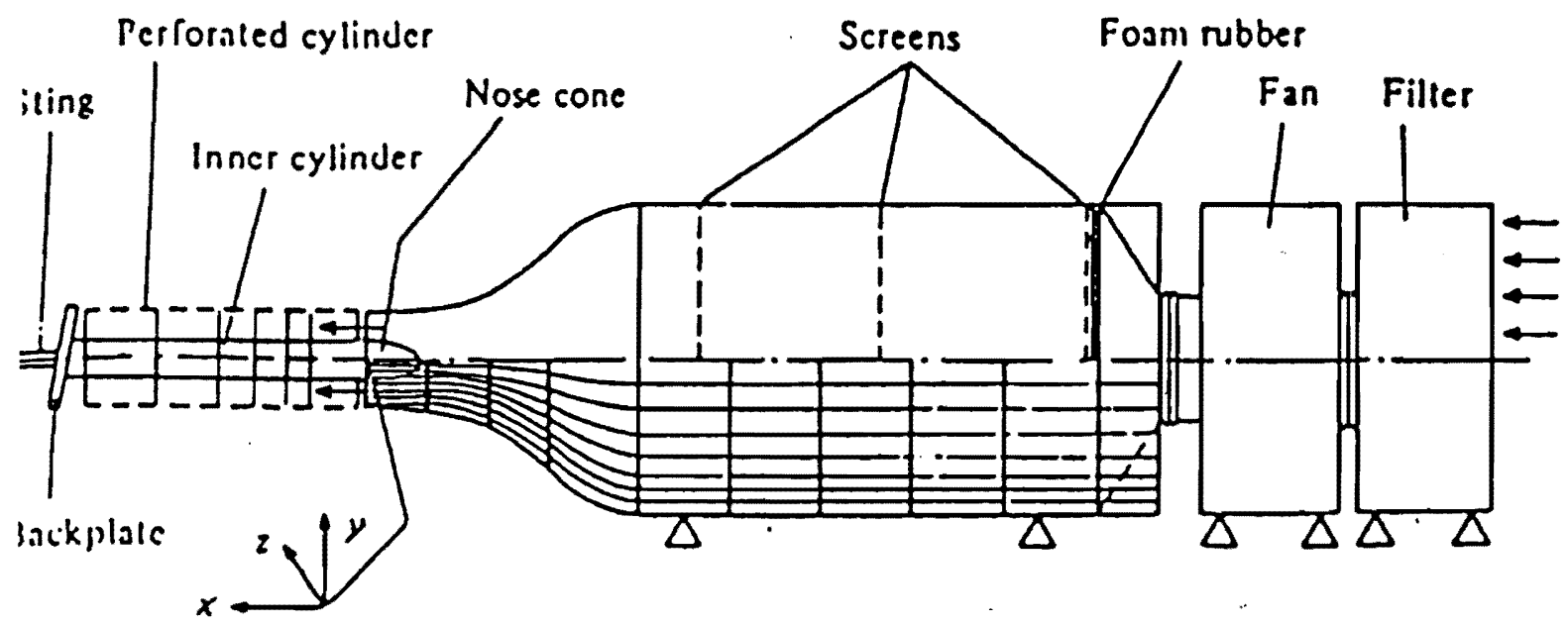

Figure 93. Schematic figure of Fernholz-Vagt experimental set up. 


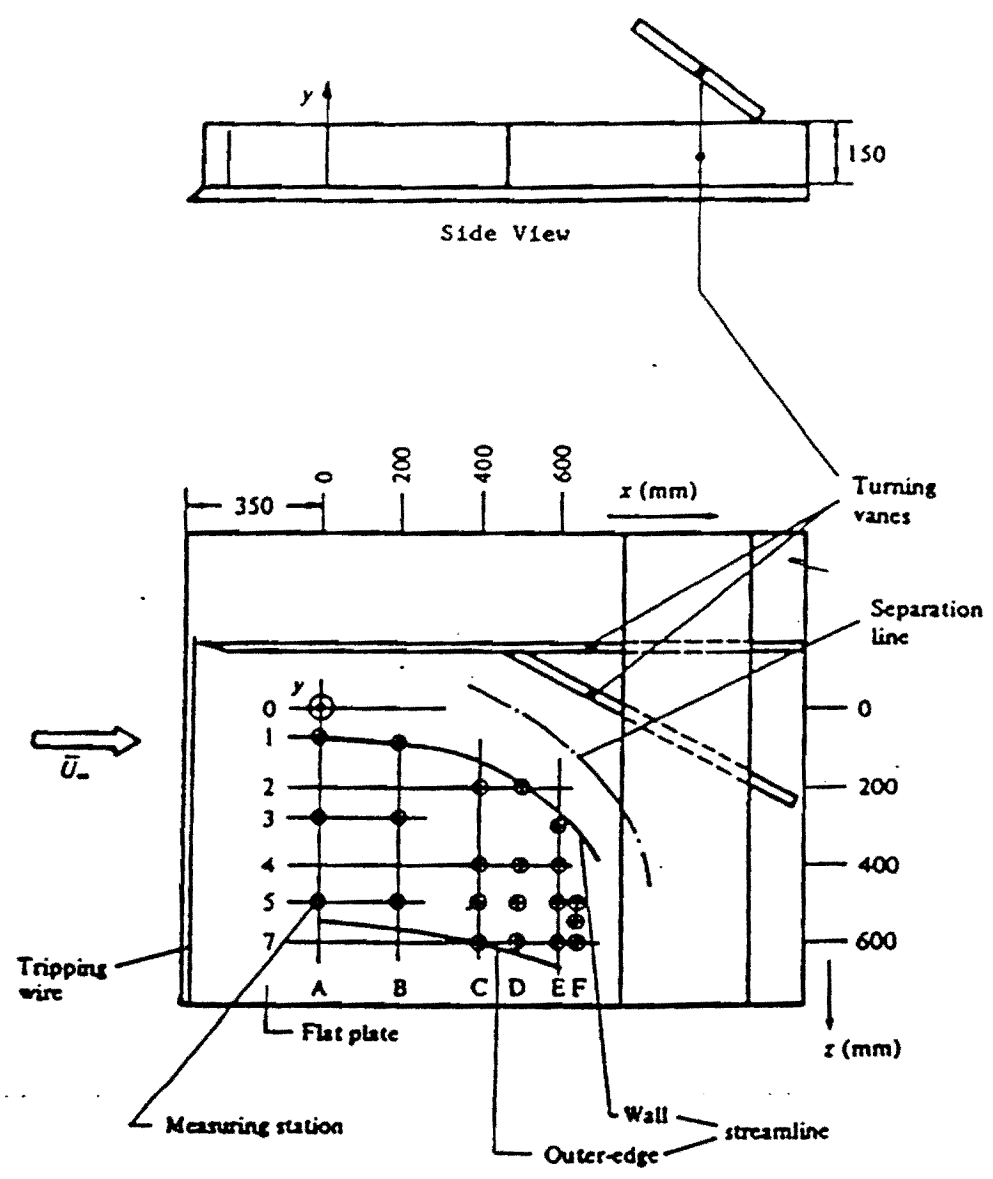

Top V1es

Figure 94. Schematic figure of Muller's experimental set up. 

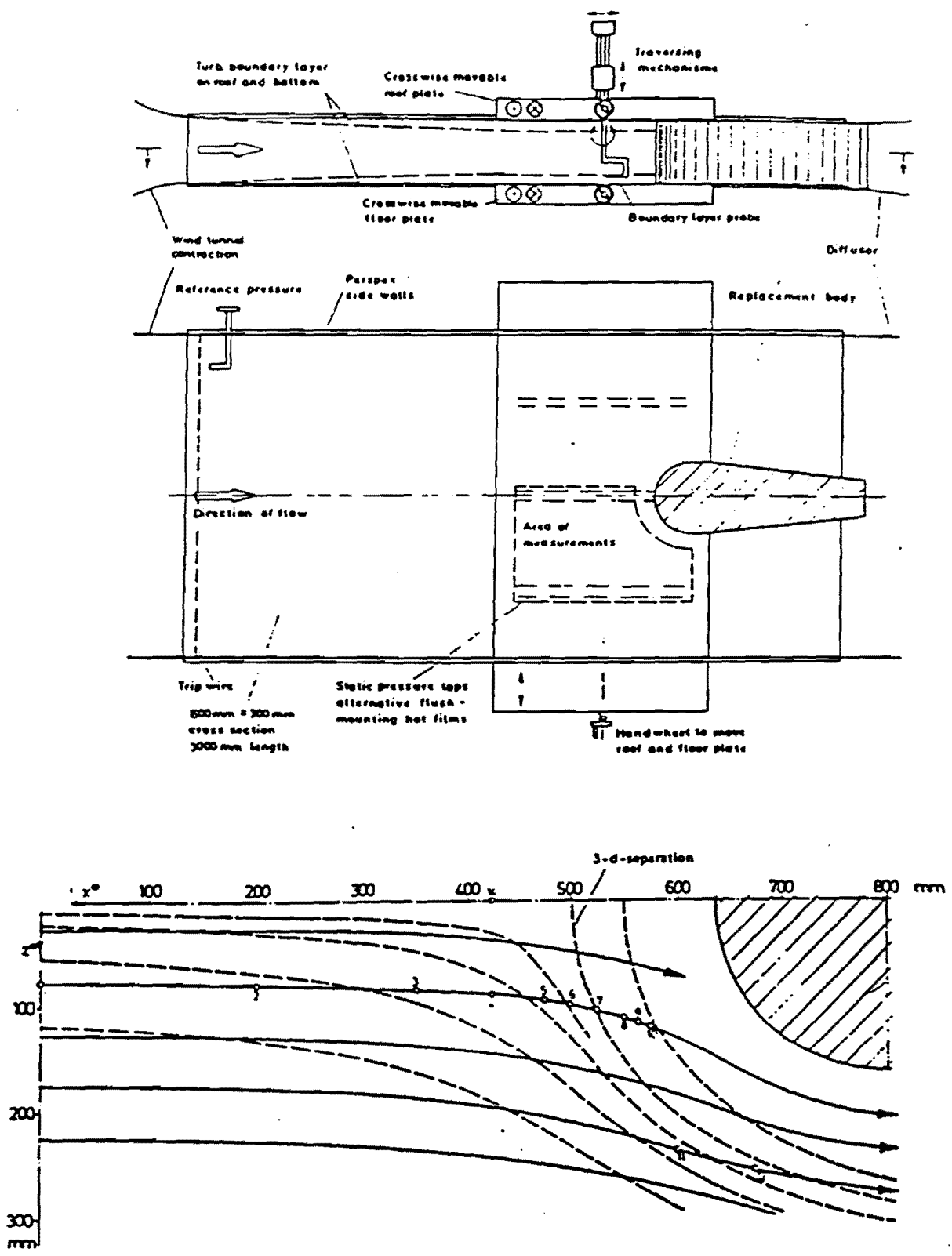

Figure 95. Schematic figure of Dechow-Felsch experimental set up. 


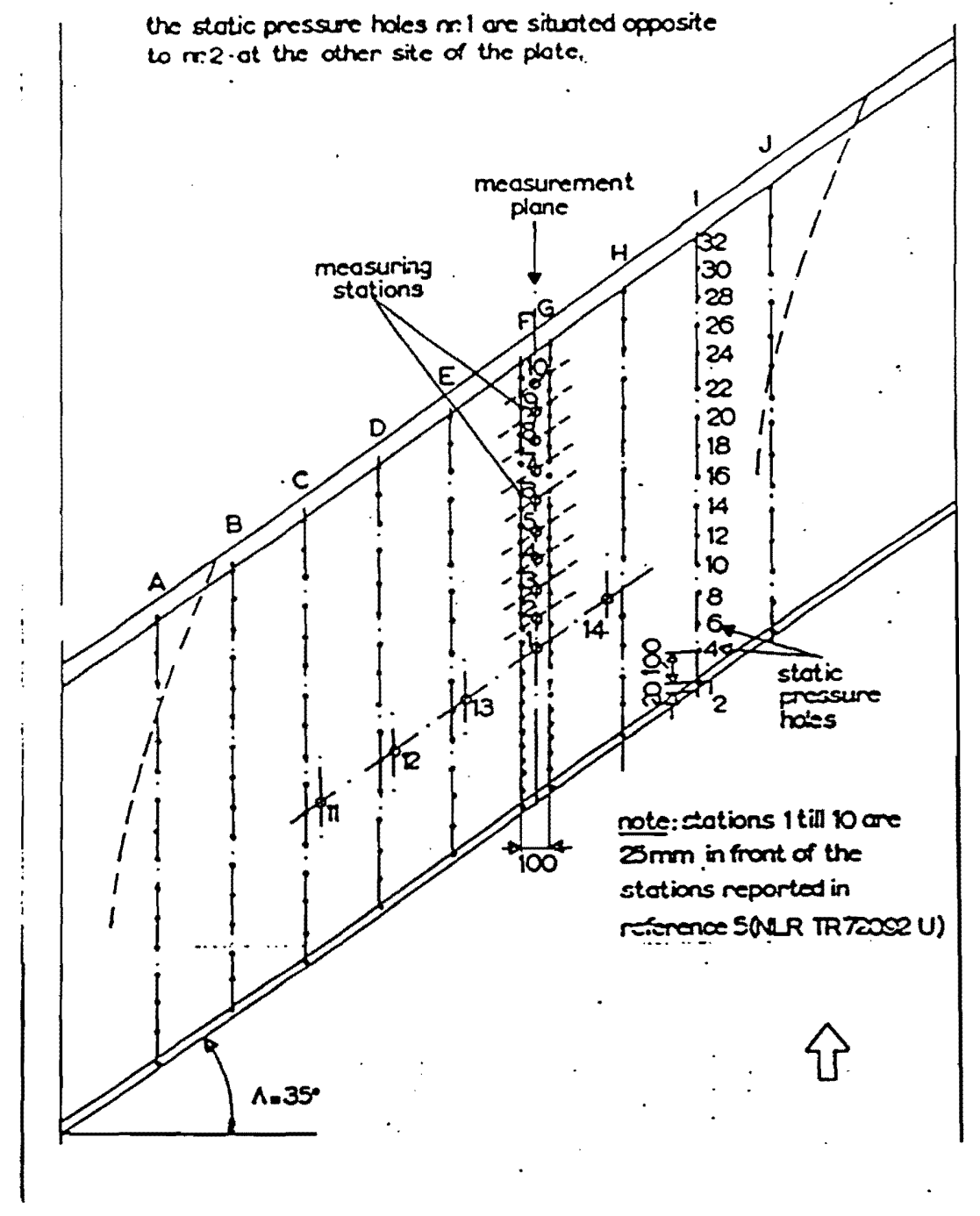

Figure 96. Schematic figure of Elsenaar-Boelsma experimental set up. 


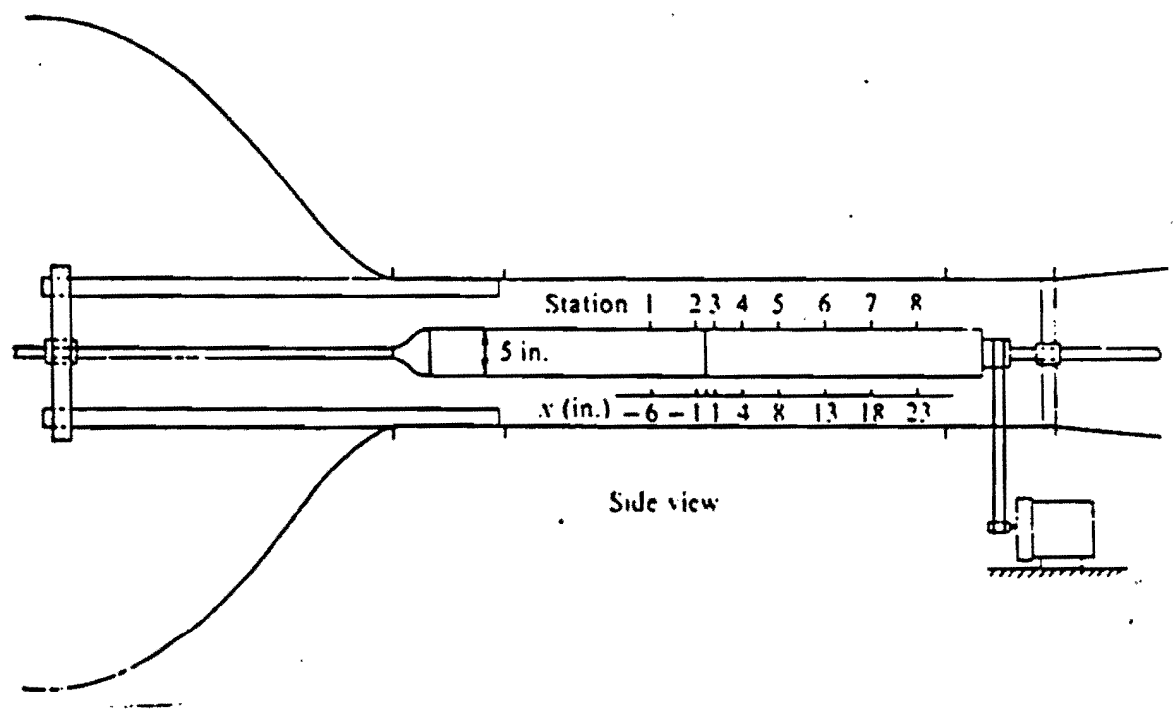

Figure 97. Schematic figure of Bissonnette-Mellor experimental set up. 


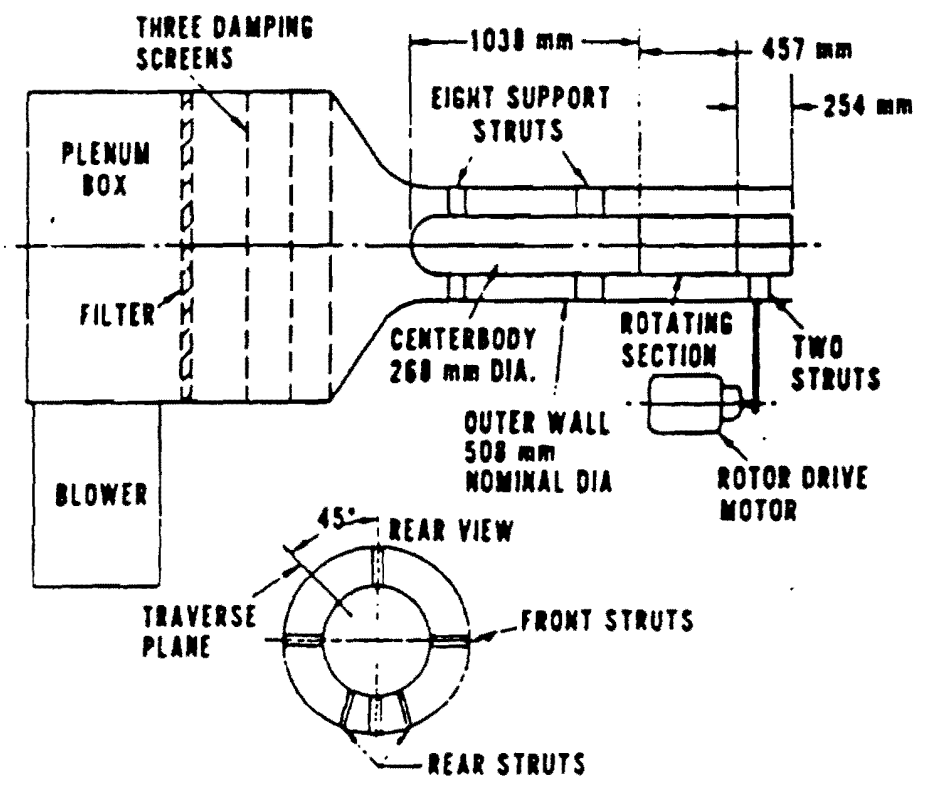

Figure 98. Schematic figure of Lohmann's experimental set up. 


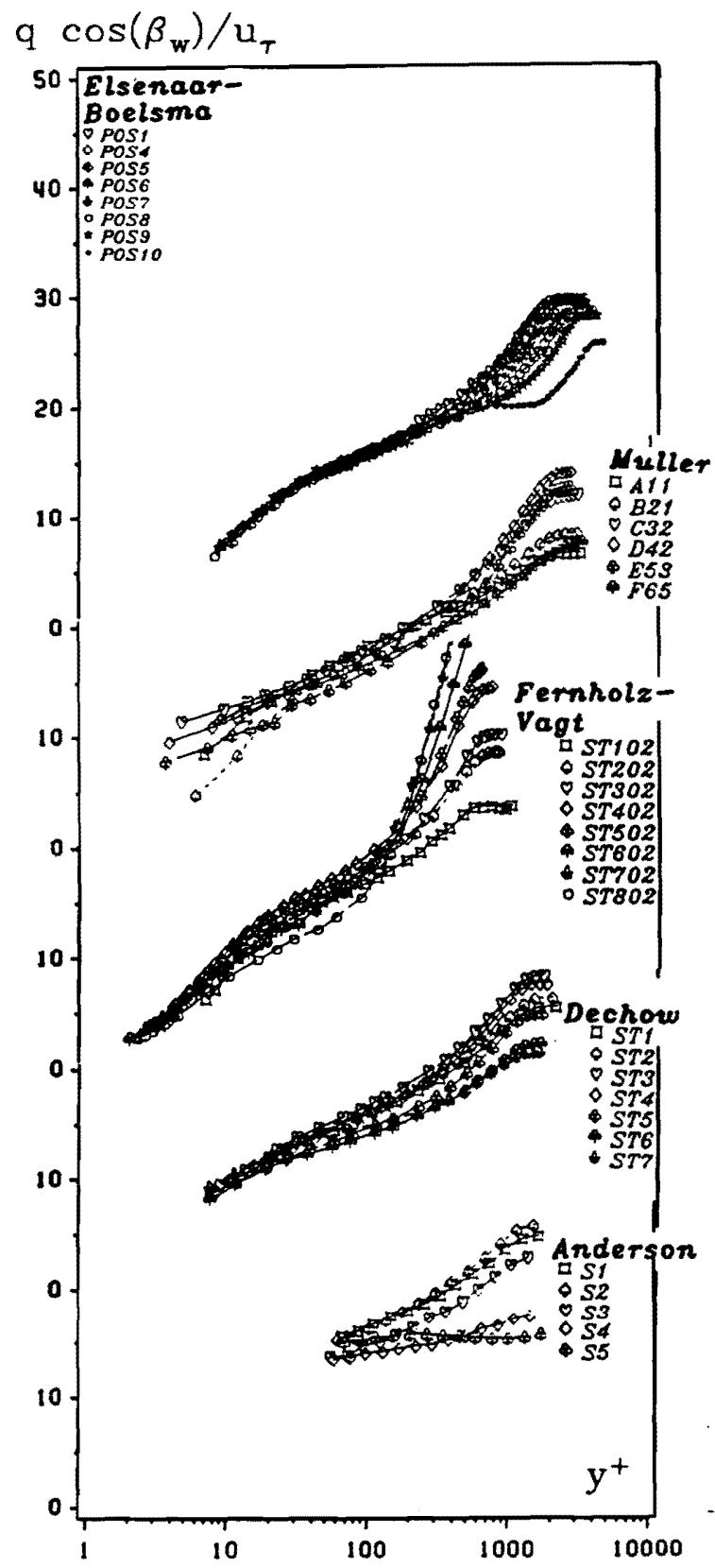

Figure 99a. Pressure-driven data in the Law-of-the-Wall coordinate system of Coles. 


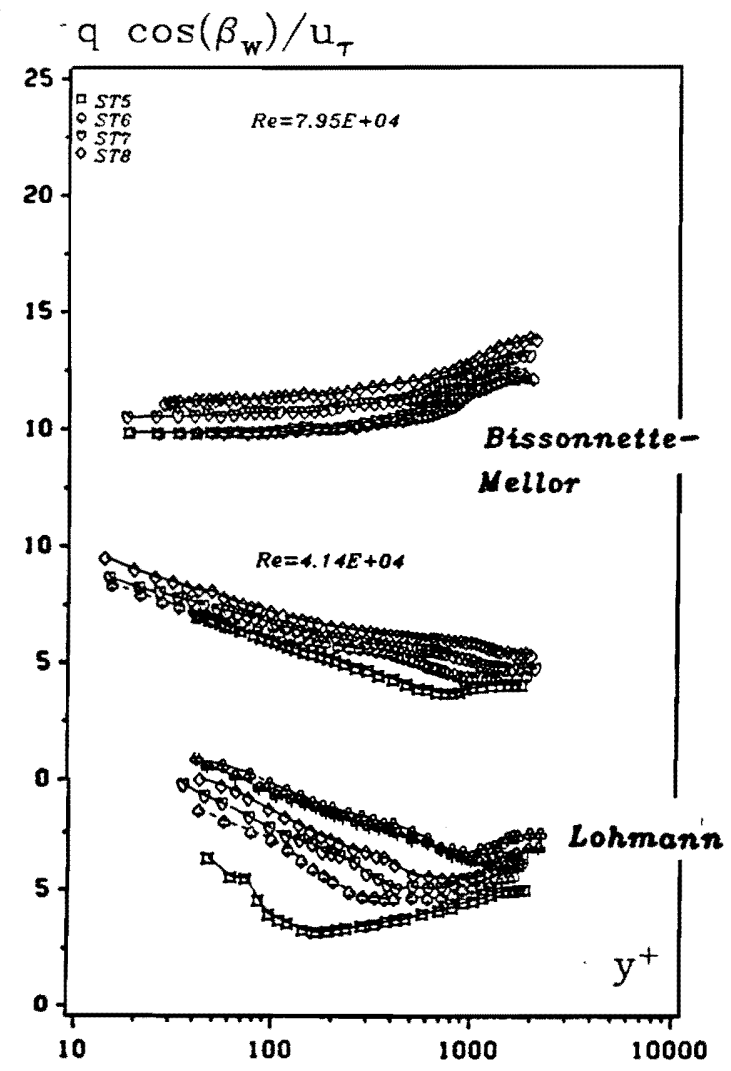

Figure 99b. Shear-driven data in the Law-of-the-Wall coordinate system of Coles. 


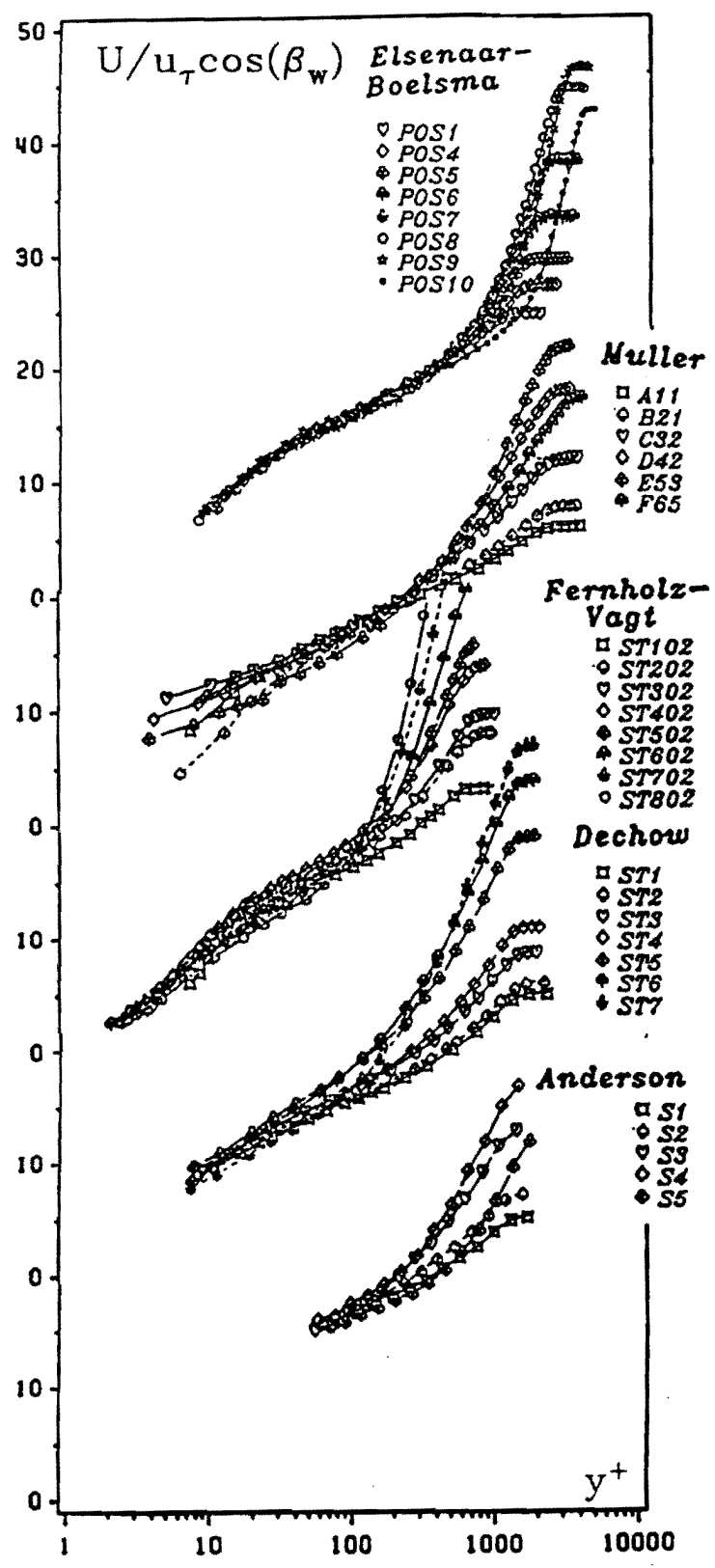

Figure 100a. Pressure-driven data in the Law-of-the-Wall coordinate system of Johnston. 


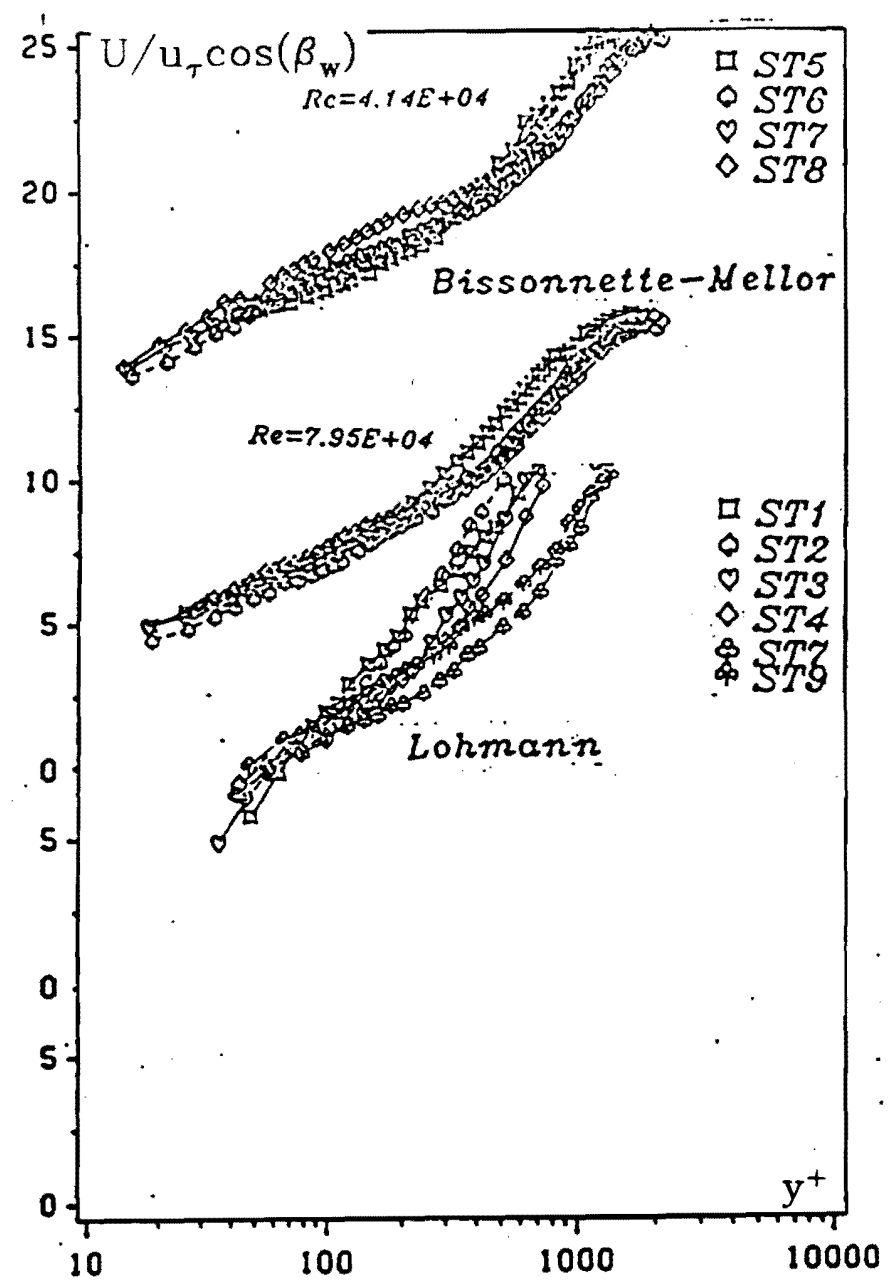

Figure 100b. Shear-driven data in the Law-of-the-Wall coordinate system of Johnston. 


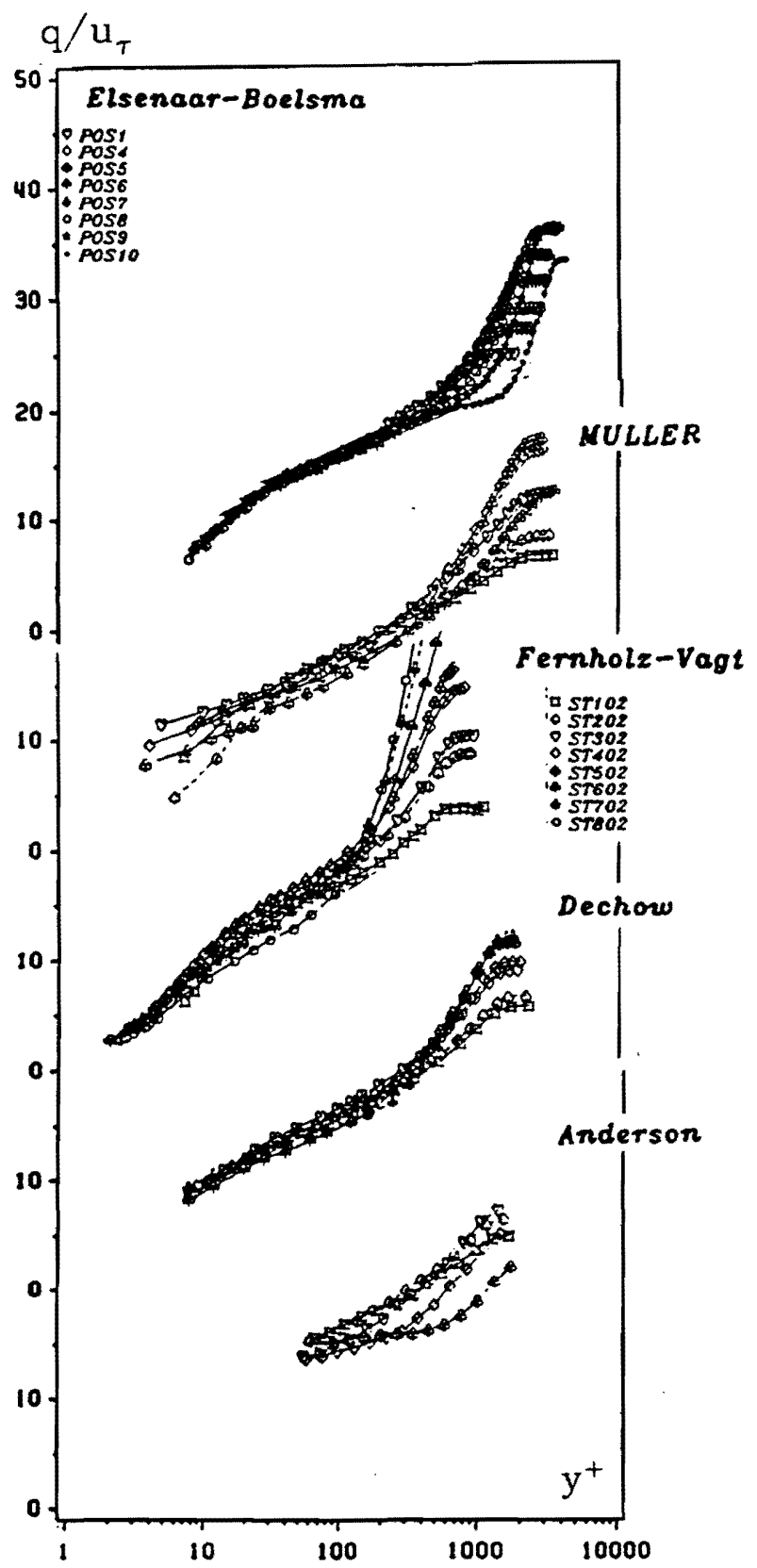

Figure 101a. Pressure-driven data in the Law-of-the-Wall coordinate system of Hornung-Joubert. 


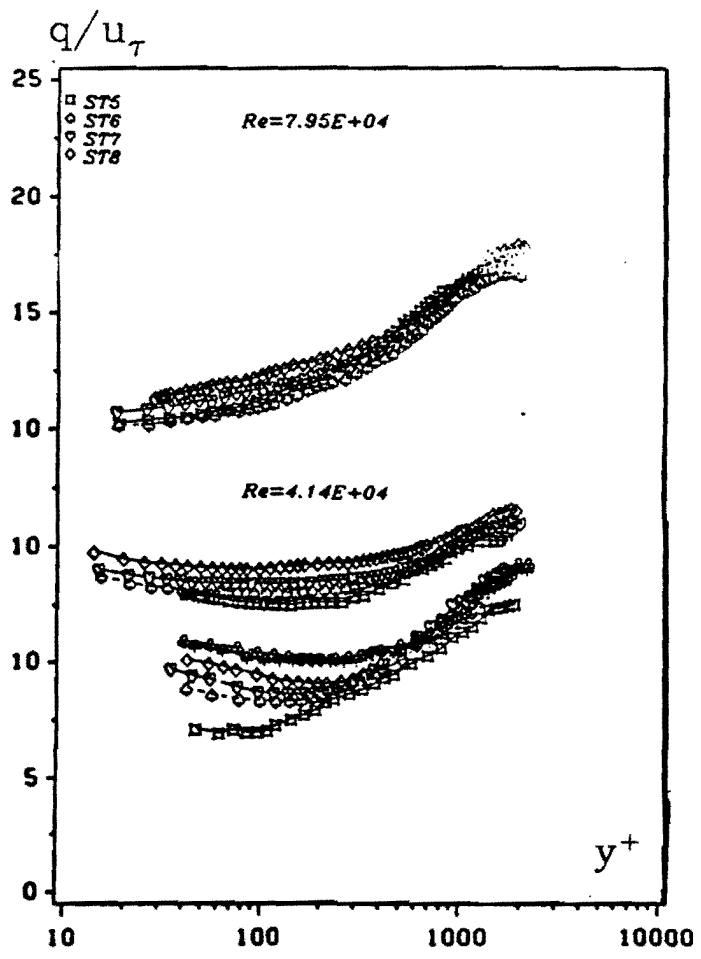

Figure 10lb. Shear-driven data in the Law-of-the-Wall coordinate system of Hornung-Joubert. 


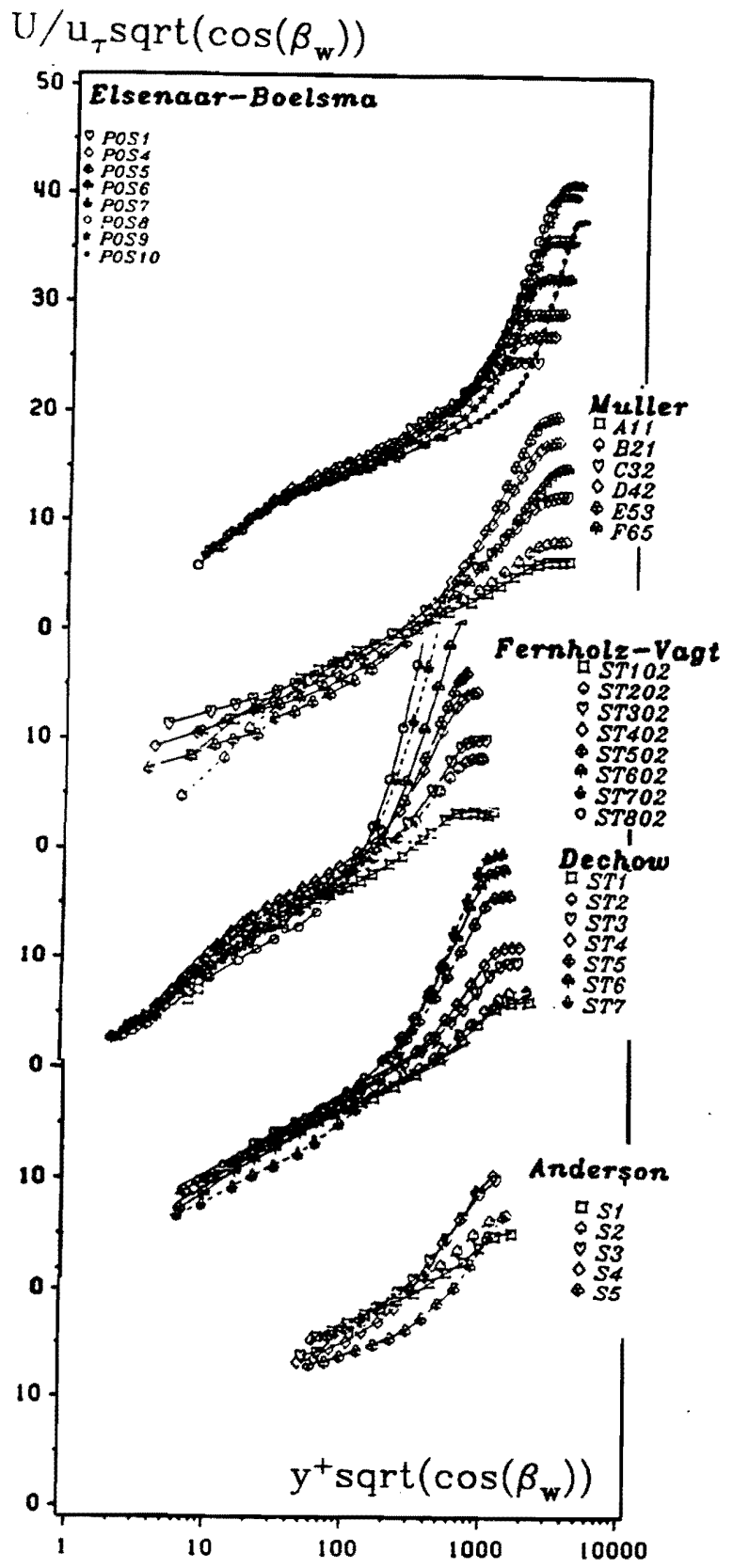

Figure 102a. Pressure-driven data in the Law-of-the-Wall coordinate system of Pierce-Krommenhoek, Chandrashekhar-Swamy. 


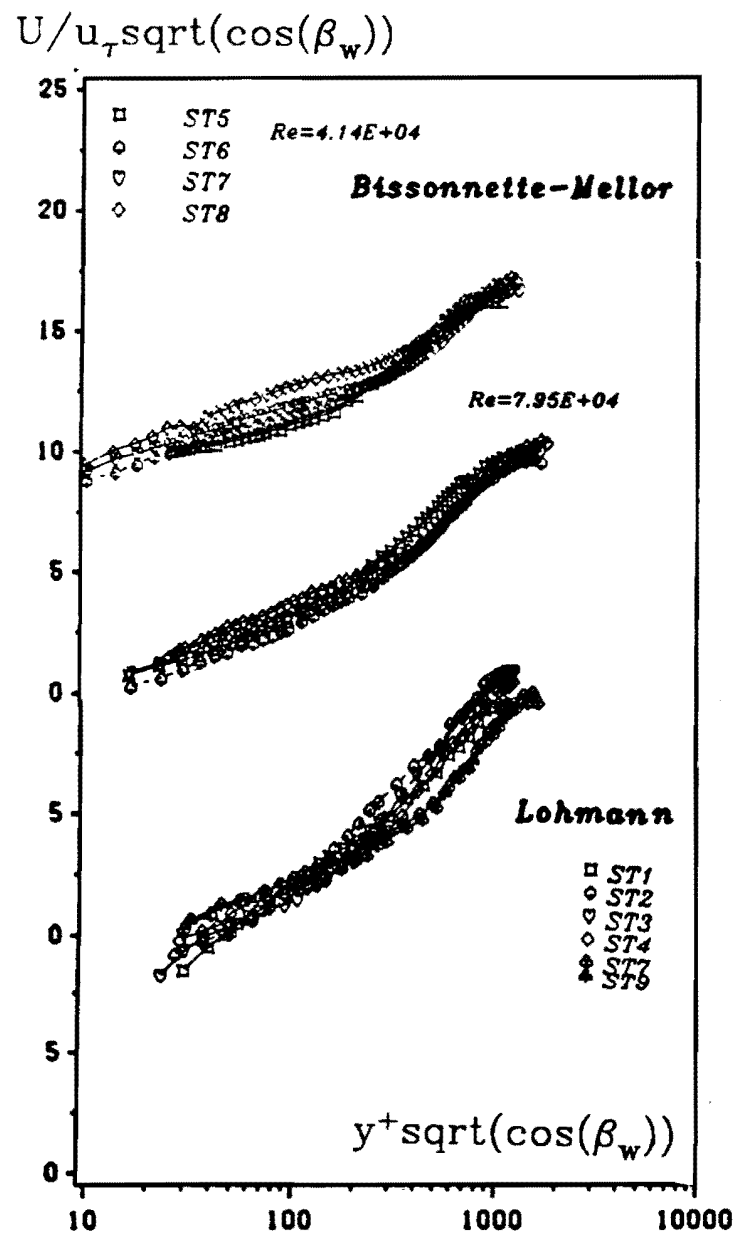

Figure 102b. Shear-driven data in the Law-of-the-Wall coordinate system of Pierce-Krommenhoek, Chandrashekhar-Swamy. 


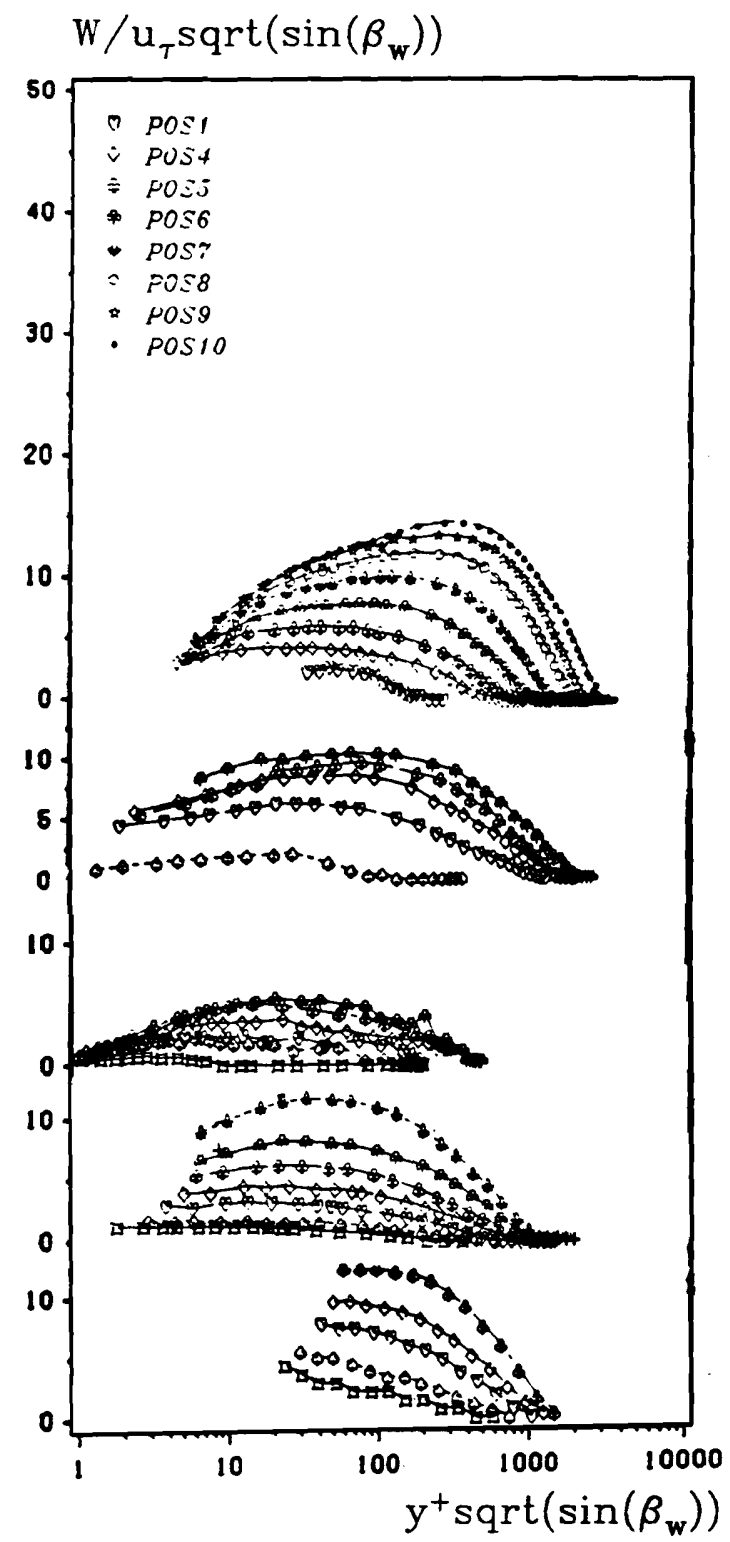

Figure 103a. Pressure-driven data in the Law-of-the-Wall coordinate system of Chandrashekhar-Swamy for the lateral component. 


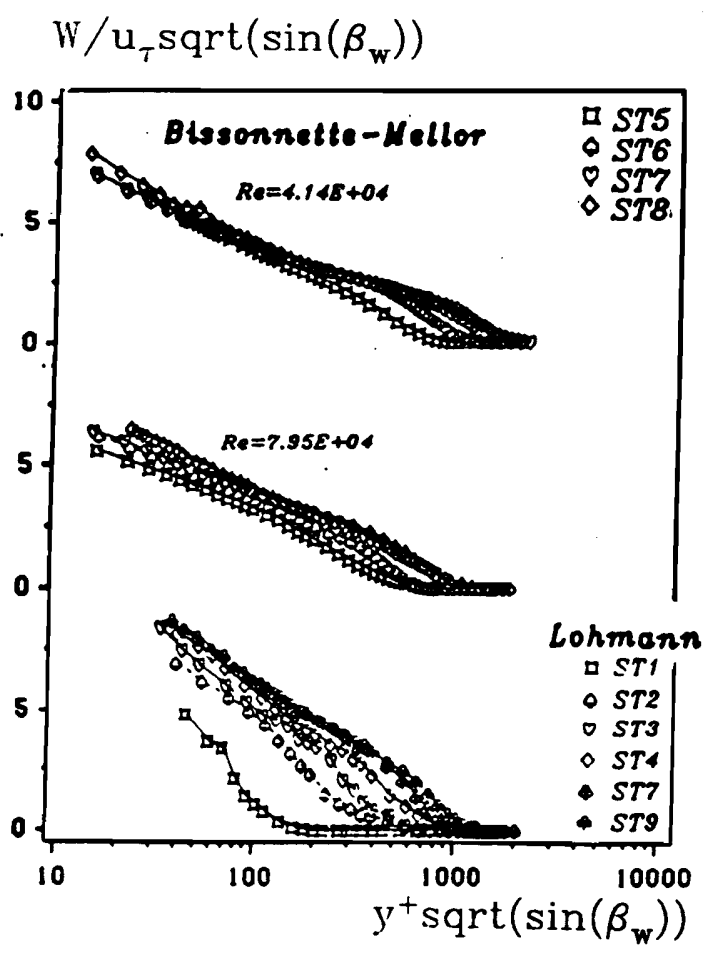

Figure 103b. Shear-driven data in the Law-of-the-Wall coordinate system of Chandrashekhar-Swamy for the lateral component. 


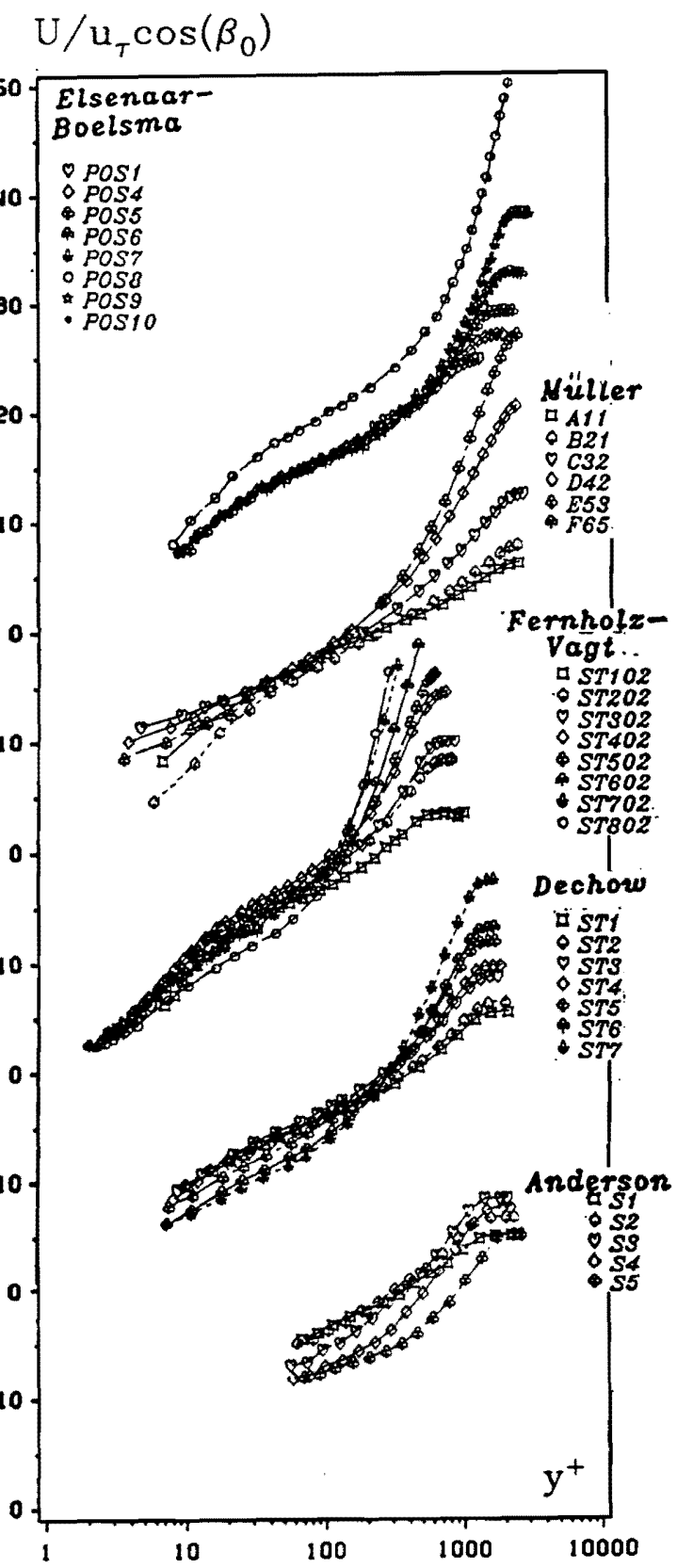

Figure 104a. Pressure-driven data in the Law-of-the-Wall coordinate system of East-Hoxey. 


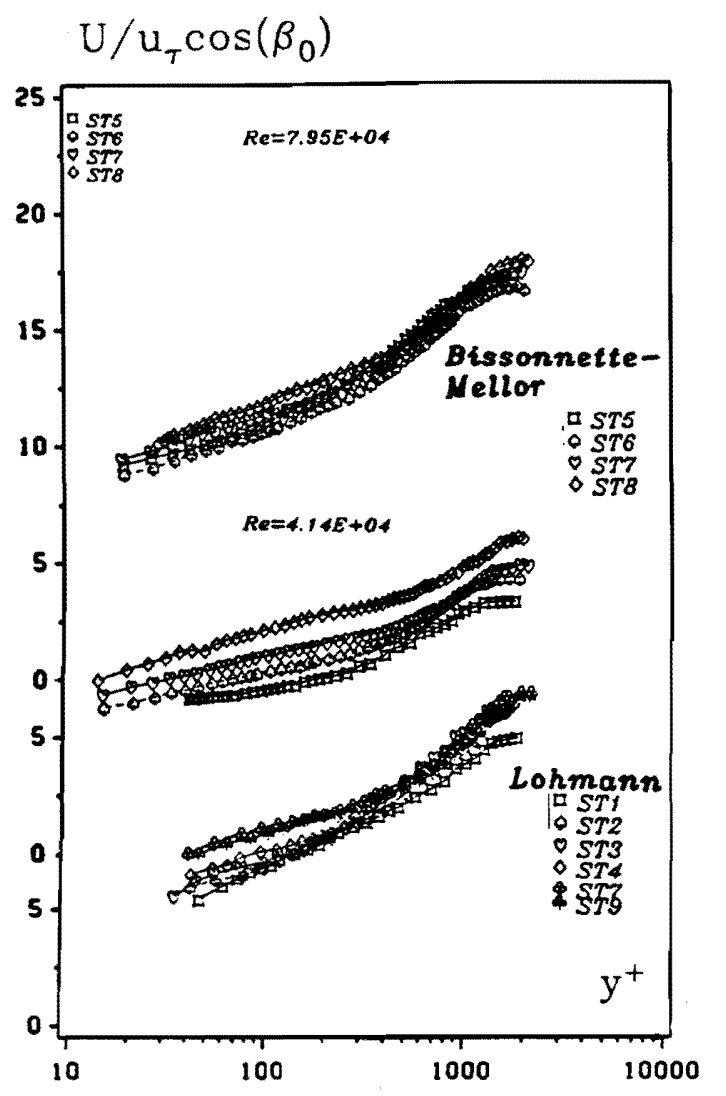

Figure 104b. Shear-driven data in the Law-of-the-Wall coordinate system of East-Hoxey. 


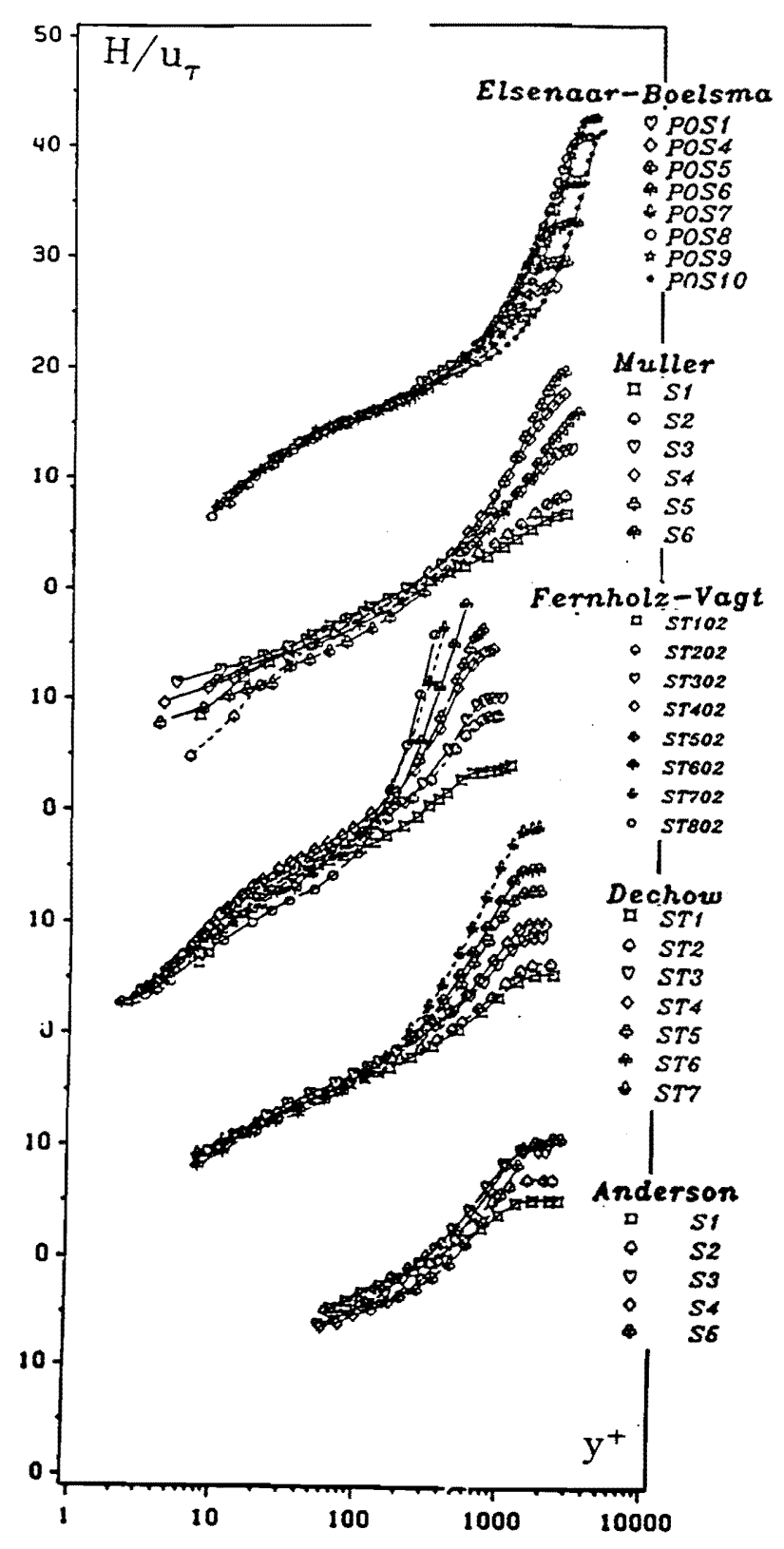

Figure 105ai. Pressure-driven data in the Law-of-the-Wall coordinate system of Perry-Joubert.: Left-hand side of the equation 8 in Chapter V. 


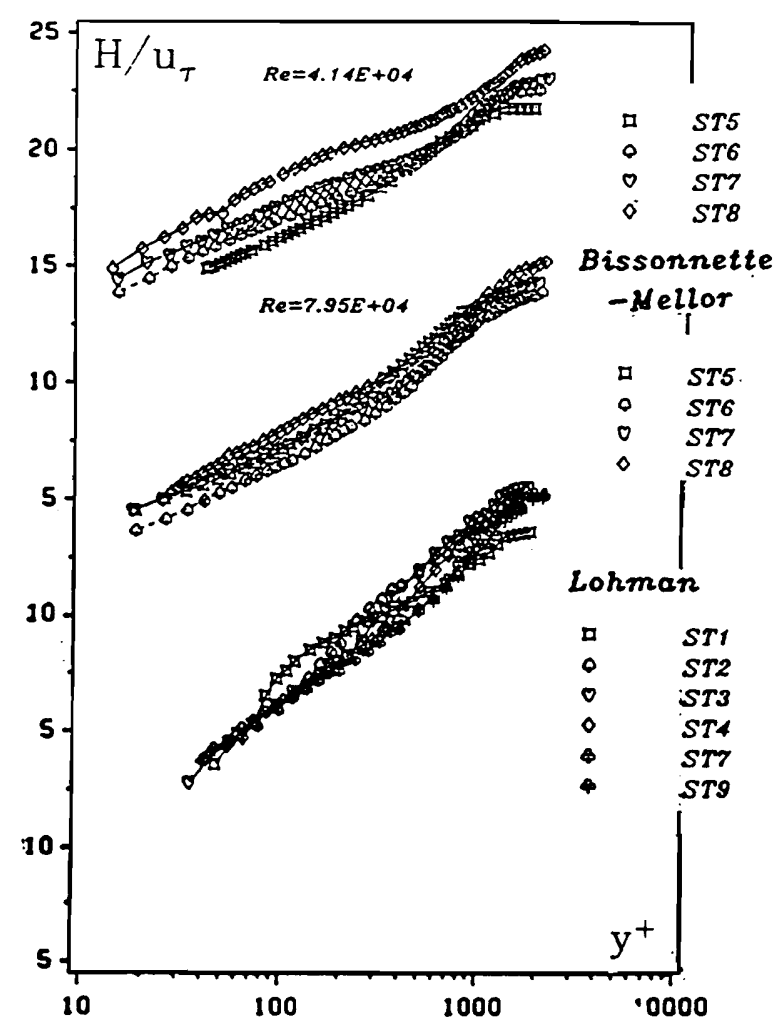

Figure 105aii. Shear-driven data in the Law-of-the-Wall coordinate system of Perry-Joubert.: Left-hand side of the equation 8 in Chapter V. 


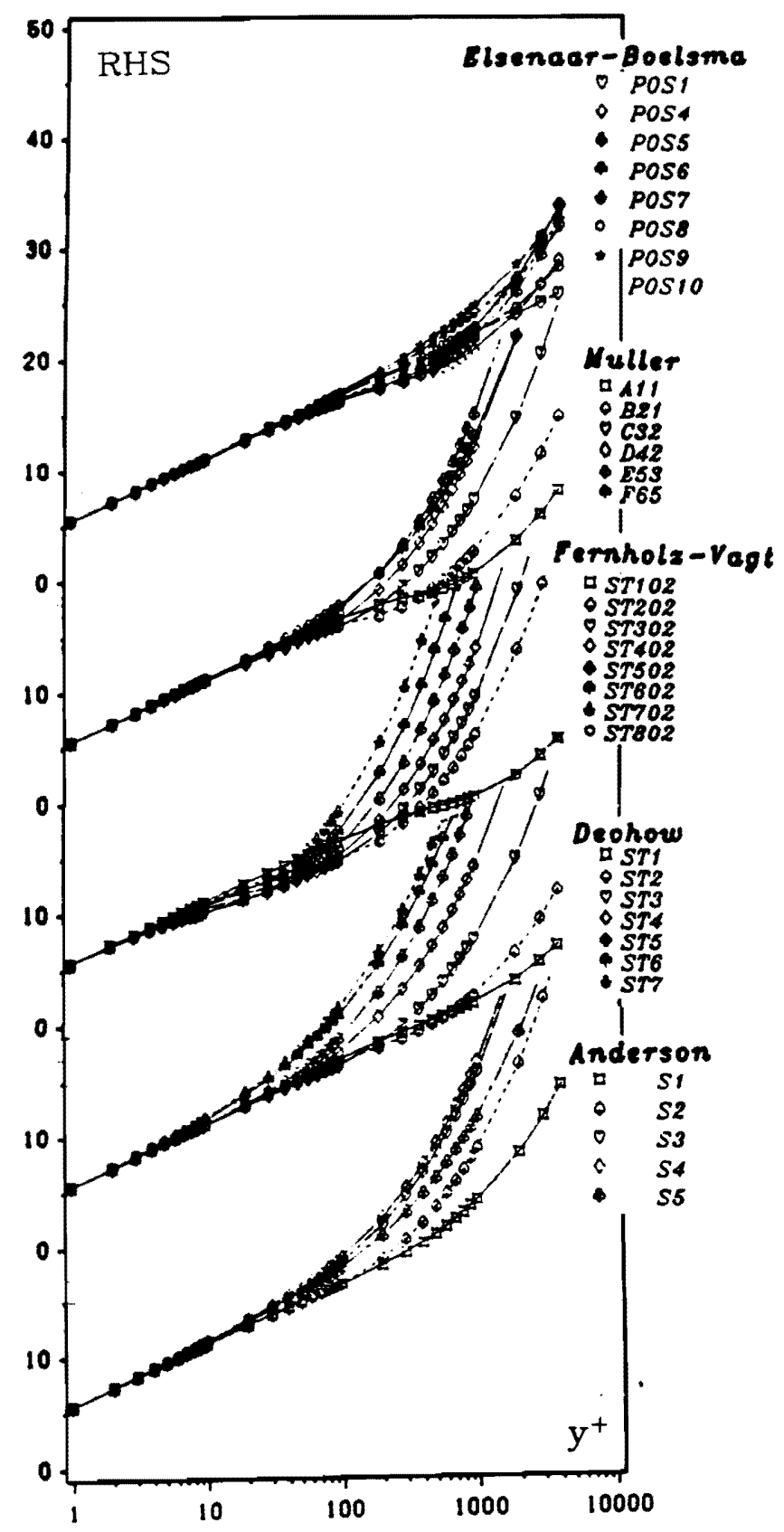

Figure 105bi. Pressure-driven data in the Law-of-the-Wall coordinate system of Perry-Joubert.: Right-hand side of the equation 8 in Chapter V. 


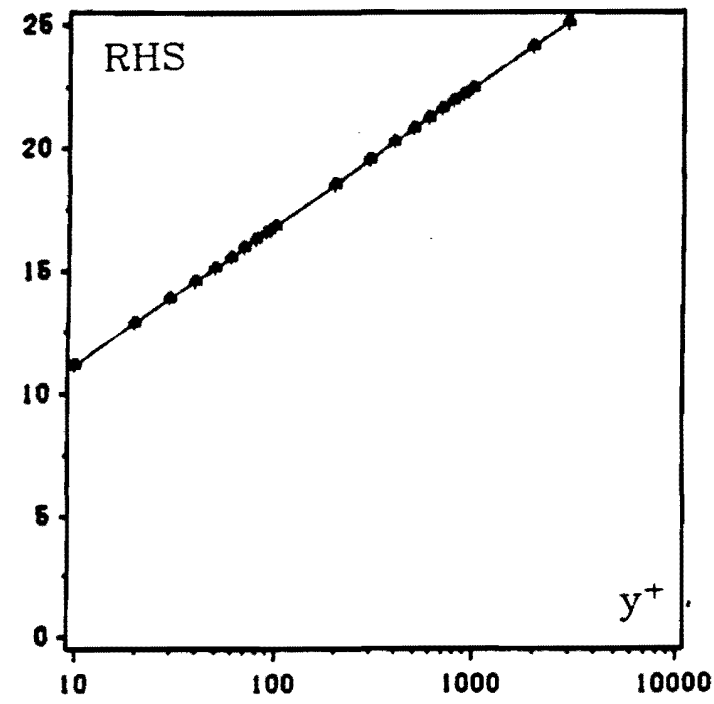

Figure 105bii. Shear-driven data in the Law-of-the-Wall coordinate system of Perry-Joubert.: Right-hand side of the equation 8 in chapter $\mathrm{V}$. 


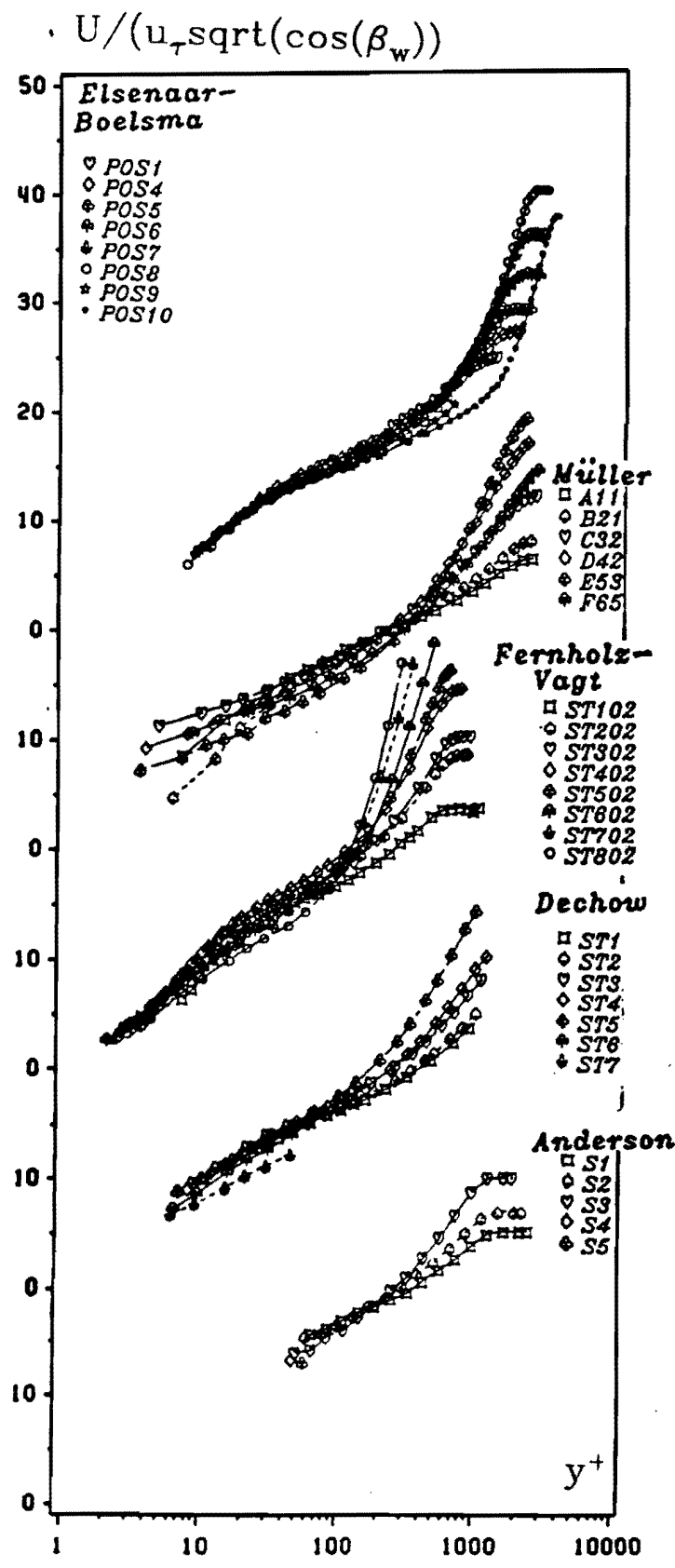

Figure 106ai. Pressure-driven data in the Law-of-the-Wall coordinate system of White-Lessmann-Christoph.: Left-hand side of the equation 11 in chapter V. Axial component of the model calculated from the data. 


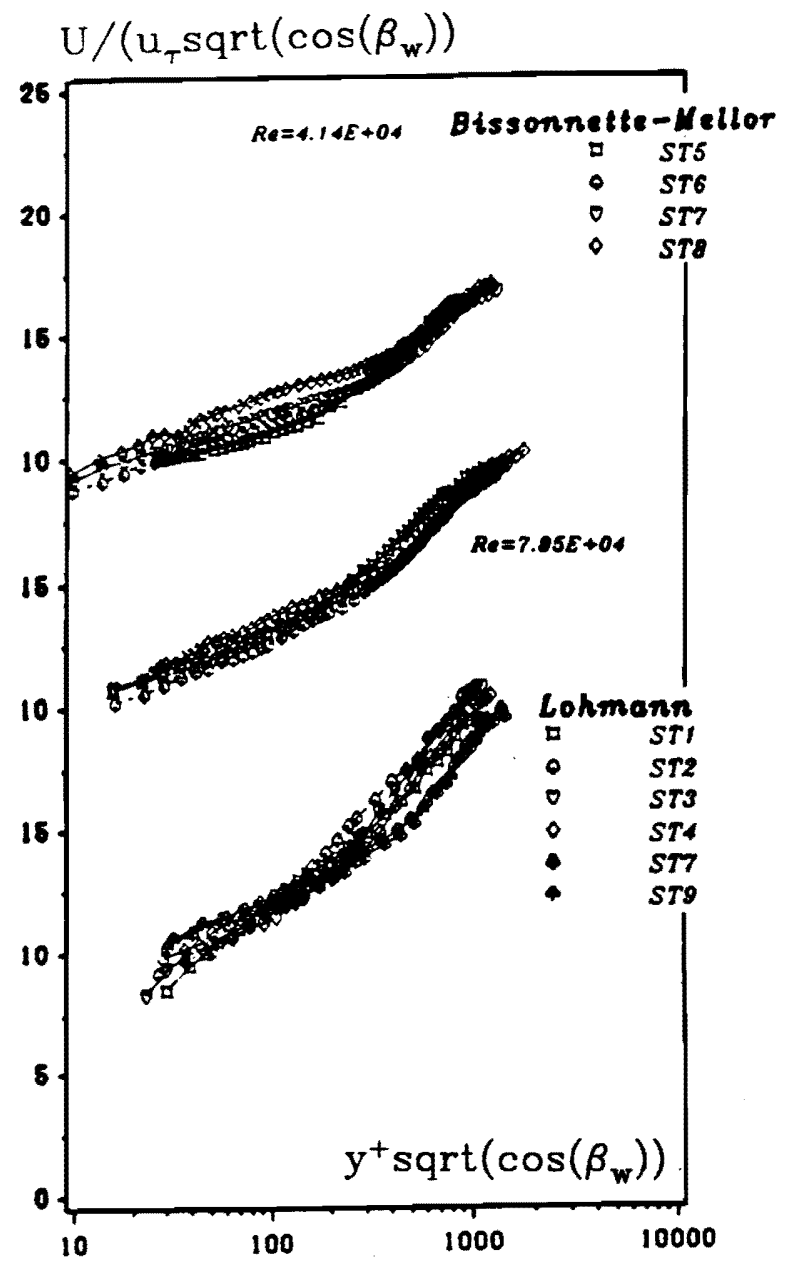

Figure 106aii. Shear-driven data in the Law-of-the-Wall coordinate system of White-Lessmann-Christoph.: Left-hand side of the equation 11 in chapter V. Axial component of the model calculated from the data. 


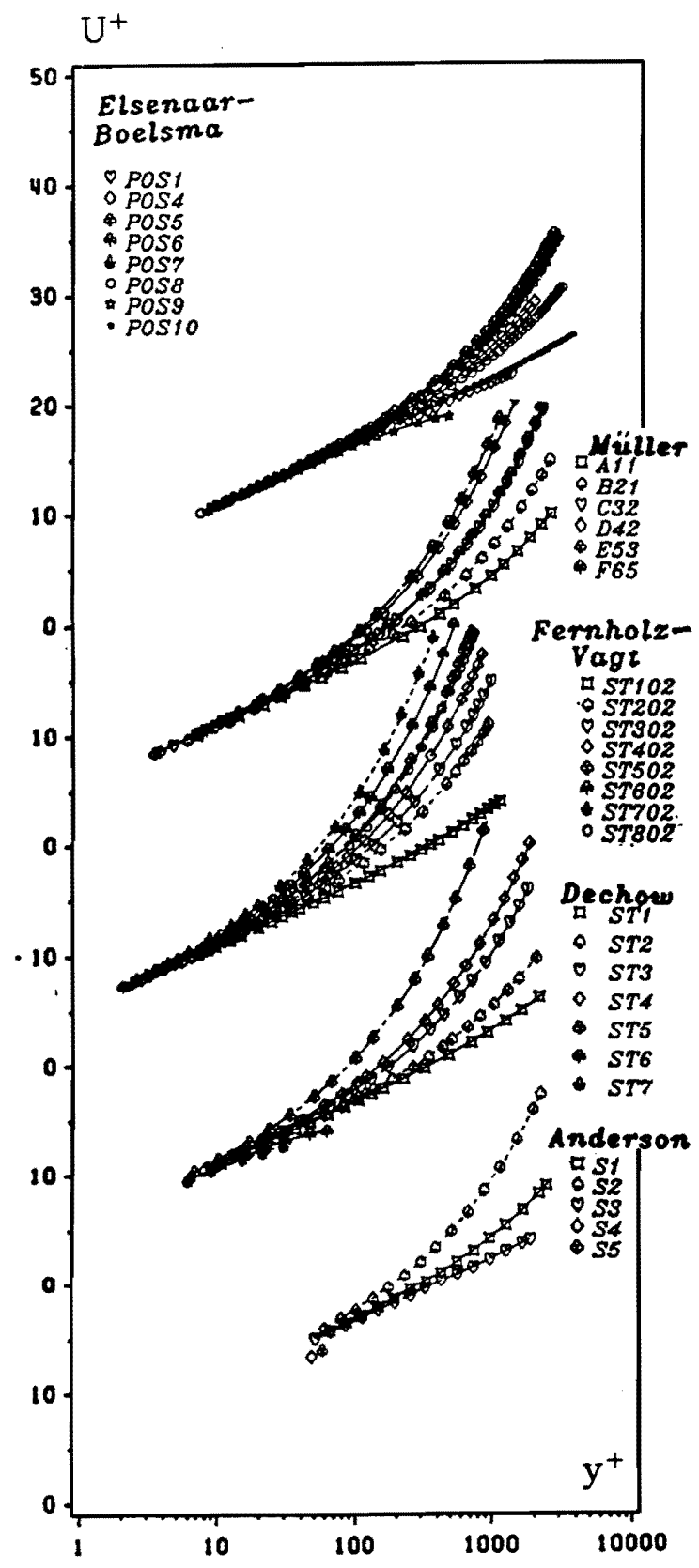

Figure 106bi. Pressure-driven data in the Law-of-the-Wall coordinate system of White-Lessmann-Christoph.: Right-hand side of the equation 11 in chapter V. Axial component of the model computed with a relation. 


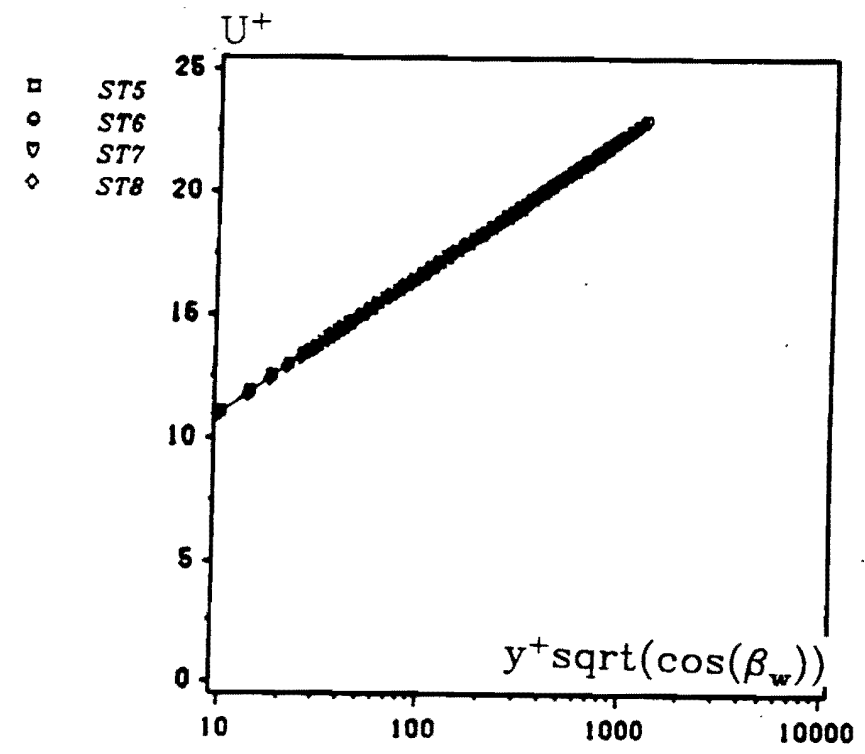

Figure 106bii. Shear-driven data in the Law-of-the-Wall coordinate system of White-Lessmann-Christoph.: Right-hand side of the equation 11 in chapter V. Axial component of the model computed with a relation. 

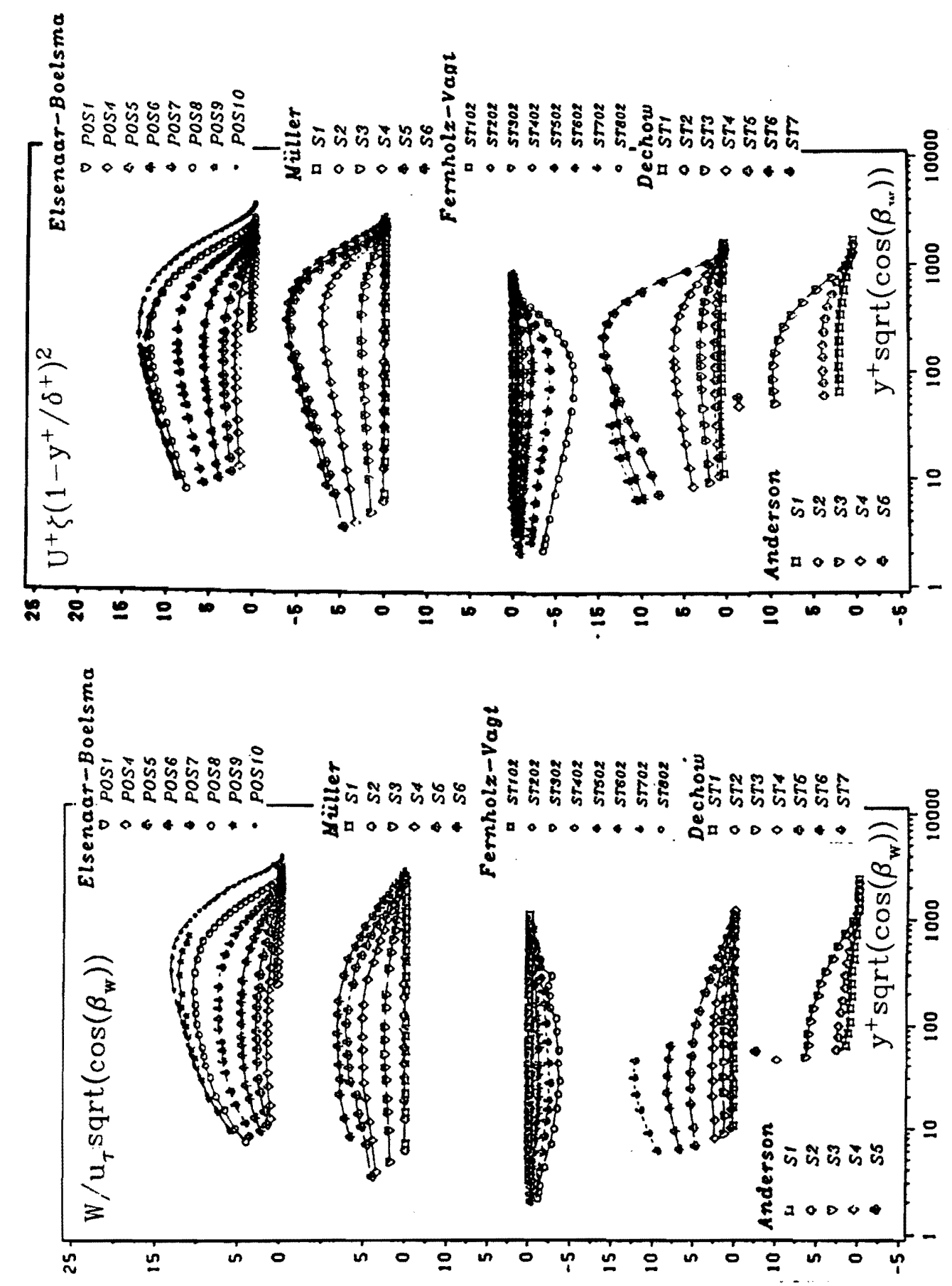

Figure 106ci106di. Pressure-driven data in the Law-of-the-Wall coordinate system of White-Lessmann-Christoph.: Left and right-hand side of the equation 12 in chapter V. Lateral component of the model calculated from the data and with a relation. 


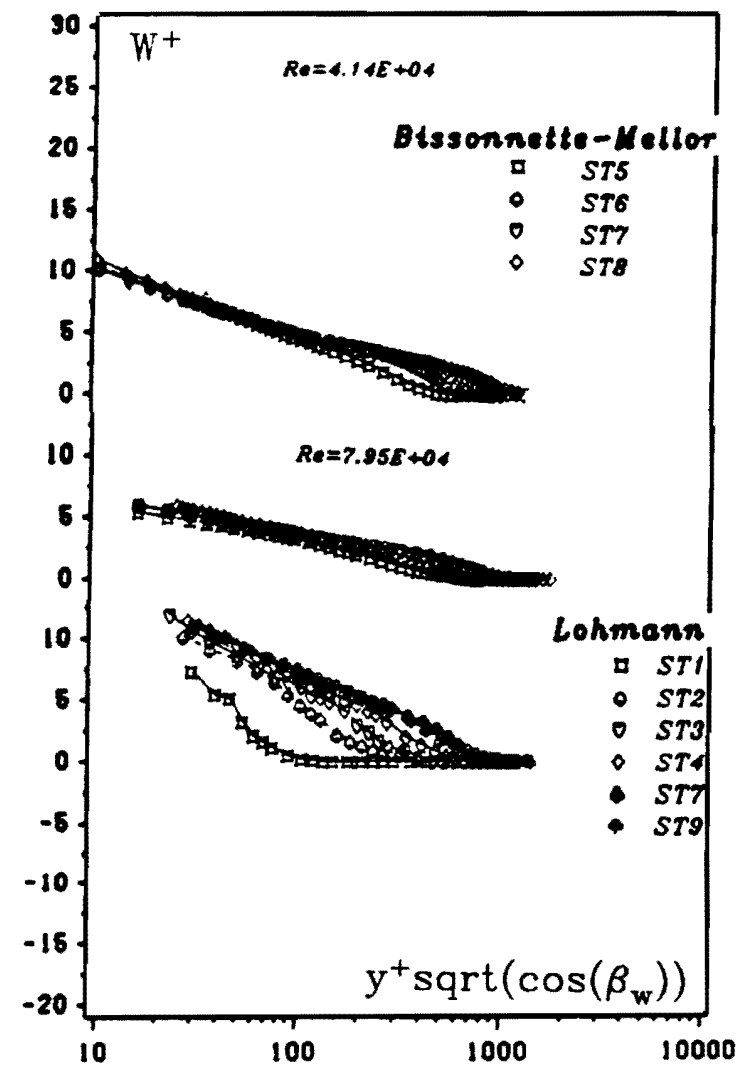

Figure 106cii. Shear-driven data in the Law-of-the-Wall coordinate system of White-Lessmann-Christoph.: Left-hand side of equation 12 in chapter V. Lateral component of the model calculated from the data. 


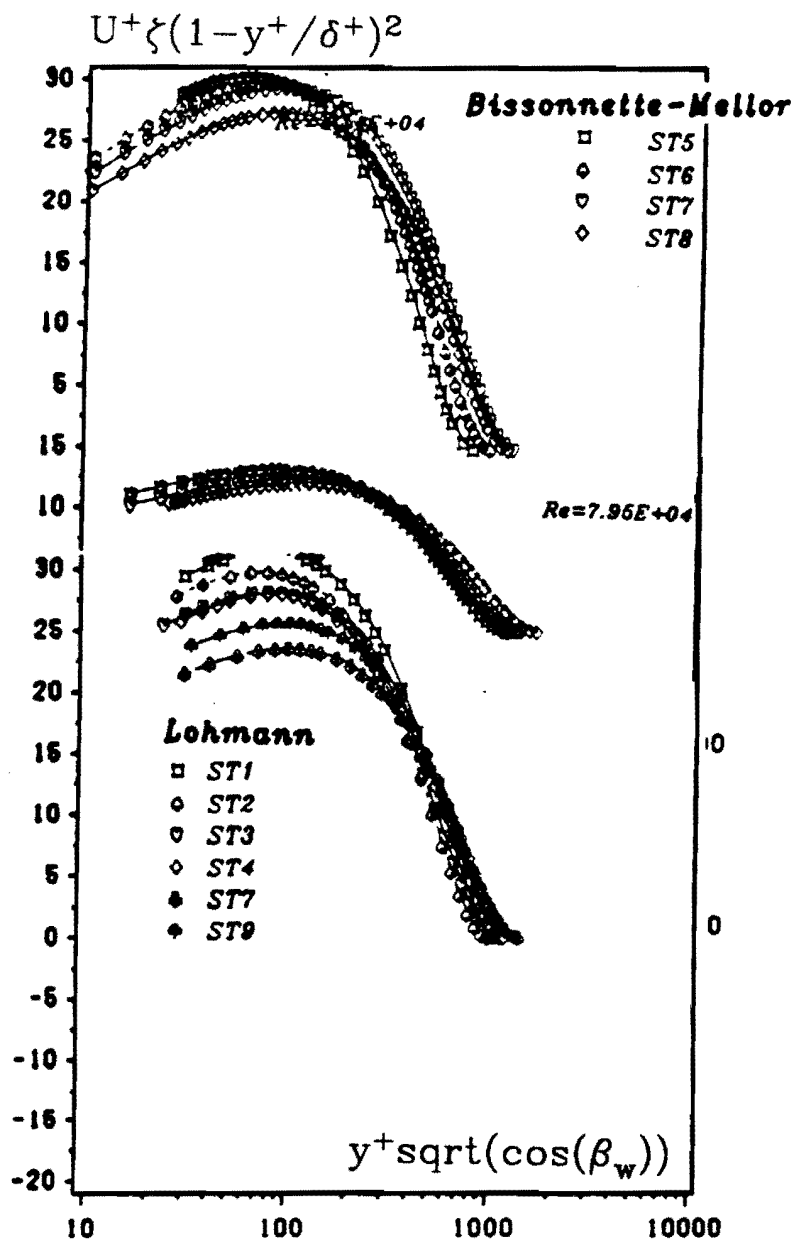

Figure 106dii. Shear-driven data in the Law-of-the-Wall coordinate system of White-Lessmann-Christoph.: Right-hand side of equation 12 in chapter V. Lateral comfonent of the model computed with a relation. 


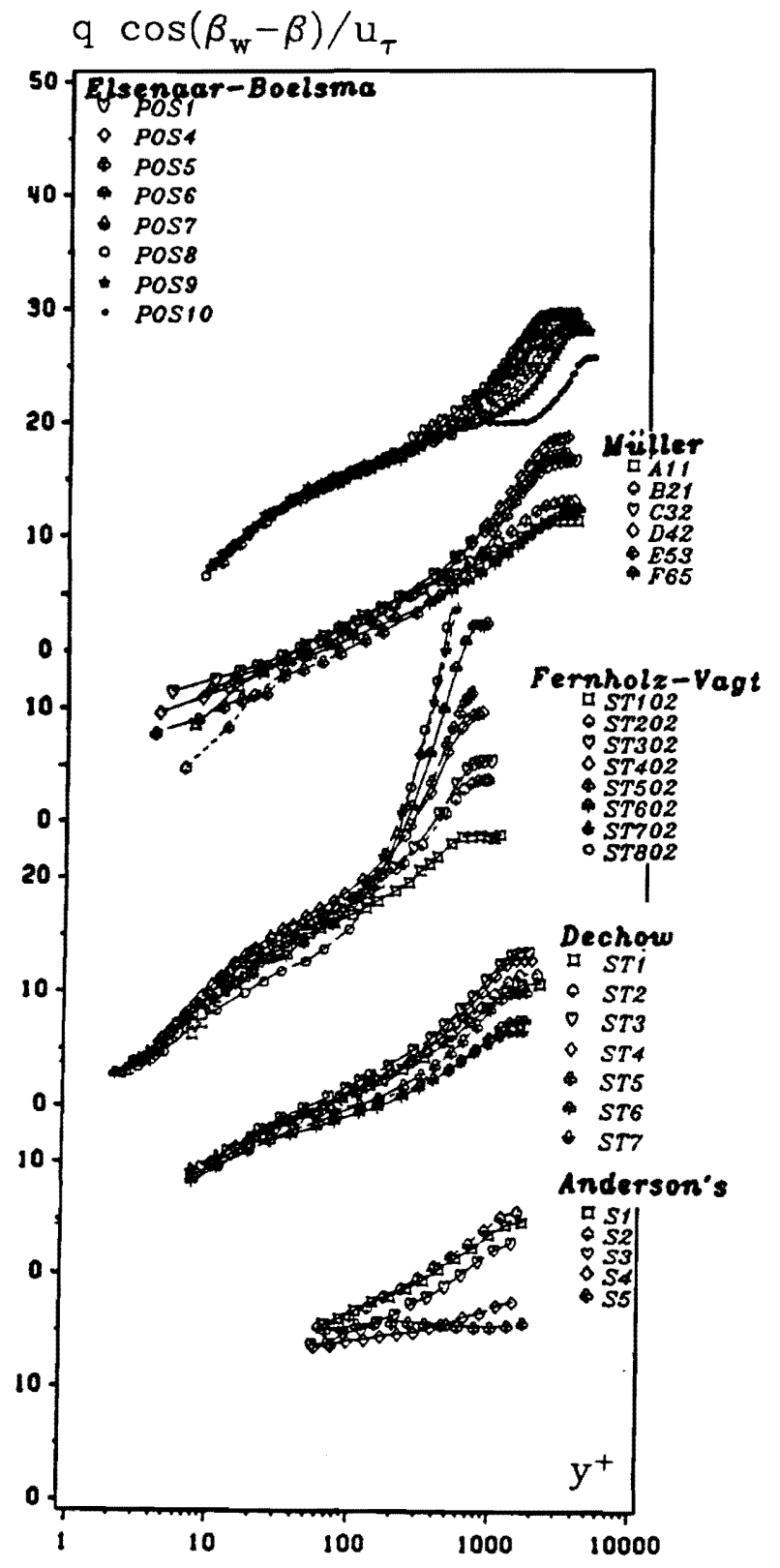

Figure 107ai. Pressure-driven data in the Law-of-the-Wall coordinate system of Van Den Berg.: Left-hand side of the equation 12 in chapter V. Axial component of the model calculated from the data. 


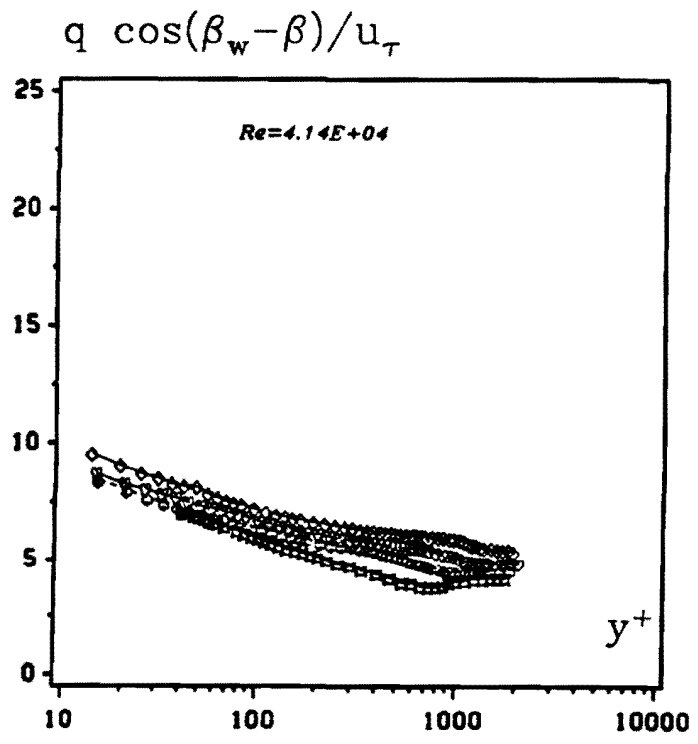

Figure 107aii. Shear-driven data in the Law-of-the-Wall coordinate system of Van Den Berg.: Left-hand side of the equation 12 in chapter V. Axial component of the model calculated from the data. 
$\mathrm{U}^{+} \mathbf{x}$

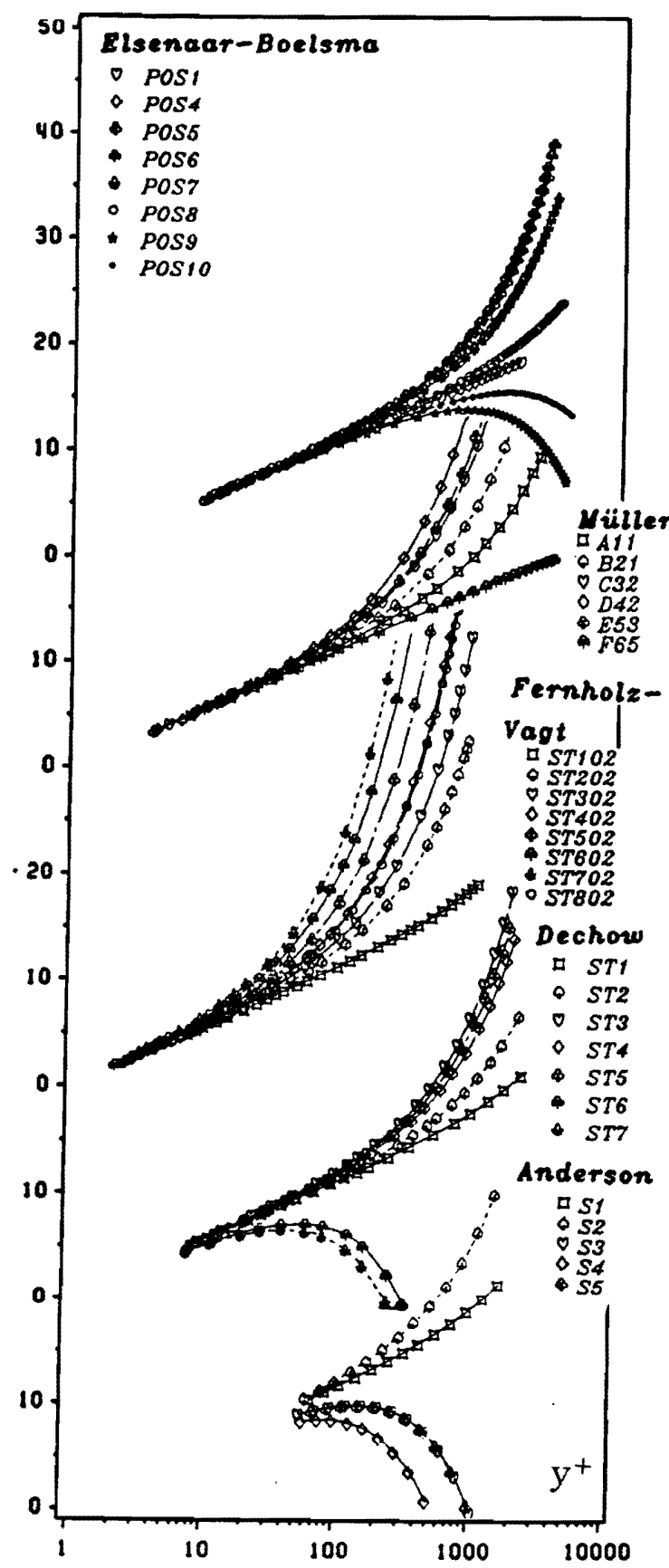

Figure 107bi. Pressure-driven data in the Law-of-the-Wall coordinate system of Van Den Berg.: Right-hand side of the equation 12 in chapter V. Axial component of the model calculated with a relation. 


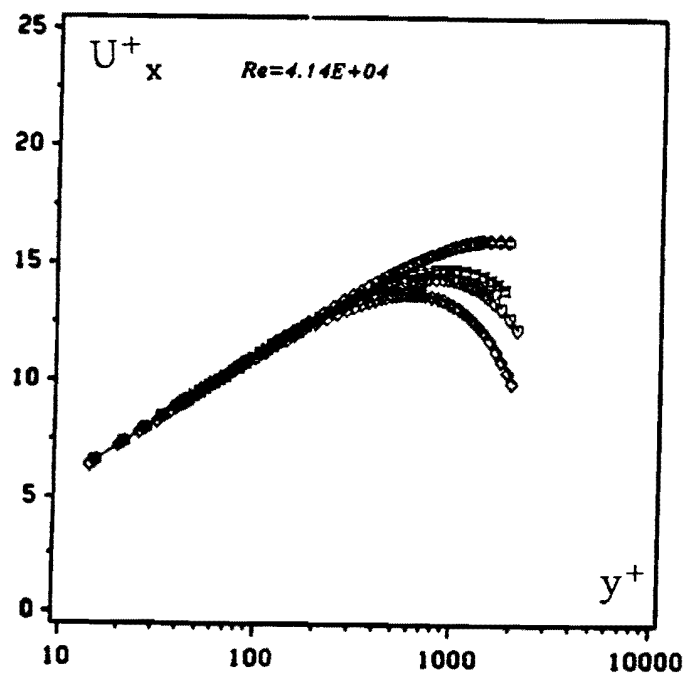

Figure 107bii. Shear-driven data in the Law-of-the-Wall coordinate system of Van Den Berg.: Right-hand side of the equation 12 in chapter V. Axial component of the model calculated with a relation. 


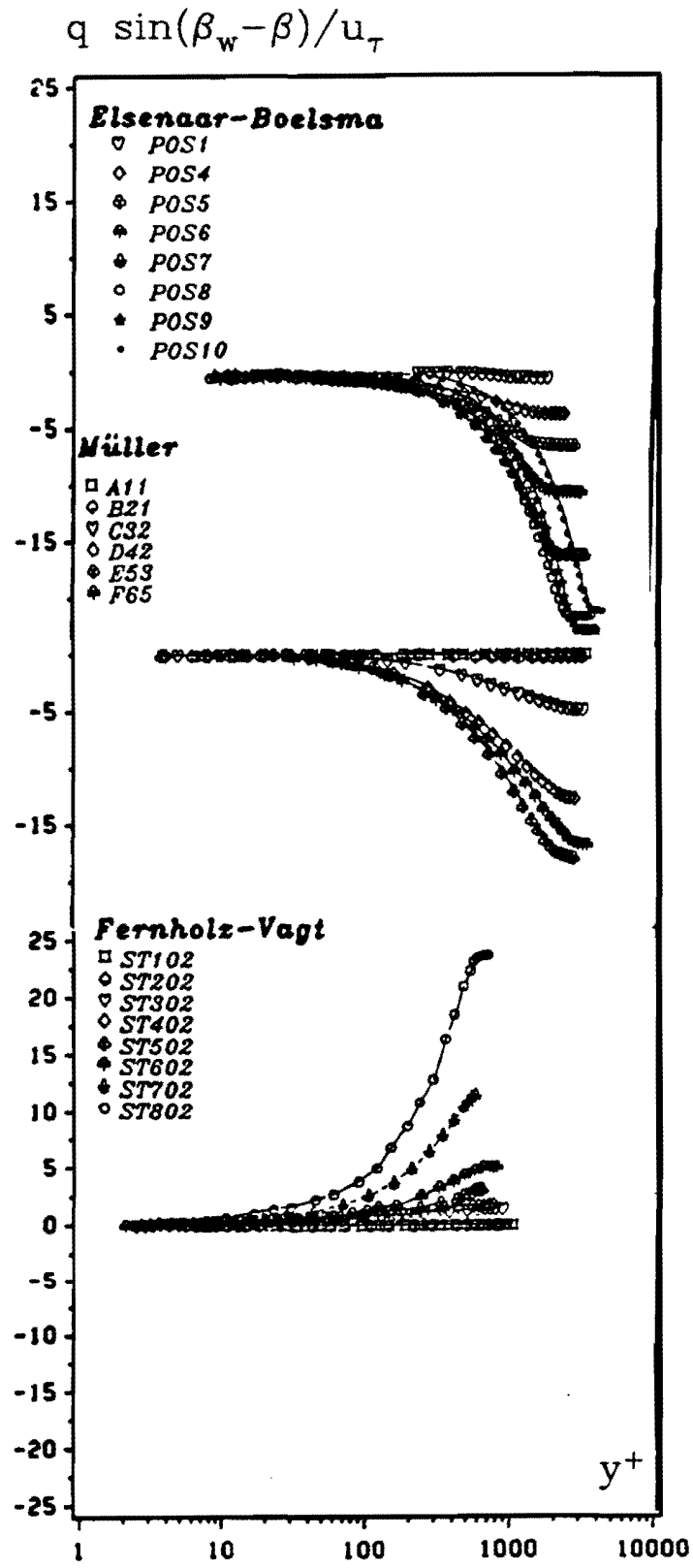

Figure 107ci. Pressure-driven data in the Law-of-the-Wall coordinate system of Van Den Berg.: Left-hand side of the equation 13 in chapter V. Lateral component of the model calculated from the data. 


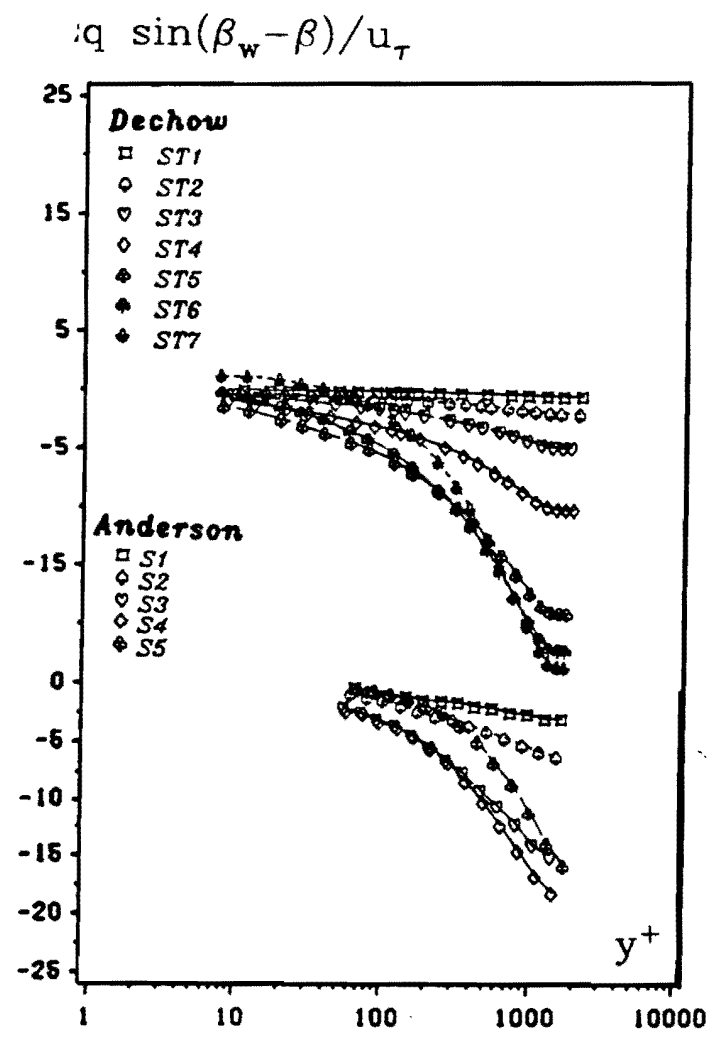

Figure 107cii. Pressure-driven data in the Law-of-the-Wall coordinate system of Van Den Berg.: Left-hand side of the equation 13 in chapter V. Lateral component of the model calculated from the data. 


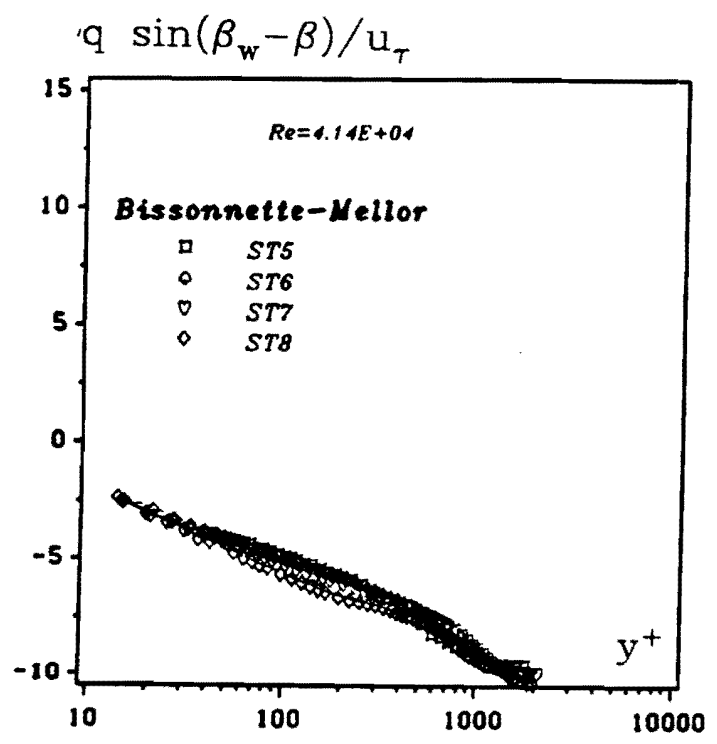

Figure 107ciii. Shear-driven data in the Law-of-the-Wall coordinate system of Van Den Berg.: Left-hand side of the equation 13 in chapter V. Lateral component of the model calculated from the data. 


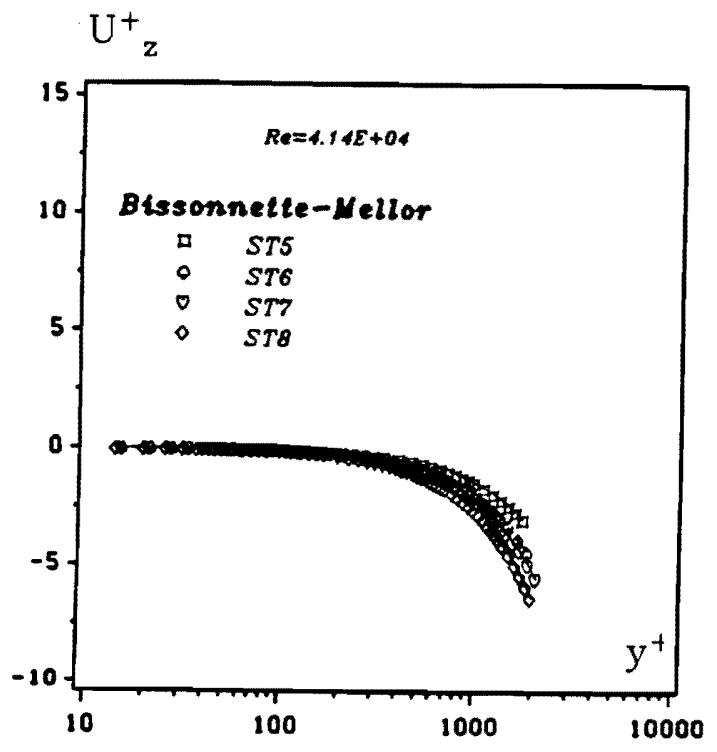

Figure 107di. Pressure-driven data in the Law-of-the-Wall coordinate system of Van Den Berg.: Right-hand side of the equation 13 in chapter V. Lateral component of the model calculated with a relation. 


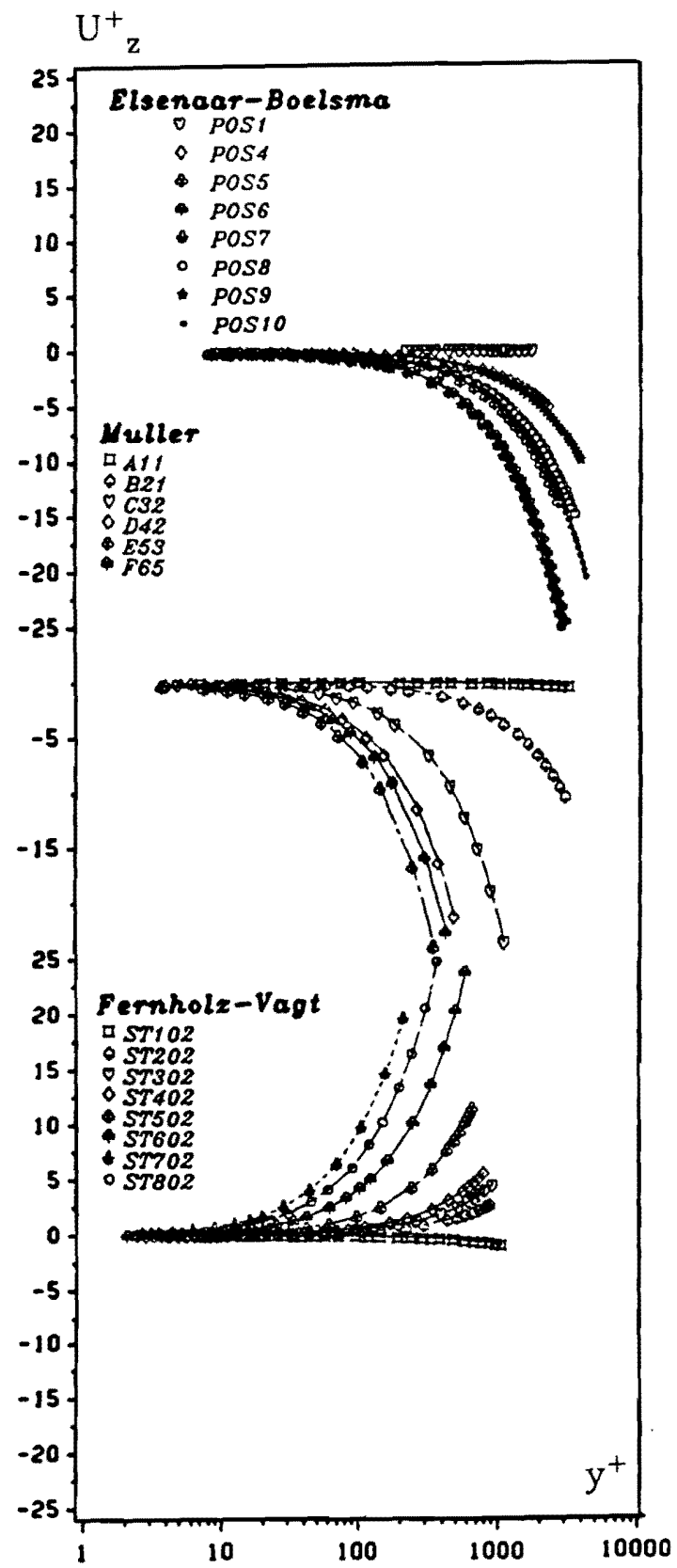

Figure 107dii. Pressure-driven data in the Law-of-the-Wall coordinate system of Van Den Berg.: Right-hand side of the equation 13 in chapter V. Lateral component of the model calculated with a relation. 


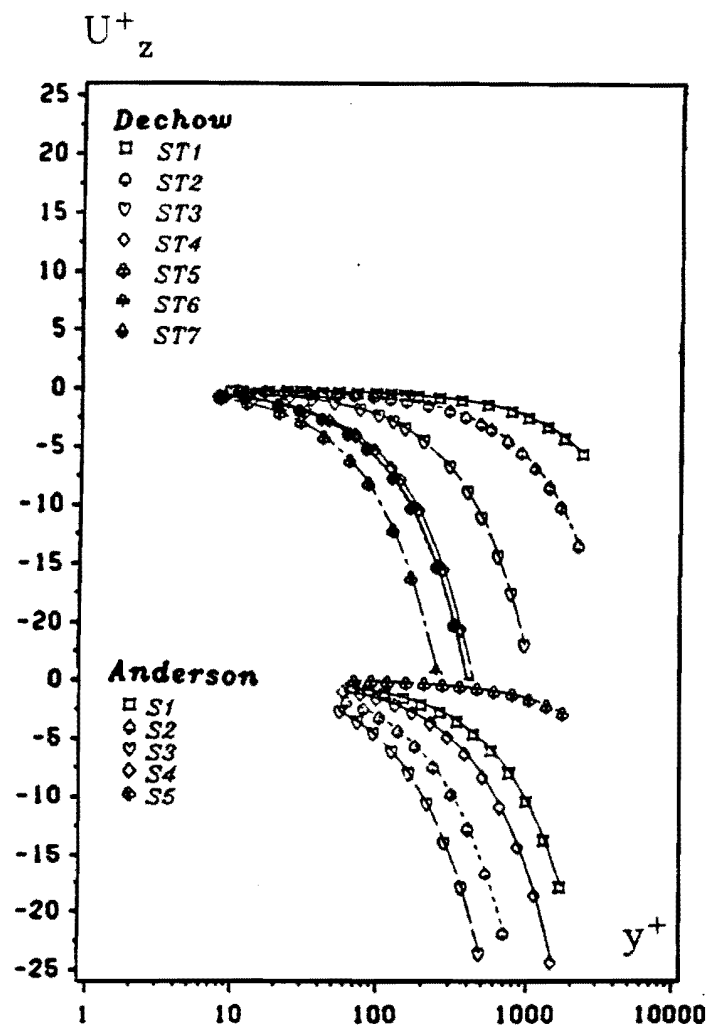

Figure 107diii. Shear-driven data in the Law-of-the-Wall coordinate system of Van Den Berg.: Right-hand side of the equation 13 in chapter V. Lateral component of the model calculated with a relation. 


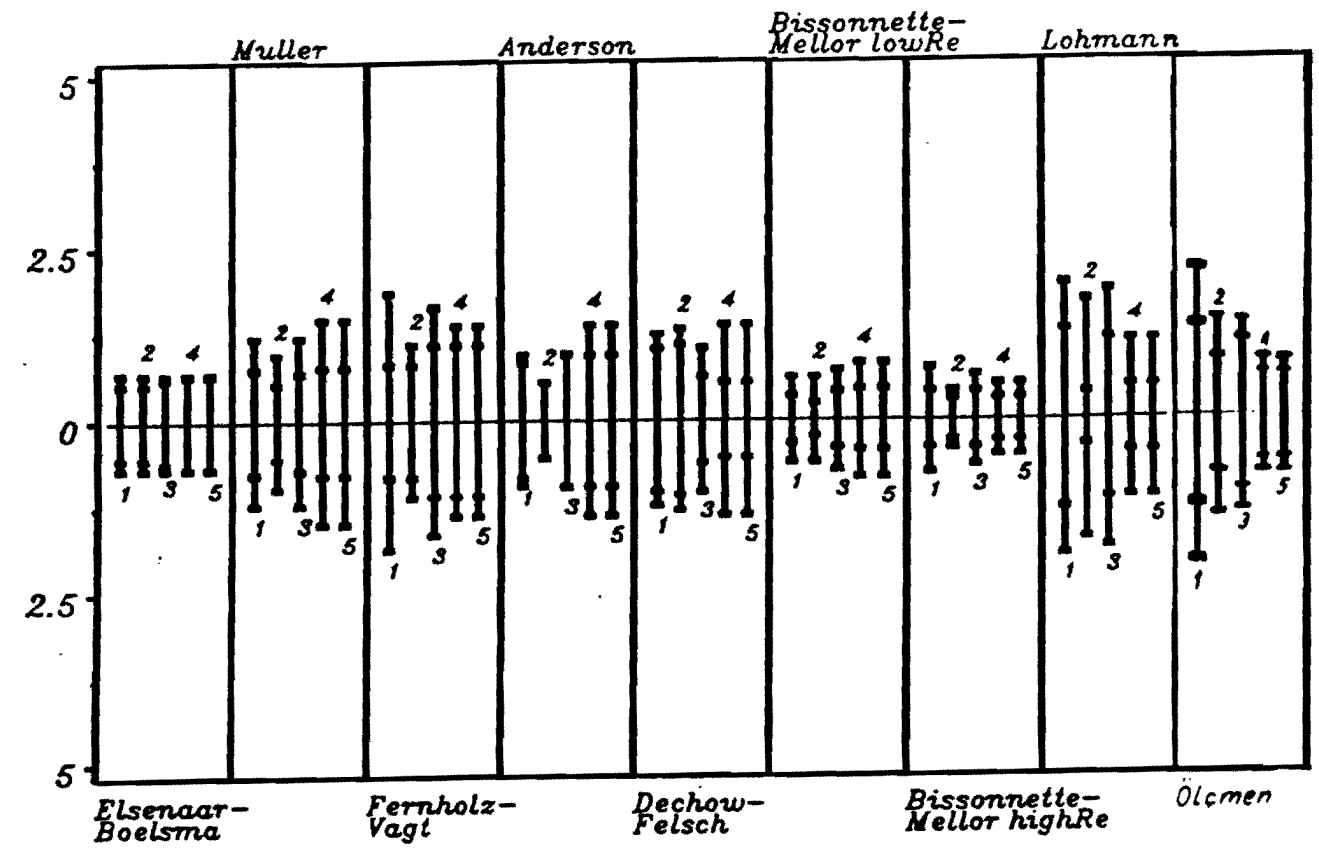

Figure 108a. $Q+$ range at $y+=70$ for various law of the wall profiles using various data
$3=$ Hornung-Joubert, sets.: $1=$ Johnston,
$4=$ Pierce-Krommenhoek, $5=$ Chandrashekhar-Swamy. 


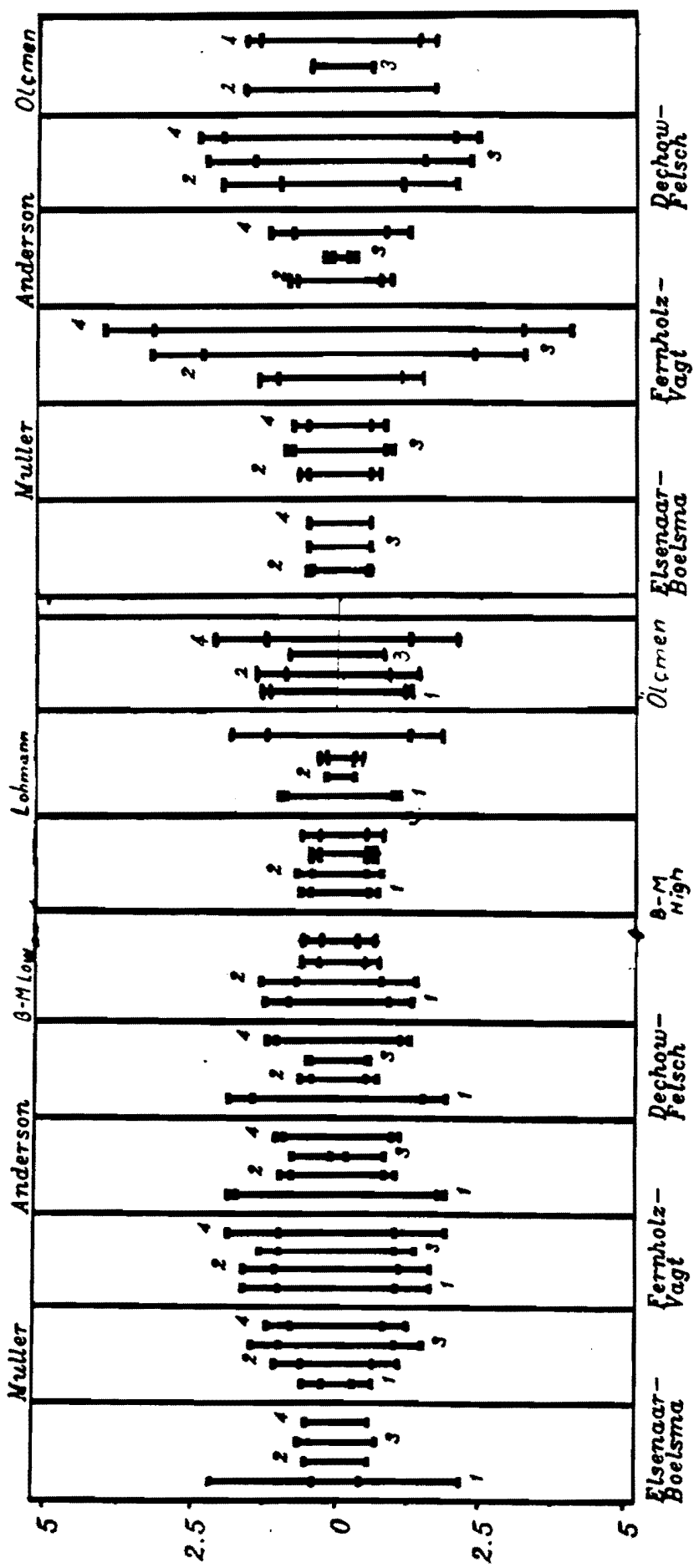

Figure 108b. $Q+$ range at $y+=70$ for various Law-of-the-Wall profiles using various data sets.: 1= East-Hoxey, 2 = Perry-Joubert,$\quad 3$ =White-Lesmann-Christoph, $4=$ Van Den Berg. 


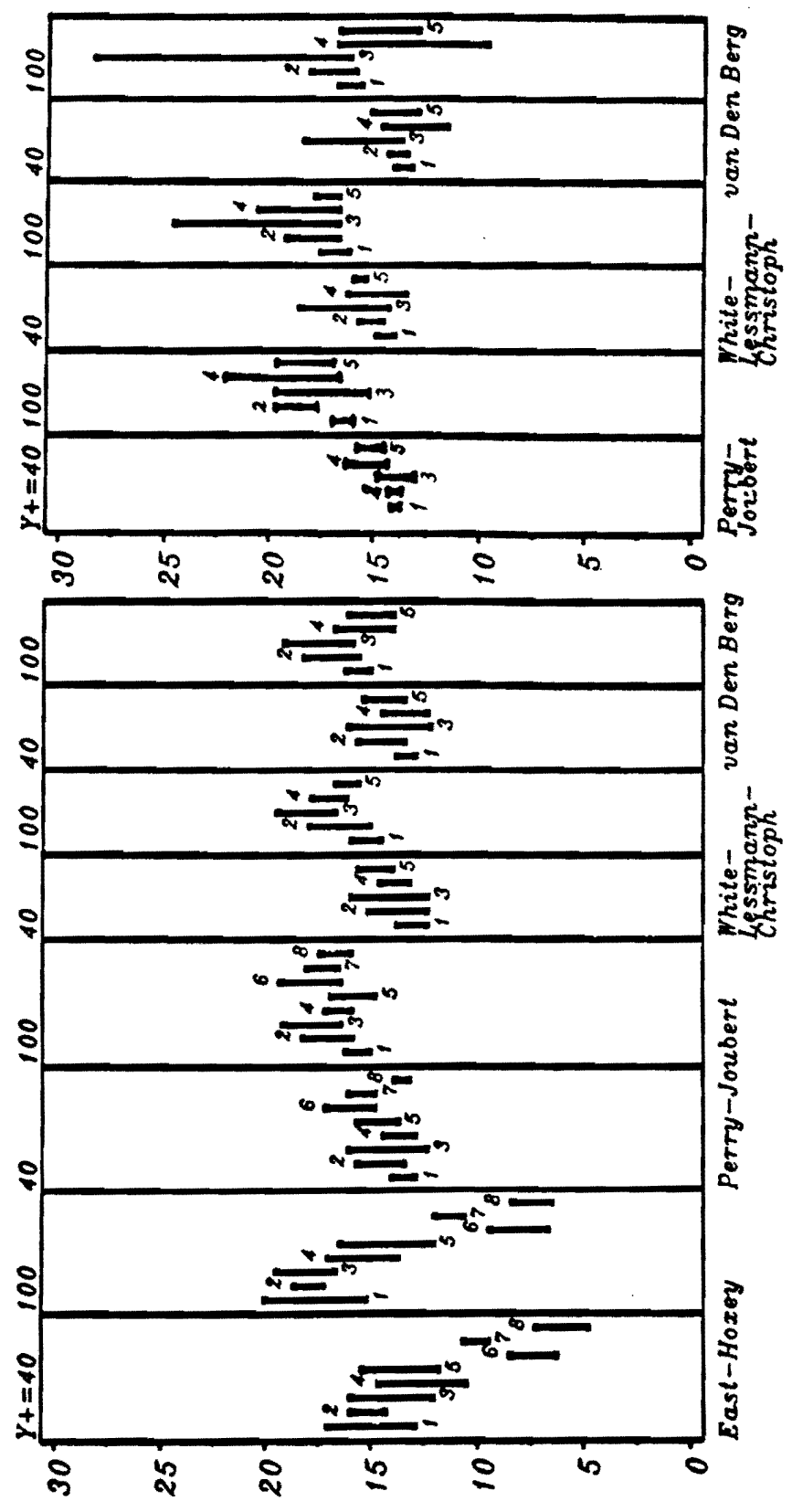

Figure 109a. $Q+$ range at $y+=40$ and $y+=100$ for various "Law-of-the-Wall" profiles using various data sets.: $1=$ Elsenaar-Boelsma, $2=$ Muller, $3=$ Fernholz-Vagt, 4 = Dechow, $5=$ Anderson, $6=$ Bissonnette-Mellor low Re\#, $7=$ Bissonnette-Mellor high Re\#, $8=$ Lohmann 


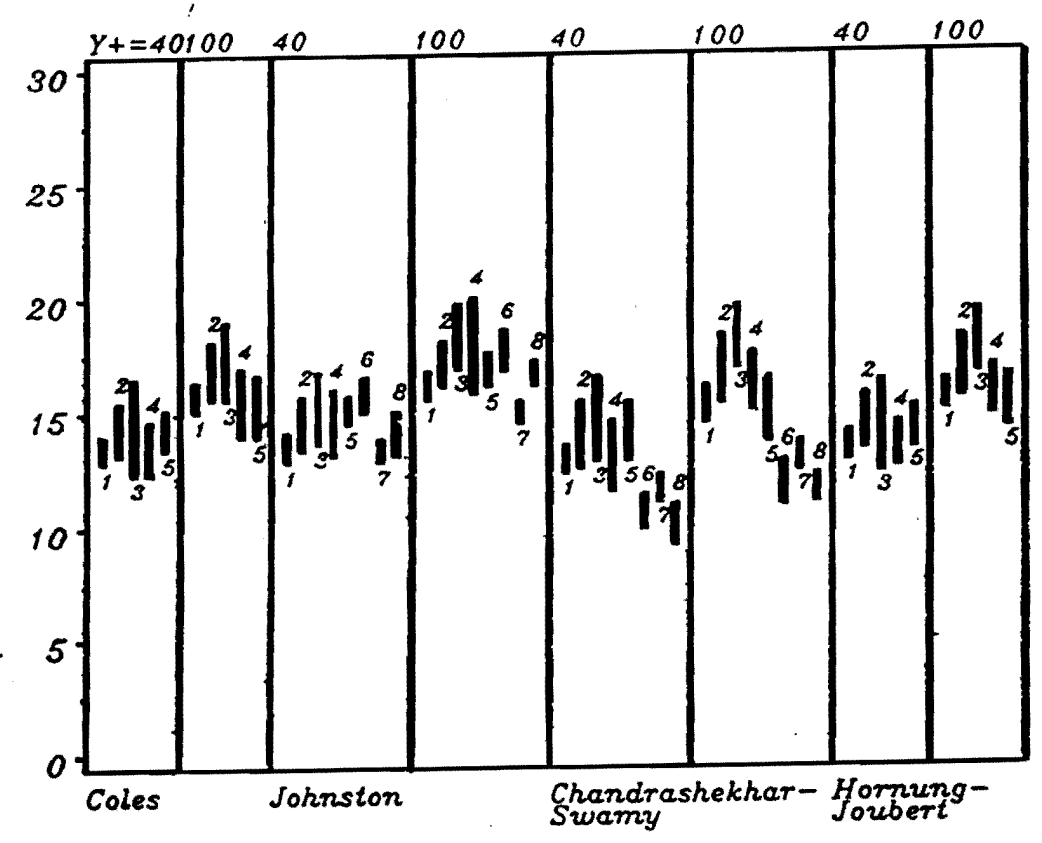

Figure 109b. $Q+$ range at $y+=40$ and $y+=100$ for various "Law-of-the-Wall" profiles using various data sets.: $1=$ Elsenaar-Boelsma, $2=$ Muller, $3=$ Fernholz-Vagt, $4=$ Dechow, $\quad 5=$ Anderson, $6=$ Bissonnette-Mellor low Re\#, $7=$ Bissonnette-Mellor high Re\#, $8=$ Lohmann 


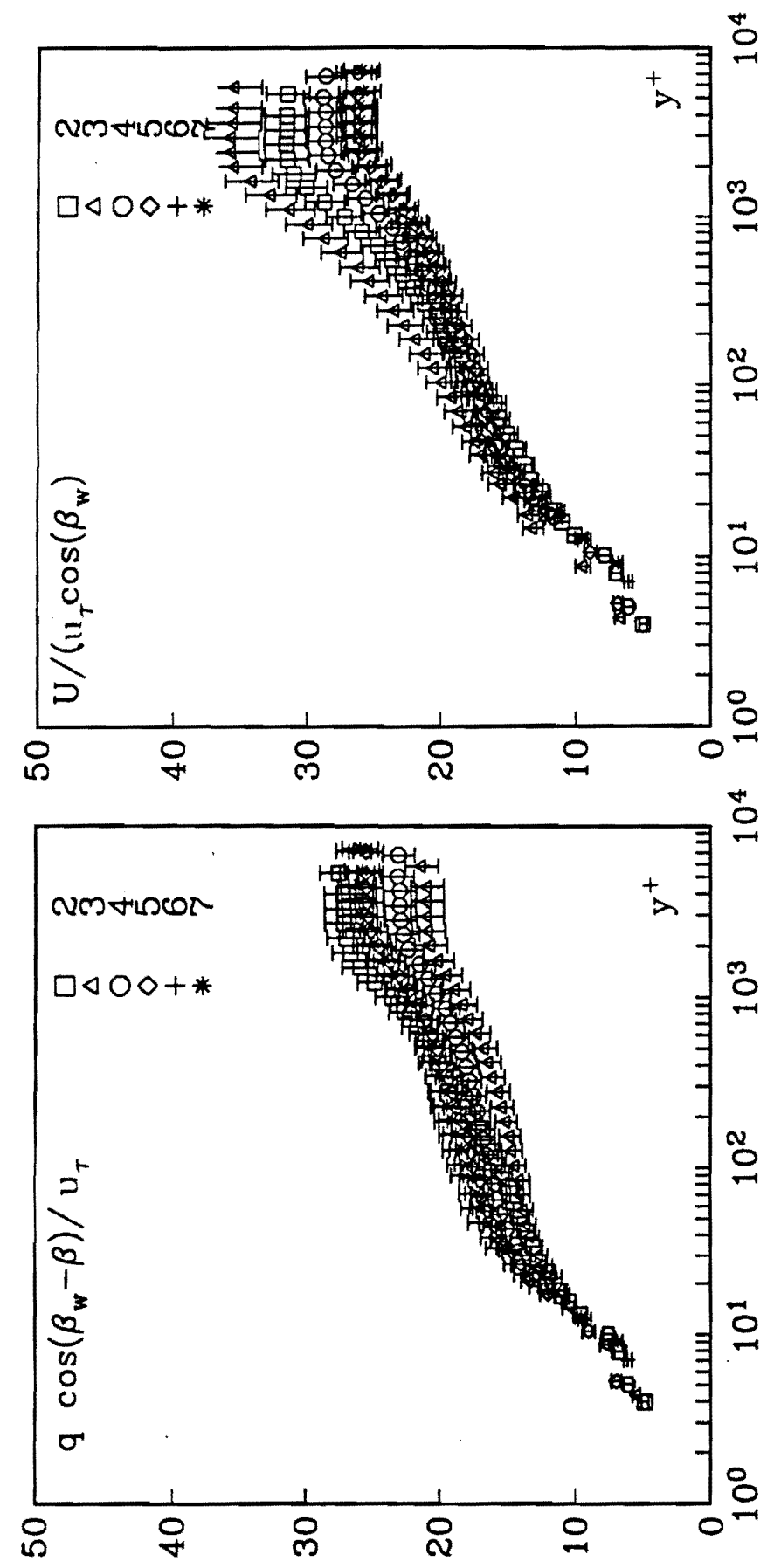

Figure 110a. Present data in the Law-of-the-Wall coordinate systems of Coles (left ) and Johnston.: Bars represent the uncertainty bands. 


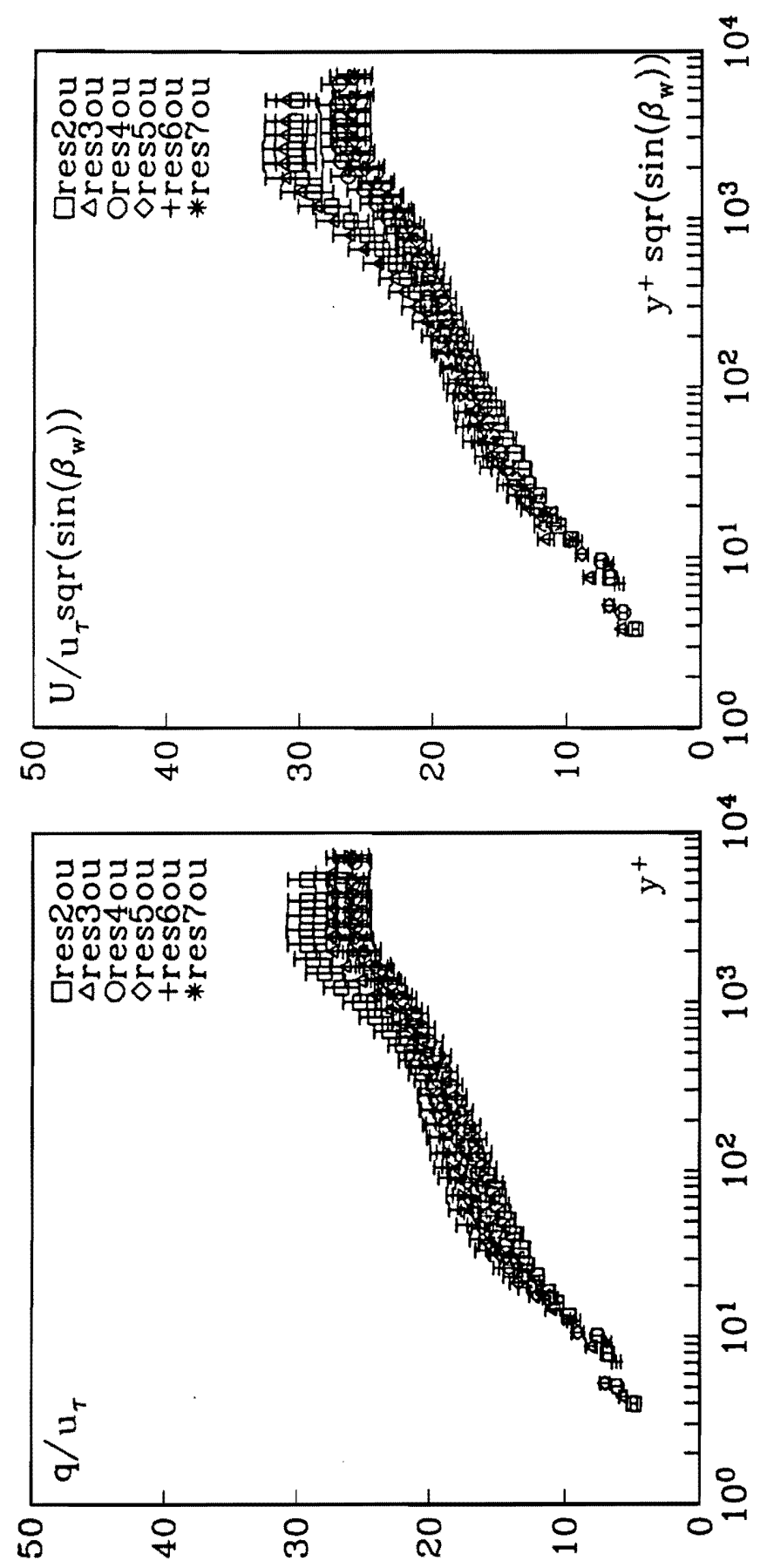

Figure 110b. Present data in the Law-of-the-Wall coordinate systems of Hornung-Joubert (left ) and Pierce-Krommenhoek and Chandrashekhar-Swamy (right ).: Bars represent the uncertainty bands. 


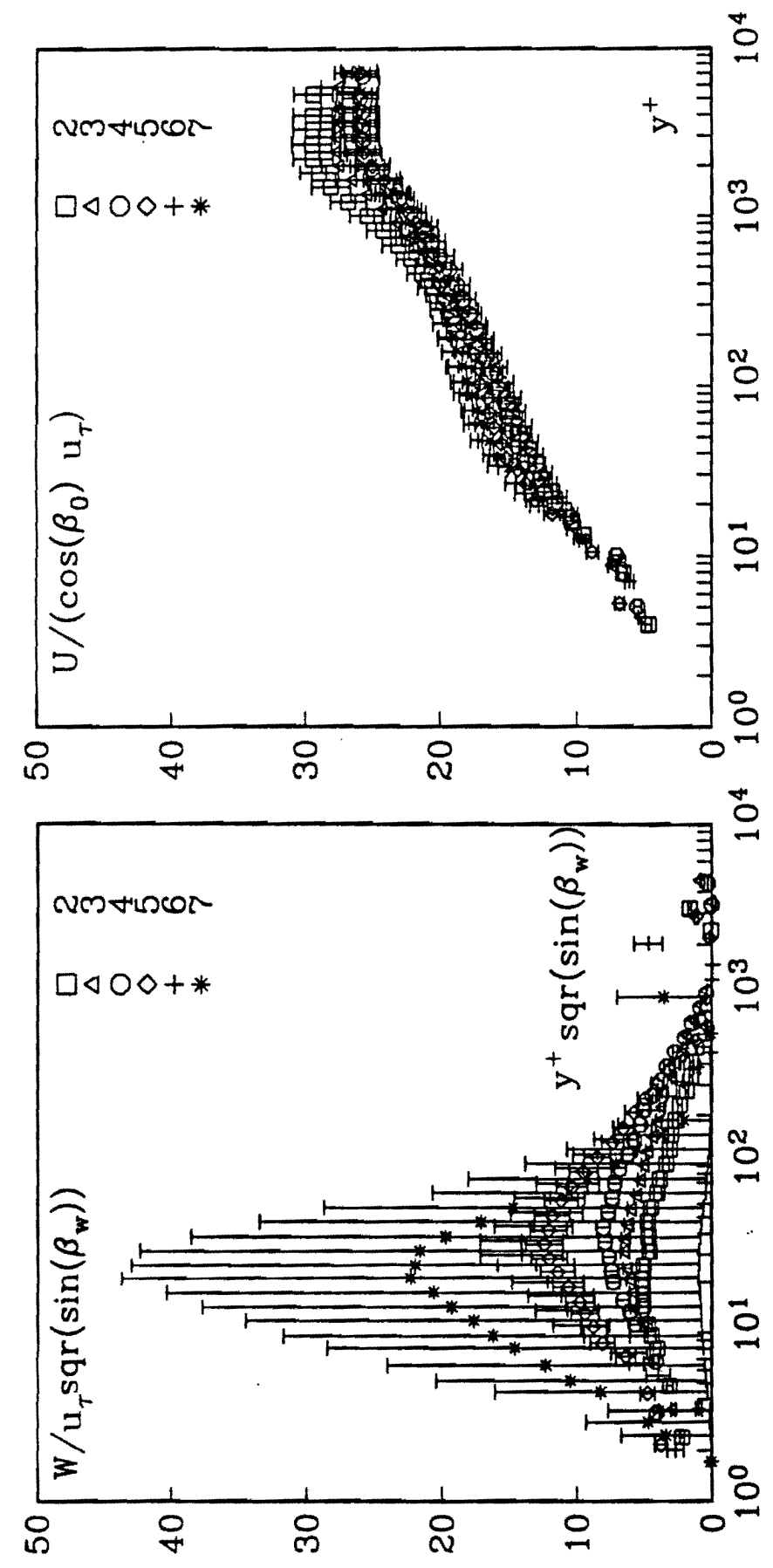

Figure 110c. Present data in the Law-of-the-Wall coordinate systems of Chandrashekhar-Swamy ( left, lateral component) and East-Hoxey ( right ).: Bars represent the uncertainty bands. 


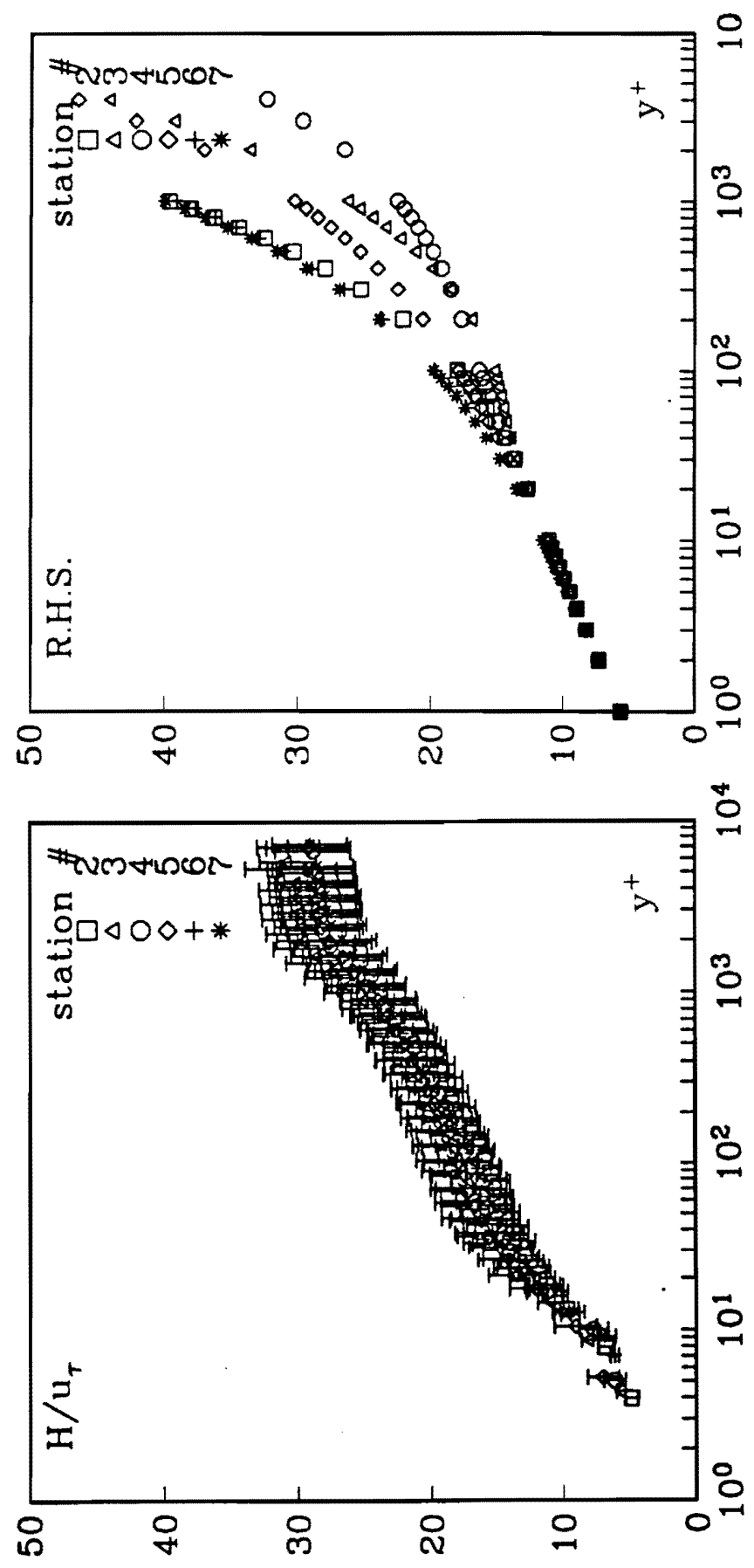

Figure 110d. Present data in the Law-of-the-Wall coordinate system of Perry-Joubert.: Left-hand side of equation 8 of Chapter IV calculated from the data ( left) and from a relation ( right) Bars represent the uncertainty bands. 


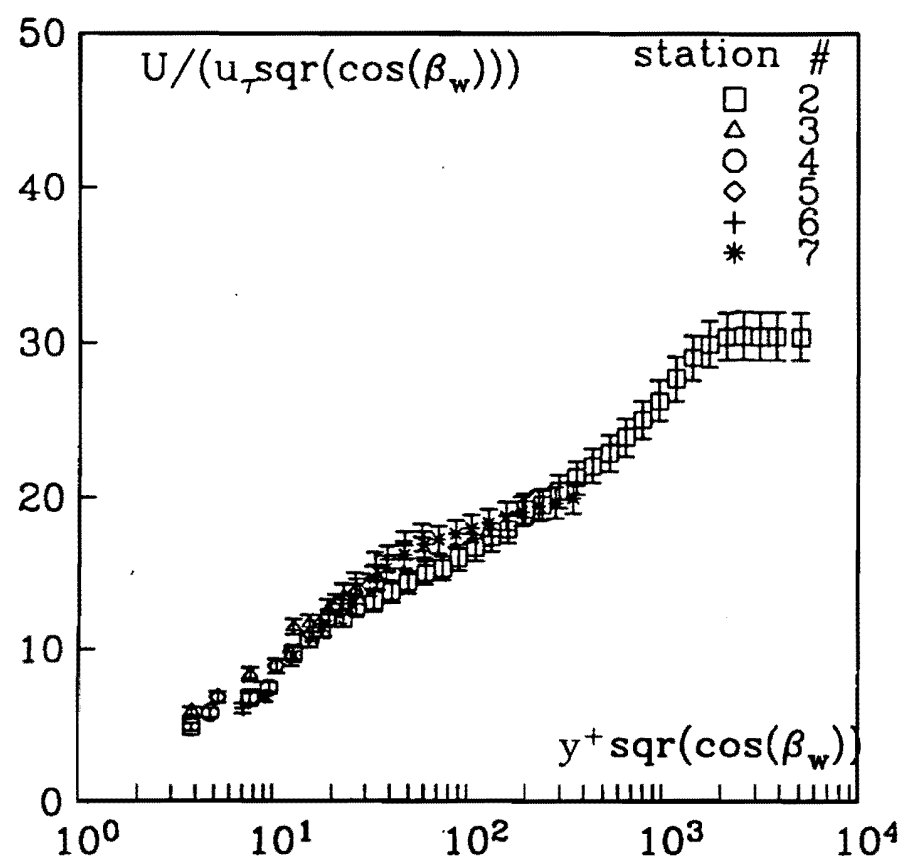

Figure 110ei. Present data in the Law-of-the-Wall coordinate system of White-Lessmann-Christoph ( Axial component ).: Left-hand side of equation 10 of Chapter IV calculated from the data

Bars represent the uncertainty bands. 


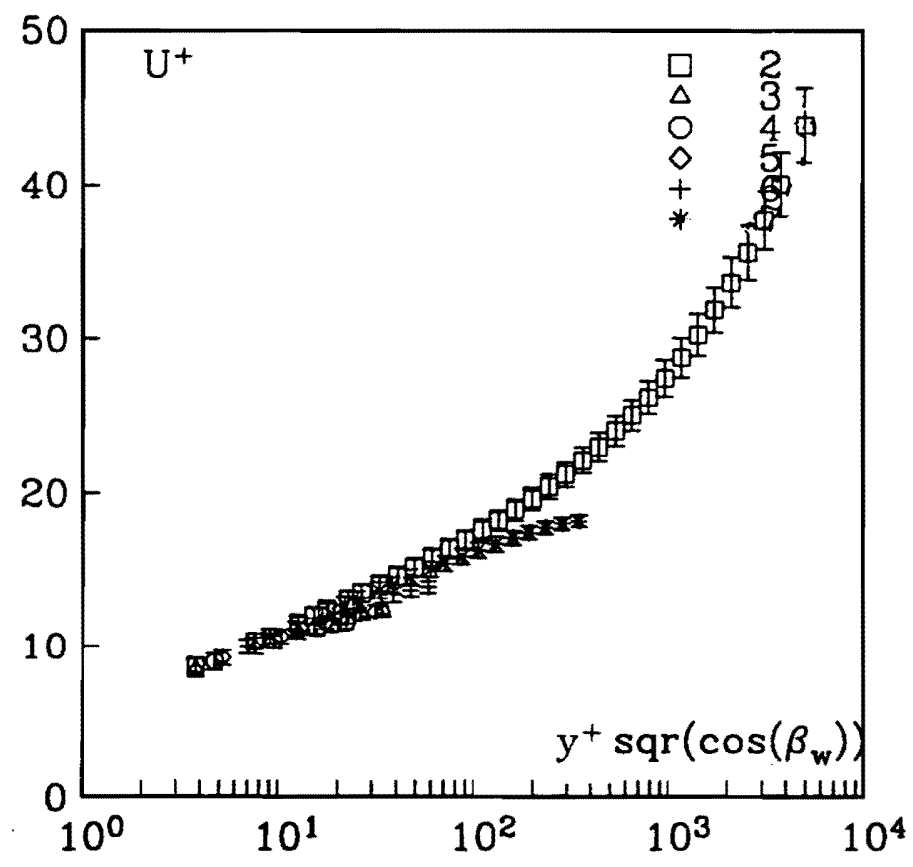

Figure 110eii. Present data in the Law-of-the-Wall coordinate system of White-Lessmann-Christoph. ( Axial component ): Right-hand side of equation 10 of Chapter IV calculated from the data

Bars represent the uncertainty bands. 


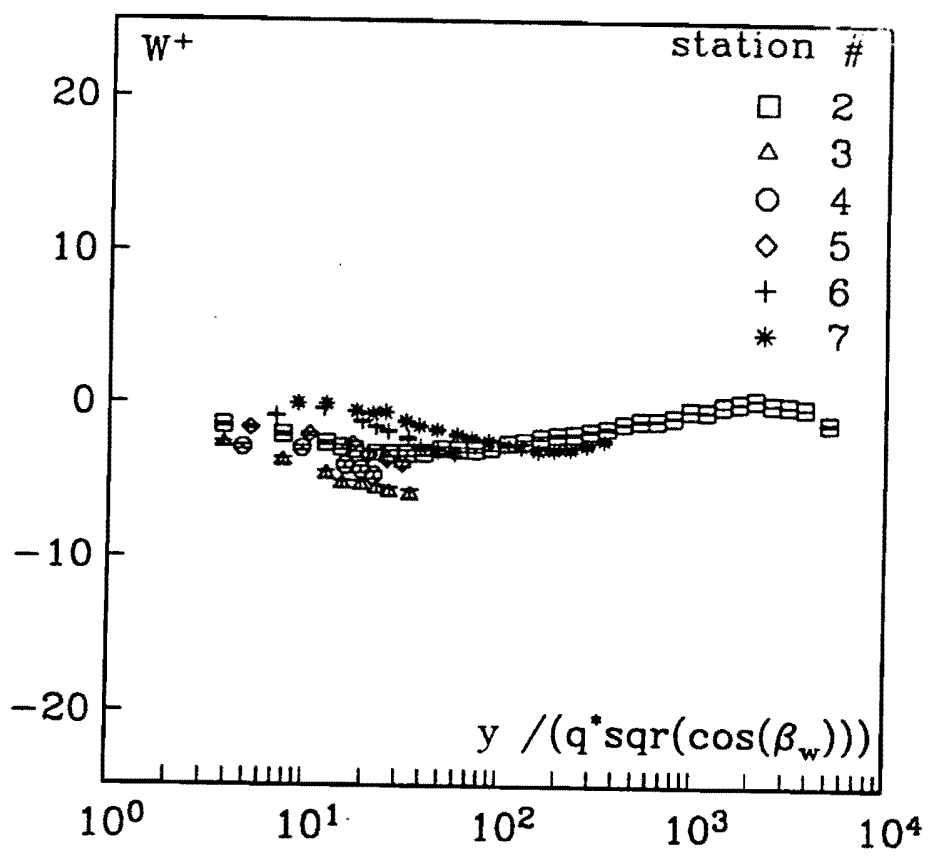

Figure 110eiii. Present data in the Law-of-the-Wall coordinate system of White-Lessmann-Christoph. ( Lateral component ): Left-hand side of equation 11 if Chapter IV calculated from the data

Bars represent the uncertainty bands. 


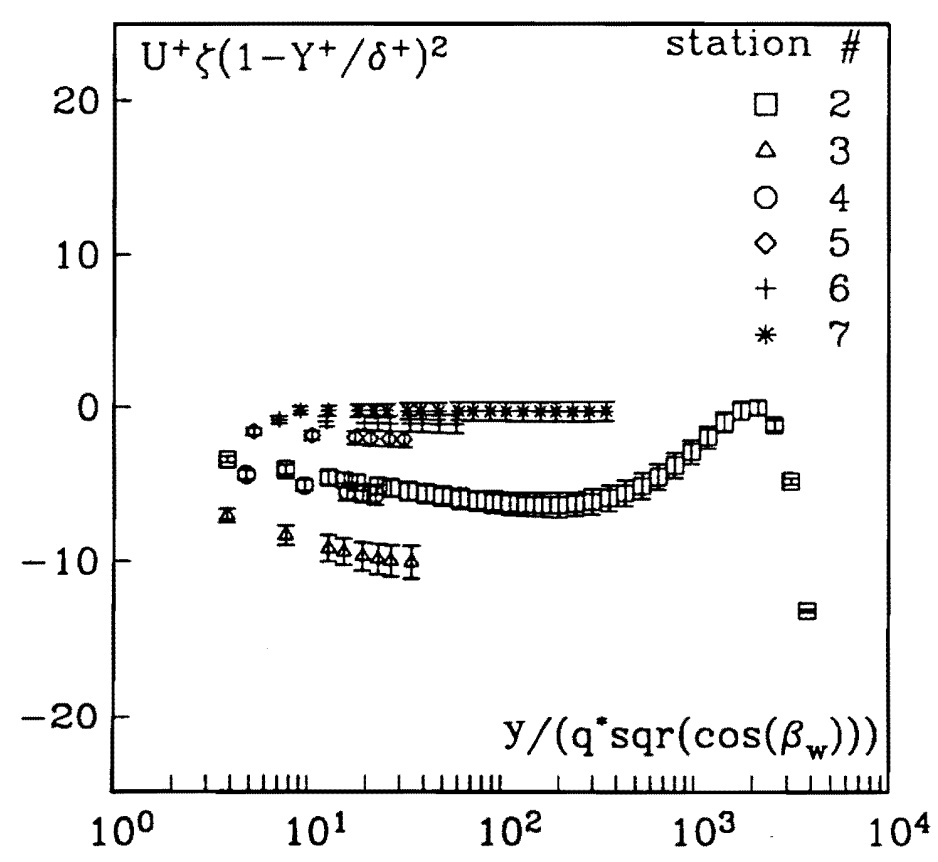

Figure 110eiiii. Present data in the Law-of-the-Wall coordinate system of White-Lessmann-Christoph. ( Lateral component ): Right-hand side of equation 11 of Chapter IV calculated

Bars represent the uncertainty bands. 


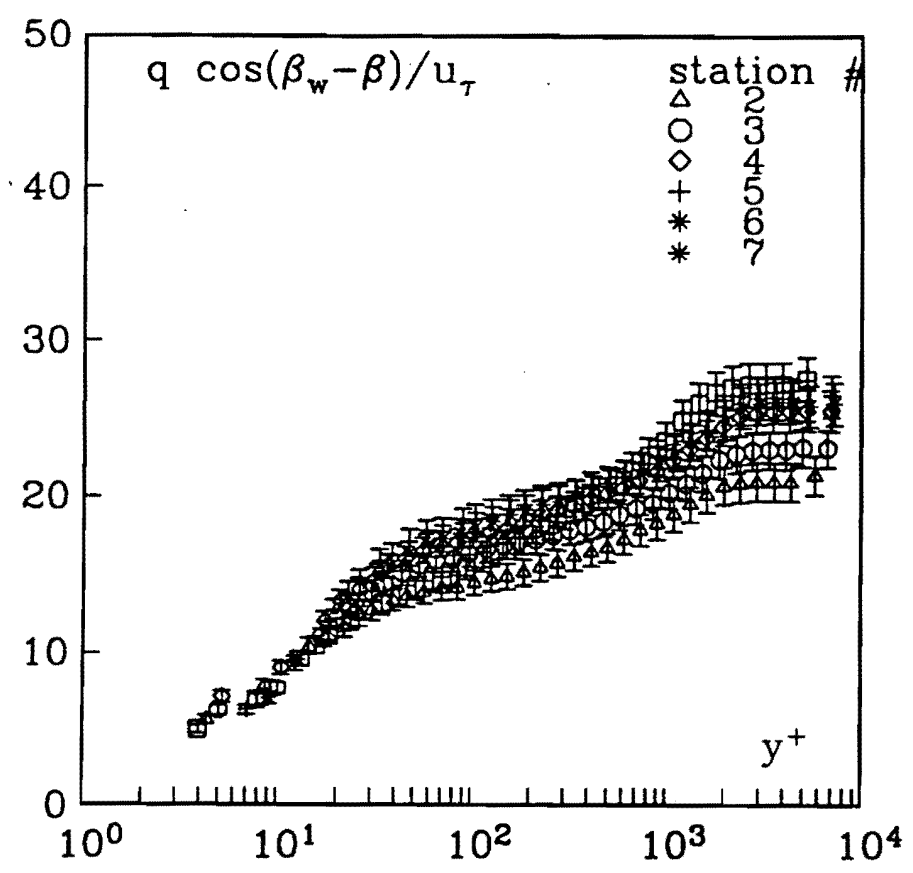

Figure 110fi. Present data in the Law-of-the-Wall coordinate system of Van Den Berg. ( Axial component ): Left-hand side of equation 12 of Chapter IV calculated from the data Bars represent the uncertainty bands. 


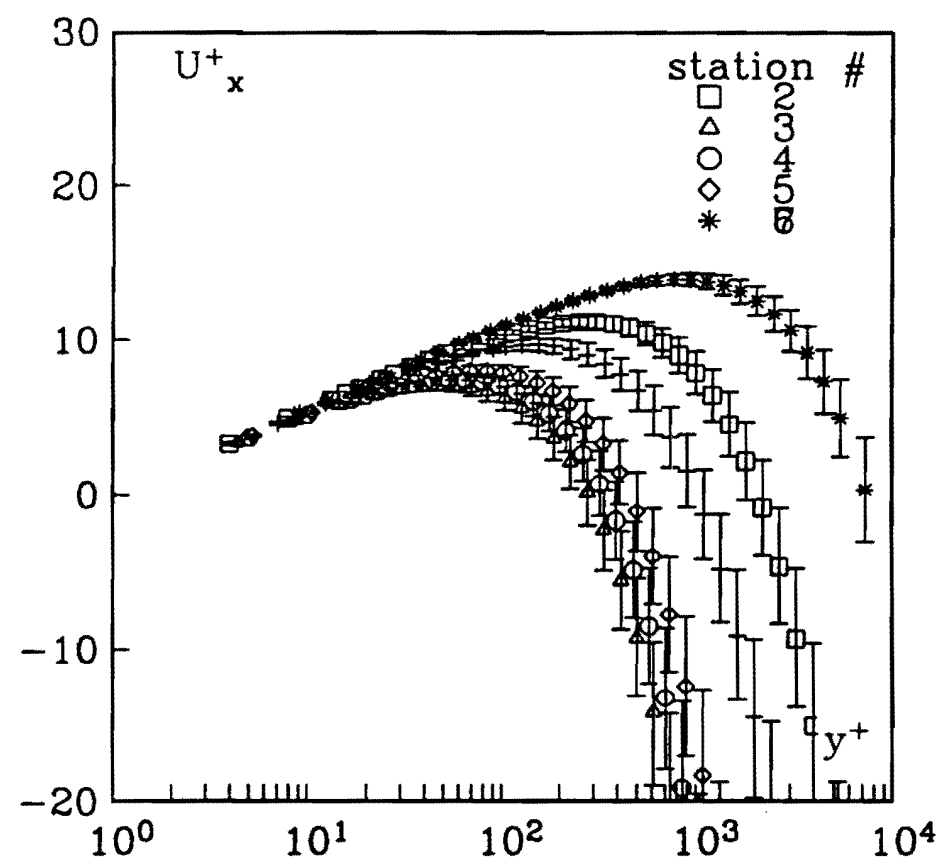

Figure 110fii. Present data in the Law-of-the-Wall coordinate system of Van Den Berg. ( Axial component ): Right-hand side of equation 12 of Chapter IV calculated from the data

Bars represent the uncertainty bands. 


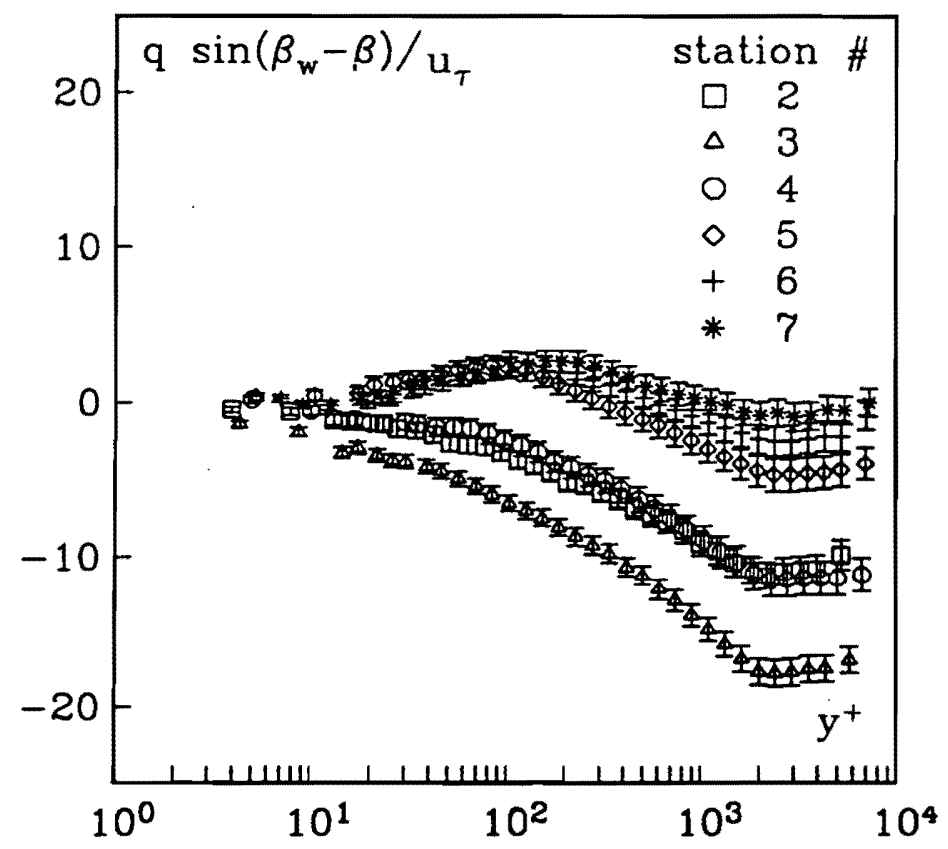

Figure 110fiii. Present data in the Law-of-the-Wall coordinate system of Van Den Berg. ( Lateral component ): Left-hand side of equation 13 of Chapter IV calculated from the data uncertainty bands.

Bars represent the 


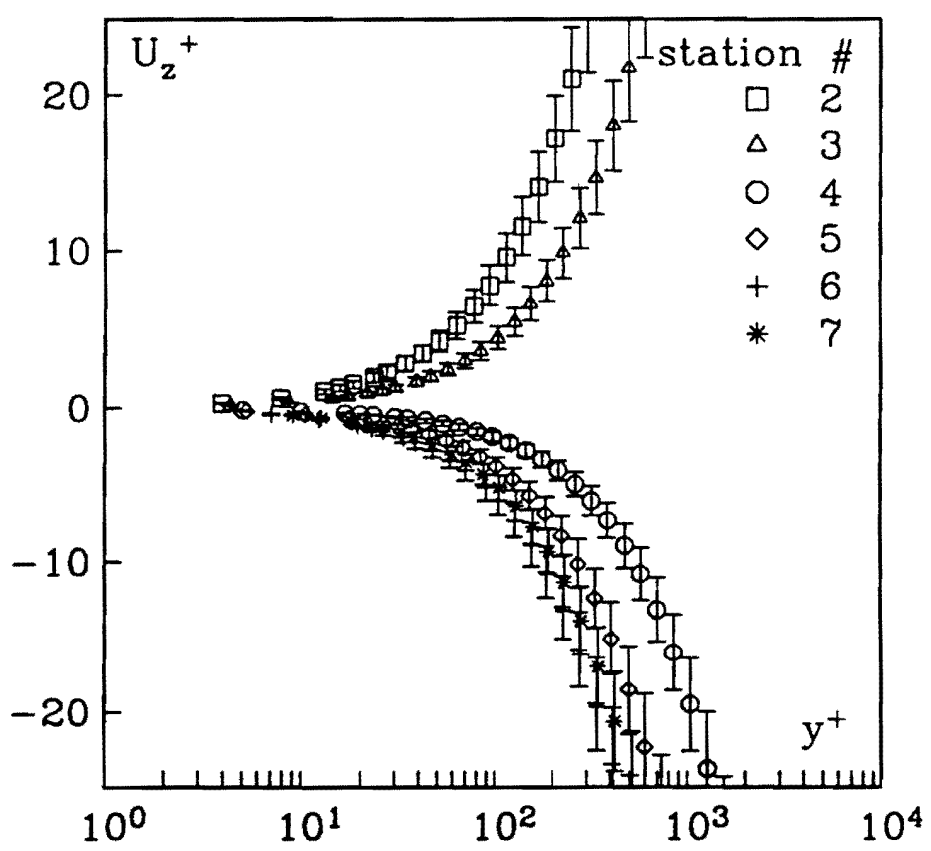

Figure 110fiiii. Present data in the Law-of-the-Wall coordinate system of Van Den Berg. ( Lateral component ): Right-hand side of equation 13 of Chapter IV calculated from the data Bars represent the uncertainty bands. 


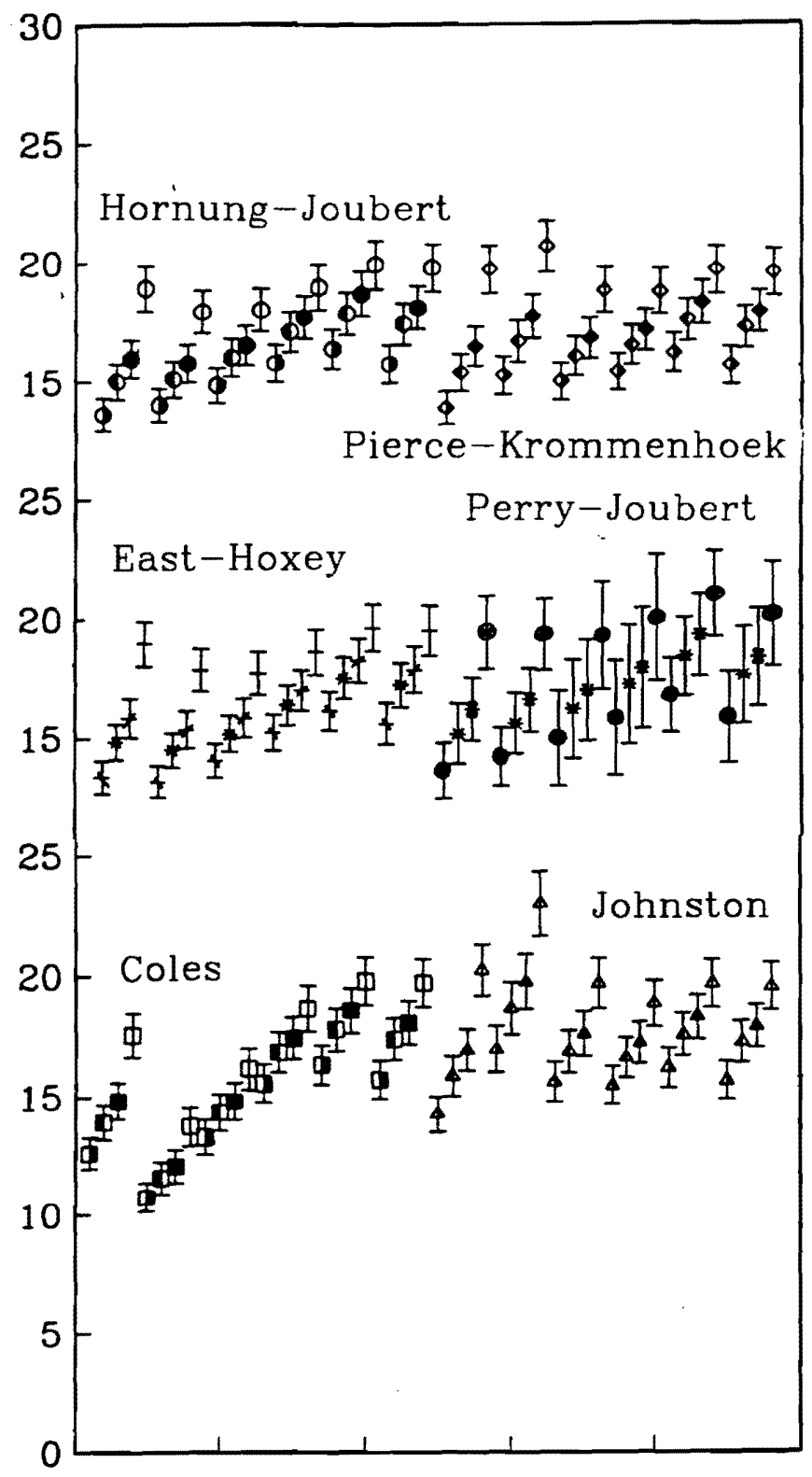

Figure 111. Computed values for different various laws of the wall for Present data at $y+=40,70,100,250$, with the uncertainty bands. 

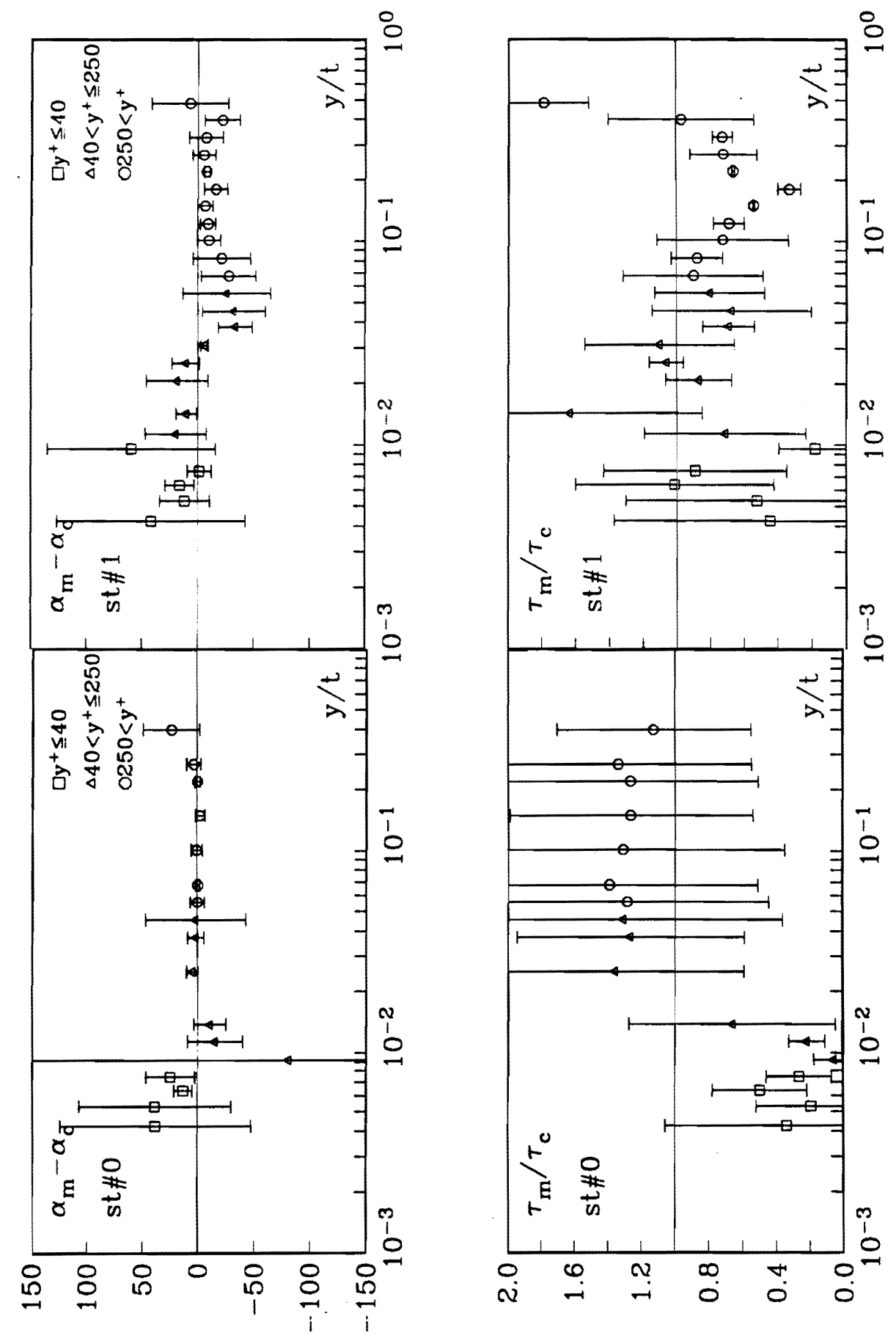

Figure 112a. Present data in Cebeci-Smith model:: Station numbers are given in the figures. Bars show the uncertainty bands. 

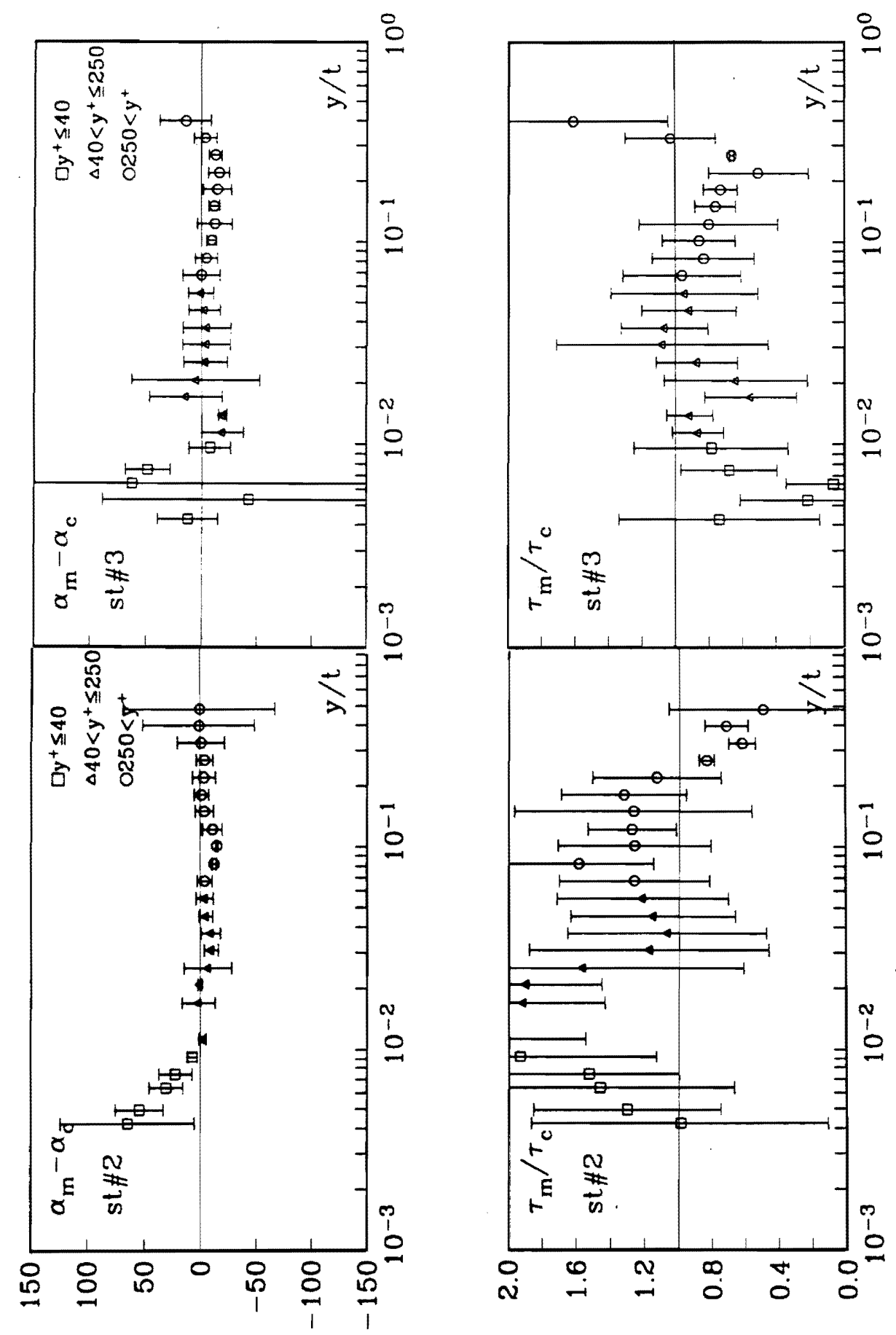

Figure 112b. Present data in Cebeci-Smith model.: Station numbers are given in the figures. Bars show the uncertainty bands. 

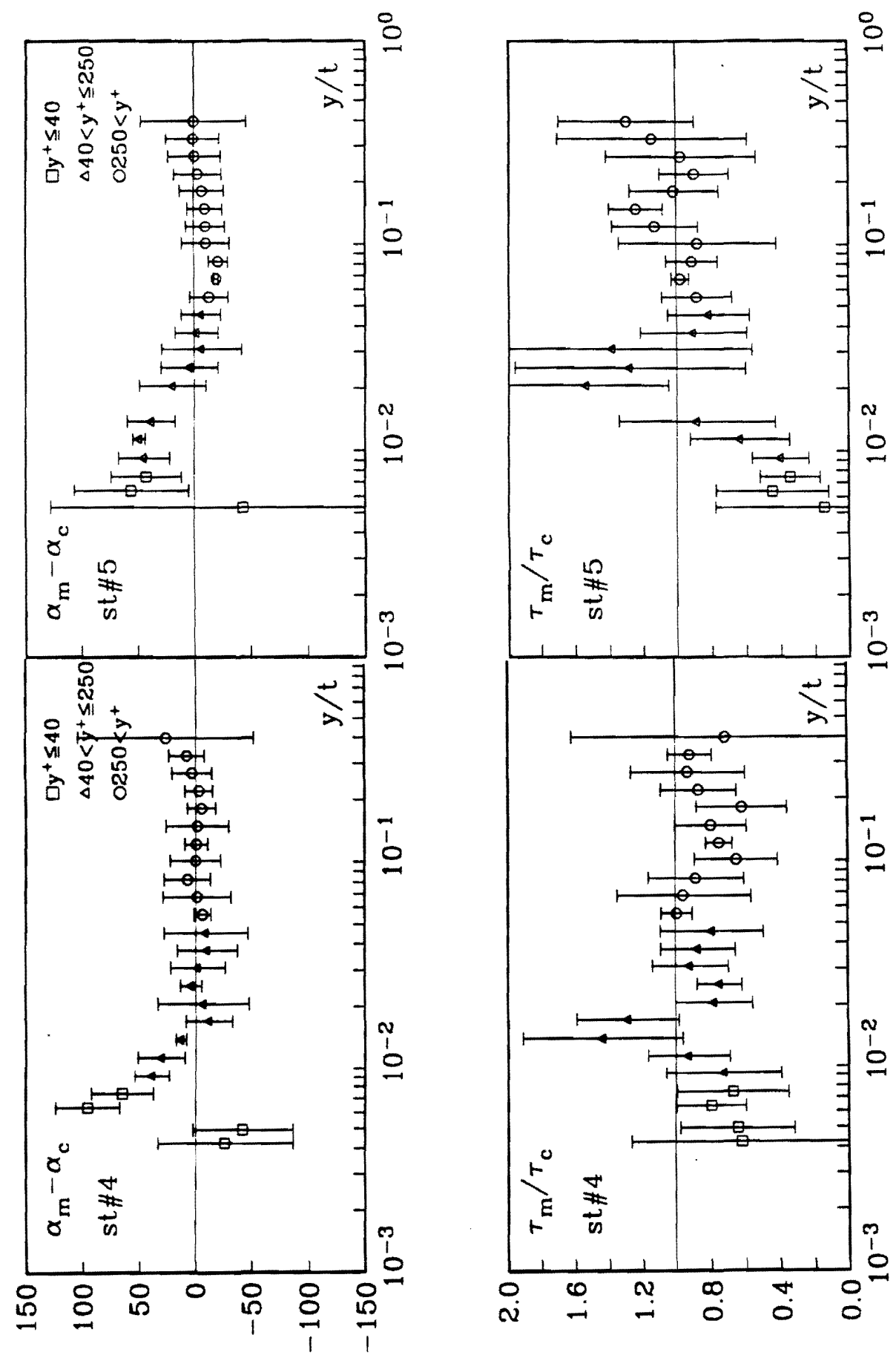

Figure 112c. Present data in Cebeci-Smith model.: Station numbers are given in the figures. Bars show the uncertainty bands. 

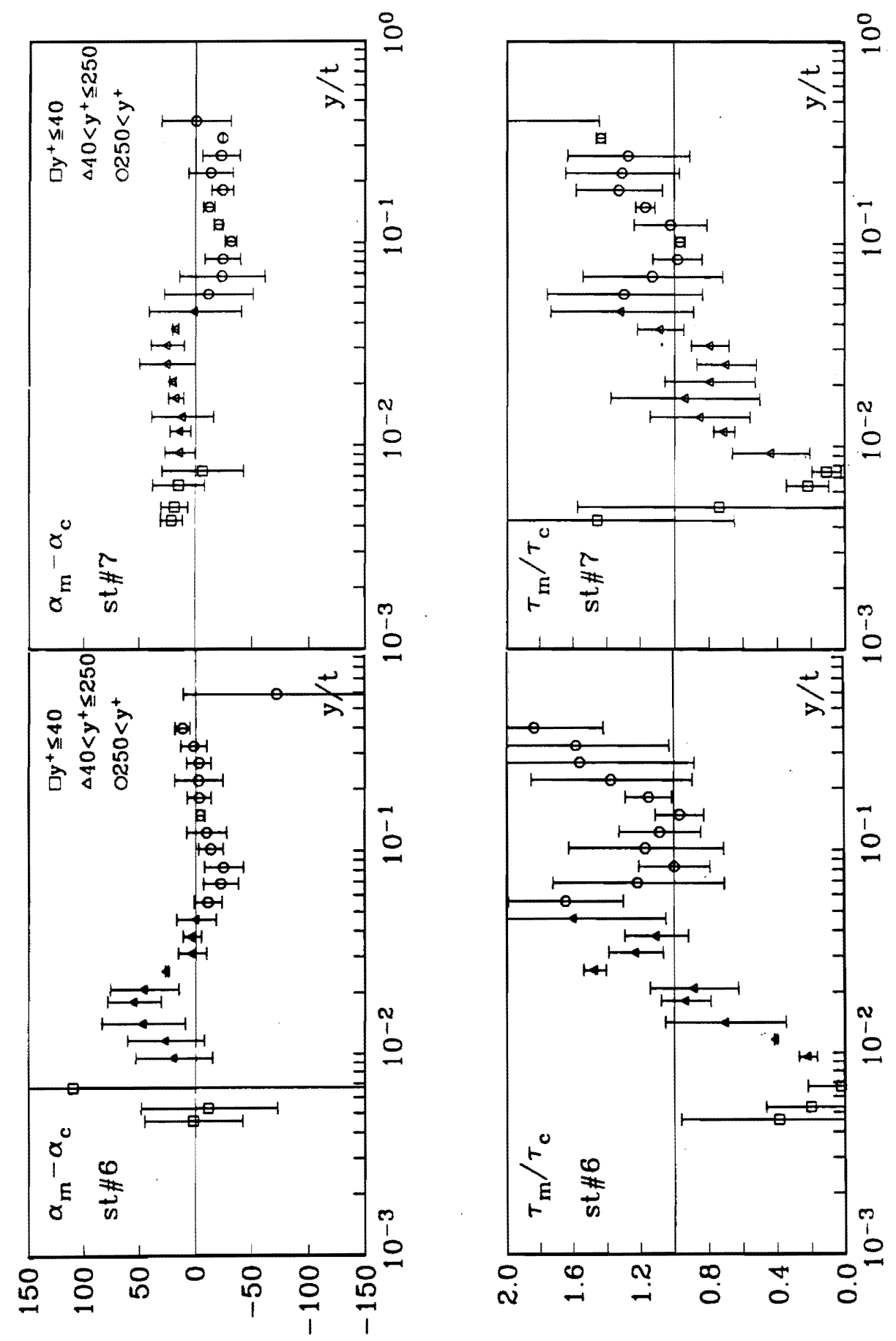

Figure 112d. Present data in Cebeci-Smith model.: Station numbers are given in the figures. Bars show the uncertainty bands. 

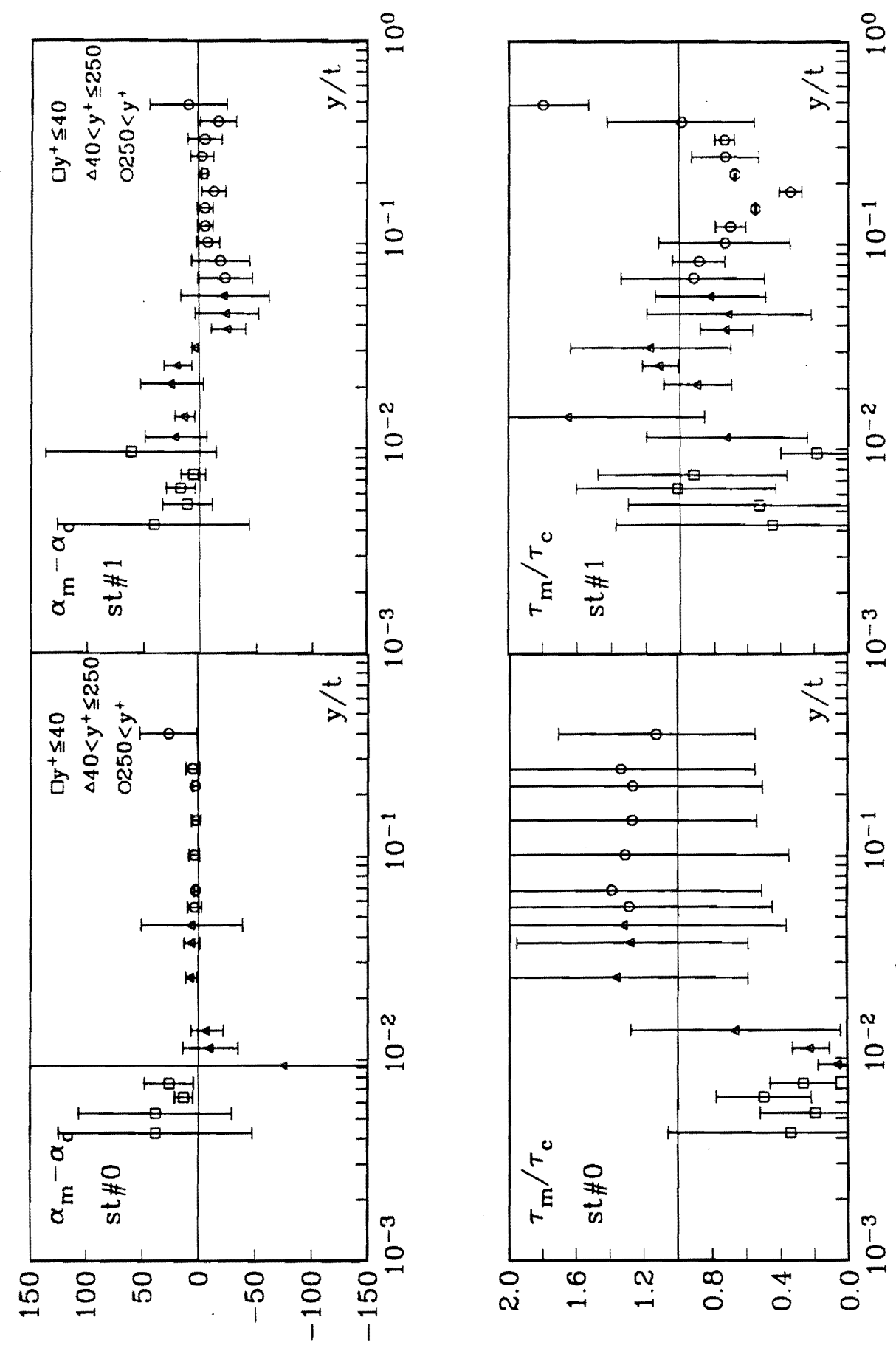

Figure 113a. Present data in Rotta's model with anisotropy constant $T=0.3$.: Station numbers are given in the figures. Bars show the uncertainty bands. 

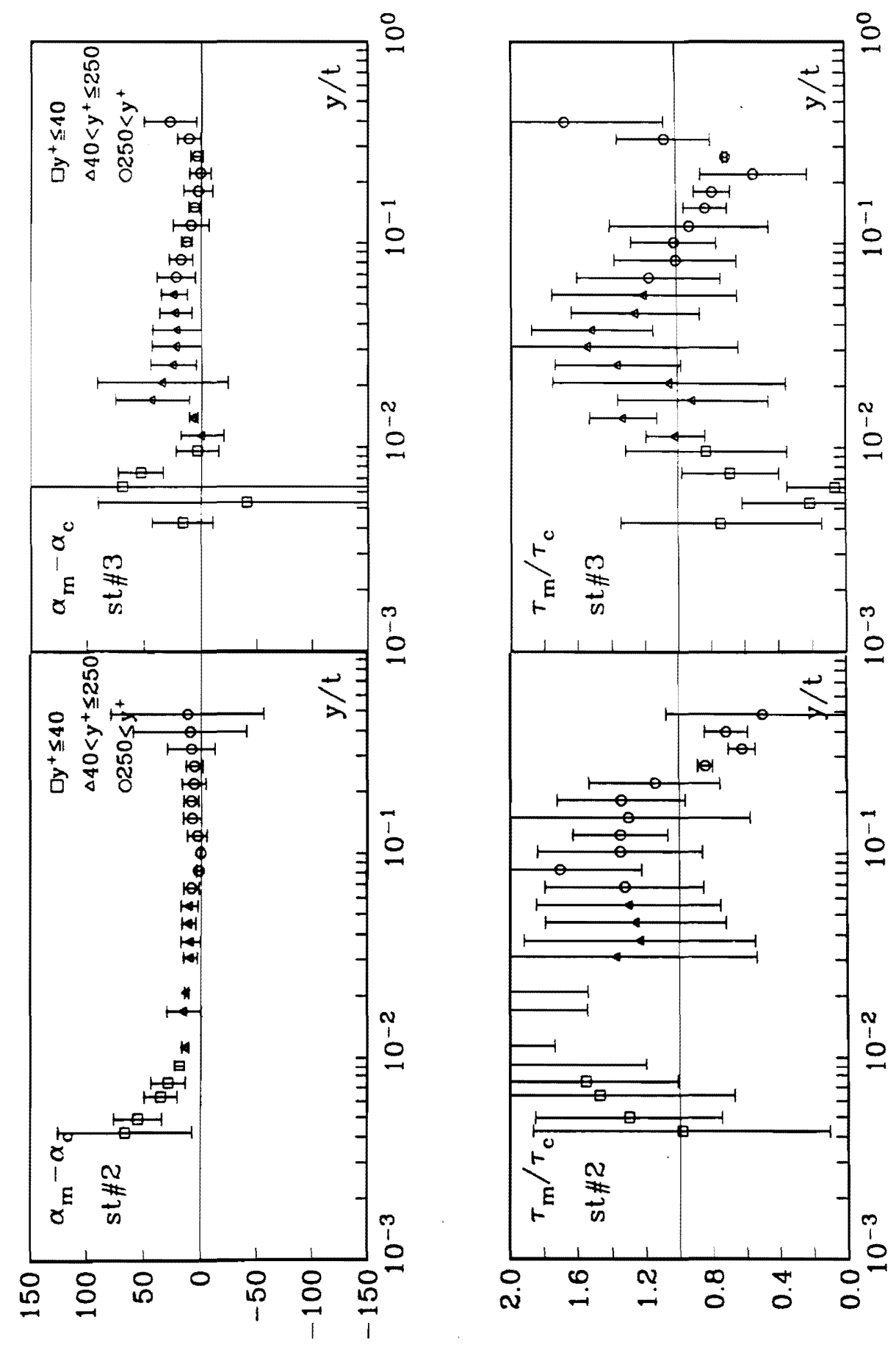

Figure 113b. Present data in Rotta's model with anisotropy constant $T=0.3$. Station numbers are given in the figures. Bars show the uncertainty bands. 

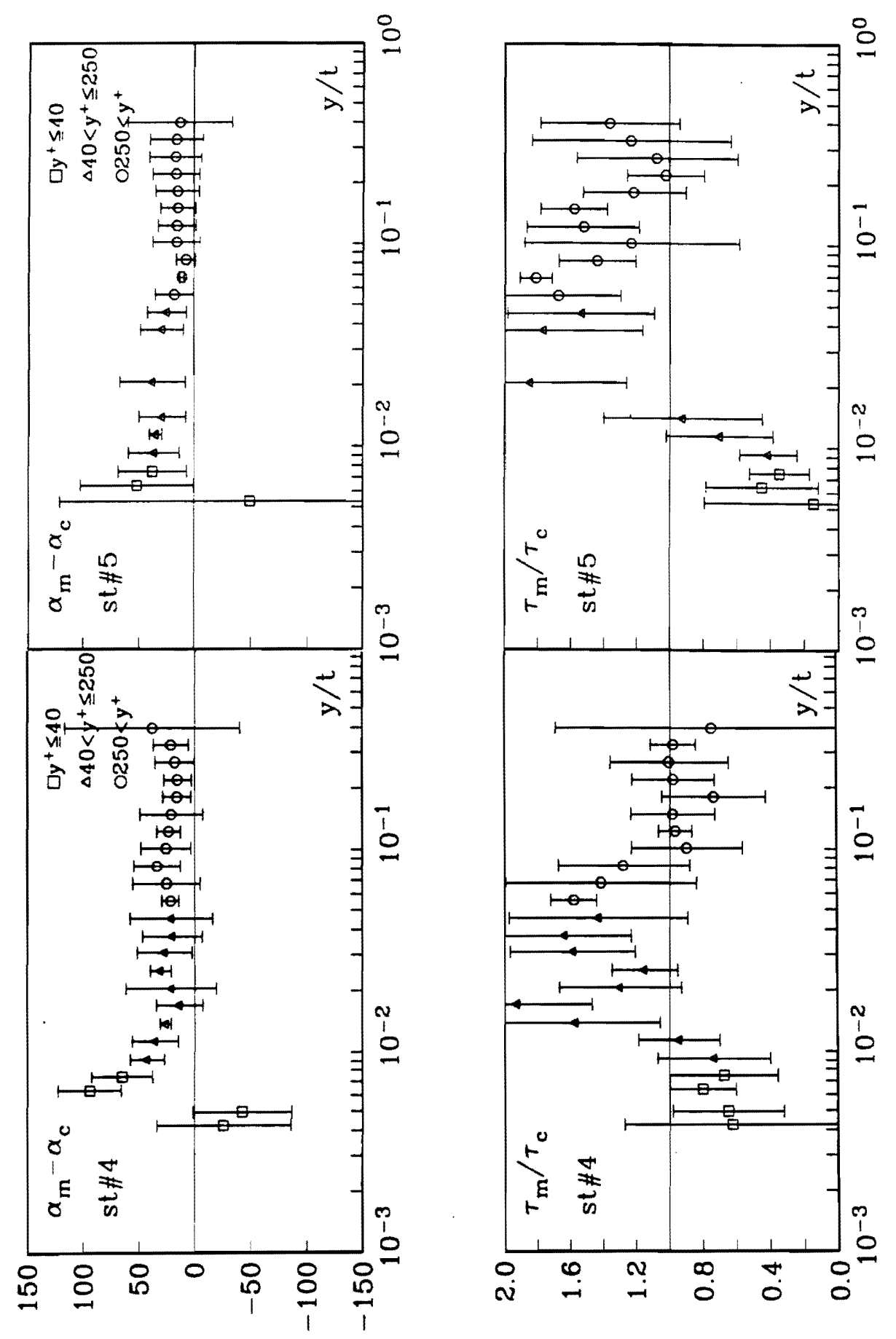

Figure 113c. Present data in Rotta's model with anisotropy constant $T=0.3$. Station numbers are given in the figures. Bars show the uncertainty bands. 

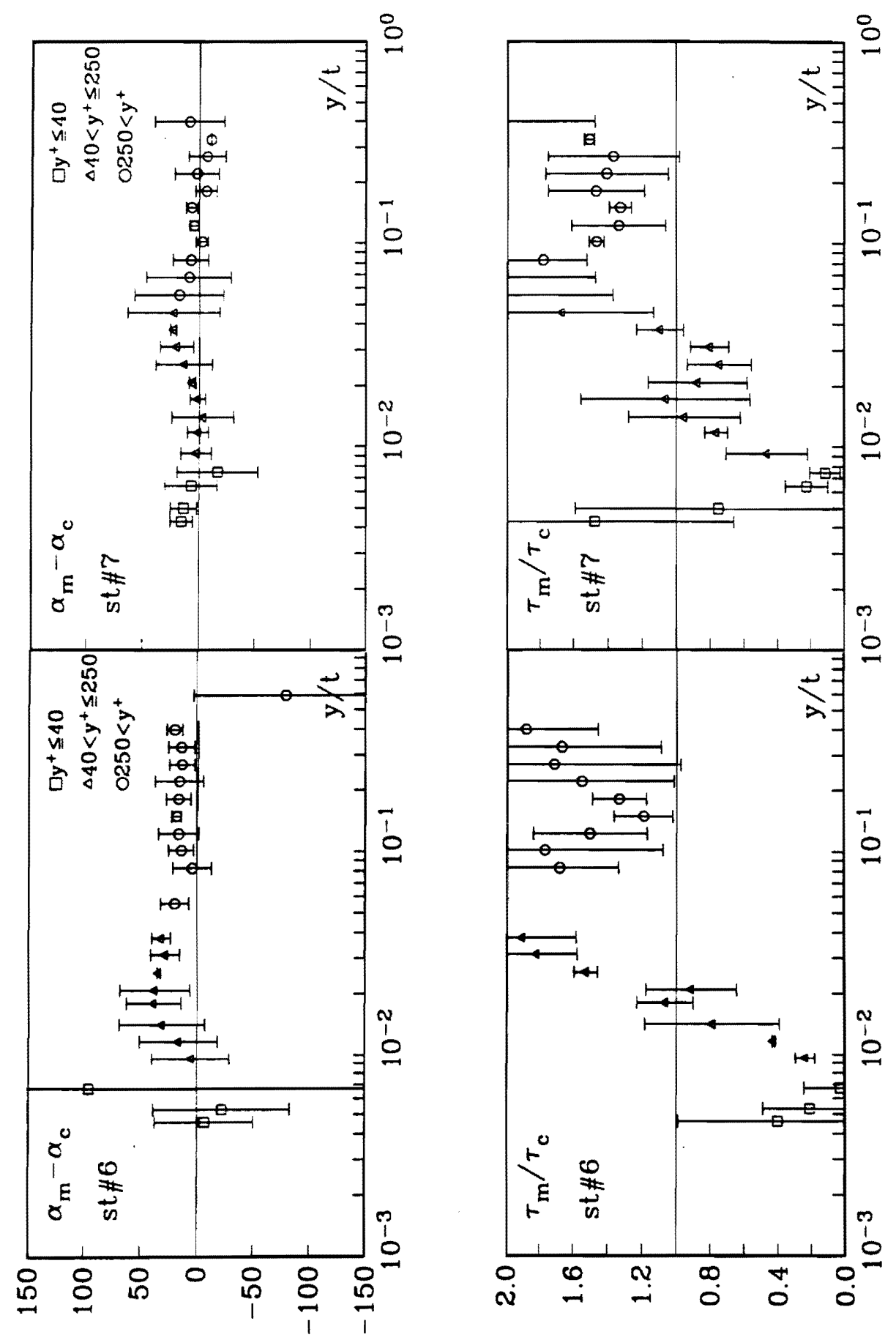

Figure 113d. Present data in Rotta's model with anisotropy constant $T=0.3$. Station numbers are given in the figures. Bars show the uncertainty bands. 

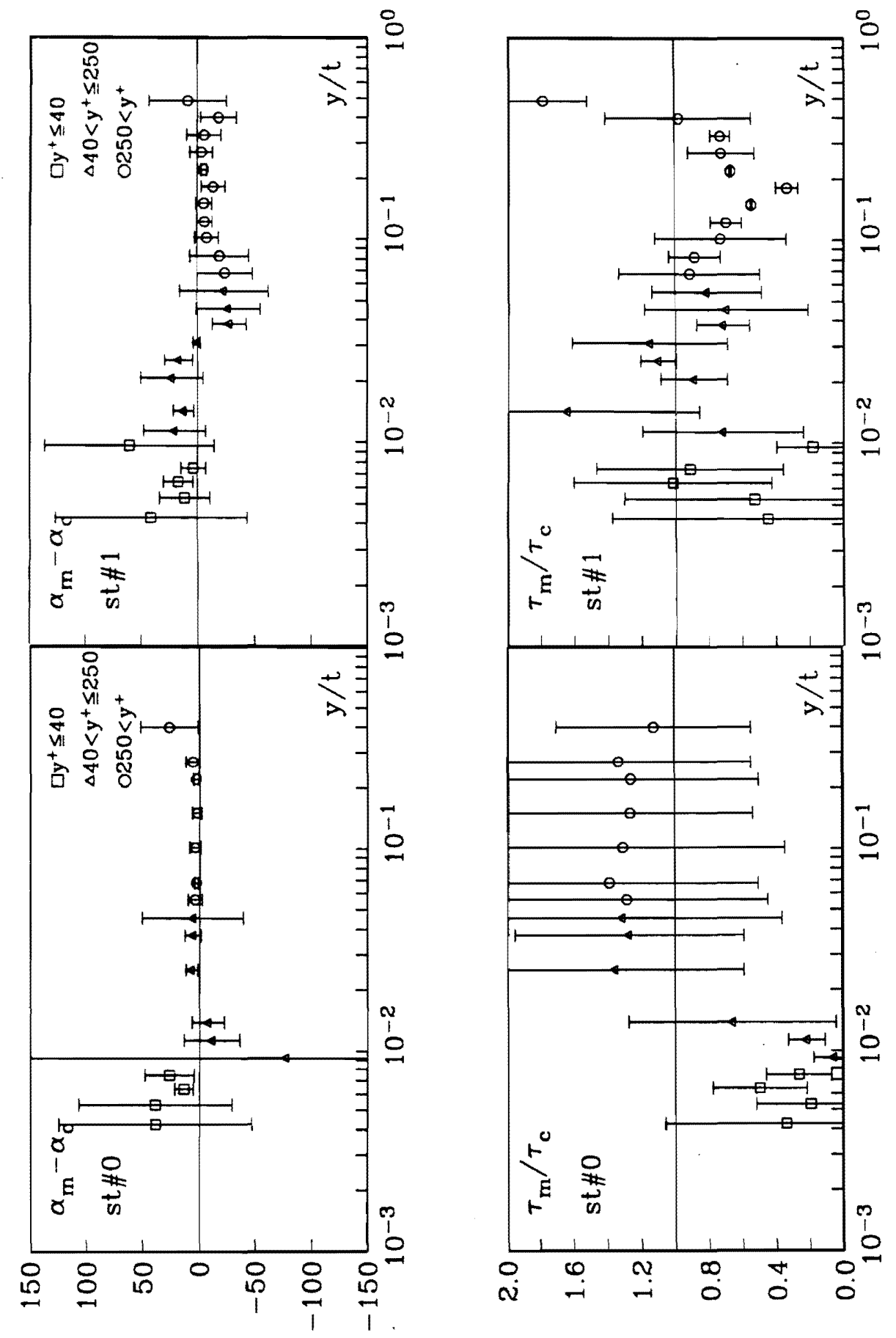

Figure 114a. Present data in Rotta's model with anisotropy constant $T=0.5$.: Station numbers are given in the figures. Bars show the uncertainty bands. 

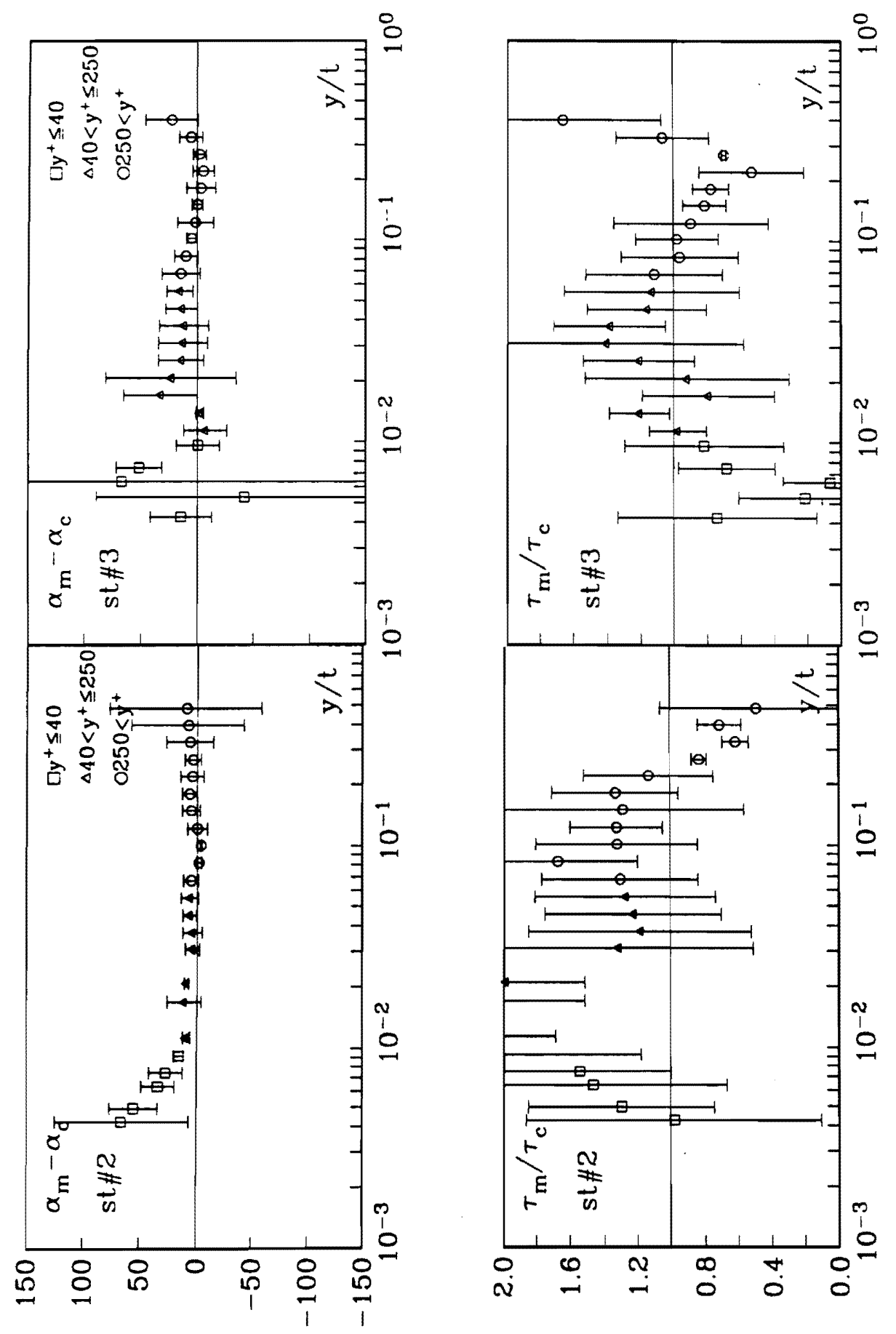

Figure 114b. Present data in Rotta's model with anisotropy constant $T=0.5$. Station numbers are given in the figures. Bars show the uncertainty bands. 

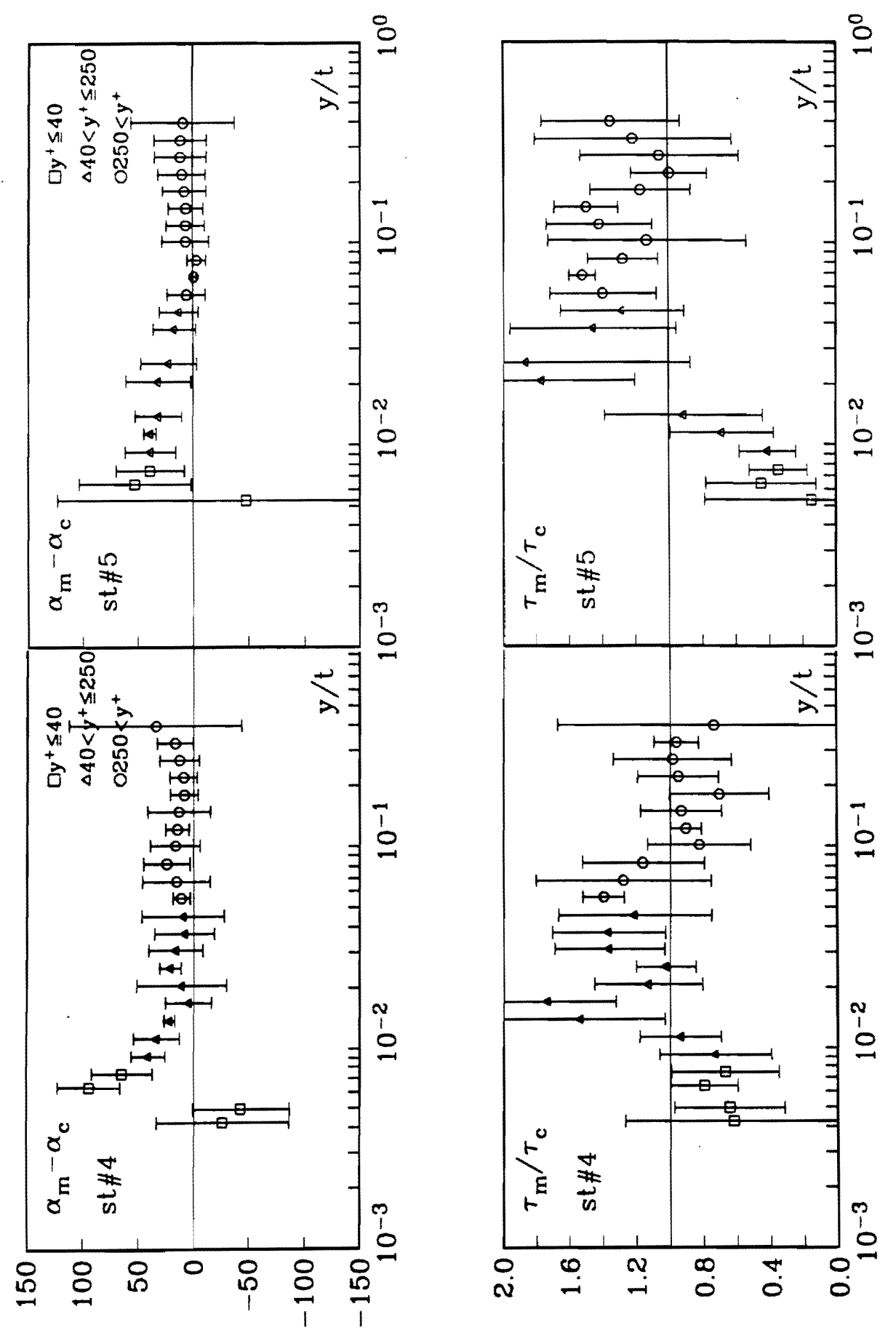

Figure 114c. Present data in Rotta's model with anisotropy constant $T=0.5$. Station numbers are given in the figures. Bars show the uncertainty bands. 

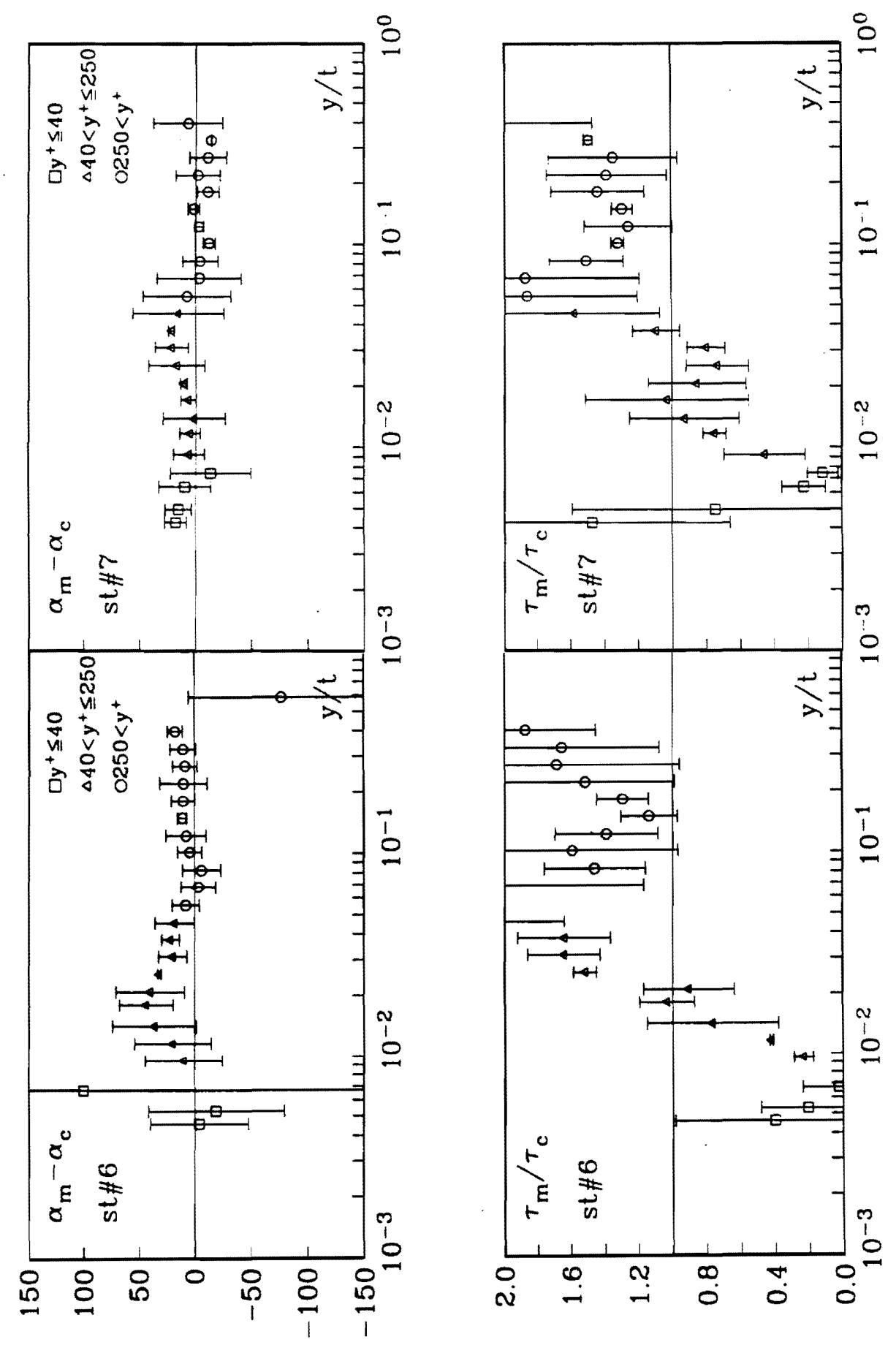

Figure 114d. Present data in Rotta's model with anisotropy constant $T=0.5$.: Station numbers are given in the figures. Bars show the uncertainty bands. 

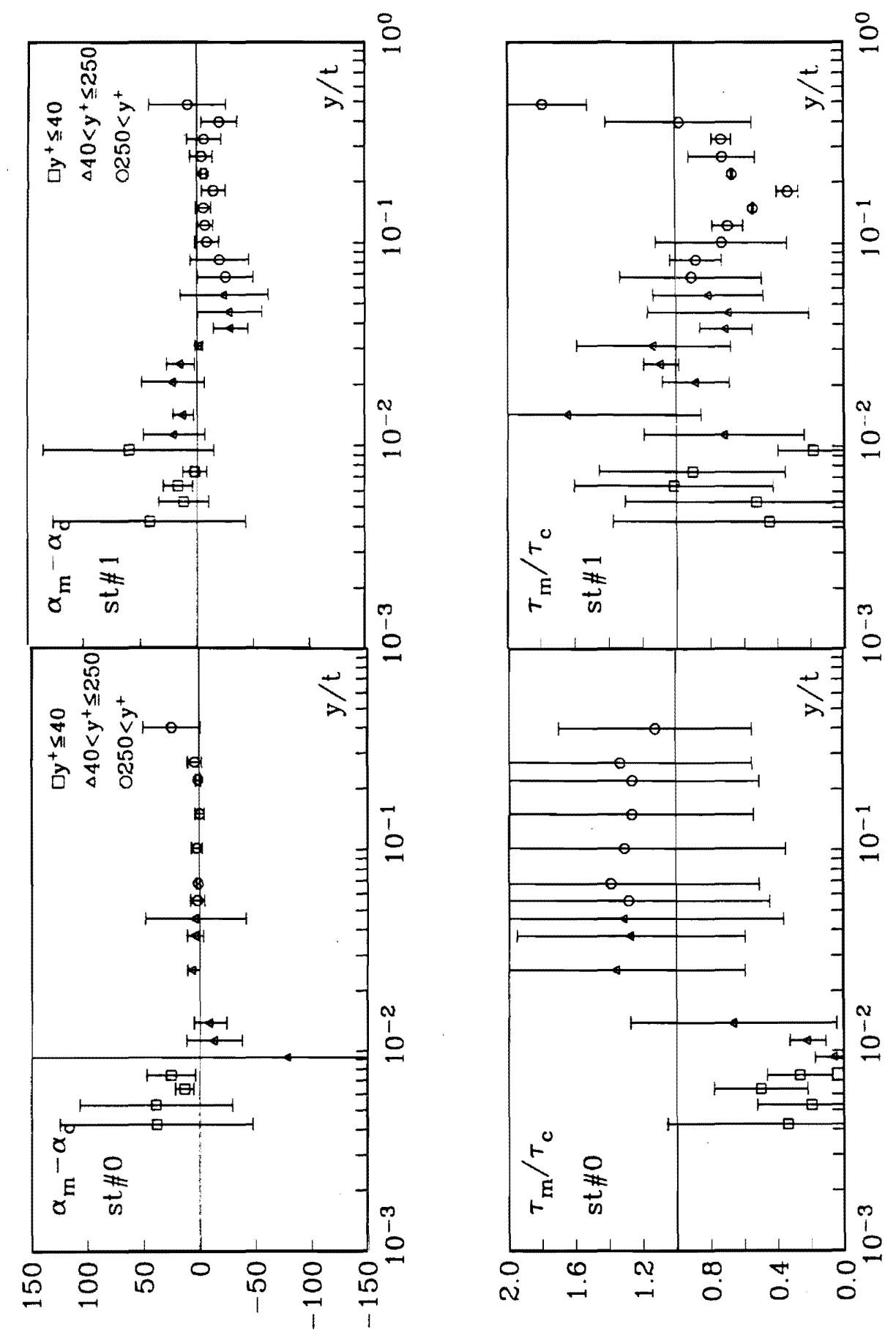

Figure 115a. Present data in Rotta's model with anisotropy constant $T=0.7$. Station numbers are given in the figures. Bars show the uncertainty bands. 

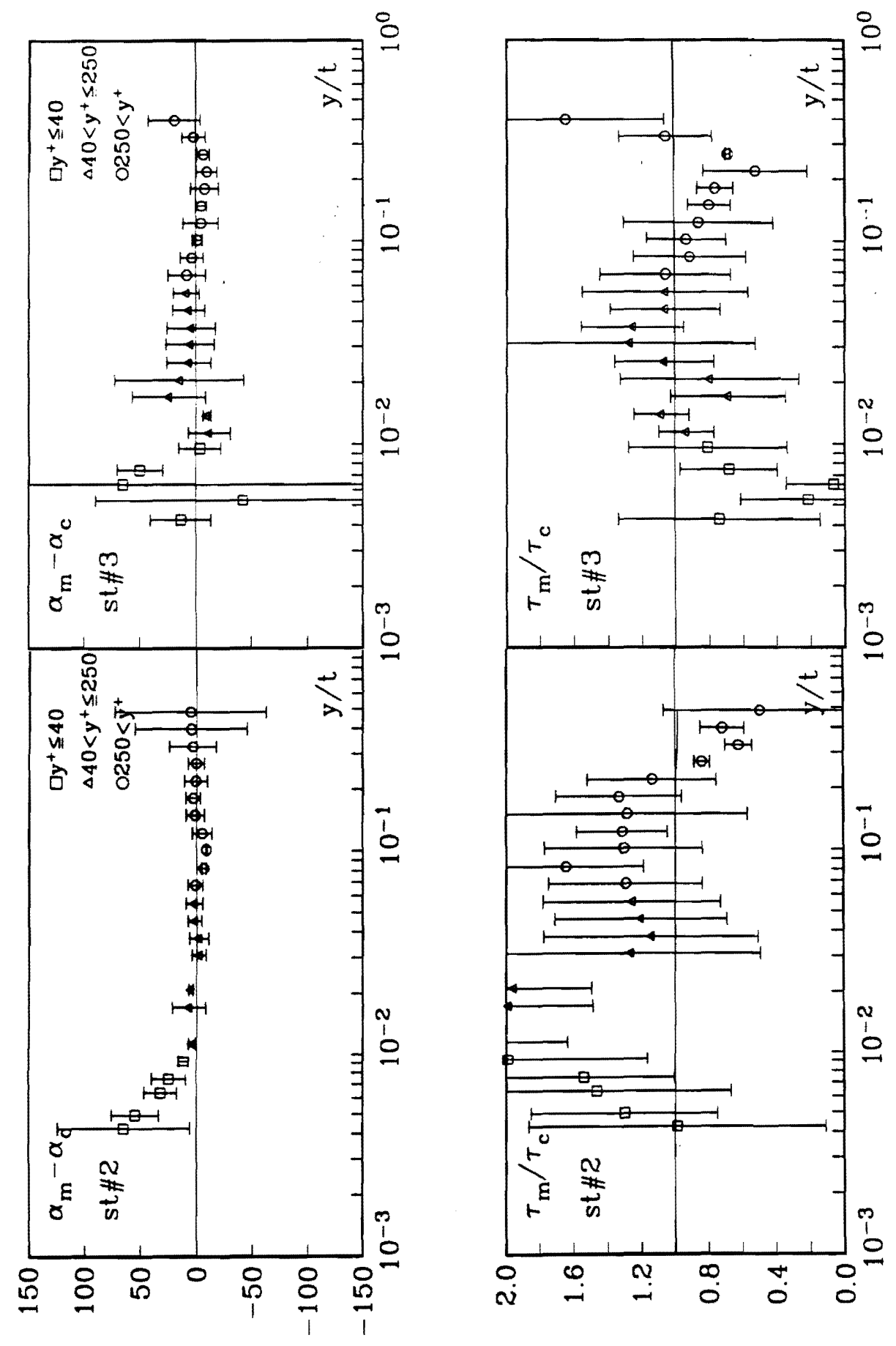

Figure 115b. Present data in Rotta's model with anisotropy constant $T=0.7 .:$ Station numbers are given in the figures. Bars show the uncertainty bands. 

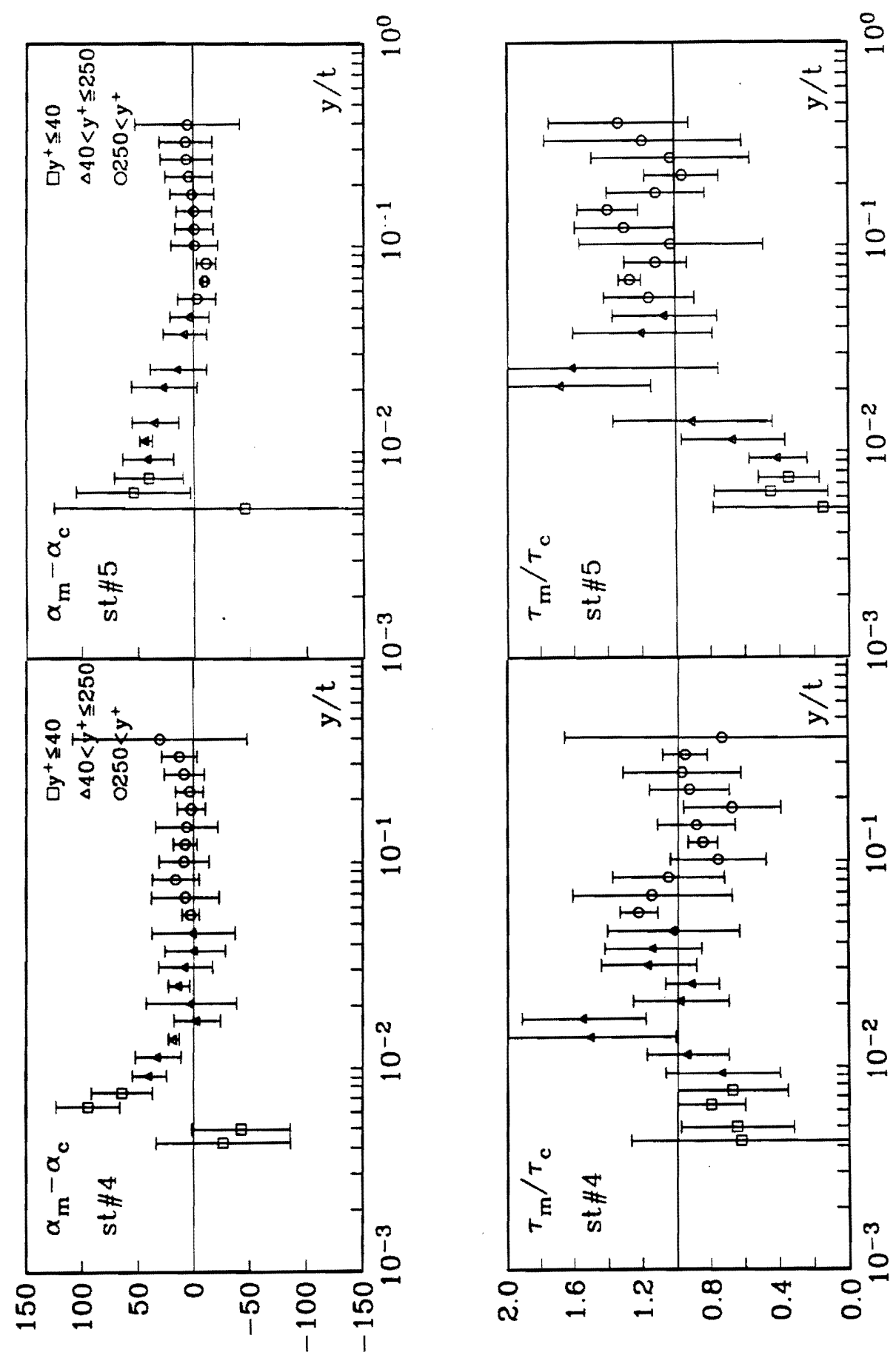

Figure 115c. Present data in Rotta's model with anisotropy constant $T=0.7$. Station numbers are given in the figures. Bars show the uncertainty bands. 

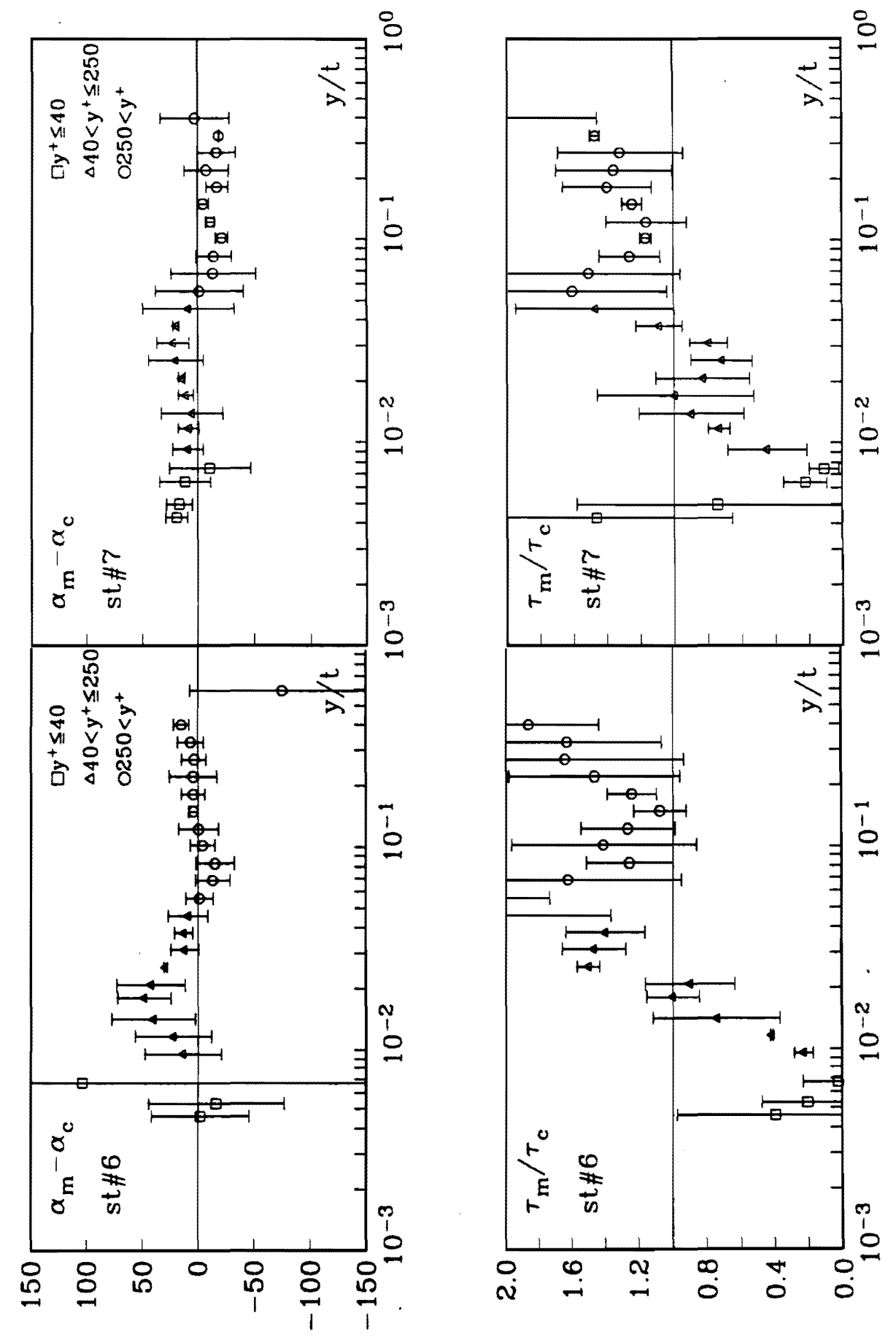

Figure 115d. Present data in Rotta's model with anisotropy constant $\mathrm{T}=0.7$. : Station numbers are given in the figures. Bars show the uncertainty bands. 

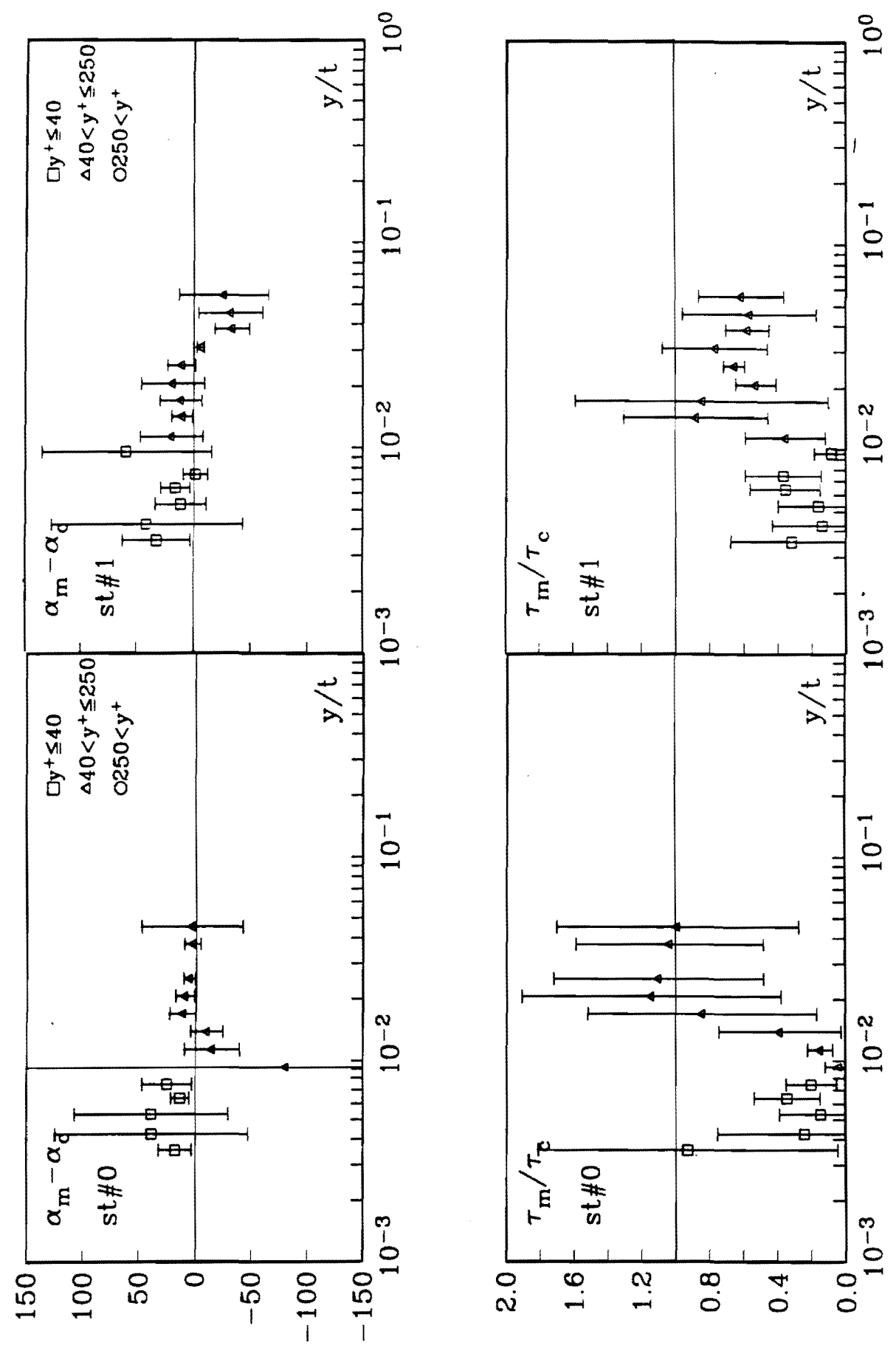

Figure 116a. Present data in Patel's model.: Station numbers are given in the figures. Bars show the uncertainty bands. 

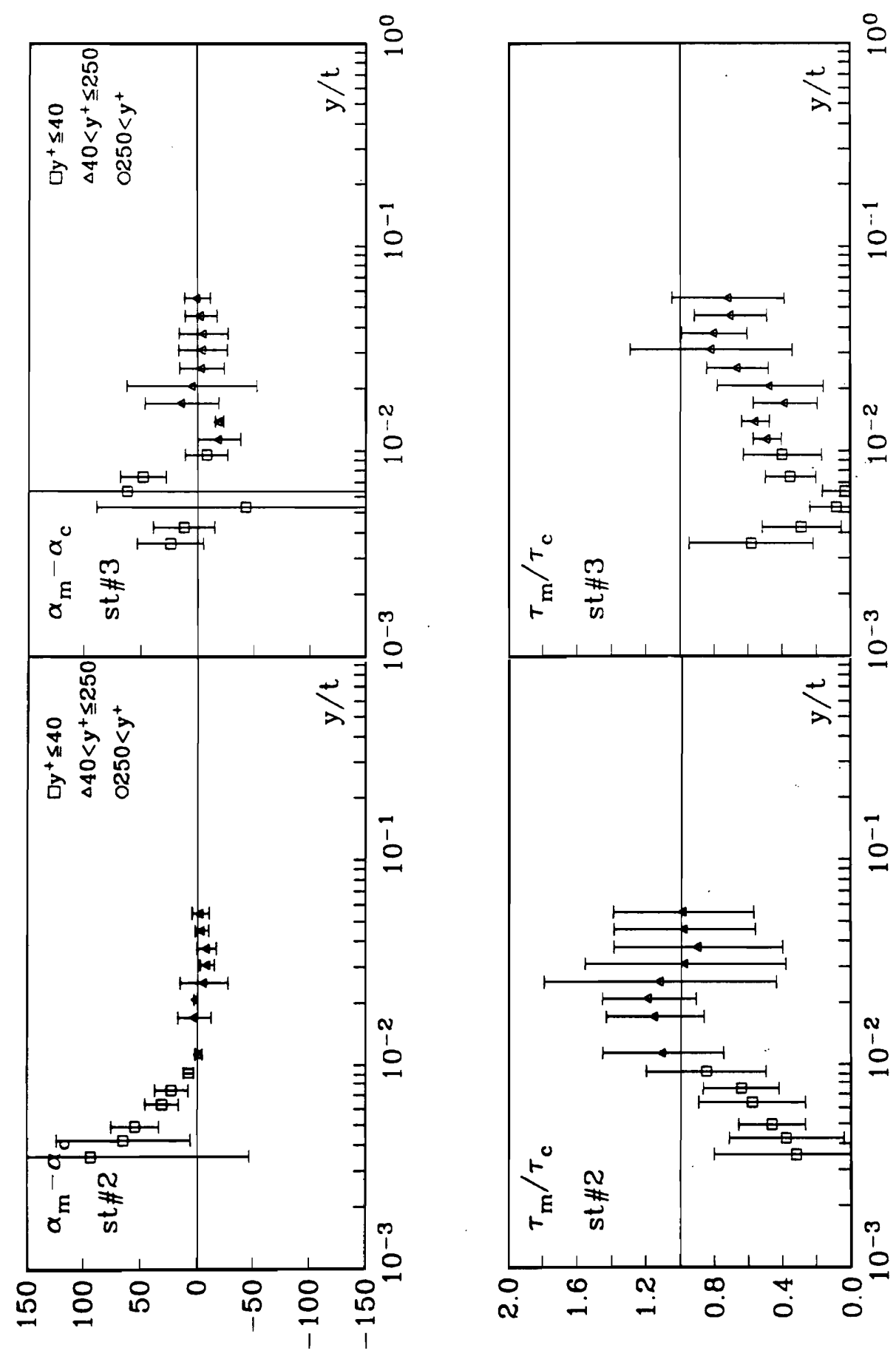

Figure 116b. Present data in Patel's model.: Station numbers are given in the figures. Bars show the uncertainty bands. 

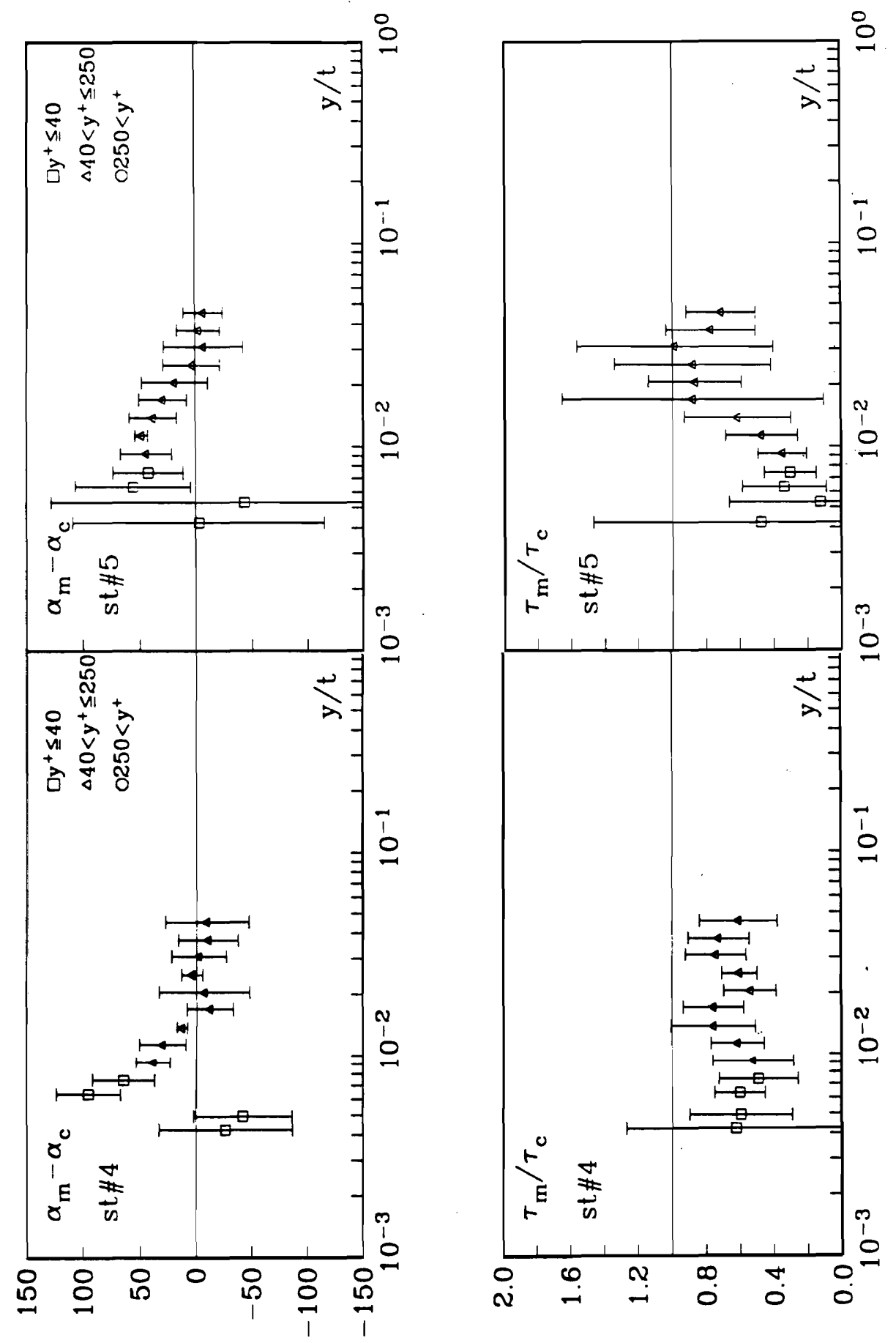

Figure 116c. Present data in Patel's model.: Station numbers are given in the figures. Bars show the uncertainty bands. 

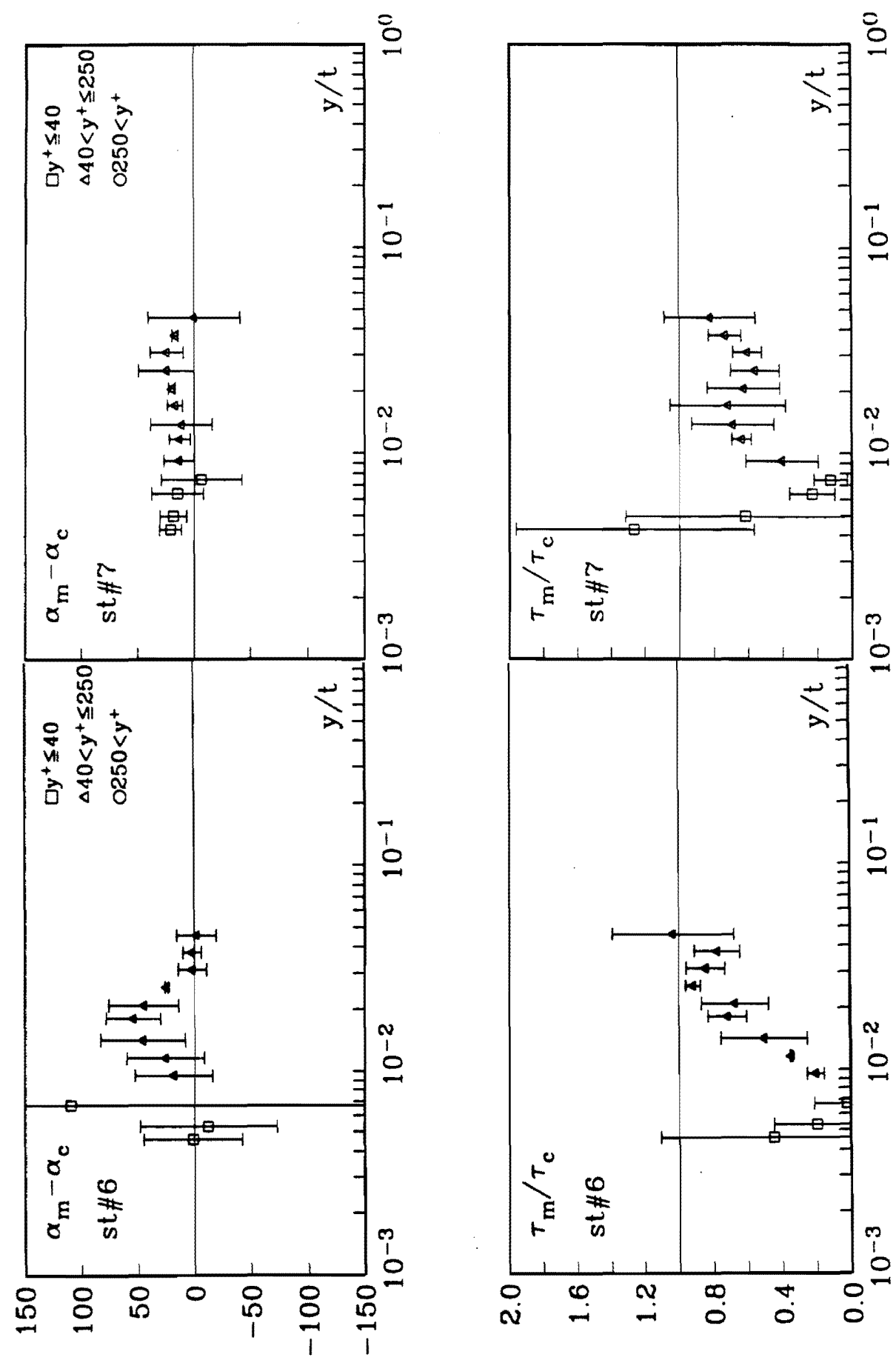

Figure 116d. Present data in Patel's model.: Station numbers are given in the figures. Bars show the uncertainty bands. 

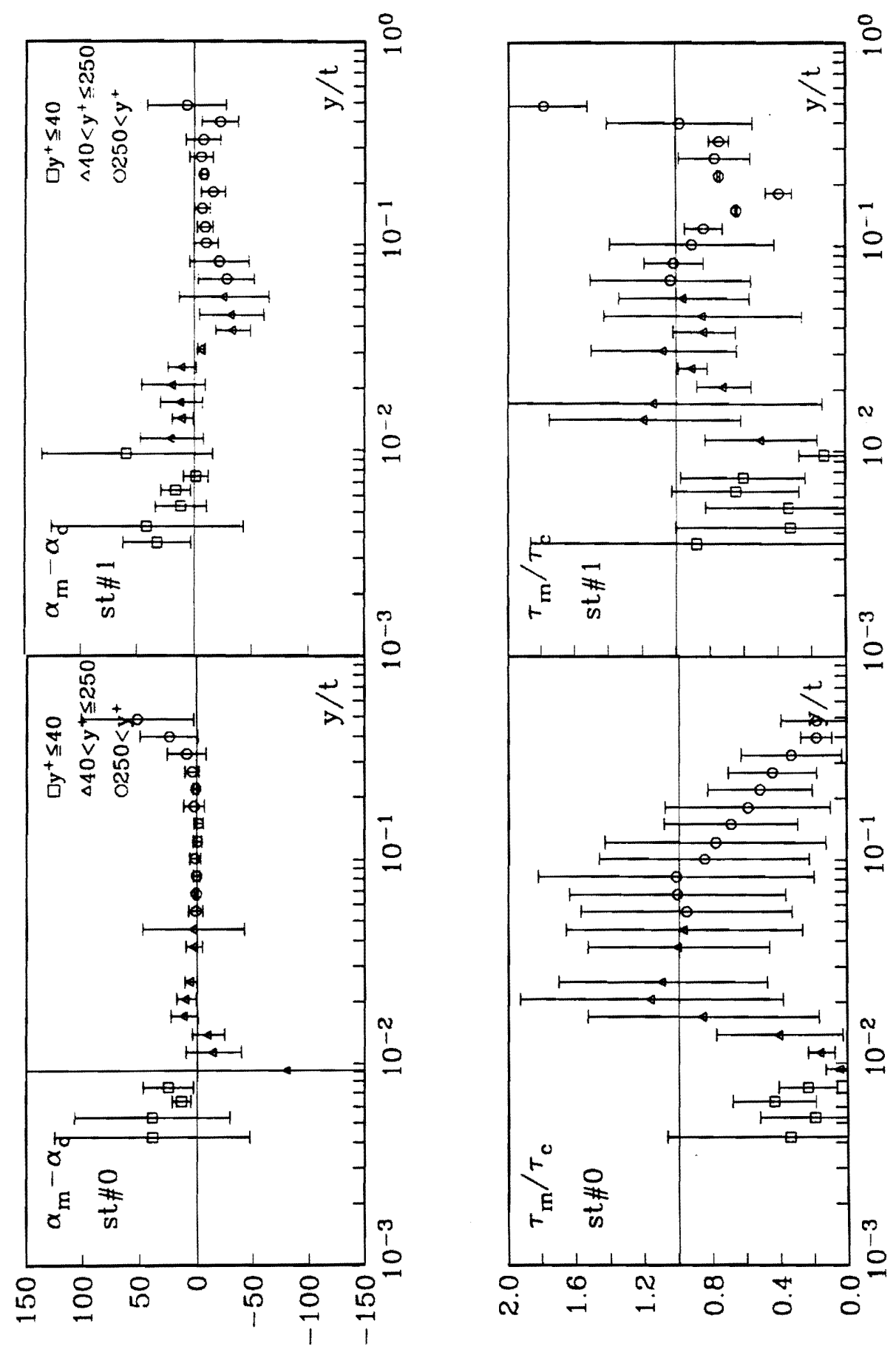

Figure 117a. Present data in Johnson-King model.: Station numbers are given in the figures. Bars show the uncertainty bands. 

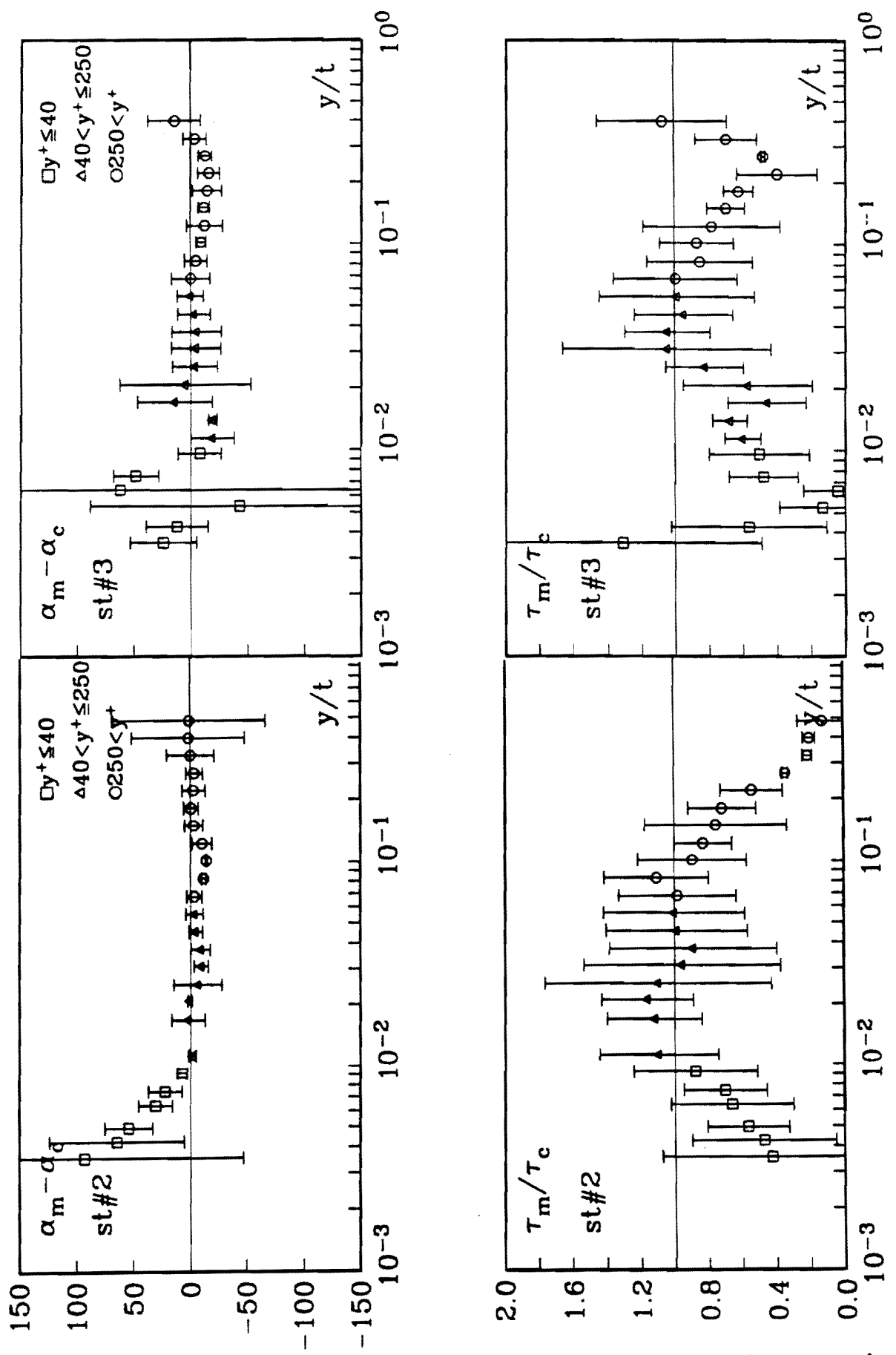

Figure 117b. Present data in Johnson-King model.: Station numbers are given in the figures. Bars show the uncertainty bands. 

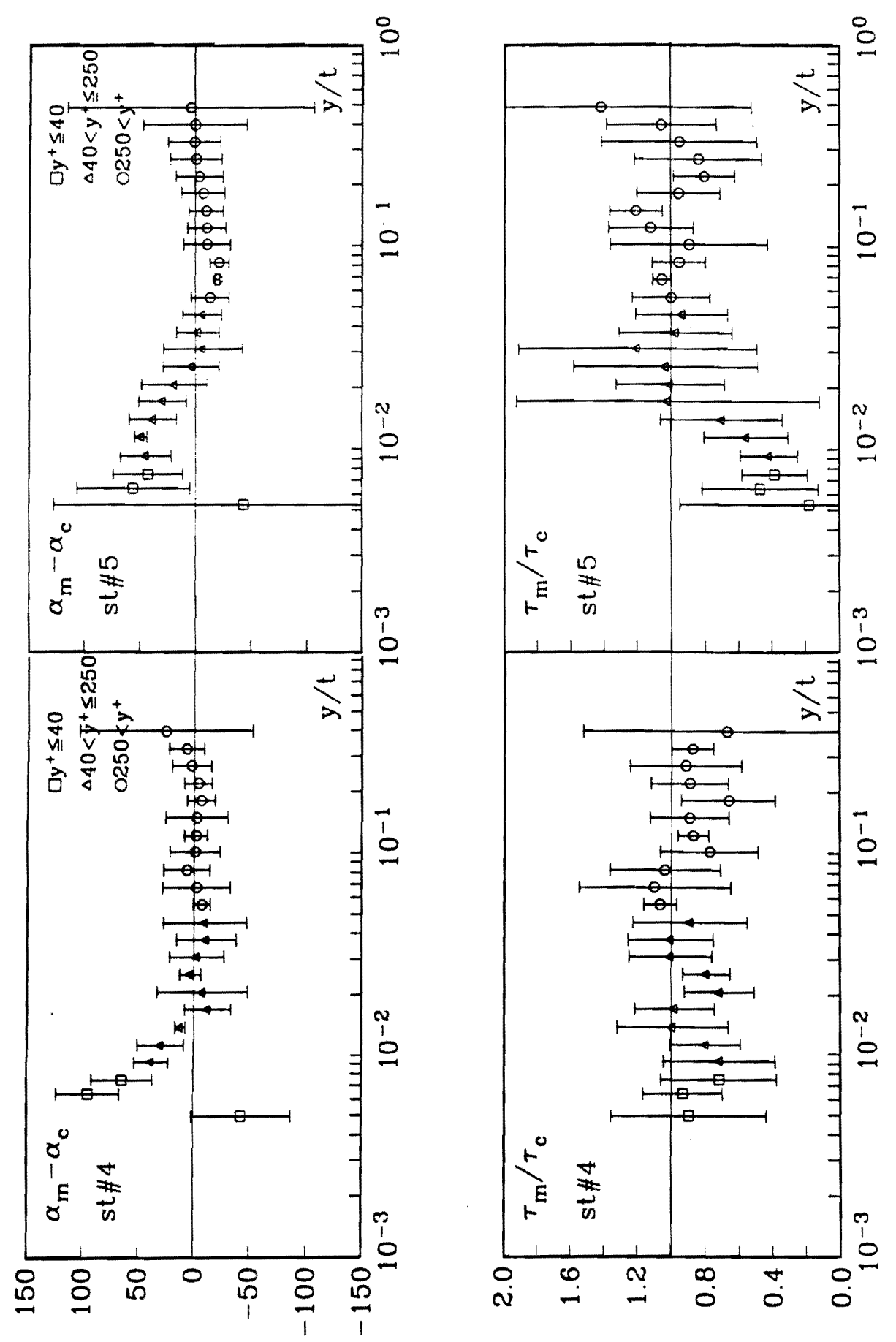

Figure 117c. Present data in Johnson-King model.: Station numbers are given in the figures. Bars show the uncertainty bands. 

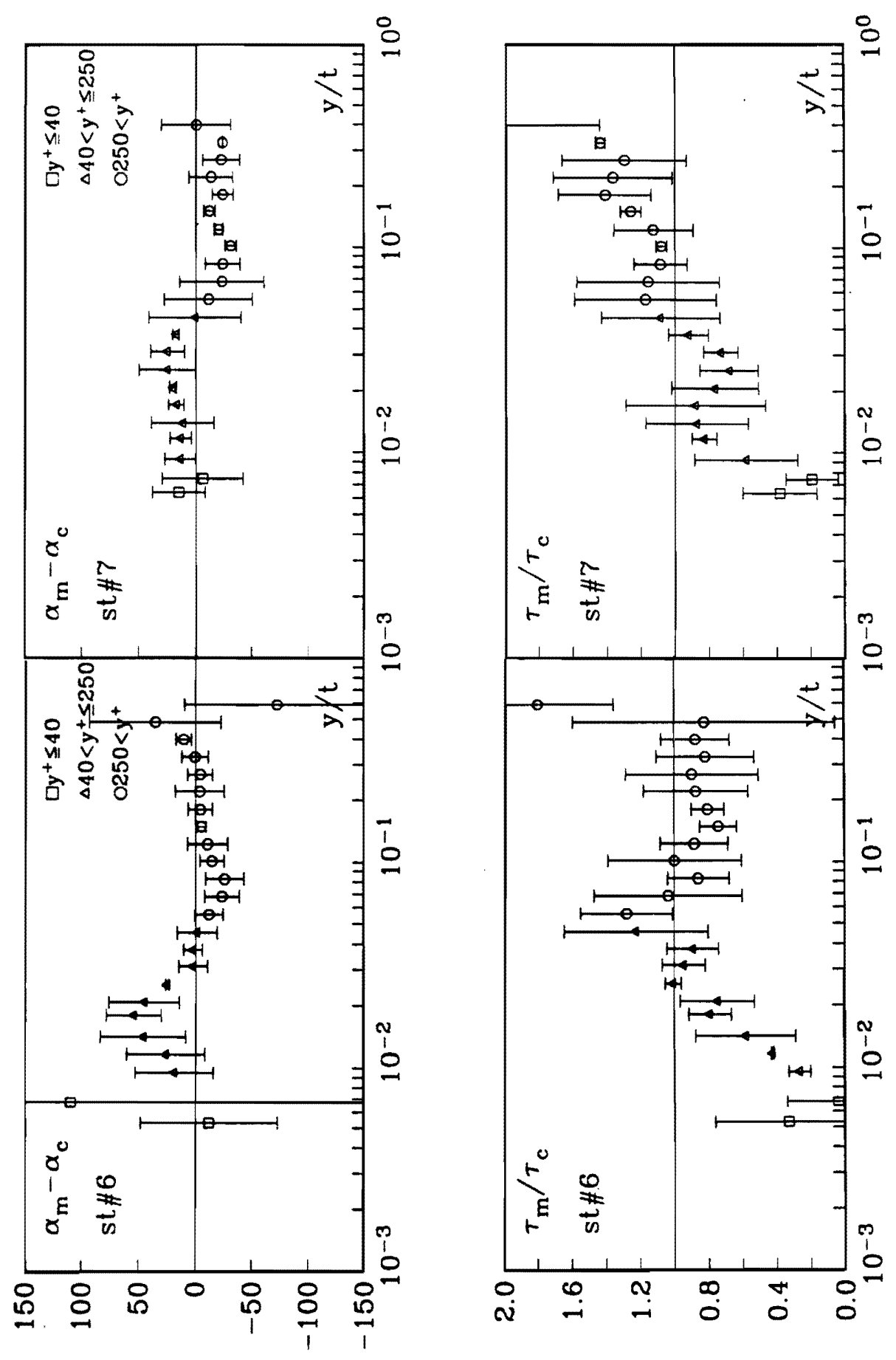

Figure 117d. Present data in Johnson-King model.: Station numbers are given in the figures. Bars show the uncertainty bands. 


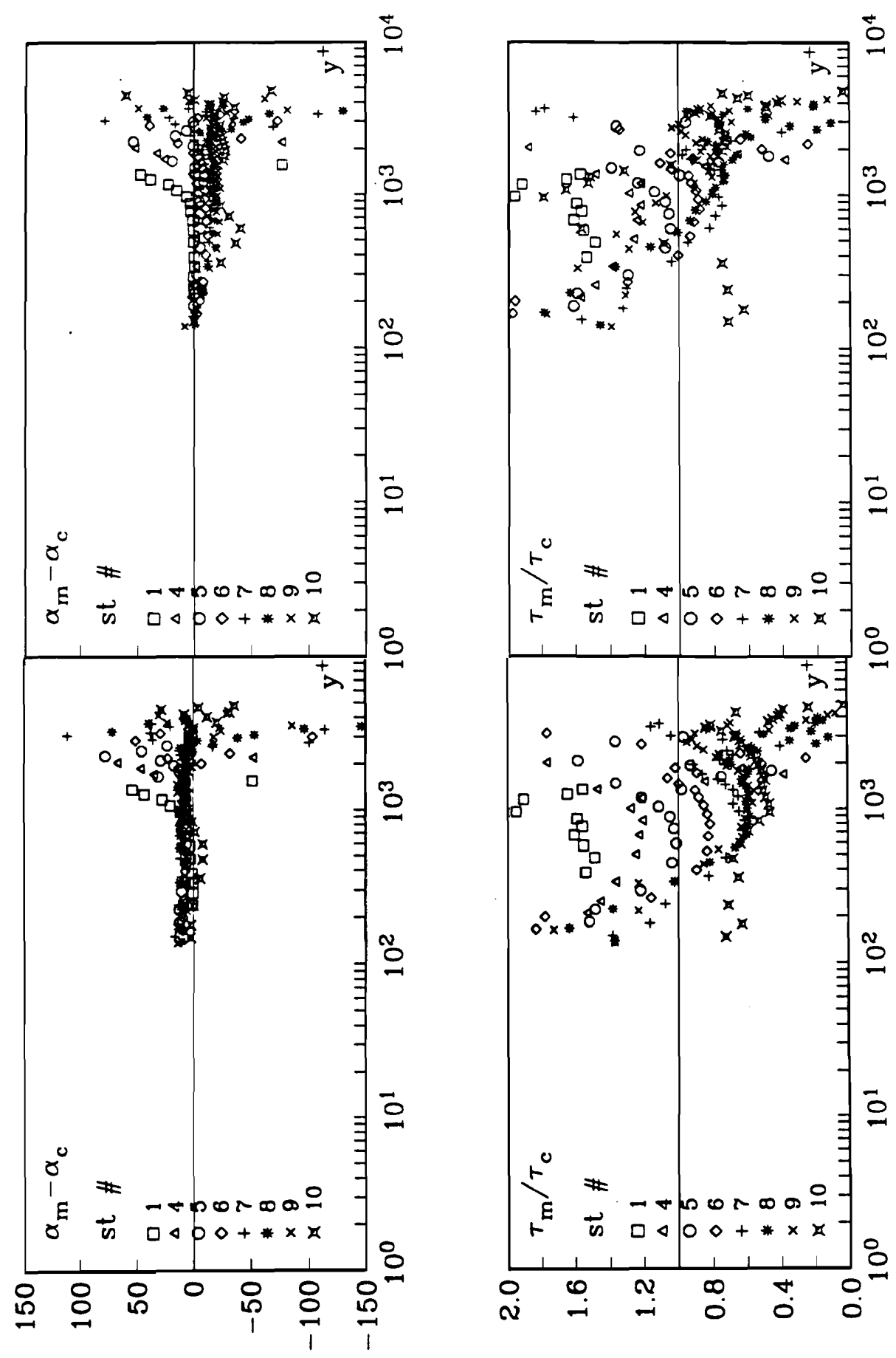

Figure 118a. Elsenaar-Boelsma data in Cebeci-Smith model ( left ) and in Rotta's model with anisotropy constant $\mathrm{T}=0.3$ ( right ). 

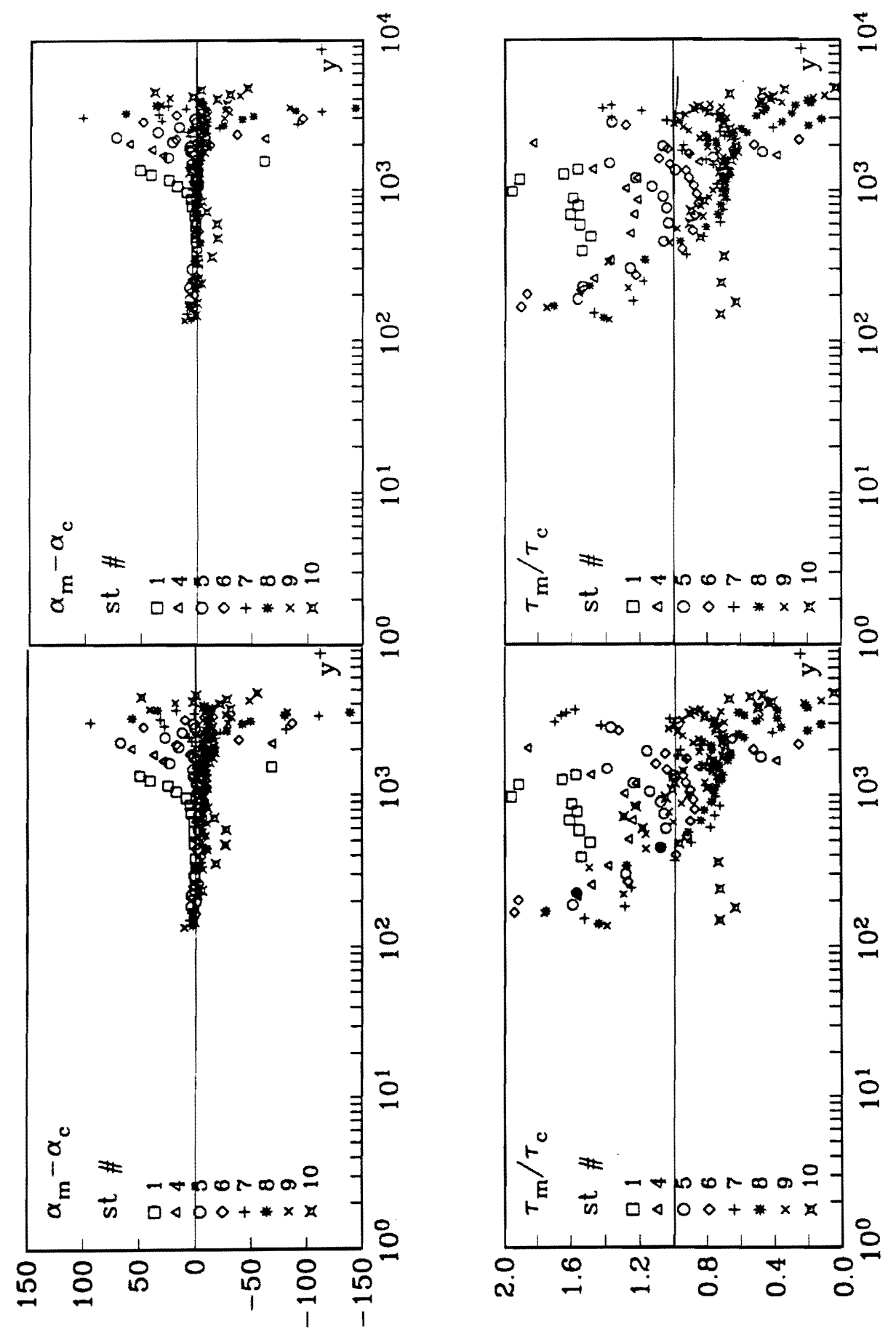

Figure 118b. Elsenaar-Boelsma data in Rotta's model with anisotropy constant $T=0.5$ (left ) and with anisotropy constant $\mathrm{T}=0.7$ ( right). 


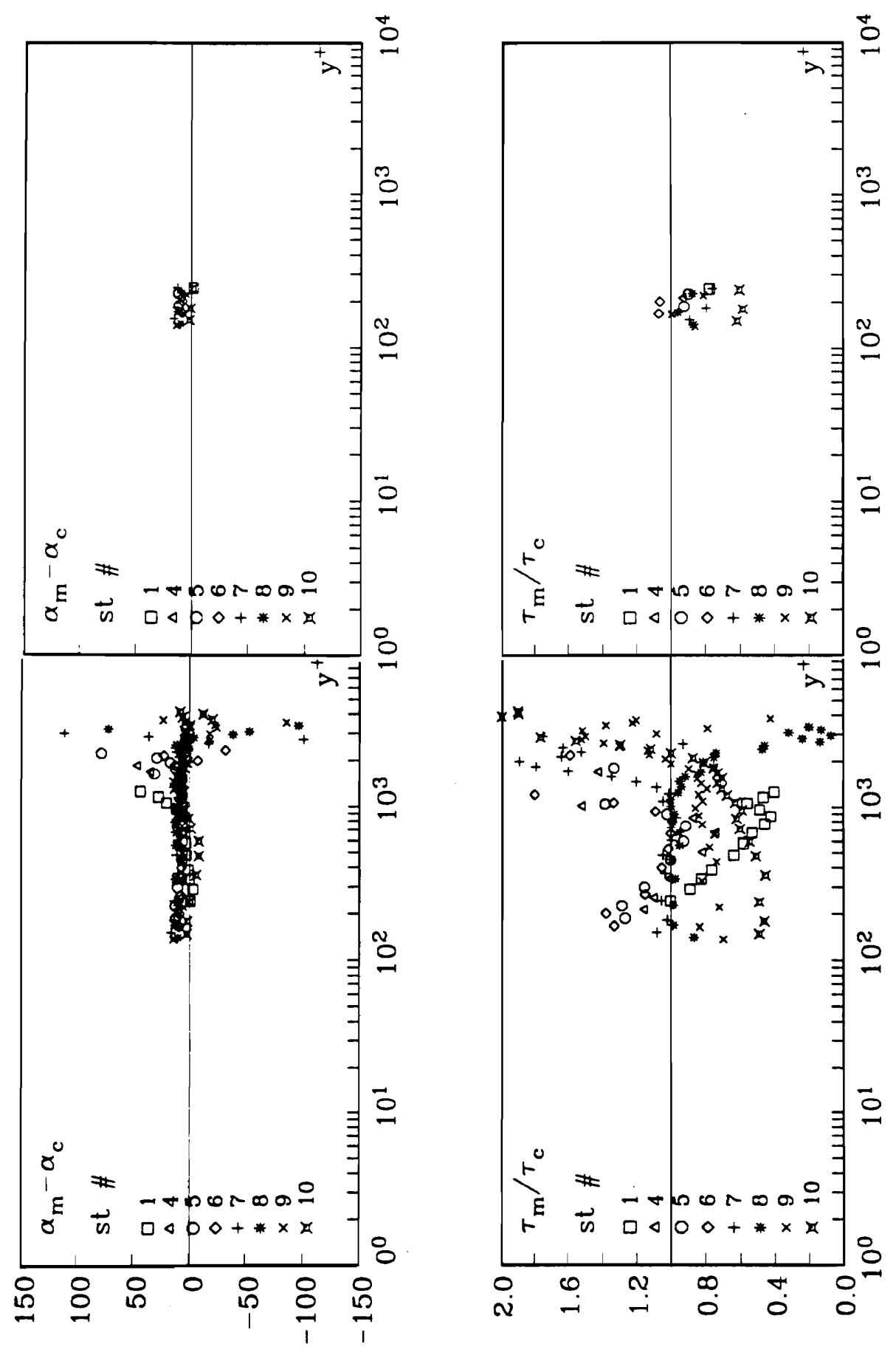

Figure 118c. Elsenaar-Boelsma data in Johnson-King ( left ) and in Patel's ( right ) models. 


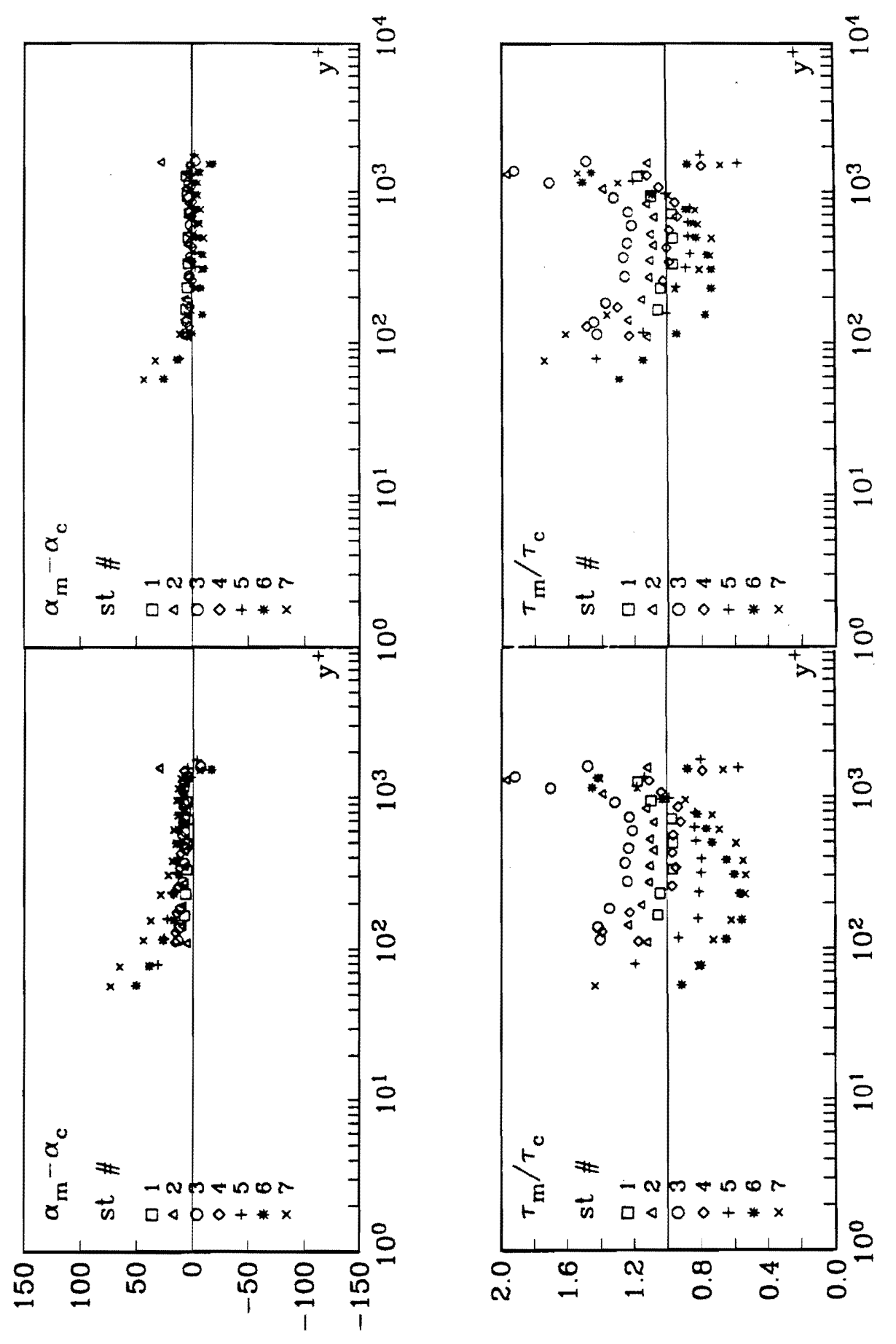

Figure 118d. Dechow's data in Cebeci-Smith model ( left) and in Rotta's model with anisotropy constant $\mathrm{T}=0.3$ ( right ). 

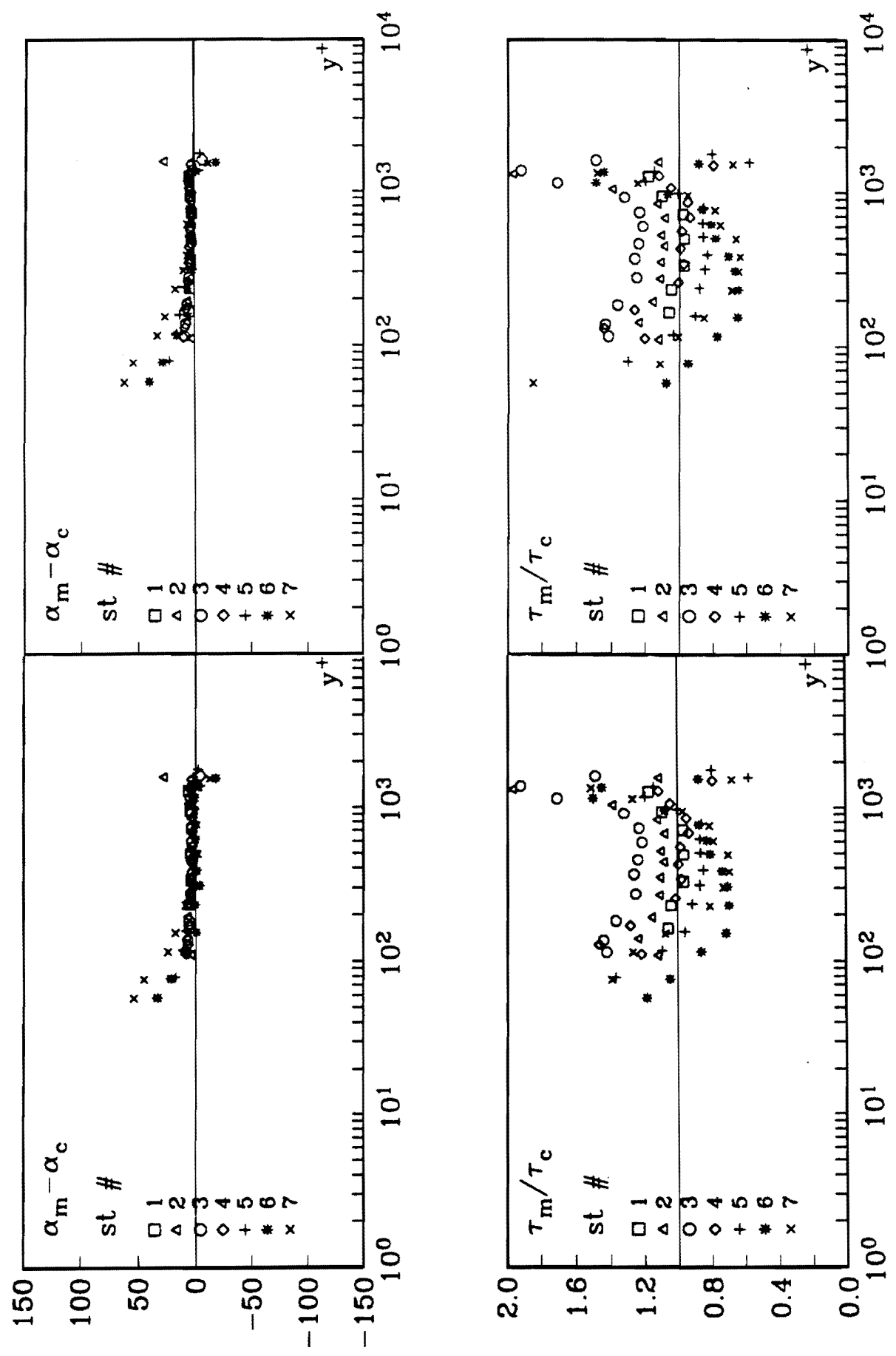

Figure 119a. Dechow's data in Rotta's model with anisotropy constant $T=0.5$ ( left ) and with anisotropy constant $\mathrm{T}=0.7$ ( right). 

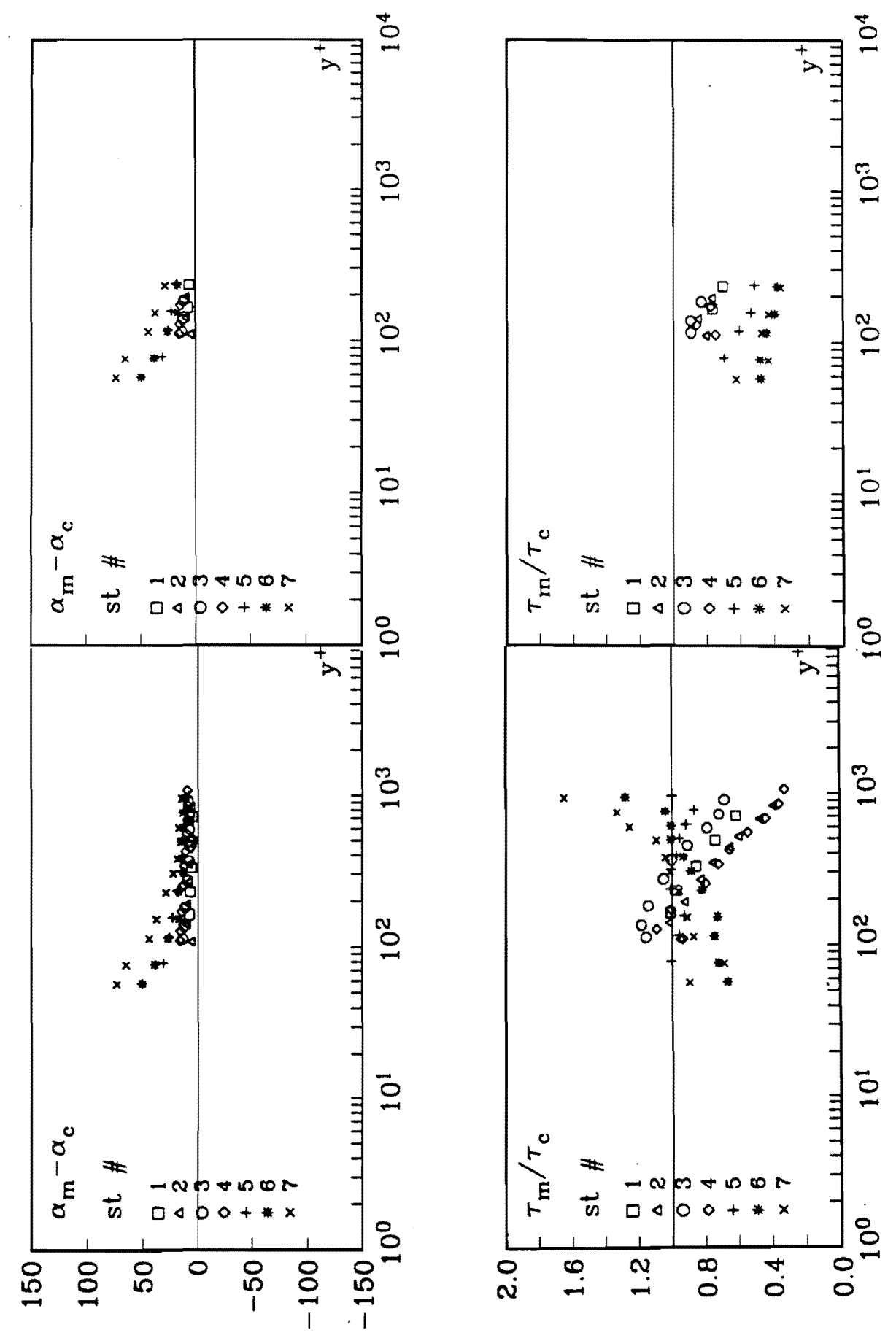

Figure 119b. Dechow's data in Johnson-King (left) and in Patel's (right) models. 


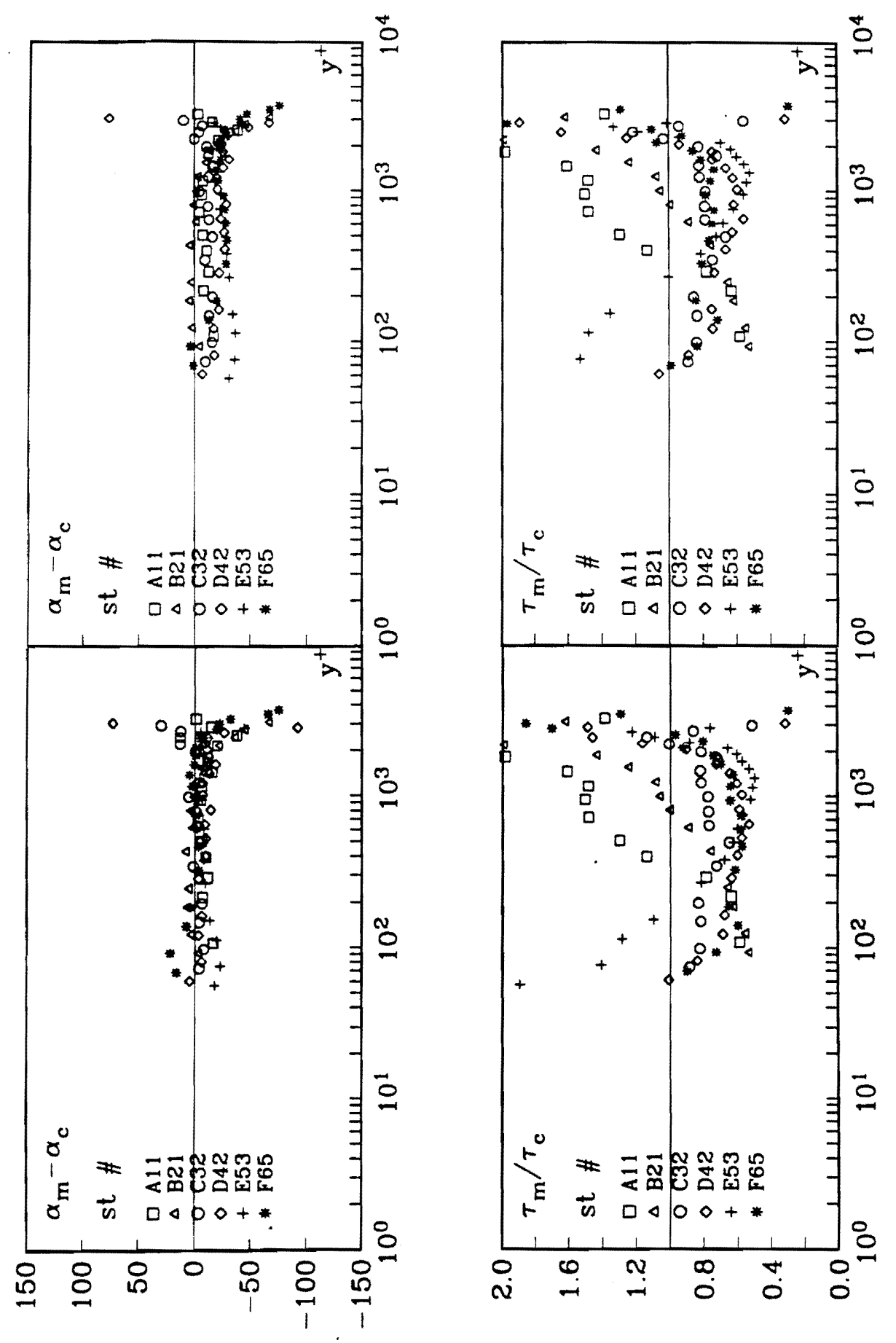

Figure 120a. Muller's data in Cebeci-Smith model ( left ) and in Rotta's model with anisotropy constant $\mathrm{T}=0.3$ ( right). 

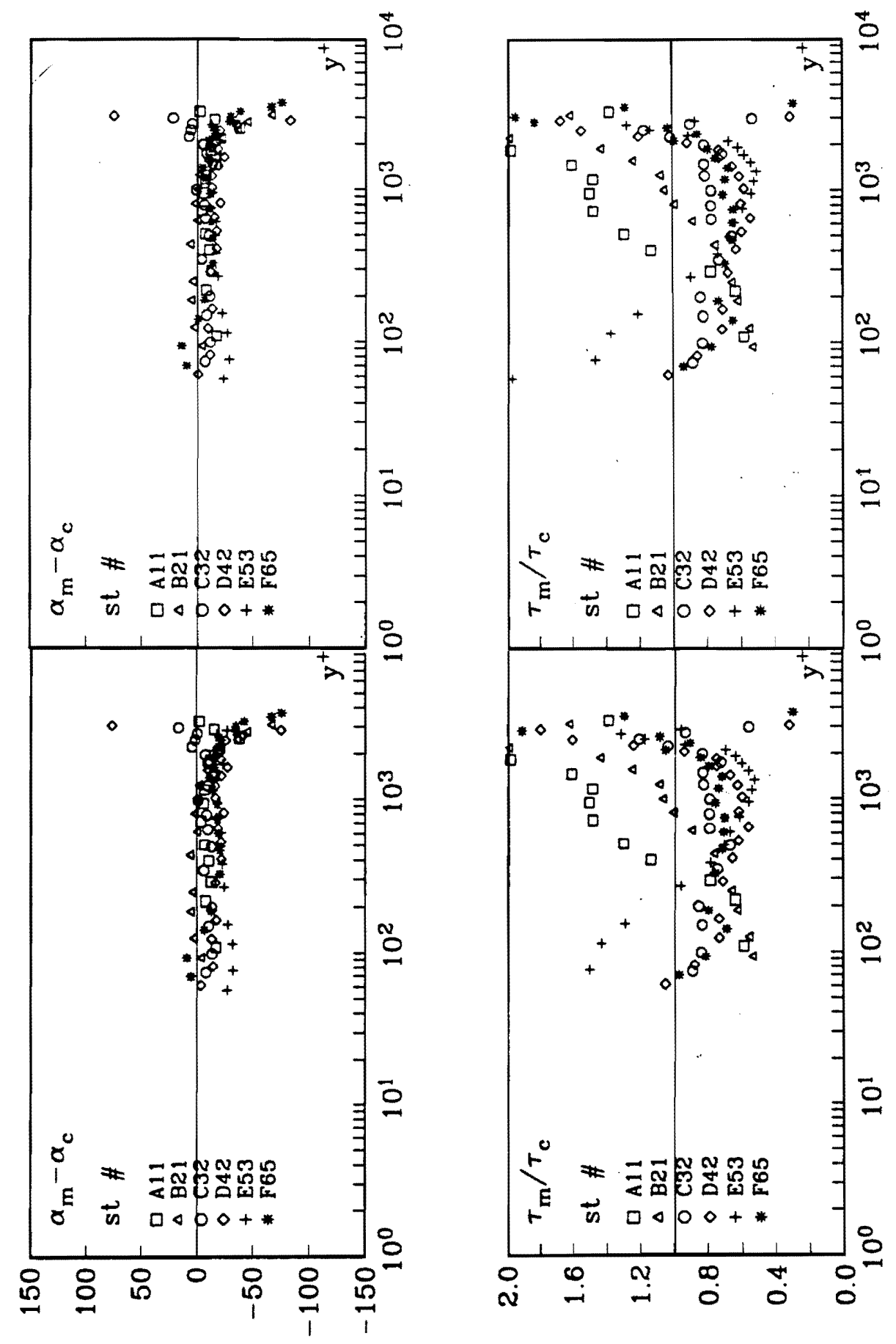

Figure 120b. Mulker's data in Rotta's model with anisotropy constant $T=0.5$ (left ) and with anisotropy constant $T=0.7$ ( right ). 


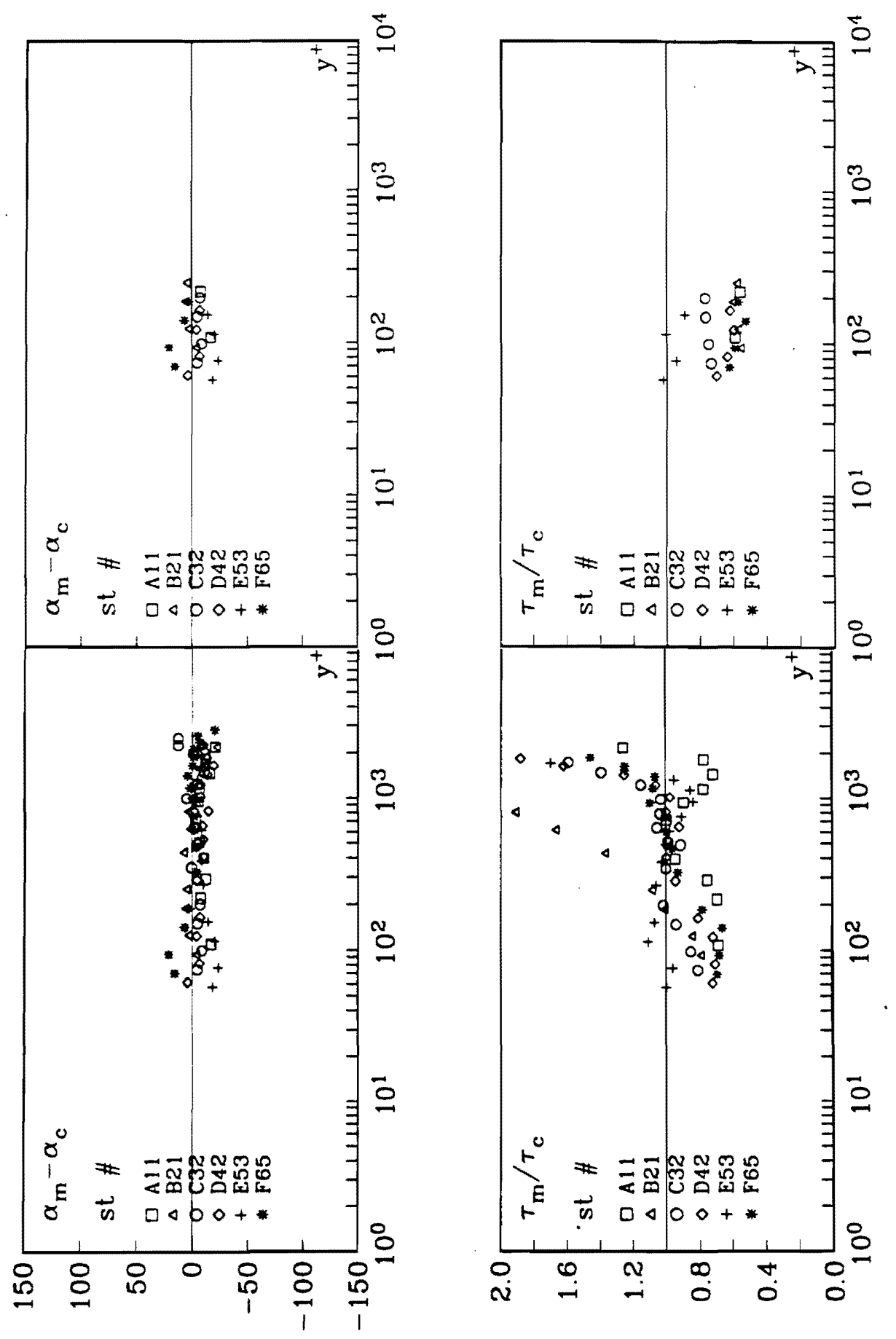

Figure 120c. Muller's data in Johnson-King ( left ) and in Patel's ( right ) models. 

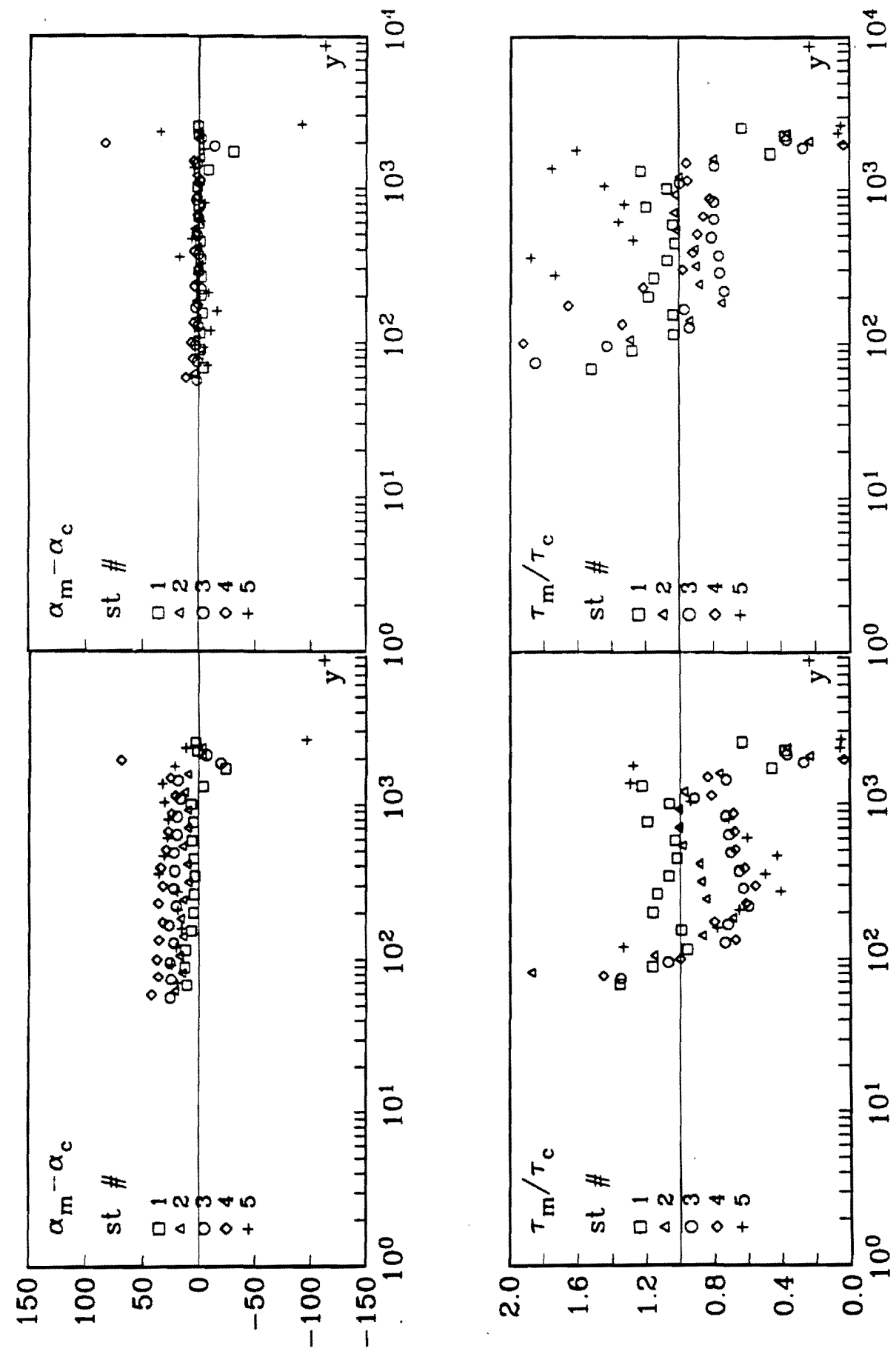

Figure 12la. Anderson's data in Cebeci-Smith model ( left) and in Rotta's model with anisotropy constant $\mathrm{T}=0.3$ ( right). 

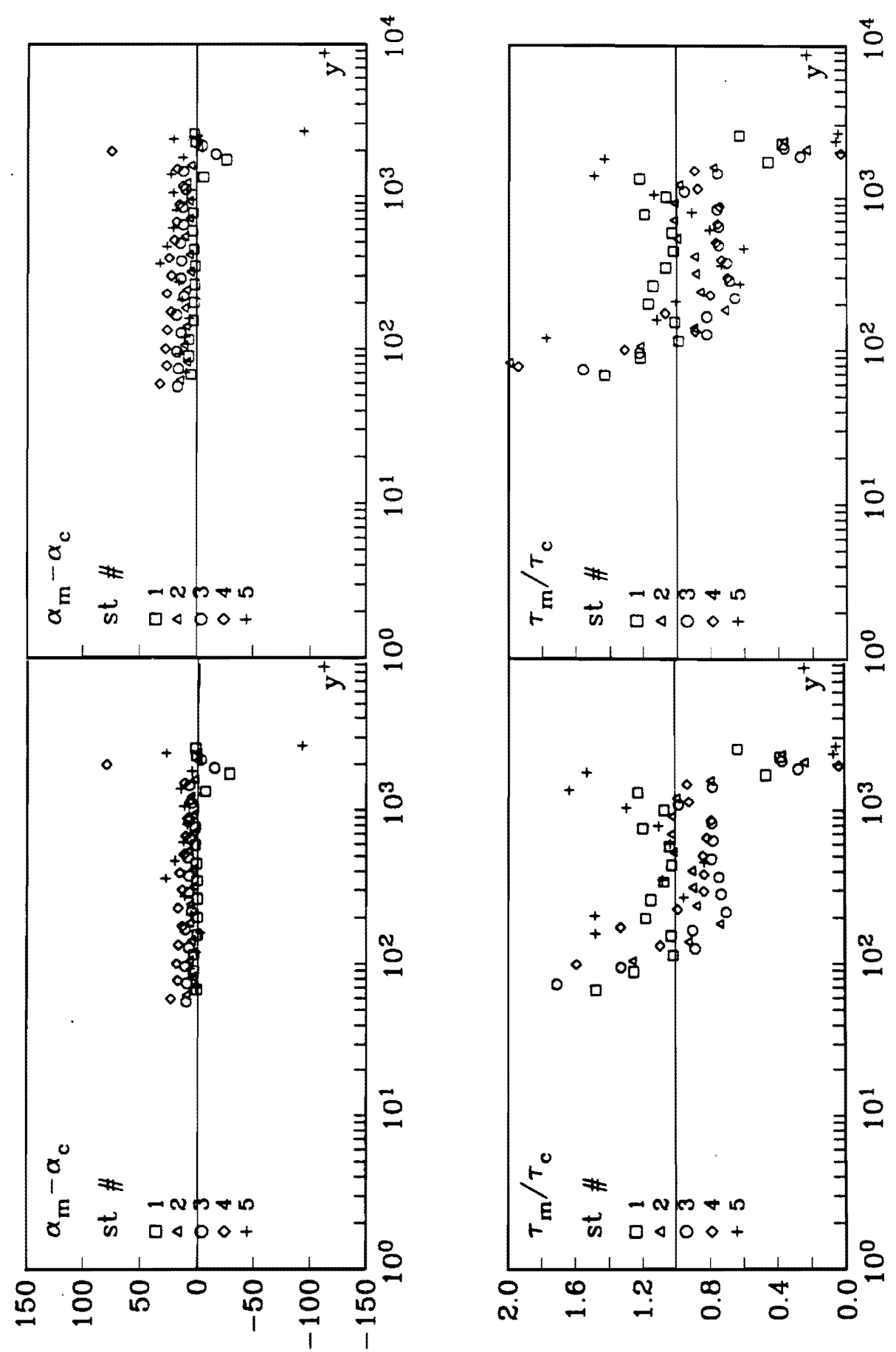

Figure 121b. Anderson's data in Rotta's model with anisotropy constant $T=0.5$ (left) and with anisotropy constant $T=0.7$ ( right ). 

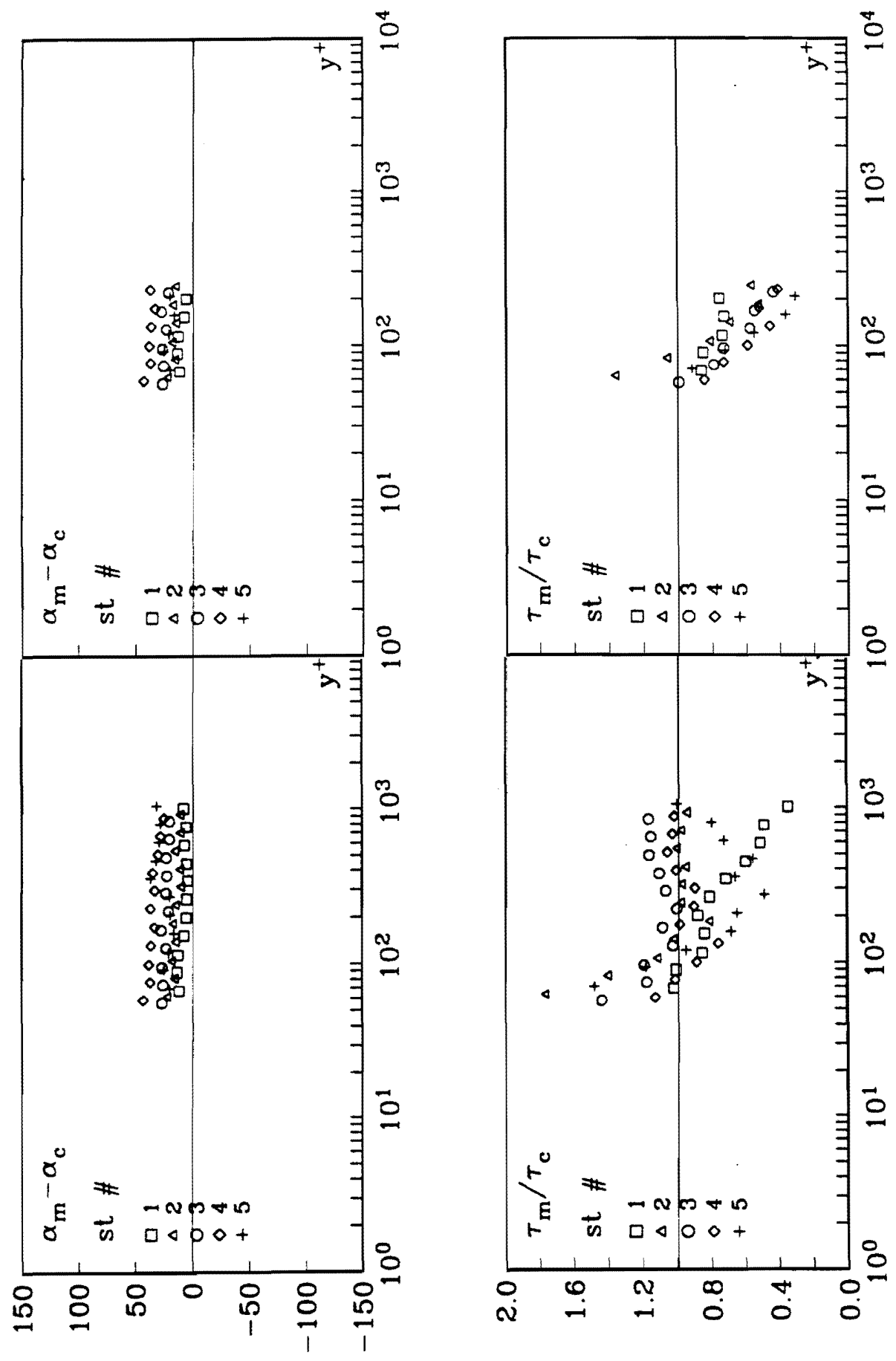

Figure 121c. Anderson's data in Johnson-King ( left ) and in Patel's ( right) models. 

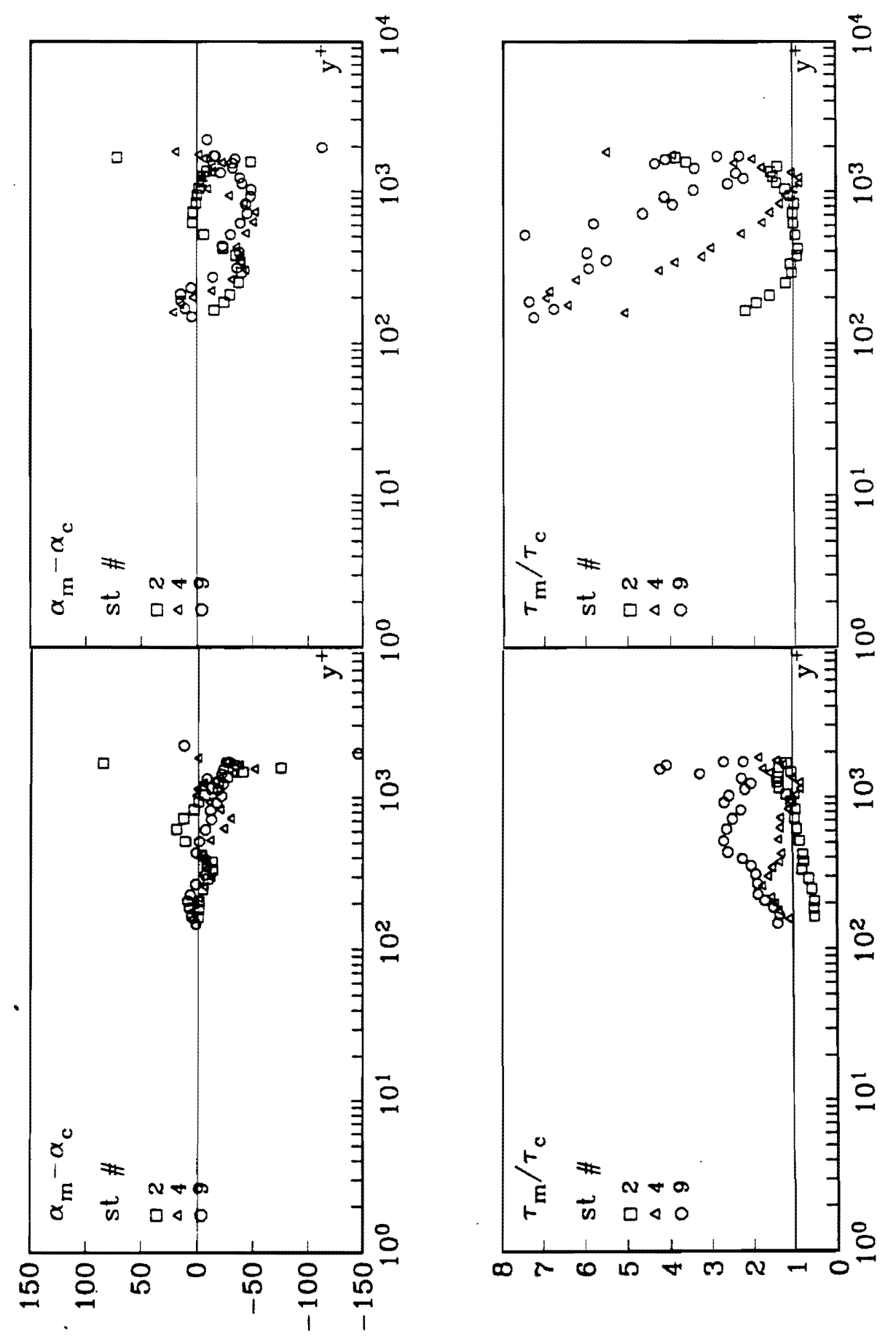

Figure 122a. Lohmann's data in Cebeci-Smith model ( left ) and in Rotta's model with anisotropy constant $\mathrm{T}=0.3$ ( right ). 

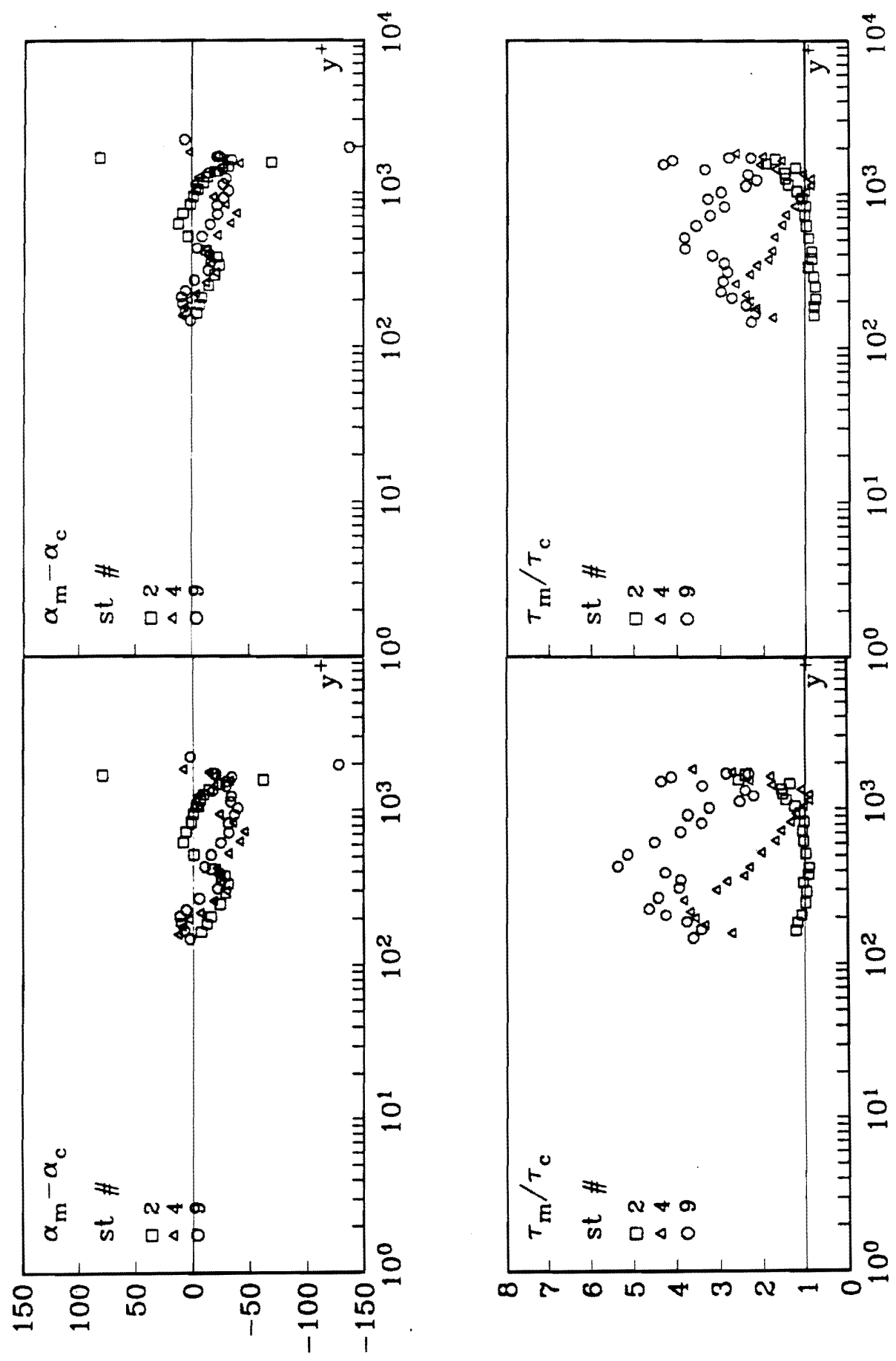

Figure 122b. Lohmann's data in Rotta's model with anisotropy constant $T=0.5$ (left) and with anisotropy constant $\mathrm{T}=0.7$ ( right). 

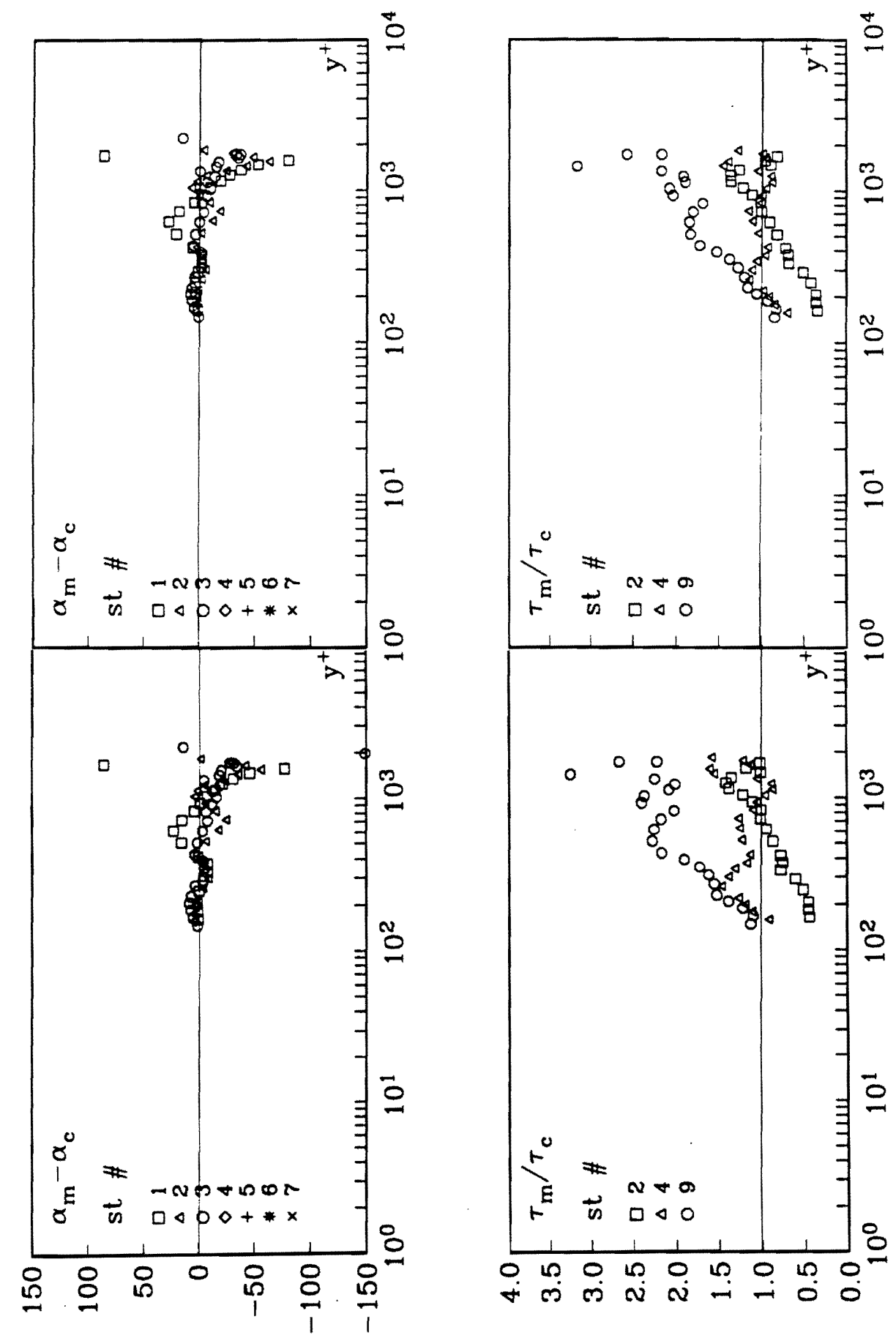

Figure 122c. Lohmann's data in Rotta's model with anisotropy constant $T=1.2$ (left) and with anisotropy constant $\mathrm{T}=1.5$ ( right ). 


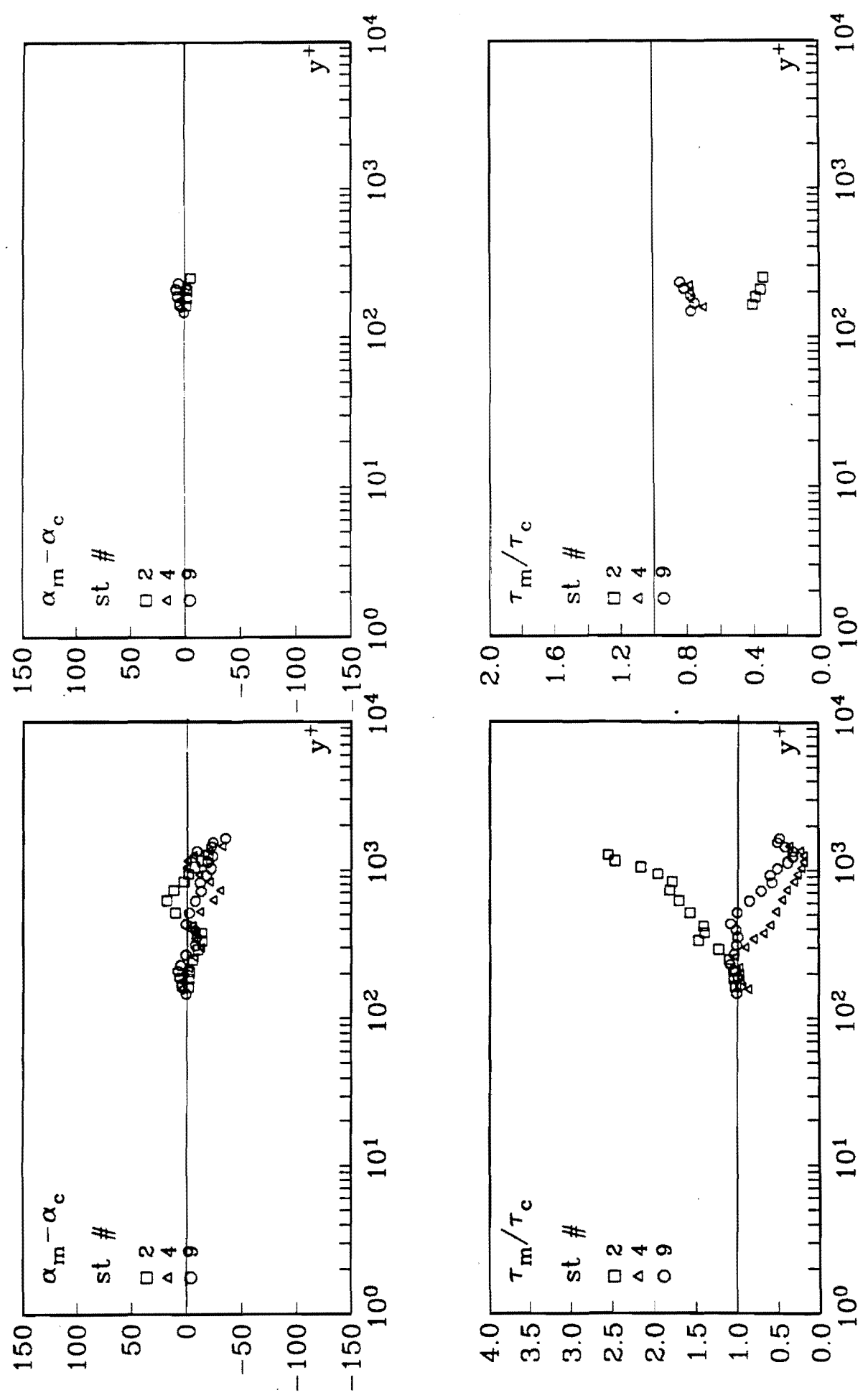

Figure 122d. Lohmann's data in Johnson-King (left ) and in Patel's (right) models. 

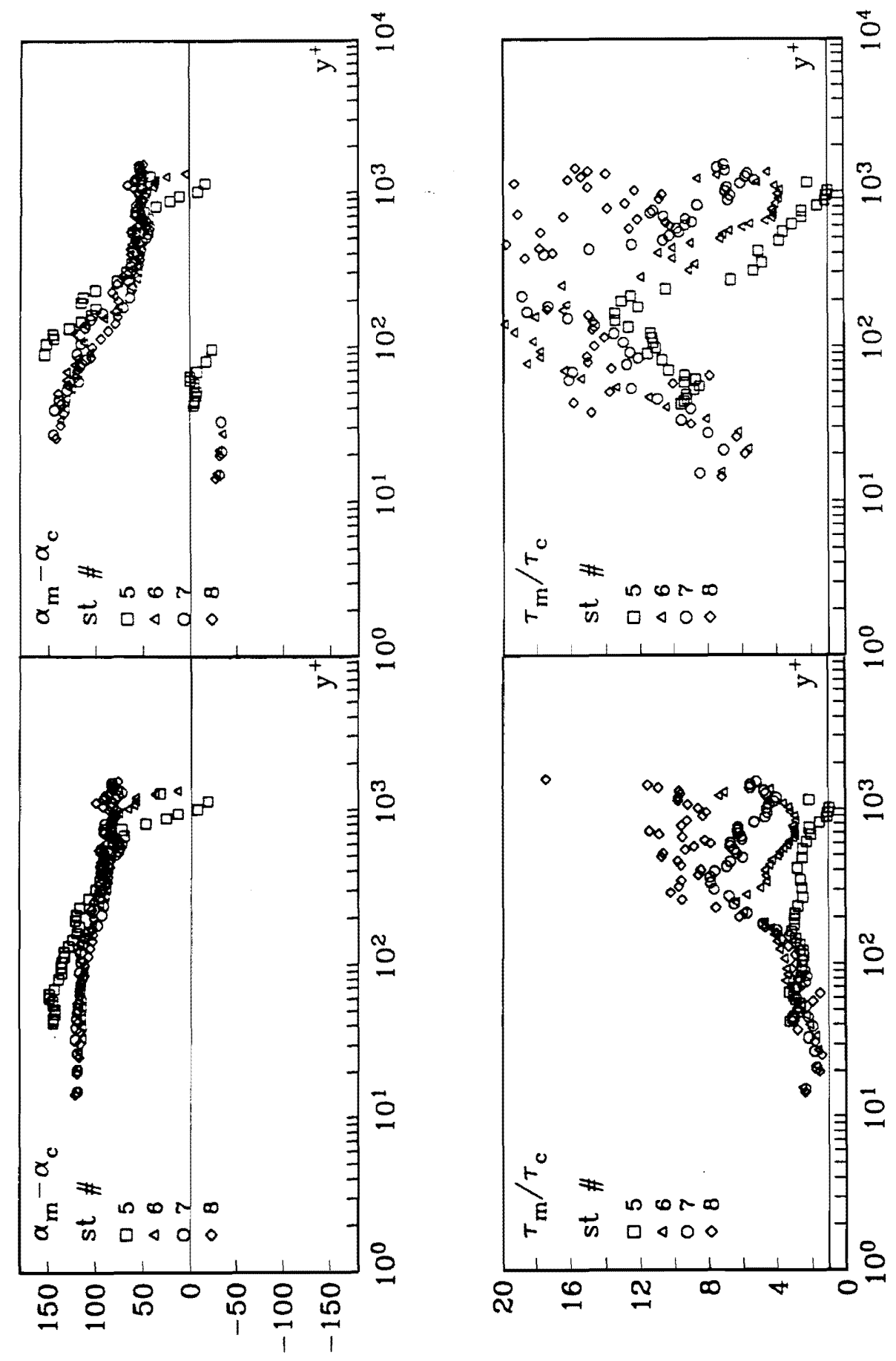

Figure 123a. Bissonnette-Mellor low Reynolds number data in Cebeci-Smith model (left ) and in Rotta's model with anisotropy constant $T=0.3$ ( right ). 

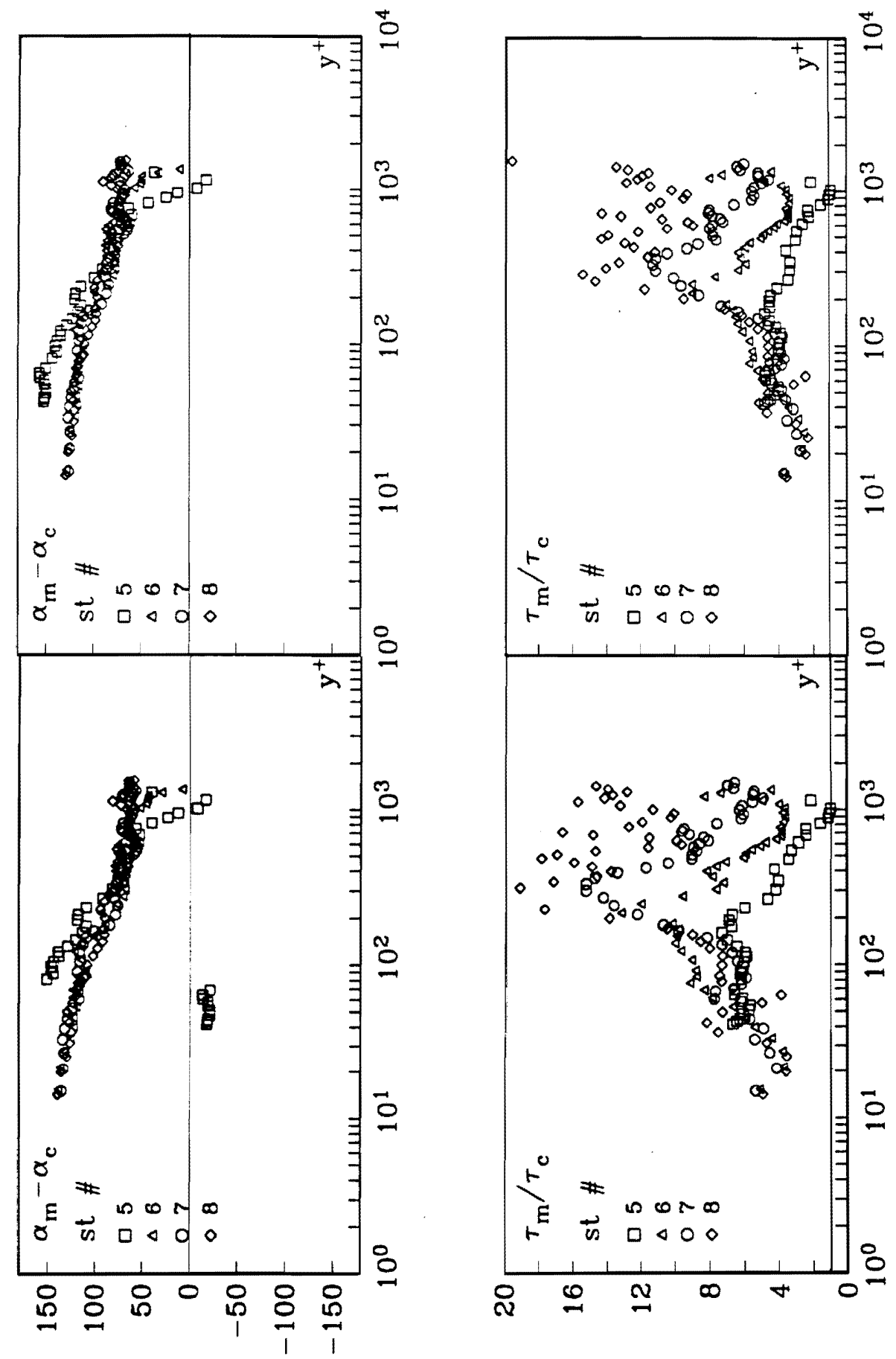

Figure 123b. Bissonnette-Mellor low Reynolds number data in Rotta's model with anisotropy constant $T=0.5$ ( left) and with anisotropy constant $T=0.7$ ( right ). 

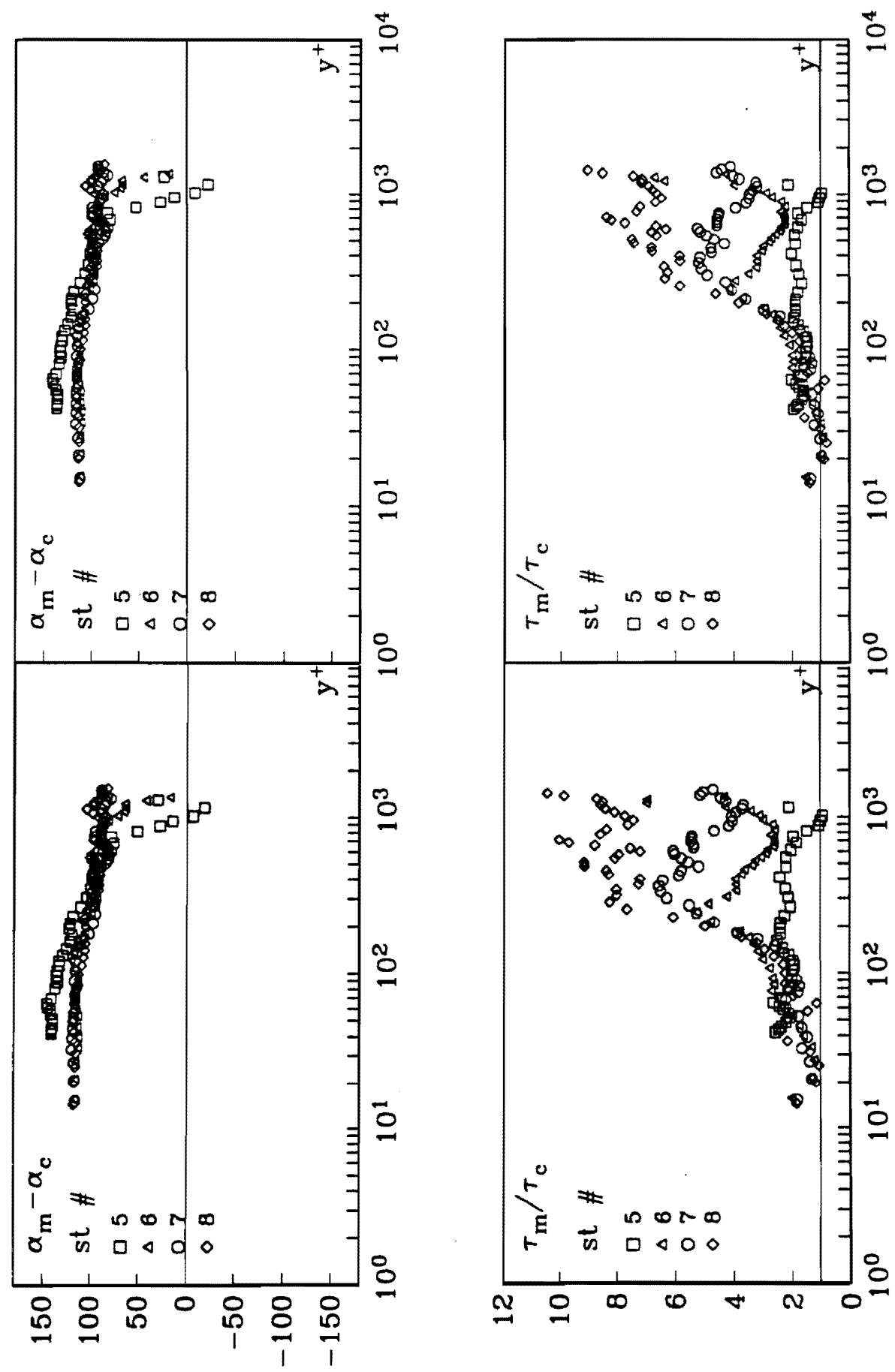

Figure 123c. Bissonnette-Mellor low Reynolds number data in Rotta's model with anisotropy constant $T=1.2$ (left) and with anisotropy constant $T=1.5$ ( right ) . 

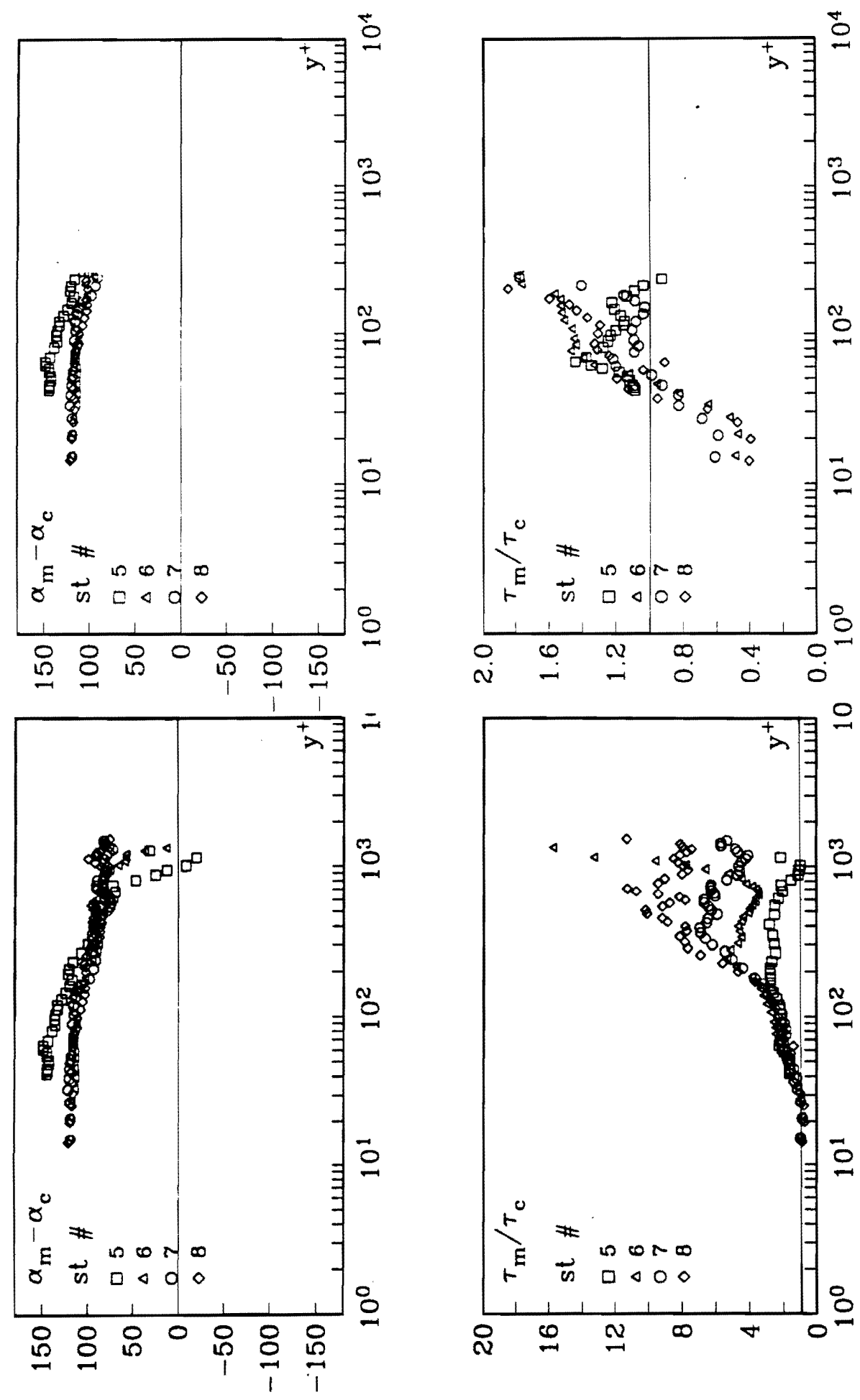

Figure 123d. Bissonnette-Mellor low Reynolds number data in Johnson-King ( left ) and in Patel's ( right ) models. 


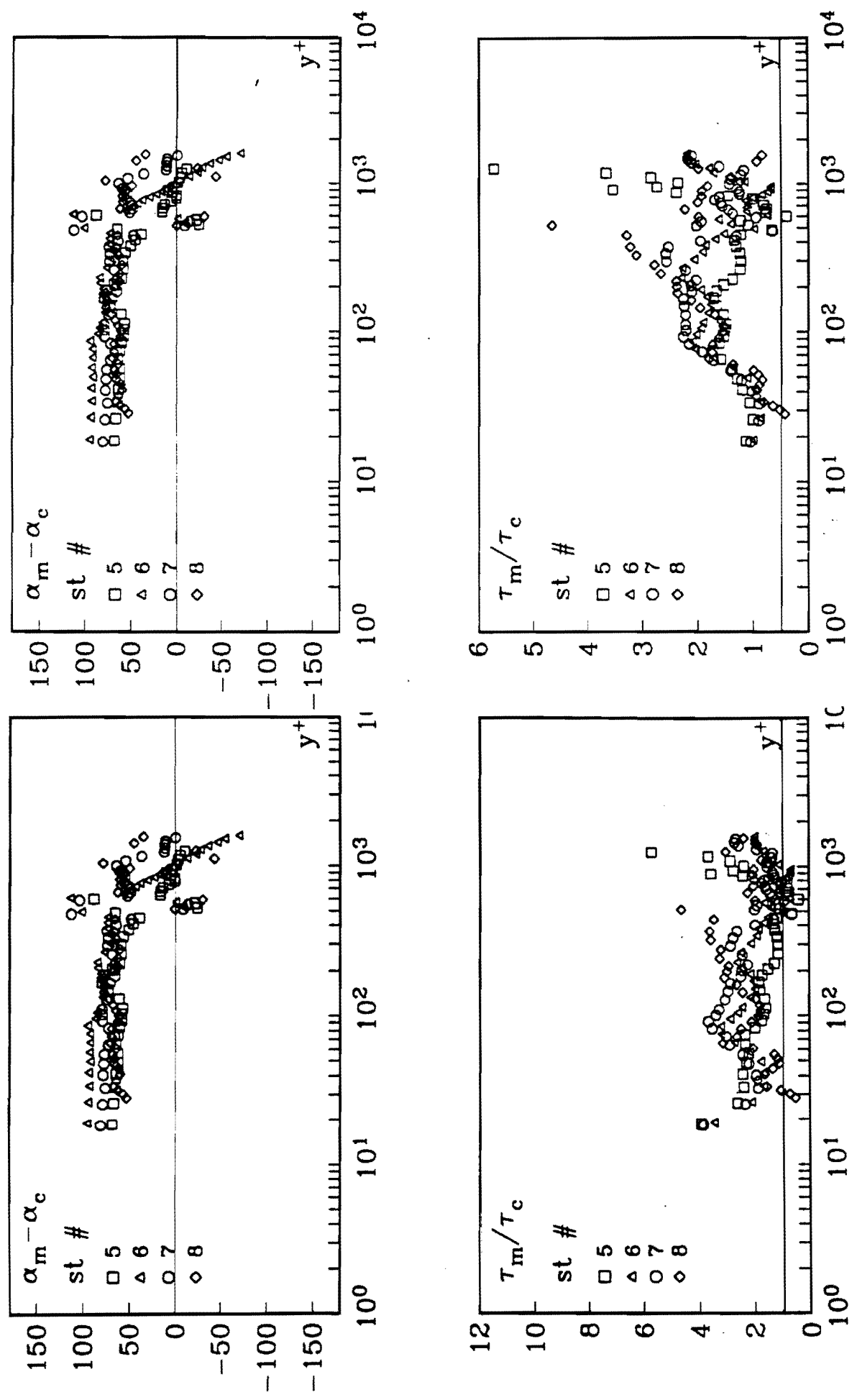

Figure 124a. Bissonnette-Mellor high Reynolds number data in Cebeci-Smith ( left ; and in Johnson-King (right ) models. 


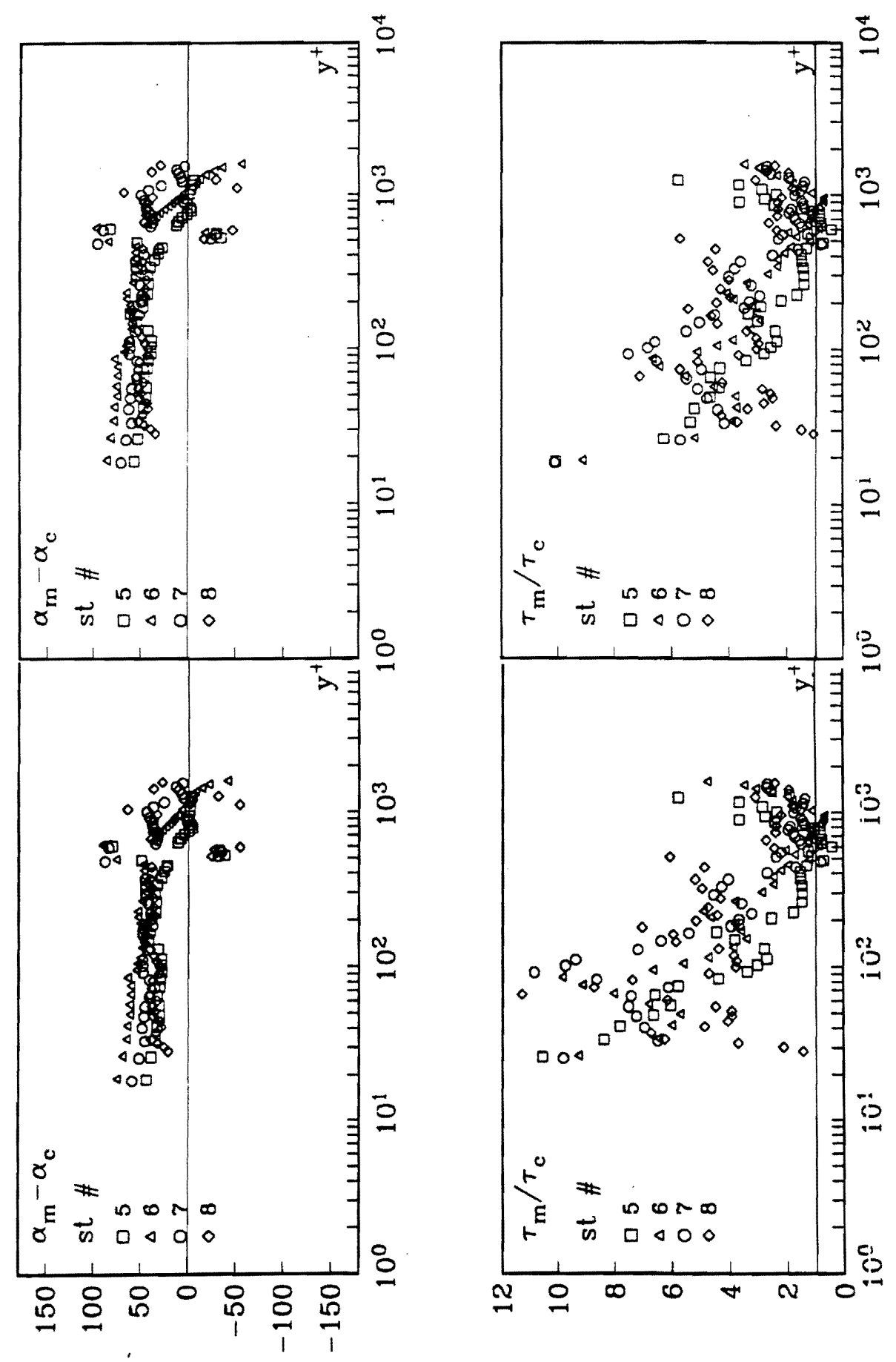

Figure 124b. Bissonnette-Mellor high Reynolds number data in Rotta's model with anisotropy constant $T=0.3$ (left) and with anisotropy constant $T=0.5$ ( right ). 

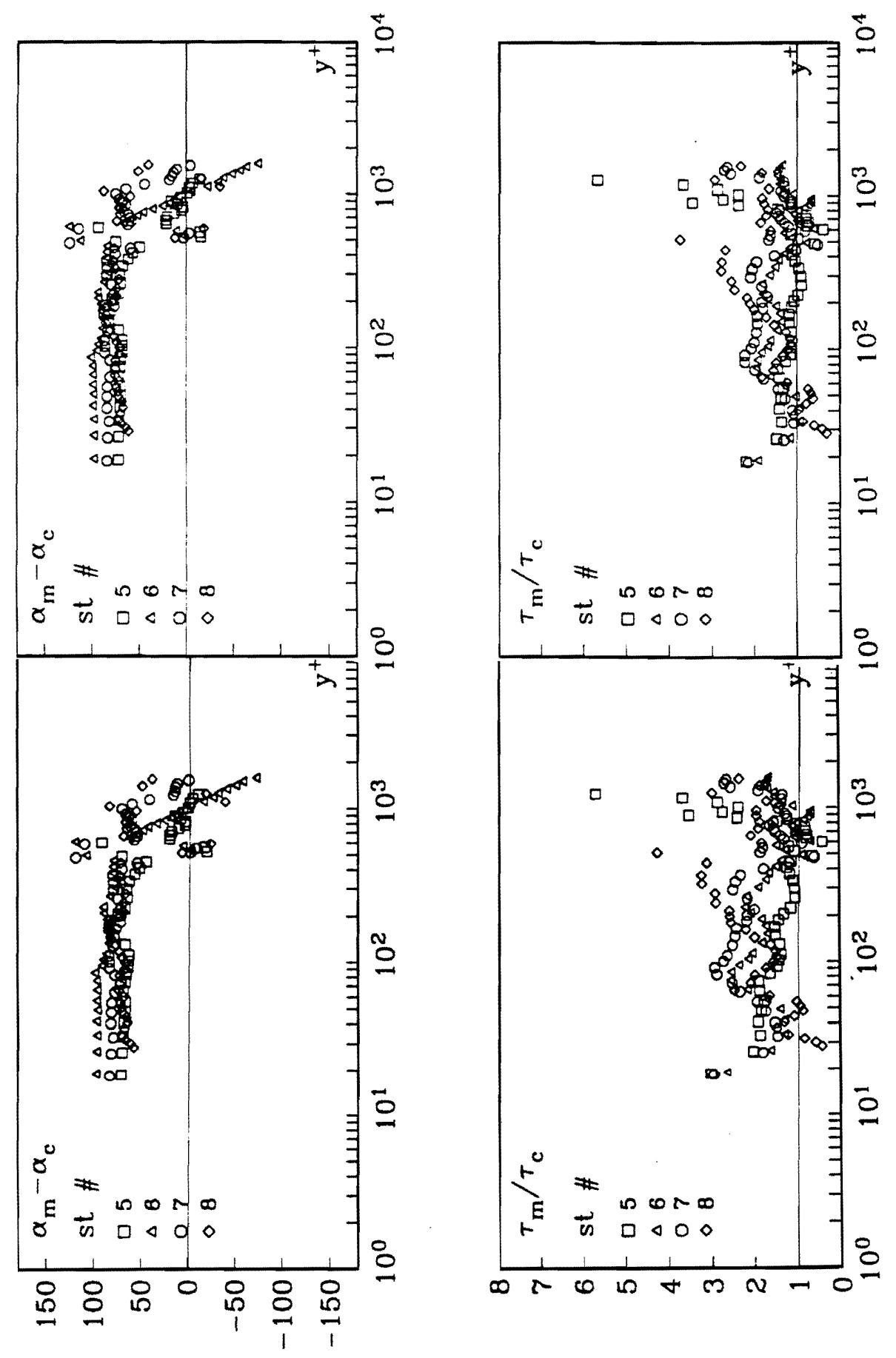

Figure 124c. Bissonnette-Mellor high Reynolds number data in Rotta's model with anisotropy constant $T=1.2$ (left) and with anisotropy constant $T=1.5$ ( right ). 


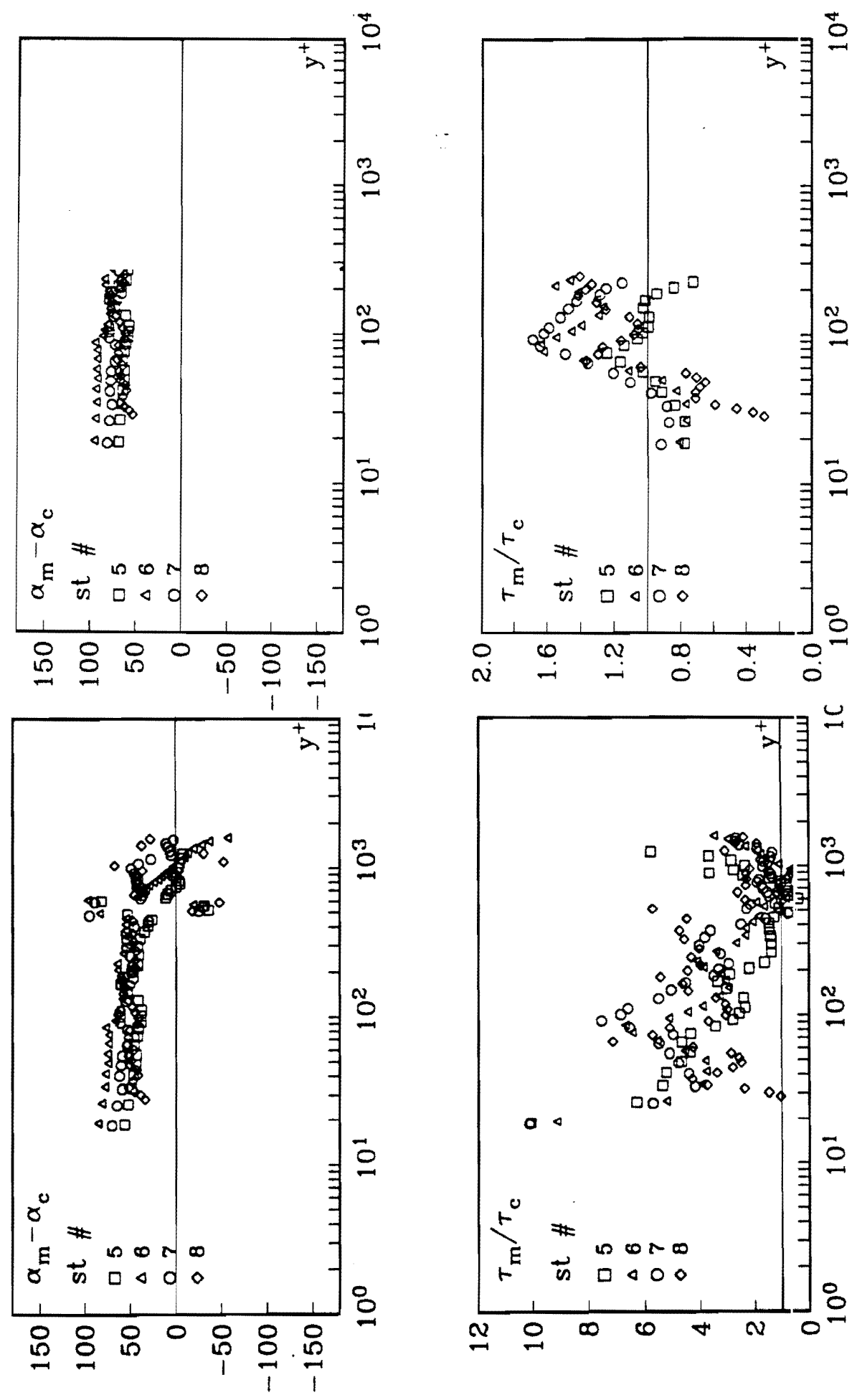

Figure 124d. Bissonnette-Mellor high Keynolds number data in Rotta's model with anisotropy constant $T=0.7$ ( left) and in Patel's ( right) models. 


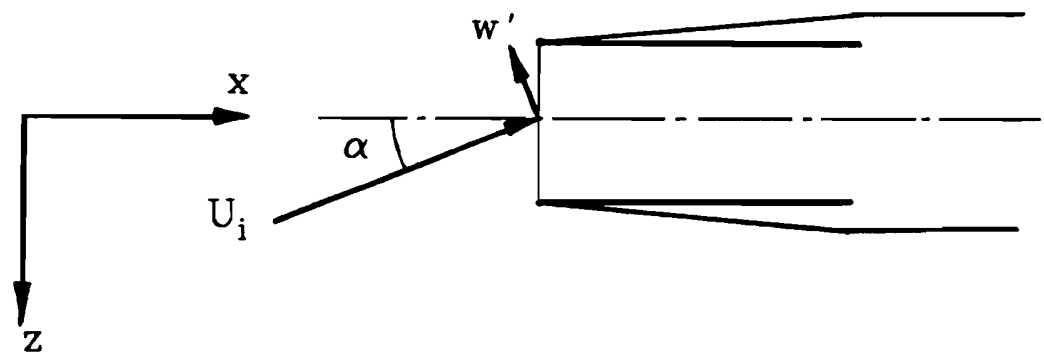

Figure 125. Single hot-wire in the $\mathrm{x}, \mathrm{z}$ plane at an angle of $\alpha$ with respect to the local instantaneous velocity projection in the same plane. 


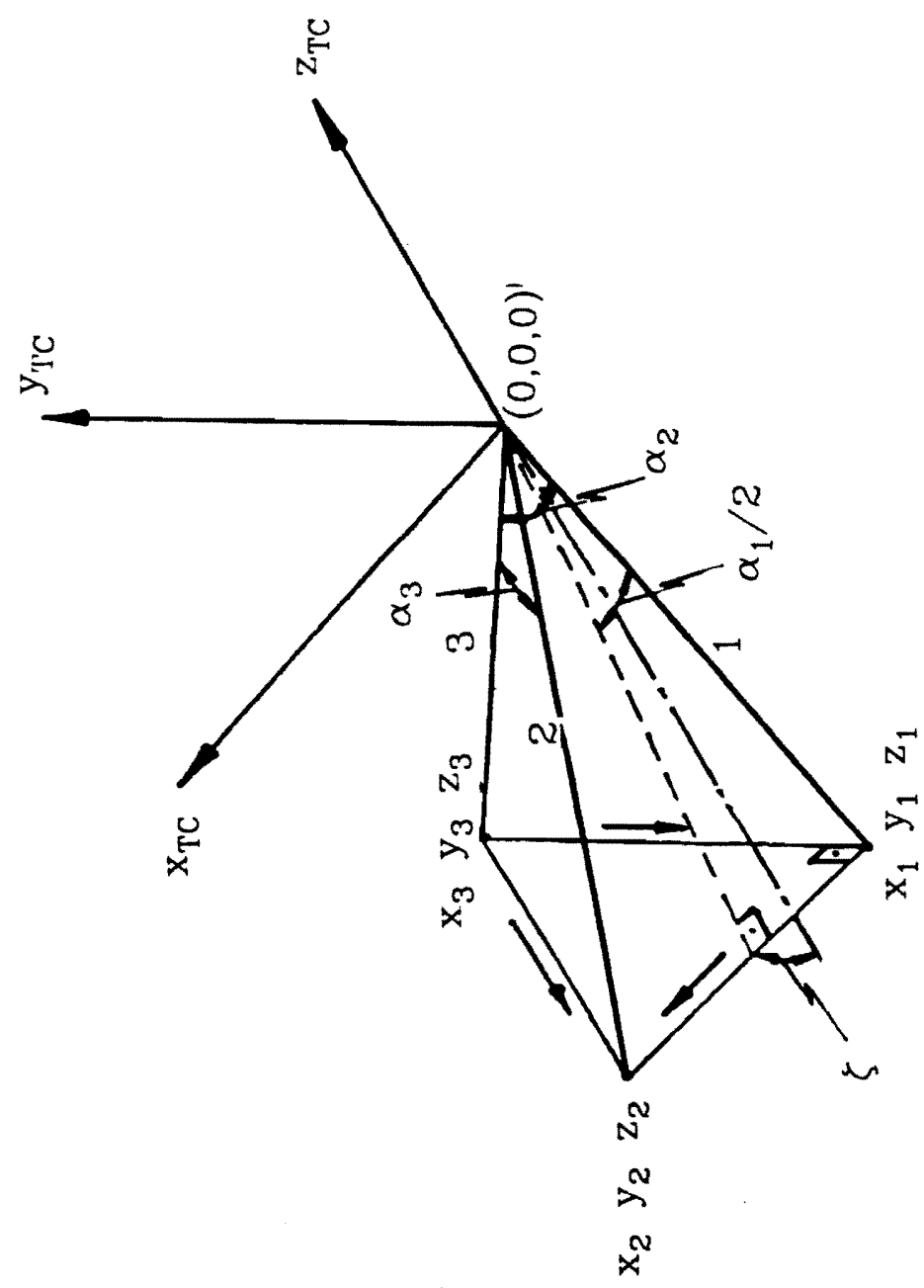

Figure 126. Schematic figure of UV system beams of the LDV set up.: Definition of the angles and conceptual points used in the reduction. 


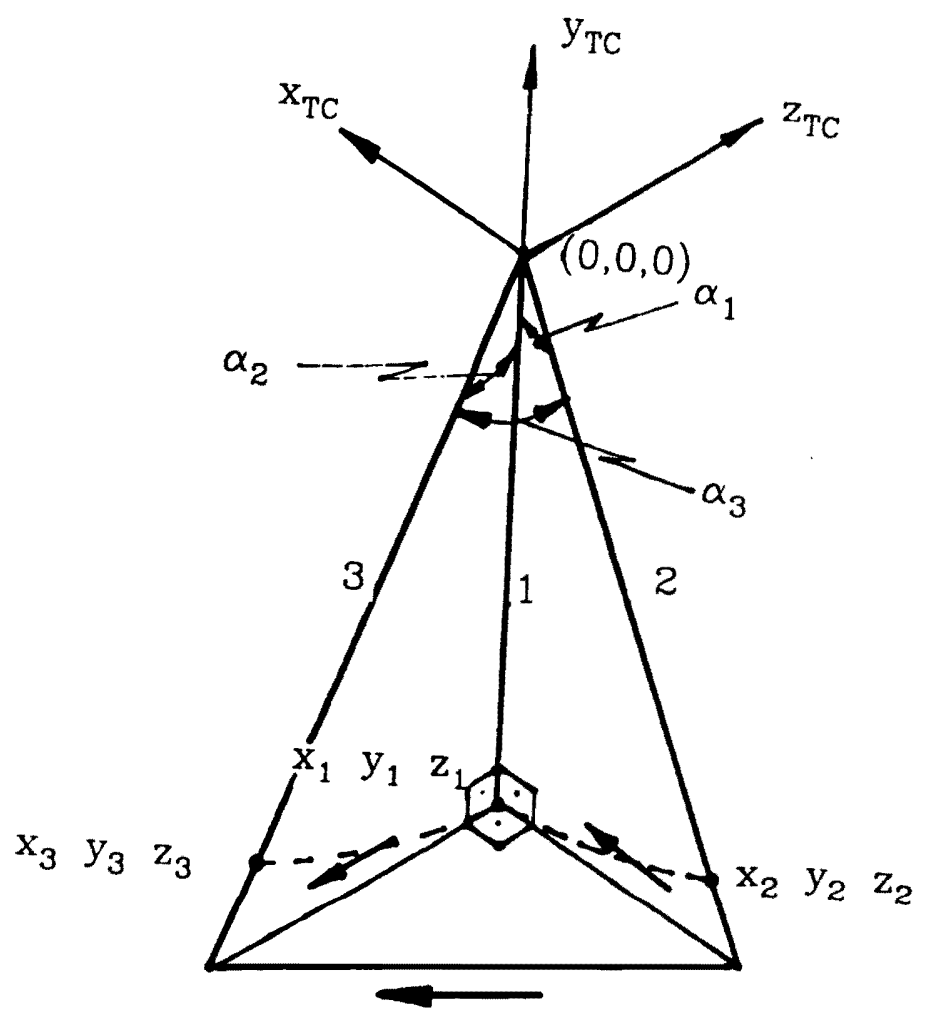

Figure 127. Schematic figure of UW system beams of the LDV set up and: Definition of the angles and conceptual points used in the reduction. 


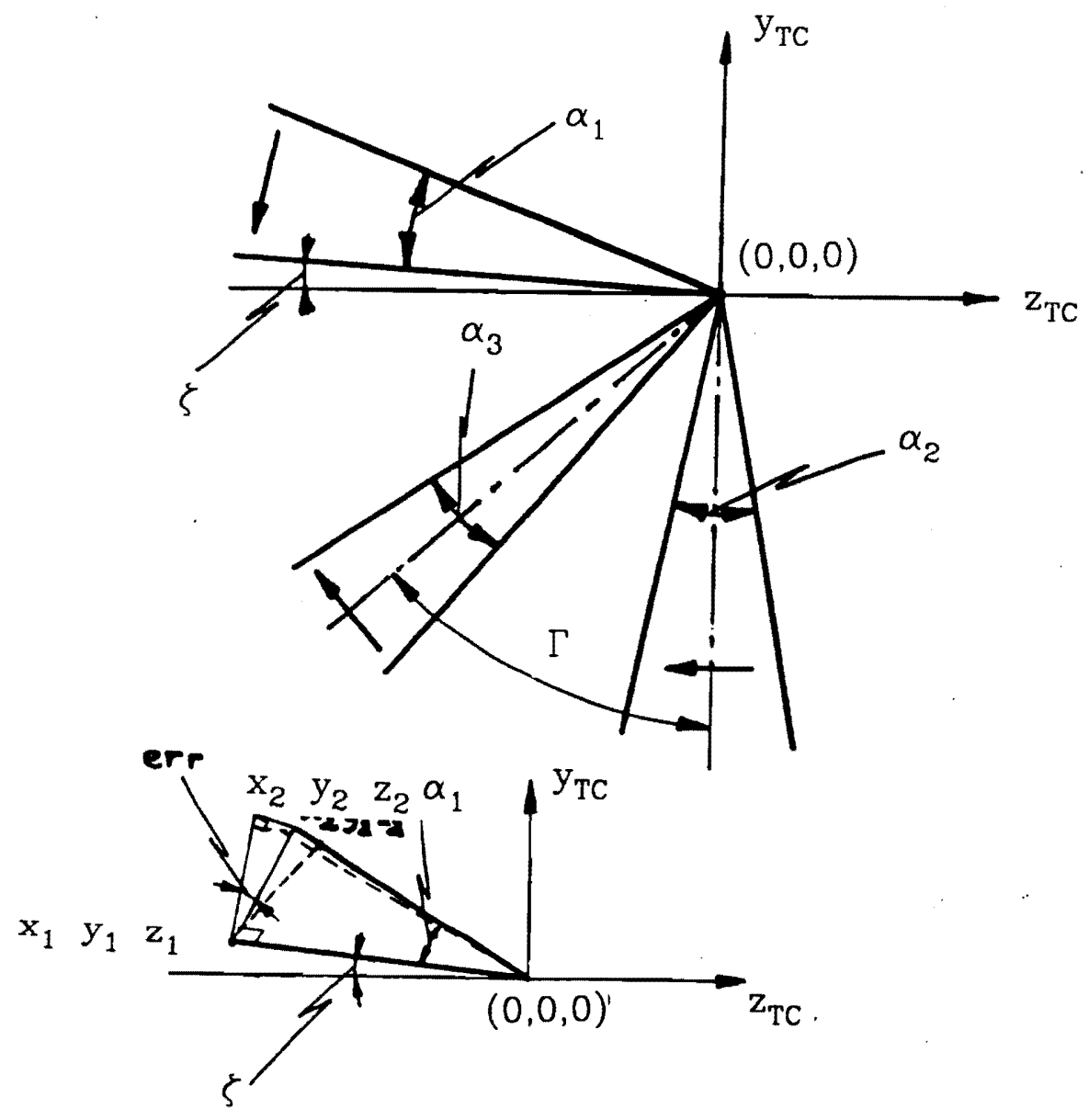

Figure 128. Schematic figure of UW system beams of the LDV set up and: Definition of the angles and conceptual points used in the reduction. 


\section{Appendix A. DATA REDUCTION FORMULAE}

\section{A.1 HOT-WIRE DATA REDUCTION EQUATIONS}

Fig 125 shows a single hot-wire in $x, z$ plane at an angle of $\alpha$ with respect to the local instantaneous velocity projection in the same plane. The effective velocity, which cools the wire may be written as

$$
U_{\text {eff }}=\sqrt{\left(U_{i n s} \cos \alpha-w \sin \alpha\right)^{2}+h^{2}\left(U_{i n s} \sin \alpha+w \cos \alpha\right)^{2}}
$$

where,

$\mathrm{h}=$ multiplication factor, since the flow tangential to the wire cools the wire less than the flow normal to it. $\mathrm{h}$ used in this study was $\mathbf{0 . 1 3}$.

Instantaneous $U_{i}$ velocity may be written as $U_{i}=U_{i m}+u_{i n s}$. Expanding the squares in the root and aissuming that absolute value of the sum of the turbulence quantities divided by $U_{i m}$ and multiplied with a factor is always less than 1 , makes it possible to use the binomial expansion. The result, after neglecting the third order velocity fluctuations can be written as,

$$
U_{e f f}=U_{i m} \sqrt{A}\left(1+\frac{1}{2}\left(P^{2}+2 P+\frac{F}{A} Q^{2}+\frac{G}{A} Q\right)\right)
$$


where,

$$
\begin{aligned}
& P=\frac{u_{i n s}}{U_{i m}} ; \quad G=\sin (2 \alpha)\left(h^{2}-1\right) \\
& Q=\frac{w}{U_{i m}} ; \quad A=\cos ^{2} \alpha+h^{2} \sin ^{2} \alpha \\
& F=\sin ^{2} \alpha+h^{2} \cos ^{2} \alpha
\end{aligned}
$$

Furthermore, $U_{\text {eff }}$ may be written as ,

$$
U_{\text {eff }}=\left(U_{\text {eff }}\right)_{m}+u_{e f f}
$$

Time averaging of $U_{\text {eff }}$ gives $\left(U_{\text {eff }}\right)_{m}$,

$$
\left(U_{e f f}\right)_{m}=U_{i m} \sqrt{A}\left(1+\frac{1}{2} P^{2}+\frac{F}{A} Q^{2}\right)
$$

in this equation the terms including $P^{2}$ and $Q^{2}$ may still be neglected, since they are much less than 1.0 . This leaves,

$$
\left(U_{e f}\right)_{m}=U_{i m} \sqrt{A}
$$

Subtraction of $\left(U_{\text {eff }}\right)_{m}$ (eq 1 ) from $U_{\text {eff }}$ gives $u_{e f f}$

$$
u_{e f f}=U_{i m} \sqrt{A}\left(\frac{1}{2}(2 P+G / A Q)\right)
$$

which may be squared to yield,

$$
\begin{aligned}
u_{e f f}^{2} & =U_{i m}^{2} A\left(\frac{1}{4}\left(4 P^{2}+4 \frac{G}{A} P Q+\frac{G^{2}}{A^{2}} Q^{2}\right)\right) \\
& =A u_{i n s}^{2}+G u_{i n s} w+\frac{G^{2}}{4 A} w^{2}
\end{aligned}
$$

To find $\overline{u^{2}}, \overline{w^{2}}, \overline{u w}$ at the same point, three successive measurements with different $\alpha$ angles were done. First two of these measurements were used to find the flow angle, and the mean velocity was measured after the probe was aligned to this angle. 


\section{A.2 CONFIGURATION OF LDV BEAMS}

The UV system which nominally measures the $U$ and $V$ components of the mean velocity vector and $\overline{u^{2}}, \overline{v^{2}}, \overline{u v}$ terms of the Reynolds stress tensor was formed by intersecting the three beams of the system which were accessed into the tunnel from the side window of the tunnel with a $90.61 \mathrm{~cm}$ focal length lens. The design of the system is such that the beams travel the same distance from the focusing lens to tie probe volume. The conceptual points which are used later on to define the measurement direction and the bisector direction and the perpendicular direction for each pair of beam are shown in Fig 126. Fig 126 also shows the directions of the positively measured quantities due to shifting by Bragg cells, which are indicated by arrows.

The UW system nominally measures the U,W components of the mean velocity and $\overline{u^{2}}, \overline{w^{2}}, \overline{u w}$ cornponents of the Reynolds stress tensor. The access to the tunnel is through the plexiglass floor of the tunnel and the lens used has $26.54 \mathrm{~cm}$ focal length. Fig 127 shows the conceptual points and positive measurement directions for each pair of beam.

Similarly, the VW system nominally measures the $\mathrm{V}, \mathrm{W}$ components of the mean velocity and $\overline{v^{2}}, \overline{w^{2}}, \overline{v w}$ components of the Reynolds stress tensor. This system uses the UV system's V component meastring pair of beams, the UW system's $W$ component measuring pair of beams and a third pair which is necessary to extract the vw shear stress and which are focused by a $26.002 \mathrm{~cm}$ focal lengthed lens. Originally the VW system's all beams were designed to be on the $y, z$ plane, but during the reduction of the velocities and stresses it was observed that very small error due to one beam of the $\mathrm{V}$ component measuring pair being out of plane resulted in different results between UV, VW systems. To take into account this error, VW system equations were rewritten by assuming that one of the $\mathrm{V}$ measuring pair of beams was out of plane. Fig 128 shows the original design and $\mathrm{V}$ measuring beams with including this error.

The probe volume for each pair of beams form an ellipsoid and the dimensions of it can be compured using,

$$
\sigma=\frac{1}{\pi} \frac{f_{0} \lambda}{d} ; \sigma_{1}=\frac{\sigma}{\cos \phi} ; \sigma_{2}=\frac{\sigma}{\sin \phi} ; \sigma_{3}=\sigma
$$

where, 
$f_{0}=$ focal length of the focusing lens

$\lambda=$ wave length of the laser

$d=$ distance between the $1 / e^{2}$ intensity points of the beam at the lens

$\phi=$ half of the angle between the two intersecting beams ( Fig 18 ), (Durst, Melling, Whitelaw, $19: 31$ ).

In reality, use of the equations prove to be difficult due to necessity to measure the beam diametor before focusing.

\section{A.3 LDV DATA REDUCTION EQUATIONS}

Once the configuration of the beams are well defined the measurement direction unit vectors can easily be found. This also makes it easier to take into account the sign judgment of the measured quantities which occur due to shifting of the beams. Unit vectors multiplied by the velocity vector $\overrightarrow{U i}+\overrightarrow{V j}+W \vec{k}$ give equations for intantaneous measurements for each pair of beam. These equations are as follows,

UV system ( Fig 126) instantaneously measures;

(2-1) pair

$$
U_{i}=\frac{\lambda / 2}{\sin \left(\alpha_{1} / 2\right)} f_{D}
$$

(1-3) pair

$$
\frac{V_{i}\left(\sin \zeta-y_{\text {new }}\right)-W_{i}\left(\cos \zeta+z_{\text {new }}\right)}{\sqrt{2-2 y_{\text {new }} \sin \zeta+2 \cos \zeta z_{\text {new }}}}=\frac{\lambda / 2}{\sin \left(\alpha_{2} / 2\right)} f_{D}
$$

(2-3) pair 


$$
\frac{2 U_{i} \tan \left(\frac{\alpha_{1}}{2}\right)+V_{i}\left(\sin \zeta-y_{n e w}\right)-W_{i}\left(\cos \zeta+z_{n e w}\right)}{\sqrt{2-2 \sin \zeta y_{n e w}+2 \cos \zeta z_{n e w}+4 \tan ^{2}\left(\frac{\alpha_{1}}{2}\right)}}=\frac{\lambda / 2}{\sin \left(\alpha_{3} / 2 f_{D}\right.}
$$

UW system ( Fig 127)

(1-2) pair

$$
\frac{U_{i} \sin \alpha_{1}+V_{i}\left(\cos \alpha_{1}-1\right)}{\sqrt{2-2 \cos \alpha_{1}}}=\frac{\lambda / 2}{\sin \left(\alpha_{1} / 2\right)} f_{D}
$$

(3-1) pair

$$
\frac{V_{i}\left(1-\cos \alpha_{2}\right)-W_{i} \sin \alpha_{2}}{\sqrt{2-2 \cos \alpha_{2}}}=\frac{\lambda / 2}{\sin \left(\alpha_{2} / 2\right)} f_{D}
$$

(2-3) pair

$$
\frac{U_{i} \sin \alpha_{1}+V_{i}\left(\cos \alpha_{1}-\cos \alpha_{2}\right)-W_{i} \sin \alpha_{2}}{\sqrt{2-2 \cos \alpha_{1} \cos \alpha_{2}}}=\frac{\lambda / 2}{\sin \left(\alpha_{3} / 2\right)} f_{D}
$$

VW system (Fig 128)

1 pair

$$
\frac{U_{i} \sin \alpha_{1}(\sin (e r r))+V_{i}(\sin \zeta-\sin (\phi+\zeta))+W_{i}(\cos (\phi+\zeta)-\cos \zeta)}{\sqrt{2-2 \cos \phi+\sin ^{2} \alpha_{1}-\sin ^{2} \phi}}=\frac{\lambda / 2}{\sin \left(\alpha_{1} / 2\right)} f_{D}
$$

2 pair

$$
V_{i} \sin \left(\frac{\alpha_{2}}{2}\right)-W_{i} \cos \left(\frac{\alpha_{2}}{2}\right)=\frac{\lambda / 2}{\sin \left(\alpha_{2} / 2\right)} f_{D}
$$

3 pair 


$$
W_{i} \cos \Gamma-V_{i} \sin \Gamma=\frac{\lambda / 2}{\sin \left(\alpha_{3} / 2\right)} f_{D}
$$

Mean velocities are reduced from the first two equations of each system. After time averaging the equations keep the same form. The first two letters, UV, UW, VW on the left-hand side of the following equations show the optical systems used and U1, V1, W1 denote the nominal mean quantities measured by these systems.

UV system

$$
\begin{aligned}
& U V U 1=U \\
& U V V 1=\frac{V\left(\sin \zeta-y_{\text {new }}\right)-W\left(\cos \zeta+z_{\text {new }}\right)}{\sqrt{2-2 y_{\text {new }} \sin \zeta+2 \cos \zeta z_{\text {new }}}}
\end{aligned}
$$

UW system

$$
\begin{aligned}
& U W U 1=\frac{U \sin \alpha_{1}+V\left(\cos \alpha_{1}-1\right)}{\sqrt{2-2 \cos \alpha_{1}}} \\
& U W W 1=\frac{V\left(1-\cos \alpha_{2}\right)-W \sin \alpha_{2}}{\sqrt{2-2 \cos \alpha_{2}}}
\end{aligned}
$$

VW system

$$
\begin{aligned}
V W V 1 & =\frac{U \sin \alpha_{1}(\sin (e r r))+V(\sin \zeta-\sin (\phi+\zeta))+W(\cos (\phi+\zeta)-\cos \zeta)}{\sqrt{2-2 \cos \phi+\sin ^{2} \alpha_{1}-\sin ^{2} \phi}} \\
V W W 1 & =V \sin \left(\frac{\alpha_{2}}{2}\right)-W \cos \left(\frac{\alpha_{2}}{2}\right)
\end{aligned}
$$

Eventhough these equations can be solved simultaneously by only taking 3 of these equations which include 3 unknowns, this would be assuming beforehand that in each traverse of the seperate systems the probe volumes were coinciding and were exposed to the same velocity. To overcome this assumption the necessary information to be able to solve each two equation was supplied. $U$ velocity component for $V W$ system was taken as UV system's $U$ velocity component, thus $V$ and $W$ components from VW system could be computed. These computed values were taken as the inputs to the UV and UW systems. Therefore UV system uses $W$ velocity computed by VW and UW system uses $\mathrm{V}$ velocity computed from VW system. This was necessitated since the original design of the systems were done with the assumption that each system would measure the mean velocities it was designed for. These equations written here were derived after the data was taken. 
After the reduction, it was seen that it was necessary to shif some of the UW system profiles up to have consistent results. UV system was taken as the base for these shifts since it was much easier to locate the probe volume on the wall with the naked eye. The maximum shifts necessary was observed to be $0.152 \mathrm{~nm}$.

Reynolds stress components were computed with the same idea in mind, such that only necessary unknowns to solve the unknowns of each system were supplied from other systems. The instantaneous velocties in the 9 equations previously written were seperated into mean and fluctuating parts and the squares of the equations were taken and Reynolds averaging was applied. After neglecting only values such as $0.2 \%$ of the mainly interested quantity that is being considered in that particular equation by the assumption that all fluctuations are on the same order of magnitude, the equations may be written as follows.

UV system

(2-1) pair

$$
U V U 2=\overline{u^{2}}
$$

$(1-3)$ pair

$$
U V V 2=A_{1} v^{\overline{2}}+A_{2} \overline{\nu w}
$$

(2-3) pair

$$
U V U V=B_{1} \bar{u}^{\overline{2}}+B_{2} \bar{v}^{\overline{2}}+B_{3} \overline{u \nu}+B_{4} \overline{u w}+B_{5} \overline{v w}
$$

where, 


$$
\begin{aligned}
A_{1} & =\frac{\left(\sin ^{2} \zeta-2 \sin \zeta y_{\text {new }}+y_{\text {new }}{ }^{2}\right)}{\left(2-2 y_{\text {new }} \sin \zeta+2 \cos \zeta z_{\text {new }}\right)} \\
A_{2} & =\frac{-2\left(\sin \zeta \cos \zeta+\sin \zeta z_{\text {new }}-y_{\text {new }} \cos \zeta-y_{\text {new }}{ }^{2} \text { new }\right)}{\left(2-2 y_{\text {new }} \sin \zeta+2 \cos \zeta z_{\text {new }}\right)} \\
D I V & =2-2 \sin \zeta y_{\text {new }}+2 \cos \zeta z_{\text {new }}+4 \tan ^{2}\left(\frac{\alpha_{1}}{2}\right) \\
B_{1} & =\frac{4 \tan ^{2}\left(\frac{\alpha_{1}}{2}\right)}{D I V} \\
B_{2} & =\frac{\left(\sin ^{2} \zeta-2 \sin \zeta y_{\text {new }}+y^{2} \text { new }\right)}{D I V} \\
B_{3} & =\frac{4\left(\tan \left(\frac{\alpha_{1}}{2}\right) \sin \zeta-\tan \left(\alpha_{1} / 2\right) y_{\text {new }}\right)}{D I V} \\
B_{4} & =\frac{-4\left(\tan \left(\frac{\alpha_{1}}{2}\right) \cos \zeta+\tan \left(\alpha_{1} / 2\right) z_{\text {new }}\right)}{D I V} \\
B_{5} & =\frac{-2\left(\sin \zeta \cos \zeta+\sin \zeta z_{\text {new }}-y_{\text {new }} \cos \zeta-y_{\text {new }} z_{\text {new }}\right)}{D I V}
\end{aligned}
$$

UW system

(1-2) pair

$$
U W U 2=A_{1} \overline{u^{2}}+A_{2} \overline{u v}
$$

(1-3) pair

$$
U W W 2=B_{1} \overline{w^{2}}+B_{2} \overline{v W}
$$

(3-2) pair

$$
U W U W=C_{1} \overline{u^{2}}+C_{2} \overline{w^{2}}+C_{3} \overline{u v}+C_{4} \overline{u w}+C_{5} \overline{v w}
$$

where, 


$$
\begin{aligned}
A_{1} & =\frac{\sin ^{2}\left(\alpha_{1}\right)}{\left(2-2 \cos \left(\alpha_{1}\right)\right)} \\
A_{2} & =\frac{2 \sin \alpha_{1}\left(\cos \alpha_{1}-1\right)}{\left(2-2 \cos \alpha_{1}\right)} \\
B_{1} & =\frac{\sin ^{2}\left(\alpha_{2}\right)}{\left(2-2 \cos \alpha_{2}\right)} \\
B_{2} & =\frac{-2 \sin \alpha_{2}\left(1-\cos \alpha_{2}\right)}{\left(2-2 \cos \alpha_{2}\right)} \\
D I V & =2-2 \cos \alpha_{1} \cos \alpha_{2} \\
C_{1} & =\frac{\sin ^{2}\left(\alpha_{1}\right)}{D I V} \\
C_{2} & =\frac{\sin ^{2}\left(\alpha_{2}\right)}{D I V} \\
C_{3} & =\frac{2 \sin \alpha_{1}\left(\cos \alpha_{1}-\cos \alpha_{2}\right)}{D I V} \\
C_{4} & =\frac{-2 \sin \alpha_{1} \sin \alpha_{2}}{D I V} \\
C_{5} & =\frac{-2\left(\sin \alpha_{2}\left(\cos \alpha_{1}-\cos \alpha_{2}\right)\right)}{D I V}
\end{aligned}
$$

VW system

1st pair

$$
V W V 2=A_{1} v^{\overline{2}}+A_{2} \overline{u \nu}+A_{3} \overline{\bar{w}}
$$

2nd pair

$$
V W W 2=B_{2} w^{2}+B_{3} \overline{v w}
$$

3rd pair

$$
V W V W=C_{1} v^{\overline{2}}+C_{2} \bar{w}^{\overline{2}}+C_{3} \overline{v w}
$$

where, 


$$
\begin{aligned}
& A_{1}=\frac{(\sin \zeta-\sin (\phi+\zeta))^{2}}{\left(2-2 \cos \phi+\sin ^{2}\left(\alpha_{1}\right)-\sin ^{2} \phi\right)} \\
& A_{2}=\frac{2 \sin \alpha_{1} \sin (e r r)(\sin \zeta-\sin (\phi+\zeta))}{\left(2-2 \cos \phi+\sin ^{2}\left(\alpha_{1}\right)-\sin ^{2} \phi\right)} \\
& A_{3}=\frac{2\left(\sin \alpha_{1}-\sin (\phi+\zeta)\right)(\cos (\phi+\zeta)-\cos \zeta)}{\left(2-2 \cos \phi+\sin ^{2}\left(\alpha_{1}\right)-\sin ^{2} \phi\right)} \\
& B_{2}=\cos ^{2}\left(\frac{\alpha_{2}}{2}\right) \\
& B_{3}=-2 \sin \left(\frac{\alpha_{2}}{2}\right) \cos \left(\frac{\alpha_{2}}{2}\right) \\
& C_{1}=\sin ^{2} \Gamma \\
& C_{2}=\cos ^{2} \Gamma \\
& C_{3}=-2 \sin \Gamma \cos \Gamma
\end{aligned}
$$

The left-hand sides of the equations may be used to indicate which quantity is being computed from which system such as UVV2, $\overline{v^{2}}$ of the UV system measurements and VWVW, $\overline{v w}$ from VW system measurements. They also show the quantity measured by the systems. In each system's equations 1 st and 2 nd serve as input to the third equation. Yet, since these 1st and 2 nd equations involve unknowns in them which have to be supplied from the solutions of the other systems, an iteration process was applied. It was observed that, a constant value for each stress term was obtained after 6 iterations. In these iterative solutions, first $\overline{v w}$ was computed assuming that $\overline{u \nu}=0$. This was put as input to UV system which further needed $\overline{u w}$, which was assumed to be zero in the first iteration. These computed $\overline{u v}$ and $\overline{v w}$ values were used as inputs to compute $\overline{u w}$. The normal stresses from each of the three systems used were therefore found individually. Likewise the $U, V, W$ mean velocity components $\overline{u^{2}}, \overline{v^{2}}, \overline{w^{2}}$, normal stress components are obtained two times, from different systems and $\overline{u v}, \overline{u w}, \overline{v w}$, are obtained once. 


\section{Appendix B. GRADIENT AND FINITE}

\section{TRANSIT TIME BROADENING}

\section{CORRECTIONS}

\section{B.1 GRADIENT BROADENING}

The existency of velocity gradient in the probe volume deviates the measured flucuating and mean quantities from the quantities that would be measured at the center of the probe volume (George and Lurnley, 1973 ) .

The formulation given by George and Lumley ( 1973 ) assumes that only one velocity component is being measured or the orientation of probe volume is such that it is exposed to only one velocity gradient which is along the major axis of the ellipsoidal probe volume. Even though it was discussed by Durst, Mlelling and Whitelaw ( 1981 ) that the correction in this direction for gradient broadening would be sufficient; it was observed that the formulation was neglecting some of the important correction terms necessary such as $\frac{\partial U}{\partial y}$ in UV system measurements. 
The equations for the gradient broadening were rewritten following the same assumptions they made except that the velocity vector had gradients with in the probe volume. The formulation is as follows: The velocity vector within the probe volume around the center of the ellipsoid may be approximated by,

$$
\vec{V}=\vec{V}_{0}+\left.\frac{\partial \vec{V}}{\partial x}\right|_{0} d x+\left.\frac{\partial \vec{V}}{\partial y}\right|_{0} d y+\left.\frac{\partial \vec{V}}{\partial z}\right|_{0} d z
$$

or this may be further written as

$$
\vec{V}=\vec{V}_{0}+\frac{\partial \vec{V}}{\partial x} l_{0}\left(x-x_{0}\right)+\frac{\partial \vec{V}}{\partial y} l_{0}\left(y-y_{0}\right)+\left.\frac{\partial \vec{V}}{\partial z}\right|_{0}\left(z-z_{0}\right)
$$

where $x_{0}, y_{0}, z_{0}$ are the center of the ellipsoid and $x, y, z$ are the coordinate axes which run along the axes of the ellipsoid where major axes is in the y direction (Fig 18 ).

The light intensity seen by the photomultiplier for example from a single particle passing from different zones of the probe volume would differ due to the Gaussian distribution of the light intensity with in the probe volume. This fact results in that in the case of say two particles having same velocity passing through the probe volume at the same time, the particle which has a path closer to the center of the ellipsoid vould have a higher probability for the detection by the signal processing unit. The intensity distribution in the probe volume can be written as

$$
\frac{I(x, y, z)}{I_{\text {center }}}=\frac{1}{(2 \pi)^{3 / 2} \sigma_{1} \sigma_{2} \sigma_{3}} \exp \left[-\frac{\left(x-x_{0}\right)^{2}}{2 \sigma_{1}^{2}}-\frac{\left(y-y_{0}\right)^{2}}{2 \sigma_{2}^{2}}-\frac{\left(z-z_{0}\right)^{2}}{2 \sigma_{3}^{2}}\right]
$$

where $\sigma_{1}, \sigma_{2}, \sigma_{3}$ are the probe volume dimensions in the $x, y, z$ directions respectively The space averaged mean velocity now may be written as

$$
V_{\text {mean }}^{-}=\int_{-\infty}^{+\infty} \int_{-\infty}^{+\infty} \int_{-\infty}^{+\infty} \vec{V}(x, y, z) \frac{I(x, y, z)}{I_{\text {center }}} d x d y d z
$$

and the variance as 


$$
<\left[\vec{V}-<\vec{V}_{0}>\right]^{2}>=\int_{-\infty}^{+\infty} \int_{-\infty}^{+\infty} \int_{-\infty}^{+\infty}\left[\vec{V}-<\vec{V}_{\text {mean }}>\right]^{2} \frac{I(x, y, z)}{I_{\text {center }}} d x d y d z
$$

Since the approximation for the velocity vector used is first order, the mean velocity is not effected by the gradient broadening.

The integrations result in

$$
\vec{V}_{\text {mean }}=\vec{V}_{0}
$$

The variance however is effected by the gradients,

$$
\text { variance } \simeq\left(\frac{\partial \vec{V}}{\partial x}\right)_{0}^{2} \sigma_{1}^{2}+\left(\frac{\partial \vec{V}}{\partial y}\right)_{0}^{2} \sigma_{2}^{2}+\left(\frac{\partial \vec{V}}{\partial z}\right)_{0}^{2} \sigma_{3}^{2}
$$

The formula shows that if the velocity measured was only $U$ which is in the direction of $x$, the troadening of variance would be

$$
\text { variance broadening } \simeq\left(\frac{\partial U}{\partial x}\right)_{0}^{2} \sigma_{1}^{2}+\left(\frac{\partial U}{\partial y}\right)_{0}^{2} \sigma_{2}^{2}+\left(\frac{\partial U}{\partial z}\right)_{0}^{2} \sigma_{3}^{2}
$$

Since LDV measures quantities perpendicular to the fringes, the formula derived was used to sorrect the turbulence quantities measured by each systems 3 pair of beams.

To be able to make most use of the gradients that can be computed and at the same time to know which terms are neglected; first of all the complete broadening correction terms were written and afterwards, the gradients different than in y direction were neglected. This was done by writing the unit vector in the bisector, in the measurement direction and in the direction perpendicular to these unit vectors for the 9 pair of beams used and using the relation

$$
\frac{\partial \phi}{\partial n}=\nabla \phi \cdot \vec{n}
$$


where $\phi$ stands for the measured mean velocity by each pair and $\vec{n}$ for the unit vector in considerat on. Once three gradient terms for each pair of beams were computed they were multiplied with the necessary dimensions which apply to the each direction in consideration to find the gradient broadenings in variances. The unit vectors for each direction could be written with the conceptual points defined before.

The probe volume dimensions, since there was not a prescribed formula to compute the focused beam diameter accurately was defined by trying the available discussions and deciding on the beam diameter to be the most consistent one with in the data sets.

\section{B.2 FINITE TRANSIT TIME BROADENING}

\section{CORRECTION}

Finite transit time brodaening results in due to the finite size of the probe volume; and each particle need to stay in the probe volume only the time to traverse it. The signal generated by the particles passing the probe volume becomes uncorrelated to the signal first generated when all the particles pass through the volume. This results in a broadening (George and Lumley).

Broadening correction described in Durst-Melling-Whitelaw 1981, assumes that the flow directicn is along the minor axis $x 1$ of the probe volume and the broadening in the Doppler frequency of the signal accordingly was given as

$$
\sigma_{F}=\frac{U_{1}}{\sqrt{2} \sigma_{1}}
$$

In the present study this broadening was seen to be not enough to explain the broadening existed at the boundary layer edge especially for the UW and VW systems' $u^{\prime}$ and $w^{\prime}$ component data. 
The necessary corrections to be applied to the profiles were found after the probe volume dimensions for each pair of beams were found. The correction suggested by George and Lumley was replaced by an empirical formula in the

$$
\sigma_{F=} \frac{\left(U^{2}+V^{2}+W^{2}\right)^{1 / 2}}{\sqrt{\sigma_{1}^{2}+\sigma_{2}^{2}+\sigma_{3}^{2}}} \times(\text { constant })
$$

form, since the transit time shortens with the other components of the velocity vector.

The constant multiplier in the broadening term was found by matching the fluctuating velocity and shear stresses of hot-wire and LDV data at the last point of the hot-wire profiles. Once the constant was found it was kept same further down in each profile. Since the HW measured $\overline{u^{2}}, \overline{w^{2}}, \overline{u w}$ stresses the constants for the UW system data were easily found. The constant for the $\overline{v w}$ stress measured with the VW system was kept same as the constant for $\overline{u^{2}}$ of the UW system. 


\section{Appendix C. TENSOR TRANSFORMATION AND PARABOLA FITTING PROGRAMS}

\section{C.1 TENSOR TRANSFORMATION}

The tensor transformation used to find the shear stresses in different coordinate systems in this study is the one described in Frederick and Chang, 1972. The direction cosine matrix used to obtain the components of the tensor in the free-stream and normal stress coordinates is given by

$$
a_{i j}=\left(\begin{array}{ccc}
\cos (\alpha) & 0 & \cos (\pi / 2+\alpha) \\
0 & 0 & 0 \\
\cos (\pi / 2-\alpha) & 0 & \cos (\alpha)
\end{array}\right)
$$

and the components of the tensor in these coordinates is found by

$$
T_{r s}^{r}=a_{n} a_{s j} T_{i j}
$$

in which $\alpha$ positive rotation is in the counter-clockwise direction.

The program used is as follows: 
Tensor transformation

Asignment of the rotation angle

INPUT "FREE-STREAM ANGLE (positive for the RHS profiles) ",ALFA

Forming the direction cosine matrix

$A(1,1)=\operatorname{COS}(A L F A * P I)$

$A(1,2)=0$ !

$\mathrm{A}(1,3)=\operatorname{COS}((90+\mathrm{ALFA}) * \mathrm{PI})$

$\mathrm{A}(2,1)=0$ !

$\mathrm{A}(2,2)=1$ !

$\mathrm{A}(2,3)=0$ !

$\mathrm{A}(3,1)=\operatorname{COS}((90-\mathrm{ALFA}) * \mathrm{PI})$

$\mathrm{A}(3,2)=0$ !

$\mathrm{A}(3,3)=\operatorname{COS}(\mathrm{ALFA} * \mathrm{PI})$

$\mathrm{T}(1,1)=\mathrm{U} 2(\mathrm{~K})$

Forming the initial tensor to be transformed

$\mathrm{T}(1,2)=\mathrm{UV}(\mathrm{K})$

$\mathrm{T}(1,3)=\mathrm{UW}(\mathrm{K})$

$\mathrm{T}(2,1)=\mathrm{UV}(\mathrm{K})$

$\mathrm{T}(2,2)=\mathrm{V} 2(\mathrm{~K})$

$\mathrm{T}(2,3)=\mathrm{VW}(\mathrm{K})$

$\mathrm{T}(3,1)=\mathrm{UW}(\mathrm{K})$

$\mathrm{T}(3,2)=\mathrm{VW}(\mathrm{K})$

$\mathrm{T}(3,3)=\mathrm{W} 2(\mathrm{~K})$

tensor transformation

FOR I $=1$ TO 3

FOR $J=1$ TO 3

SUM $=0$ !

FOR $\mathrm{L}=1 \mathrm{TO} 3$

FOR $M=1$ TO 3

$S U M=S U M+A(I, L) * A(J, M) * T(L, M)$

NEXT $M$

NEXT L

TT is the transformed tensor

$T T(I, J, K)=S U M$

NEXT J

NEXT I 


\section{C.2 PARABOLA FITTING}

Least squares parabola fitting routine used in several flow gradient and data smoothing computations is given below. Program fits a parabola to 5 points in the $y=a x^{2}+b x+c$ form and computes the $a, b$ and $c$ coefficients.

Least-squares parabola fitting program

$$
\begin{aligned}
& \mathrm{V} 1=0: \mathrm{V} 2=0: \mathrm{V} 3=0: \mathrm{V} 4=0: \mathrm{R} 1=0: \mathrm{R} 2=0: \mathrm{R} 3=0 \\
& \mathrm{FOR} \mathrm{I}=1 \text { TO } 5 \\
& \mathrm{~V} 1=\mathrm{V} 1+X P A R\left(I^{4}\right. \\
& \mathrm{V} 2=\mathrm{V} 2+X P A R\left(I^{3}\right. \\
& \mathrm{V} 3=\mathrm{V} 3+X P A R(I)^{2} \\
& \mathrm{~V} 4=\mathrm{V} 4+\mathrm{XPAR}(\mathrm{I}) \\
& \mathrm{R} 1=\mathrm{R} 1+\mathrm{YPAR}(\mathrm{I})^{*} X P A R(I)^{2} \\
& \mathrm{R} 2=\mathrm{R} 2+\mathrm{YPAR}(\mathrm{I})^{*} \mathrm{XPAR}(\mathrm{I}) \\
& \mathrm{R} 3=\mathrm{R} 3+\mathrm{YPAR}(\mathrm{I}) \\
& \mathrm{NEXT} \mathrm{I}
\end{aligned}
$$

\section{NEXT I}

IF V4 $=0$ OR R3 $=0$ THEN PRINT "THERE IS DIVISION WITH ZERO"

IF $V 4=0$ OR R3 $=0$ THEN GOTO (RETURN STATEMENT LINE NUMBER )

$\mathrm{CC}=\left(\left(\mathrm{R} 3{ }^{*} \mathrm{~V} 2-\mathrm{R} 2 * \mathrm{~V} 3\right)^{*}(\mathrm{~V} 1 * \mathrm{~V} 3-\mathrm{V} 2 * \mathrm{~V} 2)-(\mathrm{V} 1 * \mathrm{R} 2-\mathrm{V} 2 * \mathrm{R} 1)^{*}\right.$

(V2*V4-V3*V3))/((V1*V3-V2*V2)*(V2*5-V4*V3)

$-(\mathrm{V} 2 * \mathrm{~V} 4-\mathrm{V} 3 * \mathrm{~V} 3) *(\mathrm{V1} * \mathrm{~V} 4-\mathrm{V} 2 * \mathrm{~V} 3))$

$\mathrm{BB}=(\mathrm{R} 2 * \mathrm{~V} 1-\mathrm{R} 1 * \mathrm{~V} 2-\mathrm{CC} *(\mathrm{~V} 4 * \mathrm{~V} 1-\mathrm{V} 3 * \mathrm{~V} 2)) /(\mathrm{V} 1 * \mathrm{~V} 3-\mathrm{V} 2 * \mathrm{~V} 2)$

$\mathrm{AA}=(\mathrm{R} 1-\mathrm{BB} * \mathrm{~V} 2-\mathrm{CC} * \mathrm{~V} 3) / \mathrm{N} 1$

RETURN 


\section{Appendix D. DATA FOR PRESENT WORK}

The data of mean velocity, stress and pressure obtained in the present study are presented in twc $5.5^{\circ}, 360$ KB diskettes. The first diskette of the two data diskettes contains the laser-Doppler-velocimeter clata in the LDV directory and the time mean static pressure coefficient data in the PRESSURE directory.

The LDV data, as explained in Chapter II, are obtained by the Swept Spectrum Analyzer and Burst Spectrum Analyzers. The results of the uncertainty analysis which was explained in Chapter III are also presented as part of the data.

The files are named starting with " LDV " to denote the data are obtained with LDV. The subsequent numbers show the stations the data is taken. The extensions "TC", "FS", "NS", designate the different coordinate systems in which the data is presented. TC, FS, NS stand for the Tunnel coordinates, the Free-stream coordinates and for the Normal stress coordinates, respectively.

In the files, at the first 15 lines several parameters deduced from the data and the parameters of the measurement conditions are given. 16th line was left blank to separate the data from these parameters.

The data at each point of the profiles consist of the raw data and the derived quantites from the raw data. First line for each y location point in the profile contains

$y, U, V, W, U$ uncertainty, $V$ uncertainty, W uncertainty, $\overline{u^{2}}, \overline{v^{2}}, \overline{w^{2}}, \overline{u \nu}, \overline{u w}, \overline{v w}, u^{\prime}$ uncertainty, $v^{\prime}$ uncertainty, $w^{\prime}$ uncertainty, $\overline{u \nu}$ uncertainty, $\overline{u w}$ uncertain!:. $\overline{v w}$ uncertainty 
$y$ location in each profile is given in inches.

$\mathrm{U}, \mathrm{V}, \mathrm{W}$, mean velocity components and the uncertainties on these quantities are given as nondimensionalized with the reference velocities.

$\overline{u^{2}}, \overline{v^{2}}, \overline{w^{2}}, \overline{u v}, \overline{u w}, \overline{v w}$ Reynolds' stresses and the uncertainties on the shear stresses only are nondimensionalized with the square of the reference velocities.

Instead of defining the uncertainties for the normal stresses, the uncertainties for the fluctuating ve ocities are given. The uncertainties for the normal stresses can be found by using

$$
\text { uncertainty of } \overline{u^{2}}=\left[\left(\sqrt{\overline{u^{2}}}+u^{2}\right)+\left(\sqrt{u^{2}}-u^{\prime}\right)\right] / 2
$$

The uncertainties of the fluctuating velocities are nondimensionalized with the reference velocities.

The second line of the data for the same y point contains the $\mathrm{N}$, anisotropy constant, $A_{1}$, Townsend's Structural Parameter, and also the Flow angle, the Flow Gradient Angle and the Shear Stres:s Angle in degrees; in the order they are mentioned. These definition and discussion on these parameters are presented in Chapter III of the present study.

The directory 'PRESSURE' contains the time mean pressure coefficient data taken with Scanivalve and an inclined manometer. The 4 files in the directory are named as, "ON", "WALL", "FAR", and "NOSE", to denote the measurements on the wing, on the wall where the body sits, on a line at $x / t=3.17$ to see the blockage effect of the body, and detailed measurements close to the nose of the body on the wall, respectively. The extension " $\mathrm{CP}$ " is used to indicate that the data files are containing pressure data.

In the files, in the first 5 lines some information about the flow conditions that the measurements are carried out are given. Next line is left emtpy. 7th line give the title of the parameters measured. Next lines contain the data in $x / t, y / t, z / t, C p$, order. The term ' $t$ ' denote the maximum body thickness $(t=2.824$ inches $)$. The pressure coefficients were computed using the $C_{p}=p-p_{\text {ref }} / p_{0}-p_{\text {ref }}$ relation, as described in the Chapter II. The discussion about the pressure data may be found in the Chapter III of the present study. 
The data presented in the second diskette are of the hot wire measurements made on both sides and upstream of the wing.

The files are named beginning either with " LHS " or " RHS " or " UP " to denote the location of the data taken. "LHS " denote the left-hand side of the wing, and " RHS " denote the right-hand side of the wing and " UP " denote the 0.75 chord upstream of the wing measurements. The subsequent numbers show the station numbers where the data are taken. The lefthand side files are numbered in increasing order proceeding upstream, and the right-hand side files are numbered in increasing order proceeding downstream. The 0.75 chord upstream profile numbers increase in the $+z$ direction. The right-hand side hot wire measurements were made at the same station locations of the Laser Doppler Velocimeter measurements. The same numbers for the right-hand side and for the LDV files show the same station locations. The data for each group are presented in the same named directories. The extensions " TC ", "FS ", "NS ", are used to designate the coordinate system the data are presented in.

In the files first 6 lines give the location of the measurements and the flow conditions. Next 2 lines show the title of the measured quantities. In the rest of the files, data are given. The data consist of the $y$ location of the measurement in inches, $U$ and $W$ components of the mean velocity vector nondimensionalize $A$ with the daily reference velocity, the Flow Angle in degrees, and the $\overline{u^{2}}, \overline{w^{2}}$ normal stresses and $\overline{u w}-(1)^{*}$ kinematic shear stress nondimensionalized with the square of the reference velocity. Detailed discussion on these quantities may be found in the Chapter III of the present study. 


\section{The vita has been removed from the scanned document}

